Ronja Kürten, Gilbert Greefrath, Marcus Hammann (Hrsg.)

\title{
Komplexitätsreduktion in Lehr-Lern-Laboren
}

Innovative Lehrformate in der Lehrerbildung zum Umgang mit Heterogenität und Inklusion

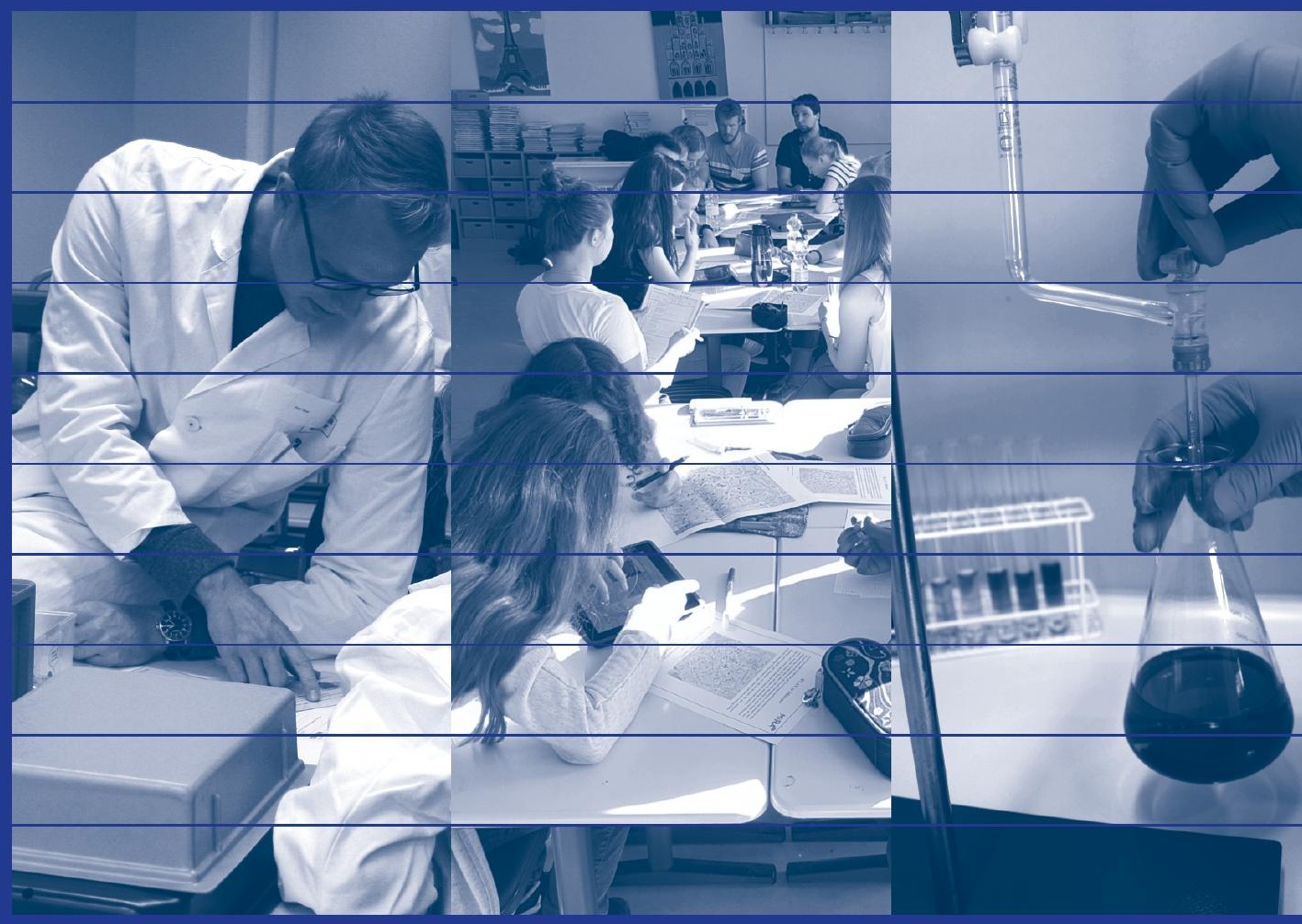




\section{Begabungsförderung}

Individuelle Förderung und Inklusive Bildung

herausgegeben von

Christian Fischer

Band 8 
Ronja Kürten, Gilbert Greefrath, Marcus Hammann (Hrsg.)

\section{Komplexitätsreduktion in Lehr-Lern-Laboren}

Innovative Lehrformate in der Lehrerbildung zum Umgang mit Heterogenität und Inklusion

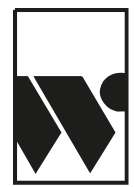

Waxmann 2020

Münster • New York 
Der Herausgeberband „Komplexitätsreduktion in Lehr-Lern-Laboren. Innovative Lehrformate in der Lehrerbildung zum Umgang mit Heterogenität und Inklusion“" aus dem Teilprojekt „Lehr-Lern-Labore, Lernwerkstätten, Learning-Center“ des Projekts „Dealing with Diversity. Kompetenter Umgang mit Heterogenität durch reflektierte Praxiserfahrung“ der WWU Münster wird im Rahmen der gemeinsamen „Qualitätsoffensive Lehrerbildung“ von Bund und Ländern aus Mitteln des Bundesministeriums für Bildung und Forschung unter dem Förderkennzeichen o1JA1621 gefördert.

GEFÖRDERT VOM

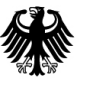

Bundesministerium

für Bildung

und Forschung

Gefördert mit Mitteln aus dem Open-Access-Fonds der ULB Münster.

Bibliografische Informationen der Deutschen Nationalbibliothek

Die Deutsche Nationalbibliothek verzeichnet diese Publikation in

der Deutschen Nationalbibliografie; detaillierte bibliografische

Daten sind im Internet über http://dnb.dnb.de abrufbar.

Begabungsförderung: Individuelle Förderung und Inklusive Bildung, Band 8

Print-ISBN 978-3-8309-3990-0

E-Book-ISBN 978-3-8309-8990-5 (open access)

doi: https://doi.org/10.31244/9783830989905

Creative Commons-Lizenz Namensnennung - Nicht-kommerziell Weitergabe unter gleichen Bedingungen CC BY-NC-SA 4.0

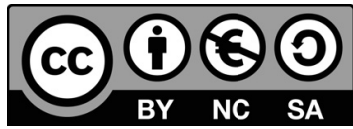

www.waxmann.com

info@waxmann.com

Umschlaggestaltung: Anne Breitenbach, Münster

Umschlagabbildungen: links: (c) Victoria Kuinke; mitte: (CRaphael Wess;

rechts: () Yvonne Rath

Satz: Roger Stoddart, Münster 


\section{Inhalt}

Gilbert Greefrath und Marcus Hammann

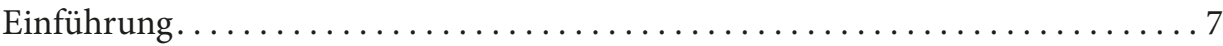

Annette Marohn, Gilbert Greefrath, Marcus Hammann,

Michael Hemmer, Ronja Kürten und Anna Windt

Komplexitätsreduktion in Lehr-Lern-Laboren.

Ein Planungs- und Reflexionsmodell........................... 17

Johannes C. S. Zang und Marcus Hammann

Kompetenzförderung mit dem Linsenmodell.

Diagnose von Schülervorstellungen im biologiedidaktischen

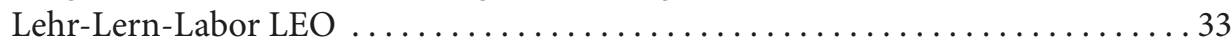

Jens Steinwachs und Helge Gresch

Professionalisierung der Unterrichtswahrnehmung

mithilfe von Videovignetten im Themenfeld Evolution.

Bearbeitung der Sachantinomie in der biologiedidaktischen Lehrerbildung. .... 57

Yvonne Rath und Annette Marohn

Stolpersteine im Lehrerhandeln: Aufbau eines Handlungsrepertoires

im Kontext Schülervorstellungen.

Das chemiedidaktische Lehr-Lern-Labor C $(\text { LE })^{2}$ VER ................. 79

Anna Grabosch und Ewald Terhart

Positive Einflussfaktoren im Zertifikat lehren.lernen.

Entwicklung beruflicher Handlungskompetenz von Lehramtsstudierenden

in der Erziehungswissenschaftlichen Lehr- und Forschungswerkstatt (ELF). . . . 105

Andreas Feindt, Christian Fischer und Horst Zeinz

Schulischen Umgang mit Vielfalt verstehen und gestalten.

Konzentration auf Komplexität im erziehungswissenschaftlichen

Lehr-Lern-Labor . . . . . . . . . . . . . . . . . . . . . . . . . . 121

Nadine Rosendahl, Michael Hemmer und Gabriele Schrüfer

Mit Vielfalt experimentieren.

Professionalisierung angehender Lehrkräfte im GEO-Lehr-Lern-Labor 
Raphael Wess und Gilbert Greefrath

Komplexitätsreduktionen im Bereich Medien und Materialien.

Entwicklung förderdiagnostischer Kompetenz im mathematik-

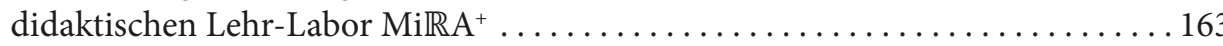

Christoph Holz und Susanne Heinicke

Unsichere Daten beim Experimentieren.

Förderung des Lehrerhandelns in ungeplanten Situationen

im physikdidaktischen Lehr-Lern-Labor La:gune .

Annika Rochholz, Katharina Fricke und Anna Windt

Naturwissenschaftlichen Unterricht planen lernen.

Professionalisierung durch Unterrichtserprobungen im

Lehr-Lern-Labor der Sachunterrichtsdidaktik .

Susanne Heinicke, Ronja Kürten, Christoph Holz und Raphael Wess

Professionalisierung von Studierenden des Lehramts

durch Komplexitätsreduktion in Lehr-Lern-Laboren . . . . . . . . . . . . . . . . 227

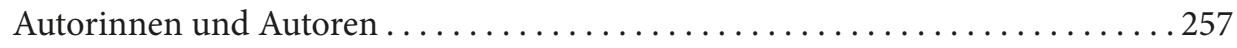




\section{Gilbert Greefrath und Marcus Hammann}

\section{Einführung}

\section{Dealing with Diversity}

Das Projekt Dealing with Diversity an der Westfälischen Wilhelms-Universität (WWU) Münster zielt darauf ab, angehende Lehrkräfte auf einen produktiven Umgang mit Heterogenität durch reflektierte Praxiserfahrung vorzubereiten. Dealing with Diversity wird im Rahmen der gemeinsamen „Qualitätsoffensive Lehrerbildung"von Bund und Ländern aus Mitteln des Bundesministeriums für Bildung und Forschung gefördert. Im Projekt werden curriculare Voraussetzungen geschaffen und innovative Lehrformate erprobt, um die Lehrerbildung inhaltlich und strukturell verstärkt auf einen produktiven Umgang mit heterogenen Lerngruppen auszurichten. Einerseits werden bestehende Lehrformate in struktureller und curricularer Hinsicht gebündelt und weiterentwickelt. Andererseits werden neue Lehrformate entwickelt und erprobt. Das Projekt wird systematisch evaluiert und auf eine größere Zahl von Disziplinen ausgeweitet. Thematisch werden dazu verschiedene Praxiselemente in der Lehrerbildung auf die Aufgabenstellung eines konstruktiven Umgangs mit der wachsenden Heterogenität der Schülerschaft ausgerichtet.

Neben curricularen Maßnahmen, die Heterogenität als durchgängiges Thema in der der Lehre verankern (siehe Rott, Zeuch, Fischer, Souvignier \& Terhart, 2018), werden auch strukturelle Maßnahmen geschaffen, die reflektierte Praxiserfahrung zum Umgang mit Schülerheterogenität ermöglichen und begleiten. Dazu gehören neben videobasierten Lehrmodulen und Praxisprojekten in Kooperationsschulen die in diesem Band dargestellten Lehr-Lern-Labore. Sie sind ein innovatives Lehrformat in der Lehrerbildung.

\section{Umgang mit Heterogenität von Schülerinnen und Schülern}

Das Projekt setzt grundlegend an den Einstellungen angehender Lehrkräfte zur Heterogenität von Schülerinnen und Schülern an. Positive Einstellungen zur Heterogenität sollen gefördert werden, denn es gibt einen engen Zusammenhang zwischen dem angemessenen Umgang mit der Heterogenität von Schülerinnen und Schülern und den entsprechenden Einstellungen der Lehrerinnen und Lehrer (Wischer, 2009). Allerdings schätzen einige Lehrkräfte die Heterogenität von Schülerinnen und Schülern in Lerngruppen negativ ein. Es zeigten sich etwa Einstellungen, dass die Leistungen in homogenen Lerngruppen besser sind und Lernschwache in Sondereinrichtungen besser gefördert werden könnten. Ein wichtiges Ziel aller Phasen der Lehrerbildung ist daher der potentialorientierte Umgang mit 
Heterogenität. Hier ist besonders die individuelle Förderung als Schlüsselstrategie zu nennen (Fischer, Rott, Veber, Fischer-Ontrup \& Gralla, 2014).

Neben den Einstellungen hängt der potentialorientierte Umgang mit Heterogenität auch von den Selbstwirksamkeitserwartungen der Lehrerinnen und Lehrer ab. So gestalten Lehrkräfte mit einer hohen Selbstwirksamkeitserwartung einen anspruchsvolleren Unterricht und bieten den Schülerinnen und Schülern dabei insbesondere ein hohes Unterstützungspotential (Schwarzer \& Jerusalem, 2002). Dies ist ein wichtiger Ansatzpunkt für die Lehrerbildung, da Lehramtsstudierende beim Übergang in die Schule häufig einen Bruch zwischen Theorie und Praxis erleben, welcher ein Absinken der Selbstwirksamkeitserwartung zur Folge hat. Lehrveranstaltungen mit reflektierter Praxiserfahrung können die Verknüpfung von Theorie und Praxis stärken und den wahrgenommenen Bruch abmindern (Korthagen, 2001). Ein Lehrformat, das die Theorie-Praxis-Verknüpfung in der Lehrerbildung in besonderer Weise fokussiert, ist das Lehr-Lern-Labor, das in diesem Projekt verstärkt in universitäre Lehrveranstaltungen eingebunden wird (Kürten, Wess \& Greefrath, 2018).

\section{Lehr-Lern-Labore für die Lehrerbildung}

Die Implementation von Lehr-Lern-Laboren ermöglicht es, Praxiselemente zu einem frühen Zeitpunkt in das Lehramtsstudium einzubeziehen. Ein wichtiges Ziel von Lehr-Lern-Laboren in der Lehrerbildung ist die Professionalisierung angehender Lehrkräfte durch eine gemeinsame Reflexion über Lehr-Lern-Prozesse (Putnam \& Borko, 200o). Im Hinblick auf die unterschiedlichen Heterogenitätsdimensionen bieten sich solche Lerngelegenheiten insbesondere dazu an, Erfahrungen im Umgang mit individuell unterschiedlichen Leistungsvoraussetzungen zu machen, indem etwa differenzierende Lernmaterialien oder instruktionale Maßnahmen erprobt werden. Lehr-Lern-Labore stellen einerseits komplexitätsreduzierte und andererseits authentische Situationen bereit, in denen die Studierenden eigene und fremde Unterrichtssituationen theoriegeleitet planen, reflektieren und analysieren können (Haupt et al., 2013). Sie unterstützen auf diese Weise Studierende darin, praktische Erfahrungen zu sammeln, Lehr-Lern-Prozesse umfassend $\mathrm{zu}$ verstehen und erfolgreich zu gestalten (Krofta, Fandrich \& Nordmeier, 2013; Kürten et al., 2018).

Lehr-Lern-Labore für die Lehrerbildung können unterschiedlich charakterisiert werden (vgl. Brüning, 2018, S. 138 ff.). Während in klassischen Schülerlaboren die teilnehmenden Schülerinnen und Schüler die zentrale Zielgruppe sind, wird in ihrer Ausweitung zu Lehr-Lern-Laboren für die Lehrerbildung zugleich die Ausund Weiterbildung von Lehrkräften in den Blick genommen (Dohrmann \& Nordmeier, 2015). Insbesondere der Theorie-Praxis-Bezug und der Erwerb von professionellen Kompetenzen spielen in der Lehrerbildung eine wichtige Rolle. Ein zentrales Element von Lehr-Lern-Laboren in der Lehrerbildung ist das theoriegeleitete Erproben praktischen unterrichtlichen Handelns. Auch die anschließende Analyse 
und Reflexion der Lehr-Lern-Prozesse ist ein essentielles Merkmal. Es soll die „forschend-reflexive Haltung durch theoriegeleitete Planung, Durchführung und Beobachtung von Unterricht mit anschließender Reflexion der Lehr-Lern-Prozesse gefördert werden" (Krofta, Fandrich \& Nordmeier, 2012, S. 2). Des Weiteren stellt die Komplexitätsreduktion einen wesentlichen Faktor von Lehr-Lern-Laboren in der Lehrerbildung dar, der auf verschiedene Weisen realisierbar ist; sei es durch die Unterstützung durch andere Studierende und Lehrende, die Arbeit mit kleinen Lerngruppen, die Lokalisation der Lerngelegenheit in vertrauter außerschulischer Umgebung oder die Beschränkung der Beobachtungsaufgaben auf ausgewählte Aspekte (Dohrmann \& Nordmeier, 2015). Die Reduktion der Komplexität ist darüber hinaus ein probates Mittel, um den von Tschannen-Moran, Woolfolk Hoy und Hoy (1998) beschriebenen „Praxisschock“ zu verhindern (Greefrath \& Wess, 2018).

\section{Lehr-Lern-Labore im Projekt Dealing with Diversity}

Im ersten Förderzeitraum der Qualitätsoffensive Lehrerbildung waren die Ziele des Teilprojektes Lehr-Lern-Labore im Projekt Dealing with Diversity die Analyse und Evaluation bestehender Praxisformate, die Optimierung dieser mit Fokus auf unterschiedliche Heterogenitätsfacetten wie z. B. Leistungsheterogenität von Lernenden und die Konzeption neuer Praxisformate in weiteren Fächern auf Grundlage der Erkenntnisse zur Ausbildung professioneller Kompetenz bei angehenden Lehrkräften (Baumert \& Kunter, 2006).

In allen beteiligten Fächern gab es bereits vor Projektbeginn unterschiedliche Lehrformate mit Praxisbezug. In vier Arbeitsgruppen (Erziehungswissenschaft, Physikdidaktik, Sachunterrichtsdidaktik, Chemiedidaktik) wurden diese weiterentwickelt. Ihnen gemeinsam sind der direkte Kontakt der Studierenden mit Lernenden in komplexitätsreduzierten Lernsituationen und ein dreiphasiger Aufbau in Vorbereitungs-, Praxis- und Reflexionsphase. Die Lehrformate konnten auf die Frage des Umganges mit Heterogenität fokussiert werden. In drei weiteren Arbeitsgruppen (Mathematikdidaktik, Biologiedidaktik, Geographiedidaktik) wurden die Ergebnisse der Analyse genutzt, um neue Lehr-Lern-Labore mit vergleichbaren Rahmenkonzepten einzurichten. Für die Lehrformate wurden unterschiedliche Schwerpunkte im Kontext des Umgangs mit Heterogenität gewählt; im MINT-Bereich insbesondere durch die Betrachtung der Schwerpunkte Schülervorstellungen und Leistungsheterogenität (siehe Tab. 1). 
Tabelle 1: Einzelprojekte des Teilprojekts Lehr-Lern-Labore und ihre Schwerpunkte im Kontext des Umgangs mit Heterogenität

\begin{tabular}{ll}
\hline Einzelprojekt & Schwerpunkt im Lehr-Lern-Labor \\
\hline Biologiedidaktik I & Schülervorstellungen zur Evolution, Neurobiologie und Genetik \\
Biologiedidaktik II & Schülervorstellungen zur Evolution \\
Chemiedidaktik & Schülervorstellungen \\
Erziehungswissenschaft I & Intrapersonelle und intrakollektive Heterogenität \\
Erziehungswissenschaft II & Konstruktion von Begabung, Benachteiligung, Beeinträchtigung \\
Geographiedidaktik & Leistungsheterogenität beim Experimentieren \\
Mathematikdidaktik & Leistungsheterogenität \\
Physikdidaktik & Schülervorstellungen zu Messdaten \\
Sachunterrichtsdidaktik & Schülervorstellungen zur Statik \\
\hline
\end{tabular}

Im Rahmen des Teilprojekts Lehr-Lern-Labore wurden verschiedene Eigenschaften von Lehr-Lern-Laboren vor dem Hintergrund der Projektbedingungen diskutiert und fokussiert. Hierzu gehören neben der Berücksichtigung von Heterogenität und der Reduktion der Komplexität die Förderung professioneller Kompetenzen in authentischen Lehr-Lern-Situationen und die Förderung von Theorie-PraxisBezügen. Der vorliegende Band stellt grundlegende Überlegungen zur Komplexitätsreduktion im Lehr-Lern-Labor in den Mittelpunkt. Die Darstellung der einzelnen Lehr-Lern-Labore nimmt auf diese einführenden Überlegungen Bezug.

Die verschiedenen Lehr-Lern-Labore im Projekt Dealing with Diversity beinhalten in teilweise unterschiedlicher Schwerpunktsetzung einen Aufbau aus Vorbereitungs-, Praxis- und Reflexionsphase. Die Vorbereitungsphase besteht in den verschiedenen Einzelprojekten aus der Vermittlung theoretischer Grundlagen, die etwa videogestützt erfolgen kann, sowie der Entwicklung einer eigenen Unterrichtseinheit mit einer spezifischen Zielstellung, beispielsweise die Vermittlung von Inhalten unter Berücksichtigung von Schülervorstellungen im Sinne moderat konstruktivistischer Lerntheorien oder die Entwicklung eigener Modellierungsaufgaben bzw. binnendifferenzierter Experimentalaufgaben. Auch die Analyse von Video- und Textvignetten ist in die Vorbereitungsphase einzelner Lehr-LernLabore integriert. Die Vorbereitungsphase kann auch auf verschiedene Zeitpunkte der Lehrveranstaltung aufgeteilt werden, um die Planung und Überarbeitung einer Unterrichtseinheit durch Praxis- oder Reflexionsphasen zu unterbrechen. Im Vergleich zu den anderen beiden Phasen ist die Vorbereitungsphase in der Regel die umfangreichste Phase in den verschiedenen Lehr-Lern-Laboren.

Die Praxisphase dient der Durchführung der entwickelten Unterrichtsstunde, Experimentier- oder Modellierungsaufgaben bzw. Unterrichtsbausteine. Hier findet in der Regel auch die Beobachtung der Lehr-Lern-Prozesse unter einem festgelegten Fokus statt. Die Selbstreflexion unmittelbar nach der Durchführung kann ebenfalls in die Praxisphase integriert sein. Im Vergleich zu den anderen beiden 
Phasen ist die Praxisphase in der Regel die kürzeste Phase in den verschiedenen Lehrkonzepten.

In der anschließenden Reflexionsphase folgt kriteriengeleitet die Reflexion der ggf. videografierten Unterrichtseinheit und die Präsentation sowie Diskussion ausgewählter Unterrichtsszenen. Dies kann zur Anpassung oder Umplanung der Unterrichtseinheit genutzt werden. Ebenso können Erfahrungen aus der Praxisphase reflektiert und das verwendete Material evaluiert werden. Die Ergebnisse werden verschriftlicht und ggf. auch an die beteiligten Schulen zurückgemeldet. Auf diese Art und Weise werden angehende Lehrkräfte angeleitet, Praxiserfahrungen theoriegeleitet zu reflektieren.

Ein wichtiger Baustein der Arbeit im Teilprojekt Lehr-Lern-Labore ist die wissenschaftliche Begleitung der entwickelten Lehrkonzepte. So werden die verschiedenen gewählten Schwerpunkte zum Umgang mit Heterogenität analysiert. Hierzu werden u. a. die Selbstwirksamkeitserwartung sowie die professionellen Kompetenzen untersucht und die Beobachtungs- und Verstehensprozesse der Studierenden rekonstruiert. Methodisch werden dazu beispielsweise ein vignettengestützer PräPost-Fragebogen mit geschlossenen und offenen Antwortformaten verwendet und die videografierten Einheiten der Studierenden analysiert, oder es werden leitfadengestützte Interviews mit Studierenden, Fachleitern sowie Referendaren geführt.

Die Studierenden können theoriegeleitet eigenständig in komplexitätsreduzierten Situationen unterrichtliches Handeln planen, mit Lernenden erproben und reflektieren. In der Evaluation aller Praxisformate des Teilprojekts zeigte sich, dass die entwickelten Lehr-Lehr-Labore die Einstellungen angehender Lehrkräfte zu Praxiserfahrungen und ihre Selbstwirksamkeitserwartungen in Bezug auf Lehrhandlungen im Lehr-Lern-Labor nicht nur signifikant positiv verändern, sondern dass sich diese Entwicklungen auch signifikant von den Entwicklungen der Vergleichsgruppe ohne Praxisformate unterscheiden (Kürten, Wess \& Greefrath, im Druck).

\section{Konzepte im Teilprojekt Lehr-Lern-Labore}

In diesem Band werden die einzelnen Lehrkonzepte im Teilprojekt Lehr-LernLabore des Projekts Dealing with Diversity in Münster im ersten Förderzeitraum der Qualitätsoffensive Lehrerbildung vorgestellt sowie Aspekte der Reduktion der Komplexität diskutiert und Forschungsergebnisse vorgestellt.

Zunächst stellen wir in einem grundlegenden Beitrag zur Komplexitätsreduktion in Lehr-Lern-Laboren ein Planungs- und Reflexionsmodell vor, das im Rahmen der gemeinsamen Arbeit im Teilprojekt entstanden ist. Es wird genauer erläutert, was sich hinter dem häufig im Kontext von Lehr-Lern-Laboren verwendeten Begriff der Komplexitätsreduktion verbirgt und was genau eine komplexitätsreduzierte Lernumgebung ausmacht. Auf der Basis dieses Modells können Lehr-LernLabore geplant, reflektiert und evaluiert werden. 
Die anschließenden Beiträge beleuchten im Kontext des jeweils vorgestellten Lehr-Lern-Labors neben individuellen Fragestellungen stets auch einzelne Dimensionen des Modells und ihre Umsetzung in der Praxis.

Im Lehr-Lern-Labor LEO der Biologiedidaktik werden angehende Biologielehrkräfte durch die Förderung diagnostischer Kompetenz auf den Umgang mit Heterogenität durch reflektierte Praxiserfahrung vorbereitet. Dazu findet das Linsenmodell von Brunswik (1955) seine Verwendung. Im Lehr-Lern-Labor LEO können Studierende diagnostisches Handeln mit Schülervideos, Schülertexten und im direkten Kontakt mit Schülerinnen und Schülern erproben. Erste Ergebnisse zeigen, dass durch das Lehr-Lern-Labor konzeptuell das bestehende Seminarangebot effektiv ergänzt und der Aufbau diagnostischer Kompetenz unterstützt wird.

Das zweite Projekt der Biologiedidaktik geht von der Annahme aus, dass die Wahrnehmung von Unterricht auch auf implizitem Wissen basiert, dessen Berücksichtigung für eine evidenzbasierte Gestaltung von Lehr-Lern-Angeboten sinnvoll ist. In diesem Kontext wurde erforscht, welches implizite Wissen von Lehramtsstudierenden zu Schülervorstellungen und dem Umgang mit ihnen im Evolutionsunterricht die Wahrnehmung von Videovignetten beeinflusst. Der Einbezug des impliziten Wissens und der theoretischen Überlegungen zur Sachantinomie bietet eine komplementäre Perspektive zu fachdidaktischen Lehrveranstaltungen zum Umgang mit Schülervorstellungen, die sich auf die Förderung expliziten Professionswissens fokussieren.

Das Lehr-Lern-Labor CL(EV) ${ }^{2}$ ER der Chemiedidaktik beschäftigt sich mit dem Aufbau von Wissen und Fähigkeiten im Kontext von Schülervorstellungen sowie dem Umgang mit Stolperstein-Situationen angehender Lehrkräfte. Unter Stolperstein-Situationen werden dabei unerwartete Unterrichtssituationen verstanden, die Lehrkräfte im Moment des Handelns als herausfordernd empfinden. Sie entstehen u. a. durch eine unzureichende Antizipation des Unterrichtsgeschehens bei der Planung. Durch Reflexion von Stolperstein-Situationen soll ein entsprechendes Handlungsrepertoire zur Professionalisierung angehender Lehrkräfte angebahnt werden.

In der erziehungswissenschaftlichen Lehr- und Forschungswerkstatt ELF als praktisches Element in der universitären Lehrerbildung werden positive Einflussfaktoren auf die Entwicklung beruflicher Handlungskompetenz von Lehramtsstudierenden in den Blick genommen. Im Fokus steht das Zertifikat lehren.lernen der erziehungswissenschaftlichen Lehr- und Forschungswerkstatt. Hier können sich Studierende selbst mit fall- und handlungsorientierten Angeboten bereits in der Hochschule im Handeln erproben und reflektieren.

Das Ziel des zweiten erziehungswissenschaftlichen Lehr-Lern-Labors „Diagnose und Individuelle Förderung" ist die Entwicklung und Etablierung einer flexiblen universitären Lernumgebung, in deren Rahmen vielfältige Auseinandersetzungsprozesse mit dem Themenfeld „Heterogenität in der Schule“ stattfinden und unterstützt werden können. Hier wird konkret das Seminar „Schulischen Umgang mit Vielfalt verstehen und gestalten" durchgeführt, in dem mit der Reduktion von 
Komplexität eine Fokussierung von Einzelaspekten stattfindet, die gleichzeitig mit einer Steigerung von Komplexität einzelner Aspekte verbunden sein kann.

Im geografiedidaktischen Lehr-Lern-Labor GEO steht die Professionalisierung von Lehramtsstudierenden hinsichtlich fachlicher und fachdidaktischer Kompetenzen beim Einsatz von Experimenten im Geographieunterricht unter Berücksichtigung heterogener Lerngruppen im Vordergrund. Dabei stellt sich die Frage, wie das Lehr-Lern-Labor gestaltet werden kann, um bei Lehramtsstudierenden die situationsspezifische Selbstwirksamkeitserwartung beim Einsatz von Experimenten im Geografieunterricht zu fördern.

Im Lehr-Lern-Labor Mi $\mathbb{R} A^{+}$der Mathematikdidaktik werden Modellierungsaufgaben wegen ihrer selbstdifferenzierenden Eigenschaften für einen produktiven Umgang mit Heterogenität genutzt. Hier wird in der gymnasialen Lehrerbildung die Entwicklung professioneller Kompetenz zum Lehren mathematischen Modellierens bei angehenden Lehrkräften in komplexitätsreduzierten Lehr-Lern-Arrangements in den Blick genommen. So wird untersucht, wie sich Komplexitätsreduktionen im Bereich Medien und Materialien auf die Entwicklung bereichsspezifischer professioneller Kompetenz auswirken.

Das physikdidaktische Lehr-Lern-Labor La:gune stellt eine Lernumgebung zur Förderung des Lehrerhandelns in ungeplanten Situationen zur Verfügung. Dazu werden Unterrichtsminiaturen in mehreren Iterationen geplant, durchgeführt und anhand von Videoaufnahmen in Gruppen reflektiert. Zur Untersuchung wird die Selbstwirksamkeitserwartung bezüglich des Experimenteinsatzes im Physikunterricht erhoben und es werden leitfadengestützte Interviews mit Studierenden geführt, um insbesondere eine Einschätzung bezüglich der emotionalen Wahrnehmung der Situation zu erlangen.

Im Lehr-Lern-Labor der Sachunterrichtsdidaktik steht eine Lehrveranstaltung im Fokus der Betrachtung, in der zur Förderung der Planungskompetenz von Lehramtsstudierenden im Master die tatsächliche Erprobung einer eigenen Unterrichtsplanung im Lehr-Lern-Labor in den Seminarverlauf eingebunden wird. In der Begleitforschung wurden die Studierenden dazu interviewt, inwieweit sie einzelne Aspekte der Erprobung im Lehr-Lern-Labor wie z.B. die Wiederholung, die Aufnahme eines Videos oder das Agieren in einer Kleingruppe als hilfreich für die Weiterentwicklung ihrer Planungskompetenz einschätzen.

Darüber hinaus werden in einem abschließenden Beitrag fachübergreifende Ergebnisse zur Evaluation des vorgestellten Modells präsentiert. Ausgehend von der Wahrnehmung der umgesetzten Komplexitätsreduktion durch die Studierenden werden sowohl Veränderungen ihrer Selbstwirksamkeitserwartungen als auch die Bedeutung der Komplexitätsreduktion für die wahrgenommene Professionalisierung der Studierenden untersucht. 


\section{Literatur}

Baumert, J. \& Kunter, M. (2006). Stichwort: Professionelle Kompetenz von Lehrkräften. Zeitschrift für Erziehungswissenschaft, 9(4), 469-520. https://doi.org/10.1007/s11618oo6-0165-2

Brüning, A.-K. (2018). Das Lehr-Lern-Labor „Mathe für kleine Asse“. Untersuchungen zu Effekten der Teilnahme auf die professionellen Kompetenzen der Studierenden. Münster. WTM-Verlag.

Brunswik, E. (1955). Representative design and probabilistic theory in a functional psychology. Psychological Review, 62 (3), 193-217. https://doi.org/10.1037/hoo47470

Dohrmann, R. \& Nordmeier, V. (2015). Schülerlabore als Lehr-Lern-Labore (LLL): Ein Projekt zur forschungsorientierten Verknüpfung von Theorie und Praxis in der MINT-Lehrerbildung. PhyDid B - Didaktik der Physik - Beiträge zur DPG-Frühjahrstagung.

Fischer, C., Rott, D., Veber, M., Fischer-Ontrup, C. \& Gralla, A. (2014). Individuelle Förderung als schulische Herausforderung. Netzwerk Bildung. Berlin: Friedrich-EbertStiftung.

Greefrath, G. \& Wess, R. (2018). MiRA+: Ein mathematikdidaktisches Lehr-Labor Forschungsnahes Lehren und Lernen im Rahmen der QLB. In N. Neuber, W. Paravicini \& M. Stein (Hrsg.), Forschendes Lernen. The Wider View. Eine Tagung des Zentrums für Lehrerbildung der Westfälischen Wilhelms-Universität Münster vom 25. bis 27.09.2017 (S. 229-232). Münster: WTM-Verlag.

Haupt, O. J., Domjahn, J., Martin, U., Skiebe-Corrette, P., Vorst, S., Zehren, W. \& Hempelmann, R. (2013). Schülerlabor - Begriffsschärfung und Kategorisierung. Der mathematische und naturwissenschaftliche Unterricht, 66 (6), 324-330.

Korthagen, F. A. J. (2001). Linking Practice and Theory: The Pedagogy of Realistic Teacher Education. Paper presented at the Annual Meeting of the American Educational Research Association, Seattle, April 2001. https://doi.org/10.4324/9781410600523

Krofta, H., Fandrich, J. \& Nordmeier, V. (2012). Professionalisierung im Schülerlabor: Praxisseminare in der Lehrerbildung. PhyDid B - Didaktik der Physik - Beiträge zur DPG-Frühjahrstagung.

Krofta, H., Fandrich, J. \& Nordmeier, V. (2013). Fördern Praxisseminare im Schülerlabor das Professionswissen und einen reflexiven Habitus bei Lehramtsstudierenden? In V. Nordmeier \& H. Grötzebauch (Hrsg.), PhyDid B. Verfügbar unter: http://www.phydid.de/index.php/phydid-b/article/view/493 (letzter Zugriff o9/2014).

Kürten, R., Wess, R. \& Greefrath, G. (2018). Potentialorientierter Umgang mit Heterogenität durch reflektierte Praxiserfahrung: Professionalisierung von Lehramtsstudierenden im mathematikdidaktischen Lehr-Labor. In Fachgruppe Didaktik der Mathematik der Universität Paderborn (Hrsg.), Beiträge zum Mathematikunterricht 2018 (S. 1119-1122). Münster: WTM-Verlag.

Kürten, R., Wess, R. \& Greefrath, G. (im Druck). Überzeugungen und Selbstwirksamkeitserwartungen von Studierenden im mathematikdidaktischen Lehr-Labor MiRA ${ }^{+}$ im Rahmen der Qualitätsoffensive Lehrerbildung. In M. Zimmermann \& W. Paravicini (Hrsg.), Hanse-Kolloquium zur Hochschuldidaktik der Mathematik 2017 - Beiträge zum gleichnamigen Symposium am 7. und 8. November 2014 an der Westfälischen Wilhelms Universität Münster. Münster: WTM-Verlag. 
Putnam, R. T. \& Borko, H. (2000). What do new views of knowledge and thinking have to say about research on teacher learning? Educational Researcher, 29 (1), 4-15. https://doi.org/10.3102/0013189X029001004

Rott, D., Zeuch, N., Fischer, C., Souvignier, E. \& Terhart, E. (Hrsg.) (2018). Dealing with Diversity. Innovative Lehrkonzepte in der Lehrer ${ }^{*}$ innenbildung zum Umgang mit Heterogenität und Inklusion (Begabungsförderung: Individuelle Förderung und Inklusive Bildung, Bd. 6). Münster: Waxmann.

Schwarzer, R. \& Jerusalem, M. (2002). Das Konzept der Selbstwirksamkeit. In M. Jerusalem \& D. Hopf (Hrsg.), Selbstwirksamkeit und Motivationsprozesse in Bildungsinstitutionen (Zeitschrift für Pädagogik: Beiheft, Bd. 44, S. 28-53). Weinheim: Beltz.

Tschannen-Moran, M., Woolfolk Hoy, A. \& Hoy, W. K. (1998). Teacher Efficacy: Its Meaning and Measure. Review of Educational Research, 68 (2), 202-248. https://doi. org/10.3102/00346543068002202

Wischer, B. (2009). Umgang mit Heterogenität im Unterricht - Das Handlungsfeld und seine Herausforderungen. TIPP (Teachers in Practice and Process), Handbuch: Heterogenität ruft nach Dialog. Verfügbar unter: http://www.teachers-ipp.eu/handbuch. html/2.\%2oUmgang\%2omit\%2oHeterogenitaet\%20-\%2oDE.pdf 

Annette Marohn, Gilbert Greefrath, Marcus Hammann,

Michael Hemmer, Ronja Kürten und Anna Windt

\section{Komplexitätsreduktion in Lehr-Lern-Laboren}

Ein Planungs- und Reflexionsmodell

\section{Motivation}

Zwei Argumente werden regelmäßig angeführt, um den Nutzen von Lehr-LernLaboren für die Lehrerbildung zu begründen: Lehr-Lern-Labore bieten Studierenden die Chance, Theorie-Praxis-Bezüge herzustellen und eröffnen die Möglichkeit, in einem komplexitätsreduzierten Setting zu lernen (Dohrmann \& Nordmeier, 2015; Hascher \& Zordo, 2015). Das erste Argument erschließt sich schnell: Studierende können fachdidaktische und bildungswissenschaftliche Theorien, die sie im Verlauf ihres Studiums kennen gelernt haben, auf unterrichtliche Praxis beziehen, indem sie Lehr-Lern-Prozesse gestalten und reflektieren. Doch was genau verbirgt sich hinter dem vielzitierten Begriff der Komplexitätsreduktion? Was kennzeichnet eine komplexitätsreduzierte Lernumgebung und welche Möglichkeiten gibt es, diese zu realisieren? Diesen Fragen widmet sich der folgende Beitrag. Die Grundlage des Beitrags bilden Überlegungen aus acht Lehr-Lern-Laboren, die im Rahmen des Projekts zur Qualitätsoffensive Lehrerbildung Dealing with Diversity an der Westfälischen Wilhelms-Universität (WWU) Münster etabliert bzw. weiterentwickelt wurden. Die dort gewonnenen Erfahrungen dienen als Basis, um den Begriff der Komplexitätsreduktion auszuschärfen und ein Modell zu generieren, das als Planungshilfe für die Ausgestaltung von Lehr-Lern-Laboren genutzt werden kann.

\section{Das Gleichgewichtsprinzip}

Eine der ersten Assoziationen, die sich mit dem Begriff der Komplexitätsreduktion in Lehr-Lern-Laboren verbinden, ist die Vorstellung, dass Studierende vor geringere Anforderungen gestellt werden, als es in der alltäglichen Unterrichtspraxis von Lehrkräften der Fall ist. Ziel ist es, den von Tschannen-Moran, Woolfolk Hoy und Hoy (1998) beschriebenen „Praxisschock“ abzumildern und eine „kognitive Überlastung" zu vermeiden (Zucker \& Leuchter, 2018). So schlagen Krofta, Fandrich und Nordmeier (2012) vor, „Studierende ... in kleineren Schritten an die Herausforderungen des Lehrerberufes heranzuführen" (S. 2). Es ist zum Beispiel denkbar, dass die Studierenden zunächst keine vollständige Einheit unterrichten, sondern sich auf eine einzelne Phase konzentrieren wie etwa die Durchführung einer Diagnose oder die Betreuung eines Schülerexperiments. Anforderungen können zudem dadurch verringert werden, dass die Studierenden das Lernarrangement nicht 
eigenständig planen, sondern vorgegebene Materialien nutzen. Möglicherweise liegt der Fokus des Lehr-Lern-Labors auch nicht auf dem eigenen unterrichtlichen Handeln, sondern richtet sich zunächst lediglich auf die Beobachtung und Analyse von Unterrichtssequenzen.

Der Blick auf die Lehr-Lern-Labore an der WWU Münster zeigt jedoch, dass die Begriffsdeutung „Komplexitätsreduktion = geringere Anforderungen“ zu kurz greift. Die gestellten Anforderungen werden von den Betreuenden häufig sogar als hoch eingeschätzt (vgl. Beiträge in diesem Sammelband). Die Studierenden planen und erproben - zum Teil mehrfach hintereinander - vollständige Unterrichtssequenzen, sie reflektieren Unterricht detailliert und setzen diverse fachdidaktische oder pädagogische Theorien in Beziehung mit erlebter Praxis.

Kann angesichts dessen tatsächlich von Komplexitätsreduktion gesprochen werden? Auf welche Weise wird verhindert, dass Studierende in Überforderungssituationen geraten? Die Antwort findet sich in der konkreten Ausgestaltung der verschiedenen Lehr-Lern-Labore: Hohe Anforderungen werden stets durch verschiedene Unterstützungsmaßnahmen begleitet. Das Konstrukt der Komplexität erschließt sich somit nicht durch einen einseitigen Blick auf die gestellten Anforderungen - es erfordert einen wechselseitigen Abgleich von Anforderungs- und Unterstützungsniveau.

Ziel muss es sein, die Komplexität des Lehr-Lern-Labors adressatengerecht an das Leistungsniveau der Studierenden anzupassen. Bildlich entspricht dies der Anbahnung eines Gleichgewichts zwischen gestellten Anforderungen und angebotenen Unterstützungsmaßnahmen (vgl. Abb. 1). Im Idealfall zeigt sich dieses Gleichgewicht in sämtlichen Abschnitten des Lehr-Lern-Labors, das typischerweise Phasen der Vorbereitung, der Praxis und der Reflexion umfasst. Anforderungen und Unterstützungen können sich dabei sowohl auf inhaltlicher als auch struktureller Ebene bewegen. Im folgenden Abschnitt wird hierzu ein differenziertes Modell entwickelt.

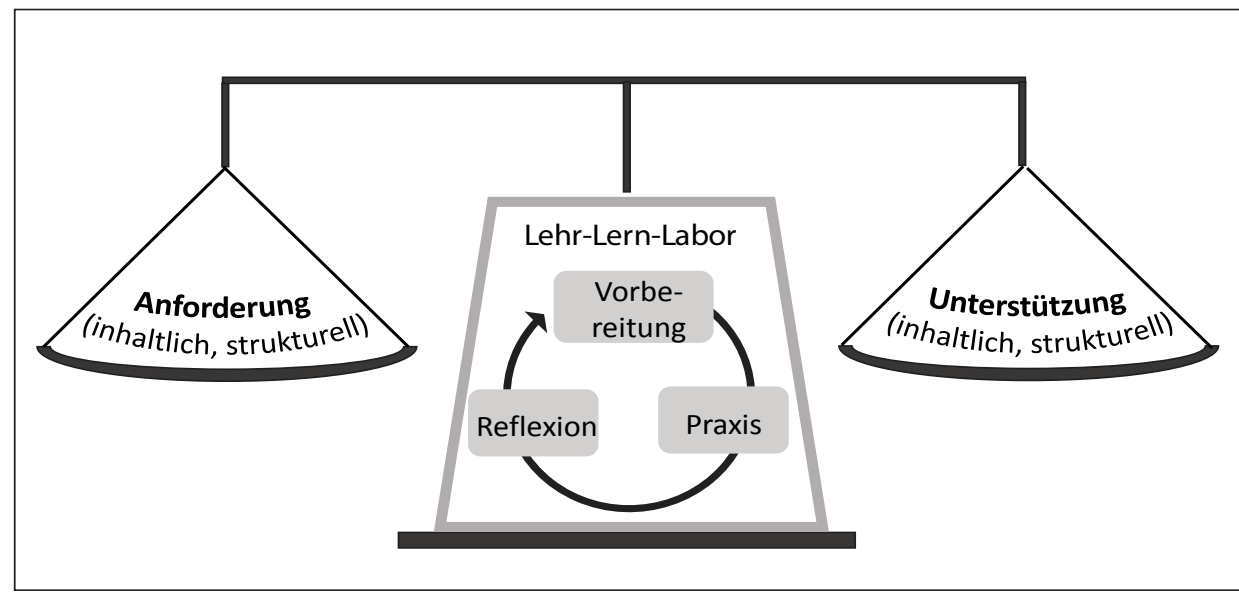

Abbildung 1: Anbahnung eines Gleichgewichts von Anforderung und Unterstützung 


\section{Komplexität in Lehr-Lern-Laboren}

Das Modell zur Komplexität in Lehr-Lern-Laboren (vgl. Abb. 2) stellt den inhaltlichen bzw. strukturellen Anforderungen, die an die Studierenden gestellt werden, entsprechende Maßnahmen zur Unterstützung gegenüber. Anforderungen und Unterstützungen sind in Form von Schiebereglern dargestellt, die zum Beispiel eine Einstellung von „gering" bis "hoch“ zulassen. Die Regler weisen keine Skalierung auf, da eine objektive, wissenschaftlich fundierte Messbarkeit der einzelnen Dimensionen nicht gegeben ist. Die Einstellung der Regler beinhaltet somit stets eine subjektive Einschätzung: Verschiedene Personen, wie etwa Betreuende oder Studierende, können das Anforderungs- und Unterstützungsniveau im selben Lehr-Lern-Labor unterschiedlich bewerten. Das Modell trägt damit ganz bewusst der subjektiven Wahrnehmung von Komplexität Rechnung; es bietet die Chance, different erlebte Komplexität zu reflektieren, um Schlussfolgerungen für eine Weiterentwicklung des jeweiligen Lehr-Lern-Labors zu ziehen.

Im Bereich der inhaltlichen Anforderungen deutet das Modell auf das Spannungsfeld zwischen quantitativen und qualitativen Anforderungen hin, indem es innerhalb der betrachteten Dimensionen zwischen "Anzahl“ und "Niveau“ unterscheidet. So kann zum Beispiel die Komplexität fachdidaktischer Theorien bedingen, das Lehr-Lern-Labor auf eine oder wenige Theorien zu fokussieren, um Überforderung zu vermeiden. Umgekehrt kann gerade die Konzentration auf eine einzelne Theorie eine intensive Auseinandersetzung ermöglichen und somit das qualitative Anspruchsniveau steigern.

Das Modell visualisiert damit eine elementare Frage, die sich hinsichtlich der Gestaltung eines jeden Lehr-Lern-Labors stellt: Möchte ich eine möglichst große inhaltliche Breite zulassen oder entscheide ich mich für eine enge inhaltliche Fokussierung? Inhaltliche Breite eröffnet die Möglichkeit für vielfältige Erfahrungen, birgt jedoch die Gefahr einer eher oberflächlichen Auseinandersetzung. Inhaltliche Fokussierung bietet wiederum die Chance der detaillierten Aufarbeitung einzelner Aspekte, lässt aber möglicherweise weitere relevante Inhalte unberücksichtigt.

Das Modell bildet somit eine Basis, um sich bereits in der Phase der Planung eines Lehr-Lern-Labors der Auswirkungen von Entscheidungen bewusst zu werden und mögliche Einflussfaktoren auf die Komplexität des Settings anhand der aufgeführten Dimensionen in den Blick zu nehmen. Durch einen wechselseitigen Abgleich der Anforderungs- und Unterstützungsregler eröffnet es zudem die Möglichkeit, passgenaue Unterstützungsmaßnahmen zu planen.

Die folgenden Abschnitte beleuchten die einzelnen Felder des Modells im Detail. 


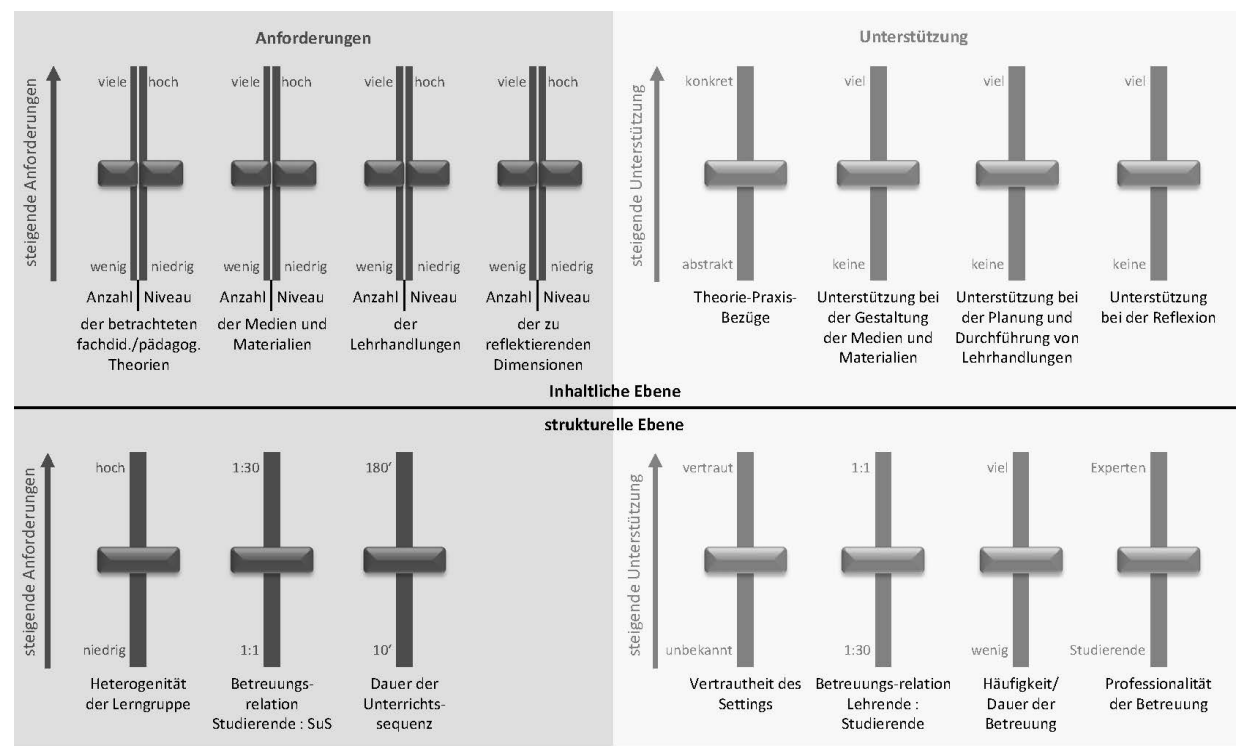

Abbildung 2: Komplexität in Lehr-Lern-Laboren

\subsection{Inhaltliche Anforderungen}

Die inhaltlichen Anforderungen eines Lehr-Lern-Labors ergeben sich im Wesentlichen aus vier Dimensionen: den betrachteten Theorien, den Lehrhandlungen, den zu erstellenden Medien und Materialien sowie der Breite und Tiefe der vorgenommenen Reflexion.

- Theorie und Praxis in Beziehung zu setzen, bildet ein zentrales Ziel von LehrLern-Laboren (Dohrmann \& Nordmeier, 2015). Eine aktuelle Veröffentlichung $\mathrm{zu}$ „Theorien in der naturwissenschaftsdidaktischen Forschung“" (Krüger, Parchmann \& Schecker, 2018) führt allein für den Bereich der Naturwissenschaften 17 für die Lehrerbildung relevante Themenfelder auf wie etwa Argumentieren, Modellieren, Experimentieren, Kontextorientiertes Lernen, Nature of Science oder Schülervorstellungen. In jedem dieser Bereiche existiert wiederum eine Vielzahl - zum Teil sehr diverser - theoretischer Rahmungen. Dass Studierende sämtliche Theorien bei der Analyse oder Planung von Unterricht nutzen, ist undenkbar. Die Anforderungen des Lehr-Lern-Labors ergeben sich somit ganz wesentlich aus der hier vorzunehmenden Fokussierung, sowohl in Bezug auf die Anzahl der betrachteten Theorien als auch das qualitative Niveau, mit der diese betrachtet werden. So lassen sich etwa conceptual change-Theorien in sämtlichen Ausdifferenzierungen behandeln, zum Beispiel als eine Veränderung von Seinskategorien, von Rahmentheorien, oder phenomenological primitives (vgl. Gropengießer \& Marohn, 2018); sie lassen sich jedoch ebenso auf unterrichtspragmatische Aspekte fokussieren wie etwa die Gestaltung kognitiver Konflikte. 
Möglicherweise werden einzelne Theorien auch als komplexer wahrgenommen als andere. Im Bereich der Naturwissenschaftsdidaktik lässt sich dieses Phänomen zum Beispiel anhand von Theorien im Bereich der Bewertungskompetenz beobachten, die von angehenden Lehrkräften häufig als schwieriger wahrgenommen werden als theoretische Ansätze zur Experimentierkompetenz. Hier mag eine Rolle spielen, dass Studierende umfangreichere Erfahrungen im Bereich der Experimentierkompetenzen mitbringen. Auch diese Effekte können das subjektiv empfundene Anforderungsniveau eines Lehr-Lern-Labors steigern und sollten von den Betreuenden berücksichtigt werden.

Ein Blick auf die Lehr-Lern-Labore an der WWU Münster zeigt, dass häufig eine Fokussierung auf eine einzelne Theorie erfolgt, diese jedoch in großer qualitativer Tiefe durchdrungen wird. Beispiele hierfür bilden z. B. die Fokussierung auf die Modellierungskompetenz (mit Vertiefungen in den Bereichen Erkennen von Phasen im Modellierungsprozess, Diagnose von Schwierigkeiten und Formulierung von Förderzielen), oder die Fokussierung auf Schülervorstellungen (mit Vertiefungen in den Bereichen Ursachen von Schülervorstellungen, Diagnosemöglichkeiten und Initiierung von Veränderungen).

Neben fachdidaktischen und pädagogischen Theorien spielen zudem die betrachteten fachwissenschaftlichen Theorien eine Rolle. Das Anforderungsniveau steigt, je komplexer die Fachinhalte sind, die von den Studierenden für die beteiligten Schülerinnen und Schüler im Lehr-Lern-Labor didaktisch elementarisiert werden müssen.

- Der zweite Schieberegler fokussiert die Lehrhandlungen. Unterrichten umfasst eine Vielzahl diverser Lehrhandlungen, wie zum Beispiel Diagnostizieren oder Experimentieren, aber auch Fragen, Erklären oder Strukturieren. Die Anforderungen im Lehr-Lern-Labor steigen mit der Zahl an Handlungen an, die die Studierenden planen und durchführen müssen. Dies gilt umso mehr, da mit jeder Handlung eigene fachdidaktische bzw. pädagogische Theorien zu verknüpfen sind.

Darüber hinaus können Handlungen mit einem unterschiedlichen Anspruchsniveau verbunden sein. So erscheint es weniger komplex, eine Gruppenarbeit durch einen Arbeitsauftrag zu initiieren als die Ergebnisse der Gruppenarbeit im Plenum zu reflektieren, um gemeinsam weitere Handlungsoptionen daraus abzuleiten. Gleiches gilt für die Betreuung von Schülerexperimenten, die per Versuchsvorschrift durchgeführt werden, im Vergleich zur Betreuung von offenen Experimentiersituationen.

Weiterhin kann das Anforderungsniveau mit der „Realitätsnähe“ des Kontextes ansteigen, in dem die Handlung erfolgt: Eine Diagnose anhand einer Videovignette wird vielleicht als weniger anfordernd empfunden als eine Diagnose, die mit „realen“ Schülerinnen und Schülern durchgeführt wird.

Neben geplanten Handlungen können im Unterricht zudem unerwartete Situationen auftreten, die ein spontanes Agieren notwendig machen (Mühl- 
hausen, 1994). Hierzu zählen zum Beispiel unerwartete Schüleraussagen oder Experimentalergebnisse. Diese Momente werden aufgrund des in der Situation gegebenen Entscheidungs- und Handlungsdrucks gerade von Berufsanfängern als besonders herausfordernd erlebt (Wahl, 1991). Das Anforderungsniveau steigt demnach auch mit der Offenheit der Lernumgebung an, die die Studierenden durchführen: Je mehr Raum für unerwartete Situationen das Setting zulässt, umso häufiger sehen sich die Studierenden der Notwendigkeit spontaner Lehrhandlungen ausgesetzt.

- Nicht nur Lehrhandlungen, auch Medien und Materialien können das Anforderungsniveau im Lehr-Lern-Labor beeinflussen. Die Zahl und Diversität nutzbarer Materialien und Medien für den Unterricht erscheint nahezu unbegrenzt. Es bedarf wohl keiner Begründung, dass die Anforderungen steigen, je vielfältiger Medien und Materialien sind, die von den Studierenden gestaltet und eingesetzt werden sollen. Zudem gilt hier Ähnliches wie im Bereich der Lehrhandlungen: Die Nutzung von Medien und Materialien erfordert jeweils spezifisches Professionswissen, zum Beispiel in Bezug auf den Einsatz digitaler Medien, die Nutzung von Modellen oder Experimenten, die Gestaltung von Arbeitsblättern oder die Einbindung von Texten und Abbildungen.

Darüber hinaus kann sich das Anforderungsniveau je nach Material deutlich unterscheiden: Die Gestaltung eines Lückentextes zur Sicherung von Wissen erscheint weniger komplex als die Generierung einer Modellierungsaufgabe oder eines geschlossenen Diagnoseformats. Die Anforderungen, die mit der Gestaltung von Materialien verbunden sind, hängen somit auch vom Anspruch der Ziele ab, die mit dem Einsatz dieser Materialien verfolgt werden: Fordert das Material lediglich zur Reproduktion von Fachwissen auf, oder soll es Schülerinnen und Schüler dazu anregen, ihr Wissen in argumentativen Bewertungsprozessen zu nutzen? Verwenden alle Lernenden dasselbe Material, oder sollen sie durch differenzierte Materialien individuell gefördert werden?

- Reflexion sowie die Bereitschaft zur Reflexion gelten als zentrale Elemente professioneller Handlungskompetenz (z.B. Berndt, Häcker \& Leonhard, 2017; Denner \& Gesenhues, 2013; Roters, 2012). Sie bilden eine notwendige Voraussetzung, um Theorie und Praxis in wechselseitige Beziehung setzen zu können und bewusstes Handeln zu ermöglichen. Reflexion stellt damit einen unabdingbaren Bestandteil des Lernens der Studierenden im Lehr-Lern-Labor dar (Krofta, Fandrich \& Nordmeier, 2013; Saathoff \& Hößle).

Allerdings existiert bislang keine einheitliche Begriffsbestimmung und Modellierung des Konstrukts (Abels, 2011). Reflexionen im Lehr-Lern-Labor können somit verschiedenen Ansätzen und Strukturierungen folgen. Nach Dewey (1951) resultiert Reflexion aus einem Moment der Verunsicherung, der einen Lösungsprozess nach sich zieht. Gemäß dem Ansatz der strukturierten Refle- 
xion (Mamede et al., 2012) vollzieht sich Reflexion auf Basis eines subjektiven Initialurteils, das durch weitere Informationen gestützt oder geschwächt wird.

Weitere Ansätze orientieren sich an immer wiederkehrenden Abfolgen, wie etwa der Beschreibung einer Situation, der Identifizierung von Ursachen und Entwicklung von Handlungsoptionen, die in Form von Kreisprozessen (u.a. Korthagen, 2002; Krieg \& Kreis, 2014) oder in Form von Stufenmodellen (u.a. Hatton \& Smith, 1995; Wyss, 2013) dargestellt werden.

Das Anforderungsniveau der Reflexion ergibt sich zum einen daraus, wie viele Dimensionen bzw. Theorien anhand einer Unterrichtssequenz in den Blick genommen werden; diese ergeben sich in der Regel aus der inhaltlichen Fokussierung des Lehr-Lern-Labors. Zum anderen ist bedeutsam, in welcher Tiefe die Reflexion erfolgt: Liegt das Hauptaugenmerk zum Beispiel nur auf den verbalen Äußerungen von Lehrenden und Lernenden oder werden Handlungen, Mimik, Gestik, Körperhaltung und Stimmlage in die Reflexion einbezogen? Werden unterschiedliche Interpretationen einer Situation durch die Studierenden kontrovers diskutiert und implizites Wissen (Bohnsack, NentwigGesemann \& Nohl, 2007) sowie subjektive Theorien (Mandl \& Huber, 1983) als handlungsleitende Elemente bewusst gemacht? Werden lediglich mögliche Ursachen für einen Handlungsverlauf rekonstruiert, oder auch Planungs- und Handlungsalternativen entwickelt?

Für das subjektiv empfundene Anforderungsniveau spielt zudem eine Rolle, ob die Studierenden eigene Lehrhandlungen reflektieren oder fremde Handlungen, z.B. von Kommilitoninnen und Kommilitonen, analysieren, da die Auseinandersetzung mit der eigenen Person im Kontext einer universitären Veranstaltung mit Unsicherheiten oder Versagensängsten behaftet sein kann.

\subsection{Inhaltliche Unterstützung}

Die Komplexität, die sich aus den aufgeführten inhaltlichen Anforderungen ergibt, lässt sich durch diverse Unterstützungsmaßnahmen verringern.

- Wesentliche Elemente bilden hierbei eine präzise Passung zwischen der theoretischen Vorbereitung der Studierenden und der anschließenden Praxisphase sowie eine möglichst konkrete Auseinandersetzung mit den theoretischen Inhalten. Dies bedeutet, dass die Studierenden im Rahmen der Vorbereitung das notwendige Professionswissen erwerben bzw. vertiefen und konkretisieren, das für die Durchführung der geforderten Lehrhandlungen in der Praxisphase erforderlich ist. Dabei hat sich im Rahmen der Lehr-Lern-Labore an der WWU Münster bewährt, schon sehr früh - etwa mit Hilfe von Textvignetten oder Videografien - Theorie-Praxis-Bezüge herzustellen, um die Relevanz des behandelten Professionswissens für die unterrichtliche Praxis bewusst zu machen. Als komplexitätsreduzierendes Element hat sich hierbei eine Fokussierung auf ausgewählte Aspekte erwiesen; diese Fokussierung kann zum Beispiel mit Hilfe kurzer Videovignetten 
erfolgen, die konkret nur eine Schülervorstellung, eine Experimentier- oder Modellierungssituation oder eine bestimmte Lehrhandlung zeigen. Die Anforderungen können dabei sukzessiv über die Länge der Videoausschnitte sowie die Komplexität der gezeigten Situation gesteigert werden.

- Auch die Planung von Lehrhandlungen kann unterstützt werden, zum Beispiel indem die Studierenden didaktische Konzepte und Methoden kennen lernen, die sie zur Einbettung der Lehrhandlungen nutzen können. Einzelne Lehrhandlungen wie etwa Experimentierhandlungen, Erklärungen oder die Anwendung von Diagnoseinstrumenten können zudem im Verlauf der Vorbereitungsphase erprobt und eingeübt werden. Die Studierenden erfahren auf diese Weise mögliche Schwierigkeiten, die sich mit der jeweiligen Lehrhandlung verknüpfen und lernen, Lösungsmöglichkeiten zu entwickeln. Zudem können die Studierenden auf den Umgang mit unerwarteten Situationen vorbereitet werden, indem sie überraschende Momente anhand von Videosequenzen analysieren, mögliche Ursachen identifizieren und schrittweise ein Handlungsrepertoire in Form von „abrufbaren“ Strategien aufbauen.

- Darüber hinaus sind Hilfestellungen sowohl bezüglich der Phasierung von Unterricht möglich als auch der Entwicklung von Lernmaterialien. Die Unterstützung kann hierbei von einzelnen Tipps, Literaturhinweisen und Beispielmaterialien bis hin zur Bereitstellung vollständiger Materialsätze reichen, die von den Studierenden in der Praxisphase genutzt werden können. Als hilfreich erweist sich zudem die Vorgabe konkreter Kriterien für die Materialentwicklung, zum Beispiel im Hinblick auf die Gestaltung von Modellierungsaufgaben (Bruder, 1988) oder Diagnoseinstrumenten (Marohn \& Schmidt, 2003). Komplexität lässt sich zudem dadurch verringern, dass die Studierenden vorab Informationen über die Schülergruppe erhalten, mit der sie im Rahmen des Lehr-Lern-Labors arbeiten. Hierbei kann es sich um Auskünfte über bereits vorhandene Fach- und Methodenkenntnisse der Lernenden handeln oder um Informationen zu Charakteristika einzelner Schülerinnen und Schüler. Letztere gewinnen gerade vor dem Hintergrund heterogener Lerngruppen eine besondere Rolle und können die Gestaltung individualisierter Materialien erleichtern.

- Nicht zuletzt sollten auch Reflexionsprozesse eingeübt und bezüglich ihres Anforderungsniveaus schrittweise gesteigert werden. Als hilfreich kann sich hierbei die Nutzung von Reflexionsschemata (z. B. Hatton \& Smith, 1995; Korthagen, 2002; Krieg \& Kreis, 2014 Wyss, 2013) erweisen, die immer wieder in Form einer "reflection on action“ (Schön, 1983) - zum Beispiel anhand von Videografien - angewendet werden. Ziel ist es, ein Schema durch wiederholte Anwendung zunehmend $\mathrm{zu}$ verinnerlichen, sodass es möglicherweise auch im situativen Handeln als Muster zur Verfügung steht („reflection in action“); es kann damit das Treffen von Entscheidungen in der Unterrichtssituation unterstützten. Da- 
rüber hinaus können auch Gruppenreflexionen und kollegiales Feedback eigene Reflexionsprozesse begleiten und dazu beitragen, neue Perspektiven auf (eigenes) Unterrichtshandeln zu gewinnen.

\subsection{Strukturelle Anforderungen}

- Der Umgang mit Vielfalt zählt zu den besonderen Herausforderungen unterrichtlichen Handelns (Hochschulrektorenkonferenz, 2015). Der Begriff Heterogenität umfasst dabei unterschiedliche Dimensionen wie etwa Alter, Gender, ethnische Herkunft, sozioökonomischer Hintergrund, kulturelle und religiöse Orientierung, physische und kognitive Fähigkeiten sowie besondere Begabungen und Talente. Seit Anpassung der Bildungssysteme an die UN-Behindertenrechtskonvention (United Nations Educational, Scientific and Cultural Organization, 2005) haben sich diese Heterogenitätsdimensionen durch eine weitere Facette erweitert: die Inklusion von Kindern und Jugendlichen mit sonderpädagogischem Unterstützungsbedarf in die allgemeinbildenden Schulen.

Die Heterogenität von Lerngruppen bildet ein strukturelles Merkmal, welches das Anforderungsniveau des Lehr-Lern-Labors steigern kann, jedoch nicht notwendigerweise muss. So kann Vielfalt in Bezug auf Alter, Interessen und Fähigkeiten gerade im Blick auf Projektarbeiten oder offene Experimentiersituationen eine durchaus erwünschte und lernförderliche Ausgangslage darstellen. Heterogenität kann jedoch in Abhängigkeit der Zielsetzung des Lehr-Lern-Labors besondere Herausforderungen generieren, etwa wenn individuelle Förderungen angestrebt, oder gemeinsame Lernsituationen von Kindern mit unterschiedlichem Leistungsniveau und Unterstützungsbedarf gestaltet werden sollen.

Der Umgang mit Vielfalt erfordert je nach Fokus des Labors und den Heterogenitätsdimensionen der Lerngruppe eine Auseinandersetzung mit entsprechenden pädagogischen oder fachdidaktischen Theorien, zum Beispiel in Bezug auf Differenzierung (Klafki \& Stöcker, 1976), Scaffolding (Wood, Bruner \& Ross, 1976), konstruktivistische Lehr-Lern-Theorien (Riemeier, 2007) oder den Ansatz der Zone der proximalen Entwicklung (Vygotski, 1964). Heterogenität generiert zudem spezielle Anforderungen im Bereich der Materialgestaltung, etwa bei der Gestaltung sprachsensiblen Lernmaterials (Leisen, 2015) oder der Nutzung von Mitteln der unterstützten Kommunikation (Gesellschaft für Unterstützte Kommunikation e. V., 2005).

- Strukturelle Anforderungen ergeben sich zudem aus der Betreuungsrelation von Studierenden zu Schülerinnen und Schülern. Die Betreuungsrelation kann von einer 1:1-Betreuung bis hin zum Unterrichten einer vollständigen Schulklasse reichen. Kleine Lerngruppen werden gerade von Studierenden mit wenig Lehrerfahrungen als entlastend empfunden. Insbesondere vor dem Hintergrund 
heterogener Lerngruppen erleichtert die Fokussierung auf wenige Schülerinnen und Schüler das adressatengerechte Planen und Handeln. Bei einem zyklischen Aufbau des Lehr-Lern-Labors mit mehreren Praxis-Durchgängen kann die Komplexität durch eine sukzessiv zunehmende Größe der Lerngruppen schrittweise gesteigert werden.

Eine häufig praktizierte Form in Bezug auf die Ausgestaltung der Betreuungsrelation bildet das Teamteaching (Huber, 2000): Studierende unterrichten Schülerinnen und Schüler in Zweier- oder sogar Dreierteams. Diese eigentlich als Erleichterung gedachte Maßnahme kann sich aus Studierendensicht allerdings als zusätzliche Anforderung darstellen; dies ist gerade dann der Fall, wenn keine Vorerfahrungen im Teamteaching vorliegen, oder wenn Lehrerpersönlichkeiten und Einstellungen der beteiligten Studierenden stark differieren. Die Arbeit in Studierendengruppen birgt zudem die Gefahr, dass diese aufgrund von pragmatischen Gründen weniger im Team als vielmehr arbeitsteilig erfolgt. Erfahrungen im Rahmen der Lehr-Lern-Labore in Münster deuten darauf hin, dass eine solche Arbeitsteilung das Zutrauen in die eigene Kompetenz behindern kann.

- Einen weiteren möglichen Einflussfaktor auf die Komplexität bildet die Dauer der Unterrichtssequenz. Mit größerer Dauer nimmt in der Regel die Zahl der zu planenden und durchzuführenden Lehrhandlungen zu, wie auch die Anzahl der genutzten Materialien. Beides kann das Anforderungsniveau steigern. In Bezug auf die Analyse bzw. Reflexion von Lehr-Lern-Situationen ist allerdings eine ähnliche Unterscheidung zwischen quantitativen und qualitativen Anforderungen zu treffen wie bei den inhaltlichen Schiebereglern: Eine Fokussierung auf wenige Schülerinnen und Schüler bzw. einen kurzen Unterrichtsausschnitt kann durchaus mit einer besonderen qualitativen Tiefe der Reflexion einhergehen, was das Anforderungsniveau wiederum erhöhen würde.

\subsection{Strukturelle Unterstützung}

Auch strukturelle Maßnahmen können dazu beitragen, Studierende in ihrem Lernprozess zu unterstützen und damit die Komplexität des Lehr-Lern-Labors zu verringern.

- Eine wesentliche Bedeutung kommt hierbei der Vertrautheit des Settings zu. Der Begriff der Vertrautheit kann sich sowohl auf räumliche, mediale, materielle als auch personelle Aspekte beziehen. So erleben es Studierende als hilfreich, wenn sie die räumlichen Gegebenheiten sowie die mediale Ausstattung vorab kennen, da dies die Gefahr von unvorhergesehenen Situationen verringert. Vor allem im Rahmen von experimentellen Lehr-Lern-Settings spielt die Vertrautheit mit räumlichen und materiellen Ausstattungen eine zentrale Rolle: Wie bediene ich 
die Gaszufuhr? Wo befinden sich Sicherheitseinrichtungen wie Notausschalter oder Feuerlöscher? Welche Materialien existieren in welcher Anzahl?

Daneben erweist sich die Vertrautheit mit der Lerngruppe als wesentlicher Einflussfaktor auf die Wahrnehmung von Komplexität. Einen ersten Schritt bildet - wie oben erwähnt - die Bereitstellung von Informationen über den Kenntnisstand oder besondere Charakteristika der Schülerinnen und Schüler. Eine größere Vertrautheit und damit stärkere Komplexitätsreduktion wird verständlicherweise dann hergestellt, wenn Studierende und Lerngruppe in einem zyklischen Prozess mehrfach miteinander arbeiten. Denkbar ist auch, dass die Studierenden ausgehend von einem vertrauten Setting an zunehmend fremdere Situationen herangeführt werden; so können sie zum Beispiel eine Lehr-Lern-Situation zunächst mit Studierenden derselben Veranstaltung oder vertrauten Personen des persönlichen Umfeldes erproben, um auf diese Weise ausreichend Sicherheit für die Durchführung der Handlungen in einer fremden Lerngruppe zu gewinnen.

- Intensität und Qualität der Unterstützung macht sich auf struktureller Ebene zudem an äußeren Rahmenbedingungen der Betreuung fest. Hierbei spielen drei Parameter eine Rolle: die Relation von Betreuenden zu Studierenden, die Häufigkeit und Dauer der Betreuung sowie deren Professionalität.

Eine hohe Zahl an Betreuenden im Verhältnis zur Studierendenanzahl eröffnet die Option einer stark individualisierten Betreuung, die auf unterschiedliche Wissensstände, individuelle Fragen oder Sorgen der Lernenden eingehen kann. Häufige und langandauernde Betreuung über sämtliche Phasen des LehrLern-Labors hinweg ermöglicht es, den Lernprozess der Studierenden permanent zu begleiten und individuelle Scaffolding-Maßnahmen an die jeweiligen Lernfortschritte anzupassen.

Neben den quantitativen Parametern Betreuungsrelation und Häufigkeit bestimmen vor allem die personalen Merkmale die Qualität der Betreuung. Die erforderliche Professionalität der Betreuenden misst sich dabei am jeweiligen Fokus der Unterstützung: Die theoretische Vorbereitung der Studierenden sowie die Initiierung von Theorie-Praxis-Bezügen setzt in jedem Fall einen Wissensvorsprung im Bereich des fachdidaktischen und pädagogischen Professionswissens voraus. Eine Unterstützung in der Planung von Unterrichtssequenzen erfordert über das Professionswissen hinaus zudem Erfahrungswissen; einige Lehr-Lern-Labore binden hierzu neben den Dozentinnen und Dozenten der Universität auch Lehrkräfte in den Betreuungsprozess mit ein. Die Planung von Experimenten kann zudem durch Labormitarbeitende oder Technikerinnen und Techniker begleitet werden, die spezifisches Wissen im Bereich der Experimentierkompetenzen beitragen.

Die Durchführung der geplanten Lehrhandlungen erfordert weniger inhaltliche als organisatorische Unterstützung; diese kann zum Beispiel über studentische Hilfskräfte erfolgen, die bei der Bereitstellung von Materialien helfen 
oder Verantwortung für die Videografie der Lehrer-Schüler-Interaktionen übernehmen.

Unterstützung im Bereich der Reflexion kann nicht nur von Seiten der Betreuenden geleistet werden, sondern auch im Rahmen von peer groups und kollegialem Feedback der Studierenden erfolgen. Einzelne Reflexionsansätze zielen sogar bewusst auf Interaktionsprozesse der Studierenden und eine Kontrastierung verschiedener Perspektiven und Interpretationen.

Neben professionellem Wissen und Erfahrungen bilden natürlich auch Aspekte wie Empathie oder die Fähigkeit zur positiven Bestärkung Kennzeichen einer professionellen Betreuung und tragen nicht unwesentlich zum Erfolg der Unterstützungsmaßnahmen bei.

\section{4. $\quad$ Fazit}

Der Beitrag beschreibt ein Modell zur Komplexität in Lehr-Lern-Laboren, das „aus der Praxis für die Praxis“ generiert wurde. Das Modell kann dazu dienen, eine überhöhte Komplexität bei der Ausgestaltung von Lehr-Lern-Laboren zu vermeiden, indem es Möglichkeiten aufzeigt, den jeweiligen Anforderungen ein adäquates „Gegengewicht“ an Unterstützungsmaßnahmen gegenüberzustellen.

Das Modell bietet zudem eine Grundlage, um Einflussfaktoren auf die Komplexität aus der Perspektive von Dozierenden und Studierenden in einem retrospektiven Reflexionsprozess zu bewerten und zu kontrastieren. Die individuelle Wahrnehmung von Komplexität kann sich hierbei grundlegend unterscheiden und Anlass bieten, einzelne Gestaltungselemente in den nachfolgenden Durchgängen des Lehr-Lern-Labors zu verändern.

Neben dem Nutzen als Planungs- und Reflexionsmodell soll das Modell zudem die breite Varianz visualisieren, in der sich Lehr-Lern-Labore ausgestalten lassen. Entgegen typischer Assoziationen eines aufwändig ausgestatteten, materiellen Raums, in dem Studierende vollständige Unterrichtssequenzen mit ganzen Schulklassen durchführen, weitet das Modell den Blick auf deutlich kleinere Optionen: So kann sich die Interaktion ebenso auf eine ausgewählte Lehrhandlung beschränken, die ein Studierender anhand eines einzelnen Schülers erprobt. Das Modell eröffnet damit vielfältige Möglichkeiten, auch angesichts schwieriger Rahmenbedingungen in Bezug auf Räumlichkeiten oder personelle und finanzielle Ressourcen, Praxiserfahrungen in die Lehrerbildung zu integrieren und Studierenden die Chance zu eröffnen, Theorie und Praxis gewinnbringend miteinander zu verzahnen. 


\section{Literatur}

Abels, S. (2011). LehrerInnen als „Reflective Practitioner“. Reflexionskompetenz für einen demokratieförderlichen Naturwissenschaftsunterricht. Wiesbaden: VS Verlag für Sozialwissenschaften. https://doi.org/10.1007/978-3-531-92735-O

Berndt, C., Häcker, T. H. \& Leonhard, T. (Hrsg.) (2017). Reflexive Lehrerbildung revisited. Traditionen - Zugänge - Perspektiven (Studien zur Professionsforschung und Lehrerbildung). Bad Heilbrunn: Julius Klinkhardt.

Bohnsack, R., Nentwig-Gesemann, I. \& Nohl, A.-M. (2007). Die dokumentarische Methode und ihre Forschungspraxis. Wiesbaden: VS Verlag für Sozialwissenschaften. https://doi.org/10.1007/978-3-531-90741-3

Bruder, R. (1988). Grundfragen mathematikmethodischer Theoriebildung unter besonderer Beruecksichtigung des Arbeitens mit Aufgaben. Dissertation, Pädagogische Hochschule Potsdam. Potsdam.

Denner, L. \& Gesenhues, D. (2013). Professionalisierungsprozesse im Lehramtsstudium eine explorative Studie zu Analyse, Interpretation und Handlungsoption. In R. Bolle (Hrsg.), Professionalisierung im Lehramtsstudium. schulpraktische Kompetenzentwicklung und theoriegeleitete Reflexion (S. 59-119). Leipzig: Leipziger Universitätsverlag.

Dewey, J. (1951). Wie wir denken. eine Untersuchung über die Beziehung des reflektiven Denkens zum Prozeß der Erziehung. Zürich: Morgarten.

Dohrmann, R. \& Nordmeier, V. (2015). Schülerlabore als Lehr-Lern-Labore (LLL). Ein Projekt zur forschungsorientierten Verknüpfung von Theorie und Praxis in der MINT-Lehrerbildung. PhyDid B - Didaktik der Physik - Beiträge zur DPG-Frühjahrstagung. Verfügbar unter http://phydid.physik.fu-berlin.de/index.php/phydid-b/ article/view/658.

Gesellschaft für Unterstützte Kommunikation e. V. (Hrsg.) (2005). Handbuch der unterstützten Kommunikation (2. Aufl.). Karlsruhe: Loeper Literaturverlag.

Gropengießer, H. \& Marohn, A. (2018). Schülervorstellungen und Conceptual Change. In D. Krüger, I. Parchmann \& H. Schecker (Hrsg.), Theorien in der naturwissenschaftsdidaktischen Forschung (Springer Spektrum, S. 49-68). Berlin: Springer. https://doi.org/10.1007/978-3-662-56320-5_4

Hascher, T. \& Zordo, L. de (2015). Langformen von Praktika. Ein Blick auf Österreich und die Schweiz. Journal für LehrerInnenbildung, 15 (1), 22-32. https://doi.org/10. 7892 /boris.74667.

Hatton, N. \& Smith, D. (1995). Reflection in teacher education. Towards definition and implementation. Teaching and Teacher Education, 11 (1), 33-49. https://doi.org/ 10.1016/0742-051X(94)00012-U.

Hochschulrektorenkonferenz (Hrsg.) (2015). Lehrerbildung für eine Schule der Vielfalt. Gemeinsame Empfehlung von Hochschulrektorenkonferenz und Kultusministerkonferenz. (Beschluss der Kultusministerkonferenz vom 12.03.2015/ Beschluss der Hochschulrektorenkonferenz vom 18.03.2015).

Huber, B. (2000). Team-Teaching - Bilanz und Perspektiven. Eine empirische Untersuchung im Kärntner Volksschulbereich / Integrationsklassen (Schuljahr 1998/99) zur Thematik/Problematik der Zusammenarbeit im Zweierteam (Europäische Hochschulschriften. Reihe VI, Psychologie Publications universitaires européennes. Série VI, Psychologie European university studies. Series VI, Psychology, vol. 657). Frankfurt am Main: P. Lang. 
Klafki, W. \& Stöcker, H. (1976). Innere Differenzierung des Unterrichts. Zeitschrift für Pädagogik, 22 (4), 497-523.

Korthagen, F. A. J. (2002). Eine Reflexion über Reflexion. In J. Kessels, B. Koster, B. Lagerwerf \& T. Wubbels (Hrsg.), Schulwirklichkeit und Lehrerbildung. Reflexion der Lehrertätigkeit (S. 55-73). Hamburg: EB-Verlag.

Krieg, M. \& Kreis, A. (2014). Reflexion in Mentoringgesprächen - ein Mythos? Zeitschrift für Hochschulentwicklung, 9 (1), 103-117. https://doi.org/10.3217/zfhe-9-01/11

Krofta, H., Fandrich, J. \& Nordmeier, V. (2012). Professionalisierung im Schülerlabor. Praxisseminare in der Lehrerbildung. PhyDid B - Didaktik der Physik - Beiträge zur $D P G$-Frühjahrstagung. Verfügbar unter phydid.physik.fu-berlin.de/index.php/phydidb/article/viewFile/412/550.

Krofta, H., Fandrich, J. \& Nordmeier, V. (2013). Fördern Praxisseminare im Schülerlabor das Professionswissen und einen reflexiven Habitus bei Lehramtsstudierenden? PhyDid B - Didaktik der Physik - Beiträge zur DPG-Frühjahrstagung. Verfügbar unter http://www.phydid.de/index.php/phydid-b/article/view/493.

Krüger, D., Parchmann, I. \& Schecker, H. (Hrsg.) (2018). Theorien in der naturwissenschaftsdidaktischen Forschung (Springer Spektrum). Berlin: Springer. https://doi. org/10.1007/978-3-662-56320-5

Leisen, J. (2015). Fachlernen und Sprachlernen! Bringt zusammen, was zusammen gehört! MNU Journal, 68 (3), 132-137.

Mamede, S., van Gog, T., Moura, A. S., Faria, R. M. D. de, Peixoto, J. M., Rikers, R. M. J. P. \& Schmidt, H. G. (2012). Reflection as a strategy to foster medical students' acquisition of diagnostic competence. Medical education, 46 (5), 464-472. https://doi. org/10.1111/j.1365-2923.2012.04217.x.

Mandl, H. \& Huber, G. L. (1983). Subjektive Theorien von Lehrern. Psychologie in Erziehung und Unterricht, 30, 98-112.

Marohn, A. \& Schmidt, H.-J. (2003). Mehrfachwahlaufgaben als Instrument zur Erforschung von Schülervorstellungen. Chimica didactica, 29 (91), 38-51.

Mühlhausen, U. (1994). Überraschungen im Unterricht. Situative Unterrichtsplanung (Beltz Praxis). Weinheim: Beltz.

Riemeier, T. (2007). Moderater Konstruktivismus. In D. Krüger \& H. Vogt (Hrsg.), Theorien in der biologiedidaktischen Forschung. Ein Handbuch für Lehramtsstudenten und Doktoranden (S. 69-79). Berlin: Springer. https://doi.org/10.1007/978-3-540-681663_7

Roters, B. (2012). Professionalisierung durch Reflexion in der Lehrerbildung. Eine empirische Studie an einer deutschen und einer US-amerikanischen Universität (Studien zur international und interkulturell vergleichenden Erziehungswissenschaft, Bd. 12). Münster: Waxmann.

Saathoff, A. \& Hößle, C. (2018) Professionalisierung durch Lehr-Lern-Labore. Lehramtsstudierende der Biologie reflektieren ihren Unterricht. In M. Hammann \& M. Linder (Hrsg.), Biologieddaktik als Wissenschaft (Lehr- und Lernforschung in der Biologiedidaktik, Bd. 8, S. 287-301). 21. Internationale Tagung der Fachsektion Didaktik der Biologie im VBIO, Martin-Luther-Universität Halle-Wittenberg. Innsbruck, Wien, Bozen: StudienVerlag.

Schön, D. A. (1983). The reflective practitioner. How professionals think in action. New York: Basic Books. 
Tschannen-Moran, M., Woolfolk Hoy, A. \& Hoy, W. K. (1998). Teacher Efficacy: Its Meaning and Measure. Review of Educational Research, 68 (2), 202-248. https://doi. org/10.3102/00346543068002202.

United Nations Educational, Scientific and Cultural Organization (Hrsg.) (2005). Guidelines for Inclusion. Ensuring Access to Education for All, Paris.

Vygotski, L. S. (1964). Denken und Sprechen. Berlin: Akademie-Verlag.

Wahl, D. (1991). Handeln unter Druck. Der weite Weg vom Wissen zum Handeln bei Lehrern, Hochschullehrern und Erwachsenenbildern. Weinheim: Deutscher Studien Verlag.

Wood, D., Bruner, J. S. \& Ross, G. (1976). The role of tutoring in problem solving. Journal of Child Psychology and Psychiatry, 17 (2), 89-10o. https://doi.org/10.1111/j.14697610.1976.tboo381.x.

Wyss, C. (2013). Unterricht und Reflexion. Eine mehrperspektivische Untersuchung der Unterrichts- und Reflexionskompetenz von Lehrkräften (Empirische Erziehungswissenschaft, Band 44). Münster: Waxmann.

Zucker, V. \& Leuchter, M. (2018). Lehr-Lern-Labore als Orte der fachdidaktischen MINT-Lehramtsausbildung. Förderung von Kompetenzen Lehramtsstudierender hinsichtlich des Diagnostizierens und Rückmeldens. MNU Journal, 71 (6), 364-369. 

Johannes C. S. Zang und Marcus Hammann

Kompetenzförderung mit dem Linsenmodell

\section{Diagnose von Schülervorstellungen im biologiedidaktischen Lehr-Lern-Labor LEO}

LEO ist das Lehr-Lern-Labor am Zentrum für Didaktik der Biologie der Westfälischen Wilhelms-Universität (WWU) Münster. Es wird im Rahmen des Projekts "Dealing with Diversity“ der Qualitätsoffensive Lehrerbildung an der Universität Münster entwickelt. Ziel des Lehr-Lern-Labors LEO ist es, angehende Biologielehrkräfte durch die Förderung diagnostischer Kompetenz auf den Umgang mit Heterogenität durch reflektierte Praxiserfahrung vorzubereiten. LEO basiert auf verschiedenen Erkenntnissen zur kognitiven Informationsverarbeitung im Kontext diagnostischer Urteilsbildung. Inhaltlich steht eine Auseinandersetzung mit Aspekten kognitiver Komplexitätsreduktion im Vordergrund. Die Entwicklung eines reflektierten diagnostischen Blickes soll durch strukturelle De-Automatisierung und Förderung einer reflexiven Distanz zur eigenen Urteilsbildung unterstützt werden. Dazu findet das Linsenmodell von Brunswik (1955) seine Verwendung. LEO umfasst insgesamt 30 Semesterstunden, in denen Studierende diagnostisches Handeln mit Schülervideos, Schülertexten und wiederholt im direkten Kontakt mit Schülerinnen und Schülern erproben. Erste Pilotierungsergebnisse verweisen darauf, dass LEO konzeptuell das bestehende Seminarangebot effektiv ergänzt und den Aufbau diagnostischer Kompetenz unterstützt.

Schlüsselwörter: Diagnostik, kognitive Komplexitätsreduktion, Reflexion

\section{Einführung}

Dieser Artikel stellt LEO vor, das Lehr-Lern-Labor am Zentrum für Didaktik der Biologie der Westfälischen Wilhelms-Universität (WWU) Münster. LEO wurde im Kontext der Qualitätsoffensive Lehrerbildung entwickelt. Übergeordnetes Ziel ist es, angehende Biologielehrkräfte auf den Umgang mit Heterogenität im Klassenzimmer durch reflektierte Praxiserfahrung vorzubereiten. Spezifisch unterstützt LEO die Ausbildung diagnostischer Kompetenz, die als maßgebliche Voraussetzung für Individualisierung und adaptive Unterrichtsgestaltung gilt. Instruktional basiert LEO auf den theoretischen Prinzipien von Lehr-Lern-Laboren. Die theoriegeleitete Erprobung eigenen diagnostischen Handelns in komplexitätsreduzierten Situationen sowie die Reflexion eigener Praxiserfahrung stellen damit zentrale Elemente des Lehr-Lern-Labors LEO dar. 
Inhaltlich orientiert sich LEO an verschiedenen psychologischen, bildungswissenschaftlichen und fachdidaktischen Überlegungen zur Förderung diagnostischer Kompetenz.

Der erste Teil des vorliegenden Artikels skizziert den übergeordneten theoretischen Rahmen. Ausgehend von der Beschreibung des NeDiKo-Arbeitsmodells (Herppich et al., 2017), als Grundlage der Konzeptualisierung diagnostischer Kompetenz in LEO, wird die Beziehung von diagnostischer Urteilsbildung und Prozessen kognitiver Informationsverarbeitung aufgezeigt. Im Vordergrund steht dabei die Problematisierung kognitiver Komplexitätsreduktion durch automatisierte Prozesse heuristischer Informationsverarbeitung. Die Ableitung konkreter Ziele und Maßnahmen LEOs erfolgt anschließend ausgehend von der hier erfolgten Betrachtung unterschiedlicher empirischer Ansatzpunkte, die einen Bezug zur Förderung diagnostischer Kompetenz durch die Berücksichtigung kognitiver Informationsverarbeitungsprozesse aufweisen.

Der zweite Teil des vorliegenden Artikels beschreibt die Realisierung vorangegangener theoretischer Überlegungen in LEO. Eine besondere Rolle spielt dabei die methodische Verwendung des Linsenmodells nach Brunswik (1955), das als zentrales strukturierendes Element und integratives Instrument zur Förderung diagnostischer Kompetenz in LEO eingesetzt wird. Neben der Darstellung der übergeordneten Ziele und der daraus resultierenden Seminarstruktur beschäftigt sich ein Unterkapitel mit den in LEO realisierten Maßnahmen zur didaktischen Komplexitätsreduktion, die als wesentliche Charakteristika von Lehr-Lern-Laboren der Unterstützung des Lernprozesses dienen. Der Artikel schießt mit einer kurzen Darstellung erster Evaluationsergebnisse und gibt einen Ausblick auf weitere Entwicklungen.

\section{Diagnostische Kompetenz: theoretischer Hintergrund und konzeptuelle Verortung}

\subsection{Diagnostische Kompetenz als Voraussetzung für individuelle Förderung}

Gesellschaftlicher Wandel, Globalisierung, Migrationsbewegungen, internationaler Austausch sowie bildungspolitische Entscheidungen resultieren in einer zunehmenden Vielfalt der Lernenden im Klassenzimmer. Die Auseinandersetzung mit Vielfalt und Diversität vollzieht sich im Bildungsbereich anhand des Begriffes der Heterogenität. Vor dem Hintergrund des schulischen Bildungsauftrages wird Heterogenität verstärkt unter dem Gesichtspunkt der individuellen Lernvoraussetzungen diskutiert und stellt nach Vock und Gronostaj (2017) nicht nur schulische Realität, sondern auch eine Herausforderung für das Lernen und die Gestaltung von Unterricht dar (siehe auch Terhart, 2010). Diese Herausforderung ergibt sich aus dem pädagogischen Anspruch, eine optimale Förderung im Unterricht durch die Passung von Lehrangebot und individuellen Lernvoraussetzungen zu erzielen 
(Helmke, 2013). Mit zunehmender Heterogenität steigt die Bedeutung diagnostischer Kompetenz als zentraler Aspekt der Lehrerprofessionalität. Erst das Erkennen und differenzierte Einschätzen individueller Lernvoraussetzungen erlaubt die optimale Förderung der Schülerinnen und Schüler im Unterricht. Diagnostische Kompetenz kann damit als Voraussetzung für adaptiven Unterricht angesehen werden (Beck, 2008).

\subsection{Diagnostische Kompetenz als mehrdimensionales Konstrukt}

Diagnostische Kompetenz gilt als wichtiger Aspekt der professionellen Kompetenz von Lehrkräften (Bromme, 1997; Weinert, 2000). In verschiedenen Kompetenzmodellen lässt sich diagnostische Kompetenz implizit (Shulman, 1986) oder auf Grundlage des COACTIV-Modells zur professionellen Handlungskompetenz (Baumert \& Kunter, 2006; Kunter, Klusmann \& Baumert, 2009) explizit (Krauss et al., 2004) als Aspekt fachdidaktischen Wissens (pedagogical content knowledge) verorten. Nach Brunner, Anders, Hachfeld und Krauss (2011) stellt diagnostische Kompetenz ein mehrdimensionales Konstrukt dar, das nach der Integration fachdidaktischen und pädagogisch-psychologischen (pedagogical knowledge) Wissens verlangt.

Dass die Befähigung zur differenzierten Diagnose sowohl in die Standards für die Lehrerbildung der Kultusministerkonferenz (KMK, 2004) als auch der Gesellschaft für Fachdidaktik (GFD, 2005) Eingang gefunden hat, unterstreicht ihre Bedeutung. Diese spiegelt sich auch in der regen psychologischen, bildungswissenschaftlichen und fachdidaktischen Auseinandersetzung mit dem Konstrukt der diagnostischen Kompetenz von Lehrkräften wider. Dabei lässt sich ein zunehmender Wandel in der Konzeptualisierung diagnostischer Kompetenz verzeichnen. Während diagnostische Kompetenz zunächst maßgeblich anhand der Urteilsgenauigkeit beschrieben wurde (Karst, 2017; Schrader, 1989; Schrader \& Helmke, 1987), berücksichtigen Ansätze zur Operationalisierung zunehmend auch prozessbezogene Aspekte diagnostischer Urteilsbildung (Klug, Bruder, Kelava, Spiel \& Schmitz, 2013; Klug, Bruder, Keller \& Schmitz, 2012; Südkamp \& Möller, 2009).

Eine integrative Perspektive auf diagnostische Kompetenz liegt dem durch das wissenschaftliche Netzwerk zur diagnostischen Kompetenz von Lehrkräften (NeDiKo) vorgeschlagenem Arbeitsmodell zu Grunde (Herppich u.a., 2017). Nach diesem Ansatz ist diagnostische Kompetenz „eine kognitive Leistungsdisposition, die es Lehrkräften ermöglicht relativ stabil und relativ konsistent sowie quantifizierbar pädagogisch-diagnostische Anforderungen in verschiedenen pädagogischen Handlungssituationen zu meistern" (Herppich et al., 2017, S. 80). Diagnostisches Handeln wird dabei als Prozess beschrieben, der in Abhängigkeit von Zielsetzungen, situativen Anforderungen sowie kognitiven und sonstigen Leistungsdispositionen in unterschiedlichem Ausmaß kontrolliert oder automatisiert abläuft. Als zentrales Element des NeDiKo-Modells umfassen kognitive Leistungsdisposi- 
tionen konzeptuelle, situationale, prozedurale und strategische Wissensbestände. Damit lassen sich fachdidaktisches, pädagogisches, und fachwissenschaftliches Wissen im NeDiKo-Modell als Aspekte diagnostischer Kompetenz verorten und stellen gleichermaßen Ansatzpunkte zur Förderung diagnostischer Kompetenz dar (Herppich et al., 2017). Inwiefern kognitive Leistungsdispositionen in konkreten diagnostischen Handlungssituationen wirksam werden, hängt im NeDiKo-Modell von sonstigen Leistungsdispositionen, wie bspw. der Motivation oder dem diagnoserelevanten Selbstkonzept, ab. Auf Grundlage empirischer Befunde zum Einfluss der Situation auf die Diagnosekompetenz (Karst, 2017, S. 28) wird die Stabilität diagnostischer Kompetenz im NeDiKo-Modell als relativ beschrieben. In Abhängigkeit von der jeweiligen Zielsetzung lassen sich darüber hinaus sowohl summative (assessment of learning) als auch formative (assement for learning) (Bennett, 2011; Black \& Wiliam, 2009; Dunn \& Mulvenon, 2009) Diagnosehandlungen mit Hilfe des NeDiKo-Modells konzeptualisieren. Unterschiedliche diagnostische Prozesse variieren dabei in Formalisierung, Kontrolliertheit und Automatisierung. Die Beziehung zwischen diagnostischer Urteilsbildung und den Prozessen kognitiver Informationsverarbeitung (Ferreira, Garcia-Marques, Sherman \& Sherman, 2006) nimmt besonders in eher unstrukturierten diagnostischen Handlungssituationen eine bedeutende Rolle ein.

\subsection{Diagnostische Urteilsbildung als kognitive Informationsverarbeitung}

Ausgehend von Dual-Process-Theorien (Gawronski \& Creighton, 2013) lassen sich verschiedene Strategien zur diagnostischen Urteilsbildung als schnelle, autonome, unbewusste und assoziative (Typ 1) oder langsame, steuerbare, bewusste und regelbasierte (Typ 2) Prozesse kognitiver Informationsverarbeitung (Kahneman, 2011; Kahneman \& Frederick, 2012) beschreiben. Hetmanek und van Gog (2017) verwenden die Begriffe der „Typ 1-Diagnose“ und „Typ 2-Diagnose“ (S. 211), die als unterschiedliche Modi je nach situativer Anforderung (Unterrichtsgeschehen oder Übergangsentscheidung) in der Praxis Anwendung finden. Nach (KrolakSchwerdt, Böhmer \& Gräsel, 2013) stellt dabei das Maß an Verantwortlichkeit für das Urteil einen wichtigen Moderator für den Diagnoseprozess dar (siehe auch Krolak-Schwerdt, Böhmer \& Gräsel, 2009). Während kontrollierte Typ 2 Prozesse mit genaueren Urteilen (Ferreira et al., 2006) assoziiert sind, werden Urteile auf Basis einer Typ 1-Diagnose zwar als in vielen Alltagssituationen hinreichend genau (Gigerenzer \& Goldstein, 1996) aber auch anfällig für Urteilsverzerrungen beschrieben (Evans \& Stanovich, 2013; Kahneman, 2011). Urteilsverzerrungen wie zum Beispiel der Halo-Effekt (Thorndike, 1920) können dabei als Ergebnisse kognitiver Komplexitätsreduktion aufgefasst werden. Der Halo-Effekt beschreibt einen Urteilsfehler, der darauf beruht, dass voneinander unabhängige Merkmale als korreliert wahrgenommen werden. Die Ausprägung eines oft herausragenden 
Merkmals (Hesse \& Latzko, 2011) wirkt sich dabei auf die Beurteilung weiterer Merkmale aus.

Die Reduktion von Komplexität auf kognitiver Ebene wird durch den unbewussten Rückgriff auf vereinfachende Urteilsstrategien erklärt. Diese helfen in komplexen Situationen ausreichende, aber oft fehlerbehaftete Antworten zu generieren und werden als Heuristiken bezeichnet (Kahneman, 2011). Urteilsbildung auf der Basis von Heuristiken als Resultat einer automatisierten assoziativen Informationsverarbeitung nach Typ 1 wird von Südkamp, Praetorius und Spinath (2018) neben der Anwendung von Schemata nach Judd und Kulik (1980) und Protoypen (Cantor \& Mischel, 1977) als eine Form komplexitätsreduzierender Strategien angeführt, die zu einer Konfundierung von Lehrerurteilen beitragen kann.

Das Urteil einer Lehrkraft hinsichtlich eines bestimmten Schülermerkmals wird von den Autorinnen als „multi-determined“ beschrieben, wenn es von der Ausprägung weiterer Schülermerkmale beeinflusst wird. Basierend auf der Arbeit mit konsistenten und inkonsistenten Schülerprofilen berichten Südkamp et al. (2018), dass es Lehrenden schwerfällt, unterschiedliche Merkmale von Lernenden akkurat und voneinander separat zu beurteilen. Die Sensibilisierung für eigene Tendenzen zur heuristischen Informationsverarbeitung, ein Wissen um Urteilsfehler, die Ausbildung eines Bewusstseins für heterogene inkonsistente Schülerprofile und Wissen über lernrelevante Schülermerkmale stellen den Autorinnen zu Folge Ansatzpunkte zur Förderung diagnostischer Kompetenz in der Lehrerbildung dar.

\subsection{Reflexion: Mittel und Ziel der Förderung diagnostischer Kompetenz}

Ein Bewusstsein für unterschiedliche Modi der Urteilsbildung kann auch nach Hetmanek und van Gog (2017) als ein zentraler Aspekt diagnostischer Kompetenz angesehen werden. Vor dem Hintergrund der dualen Struktur menschlicher Wahrnehmungsprozesse drückt sich diagnostische Kompetenz in der Auswahl des geeigneten Diagnose-Typs (Typ 1 oder Typ 2) aus. Die Möglichkeit zur Wahl setzt die Kenntnis verschiedener Urteilsmodi voraus und verlangt nach Kontrolle spontan ablaufender Informationsverarbeitungsprozesse. Eine systematische De-Automatisierung diagnostischer Urteilsbildung nach Typ 1 stellt nach Hetmanek und van Gog (2017) eine konkrete Strategie zur Förderung diagnostischer Kompetenz dar. Die Autoren beziehen sich hier auf den Ansatz der strukturierten Reflexion (Mamede et al., 2012), der zur Verbesserung diagnostischer Kompetenz von Medizinstudierenden entwickelt wurde. Im Ansatz der strukturierten Reflexion erfolgt die Initiation von Reflexion ausgehend von einer fallbasierten Initialdiagnose. Aufgabe ist dann, die vorliegende diagnostische Information reflektiert auf die gestellte Initialdiagnose zu beziehen und herauszuarbeiten, welche Informationen die Diagnose stützen und welche dagegensprechen. Zentral ist dabei die Auseinandersetzung mit den Beziehungen von vorliegender diagnostischer Information und abgegebenem diagnostischen Urteil. In weiteren Schritten werden die Studieren- 
den dazu aufgefordert, alternative Diagnosen zu formulieren und diese gegeneinander abzuwägen (Mamede et al., 2012). Damit wird im Prozess der Aufbau einer reflexiven Distanz zum vorläufigen Initialurteil gefördert. Die Bedeutung eines Bewusstseins für die mögliche Vorläufigkeit der eigenen diagnostischen Urteile im Unterricht wird auch von Weinert und Schrader (1986) betont.

Die Initiation von Reflexion erfolgt im Diagnosezyklus nach Helmke (2009) zum Training der Diagnosekompetenz anhand des Vergleichs von subjektivem Initialurteil und den Ergebnissen eines Diagnoseinstrumentes. Eine Analyse bestehender Diskrepanzen bietet dann die Gelegenheit, den eigenen pädagogischen Blick zu schärfen (Helmke, 2009). Während Reflexion als Aspekt diagnostischen Handelns sich im Prozessmodell von Klug und Kollegen (2013) indirekt aus der zyklischen Abfolge ineinandergreifender Phasen ergibt, modellieren Ohle, McElvany, Horz und Ullrich (2015) Selbstreflexion als eigenständige Facette diagnostischer Kompetenz. Diese wird mit Bezug auf Shulman und Shulman (2004) dem Bereich selbstbezogener Kognitionen zugeordnet.

Insgesamt nimmt Reflexion im Kontext diagnostischer Kompetenz damit eine zweifache Rolle ein: Während die Initiation von Reflexionsprozessen dem Erwerb diagnostischer Kompetenz dient, kann die Förderung von Reflexionsfähigkeit im Kontext diagnostischer Urteilsbildung als Ziel solcher kompetenzfördernden Maßnahmen verstanden werden.

\section{LEO: ein Ansatz zur Förderung diagnostischer Kompetenz angehender Biologielehrkräfte}

Übergeordnetes Ziel des Lehr-Lern-Labors LEO ist es, künftige Biologielehrkräfte durch den Erwerb diagnostischer Kompetenz auf den Umgang mit Heterogenität durch reflektierte Praxiserfahrung vorzubereiten. Vor dem Hintergrund schulischer Praxis steht die Entwicklung eines reflektierten „diagnostischen Blickes“ (Paradies, Linser \& Greving, 2009) durch authentische und komplexitätsreduzierte Praxiserfahrung im Vordergrund. In LEO erfolgt dazu eine Sensibilisierung für Tendenzen zur heuristischen Urteilsbildung (Diagnose-Typ 1). Durch strukturelle De-Automatisierung von Urteilsprozessen soll die Förderung einer reflexiven Distanz zur eigenen diagnostischen Urteilsbildung unterstützt werden. Angesichts beschriebener Urteilskonfundierungen (Südkamp et al., 2018) liegt ein besonderer Fokus auf der bewussten Auseinandersetzung mit der Beziehung von Merkmalen, verfügbarer diagnostischer Information und resultierendem Urteil. Die Frage „Woran habe ich das eigentlich erkannt?“ kann damit als leitendes Prinzip des Lehr-Lern-Labors LEO gelten, das durch die Verwendung des Linsenmodells nach Brunswik (1955) im Seminar umgesetzt wird. Die Förderung einer reflexiven Distanz zur eigenen Urteilsbildung lässt sich im NeDiKo-Modell als Aspekt metakognitiven Wissens und in Bezug auf die unterrichtliche Umsetzung als Bestandteil strategischen Wissens verorten (Herppich et al., 2017). Der Aufbau strukturellen 
Wissens als Element der Diagnosekompetenz erfolgt im Seminar anhand der domänenspezifischen Auseinandersetzung mit unterschiedlichen fachdidaktischen Theoriefeldern und geht mit dem Erwerb deklarativen Wissens einher. Durch die Auseinandersetzung mit multiplen fachdidaktischen Inhalten werden im Seminar die empirischen Befunde zur Situationsabhängigkeit diagnostischer Resultate aufgegriffen, und es wird die Herstellung von Theorie-Praxisbezügen in unterschiedlichen Kontexten ermöglicht.

\subsection{Das Linsenmodell als Perspektive}

Das Linsenmodell nach Brunswik (1955) (vgl. Abb. 1) stellt eine Perspektive auf den Prozess der Urteilsbildung dar. Als theoretisches Rahmenmodell erlaubt es die plausible Erklärung von Urteilsfehlern und Verzerrungen (Hesse \& Latzko, 2011). Die zentrale Aussage des Linsenmodells besteht darin, dass eine Beurteilung nicht beobachtbarer Merkmale anhand der Verwendung beobachtbarer Signale (cues) erfolgt. Der Urteilsbildung liegt damit ein Konstruktionsprozess zu Grunde. Nicht beobachtbare Merkmale wie etwa Schülervorstellungen, der aktuelle Leistungsstand, Vorwissen, Intelligenz oder Motivation werden erst durch die „Linse“ beobachtbarer Signale sichtbar. Als Signale können beispielsweise Verhaltensweisen, Mimik, Gestik und Körperhaltung, Kleidungsstil, Stimmlage, oder auf inhaltlicher Ebene einzelne Begriffe, Sätze, oder Zeichnungen dienen (Schmidt-Atzert \& Amelang, 2012).

Die Genauigkeit eines Urteils hängt dabei von der Validität der vorliegenden Signale (Steht das Signal tatsächlich in einem Zusammenhang mit dem Merkmal, das ich beurteilen will?) und der Nutzung vorhandener Signale durch den Urteilenden (Gehen nur valide Signale in die Urteilsbildung ein?) ab. Valide Signale zur Diagnose einer vorliegenden Schülervorstellung müssen bspw. durch diagnostische Fragen (Wie ist die Giraffe zu ihrem langen Hals gekommen?) sichtbar gemacht werden. Die Antwort, „Die Giraffe hat sich immer gestreckt“ beinhaltet dann Signale (aktives Handeln, Strecken), die auf das Vorliegen einer Schülervorstellung schließen lassen. Der aktuelle Leistungsstand im Schulfach Sport steht dagegen in keinem Zusammenhang mit vorliegenden Vorstellungen zur evolutionären Merkmalsentwicklung und kann als Beispiel für ein nicht valides Signal dienen. Das Ausmaß, zu dem ein Urteilender auf valide Signale zurückgreift, wird als Sensitivität bezeichnet. Akkurate Urteile resultieren nach dem Linsenmodell dann, wenn valide Signale konsistent zur Urteilsbildung herangezogen werden (Förster \& Böhmer, 2017). 


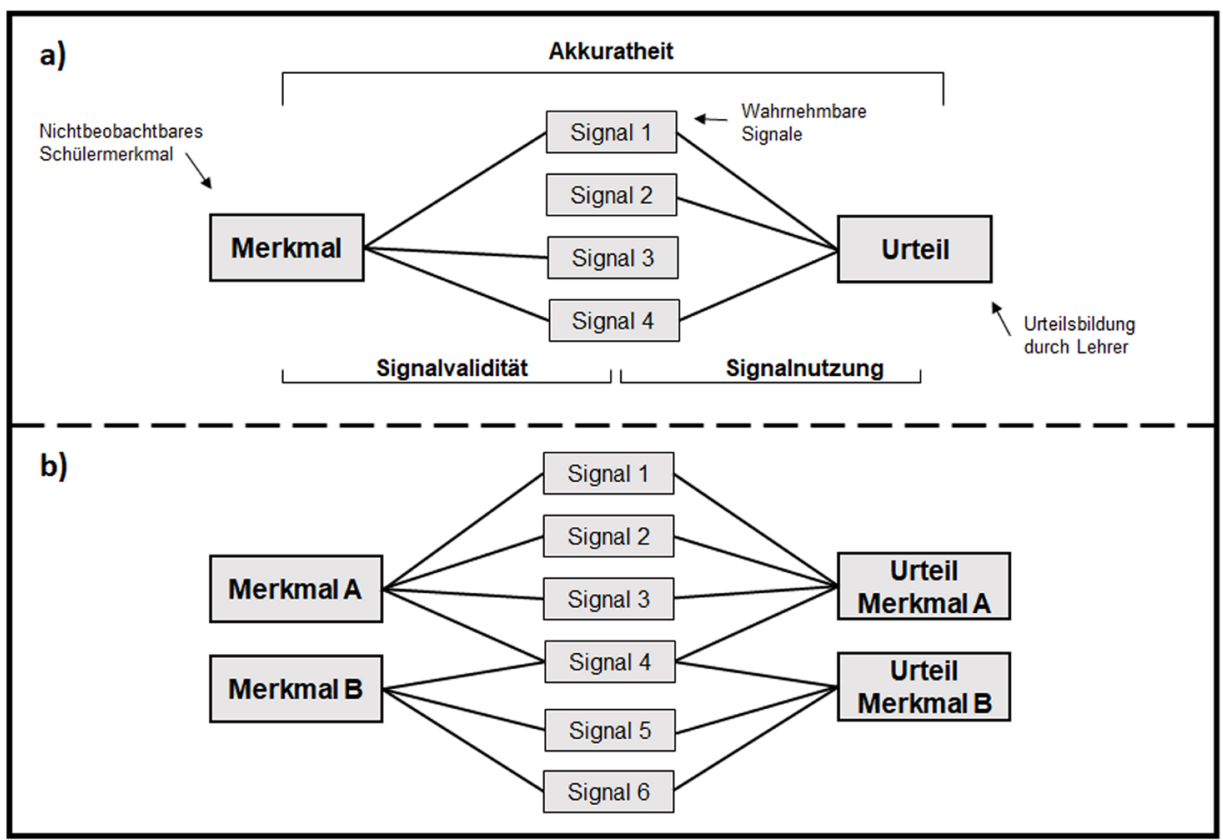

Abbildung 1: Das Linsenmodell nach Brunswik (Abbildung adaptiert nach Nestler \& Back (2013) sowie Förster \& Böhmer (2017)).

Darstellung der Urteilsbildung nach dem Linsenmodell (a). Nichtbeobachtbare Merkmale werden anhand beobachtbarer Signale beurteilt. Genaue Urteile resultieren bei der Verwendung valider Signale (1 und 4). Das valide Signal 3 wird nicht zur Urteilsbildung genutzt. Signal 4 zeigt keine Beziehung zum Merkmal, fließt aber in die Urteilsbildung mit ein. Anhand eines erweiterten Linsenmodells (b) lassen sich Signal-Merkmalsbeziehungen bei der Beurteilung unterschiedlicher Merkmale differenziert abbilden.

\subsection{Das Linsenmodell als Instrument der Kompetenzförderung}

In LEO erstellen angehende Biologielehrkräfte eigenständig Linsenmodelle, um anhand dieser den Prozess diagnostischer Urteilsbildung nachzuvollziehen. Im Vordergrund stehen dabei die Identifikation und Zuordnung valider Signale. Die Linsenmodelle können als visuell strukturierte Antwort auf die Frage „Woran genau habe ich das eigentlich erkannt?" verstanden werden. Durch die Verwendung der Linsenmodelle soll im Seminar eine de-automatisierte und reflektierte Betrachtung eigener Urteilsprozesse angeregt werden. Die Erweiterung bestehender Linsenmodelle (Nestler \& Back, 2013) ermöglicht eine differenzierte Betrachtung der Signal-Merkmalsbeziehungen bei der Beurteilung verschiedener Merkmale (vgl. Abb. 1b). Eine akkurate und separate Merkmalseinschätzung (Südkamp et al., 2018) setzt voraus, dass Signale entsprechend ihrer Validität ausschließlich als Indikatoren für das jeweilige Merkmal verwendet werden. In LEO stellt die Ausarbeitung eines Linsenmodells den Ausgangspunkt jeder geplanten diagnostischen 
Handlung dar. Im Vordergrund steht dabei weniger die Akkuratheit entstehender Modelle, sondern der Prozess der reflektierten Auseinandersetzung mit der diagnostischen Urteilsbildung selbst.

Die konsequente Verwendung des Linsenmodells als Instrument dient in LEO der Förderung diagnostischer Kompetenz in zweierlei Hinsicht. Zunächst fördert der strukturelle Aufbau des Linsenmodells eine reflektierte Auseinandersetzung mit der Beziehung von wahrnehmbaren Signalen und resultierenden Urteilen. Die Verwendung des Modells als Perspektive auf die eigene Wahrnehmung soll den Aufbau metakognitiven Wissens fördern, und zu einer reflexiven Distanzierung gegenüber der eigenen Wahrnehmung und diagnostischen Urteilsbildung anregen. Darüber hinaus fördert die aktive Konstruktion unterschiedlicher Linsenmodelle im Seminar die Vertiefung fachdidaktischer und fachlicher Wissensbestände, die nach dem NeDiKo-Modell Aspekte kognitiver Leistungsdispositionen und diagnostischer Kompetenz darstellen. Die Identifikation valider Signale im Konstruktionsprozess verlangt nach einer Integration beider Wissensbestände, die zur diagnostischen Beurteilung vorliegender Schülervorstellungen aufeinander bezogen werden müssen. Die vertiefte und strukturierte Auseinandersetzung mit SignalMerkmalsbeziehungen unter Rückgriff auf bestehende Fachliteratur soll damit den Aufbau strukturierten und vernetzten Wissens fördern. Diese Überlegungen schließen sich an Mamede et al. (2012) an, die eine Reorganisation und Integration von Wissensbeständen durch den Prozess strukturierter Reflexion beschreibt. Durch die strukturierte Visualisierung von Signal-Merkmalsbeziehungen weist der Aufbau eines Linsenmodells darüber hinaus Parallelen zum Concept Mapping auf, einer Methode, die als effektive Strategie zum Aufbau vernetzter Wissensbestände gilt (Hay, Kinchin \& Lygo-Baker, 2008; Nesbit \& Adesope, 2006). Der Einsatz externaler Visualisierungen komplexer Zusammenhänge scheint sich zudem förderlich auf den Prozess der Urteils- und Entscheidungsfindung im klinischen Kontext auszuwirken (Wu, Wang, Grotzer, Liu \& Johnson, 2016). Zusammenfassend wird das Linsenmodell in LEO als ein integratives Instrument verwendet, das zur Förderung diagnostischer Kompetenz durch die Vertiefung und Strukturierung fachlicher und fachdidaktischer Wissensbestände im Rahmen der Ausgestaltung eines diagnostischen Urteilmodels beitragen soll.

\subsection{LEO: Seminarstruktur und Ablauf}

Zum Zeitpunkt gegenwärtiger Entwicklung umfasst LEO drei Einheiten mit einem Gesamtumfang von 30 Semesterstunden. LEO richtet sich an angehende Biologielehrkräfte im Masterstudiengang. Das Lehr-Lern-Labor liegt zeitlich vor dem Praxissemester. Vor dem Hintergrund einer reflektierten Auseinandersetzung mit der eigenen diagnostischen Urteilsbildung und mit theoretischen Aspekten diagnostischen Handelns anhand der Arbeit mit Schülervideos und Schülertexten (Einheit 1) vollzieht sich in LEO eine Hinführung zur wiederholten reflektierten 
Praxiserfahrung (Einheit 2 und 3). Diese umfasst das Kennenlernen von Unterrichtsversuchen, die eigenständige Vorbereitung von Unterrichtseinheiten, die Durchführung der erarbeiteten Unterrichtseinheiten sowie die anschließende Reflexion individueller Erfahrungen (vgl. Abbildung 2). Ein besonderer Fokus liegt dabei auf der Diagnose bestehender Schülervorstellungen als Facette der Lernausgangslage, sowie der Überprüfung des Lernziels nach Durchführung der Unterrichtseinheit. Die modulare Struktur LEOs reflektiert unterschiedliche inhaltliche Schwerpunkte, die es ermöglichen, diagnostisches Handeln in verschiedenen Themenfeldern zu erproben. Damit werden den Studierenden multiple Kontexte zum Erwerb diagnostischer Kompetenz bereitgestellt, die angesichts der beschriebenen Situationsabhängigkeit diagnostischer Kompetenz die flexible Anwendung erworbenen Wissens fördern soll (Mandl, Gruber \& Renkl, 2002)

\subsubsection{Einheit 1: LEO-Evolution}

LEO-Evolution gliedert sich in eine Einführungsphase und eine Phase mit dem Schwerpunkt der Modellbildung und Reflexion, die im Seminarverlauf sukzessive ineinander übergehen.

Ziele der Einführungsphase sind der Erwerb deklarativen Wissens und die zunehmende Ausbildung eines Bewusstseins für Wahrnehmungs- und Urteilsprozesse (metakognitives Wissen). Beide Wissensarten lassen im NeDiKo-Modell als Elemente diagnostischer Kompetenz verorten.

Im Seminar beschäftigen sich die Studierenden mit der Relevanz diagnostischer Urteilsbildung für den Umgang mit Heterogenität und adaptiver Unterrichtsgestaltung, verschiedenen Modi und Zielen diagnostischer Urteilsbildung im Unterricht, dem diagnostischen Prozess und verschiedenen Bezugsnormen diagnostischer Urteilsbildung. Ein Fokus liegt auf der Betrachtung typischer Urteilsfehler und Wahrnehmungstendenzen wie bspw. dem Halo-Effekt (Thorndike, 1920) oder dem Einfluss implizierter Persönlichkeitstheorien (Rosenthal, Jacobson, Brinkmann \& Weinert, 1971) auf die Urteilsbildung. Im Vordergrund steht dabei nicht nur die theoretische Auseinandersetzung mit Urteilsfehlern, sondern auch die Frage danach, wo die eigene Wahrnehmung und Urteilsbildung solchen Urteilsfehlern und Tendenzen unterliegt.

Die Initiation von Reflexionsprozessen wird zu Beginn des Seminars durch die Verwendung zweier Videosequenzen unterstützt und folgt methodisch einer Variation der Methode „Predict-Observe-Explain“ (White \& Gunstone, 1992). Die eingesetzten Videos zeigen zwei inhaltlich inkonsistente Antworten eines Schülers auf unterschiedliche diagnostische Fragen zur Erfassung von Schülervorstellungen zur Evolution.

In einer sequentiellen Abfolge werden die Studierenden zunächst darum gebeten, Schülerantworten auf die gegebenen Fragestellungen begründet vorherzusagen und nach der Betrachtung der Videos offene Fragen zur vorliegenden Schülerant- 
wort zu beantworten. Die zugängliche Information zur Urteilsbildung stellt dabei zunächst ein Bild des Schülers sowie Alter und Klassenstufe dar. Im weiteren Verlauf liegt zusätzlich die Antwort des Schülers auf die erste Frage als diagnostische Information vor.

Sowohl Vorhersage der Schülerantwort als auch die Beantwortung der Fragestellung erfolgen schriftlich in Einzelarbeit und werden anhand eines Begleitbogens strukturiert. Der Vergleich von erwarteter und im Video gegebener Schülerantwort löst im Seminar ein zuverlässiges Überraschungsmoment aus. Die von den Studierenden erwartete Antwort unterscheidet sich oft deutlich von der gegebenen Antwort. Diese Diskrepanz wird als Reflexionsanlass (Schön, 1987) zur weiteren thematischen Vertiefung genutzt. Ein besonderer Fokus liegt dabei auf der Frage, auf welche Art und Weise die jeweils vorliegende Information die nachfolgende Erwartungshaltung beeinflusst hat.

Im weiteren Seminarverlauf dient die Arbeit mit dem Linsenmodell dazu, die Entwicklung eines reflektierten diagnostischen Blickes zu fördern. In den Phasen der Modellbildung und Reflexion erstellen die Studierenden Linsenmodelle zur Diagnose von Schülervorstellungen zur Evolution. Die Modellbildung erfolgt zunächst anhand vorliegender Schülertexte. Diese stellen authentische, handschriftliche Antworten auf verschiedene diagnostische Fragen zur Diagnose von Schülervorstellungen zur Evolution dar. Aufgabe ist es zunächst in den Texten Signale $\mathrm{zu}$ identifizieren, die für das Vorliegen einer bestimmten Vorstellung sprechen. Unter Rückgriff auf bestehende Fachliteratur (Hammann \& Asshoff, 2017) werden weitere Signale formuliert und in das Linsenmodell integriert. Das so entstehende Modell dient als Grundlage weiteren diagnostischen Handelns und wird im Seminarverlauf anhand der Arbeit mit Schülervideos getestet und weiterentwickelt.

Der Einsatz videografierter Schülerantworten schafft ein hohes $\mathrm{Maß}$ an $\mathrm{Au}$ thentizität und erlaubt die kontrollierte, standardisierte und wiederholte Analyse gegebener diagnostischer Information. Die verwendeten Videosequenzen unterscheiden sich hinsichtlich ihrer Länge, der enthaltenen Schülervorstellungen und der vorliegenden Signaldichte. Sie geben die Antworten einzelner oder mehrerer Schülerinnen und Schüler wieder, die sich in Alter, Geschlecht, Vorwissen und besuchter Schulform unterscheiden, und bilden ein breites Spektrum bekannter Vorstellungen zur Evolution ab.

Neben der Identifikation inhaltlicher Signale zur Diagnose vorliegender Schülervorstellungen dienen die Schülervideos der reflektierten Auseinandersetzung mit dem Einfluss weiterer Signalkategorien auf die diagnostische Urteilsbildung. Zur differenzierten Auseinandersetzung mit inhaltlichen, verbalen, paraverbalen und nonverbalen Signalen erfolgt im Seminar die isolierte Betrachtung unterschiedlicher Signalebenen. Dazu werden auf Grundlage einer verschriftlichten Schülerantwort bestehende Schülervorstellungen anhand vorliegender Signale diagnostiziert. Anschließend wird die Schülerantwort auditiv eingespielt, um eine fokussierte Auseinandersetzung mit verbalen und paraverbalen Signalen anzuregen. Es folgt die Beobachtung der Videosequenz unter Tonausschluss, um gestische 
und mimische Signale zur Verfügung zu stellen und anschließend eine integrierte Betrachtung aller Signalebenen der Videosequenz. Nach Einführung jeder neuen Signalebene steht die Frage im Vordergrund, wie sich die zusätzliche Information auf das diagnostische Urteil auswirkt.

\section{LEO: Das Lehr-Lernlabor der Biologiedidaktik Münster}

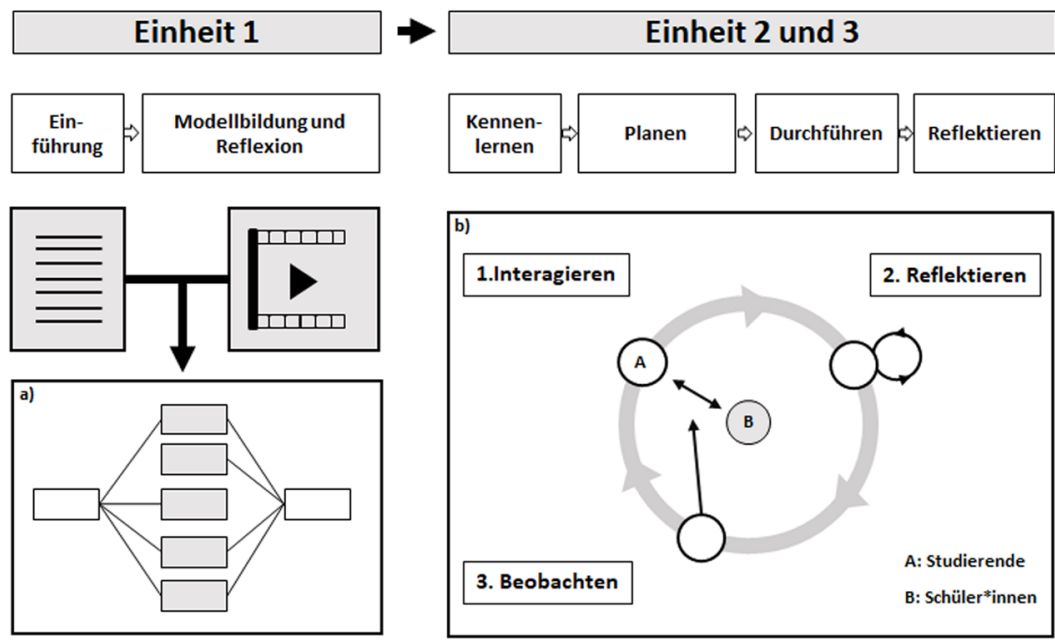

Abbildung 2: Strukturelle Darstellung LEO

LEO gliedert sich in drei Einheiten. Die erste Einheit dient der Einführung und Modellbildung. Im Seminar diagnostizieren die Studierenden Schülervorstellungen zur Evolution. Anhand vorliegender Schülertexte und Schülervideos werden dazu Signale identifiziert, unter Rückgriff auf bestehende Forschungsliteratur validiert und in ein Linsenmodell (a) integriert. Die Einheiten 2 und 3 dienen der Erprobung diagnostischen Handelns in der Interaktion mit Schülerinnen und Schülern. Im Seminar lernen die Studierende zwei Unterrichtsversuche kennen, bereiten in Teams zwei Unterrichtssequenzen vor, führen diese mit einer Gruppe von Schülerinnen und Schülern durch und reflektieren die Durchführung. Während der Durchführung nehmen die Studierenden unterschiedliche Rollen ein, in denen sie konkret mit den Schülerinnen und Schülern interagieren, ihre Erfahrung reflektieren oder die Interaktion ihrer Peers beobachten (b). 


\subsubsection{Einheiten 2 und 3: LEO-Neurobiology und LEO-Genetics}

Während in Einheit 1 einführend die Diagnose von Schülervorstellungen zur Evolution anhand von Schülertexten und Schülervideos im Vordergrund steht, erproben die Studierenden in Einheit 2 (Leo-Neurobiology) und in Einheit 3 (Leo-Genetics) diagnostisches Urteilen und Handeln im wiederholten Kontakt mit Schülerinnen und Schülern. Die beiden Einheiten folgen im Seminarverlauf sukzessive aufeinander und sind strukturgleich aufgebaut. Sie gliedern sich in insgesamt vier aufeinanderfolgende Phasen (vgl. Abb. 2).

In der ersten Phase steht das Kennenlernen eines Schulversuchs im Vordergrund. Dabei handelt es sich um ein Experiment zur Wirkung von Neuroenhancern (Koffein oder Nikotin) auf die Herzschlagfrequenz des Modellorganismus Großer Wasserfloh (Daphnia magna) (LEO-Neurobiology) und um einen Versuch zur Extraktion von DNA mittels Ethanol-Fällung (LEO-Genetics). Während der ersten Phase führen die Studierenden den jeweiligen Versuch unter Anleitung im Seminar durch.

Ein begleitender Fragebogen regt zur Auseinandersetzung mit eigenen Verständnisschwierigkeiten an und fordert dazu auf, mögliche Herausforderungen und Probleme in der konkreten Durchführung des Versuches mit Schülerinnen und Schülern zu identifizieren.

In der zweiten Phase planen die Studierenden in Teams eigenständig eine individuelle Unterrichtseinheit, die den jeweiligen Schulversuch sinnvoll rahmt und formulieren ein übergeordnetes Lernziel. Dies kann sich bei der Durchführung des Daphnienversuchs bspw. auf den Erwerb fachlichen Wissens zur neuronalen Signaltransduktion, die molekularen Mechanismen der Wirkung von Neuroenhancern oder auf die Förderung experimenteller Kompetenzen beziehen. Ausgehend von theoretischer Ausrichtung und Lernziel erarbeiten die Studierenden zwei Linsenmodelle zur Diagnose relevanten Vorwissens sowie bestehender Schülervorstellungen zu Beginn und zur Überprüfung des Lernziels nach Durchführung der Unterrichtseinheit. Die Linsenmodelle dienen dann der Auswahl geeigneter Erhebungsmethoden (bspw. diagnostische Fragestellungen, Lückentexte, ConceptMaps).

Die dritte Phase dient der Durchführung der vorbereiteten Unterrichtseinheiten mit Schülerinnen und Schülern einer Kooperationsschule. Orte der Durchführung sind zunächst die Kursräume des Zentrums für Didaktik der Biologie (Leo-Neurobiology). Die Durchführungsphase der zweiten Einheit (Leo-Genetics) vollzieht sich zur Maximierung der situativen Authentizität in den Biologieräumen der Kooperationsschule. Während der Durchführungsphase arbeiten die Studierendenteams mit einer Gruppe von Schülerinnen und Schülern.

Ziel ist die Durchführung der vorbereiteten Unterrichtseinheit sowie die individuelle, schülerbezogene Diagnose der Lernausgangslage und des Lernziels. Die Reflexion eigenen Handelns sowie die kritische Auseinandersetzung mit der entwickelten Unterrichtseinheit und mit den gewählten diagnostischen Strategi- 
en sind fester Bestandteil der Einheiten 2 und 3 in LEO und strukturell in der Durchführungsphase angelegt. Während der Durchführungsphase nehmen die Studierenden nacheinander unterschiedliche Rollen ein, indem sie entweder direkt mit den Schülerinnen und Schülern interagieren, die vorangegangene Interaktion reflektieren oder ihre Kommilitonen bei der Interaktion beobachten (vgl. Abb. 2). Die Ergebnisse der Reflexion und Beobachtung werden schriftlich mittels zuvor ausgearbeiteter Protokollbögen festgehalten und dienen als Grundlage der Auseinandersetzung mit den gemachten Praxiserfahrungen während der vierten Phase.

Die vierte Phase dient der Reflexion des vorangegangenen Schülerkontaktes. Im Seminar wird dabei der schrittweise Übergang von der Selbstreflexion zur Peerund Gruppenreflexion gestaltet. Gegenstände der Reflexion stellen Erfahrungen mit den Schülerinnen und Schülern und dem diagnostischen Handeln dar. Nach Schön (1987) vollzieht sich damit eine „reflexion on action“, die retrospektiv durch die vorliegenden Protokollbögen unterstützt wird. Auf Individualebene besteht dabei das Ziel, die Auseinandersetzung mit dem eigenen Handeln während der Interaktionsphase und der eigenen diagnostischen Einschätzung der Schülerinnen und Schülern anzuregen (Selbstreflexion). Dazu vollzieht sich der Reflexionsprozess anhand strukturierender Fragestellungen zunächst in Einzelarbeit. Die vorliegenden Selbstreflexionsbögen dienen dazu, zentrale Praxiserfahrungen herauszuarbeiten und sich reflexiv mit den eigenen diagnostischen Urteilen der individuellen Schülerinnen und Schüler auseinanderzusetzen. Die Frage: „Woran habe ich das eigentlich erkannt?" nimmt hier erneut einen zentralen Stellenwert ein. In einer sich anschließenden Gruppenphase werden die individuellen Erfahrungen behandelt. Die Fremdbeobachtungsbögen erlauben es an dieser Stelle den individuellen Bezugsrahmen um weitere Perspektiven zu ergänzen (Peer-Reflexion).

Zur kritischen Reflexion der ausgearbeiteten diagnostischen Strategien zur Erhebung von Vorwissen und Schülervorstellungen schätzen die Studierenden gemeinsam alle Schülerinnen und Schüler anhand der vorwissens- und lernzielbezogenen Dimensionen ein. Die diagnostische Urteilsbildung wird durch die Ergebnisse der gewählten Erhebungsmethoden und durch die protokollierten Beobachtungen informiert. Unter Rückgriff auf die ausgearbeiteten Linsenmodelle steht die Frage nach vorliegenden Signal-Merkmalsbeziehungen im Vordergrund. Ausgehend von der diagnostischen Abklärung des Lernziels und von den eigenen Erfahrungen beschäftigen sich die Studierenden in den Reflexionsphasen darüber hinaus kritisch mit der entworfenen Unterrichtssequenz. Wesentlich ist in Einheit 2 dabei die Frage, welche Aspekte bei der Planung der nächsten Unterrichtssequenz stärker berücksichtigt werden sollten. In Einheit 3 gilt es dann zu überlegen, ob es gelungen ist, die Unterrichtssequenz auf die Bedürfnisse der Lerngruppe zuzuschneiden. 


\subsubsection{LEO als Lehr-Lern-Labor: Überlegungen zur Komplexitätsreduktion}

LEO basiert instruktional auf den Prinzipien von Lehr-Lern-Laboren, die sich als Format ausgehend von der Integration bestehender Schülerlabore als außerschulische Lernorte in die universitäre Ausbildung angehender Lehrkräfte entwickelt haben (Haupt et al., 2013). Als zentraler Aspekt des Lehr-Lern-Labors kann die wiederholte und reflektierte Praxiserfahrung gelten (Dohrmann \& Nordmeier, 2015), die es Studierenden ermöglicht durch die Interaktion mit Schülerinnen und Schülern eigenes Handeln zu erproben und Theorie-Praxisbezüge aktiv herzustellen. Lehr-Lern-Labore können als situierte Instruktionsformen (Mandl et al., 2002) beschrieben werden, die den Erwerb anwendungsbezogenen Wissens in authentischen Situationen unterstützen. Die im Lehr-Lern-Labor gestalteten Situationen zeichnen sich dabei durch reduzierte Komplexität aus (Brüning, 2018). Während die Tendenz zur Komplexitätsreduktion auf Ebene der kognitiven Informationsverarbeitung ein zentrales Argument für die Bedeutung diagnostischer Kompetenz darstellt, dient die Reduktion von Komplexität im Lehr-Lern-Labor der Förderung des Lernprozesses (Dohrmann \& Nordmeier, 2015). In LEO erproben Studierende diagnostisches Handeln in unterschiedlichen unterrichtsrelevanten Situationen. Die Reduktion von Komplexität wird dabei durch verschiedene Maßnahmen realisiert, die im Folgenden auch unter Rückgriff auf das Modell der Komplexitätsreduktion in Lehr-Lern-Laboren (Marohn, Greefrath, Hammann, Hemmer, Kürten \& Windt, in diesem Band) dargestellt werden (vgl. Abb. 3).

Auf inhaltlicher Ebene erfolgt die Hinführung zur Thematik des diagnostischen Urteilens und Handelns ausgehend von der Auseinandersetzung mit ausgewählten Schülervorstellungen zur Evolution. Diese stellen eine kognitive Lernvoraussetzung dar und sind aus den einführenden Fachdidaktik-Veranstaltungen bekannt. Die Studierenden können so auf Vorwissen zurückgreifen. Die Konzentration auf ausgewählte Schülervorstellungen in einem Themengebiet stellt eine komplexitätsreduzierende Maßnahme dar, die den cognitive load beim Wissenserwerb verringert. Diagnostisches Urteilen und Handeln werden ferner anhand bereits verfügbarer diagnostischer Information in Form von Schülertexten oder Videos eingeübt. Damit entfallen zunächst die im diagnostischen Prozess vorgeschalteten Schritte, die der methodischen Sammlung von relevanter diagnostischer Information dienen. Im weiteren Seminarverlauf erhöht sich die Komplexität durch das Planen und Durchführen eigener Diagnoseprozesse. Damit steigt auch die Anzahl betrachteter Inhalte und Theorien (vgl. Abb. 3).

Der Einsatz von Schülervideos und Texten zu Beginn des Seminars erlaubt einen komplexitätsreduzierten und didaktisch kontrollierten Einstieg in die Thematik diagnostischen Urteilens und Handelns. Durch die Arbeit mit Videos und Schülertexten lässt sich die Reduktion von Komplexität in mehrerlei Hinsicht realisieren. Komplexität wird durch die gezielte Auswahl einzelner Videosequenzen reduziert, die sich in ihrer Länge, inhaltlichen Dichte oder Anzahl der interagierenden Schülerinnen und Schüler unterscheiden. In der praktischen Arbeit mit Schülervideos 
und Texten erweist sich die Möglichkeit zur Wiederholung als komplexitätsreduzierend. So lassen sich Texte mehrmals lesen und Schülervideos wiederholt betrachten. Gleichzeitig entfällt eine unmittelbare situative Handlungsaufforderung, die bspw. mit konkreten Interaktionssituationen einhergeht. Die Studierenden können sich so ganz auf die reflektierte Verarbeitung der jeweiligen Inhalte konzentrieren.

In LEO arbeiten die Studierenden in Teams und führen eigene Unterrichtseinheiten mit einer Gruppe von Schülerinnen und Schülern durch. Die Arbeit in Teams reduziert die Komplexität der Situation für den Einzelnen. Gleichzeitig müssen die Studierenden nicht den ganzen Klassenverband im Auge behalten und können sich verstärkt auf einzelne Schülerinnen und Schüler konzentrieren. Die Studierenden werden dabei sowohl während der Vorbereitung der einzelnen Unterrichtssequenzen als auch in den Reflexionsphasen unterstützt. Insgesamt ergibt sich damit eine hohe Betreuungsrelation (vgl. Abb. 3).

Darüber hinaus variiert in LEO die Vertrautheit des Settings zur Unterstützung des Lernprozesses. In LEO erproben die Studierenden diagnostisches Urteilen und Handeln im wiederholten Kontakt mit Schülerinnen und Schülern. Der erste Kontakt mit Schülerinnen und Schülern (Durchführung, Einheit 2) findet am Zentrum für Didaktik der Biologie statt. Dies hat sich als sinnvoll erweisen, da die erste Praxiserfahrung bei den Studierenden auch mit einem Gefühl der Unberechenbarkeit einhergeht. Dieses Gefühl scheint aus einer fehlenden Praxisroutine einerseits sowie der Unbekanntheit der Schülergruppe andererseits zu resultieren. Die Arbeit in einer für die Studierenden vertrauten räumlichen Umgebung am Zentrum für Didaktik vermeidet an dieser Stelle weitere Komplexität, die mit unbekannten Räumlichkeiten einhergehen würde. Die kontextuelle Sicherheit ergibt sich dabei aus Faktoren, die die Wahrnehmung von Planbarkeit und situativer Kontrolle erhöhen. So sind die Studierenden mit den Räumlichkeiten vertraut und wissen bspw., wo die Mikroskope und Materialien zu finden sind. Während des ersten Kontakts besteht damit eine hohe Vertrautheit des Settings, bei gleichzeitiger Unbekanntheit der Schülerinnen und Schüler. Der zweite Kontakt mit Schülerinnen und Schülern (Durchführung, Einheit 3) findet in der Schule statt. Während der Arbeit mit Schülerinnen und Schülern können die Studierenden auf reflektierte Vorerfahrungen zurückgreifen und das eigene pädagogische Handeln zielgruppenspezifisch ausrichten. Das Klassenzimmer ermöglicht als Ort der Begegnung größtmögliche kontextuelle Authentizität. Gleichzeitig stellt es die Studierenden aber hinsichtlich der Planung und Vorbereitung vor neue Herausforderungen. Im Verlauf des Lehr-Lern-Labors LEO vollzieht sich damit eine Reduktion von Komplexität auf sozialer Ebene, während die räumliche Vertrautheit des Settings abnimmt. Dabei scheint es den Studierenden leichter zu fallen, mit der Unbekanntheit der Lernumgebung des Klassenzimmers umzugehen als mit der Unbekanntheit der sozialen Situation. 


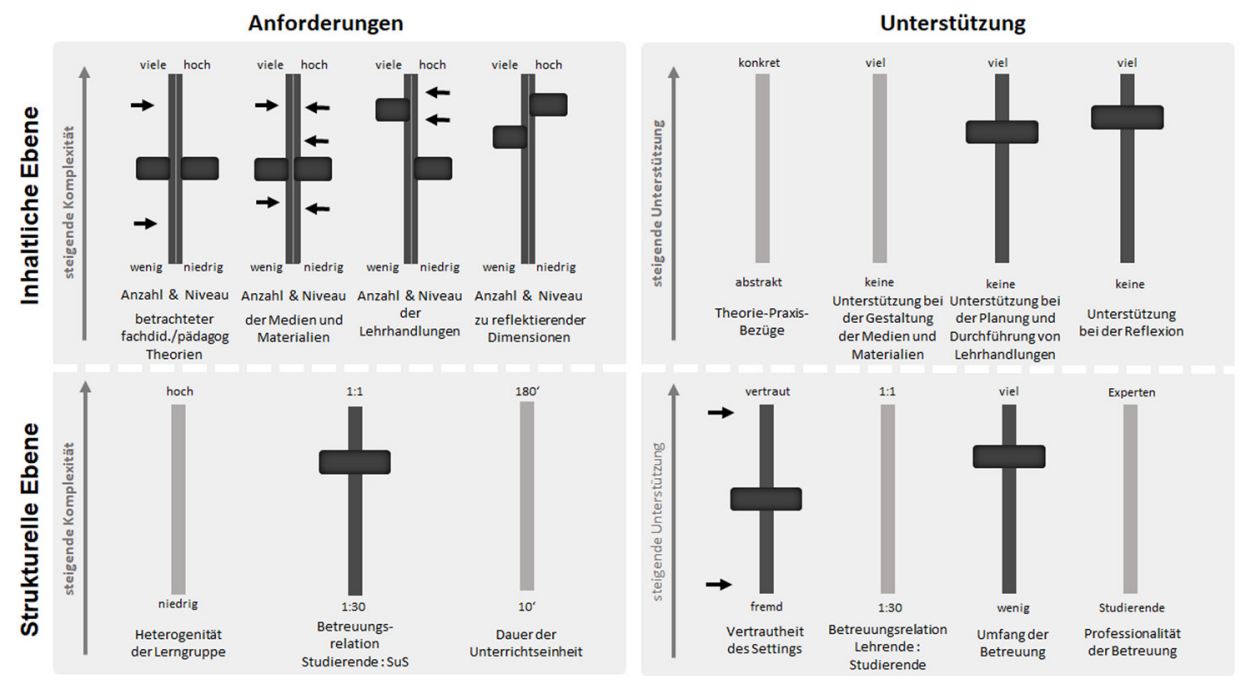

Abbildung 3: Komplexitätsreduktion in LEO

Darstellung ausgewählter Dimensionen der Komplexitätsreduktion in LEO nach dem Modell der Komplexitätsreduktion in Lehr-Lern-Laboren (Marohn et al., in diesem Band). Die Pfeile sollen die Dynamik des Seminarverlaufs verdeutlichen, in dessen Zuge betrachtete Theorien, die Verwendung von Medien und Materialien, Anzahl und Niveau der Lehrerhandlungen, Anzahl und Niveau der zu reflektierenden Dimensionen sowie die Vertrautheit des Settings variieren. Als eher stabil können die im Seminar gewährleistete Unterstützung bei der Ausarbeitung und Durchführung der Lehrerhandlung, sowie die Unterstützung bei der Reflexion und der Umfang der Betreuung gelten. Auf struktureller Ebene ergibt sich die Betreuungsrelation von Studierenden und Schülerinnen und Schülern aus den jeweiligen Klassen und Kursgrößen, kann aber als eher hoch beschrieben werden.

\section{LEO: Entwicklung und Evaluation}

LEO zielt darauf ab, Studierende bei der Entwicklung eines reflektierten diagnostischen Blickes zu unterstützten und auf den Umgang mit Heterogenität vorzubereiten. Das Seminarformat wird dabei kontinuierlich evaluiert und entwickelt. Exemplarisch werden an dieser Stelle ausgewählte Ergebnisse einer ersten Pilotierungsphase kurz dargestellt. Während der Pilotierungsphase wurde LEO-Evolution (Einheit 1) an zwei Terminen mit einem Umfang von insgesamt 8 Stunden in ein bestehendes Seminarkonzept mit einem Umfang von insgesamt 9 Kurstagen integriert, in dem die praxisnahe Auseinandersetzung mit Unterrichtsversuchen (UV) im Vordergrund steht. Die Stichprobe umfasst insgesamt 85 Studierende $(58$ weiblich), die das Seminar in vier Kursen $(20 \leq n \leq 23)$ am Zentrum für Didaktik der Biologie durchliefen. Die Pilotierung folgte einem Switching-Replications-Design 
(Kline, 2008), bei dem der Zeitpunkt der beiden LEO-Kurstage im Seminarverlauf für die Hälfte der Kurse variierte (vgl. Abbildung $4 \mathrm{~A}$ und C).

Nach den einzelnen Seminarsitzungen $(\mathrm{N}=9)$ wurden die Studierenden darum gebeten, verschiedene Seminarparameter anhand einer kurzen Fragebogenbatterie auf einer siebenstufigen Likert-Skala einzuschätzen. Hohe Skalenwerte beschreiben eine Zustimmung der Studierenden. Die Antworten der Studierenden weisen darauf hin, dass die LEO-Seminare unabhängig vom Zeitpunkt der Durchführung oder dem jeweiligen Kurs deutlich mit der Förderung diagnostischer Kompetenz assoziiert werden (vgl. Abbildung 4 A). Übergreifend zeigt sich ein signifikanter Effekt des Kursinhaltes ((LEO vs. UV) $\left.F(1,81)=617.68, p<.001, \eta^{2}=.76\right)$ auf die Einschätzung der Studierenden (vgl. Abbildung 4 B). Ein Einfluss der Kurszugehörigkeit (Kurs 1-4) auf das Antwortverhalten lässt sich dagegen nicht verzeichnen $(F(3,81)=1.34, p=.27)$. Der leichte Peak zum Zeitpunkt der zweiten Seminareinheit (vgl. Abbildung 4 A) reflektiert inhaltlich die Analyse von Schülererzeugnissen während der Seminareinheit.

Der Bezug zur Thematik der Heterogenität wird in den LEO-Seminaren $(M=$ 5.55, $S D=1.21$ ) insgesamt als signifikant ausgeprägter wahrgenommen als in den UV-Seminaren $\left(M=2.23 S D=1.08, F(1,81)=440.66, p<.001, \eta^{2}=.68\right.$, vgl. Abbildung 4 D). Obwohl sich über den Verlauf der beiden LEO-Einheiten kursübergreifend ein leichter Rückgang des wahrgenommenen Heterogenitätsbezugs verzeichnen lässt, besteht weiterhin ein deutlicher Unterschied zwischen den LEO- und UV-Seminaren (vgl. Abbildung 4 C). Ein genereller Einfluss der Kurszugehörigkeit auf die Einschätzung des Heterogenitätsbezuges scheint nicht zu bestehen $(F(3$, $81)=1.63, \mathrm{p}=.64$ ). Nach Angabe der Studierenden unterstützen die LEO-Seminare den Erwerb von Strategien zur reflektierten Urteilsbildung (vgl. Abbildung 4 E) deutlich stärker $(M=6.21 S D=0.81)$ als die UV-Seminare $(M=2.15 S D=0.94, F(1$, $\left.84)=1260.04, p<.001, \eta^{2}=.84\right)$. Darüber hinaus stimmen $88 \%$ der Studierenden der Aussage zu (Rating $>4$ ), dass die LEO-Seminare zur Selbstreflexion anregen $(M=5.58 S D=1.14)$ (vgl. Abbildung $4 \mathrm{~F})$. Auch wenn sich an dieser Stelle ein signifikanter Unterschied zwischen den LEO und den UV-Seminaren $(M=3.85 S D=$ 1.26) zeigt $\left(F(1,84)=158.93, p<.001, \eta^{2}=.35\right)$, lässt sich eine im Vergleich zu den übrigen Parametern etwas geringer ausfallende Effektstärke verzeichnen. Die während der Pilotierungsphase erhobenen Daten weisen insgesamt darauf hin, dass sich die LEO-Einheiten in ihrer thematischen Orientierung stark von den übrigen Seminaren unterscheiden. Insgesamt attestieren Studierende den LEO-Seminaren einen vergleichsweise hohen Bezug zur Thematik der Heterogenität und verschiedenen Aspekten diagnostischer Kompetenz. Den Einschätzungen der Studierenden nach scheinen die LEO-Seminare damit einen Beitrag zur Entwicklung eines reflektierten diagnostischen Blickes zu leisten. Im Verlauf der weiteren Entwicklung von LEO dienten diese ersten Ergebnisse dazu, verschiedene Aspekte der LEOEinheiten weiterzuentwickeln, um etwa den Bezug zur Selbstreflexion in den LEOEinheiten noch stärker zu akzentuieren. 
A Förderung diagnostischer kompetenz

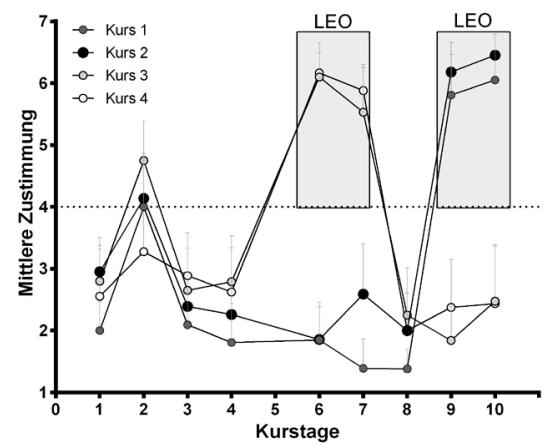

C

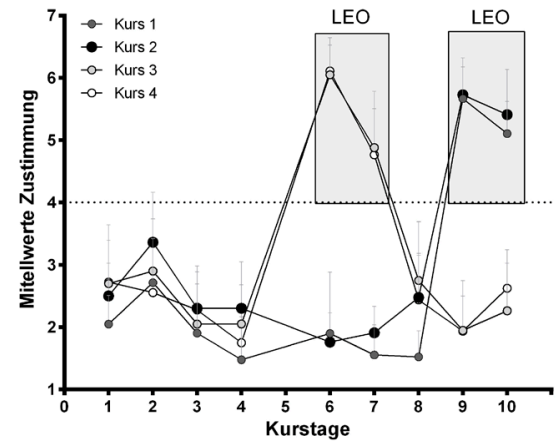

E

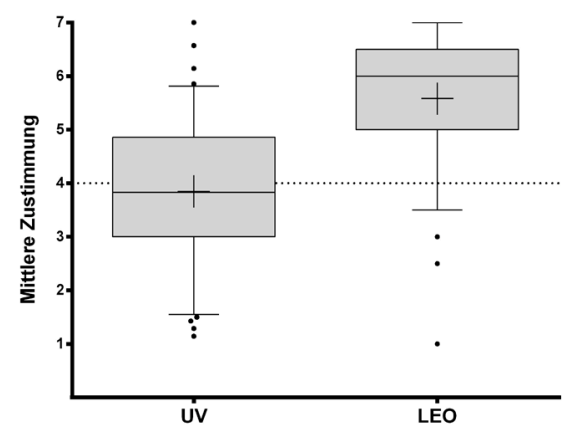

B Förderung diagnostischer Kompetenz

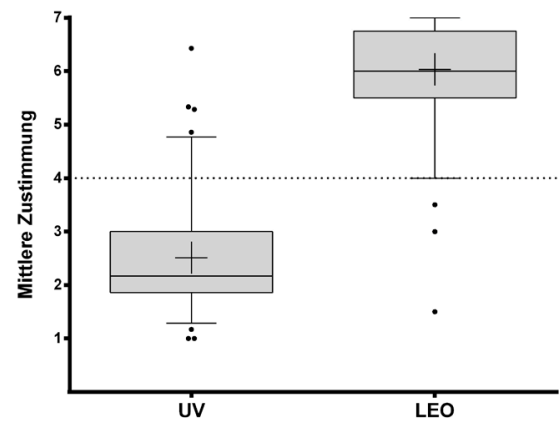

D

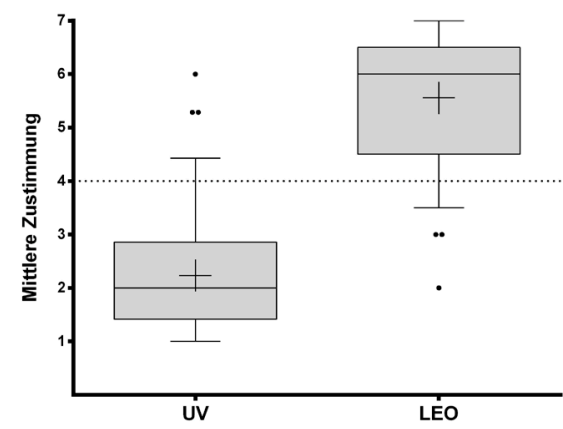

F Strategien reflektierte Urteilsbildung

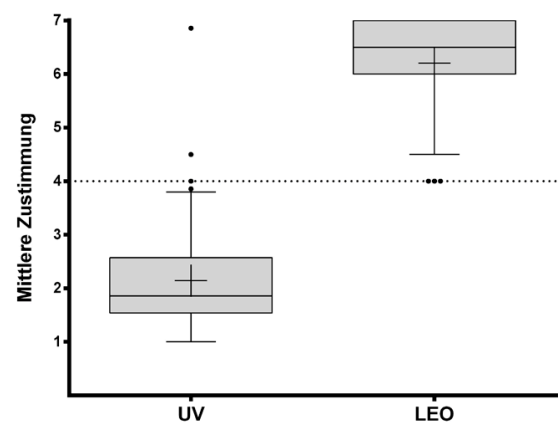

Abbildung 4: Ausgewählte Ergebnisse der Pilotierungsphase

Grafische Darstellung der Einschätzung verschiedener Parameter durch Studierende separiert nach Kurs und Seminarsitzung (A \& C) oder aggregiert nach inhaltlicher Kategorie (B, D, E \& F). Hohe Skalenwerte repräsentieren eine hohe wahrgenommene Ausprägung der entsprechenden Parameter. Die Fehlerbalken innerhalb der Zeitreihen (A \& C) geben das 95\%-Konfidenzintervall an, die Zeitpunkte der LEO-Durchführung sind grau hinterlegt. Die Whisker der Box Plots indizieren den 5-95 Perzentilrange, der Median wird durch einen horizontalen Balken, das arithmetische Mittel durch ein Kreuz angegeben. 


\section{LEO: Resumé und Ausblick}

Mit LEO entstand im Kontext der Qualitätsoffensive Lehrerbildung „Dealing with Diversity“ erstmals ein Lehr-Lern-Labor am Zentrum für Didaktik der Biologie der WWU Münster. LEO zielt darauf $a b$, angehende Biologielehrkräfte auf den Umgang mit Heterogenität durch reflektierte Praxiserfahrung vorzubereiten. Dabei steht die Förderung diagnostischer Kompetenz im Vordergrund, die als Voraussetzung für Individualisierung und adaptive Unterrichtsgestaltung gelten kann. Instruktional ist LEO als Lehr-Lern-Labor gestaltet und folgt einem multimodalen Ansatz. In LEO arbeiten Studierende mit Schülervideos, Schülertexten und erproben diagnostisches Handeln im direkten und wiederholten Kontakt mit Schülerinnen und Schülern. Der Lernprozess wird dabei durch verschiedene Maßnahmen zur Reduktion von Komplexität unterstützt. Während die Reduktion von Komplexität ein zentrales Kriterium von Lehr-Lern-Laboren darstellt, nimmt sie in LEO eine zweifache Funktion ein und dient gleichermaßen didaktischen Zielen sowie einer theoretischen und methodischen Begründung des entwickelten Konzeptes. Aus Perspektive der kognitiven Informationsverarbeitung lässt sich die Reduktion von Komplexität durch heuristische Informationsverarbeitung mit Urteilsfehlern und Wahrnehmungsverzerrungen assoziieren. Dem folgend greift LEO verschiedene theoretische Überlegungen zur Förderung diagnostischer Kompetenz auf. Die Entwicklung eines reflektierten diagnostischen Blickes soll dabei maßgeblich durch strukturelle De-Automatisierung und Förderung einer reflexiven Distanz zur eigenen Urteilsbildung unterstützt werden. Methodisch finden diese Überlegungen Ausdruck in der Verwendung des Brunswikschen Linsenmodells, das als Perspektive auf den diagnostischen Prozess und Instrument im Seminar Verwendung findet. Erste Pilotierungsergebnisse verweisen darauf, dass LEO in thematischer Ausrichtung und inhaltlicher Akzentuierung das Spektrum bestehender Seminare ergänzt. Entwicklungsmöglichkeiten bestehen in der zunehmenden Spezifizierung und Konkretisierung diagnostischer Handlungen, in der stärkeren Vernetzung evolutionsbiologischer und genetischer Seminarinhalte sowie in der zunehmenden Realisierung von Digitalisierungsaspekten im Seminarverlauf.

\section{Literatur}

Baumert, J. \& Kunter, M. (2006). Stichwort: Professionelle Kompetenz von Lehrkräften. Zeitschrift Für Erziehungswissenschaft, 9 (4), 469-520. https://doi.org/10.1007/s11618oo6-0165-2

Beck, E. (Hrsg.) (2008). Adaptive Lehrkompetenz: Analyse und Struktur, Veränderbarkeit und Wirkung handlungssteuernden Lehrerwissens. (Pädagogische Psychologie und Entwicklungspsychologie, Bd. 63.) Münster: Waxmann.

Bennett, R. E. (2011). Formative assessment: a critical review. Assessment in Education: Principles, Policy \& Practice, 18 (1), 5-25. https://doi.org/10.1080/096959 4X.2010.513678 
Black, P. \& Wiliam, D. (2009). Developing the theory of formative assessment. Educational Assessment, Evaluation and Accountability, 21 (1), 5-31. https://doi.org/10.1007/ s11092-008-9068-5

Bromme, R. (1997). Kompetenzen, Funktionen und unterrichtliches Handeln des Lehrers. In F.E. Weinert (Hrsg.), Psychologie des Unterrichts und der Schule (S. 177-212). Göttingen: Hogrefe.

Brüning, A.-K. (2018). Das Lehr-Lern-Labor „Mathe für kleine Asse“: Untersuchungen zu Effekten der Teilnahme auf die professionellen Kompetenzen der Studierenden (Schriften zur mathematischen Begabungsforschung: Vol. 10.). Münster: WTM-Verlag.

Brunner, M., Anders, Y., Hachfeld, A. \& Krauss, S. (2011). Diagnostische Fähigkeiten von Mathematiklehrkräften. In M. Kunter, J. Baumert, W. Blum, U. Klusmann, S. Krauss \& M. Neubrand (Hrsg.), Professionelle Kompetenz von Lehrkräften - Ergebnisse des Forschungsprogramms COACTIV (S. 215-234). Münster: Waxmann.

Brunswik, E. (1955). Representative design and probabilistic theory in a functional psychology. Psychological Review, 62 (3), 193-217. https://doi.org/10.1037/hoo47470

Cantor, N. \& Mischel, W. (1977). Traits as prototypes: Effects on recognition memory. Journal of Personality and Social Psychology, 35 (1), 38-48. https://doi.org/10.1037/ 0022-3514.35.1.38

Dohrmann, R. \& Nordmeier, V. (2015). Schülerlabore als Lehr-Lern-Labore (LLL): Ein Projekt zur forschungsorientierten Verknüpfung von Theorie und Praxis in der MINTLehrerbildung. Vortrag auf der Frühjahrstagung der Didaktik der Physik, Wuppertal.

Dunn, K. E. \& Mulvenon, S. W. (2009). A critical review of research on formative assessment: The limited scientific evidence of the impact of formative assessment in education. Practical Assessment, Research and Evaluation, (14), 1-11.

Evans, J. S. B. T. \& Stanovich, K. E. (2013). Dual-Process Theories of Higher Cognition: Advancing the Debate. Perspectives on Psychological Science: a Journal of the Association for Psychological Science, 8 (3), 223-241. https://doi.org/10.1177/1745691612460685

Ferreira, M. B., Garcia-Marques, L., Sherman, S. \& Sherman, J. (2006). Automatic and controlled components of judgment and decision making. Journal of Personality and Social Psychology, 91 (5), 797-813. https://doi.org/10.1037/0022-3514.91.5.797

Förster, N. \& Böhmer, I. (2017). Das Linsenmodell - Grundlagen und exemplarische Anwendungen in der pädagogisch-psychologischen Diagnostik. In A. Südkamp \& A. K. Praetorius (Hrsg.), Diagnostische Kompetenz von Lehrkräften. Theoretische und methodische Weiterentwicklungen (S. 46-50). Münster: Waxmann.

Gawronski, B. \& Creighton, L. A. (2013). Dual process theories. In D. E. Carlston (Hrsg.), Oxford library of psychology. The Oxford handbook of social cognition (S. 282312). New York: Oxford University Press. https://doi.org/10.1093/oxfordhb/978019 9730018.013.0014

GFD. (2005). Fachdidaktische Kompetenzbereiche, Kompetenzen und Standards für die 1. Phase der Lehrerbildung. Abgerufen von http://www.fachdidaktik.org/cms/download. php?cat=40_Ver\%C3\%B6ffentlichungen \&file=Publikationen_zur_LehrerbildungAnlage_1.pdf

Gigerenzer, G. \& Goldstein, D. G. (1996). Reasoning the fast and frugal way: models of bounded rationality. Psychological Review, 103 (4), 650-669. https://doi.org/10.1037/ 0033-295X.103.4.650

Hammann, M. \& Asshoff, R. (2017). Schülervorstellungen im Biologieunterricht: Ursachen für Lernschwierigkeiten (3. Auflage). Seelze: Klett/Kallmeyer. 
Haupt, O. J., Domjahn, J., Martin, U., Skiebe-Corrette, P., Vorst, S., Zehren, W., \& Hempelmann, R. (2013). Schülerlabor - Begriffsschärfung und Kategorisierung. Der mathematische und naturwissenschaftliche Unterricht, 66(6), 324-330.

Hay, D., Kinchin, I. \& Lygo-Baker, S. (2008). Making learning visible: the role of concept mapping in higher education. Studies in Higher Education, 33 (3), 295-311. https://doi. org/10.1080/03075070802049251

Helmke, A. (2009). Unterrichtsqualität und Lehrerprofessionalität: Diagnose, Evaluation und Verbesserung des Unterrichts; Franz Emanuel Weinert gewidmet. Stuttgart, SeelzeVelber: Klett; Klett Kallmeyer. Abgerufen von http://www.socialnet.de/rezensionen/ isbn.php?isbn=978-3-7800-1009-4

Helmke, A. (2013). Individualisierung: Hintergrund, Missverständnisse, Perspektiven. PÄDAGOGIK, (2), 34-37.

Herppich, S., Praetorius, A.-K., Hetmanek, A., Glogger-Frey, I., Ufer, S., Leutner, D., ... Südkamp, A. (2017). Ein Arbeitsmodell für die empirische Erforschung der diagnostischen Kompetenz von Lehrkräften. In A. Südkamp \& A. K. Praetorius (Hrsg.), Diagnostische Kompetenz von Lehrkräften. Theoretische und methodische Weiterentwicklungen (S. 75-93). Münster: Waxmann.

Hesse, I. \& Latzko, B. (2011). Diagnostik für Lehrkräfte (2. Auflage). Opladen: Verlag Barbara Budrich (UTB).

Hetmanek, A. \& van Gog, T. (2017). Förderung diagnostischer Kompetenz: Potenziale von Ansätzen aus der medizinischen Forschung. In A. Südkamp \& A. K. Praetorius (Hrsg.), Diagnostische Kompetenz von Lehrkräften. Theoretische und methodische Weiterentwicklungen (S. 209-216). Münster: Waxmann.

Judd, C. M. \& Kulik, J. A. (1980). Schematic effects of social attitudes on information processing and recall. Journal of Personality and Social Psychology, (38), 569-578. https://doi.org/10.1037//o022-3514.38.4.569

Kahneman, D. (2011). Thinking, fast and slow (First edition). New York: Farrar Straus and Giroux.

Kahneman, D. \& Frederick, S. (2012). Representativeness Revisited: Attribute Substitution in Intuitive Judgment. In T. Gilovich, D. Griffin \& D. Kahneman (Hrsg.), Heuristics and Biases (S. 49-81). Cambridge University Press. https://doi.org/10.1017/ CBO9780511808098.004

Karst, K. (2017). Akkurate Urteile - die Ansätze von Schrader (1989) und McElvany et al. (2009). In A. Südkamp \& A. K. Praetorius (Hrsg.), Diagnostische Kompetenz von Lehrkräften. Theoretische und methodische Weiterentwicklungen (S. 21-29). Münster: Waxmann.

Kline, R. B. (2008). Becoming a Behavioral Science Researcher: A Guide to Producing Research That Matters: Guilford Publications. Abgerufen von https://books.google.de/ books?id=ppJg1QIncRYC

Klug, J., Bruder, S., Kelava, A., Spiel, C. \& Schmitz, B. (2013). Diagnostic competence of teachers: A process model that accounts for diagnosing learning behavior tested by means of a case scenario. Teaching and Teacher Education, 30, 38-46. https://doi. org/10.1016/j.tate.2012.10.004

Klug, J., Bruder, S., Keller, S. \& Schmitz, B. (2012). Hängen Diagnostische Kompetenz und Beratungskompetenz von Lehrkräften zusammen? Psychologische Rundschau, 63 (1), 3-10. https://doi.org/10.1026/0033-3042/a0oo104 
KMK. (2004). Sekretariat der Ständigen Konferenz der Kultusminister der Länder in der Bundesrepublik Deutschland. Standards für die Lehrerbildung. Bildungswissenschaften. Beschluss der KMK vom 16.12.2004. Abgerufen von http://www.kmk.org/fileadmin/ Dateien/veroeffentlichungen_beschluesse/2004/2004

Krauss, S., Kunter, M., Brunner, M., Baumert, J., Blum, W., Neubrand, M., ... Löwen, K. (2004). COACTIV: Professionswissen von Lehrkräften, kognitiv aktivierender Mathematikunterricht und die Entwicklung von mathematischer Kompetenz. In J. Doll \& M. Prenzel (Hrsg.), Bildungsqualität von Schule: Lehrerprofessionalisierung, Unterrichtsentwicklung und Schülerförderung als Strategien der Qualitätsverbesserung (S. 31-53). Münster: Waxmann.

Krolak-Schwerdt, S., Böhmer, M. \& Gräsel, C. (2009). Verarbeitung von schülerbezogener Information als zielgeleiteter Prozess. Zeitschrift Für Pädagogische Psychologie, 23 (34), 175-186. https://doi.org/10.1024/1010-0652.23.34.175

Krolak-Schwerdt, S., Böhmer, M. \& Gräsel, C. (2013). The impact of accountability on teachers' assessments of student performance: a social cognitive analysis. Social Psychology of Education, 16 (2), 215-239. https://doi.org/10.1007/s11218-013-9215-9

Kunter, M., Klusmann, U. \& Baumert, J. (2009). Professionelle Kompetenz von Mathematiklehrkräften: Das COACTIV-Modell. In O. Zlatkin-Troitschanskaia, K. Beck, D. Sembill, R. Nickolaus \& R. Mulder (Hrsg.), Lehrprofessionalität - Bedingungen, Genese, Wirkungen und ihre Messung (S. 153-165). Weinheim: Beltz.

Mamede, S., van Gog, T., Moura, A. S., Faria, R. M. D. de, Peixoto, J. M., Rikers, R. M. J. P. \& Schmidt, H. G. (2012). Reflection as a strategy to foster medical students' acquisition of diagnostic competence. Medical Education, 46 (5), 464-472. https://doi. $\operatorname{org} / 10.1111 /$ j.1365-2923.2012.04217.X

Mandl, H., Gruber, H. \& Renkl, A. (2002). Situiertes Lernen in multimedialen Lernumgebungen. In L. J. Issing \& P. Klimsa (Hrsg.), Information und Lernen mit Multimedia und Internet: Lehrbuch für Studium und Praxis (3. Auflage, S. 138-148). Weinheim: Beltz PVU.

Nesbit, J. C. \& Adesope, O. O. (2006). Learning With Concept and Knowledge Maps: A Meta-Analysis. Review of Educational Research, 76 (3), 413-448. https://doi. org/10.3102/00346543076003413

Nestler, S. \& Back, M. D. (2013). Applications and Extensions of the Lens Model to Understand Interpersonal Judgments at Zero Acquaintance. Current Directions in Psychological Science, 22 (5), 374-379. https://doi.org/10.1177/0963721413486148

Ohle, A., McElvany, N., Horz, H. \& Ullrich, M. (2015). Text-picture integration. Teachers' attitudes, motivation and self-related cognitions in diagnostics. Journal for Educational Research Online. 7 (2), 5-10.

Paradies, L., Linser, H. J. \& Greving, J. (2009). Diagnostizieren, Fordern und Fördern (3. Aufl.). Berlin: Cornelsen Scriptor.

Rosenthal, R., Jacobson, L., Brinkmann, I. \& Weinert, F. (1971). Pygmalion im Unterricht: Lehrererwartungen und Intelligenzentwicklung der Schüler. Beltz-Studienbuch. Weinheim: Beltz.

Schmidt-Atzert, L. \& Amelang, M. (2012). Diagnostische Verfahren. In L. Schmidt-Atzert \& M. Amelang (Hrsg.), Psychologische Diagnostik (S. 175-357). Berlin: Springer. https://doi.org/10.1007/978-3-642-17001-0_3 
Schön, D. A. (1987). Jossey-Bass higher education series. Educating the reflective practitioner: Toward a new design for teaching and learning in the professions. San Francisco, CA, US: Jossey-Bass.

Schrader, F.-W. (1989). Diagnostische Kompetenzen von Lehrern und ihre Bedeutung für die Gestaltung und Effektivität des Unterrichts. Frankfurt a.M: Lang.

Schrader, F.-W. \& Helmke, A. (1987). Diagnostische Kompetenz von Lehrern: Komponenten und Wirkungen. Empirische Pädagogik (1), 27-52.

Shulman, L. (1986). Those who understand: Knowledge growth in teaching. Educational Researcher. (15(2)), 4-14. https://doi.org/10.3102/0013189X015002004

Shulman, L \& Shulman, J. (2004). How and what teachers learn: a shifting perspective. Journal of Curriculum Studies, 36 (2), 257-271. https://doi.org/10.108o/ 0022027032000148298

Südkamp, A. \& Möller, J. (2009). Referenzgruppeneffekte im Simulierten Klassenraum. Zeitschrift Für Pädagogische Psychologie, 23(34), 161-174. https://doi.org/10.1024/10100652.23.34.161

Südkamp, A., Praetorius, A.-K. \& Spinath, B. (2018). Teachers' judgment accuracy concerning consistent and inconsistent student profiles. Teaching and Teacher Education, 76, 204-213. https://doi.org/10.1016/j.tate.2017.09.016

Terhart, E. (2010). Heterogenität der Schüler - Professionalität der Lehrer: Ansprüche und Wirklichkeiten: Nachtrag 2014. In S. L. Ellger-Rüttgardt \& G. Wachtel (Hrsg.), Pädagogische Professionalität und Behinderung: Herausforderungen aus historischer, nationaler und internationaler Perspektive (S. 89-104). Stuttgart: Kohlhammer Verlag.

Thorndike, E. L. (1920). A constant error in psychological ratings. Journal of Applied Psychology, 4 (1), 25-29. https://doi.org/10.1037/hoo71663

Vock, M. \& Gronostaj, A. (2017). Umgang mit Heterogenität in Schule und Unterricht (Schriftenreihe des Netzwerk Bildung, Bd. 40.2). Berlin: Friedrich-Ebert-Stiftung Abt. Studienförderung.

Weinert, F. (2000). Lehren und Lernen für die Zukunft - Ansprüche an das Lernen in der Schule. Pädagogische Nachrichten Rheinland-Pfalz (2), 1-16.

Weinert, F. \& Schrader, F.-W. (1986). Diagnose des Lehrers als Diagnostiker. In H. Petillon, J. W. L. Wagner \& B. Wolf (Hrsg.), Schülergerechte Diagnose. Theoretische und empirische Beiträge zur Pädagogischen Diagnostik. Festschrift zum 6o. Geburtstag von Karlheinz Ingenkamp (S. 11-29). Weinheim: Beltz.

White, R. \& Gunstone, R. (1992). Probing understanding. New York: The Falmer Press.

Wu, B., Wang, M., Grotzer, T. A., Liu, J. \& Johnson, J. M. (2016). Visualizing complex processes using a cognitive-mapping tool to support the learning of clinical reasoning. BMC Medical Education, 16 (1), 216. https://doi.org/10.1186/s12909-016-0734-X 


\title{
Professionalisierung der Unterrichtswahrnehmung mithilfe von Videovignetten im Themenfeld Evolution
}

\author{
Bearbeitung der Sachantinomie in der biologiedidaktischen \\ Lehrerbildung
}

Aus einer biologiedidaktischen Perspektive ist die Reflexion des Verhältnisses von fachlicher Norm und Schülervorstellungen von besonderer Bedeutung für die Lehrerbildung. Im Beitrag wird dieses Spannungsfeld in strukturtheoretischen Ansätzen von Lehrerprofessionalität verortet. Demnach ist der Umgang von Lehrkräften mit professionsspezifischen Antinomien ein zentrales Merkmal von Professionalität, wobei im Projekt die Sachantinomie aus einer fachdidaktischen Perspektive fokussiert wird. Ein Ziel der Lehrerbildung ist die Professionalisierung der Unterrichtswahrnehmung bezüglich eines angemessenen Umgangs mit der Vielfalt individueller Vorstellungen. Im Beitrag werden Perspektiven aufgezeigt, wie in einem fallrekonstruktiven Seminar die Sachantinomie vor dem Hintergrund des impliziten Wissens der Studierenden mithilfe von Videovignetten aus dem Unterricht im Themenfeld Evolution bearbeitet werden kann. Dabei wird diskutiert, wie im Seminar mit der Komplexität von Unterricht umgegangen werden kann und authentische Lernangebote für Studierende entwickelt werden können.

Schlüsselwörter: Sachantinomie, Schülervorstellungen, Unterrichtswahrnehmung

\section{Einleitung}

Das Teilprojekt „Lehr-Lern-Labore, Lernwerkstätten und Learning-Center“ der Qualitätsoffensive Lehrerbildung an der Universität Münster hat sich u.a. zum Ziel gesetzt, für Lehramtsstudierende komplexitätsreduzierte und authentische Lernangebote $\mathrm{zu}$ entwickeln, in denen sie eigenen und fremden Unterricht hinsichtlich des Umgangs mit Heterogenität analysieren und reflektieren können (Marohn, Greefrath, Hammann, Hemmer, Kürten \& Windt, in diesem Band). Der vorliegende Beitrag stellt Perspektiven für ein fallrekonstruktives Seminar zur Diskussion, mit dem mithilfe von Videovignetten eine Professionalisierung der Unterrichtswahrnehmung von Biologielehramtsstudierenden angestrebt wird. Der Gegenstand der Unterrichtswahrnehmung ist der Umgang mit Schülervorstellungen im Evolutionsunterricht und damit eine für das Lehren und Lernen im Biologieunterricht zentrale Heterogenitätsdimension. Im Hinblick auf die Professionalisierung von Lehrkräften beziehen wir diese fachdidaktische Fragestellung weitergehend auf den Umgang von Lehrkräften mit der Sachantinomie, d.h. dem Spannungsfeld von Sachorientierung und Personenorientierung (Helsper, 200oa). Damit nehmen 
wir bei der Gestaltung unseres Seminars schwerpunktmäßig eine strukturtheoretische Perspektive auf Lehrerprofessionalität ein und gehen davon aus, dass Lehrkräfte in ihrem Handeln mit professionsspezifischen Antinomien konfrontiert sind und der reflektierte Umgang mit diesen ein zentrales Merkmal von Professionalität darstellt (Helsper, 2016). Wir fokussieren für eine Professionalisierung der Unterrichtswahrnehmung hinsichtlich des Umgangs mit Schülervorstellungen auf die Entwicklung eines „antinomischen Blicks“ (Schlömerkemper, 2006, S. 281). In der Reflexion von Fällen aus der Unterrichtspraxis sehen wir eine vielversprechende und bildungspolitisch eingeforderte Möglichkeit (Kultusministerkonferenz, 2019), einen Beitrag im Professionalisierungsprozess von Lehramtsstudierenden zu leisten (Dzengel, 2017; Helsper, 20oob; Ohlhaver \& Wernet, 1999).

\section{Theoretischer Rahmen}

\subsection{Professionalisierung der Unterrichtswahrnehmung mithilfe von Unterrichtsvideos}

Seidel und Kolleginnen und Kollegen (Seidel, Blomberg \& Stürmer, 2010) haben den Begriff professionelle Unterrichtswahrnehmung als Übersetzung des Begriffs der Professional Vision (Goodwin, 1994; Sherin, 2007) in die deutschsprachige Diskussion zur Lehrerprofessionalität eingeführt. Darunter wird die Fähigkeit verstanden, lernrelevante Unterrichtssituationen $\mathrm{zu}$ erkennen und $\mathrm{zu}$ interpretieren (Seidel, Prenzel, Schwindt, Stürmer, Blomberg \& Kobarg, 2009; Sherin, 2007). Zu dem Konzept der professionellen Unterrichtswahrnehmung liegen mittlerweile unterschiedliche Modellierungen (Übersicht: Barth, 2017) sowie Kompetenzmodelle (z.B. Meschede, 2014) vor, auf denen viele empirische Studien basieren. Ein zentraler Ertrag dieser Studien ist es, einen Zusammenhang einer Vielzahl an professionellen Kompetenzen und Wissensbeständen mit der Unterrichtswahrnehmung herzustellen.

Die professionelle Unterrichtswahrnehmung wird als ein zentrales Element von Lehrerexpertise beschrieben (Goodwin, 1994) und als Voraussetzung für individuelle Förderung angesehen. Diese hohe Bedeutung wird einerseits damit begründet, dass Lehrende mit komplexen Unterrichtssituationen konfrontiert sind und ein professionelles Handeln besser möglich erscheint, wenn sie die besonders lernrelevanten Ereignisse im Unterricht erkennen und interpretieren können (Hammerness, Darling-Hammond \& Shulman, 2002). So können sinnvolle Entscheidungen zur Förderung einzelner Schülerinnen und Schüler erst getroffen werden, nachdem zentrale Heterogenitätsdimensionen (z.B. individuelle Schülervorstellungen) wahrgenommen wurden. In mehreren Studien wurden positive Zusammenhänge zwischen der Unterrichtswahrnehmung der Lehrkraft und dem Lernerfolg der Schülerinnen und Schüler nachgewiesen (z. B. Kersting, Givvin, Sotelo \& Stigler, 
2010; Roth, Garnier, Chen, Lemmens, Schwille, \& Wickler, 2011; Sherin \& van Es, 2009).

Zur Professionalisierung der Unterrichtswahrnehmung wird der Einsatz von Unterrichtsvideos als besonders geeignet angesehen, da sich der Prozess der Unterrichtswahrnehmung gerade in der Analyse von Unterrichtssituationen zeigt (Rehm, Bölsterli, Brovelli \& Wilhelm, 2014), sodass die Lernvoraussetzungen der Beobachtenden (hier: Studierende) besser berücksichtigt werden können. Zudem eignen sich Unterrichtsvideos für ein theorie- und fallbasiertes Nachdenken über einen lernwirksamen Unterricht (Krammer, 2014).

Dem Einsatz von Unterrichtsvideos liegen unterschiedliche theoretische Annahmen zugrunde. Viele Konzepte zur Förderung der professionellen Unterrichtswahrnehmung (z. B. Sunder, Todorova \& Möller, 2016; Seidel et al., 2009) stützen sich hierbei primär auf kompetenztheoretische Ansätze zur Lehrerprofessionalität (vgl. Baumert \& Kunter, 2006) und setzen einen rationalen Akteur voraus, der sein Professionswissen für die Unterrichtswahrnehmung nutzt.

Andererseits ist im Sinne der Wissenssoziologie nach Mannheim (1980) davon auszugehen, dass das eigene Unterrichten (Asbrand \& Martens, 2018; Neuweg, 2002) und die Unterrichtswahrnehmung auch durch implizites Wissen beeinflusst wird, welches im Sinne der Habitustheorie (Bourdieu, 1992) in kollektiven, milieuspezifischen Erfahrungsräumen erworben wird. Vielfältige Erfahrungen von Akteuren der Lehrerbildung legen die Annahme nahe, dass die Studierenden dieses Wissen durch die umfangreichen individuellen Erfahrungen mit Lehr-LernProzessen, die sie als Schülerinnen und Schüler und Studierende gemacht haben, bestimmt wird (z.B. Gautschi, 1995; Hagemann \& Rose, 1998). Dieses implizite Wissen stellt einen Orientierungsrahmen für die Handlungen der Studierenden (hier: die Unterrichtswahrnehmung) dar und wird im konzeptuellen Kontrast zu beispielsweise Überzeugungen oder subjektiven Theorien (z. B. Groeben \& Scheele, 2010; Leuchter, Pauli, Reusser \& Lipowsky, 2006) als handlungsleitend und Teil des Habitus der Lehrkraft konstruiert. Das implizite Wissen bestimmt als unbewusst vorliegendes „Wahrnehmungs-, Denk- und Handlungsschemata“ (Bourdieu, 1987, S. 101) das Verhalten und Handeln von Menschen. Die Unterrichtswahrnehmung von Studierenden ist demnach nicht nur ein Ergebnis eines zielgerichteten Bewusstseins, sondern wird auch unbewusst durch ihr implizites Wissen beeinflusst.

Es ist forschungsmethodisch eine Herausforderung, zu untersuchen, inwiefern die Wissensbestände eine handlungsleitende Funktion haben und nicht umgekehrt das Handeln im Nachhinein damit gerechtfertigt wird (Nisbett \& Wilson, 1977). Die Rekonstruktion des handlungsleitenden Wissens scheint besonders durch „eine Analyse von Denkprozessen in situ“ (Neuweg, 2011, S. 455) möglich zu sein. Derartige Denkprozesse können bspw. beim Sprechen über die eigene Unterrichtswahrnehmung stattfinden. 


\subsection{Fallbasierte Reflexion der Sachantinomie mithilfe von Videovignetten}

Im Sinne einer strukturtheoretischen Perspektive auf Lehrerprofessionalität gehen wir davon aus, dass Lehrkräfte in ihrem Handeln mit professionsspezifischen Antinomien konfrontiert sind und der reflektierte Umgang mit diesen ein zentrales Merkmal von Professionalität darstellt (Helsper, 2016).

„Die Qualität der professionellen Praxis ist damit - wenn auch nicht ausschließlich - von der reflektierten Handhabung der Antinomien abhängig, was wiederum die Fähigkeit voraussetzt, sie rekonstruktiv zu erschließen und konstruktiv unterschiedliche Möglichkeiten des Handelns in antinomischen Kontexten zu entwerfen.“ (Helsper, 2000a, S. 158)

Ein zentrales unterrichtspraktisches Problem ist aus einer strukturtheoretischen Perspektive die „systematische Unsicherheit pädagogischen Handelns" (Combe \& Kolbe, 2004, S. 834), die nicht auflösbar ist (Combe \& Helsper, 1996; Combe \& Kolbe, 2004). In vielen Ansätzen zur Lehrerprofessionalisierung wird u. a. daraus geschlussfolgert, dass Reflexivität (Fähigkeit und Bereitschaft zur Reflexion) einen zentralen Bestandteil von Professionalität darstellt (Berndt, Häcker \& Leonhard, 2017; Combe \& Kolbe, 2004; Herzog, 1995). Wir gehen von der Annahme aus, dass vor allem durch Reflexion ein angemessener Umgang mit der Sachantinomie möglich ist und sich das eigene Handeln (z.B. im Unterricht) legitimieren lässt (Helsper, 2001).

In der Biologiedidaktik ist besonders eine Reflexion der Sachantinomie hinsichtlich des Verhältnisses von fachlicher Norm und Schülervorstellungen von Bedeutung. Demnach steht das Handeln von Lehrkräften insofern in einem unauflösbaren Widerspruch, als die individuellen Besonderheiten jedes Lernenden berücksichtigt werden sollen (Personenorientierung) und zugleich curriculare sowie inhaltliche Ansprüche der Lern-Sache (Sachorientierung) umgesetzt werden müssen. Dieser Widerspruch kann in Unterrichtssituationen nicht aufgelöst werden und erfordert eine professionelle Bearbeitung der Sachantinomie (Helsper, 2016).

Im Bereich des schulischen Inhaltsfelds Evolution sind vielfältige Schülervorstellungen umfangreich empirisch nachgewiesen, sodass wesentliche Lernvoraussetzungen und Lernschwierigkeiten bekannt sind (Hammann \& Asshoff, 2014; Kattmann, 2017a). Diese Vielfalt lässt sich systematisieren, sodass mehrere Schülervorstellungen bspw. zu „allgemeinen Denkweisen“ (Hammann \& Asshoff, 2014, S. 26) zusammengefasst werden können. Für unser Seminar sind davon vor allem zwei von Bedeutung. Erstens denken Lernende, dass menschliche Eigenschaften und Denkweisen auf die Natur übertragbar wären (anthropomorphistische Vorstellungen). Dabei stehen Anthropomorphismen im Kontrast zur wissenschaftlichen Norm von Objektivität, Neutralität und Wertfreiheit (Hammann \& Asshoff, 2014). Zweitens denken Lernende, dass biologische Erklärungen über den Zweck, Zielgerichtetheit oder Anpassungsnotwendigkeit der Lebewesen sowie externe 
Designer erfolgen können (teleologische Vorstellungen), obwohl das Prinzip der Kausalität hier anzuwenden wäre (Hammann \& Asshoff, 2014; Kelemen, 2012).

Wie im Unterricht mit Schülervorstellungen umgegangen werden kann und sollte, ist zum einen Gegenstand biologiedidaktischer Kontroverse (Gresch \& Martens, 2019; Gropengießer \& Marohn, 2018; Hammann \& Asshoff, 2014; Kattmann, Duit, Gropengießer \& Komorek, 1997; Martens \& Gresch, 2018) und zum anderen eine unterrichtspraktische Herausforderung für (angehende) Lehrkräfte. So entstehen im Umgang mit Schülervorstellungen im Unterricht oftmals Entscheidungssituationen, in denen Lehrkräfte nicht allen Erwartungen gleichermaßen gerecht werden können (siehe Abb. 1). Dies wird im Bild einer Apothekerwaage symbolisch deutlich. Denn wenn die Lehrkraft in ihrem Handeln mehr Bedeutung (Gewicht) auf eine Orientierung legt (links: Personenorientierung, rechts: Sachorientierung), verliert dadurch gleichzeitig die jeweils andere Orientierung an Bedeutung. Einerseits wird beispielsweise erwartet, dass sie im Unterricht die individuellen Vorstellungen der Lernenden berücksichtigt (Differenzierung). Andererseits gilt es beispielsweise, in einer vorgegebenen Zeit die Vorgaben des Kernlehrplans zu erfüllen (Homogenisierung).

Ein professioneller Umgang mit der Sachantinomie zielt darauf ab, dass Lehrkräfte die Unauflösbarkeit der Antinomie aushalten sowie situativ rekonstruieren und unter Abwägung der Handlungsalternativen entsprechend begründete Entscheidungen für ihr Handeln im Unterricht treffen (Helsper, 2000a, 2016). Hierbei

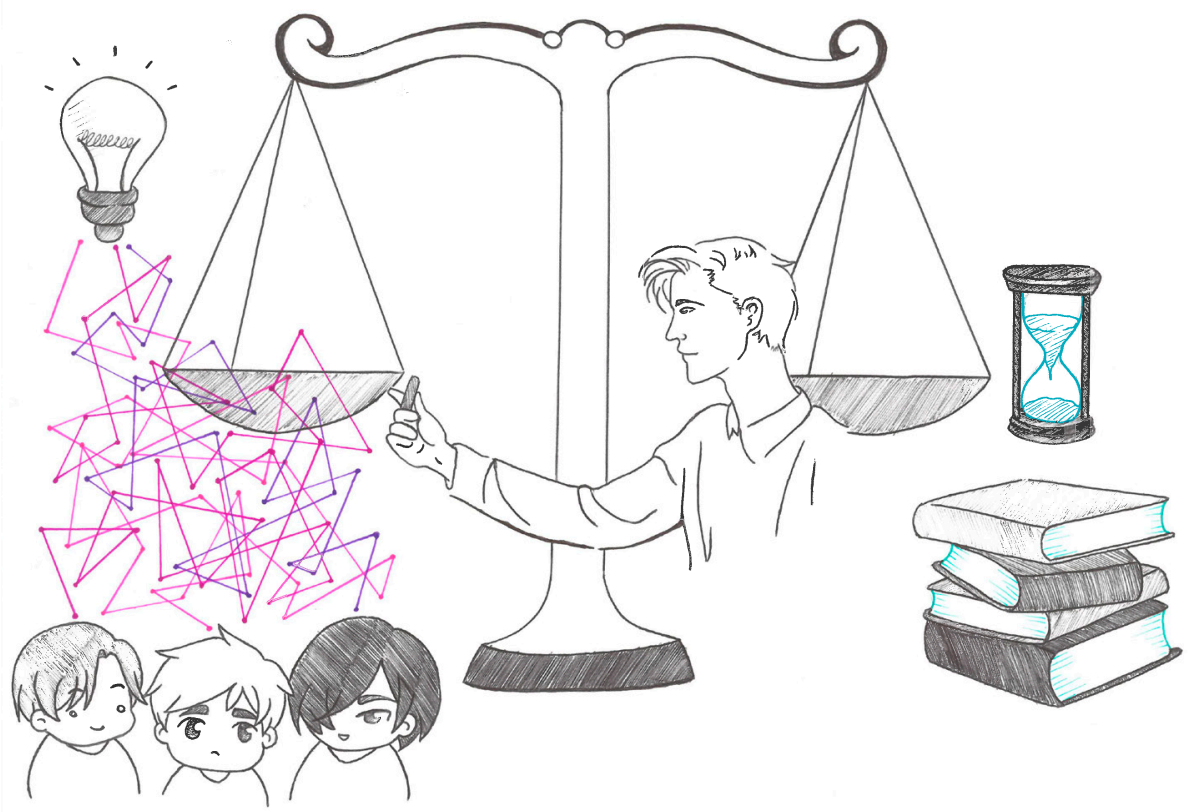

Abbildung 1: Sachantinomie in unterrichtlichen Entscheidungssituationen - Widersprüchliche Erwartungen an die Lehrkraft (๑ Anna Sonntag 2018) 
sollte in größeren unterrichtlichen Kontexten, bspw. bei der Planung einer Unterrichtsreihe, sowohl eine Personenorientierung als auch eine Sachorientierung angemessen zur Geltung kommen.

In der Literatur liegen verschiedene Verständnisse von Reflexion vor sowie entsprechende Ansätze zur Gestaltung der Reflexionsprozesse (Hatton \& Smith, 1995). Wir verstehen unter Reflexion „insbesondere die Fähigkeit, eigenes Verhalten, mentale Konzepte, Gefühle und Haltungen wahrzunehmen und in Bezug zur Umwelt kritisch zu hinterfragen" (Lutz, 2019, Absatz 1). Nach dieser Begriffsbestimmung ist ein forschender und fallorientierter Zugang $\mathrm{zu}$ den Vignetten zur Förderung der Reflexivität der Studierenden nützlich. In unserem Seminar konkretisiert sich dies bspw. derart, dass die Studierenden die Sachantinomie im Umgang mit Schülervorstellungen sowie das handlungsleitende implizite Wissen ihrer Unterrichtswahrnehmung fallbasiert rekonstruieren und vor diesem Hintergrund ihre Unterrichtswahrnehmung kritisch hinterfragen.

In internationalen und nationalen Studiengängen stellt die Arbeit an und mit Fällen eine wesentliche Lernmöglichkeit dar. Diese hat sich auch im Lehramtsstudium etabliert (z. B. Beck, Helsper, Heuer, Stelmaszyk \& Ullrich, 20oo; Pieper, Frei, Hauenschild \& Schmidt-Thieme, 2014; Syring, Bohl, Kleinknecht, Kuntze, Rehm \& Schneider, 2016). Es lassen sich verschiedene Ansätze der Fallarbeit unterscheiden (z.B. rekonstruktive und entwicklungsorientierte Fallarbeit) (Steiner, 2014).

Wir gehen davon aus, dass sich der Einsatz von Videovignetten als Fälle besonders eignet, da hier situiert und authentisch der Umgang mit den Schülervorstellungen und dadurch auch eine exemplarische Bearbeitung der Sachantinomie wahrgenommen werden kann. Wir haben uns dabei für die Fallarbeit an fremden Unterrichtsaufnahmen entschieden, wobei davon ausgegangen wird, dass durch deren Wahrnehmung bereits eine Vorbereitung auf eigenes unterrichtliches Handeln erfolgen kann (Bromme, 2014). Zwar ermöglichen Videos des eigenen Unterrichts es, die eigenen Erfahrungen zu reflektieren, jedoch bietet fremder Unterricht im Gegensatz zu eigenen Videoaufnahmen auch Vorteile, die es abzuwägen gilt: (1) Es treten keine „Rechtfertigungszwänge“ (Herzmann \& Proske, 2014, S. 34) auf, die zu einem "Reflexionshindernis“ (S. 34) führen könnten (Herzmann \& Proske, 2014; Liegmann, Racherbäumer \& Drucks, 2018). (2) Es entstehen keine „Legitimationsprobleme und -ängste" (Stelmaszyk, 2000, S. 17). (3) Es ist eine Analyse beispielhaften Umgangs mit Schülervorstellungen von erfahrenen Lehrpersonen möglich, deren Umsetzung für Studierende aufgrund der vielfältigen weiteren Handlungsanforderungen (z. B. eigene Lehrerrolle oder Gesprächsführung) eine große Herausforderung ist. 


\section{Konstruktion der Videovignette als Fall: Bearbeitung der Sachantinomie}

Im Rahmen des Forschungsprojekts zur Rekonstruktion des impliziten Wissens der Studierenden bezüglich des Umgangs mit Schülervorstellungen (Steinwachs \& Gresch, 2019) wurden 51 Schulstunden (ca. 41 Zeitstunden) Evolutionsunterricht in der Sekundarstufe I und II an verschiedenen Schulen aufgenommen. Aus diesem Videomaterial wurden bereits vier etwa 19-minütige Videovignetten entwickelt, die sowohl eine Kameraperspektive auf die Lehrkraft als auch eine auf die Schülerinnen und Schüler zeigen. Hierzu wurden Sequenzen kriteriengeleitet ausgewählt, in denen der Umgang mit Schülervorstellungen von zentraler Bedeutung ist, insbesondere hinsichtlich teleologischer und anthropomorpher Schülervorstellungen, die in besonderer Weise Lernschwierigkeiten darstellen und die Lernausgangslage charakterisieren (Hammann \& Asshoff, 2014). Das Auswahlkriterium führt automatisch auch dazu, dass eine Bearbeitung der Sachantinomie durch die Lehrkraft wahrnehmbar ist, denn gerade im Umgang mit Schülervorstellungen ergeben sich widersprüchliche Erwartungen an das Handeln von Biologielehrkräften.

Im Folgenden wird exemplarisch eine Videovignette vorgestellt, die auf Basis von acht videographierten Unterrichtsstunden zum Inhaltsfeld Evolution aus einem Leistungskurs Biologie eines Berufskollegs entwickelt wurde. Der inhaltliche Fokus ist die evolutive Anpassung unterschiedlicher Arten, was im Unterricht an unterschiedlichen Kontexten thematisiert wird, u.a. der Entstehung von Antibiotikaresistenz, den Ursachen für Vogelzug und der stammesgeschichtlichen Entwicklung der Grabbeine des Maulwurfs.

Die Lernpotentiale der Vignette und Möglichkeiten der Reflexion sollen basierend auf den Rekonstruktionen der unterrichtlichen Interaktionen (für eine Interpretation der unterrichtlichen Interaktionen siehe Gresch \& Martens, 2019) mithilfe von drei nachfolgend dargestellten Ausschnitten (siehe erstmals Steinwachs \& Gresch, 2019) aus der Videovignette exemplarisch begründet werden.

Ausschnitt 1 (Vignette: oo:20-01:18; Bach-Schule): ${ }^{1}$

L: $\quad$ Es gibt ein Beispiel fü- was sogar noch schneller geht und was uns auch, ähm, direkt betrifft und auch häufig in den Medien diskutiert wird; und zwar, äh das Thema Antibiotikaresistenz, von Bakterien; hat da jemand schon mal was von gehört; wissen sie da was drüber; (2) Antibiotika sind ja auch schon mal im im Bereich Genetik vorgekommen bei uns, (.) Gentechnik aber ${ }^{\circ}$ Antibiotikaresisten $z^{\circ}$ Lara

Lara: Ich bin mir nicht ganz sicher aber ist das nicht wenn man krank ist zum Beispiel, und die ganze Zei:t äh, Medikamente nimmt dass sich der Körper da ganz schnell dran gewöhnt und darauf dann nicht mehr anspringt also quasi äh dass die dann ihre Wirksamkeit verfehlen? (.) weil

1 Die Transkriptionen erfolgten nach dem Transkriptionssystem TiQ (siehe Przyborski und Wohlrab-Sahr, 2014, S. 167-169). 
L: $\quad$ Ja, die die Aussage dass sich der Körper dran gewöhnt die behalten wir mal im Hinterkopf, und überlegen hinterher wenn wir das gesehen haben, ähm inwieweit (.) das stimmt. nä?

Ausschnitt 2 (Vignette: 08:31-10:36; Bach-Schule):

L: $\quad$ Vielleicht noch ein Beispiel um das endgültig oder um ihnen das so ein bisschen zu erklären; ähm Phänomen; aktuelles Phänomen die Zugvögel die ziehen jetzt nach Süden; warum machen sie das, ich gebe ihnen zwei Antworten; ich geb ihnen zwei Antworten und sie sagen mir was zu den Antworten; Antwort A, (.) sie wollen Kälte und Nahrungsmangel im Winter hier ausweichen. deshalb ziehen sie nach Süden; (.) Antwort B, (.) sie haben eine hormonelle Veränderung in ihrem Körper und deshalb verspüren sie eine Zugunruhe und müssen fliegen; (7) Lara

Lara: Ich würd sagen dass A stimmt;

L: $\quad$ Erläutern sie mal,

Lara: Könnten sie noch einmal wiederholen

L: $\quad$ A war sie wollen hier Kälte und Nahrungsmangel im Winter ausweichen. deshalb fliegen sie nach Süden;

Lara: Ich glaub dass sie einfach ähm wissen dass die hier kein äh Futter und sowas bekommen und deswegen fliegen dann halt fliegen die dann halt woanders hin wo die halt wissen wo die mehr Nahrung und sowas alles bekommen,

L: $\quad$ Okay möchte jemand was zu Lara sagen; jetzt nicht was eigen-, sondern erstmal dazu was sagen. Pia.

Pia: Also das also Antwort A und das was Lara gesagt hat ähm passt so zu Lamarcks Vorstellungen; weil weil bei Lamarck war das ja auch so dass die Tiere zum Beispiel die Giraffe nen längeren Hals bekommen hat weil die an das Futter dran will und das ist ja genau das gleiche mit den Vögeln die fliegen weg um auch bessere äh um äh Futter zu bekommen,

L: $\quad \mathrm{Hmhm}$.

Lara: Das versteh dann muss ich jetzt aber was sagen weil das versteh ich jetzt nicht weil das ist ja die haben ja nicht nichts an sich verändert die fliegen ja immer nur woanders hin; also das verstehe ich nicht;

L: $\quad$ Ja ja das ich hoffe dass wir es erklären noch dazu? ah Melina

Melina: Ich glaub Tiere können gar nicht so denken oder, dass die jetzt so wissen ok äh Sommer also Winter hier ist doof ich muss in Sommer so können die glaube ich gar nicht denken ich glaube dann muss Antwort B wohl stimmen weil irgendwat irgendein Signal müssen die ja bekommen damit die wegfliegen;

L: Dazu woher sollen sie es auch wissen; woher sollen die wissen wie hier der Winter ist

Ellen: Hä wenn man den einmal durchgemacht hat;

$\mathrm{L}: \quad \mathrm{Hm}$ 
Ellen: Wenn die das früher mal öfters durchgemacht haben;

L: $\quad$ Dann haben sie es weitererzählt

[mehrere lachen]

Ausschnitt 3 (Vignette: 15:06-15:36; Bach-Schule):

L: $\quad$ Also der Begriff Anpassung wird immer zielgerichtet verwendet, ja die Mutter sagt zu ihrem Kind; nun pass dich da mal ein bisschen an ja dass man selber was ändert; dass man aktiv zielgerichtet was ändert; das ist der Punkt den wir im Kopf haben und der hier nicht passt und der uns hier auf den Holzweg führt. und äh (1) ja weil aber dieser; das ist im Grunde auch der gleiche Denkfehler den Lamarck hatte; sach ich mal Denkfehler so in Anführungszeichen; der steckt in uns allen und ist ganz schwer rauszukriegen

In der Videovignette sind ein bestimmter Umgang mit den Schülervorstellungen und damit auch eine Bearbeitung der Sachantinomie durch die Lehrkraft beobachtbar. Die strukturell bedingten widersprüchlichen Erwartungen an die Lehrkraft führen dazu, dass sie in einzelnen Unterrichtssituationen zwangsläufig der einen oder anderen Orientierung (Sach- oder Personenorientierung) nicht gerecht wird. So zeigt sich in diesen Abschnitten eine stärkere Sachorientierung und eine geringere Personenorientierung. Beispielsweise geht die Lehrkraft nicht mehr auf das explizit geäußerte Verständnisproblem von Lara ein, die trotz der mehrfachen Ablehnung intentionaler und teleologischer Erklärungen im Unterricht durch die Lehrkraft und andere Lernende die intentionale Antwort A zur Erklärung des Vogelzugs auswählt. Bei Lara zeigt sich hier eine teleologische Orientierung, die von der fachlichen Norm abweicht. Dies wird von anderen Lernenden und der Lehrkraft negativ evaluiert und als absurd bewertet, woraufhin mehrere lachen. Diese Beobachtung bietet einen didaktisch herausfordernden Anlass zur Reflexion über Handlungsmöglichkeiten. Durch die Entscheidung im Unterricht derart vorzugehen, entsteht die Notwendigkeit einer Begründung und Legitimation der Handlungsentscheidung und dabei der Begründung des Ausschließens von Handlungsalternativen. Hier bieten der theoretische Hintergrund zur Sachantinomie und fachdidaktische Ansätze zum Umgang mit Schülervorstellungen Möglichkeiten, um das unauflösbare Spannungsverhältnis von Sache und Person in seiner Widersprüchlichkeit zu reflektieren und kontrovers über Handlungsalternativen zu diskutieren.

Bei der Fallarbeit könnte als Umgang mit den Schülervorstellungen beispielsweise ein „Perspektivenwechsel“ (Kattmann, 2017b, S. 9) im Sinne metakognitiven Wissens (Gropengießer, 2008; Kattmann, 2017b) einem Konzeptwechsel gegenübergestellt werden. Hierbei wird die Perspektive, die der Schülervorstellung zugrunde liegt, mit anderen Perspektiven verglichen. Hieran könnte herausgearbeitet werden, in welchen Domänen bzw. Kontexten (z. B. Alltag vs. Biologie; Humanbiologie vs. Evolution) teleologische Erklärungen als legitim angesehen werden und 
in welchen nicht. Es könnten Handlungsmöglichkeiten diskutiert werden, die es den Lernenden ermöglichen sollen, im Sinne einer "Zweisprachigkeit“ (Combe \& Gebhard, 2007, S. 79), sowohl mit der Alltags- als auch mit der Fachsprache umgehen zu können.

In der Videovignette lässt sich rekonstruieren, dass die Lehrerin eine Differenzkonstruktion vornimmt, indem sie die als fachlich korrekt eingeführte synthetische Evolutionstheorie den als falsch dargestellten Schülervorstellungen, insbesondere finalem, teleologischem Denken, und Lamarcks Evolutionstheorie gegenüberstellt. In diese beiden Pole werden im Sinne einer Sachorientierung alle in der Vignette beobachtbaren Schüleraussagen als richtig und falsch eingeordnet. Dies wird auch durch die Vorgabe zweier zu kontrastierender Antwortoptionen (eine intentionale und eine wissenschaftlich kausale Erklärung) zu den Ursachen des Vogelzugs beobachtbar. Die Studierenden könnten über die subsumtiv-generalisierende Vorgehensweise im Umgang mit den vielfältigen Schülervorstellungen reflektieren. Hierzu könnten sie zunächst die Schüleraussagen in die "allgemeinen Denkweisen“ (Hammann \& Asshoff, 2014, S. 26) und spezifischere Kategorien einordnen und dann an konkreten Situationen aus der Vignette im Sinne einer Rekonstruktion des Einzelfalls darüber diskutieren, inwiefern eine unzulässige Typisierung stattfindet, die dem Einzelfall nicht gerecht wird. Im Sinne einer Stärkung der Sachorientierung ließe sich auch darüber diskutieren, inwiefern eine Unterscheidung in eine proximate und ultimate Erklärung für den Vogelzug und/oder der Einbezug der vier klassischen Fragen des Verhaltensbiologen Tinbergen (Naguib, 2006) zur Gestaltung der Lernprozesse der Schülerinnen und Schüler sinnvoll genutzt werden können.

In der Videovignette lässt sich auch rekonstruieren, dass die Schülervorstellungen für die Lehrkraft stabil und lernhinderlich sind. Der Umgang mit ihnen orientiert sich eher an einer defizitären Sichtweise, wonach die Vorstellungen ein Lernhindernis darstellen. Hierin dokumentieren sich homolog die Sachorientierung und die geringer ausgeprägte Personenorientierung. Auch aus der Perspektive konstruktivistischer Lerntheorien (Riemeier, 2007) erscheint eine wertschätzende Sichtweise auf Schülervorstellungen förderlich, um diese für den Prozess des fachlichen Lernens zu nutzen. Dabei scheint es von besonderer Bedeutung zu sein, den Ursachen der Schülervorstellungen nachzugehen (Gropengießer, 2008) und die Lernerperspektive einzunehmen (Dannemann, Niebert, Affeldt \& Gropengießer, 2014). Durch eine stärkere Gewichtung der Personenorientierung könnten die Studierenden hier über Handlungsalternativen diskutieren, die in Form einer „Anknüpfung“ (Kattmann, 2017b, S. 9) fachlich korrekte Aspekte in den Schülervorstellungen aufspürt und diese als Ausgangspunkt des Lernens nutzt. So könnte beispielsweise mit der Schülerin Lara an denen von ihr geäußerten Kausalmechanismen zur Entstehung der Antibiotikaresistenz weitergearbeitet werden, um die fachlich korrekten Ursache-Wirkungsbeziehungen besser zu verstehen. Die Studierenden könnten folglich darüber diskutieren, wie die Lehrkraft an die Lernausgangslage anknüpfend sinnvolle Lernangebote machen könnte (Kattmann, 2017b). 
Durch die individuell unterschiedlichen Anknüpfungspunkte sollte sich die Entwicklung von Handlungsmöglichkeiten dabei nicht nur an einer Homogenisierung, sondern gleichzeitig auch an einer Differenzierung im Sinne einer Personenorientierung orientieren. Hierbei könnten die heterogenen Schülervorstellungen und die unterschiedlichen Lernwege, die sich in der Vignette wahrnehmen lassen, mithilfe einer „konzeptuellen Landkarte“ (Zabel \& Gropengießer, 2010, S. 221), die fünf Lernhürden beim Verstehen der fachlichen Norm darstellt, visualisiert werden.

Anhand der Beobachtung einzelner Lernender über die Zeit hinweg ließe sich theoriebasiert (z.B. in Bezug auf „conceptual reconstruction“ (Gropengießer \& Marohn, 2018, S. 57) oder hinsichtlich der Koexistenz von Schülervorstellungen und fachlichen Erklärungen (Kattmann, 2017a; Martens \& Gresch, 2018)) auch kontrovers darüber diskutieren, inwiefern die fachliche Norm von allen Lernenden erreicht werden kann.

\section{Perspektiven für ein fallrekonstruktives Seminar}

Wir haben uns im Sinne unserer theoretischen Annahmen für eine rekonstruktive Fallarbeit (Beck et al., 200o) entschieden. Diese Arbeitsweise zielt auf ein „Verstehen der grundlegenden Strukturlogik" (Hummrich, 2016, S. 25), auf eine Irritation der verinnerlichten „Deutungs- und Handlungsmuster“ (S. 25) und auf die Entwicklung eines „professionalisierten Habitus“ (S. 25) ab. Sie ermöglicht unter Anwendung der Dokumentarischen Methode die Interpretation der unterrichtlichen Interaktionen (Asbrand \& Martens, 2018; Nentwig-Gesemann, 2014). Dadurch wird eine Beschreibung und das Verstehen der spezifischen unterrichtlichen Strukturen (v. a. die Sachantinomie) angestrebt, die den Umgang mit Schülervorstellungen rahmen. Diese Vorgehensweise ist gegenstandsangemessen, da der Umgang mit Schülervorstellungen keine standardisierbare Handlung von Lehrkräften darstellt.

Darüber hinaus können durch eine rekonstruktive Fallarbeit die impliziten Wissensbestände der Studierenden rekonstruiert werden (Bohnsack, 2003), die ihre Wahrnehmung des Falls beeinflussen (siehe für erste empirische Ergebnisse Steinwachs \& Gresch, 2019). Dies hilft dabei, unbewusste verinnerlichte Wahrnehmungs- und Interpretationsmuster zu explizieren, um sie der Reflexion zugänglich $\mathrm{zu}$ machen.

Neben der Fallrekonstruktion (deskriptiv) wird im Seminar auch eine Perspektive der fachdidaktischen Planung (normativ) eingenommen, die auf die LehrLern-Prozesse beim Umgang mit Schülervorstellungen im Evolutionsunterricht unter Berücksichtigung der Sachantinomie fokussiert. Hierbei werden unter Einbezug der Anforderungen an Biologielehrkräfte (z.B. schulpolitische Vorgaben) für exemplarische Unterrichtssituationen vor allem (angemessene) Handlungsstrategien entwickelt und diskutiert. 
Das fallrekonstruktive Seminar wird aktuell für den Master of Education am Zentrum für Didaktik der Biologie konzipiert und soll im Wintersemester 2020/21 erprobt und evaluiert werden. Insofern stellen die folgenden Überlegungen kein ausgereiftes Konzept dar, sondern sollen erste Perspektiven aufzeigen. Der Einbezug des impliziten Wissens der Studierenden und der theoretischen Überlegungen zur Sachantinomie stellen ein Alternativangebot zu Lehrveranstaltungen dar, die auf kompetenzorientierten Ansätze der Lehrerbildung basieren (z. B. im Projekt Observe: Seidel et al., 2009). Das Seminar bietet auch eine komplementäre Perspektive zu fachdidaktischen Lehrveranstaltungen zum Umgang mit Schülervorstellungen, die sich auf die Förderung expliziten Professionswissens (z. B. Diagnose-, Planungs- und Reflexionskompetenz) fokussieren (z. B. Dannemann et al., 2014).

Der Umgang mit der Komplexität von Unterrichtssituationen im Seminar ist Gegenstand der gemeinsamen Konzeption im Rahmen der Qualitätsoffensive Lehrerbildung an der Universität Münster (Marohn et al., in diesem Band). So erfolgt in diesem Projekt zum einen eine Fokussierung auf den Umgang mit Schülervorstellungen als eine Dimension aus der Vielzahl von Möglichkeiten im komplexen Unterrichtsgeschehen. Zum anderen wird für die Seminararbeit eine fokussierte Auswahl biologiedidaktischer (Umgang mit Schülervorstellungen: z. B. Gropengießer \& Marohn, 2018; Hammann \& Asshoff, 2014; Kattmann, 2017a; Riemeier, 2007) und pädagogischer (Sachantinomie: z. B. Helsper, 200oa) Theorien und Forschungsarbeiten herangezogen. Durch die so erfolgte Komplexitätsreduktion der Anforderungen hinsichtlich der Breite der Analyseschwerpunkte (siehe Abb. 2) kann gleichzeitig die Komplexität hinsichtlich der Tiefe der Analyse erhöht werden. Dabei soll eine systematische Unterstützung durch die Dozierenden bei der Reflexion und der Herstellung von Theorie-Praxis-Bezügen angeboten werden, um die Bewältigung der Komplexität der Unterrichtswahrnehmung zu ermöglichen. So können diese beispielsweise den studentischen Reflexionsprozess und die Erweiterung von Perspektiven durch Irritationen und Korrekturen fördern (Kunze, 2017). Eine Unterstützung bei der rekonstruktiven Fallarbeit ist besonders wichtig, da es sich um eine sehr anspruchsvolle Aufgabe handelt, die den Studierenden ohne Unterstützung vermutlich nur bedingt gelingt (Beck et al., 200o).

Das Seminar lässt sich aus einer hochschuldidaktischen Perspektive in vier Schritte untergliedern, wobei diese in der Praxis vielfältig miteinander verschränkt sind.

In einem ersten Schritt schauen sich die Studierenden in Kleingruppen eine Videovignette vollständig an und erhalten zugehörige Kontextinformationen (z. B. zur Verortung der Stunden in der Unterrichtsreihe). Die Studierenden haben dabei jederzeit die Möglichkeit, beliebige Ausschnitte erneut abzuspielen und die Kontextinformationen umfangreicher zu analysieren. Die Studierenden diskutieren anschließend über ihre Unterrichtswahrnehmungen im Rahmen einer Gruppendiskussion (Bohnsack, 2010), wobei die Vignette ohne weitere Instruktionen der Diskussionsimpuls ist. Die Diskussionen werden audiografiert, sodass diese Daten später im Seminar zur Rekonstruktion der impliziten Wissensbestände genutzt werden können. 


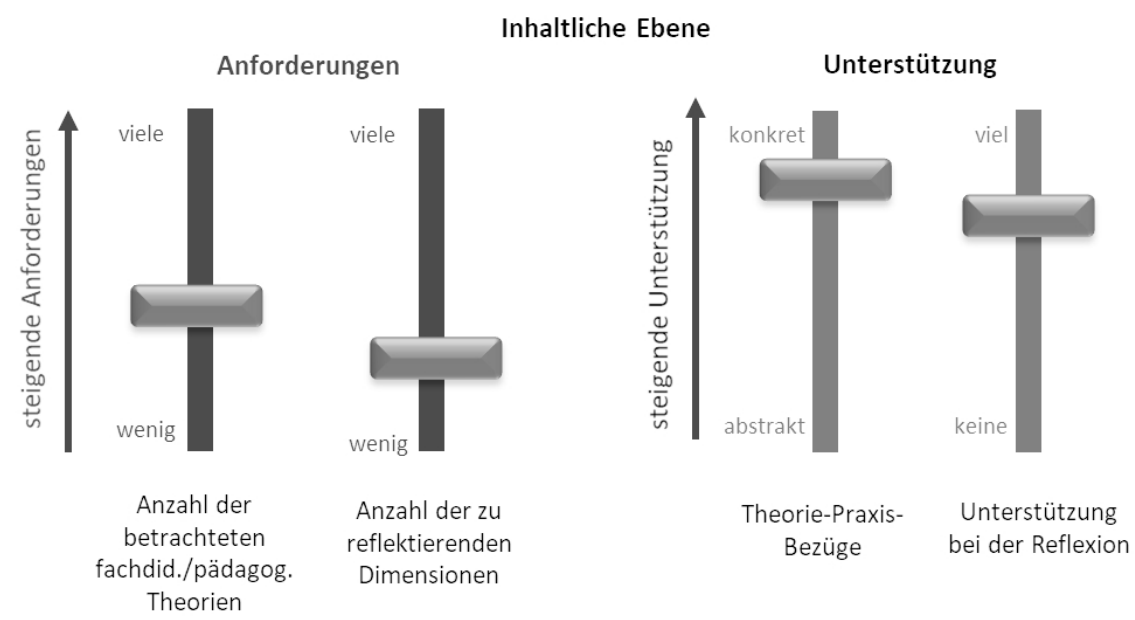

Abbildung 2: Übersicht zur Komplexitätsreduktion im fallrekonstruktiven Seminar

In einem zweiten Schritt folgt zunächst eine Einführung in die und eine Einübung der Grundlagen der hermeneutischen Textinterpretation im Sinne der Dokumentarischen Methode (Asbrand \& Martens, 2018; Bohnsack, 2003), um einen wissenschaftlich-reflexiven Zugang anzubahnen. Die Dokumentarische Methode ermöglicht hierbei auch die Rekonstruktion des impliziten Wissens der Lehrperson sowie der Lernenden und damit habitueller Strukturen, die für das Unterrichtsgeschehen handlungsleitend sind. Hierbei kann keine differenzierte Methodenkompetenz angestrebt werden. Welche Vereinfachungen beim Einsatz der Methode sinnvoll sind (z. B. kein Einbezug bestimmter nonverbaler Kommunikation), ohne den Anspruch einer tiefergehenden Fallanalyse aufzugeben, bleibt eine Herausforderung für die Seminarpraxis.

Mithilfe der Dokumentarischen Methode analysieren die Studierenden exemplarisch selbst gewählte Interaktionen zwischen der Lehrkraft und den Schülerinnen und Schülern hinsichtlich des Umgangs mit Schülervorstellungen. Bei der Fallrekonstruktion soll nicht beurteilt werden, ob der Umgang mit den Schülervorstellungen in bestimmten Situationen angemessen ist oder nicht. Die Interpretation fokussiert im Sinne der Dokumentarischen Methode vielmehr, wie im Umgang mit den Schülervorstellungen miteinander interagiert wird, welche Strukturen diesen Interaktionen zugrunde liegen (v.a. Sachantinomie) und welches implizite Wissen dabei handlungsleitend ist. Dadurch lässt sich die Normativität der Unterrichtspraxis fokussieren, sodass nicht primär die Wirkungen der Lehrangebote bei den Lernenden analysiert werden, sondern die Struktur des Lehr-LernProzesses selbst.

Die Interpretationen im Rahmen der Fallrekonstruktion sollen dann durch theoretische Perspektiven erweitert werden. Hierzu soll aus Literatur zur Schülervorstellungsforschung (z. B. Gropengießer \& Marohn, 2018; Hammann \& Asshoff, 
2014; Kattmann et al., 1997) und zur Sachantinomie (z.B. Helsper, 200oa, 2016) eine Heuristik entwickelt werden, die von den Studierenden auf die Unterrichtssituationen angelegt werden kann. So könnte bspw. mithilfe des Modells der Didaktischen Rekonstruktion (Kattmann et al., 1997) anhand exemplarischer Unterrichtssituationen ein Vergleich der Lernerperspektiven mit den fachlich angemessenen Konzepten durchgeführt werden. Durch diesen Vergleich, der auch die Unterschiede zwischen einzelnen Lernenden untereinander und zu der fachlichen Norm verdeutlicht, lässt sich die Sachantinomie u.U. differenzierter herausarbeiten. Hierbei kann die fachliche Norm in Form von schulpolitisch vorgegebenen Lernzielen bzw. Kompetenzerwartungen formuliert werden.

In einem dritten Schritt rekonstruieren die Studierenden mithilfe der Dokumentarischen Methode (Bohnsack, 2003) ihre eigene Unterrichtswahrnehmung vom Beginn des Seminars. Hierzu interpretieren sie exemplarisch selbst gewählte Transkriptstellen aus der eigenen Gruppendiskussion (siehe erster Schritt: Audioaufnahmen). Danach kontrastieren sie exemplarisch ihre eigene Wahrnehmung mit der Wahrnehmung von anderen Gruppen, die in der Vergangenheit die Vignette angesehen und darüber diskutiert haben (Steinwachs \& Gresch, 2019). Hierdurch wird angestrebt, dass den Studierenden erste implizite Wissensbestände (z.B. unbewusste Normativitätsvorstellungen und Erwartungen) bewusstwerden und sie diese als Maßstab ihrer Unterrichtswahrnehmungen explizieren und reflektieren können. Dies ermöglicht eigene Interpretationen und Selbstverständlichkeiten zu hinterfragen, sodass in vermeintlich vertrauten Unterrichtsituation eine Erklärungs- und Begründungsbedürftigkeit entsteht.

Erste Gruppendiskussionen wurden im Rahmen des Forschungsprojektes (Steinwachs \& Gresch, 2019) bereits interpretiert, sodass Transkripte sowie Rekonstruktionen des handlungsleitenden impliziten Wissens vorliegen. Das implizite Wissen lässt sich in den Gruppendiskussionen beispielsweise in den Vorstellungen von den Lernprozessen der Schülerinnen und Schüler oder in der Bewertung der Handlungen der Lehrkraft rekonstruieren. Hierin zeigt sich auch, wie die Antinomie von Sache und Person von den Gruppen bearbeitet wurde. So äußern Studierende beispielsweise in einer Gruppendiskussion explizit, dass im Biologieunterricht die Berücksichtigung der einzelnen Schülervorstellungen relevant ist. Bei der Diskussion über Handlungsalternativen und der Bedeutung der Lehrkraft lässt sich aber rekonstruieren, dass einige Studierende implizit die Sachantinomie einseitig auf die Seite der Sachorientierung auflösen (Steinwachs \& Gresch, 2019).

In einem vierten und letzten Schritt wird eine fachdidaktische Planungsperspektive (normativ) eingenommen, die auf die Lehr-Lern-Prozesse beim Umgang mit Schülervorstellungen im Evolutionsunterricht unter Berücksichtigung der Sachantinomie fokussiert. Die Studierenden entwickeln alternative Handlungsmöglichkeiten für selbst gewählte Unterrichtssituationen. Sie reflektieren fall- und theoriebasiert den Umgang mit Schülervorstellungen und erhalten somit Einblicke in die Strukturen der späteren Berufspraxis, denen eine praxisvorbereitende Bedeutung zukommt. Die Studierenden sollen, einer zentralen Annahme des 
Modells der Didaktischen Rekonstruktion folgend, durch den Vergleich zwischen „fachlicher Klärung“ und „Erfassen von Schülerperspektiven“ (Kattmann et al., 1997, S. 4) sinnvolle Lernwege überlegen, in denen Schülervorstellungen und Lernschwierigkeiten berücksichtigt werden. Hier ließe sich vor dem Hintergrund der Sachantinomie auch kritisch darüber diskutieren, inwiefern im Sinne des Modells der Didaktischen Rekonstruktion „fachliche Konzepte und Schülervorstellungen gleichwertig behandelt" (Kattmann et al., 1997, S. 6) werden können. Hier könnte mithilfe der Metapher der Apothekerwaage (siehe Abb. 1) reflektiert werden, inwiefern in größeren unterrichtlichen Kontexten (z. B. Reihenplanung) ein Gleichgewicht zwischen der Sach- und Personenorientierung hergestellt werden kann und sollte.

Die Studierenden diskutieren die Handlungsmöglichkeiten bspw. hinsichtlich ihrer Folgen für die Interaktionen im Unterricht und den implizit enthaltenen normativen Annahmen. Hier kann auch kontrovers darüber diskutiert werden, wie situationsspezifisch eine praxistaugliche Diagnose von Schülervorstellungen und deren Veränderung aussehen und wie mit der Vielfalt der Vorstellungen angemessen umgangen werden kann.

Der vierte Seminarschritt mündet unter Abwägung der Handlungsalternativen in einer Positionierung der Studierenden, indem diese sich für eine Handlungsmöglichkeit begründet entscheiden. Wie ein angemessener Umgang mit den Schülervorstellungen aussieht, beurteilen folglich die Studierenden, auch unter Berücksichtigung ihrer Voraussetzungen, selbst. Hierbei sollen die Studierenden darin unterstützt werden, ihre Positionierungen kriterienorientiert, unter Berücksichtigung kontroverser Perspektiven und unter Abwägung und Gewichtung von Argumenten zu begründen. Angestrebt wird, dass sich diese Positionierungen nicht zu dogmatischen Überzeugungen entwickeln, sondern offen für Weiterentwicklungen sind. Durch unterschiedliche Positionierungen, die offen nebeneinander stehen bleiben können, können Perspektiven auf die Unterrichtspraxis erweitert und einfache, unterkomplexe Deutungen hinterfragt werden. Hierbei geht es nicht darum, die strukturelle Unsicherheit professionellen Handelns (Combe \& Helsper, 1996) durch die Anwendung fachdidaktischer Erkenntnisse aufzulösen, sondern diese Strukturen zu reflektieren. Hierzu könnte kontrovers diskutiert werden, welche Praxiszwänge nicht haltbar und welche theoretischen Idealvorstellungen (z. B. im Sinne einer Perfektionierung einer individuellen Förderung) in antinomischen Strukturen nicht umsetzbar sind.

Die Studierenden können abschließend ihre Positionierung in Bezug zu ihrer anfänglichen Perspektive auf die Vignette bringen und über den Mehrwert der fallrekonstruktiven Seminararbeit reflektieren.

Ein zentrales hochschuldidaktisch-normatives Ziel des Seminars ist, dass die Studierenden ihre Wahrnehmungs- und Interpretationskompetenz unter Berücksichtigung des eigenen impliziten Wissens auch im Sinne eines „antinomischen Blicks" (Schlömerkemper, 2006, S. 281) professionalisieren. Dies zielt auf die Anbahnung bzw. Entwicklung einer "(selbst)reflexiven Haltung“ ab (Helsper, 2000a, 
S. 163; Schlömerkemper, 2006) und auf ein (angemessenes) Fallverstehen, welches eine Vorbereitung auf das eigene Handeln in der Praxis unter Handlungsdruck darstellt. Hierzu wird auch die Erweiterung der Perspektiven auf einen (angemessenen) Umgang mit Schülervorstellungen und der Sachantinomie angestrebt.

\section{Diskussion und Ausblick}

Bei der Gestaltung von Reflexionsprozessen in der Lehrerbildung beschreibt Häcker (2017) die Gefahr, dass die Studierenden die Reflexion nicht als Mittel, sondern als Selbstzweck erleben und die Reflexionsprozesse im Sinne einer Aufgabenerledigung abarbeiten. Die Wahrscheinlichkeit einer solchen Entwicklung steigt vermutlich, wenn die Relevanz einer fallrekonstruktiven Arbeit für die eigene Professionalisierung aberkannt (Pflugmacher, 2014) und die Sinnhaftigkeit der Vorgehensweise an sich in Frage gestellt wird (Gruschka, 2005).

Dieser Herausforderung soll vor allem dadurch begegnet werden, dass die Dozierenden bei der theoriebasierten Arbeit die Perspektive der Studierenden auf ihre zukünftige Unterrichtspraxis zum zentralen Bezugspunkt der Lehr-LernProzesse machen sowie sie bei Reflexionsprozessen und bei der Herstellung von Theorie-Praxis-Bezügen systematisch unterstützen. Der Anspruch des Seminars ist dabei sowohl wissenschaftlichen als auch berufspraktischen Ansprüchen gerecht $\mathrm{zu}$ werden. Wir gehen davon aus, dass dies den Studierenden ermöglichen kann, die Sinnhaftigkeit ihres eigenen zukünftigen unterrichtlichen Handelns inhaltlich zu begründen und gegenüber anderen Akteuren im System Schule zu legitimieren.

Wie die Studierenden im Seminar das videovignettengestützte Reflexionsangebot nutzen und inwiefern Professionalisierungsprozesse stattfinden, gilt es empirisch zu untersuchen. Hierzu sollen perspektivisch die Reflexionsprozesse beim Einsatz der Videovignette in unserem fallrekonstruktiven Seminar rekonstruiert werden, um somit die Praxis der Lehrerbildung zum Gegenstand empirischer Forschung zu machen.

Wir schätzen einen professionellen Umgang mit der Heterogenität im Unterricht als sehr anspruchsvoll ein, sodass bei einer Evaluation des Seminars auch die Frage zu klären wäre, was von (angehenden) Lehrkräften überhaupt erwartet werden darf bzw. kann. Dies ist letztlich eine hochschuldidaktische, bildungstheoretische und bildungspolitische Frage.

Wir gehen davon aus, dass eine Verbesserung des Biologieunterrichts nicht nur von den expliziten Wissensbeständen der (angehenden) Lehrkräfte abhängen wird, sondern es auch wichtig ist, dass diese ihre eigene Unterrichtspraxis reflektieren können, um das eigene Handeln zu verändern. Für Professionalisierungsprozesse ist es aus unserer Sicht daher unabdingbar, dass das eigene handlungsleitende implizite Wissen bearbeitet wird, um sich der Perspektive und Subjektivität der eigenen Wahrnehmung und Handlung bewusst zu werden. Im Seminar soll ein Beitrag dazu geleistet werden, dass die Studierenden ihr eigenes implizites Wissen 
explizieren, sodass sie neue Perspektiven auf den Umgang mit Schülervorstellungen im Evolutionsunterricht einnehmen können. Hierbei stellen die Videovignetten ein vielversprechendes Mittel dar, um gemeinsam über lernwirksame Ereignisse im Unterricht kontrovers zu diskutieren und zu reflektieren. Ein fallorientierter Zugang zu den Vignetten kann dabei zur Einübung der zukünftigen eigenen Reflexionspraxis im Sinne eines „wissenschaftlich-reflexiven Habitus“ (Helsper, 20oob, S. 35) dienen.

\section{Literatur}

Asbrand, B. \& Martens, M. (2018). Dokumentarische Unterrichtsforschung. Wiesbaden: Springer VS. https://doi.org/10.1007/978-3-658-10892-2

Barth, V. L. (2017). Professionelle Wahrnehmung von Störungen im Unterricht. Wiesbaden: Springer VS. https://doi.org/10.1007/978-3-658-16371-6

Baumert, J. \& Kunter, M. (2006). Stichwort. Professionelle Kompetenz von Lehrkräften. Zeitschrift für Erziehungswissenschaft, 9 (4), 469-520. https://doi.org/10.1007/s11618oo6-0165-2

Beck, C., Helsper, W., Heuer, B., Stelmaszyk, B. \& Ullrich, H. (Hrsg.). (20oo). Fallarbeit in der universitären LehrerInnenbildung. Professionalisierung durch fallrekonstruktive Seminare? Eine Evaluation. Wiesbaden: VS Verlag für Sozialwissenschaften. https:// doi.org/10.1007/978-3-663-10029-4

Berndt, C., Häcker, T. H. \& Leonhard, T. (Hrsg.). (2017). Reflexive Lehrerbildung revisited. Traditionen - Zugänge - Perspektiven. Bad Heilbrunn: Klinkhardt.

Bohnsack, R. (2003). Dokumentarische Methode und sozialwissenschaftliche Hermeneutik. Zeitschrift für Erziehungswissenschaft, 6 (4), 550-570. https://doi.org/10.1007/ s11618-003-0057-7

Bohnsack, R. (2010). Gruppendiskussionsverfahren und dokumentarische Methode. In B. Friebertshäuser (Hrsg.), Handbuch qualitative Forschungsmethoden in der Erziehungswissenschaft (S. 205-218). Weinheim: Juventa. https://doi.org/10.2307/j.ctvhktj dr.26

Bourdieu, P. (1987). Sozialer Sinn. Kritik der theoretischen Vernunft. Frankfurt am Main: Suhrkamp.

Bourdieu, P. (1992). Die feinen Unterschiede. In P. Bourdieu, M. Steinrücke, J. Bolder \& U. Nordmann (Hrsg.), Die verborgenen Mechanismen der Macht (S. 31-47). Hamburg: VSA.

Bromme, R. (2014). Der Lehrer als Experte. Zur Psychologie des professionellen Wissens. Münster: Waxmann.

Combe, A. \& Gebhard, U. (2007). Sinn und Erfahrung. Zum Verständnis fachlicher Lernprozesse in der Schule. Opladen: Barbara Budrich. https://doi.org/10.2307/j.ctvdfoopz

Combe, A. \& Helsper, W. (Hrsg.). (1996). Pädagogische Professionalität. Untersuchungen zum Typus pädagogischen Handelns. Frankfurt am Main: Suhrkamp.

Combe, A. \& Kolbe, F.-U. (2004). Lehrerprofessionalität: Wissen, Können, Handeln. In W. Helsper \& J. Böhme (Hrsg.), Handbuch der Schulforschung (S. 833-851). Wiesbaden: VS Verlag für Sozialwissenschaften. https://doi.org/10.1007/978-3-663-10249$6 \_34$ 
Dannemann, S., Niebert, K., Affeldt, S. \& Gropengießer, H. (2014). Fallsammlung zum Lehren und Lernen der Biologie - Entwicklung von Videovignetten. In I. Baumgardt (Hrsg.), Forschen, Lehren und Lernen in der Lehrerausbildung. Fachdidaktische Beiträge aus der universitären Praxis (S. 41-56). Baltmannsweiler: Schneider Verlag Hohengehren.

Dzengel, J. (2017). Kasuistik in der Lehrerbildung als Vermittlungsinstanz zwischen Theorie und Praxis? In T. Burger \& N. Miceli (Hrsg.), Empirische Forschung im Kontext Schule. Einführung in theoretische Aspekte und methodische Zugänge (S. 373-391). Wiesbaden: Springer VS. https://doi.org/10.1007/978-3-658-15437-0_24

Gautschi, P. (1995). Biographische Arbeit in der Lehrerbildung als Möglichkeit der Ausbildung von „Reflektierenden Praktikern“ - Ein Erfahrungsbericht. Beiträge zur Lehrerinnen- und Lehrerbildung, 13 (3), 293-299.

Goodwin, C. (1994). Professional vision. American Anthropologist, 96 (3), 606-633. https://doi.org/10.1525/aa.1994.96.3.02aoo100

Gresch, H. \& Martens, M. (2019). Teleology as a tacit dimension of teaching and learning evolution: A sociological approach to classroom interaction in science education. Journal of Research in Science Teaching, 56 (3), 243-269. https://doi.org/10.1002/ tea.21518

Groeben, N. \& Scheele, B. (2010). Das Forschungsprogramm Subjektive Theorien. In G. Mey \& K. Mruck (Hrsg.), Handbuch Qualitative Forschung in der Psychologie (S. 151-165). Wiesbaden: VS Verlag für Sozialwissenschaften. https://doi.org/10.1007/9783-531-92052-8_10

Gropengießer, H. (2008). Wie man Vorstellungen der Lerner verstehen kann. Lebenswelten, Denkwelten, Sprechwelten. Oldenburg: Didaktisches Zentrum Carl von Ossietzky-Universität.

Gropengießer, H. \& Marohn, A. (2018). Schülervorstellungen und Conceptual Change. In D. Krüger, I. Parchmann \& H. Schecker (Hrsg.), Theorien in der naturwissenschaftsdidaktischen Forschung (S. 49-68). Berlin: Springer. https://doi.org/10.1007/ 978-3-662-56320-5_4

Gruschka, A. (2005). Auf dem Weg zu einer Theorie des Unterrichtens. Die widersprüchliche Einheit von Erziehung, Didaktik und Bildung in der allgemeinbildenden Schule; Vorstudie. Frankfurt am Main: Fachbereich Erziehungswissenschaften der Johann Wolfgang Goethe-Universität.

Häcker, T. (2017). Grundlagen und Implikationen der Forderung nach Förderung von Reflexivität in der Lehrerinnen- und Lehrerbildung. In C. Berndt, T. H. Häcker \& T. Leonhard (Hrsg.), Reflexive Lehrerbildung revisited. Traditionen - Zugänge - Perspektiven (S. 21-45). Bad Heilbrunn: Klinkhardt.

Hagemann, W. \& Rose, F.-J. (1998). Zur Lehrer/innen-Erfahrung von Lehramts-Studierenden. Zeitschrift für Pädagogik, 44 (1), 7-19.

Hammann, M. \& Asshoff, R. (2014). Schülervorstellungen im Biologieunterricht. Ursachen für Lernschwierigkeiten. Seelze: Klett | Kallmeyer.

Hammerness, K., Darling-Hammond, L. \& Shulman, L. S. (2002). Toward expert thinking: How curriculum case-writing prompts the development of theory-based professional knowledge in student teachers. Teacher Education, 13, 221-245. https://doi. org/10.1080/1047621022000007594 
Hatton, N. \& Smith, D. (1995). Reflection in teacher education: Towards definition and implementation. Teaching and Teacher Education, 11 (1), 33-49. https://doi.org/10. 1016/0742-051X(94)00012-U

Helsper, W. (2000a). Antinomien des Lehrerhandelns und die Bedeutung der Fallrekonstruktion. In E. Cloer, D. Klika \& H. Kunert (Hrsg.), Welche Lehrer braucht das Land? Notwendige und mögliche Reformen der Lehrerbildung (S. 142-177). Weinheim: Juventa.

Helsper, W. (200ob). Zum systematischen Stellenwert der Fallrekonstruktion in der universitären Lehrerinnenbildung. In C. Beck, W. Helsper, B. Heuer, B. Stelmaszyk \& H. Ullrich (Hrsg.), Fallarbeit in der universitären LehrerInnenbildung. Professionalisierung durch fallrekonstruktive Seminare? Eine Evaluation (S. 29-50). Wiesbaden: VS Verlag für Sozialwissenschaften.

Helsper, W. (2001). Praxis und Reflexion. Die Notwendigkeit einer „doppelten Professionalisierung“ des Lehrers. Journal für Lehrerinnen- und Lehrerbildung (3), 7-15.

Helsper, W. (2016). Lehrerprofessionalität - der strukturtheoretische Ansatz. In M. Rothland (Hrsg.), Beruf Lehrer/Lehrerin. Ein Studienbuch (S. 103-127). Münster: Waxmann (UTB).

Herzmann, P. \& Proske, M. (2014). Unterrichtsvideographien als Medium der Beobachtung und Reflexion von Unterricht im Lehramtsstudium. Ein Forschungsbericht. Journal für LehrerInnenbildung, 14 (1), 33-38.

Herzog, W. (1995). Reflexive Praktika in der Lehrerinnen- und Lehrerbildung. Beiträge zur Lehrerbildung, 13 (3), 235-273.

Hummrich, M. (2016). Was ist der Fall? Zur Kasuistik in der Erziehungswissenschaft. In M. Hummrich, A. Hebenstreit, M. Hinrichsen \& M. Meier (Hrsg.), Was ist der Fall? Kasuistik und das Verstehen pädagogischen Handelns (S. 13-38). Wiesbaden: Springer VS. https://doi.org/10.1007/978-3-658-04340-7

Kattmann, U. (Hrsg.). (2017a). Biologie unterrichten mit Alltagsvorstellungen. Didaktische Rekonstruktion in Unterrichtseinheiten. Seelze: Klett | Kallmeyer.

Kattmann, U. (2017b). Einführung. In U. Kattmann (Hrsg.), Biologie unterrichten mit Alltagsvorstellungen. Didaktische Rekonstruktion in Unterrichtseinheiten (S. 6-13). Seelze: Klett | Kallmeyer.

Kattmann, U., Duit, R., Gropengießer, H. \& Komorek, M. (1997). Das Modell der Didaktischen Rekonstruktion. Ein Rahmen für naturwissenschaftliche Forschung und Entwicklung. Zeitschrift für Didaktik der Naturwissenschaften, 3 (3), 3-18.

Kelemen, D. (2012). Teleological minds: How natural intuitions about agency and purpose influence learning about evolution. In K. S. Rosengren (Hrsg.), Evolution challenges. Integrating research and practice in teaching and learning about evolution (S. 66-92). New York: Oxford University Press. https://doi.org/10.1093/acprof:oso/ 9780199730421.003.0004

Kersting, N. B., Givvin, K. B., Sotelo, F. \& Stigler, J. W. (2010). Teachers' analyses of classroom video predict student learning of mathematics: Further explorations of a novel measure of teacher knowledge. Journal of Teacher Education, 61 (1-2), 172-181. https://doi.org/10.1177/0022487109347875

Kultusministerkonferenz (2014). Standards für die Lehrerbildung: Bildungswissenschaften. (Beschluss der Kultusministerkonferenz vom 16.12.2004 i. d. F. vom 16.05.2019). Verfügbar unter: https://www.kmk.org/fileadmin/Dateien/veroeffentlichungen_beschluesse/ 2004/2004_12_16-Standards-Lehrerbildung-Bildungswissenschaften.pdf [06.11.2019]. 
Krammer, K. (2014). Fallbasiertes Lernen mit Unterrichtsvideos in der Lehrerinnen- und Lehrerbildung. Beiträge zur Lehrerinnen- und Lehrerbildung, 32 (2), 164-175.

Kunze, K. (2017). Reflexivität und Routine. Zur empirischen Realität kasuistischer Gruppenarbeit im Universitätsseminar. In C. Berndt, T. H. Häcker \& T. Leonhard (Hrsg.), Reflexive Lehrerbildung revisited. Traditionen - Zugänge - Perspektiven (S. 214-227). Bad Heilbrunn: Klinkhardt.

Leuchter, M., Pauli, C., Reusser, K. \& Lipowsky, F. (2006). Unterrichtsbezogene Überzeugungen und handlungsleitende Kognitionen von Lehrpersonen. Zeitschrift für Erziehungswissenschaft, 9 (4), 562-579. https://doi.org/10.1007/s11618-006-0168-Z

Liegmann, A. B., Racherbäumer, K. \& Drucks, S. (2018). Studentische Dispositionen zum Forschenden Lernen. In T. Leonhard, J. Košinár \& C. Reintjes (Hrsg.), Praktiken und Orientierungen in der Lehrerbildung. Potentiale und Grenzen der Professionalisierung (S. 175-190). Bad Heilbrunn: Klinkhardt.

Lutz, G. (2019). Reflexion, kognitionspsychologisch. In M. A. Wirtz (Hrsg.), Dorsch Lexikon der Psychologie. Verfügbar unter: https://portal.hogrefe.com/dorsch/reflexionkognitionspsychologisch/ [01.04.2019].

Mannheim, K. (1980). Strukturen des Denkens. Frankfurt am Main: Suhrkamp.

Martens, M. \& Gresch, H. (2018). Ambivalente Fachlichkeiten. Die (Re)Produktion fachlicher Vorstellungen im Biologieunterricht. In M. Martens, K. Rabenstein, K. Bräu, M. Fetzer, H. Gresch, I. Hardy \& C. Schelle (Hrsg.), Konstruktionen von Fachlichkeit. Ansätze, Erträge und Diskussionen in der empirischen Unterrichtsforschung (S. 275-288). Bad Heilbrunn: Klinkhardt.

Meschede, N. (2014). Professionelle Wahrnehmung der inhaltlichen Strukturierung im naturwissenschaftlichen Grundschulunterricht. Theoretische Beschreibung und empirische Erfassung. Berlin: Logos.

Naguib, M. (2006). Methoden der Verhaltensbiologie. Berlin: Springer.

Nentwig-Gesemann, I. (2014): Die Unterrichtssequenz Parallelogramme II - Fallrekonstruktion mit der Dokumentarischen Methode. In I. Pieper, P. Frei, K. Hauenschild \& B. Schmidt-Thieme (Hrsg.), Was der Fall ist. Beiträge zur Fallarbeit in Bildungsforschung, Lehramtsstudium, Beruf und Ausbildung (S. 123-138). Wiesbaden: Springer VS. https://doi.org/10.1007/978-3-531-19761-6_8

Neuweg, G. H. (2002). Lehrerhandeln und Lehrerbildung im Lichte des Konzepts des impliziten Wissens. Zeitschrift für Pädagogik, 48 (1), 10-29.

Neuweg, G. H. (2011). Das Wissen der Wissensvermittler - Problemstellungen, Befunde und Perspektiven der Forschung zum Lehrerwissen. In E. Terhart (Hrsg.), Handbuch der Forschung zum Lehrerberuf (S. 451-477). Münster: Waxmann.

Nisbett, R. E. \& Wilson, T. D. (1977). Telling more than we can know. Verbal reports on mental processes. Psychological Review, 84 (3), 231-259. https://doi.org/10.1037/0033295X.84.3.231

Ohlhaver, F. \& Wernet, A. (Hrsg.). (1999). Schulforschung Fallanalyse Lehrerbildung. Wiesbaden: VS Verlag für Sozialwissenschaften. https://doi.org/10.1007/978-3-32297419-8

Pflugmacher, T. (2014). Möglichkeiten und Grenzen kasuistischer Literaturdidaktik in der Deutschlehrerausbildung. In I. Pieper, P. Frei, K. Hauenschild \& B. SchmidtThieme (Hrsg.), Was der Fall ist. Beiträge zur Fallarbeit in Bildungsforschung, Lehramtsstudium, Beruf und Ausbildung (S. 183-200). Wiesbaden: Springer VS. https:// doi.org/10.1007/978-3-531-19761-6_12 
Pieper, I., Frei, P., Hauenschild, K. \& Schmidt-Thieme, B. (Hrsg.). (2014). Was der Fall ist. Beiträge zur Fallarbeit in Bildungsforschung, Lehramtsstudium, Beruf und Ausbildung. Wiesbaden: Springer VS. https://doi.org/10.1007/978-3-531-19761-6

Przyborski, A. \& Wohlrab-Sahr, M. (2014). Qualitative Sozialforschung. Ein Arbeitsbuch. München: Oldenbourg. https://doi.org/10.1524/9783486719550

Rehm, M., Bölsterli, K., Brovelli, D. \& Wilhelm, M. (2014). Entwicklung von Unterrichtsvignetten. In D. Krüger, I. Parchmann \& H. Schecker (Hrsg.), Methoden in der naturwissenschaftsdidaktischen Forschung (S. 213-225). Berlin: Springer. https://doi. org/10.1007/978-3-642-37827-0_18

Riemeier, T. (2007). Moderater Konstruktivismus. In D. Krüger \& H. Vogt (Hrsg.), Theorien in der biologiedidaktischen Forschung. Ein Handbuch für Lehramtsstudenten und Doktoranden (S. 69-79). Berlin: Springer.

Roth, K. J., Garnier, H. E., Chen, C., Lemmens, M., Schwille, K. \& Wickler, N. I. Z. (2011). Videobased lesson analysis. Effective science PD for teacher and student learning. Journal of Research in Science Teaching, 48 (2), 117-148. https://doi.org/10. 1002/tea.20408

Schlömerkemper, J. (2006). Die Kompetenz des antinomischen Blicks. In W. Plöger (Hrsg.), Was müssen Lehrerinnen und Lehrer können? (S. 281-308). Paderborn: Schöningh.

Seidel, T., Blomberg, G. \& Stürmer, K. (2010). „Observer“ - Validierung eines videobasierten Instruments zur Erfassung der professionellen Wahrnehmung von Unterricht. Projekt OBSERVE. In E. Klieme (Hrsg.), Kompetenzmodellierung. Zwischenbilanz des DFG-Schwerpunktprogramms und Perspektiven des Forschungsansatzes (S. 296-306). Weinheim: Beltz.

Seidel, T., Prenzel, M., Schwindt, K., Stürmer, K., Blomberg, G. \& Kobarg, M. (2009). LUV and Observe: Two projects using video to diagnose teachers' competence. In T. Janík \& T. Seidel (Hrsg.), The power of video studies in investigating teaching and learning in the classroom (S. 243-258). Münster: Waxmann.

Sherin, M. G. (2007). The development of teachers' professional vision in video clubs. In R. Goldman (Hrsg.), Video research in the learning sciences (S. 383-395). Mahwah NJ: Erlbaum.

Sherin, M. G. \& van Es, E. A. (2009). Effects of video club participation on teachers' professional vision. Journal of Teacher Education, 60 (1), 20-37. https://doi.org/10.1177/ O022487108328155

Steiner, E. (2014). Kasuistik - ein Fall für angehende und praktizierende Lehrpersonen. Beiträge zur Lehrerinnen- und Lehrerbildung 32 (1), 6-20.

Steinwachs, J. \& Gresch, H. (2019). Umgang mit Schülervorstellungen im Evolutionsunterricht - Implizites Wissen von Lehramtsstudierenden bei der Wahrnehmung von Videovignetten. Zeitschrift für interpretative Schul- und Unterrichtsforschung, 8, 24-39.

Stelmaszyk, B. (200o). (Rekonstruktiv-)fallorientierte Arbeiten in der Lehrerlnnenbildung - ein Überblick. In C. Beck, W. Helsper, B. Heuer, B. Stelmaszyk \& H. Ullrich (Hrsg.), Fallarbeit in der universitären LehrerInnenbildung. Professionalisierung durch fallrekonstruktive Seminare? Eine Evaluation (S. 13-23). Wiesbaden: VS Verlag für Sozialwissenschaften.

Sunder, C., Todorova, M \& Möller, K. (2016). Kann die professionelle Unterrichtswahrnehmung von Sachunterrichtsstudierenden trainiert werden? - Konzeption und 
Erprobung einer Intervention mit Videos aus dem naturwissenschaftlichen Grundschulunterricht. Zeitschrift für Didaktik der Naturwissenschaften 22 (1), 1-12. https:// doi.org/10.1007/s40573-015-0037-5

Syring, M., Bohl, T., Kleinknecht, M., Kuntze, S., Rehm, M. \& Schneider, J. (2016). Fallarbeit als Angebot - fallbasiertes Lernen als Nutzung. Empirische Ergebnisse zur kognitiven Belastung, Motivation und Emotionen bei der Arbeit mit Unterrichtsfällen. Zeitschrift für Pädagogik, 62 (1), 86-108.

Zabel, J. \& Gropengießer, H. (2010). Darwins konzeptuelle Landkarte: Lernfortschritt im Evolutionsunterricht. In U. Harms \& F. X. Bogner (Hrsg.), Lehr- und Lernforschung in der Biologiedidaktik (S. 209-224). Innsbruck: Studien Verlag. 


\title{
Stolpersteine im Lehrerhandeln: Aufbau eines Handlungsrepertoires im Kontext Schülervorstellungen
}

\author{
Das chemiedidaktische Lehr-Lern-Labor C(LE) ${ }^{2}$ VER
}

Der Beitrag stellt das chemiedidaktische Lehr-Lern-Labor $C(L E)^{2}$ VER vor: Durch den Einsatz von fremden und eigenen Videovignetten, erhalten Lehramtsstudierende einen authentischen Einblick in den Unterricht zu Schülervorstellungen und werden mit typischen Stolpersteinen im Lehrerhandeln konfrontiert. Sie erhalten die Chance eigenes Unterrichtshandeln zu erproben, indem sie eine Stunde zu Schülervorstellungen planen, durchführen und videobasiert anhand eines Reflexionsschemas reflektieren. Zur Unterstützung werden gezielte Maßnahmen eingesetzt, die die Komplexität der Anforderungssituation reduzieren. Der Beitrag präsentiert zwei Modelle $z u$ notwendigen Fähigkeiten von Lehrkräften im Kontext von Schülervorstellungen und Stolpersteinen im Lehrerhandeln; er erläutert die Struktur der Lehr-Lern-LaborVeranstaltung und erörtert in Anlehnung an das Modell zur „Komplexität in LehrLern-Laboren" Anforderungen und unterstützende Faktoren. Abschließend werden Perspektiven der Studierenden auf die Komplexität des Settings dargestellt.

Schlüsselwörter: Schülervorstellungen, Unterrichtsvideos, Stolpersteine im Lehrerhandeln

\section{Motivation}

Obwohl sich die Modelle professioneller Handlungskompetenz von Chemielehrkräften in einzelnen Dimensionen unterscheiden, so herrscht in einem Punkt Konsens: Lehrkräfte benötigen die Fähigkeit, Schülervorstellungen zu erkennen und Handlungsoptionen daraus abzuleiten (z. B. Großebrahm, 2013). Folgerichtig bildet die Vorbereitung auf den Umgang mit Schülervorstellungen ein wesentliches Ziel der universitären Lehrerbildung (Kultusministerkonferenz [KMK], 2004, 2008). Doch wie kann universitäre Lehre Studierende an diese Kompetenz heranführen? Die Vermittlung eines rein deklarativen Wissens über Schülervorstellungen und Conceptual-Change-Theorien reicht hierzu nicht aus. Fachdidaktische Theorien und Erkenntnisse empirischer Forschung müssen wechselseitig mit unterrichtlicher Praxis verknüpft werden.

Genau dies bildet das Ziel des hier vorgestellten Lehr-Lern-Labors C(LE) ${ }^{2}$ VER („Chemie lehren und lernen - Videografie ermöglicht Reflexion“): In der Vorbereitungsphase analysieren die Studierenden mit Hilfe von Videovignetten theorie- und forschungsbasiert Unterrichtssequenzen, die sich an Schülervorstellungen orientieren. Das erworbene Wissen wird in der folgenden Phase genutzt, 
um eine eigene Unterrichtseinheit zu entwickeln, mit Schülerinnen und Schülern durchzuführen und videobasiert, mit Rückbezug zu den erlernten Theorien, zu reflektieren. Ein besonderer Fokus liegt dabei auf typischen „Stolpersteinen“ im Lehrerhandeln, die im Kontext Schülervorstellungen auftreten und die es in der Unterrichtspraxis zu meistern gilt. Zur Identifikation dieser Stolpersteine wurden Videos aus vorangegangenen Lehr-Lern-Labor-Veranstaltungen inhaltsanalytisch ausgewertet und geeignete Sequenzen für den Einsatz in der Lehrerbildung ausgewählt.

Im Rahmen eines Prä-Post-Designs werden anhand von Videovignetten mögliche Veränderungen in den Bereichen „Wissen“ und „(reflexive) Fähigkeiten“ der Studierenden im Kontext Stolpersteine im Lehrerhandeln untersucht (vgl. Modell in Abb. 2). Zudem werden Einstellungen zur Videografie sowie Selbstwirksamkeitserwartungen bezüglich des Umgangs mit Schülervorstellungen und Stolpersteinen erfasst. In diesem Beitrag werden exemplarisch Ergebnisse aus Fragebögen und Interviews sowie Ausschnitte aus den Studierenden-Portfolios dargestellt; diese liefern Erkenntnisse, inwiefern die Studierenden die Arbeit im Lehr-Lern-Labor als „komplexitätsreduziert“ erleben.

Der Beitrag beschreibt zunächst theoretische Grundlagen und die Struktur des Lehr-Lern-Labors. Er fokussiert sodann Faktoren, die die Komplexität des Settings reduzieren. Mit Bezug zum Modell der Komplexitätsreduktion (Marohn, Greefrath, Hammann, Hemmer, Kürten \& Windt, in diesem Band) werden inhaltliche Anforderungen und Unterstützungsmaßnahmen erläutert und Einschätzungen der Studierenden dargestellt.

\section{Hintergrund}

Lehr-Lern-Labore bieten die Möglichkeit, fachdidaktische und pädagogische Inhalte mit eigenem Handeln in der Praxis zu verknüpfen. Den inhaltlichen Schwerpunkt des hier vorgestellten Lehr-Lern-Labor-Seminars bilden der Umgang mit Schülervorstellungen sowie die Reflexion typischer Stolpersteine, die einer Lehrkraft in diesem Rahmen begegnen können. Um diese Inhalte mit den Studierenden an authentischen Situationen erarbeiten zu können, werden Unterrichtsvideos als methodischer Zugang genutzt: Durch die Analyse fremder Videovignetten gewinnen die Studierenden Einblicke in den Kontext Schülervorstellungen und erhalten die Möglichkeit, ihre reflexiven Fähigkeiten zu verbessern. Gleichzeitig werden sie darauf vorbereitet, ihren eigenen Unterricht videobasiert zu reflektieren. Die folgenden Abschnitte erläutern, welche Wissenselemente und Fähigkeiten zu Schülervorstellungen und Stolpersteinen im Rahmen der Veranstaltung angebahnt werden; sie erörtern die Bedeutung einer reflektierten Praxis und beschreiben den Nutzen von fremden und eigenen Videovignetten in der Lehrerbildung. 


\subsection{Schülervorstellungen in der Lehrerbildung}

„Das Salz im Nudelwasser verschwindet“. „Beim Wasserkochen bilden sich Wasserstoff und Sauerstoff" (Marohn, 2008b). Jeder oder jede Lernende konstruiert individuelle Vorstellungen, indem er oder sie neue Informationen mit bestehenden Erfahrungen und Denkkonzepten verknüpft (z. B. Reinmann-Rothmeier \& Mandl, 2001; Kelly, 1963). Diese Vorstellungen können von fachwissenschaftlichen Konzepten abweichen und den Lernprozess im naturwissenschaftlichen Unterricht maßgeblich beeinflussen. Ansätze von Lehrkräften, ihren Unterricht an Schülervorstellungen zu orientieren, enden aufgrund der Fülle an Theorien und Literatur nicht selten in Überforderung (z. B. Gilbert, Justi, van Driel, Jong \& Treagust, 2004; Marohn, 2008a). Studierende sollten daher an die Anforderungen eines adäquaten Umgangs mit Schülervorstellungen herangeführt werden. Doch welches Wissen und welche Fähigkeiten setzt ein solch adäquater Umgang voraus? Zur Klärung dieser Frage wurde in einem Vorläuferprojekt ein Modell entwickelt, das wesentliche Wissenselemente und Fähigkeiten im Bereich Schülervorstellungen abbildet (siehe Tab. 1; Marohn, 2014; Marohn \& Rohrbach, 2013; RohrbachLochner \& Marohn, 2018).

Tabelle 1: Modell zu notwendigen Wissenselementen und Fähigkeiten von Lehrkräften im Kontext Schülervorstellungen (aus: Marohn \& Rohrbach, 2013).

\begin{tabular}{|c|c|c|c|c|}
\hline & Wissen & Verstehen & Anwendung & Synthese \\
\hline 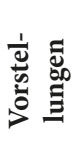 & $\begin{array}{l}\text { Typische } \\
\text { Vorstellungen } \\
\text { kennen }\end{array}$ & $\begin{array}{l}\text { Vorstellungen in } \\
\text { Schüleraussagen } \\
\text { identifizieren }\end{array}$ & $\begin{array}{l}\text { Vorstellungen } \\
\text { antizipieren }\end{array}$ & $\begin{array}{c}\text { Konsequenzen } \\
\text { für die Gestaltung } \\
\text { von Unterricht aus } \\
\text { Vorstellungen ziehen }\end{array}$ \\
\hline 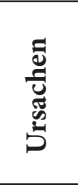 & $\begin{array}{l}\text { Ursachen von } \\
\text { Vorstellungen kennen }\end{array}$ & $\begin{array}{l}\text { Ursachen von } \\
\text { Vorstellungen } \\
\text { identifizieren }\end{array}$ & $\begin{array}{c}\text { Ursachen von } \\
\text { Vorstellungen } \\
\text { antizipieren }\end{array}$ & $\begin{array}{c}\text { Konsequenzen } \\
\text { für die Gestaltung } \\
\text { von Unterricht } \\
\text { aus Ursachen von } \\
\text { Vorstellungen ziehen }\end{array}$ \\
\hline 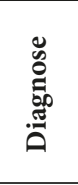 & $\begin{array}{l}\text { Diagnose- } \\
\text { instrumente und } \\
\text { methoden kennen }\end{array}$ & $\begin{array}{l}\text { Vor- und Nachteile } \\
\text { von Diagnose- } \\
\text { instrumenten } \\
\text { und -methoden } \\
\text { identifizieren }\end{array}$ & $\begin{array}{l}\text { Diagnose- } \\
\text { instrumente } \\
\text { und -methoden } \\
\text { anwenden }\end{array}$ & $\begin{array}{l}\text { Diagnoseinstrumente } \\
\text { und -methoden } \\
\text { selbstständig } \\
\text { entwickeln und } \\
\text { bewerten }\end{array}$ \\
\hline 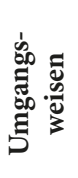 & $\begin{array}{c}\text { Beschriebene } \\
\text { Umgangsweisen mit } \\
\text { Vorstellungen kennen }\end{array}$ & $\begin{array}{l}\text { Umgangsweisen mit } \\
\text { Vorstellungen als } \\
\text { mehr oder weniger } \\
\text { geeignet identifizieren }\end{array}$ & $\begin{array}{l}\text { Umgangsweisen } \\
\text { mit Vorstellungen } \\
\text { anwenden und } \\
\text { reflektieren }\end{array}$ & $\begin{array}{l}\text { Selbstständig } \\
\text { Umgangsweisen } \\
\text { mit Vorstellungen } \\
\text { entwickeln und } \\
\text { bewerten }\end{array}$ \\
\hline
\end{tabular}

Das Modell dient als Grundlage für die Gestaltung der ersten Phase des LehrLern-Labors, der "Forschungsbasierten Vorbereitung“ (vgl. Abschnitt 3.2.1): Zunächst werden Beispiele für Schülervorstellungen im Chemieunterricht thematisiert, um darauf aufbauend verschiedene Ursachen und Diagnosemöglichkeiten 
zu behandeln. Im letzten Schritt werden Wege der Veränderung erarbeitet. Voraussetzung für die inhaltliche Auseinandersetzung mit Schülervorstellungen ist ein grundlegendes Verständnis wesentlicher Lehr-Lern-Theorien (z.B. des moderaten Konstruktivismus) und Conceptual-Change-Ansätze (Gropengießer \& Marohn, 2018).

\subsection{Stolpersteine im Lehrerhandeln}

Unterrichtshandeln von Lehrkräften ist vielfältig, häufig unvorhersehbar und von herausfordernden Momenten durchzogen (z. B. Combe \& Helsper, 1996; Doyle, 1986; Fend, 1998). Mühlhausen (2007) differenziert zwischen zwei Aktionstypen: „Handeln aufgrund vorausgegangener Planung" (S. 191) sowie „rasches, ungeplantes Reagieren, falls vom Entwurf abgewichen werden muss" (S. 192). Wenn Lehrkräfte von ihrer Planung abweichen müssen, handeln sie häufig unter Druck (Wahl, 1991). Mühlhausen (1994) spricht in diesem Zug von dem „Überrumpeltwerden von einer Situation [...], die man so nicht erwartet hat und auf die man sich einstellen muß." (S. 15).

Überraschende oder erwartungswidrige Situationen, die Lehrkräfte im Moment des Handelns als herausfordernd empfinden, werden in diesem Beitrag als Stolpersteine bezeichnet. Stolpersteine können sich aus einer unzureichenden Antizipation bzw. Planung des Unterrichtsgeschehens ergeben (z.B. der falschen Einschätzung von Lernvoraussetzungen), aus überraschenden Momenten (z. B. einem nicht funktionierenden Experiment) oder aufgrund von unerwartet agierenden Schülerinnen und Schülern (z. B. einer nicht erwarteten Schülervorstellung); sie können durch Abweichungen vom geplanten Handeln hervorgerufen werden (z. B. durch die spontane Nutzung eines anderen Modells) oder durch den Unterrichtsgegenstand selbst (z. B. aufgrund eines hohen Abstraktionsgrads des Lerngegenstandes).

Der anschauliche Begriff des Stolpersteins impliziert bereits, dass dieser unterschiedliche Folgen nach sich ziehen kann: Die Lehrkraft kann ihn in der Situation rechtzeitig erkennen und umgehen, oder ihn - nach kurzem "Stolpern“ - durch Anwendung geeigneter Strategien überwinden. Beim Fehlen adäquater Strategien kann ein Stolperstein jedoch auch zum Scheitern des Lehr-Lern-Prozesses führen (z. B. zu einem falschen Verständnis des Lerngegenstandes).

Studien zeigen, dass erfahrene Lehrkräfte besser mit unerwarteten Situationen umgehen können als Berufsanfängerinnen und -anfänger. Dies wird häufig mit größerer „Routine“ im Sinne einer unbewussten Anwendung von Schemata erklärt: „Die Situation [wird] als Repräsentant eines bekannten Typus [,wiedererkannt'] und dann ein für passend gehaltenes Muster darauf [angewendet] " (Mühlhausen, 2007, S. 195; vgl. auch Hofer, 1986; Wahl, 1991). Demgegenüber sehen andere Forschende das Handeln in unerwarteten Situationen als Resultat einer bewussten, reflektierten Entscheidung: Erfahrene Lehrkräfte verfügen demnach über ein breiteres Handlungsrepertoire, auf das sie flexibel und situationsangemessen 
zurückgreifen und das sie stetig anpassen und erweitern (Gruber, 2007; Hattie, 2010; Luhmann, 2002; Mühlhausen, 1994). Ziel des hier vorgestellten Lehrkonzeptes ist es, durch Konfrontation und Reflexion von Stolperstein-Situationen ein entsprechendes Handlungsrepertoire anzubahnen, um damit ein bewusstes Agieren in unerwarteten Momenten zu ermöglichen (Biedermann, 2011; Leonhard \& Rihm, 2011).

Dabei lassen sich zwei Kategorien von Stolpersteinen differenzieren: Situationen, die häufig auftreten und als typisch bezeichnet werden können, und Situationen, die Einzelfälle darstellen und somit kaum antizipierbar sind. Das Seminarkonzept fokussiert Stolpersteine der ersten Kategorie im Kontext Schülervorstellungen. Dazu wurden auf Basis von Videovignetten aus vier vorangegangenen Lehr-Lern-Labor-Veranstaltungen zum Thema Schülervorstellungen typische Stolperstein-Situationen identifiziert und kategorisiert.

Um angehende Lehrkräfte auf den Umgang mit herausfordernden Unterrichtssituationen vorzubereiten, wurde auf Basis vorangegangener Seminardurchläufe ein normatives Modell entwickelt, das erforderliche Kenntnisse und Fähigkeiten zur Vermeidung und Bewältigung von Stolperstein-Situationen abbildet (siehe Abb. 1). Es greift wesentliche Elemente von Reflexionsschemata auf und transferiert diese auf den Inhaltsbereich Stolpersteine: das Beschreiben von Situationen, das Erkennen potenzieller Ursachen sowie die Entwicklung von Planungs- und Handlungsalternativen (z. B. Denner \& Gesenhues, 2013; Kohler, Prinz, Schneider \& Syring, 2015; Schneider, 2016).

Das Modell dient der strukturellen Rahmung des Seminars, indem es notwendige Schritte zur Anbahnung eines Handlungsrepertoires im Umgang mit Stolpersteinen aufzeigt: Die Grundlage bildet das Wissen über typische Stolpersteine im Kontext Schülervorstellungen. Hierzu werden Unterrichtssituationen anhand von fremden Videovignetten analysiert, mögliche Ursachen für auftretende Stolpersteine ermittelt und adäquate Vermeidungs- und Bewältigungsstrategien entwickelt. Auf diese Weise entsteht eine Sammlung, auf die in der anschließenden Planung und Durchführung eigener Unterrichtseinheiten zurückgegriffen werden kann. Zusätzlich zu spezifischen Vermeidungs- und Bewältigungsansätzen werden zudem übergreifende Strategien entwickelt, die problematische Unterrichtssituationen entschleunigen können und der Lehrkraft Zeit zum Nachdenken verschaffen. Im abschließenden Reflexionsprozess werden anhand eigener Videovignetten herausfordernde Unterrichtssituationen als Lernanlass genutzt, um Rückschlüsse für weitere Planungen zu ziehen. Dabei ist es nicht das Ziel, rezeptartige Handlungsstrategien zu vermitteln, sondern vielmehr ein vertieftes Nachdenken über eigene Handlungen zu initiieren und "Reservepläne“ (Mühlhausen, 2007, S. 219) zu schaffen, die situationsadäquat eingesetzt werden können. 
Handlungsrepertoire zur Vermeidung und Bewältigung von Stolpersteinen

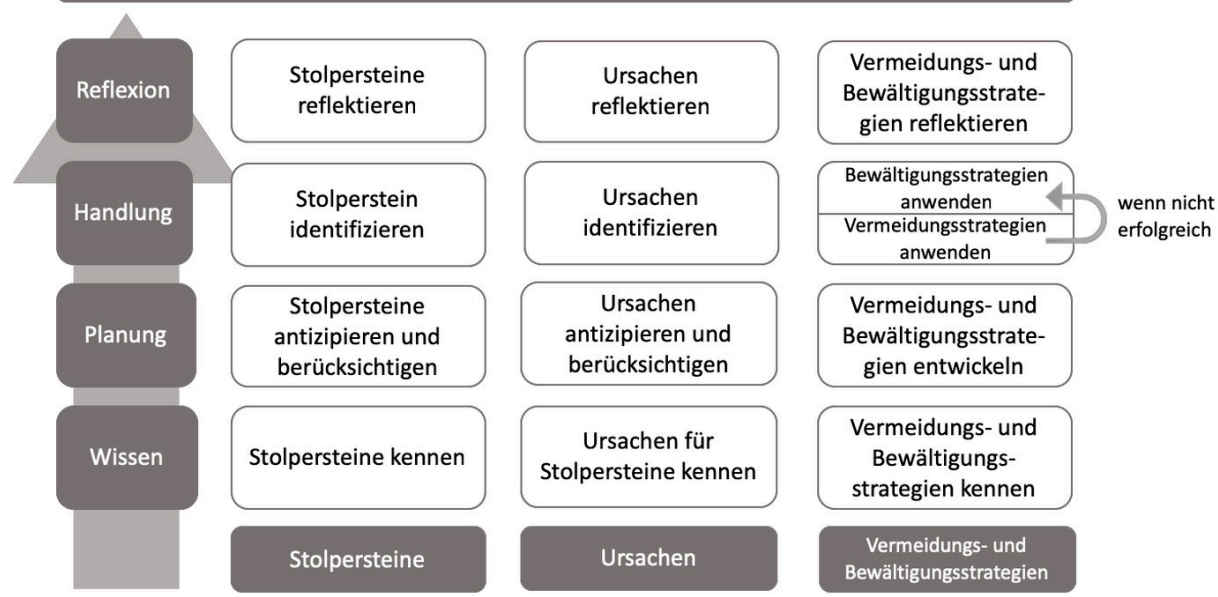

Abbildung 1: Modell „Anbahnung eines Handlungsrepertoires zur Vermeidung und Bewältigung von Stolpersteinen“.

\subsection{Reflektierte Praxis}

Im vorangegangenen Kapitel wurde die Relevanz der Reflexion von Unterrichtshandlungen für die eigene professionelle Entwicklung bereits angedeutet. Reflexionsfähigkeit ist als eine wesentliche Facette von professioneller Handlungskompetenz unumstritten (z. B. Denner \& Gesenhues, 2013; Gruber, Heid \& Bauer, 2005; Gruber \& Rehrl, 2005; Rambow \& Bromme, 2000). Day (1993) bezeichnet sie sogar als „sine qua non“ (S. 83) der professionellen Entwicklung. Eine Lehrkraft mit ausgeprägter Reflexionsfähigkeit sieht in Anforderungssituationen einen Lernanlass, in dem das eigene Handeln analysiert und Handlungsoptionen entwickelt werden können (Biederman \& Oser, 2011). Trotz dieser Bedeutsamkeit zeigt die aktuelle Forschung, dass Reflexionsfähigkeit in der Lehrerbildung immer noch unzureichend gefördert wird und Lehrkräfte über kein adäquates Verständnis von Reflexion verfügen (Wyss, 2013). Ein Appell an die universitäre Lehrerbildung lautet daher, "träges" Wissen zu vermeiden, indem geeignete Theorie-Praxis-Bezüge - z. B. in Form der Beobachtung und Analyse von Unterricht - hergestellt werden, um die komplexen Anforderungen im Lehrberuf zu reflektieren (Brouwer \& Korthagen, 2005; KMK, 2004, 2008; Terhart, 2000).

Allerdings existiert bislang keine einheitliche Begriffsbestimmung des Konstruktes Reflexion (Abels, 2011; Schneider, 2016). Aus diesem Grund wurden zunächst wesentliche Aussagen der Literatur für ein eigenes Arbeitsverständnis extrahiert. Nach Dewey (1951) steht zu Beginn der Reflexion stets ein Problem oder eine Verunsicherung, welche einen Lösungsprozess nach sich zieht. Schön (1983) erweitert in seinen Ausführungen den Reflexionsbegriff, indem er zwischen Reflexionsprozessen in der Handlung („reflection-in-action“) und Reflexionsprozessen über die Handlung („reflection-on-action“) differenziert. Er prägt zudem 
den Begriff des „Reflective Practitioner“, indem er den Fokus auf die Verknüpfung zwischen Reflexion und Handeln legt. Aus den zentralen Aussagen des Reflexionsverständnisses von Dewey und Schön ergeben sich folgende Umsetzungen in Bezug auf das Seminarkonzept:

- Die Probleme/Verunsicherungen entsprechen in diesem Seminarkonzept verschiedenen Stolpersteinen im Kontext Schülervorstellungen (siehe Kap. 2.2)

- Die Reflexion der Stolpersteine erfolgt vorrangig in Form einer reflection-onaction, indem fremdes oder eigenes Unterrichtshandeln anhand von Videos analysiert wird. Eine reflection-in-action kann im Rahmen der Durchführungsphase im Lehr-Lern-Labor ebenfalls stattfinden, wird aber nicht explizit gefordert.

- Die Reflexion vollzieht sich anhand eines Reflexionsschemas in fünf Stufen: (1) Beschreibung der Situation mit den getätigten Handlungen, (2) Benennung des Stolpersteins, (3) Erkennen potenzieller Ursachen, (4) Entwicklung von Bewältigungsstrategien, (5) Entwicklung von Vermeidungsstrategien. Die Komplexität der betrachteten Situationen nimmt dabei sukzessive zu.

- Durch die wiederholte Anwendung operationalisierter Reflexionsschritte erwerben die Studierenden Reflexionsroutinen; sie leiten zudem Erkenntnisse für das eigene Handeln im Lehr-Lern-Labor ab, das im Anschluss wiederum reflektiert wird. Auf diese Weise werden sie an die Denk- und Handlungsweise eines Reflective Practitioner herangeführt und lernen, Reflexion als lebenslangen Lernprozess zu begreifen (Copeland, Birmingham, De la Cruz \& Lewin, 1993).

\subsection{Videografie}

Unterrichtsvideos bilden ein hilfreiches Instrument, um reale Handlungsanforderungen abzubilden und reflektierte Praxiserfahrungen zu ermöglichen. Sie sind in besonderem Maße dazu geeignet, Theorie-Praxis-Bezüge herzustellen: Unterrichtssituationen werden auf Basis zuvor erworbener Theorien analysiert und umgekehrt theoretische Rückschlüsse aus beobachteten Sequenzen gezogen (Krammer \& Reusser, 2005). Darüber hinaus bieten sie weitere Vorteile: Sie wirken kognitiv und emotional aktivierend (Weidenmann, 2001), stellen die komplexen Unterrichtsvorgänge realitätsnah dar (Kittelberger \& Freisleben, 1991), haben eine hohe Authentizität, lassen sich ohne Handlungsdruck aus verschiedenen Blickwinkeln betrachten (Krammer \& Reusser, 2005) und sind beliebig oft wiederholbar (Krammer, Hugener \& Biaggi, 2012).

Trotz zahlreicher Vorteile beinhaltet der Einsatz von Videovignetten auch Grenzen: Videos bilden nur einen Ausschnitt der Realität ab und bieten zudem keine Möglichkeit, mit den Darstellenden zu interagieren. Die Auseinandersetzung mit dem Video bleibt stets auf der Ebene des Beobachtens und Interpretierens (Krammer \& Reusser, 2005). Darüber hinaus müssen Videovignetten in ein adäquates Lehr-Lern-Setting eingebettet werden; die Lernwirksamkeit des Videoeinsatzes hängt somit stets von der Qualität der didaktischen Rahmung ab. 
Der Videoeinsatz kann auf vielfältige Weise gestaltet werden: Während fremde Videos sich vor allem zur kritischen Auseinandersetzung mit Unterricht eignen und die Entwicklung alternativer Handlungsoptionen fördern, haben eigene Videos einen stärkeren Aufforderungscharakter aufgrund eines höher empfundenen Einflusses auf die eigene Praxis (Kleinknecht \& Schneider, 2013; Seidel, Stürmer, Blomberg, Kobarg \& Schwindt, 2011). Die Literatur deutet darauf hin, dass eine Kombination aus beiden Videoformen für die Förderung der professionellen Wahrnehmung besonders geeignet ist (Hellermann, 2015).

Die Förderung der Reflexions- und Analysefähigkeit durch einen adäquaten Videoeinsatz wurde bereits durch erste Studien belegt (z. B. Goeze, Hetfleisch \& Schrader, 2013; Seidel, Blomberg \& Renkl, 2013). Darüber hinaus bieten sich zahlreiche weitere Chancen für die Lehrerbildung: Videobasierte Lehr-Lern-Settings können gleichzeitig situiert und theorieorientiert stattfinden sowie den Aufbau eines flexiblen, transfer- und anschlussfähigen Wissens in Bezug auf die Unterrichtsgestaltung fördern (Krammer, Hugener \& Biaggi, 2012). Santagata und Guarino (2011) konnten in ihren Studien zum „Lesson Analysis Framework“ außerdem zeigen, dass die Beschreibung und Interpretation von Situationen sowie die Entwicklung von Handlungsoptionen durch die Arbeit mit Unterrichtsvideos zunehmend an Qualität gewannen.

Aufgrund der dargestellten Vorteile und erster empirischer Erkenntnisse zur positiven Wirkung des Einsatzes von Videos, bilden diese den methodischen Schwerpunkt des Lehr-Lern-Labors C(LE) ${ }^{2}$ VER.

\section{Das chemiedidaktische Lehr-Lern-Labor C(LE $)^{2}$ VER}

Das Lehr-Lern-Labor ermöglicht Studierenden, sich in einem komplexitätsreduzierten Setting mit heterogenen Schülervorstellungen auseinanderzusetzen. Es bietet die Chance, fremdes Unterrichtshandeln zu analysieren sowie eigenständig Unterricht zu planen, zu erproben und videobasiert zu reflektieren.

Das Format wurde auf Basis einer früheren Lehrveranstaltung zum Forschenden Lernen konzipiert (Rohrbach-Lochner \& Marohn, 2018). Es hat einen Umfang von zwei Semesterwochenstunden und bildet eine Pflichtveranstaltung im Rahmen der Chemielehrerbildung an der Universität Münster. Diese wird sowohl von Studierenden des Lehramts an Haupt-, Real-, Sekundar- und Gesamtschulen (HRSGe) als auch von Studierenden des Lehramts für Gymnasien und Gesamtschulen (Gym/Ges) sowie des Berufskollegs (BK) besucht. Die Verortung in den unterschiedlichen Studiengängen variiert zwischen dem 5. Bachelor- und 1. Mastersemester. Aufgrund seiner Besonderheiten als Lehr-Lern-Labor fungiert die Veranstaltung als Brückenseminar zwischen dem auf die Vermittlung fachlicher und fachdidaktischer Theorien ausgerichteten Bachelor und dem anschließenden Praxissemester: Die Studierenden stellen erste Theorie-Praxis-Bezüge her und werden sukzessiv an unterrichtliches Handeln herangeführt. 


\subsection{Ziele}

Die Lehr-Lern-Labor-Veranstaltung verfolgt drei Ziele:

1. Professionalisierung der Studierenden im Hinblick auf Wissenselemente und Fähigkeiten im Kontext heterogener Schülervorstellungen,

2. Förderung eines reflexiven Habitus hinsichtlich fremder und eigener Unterrichtshandlungen und -planungen,

3. Anbahnung eines situationsadäquat zu modifizierenden Handlungsrepertoires zur Vermeidung und Bewältigung von Stolperstein-Situationen im Kontext Schülervorstellungen.

\subsection{Aufbau}

Das Lehrangebot gliedert sich in vier Teilbereiche, die linear angeordnet sind: (1) Forschungsbasierte Vorbereitung, (2) Planung einer eigenen Unterrichtseinheit, (3) Durchführung der geplanten Einheit und (4) Videobasierte Selbstreflexion (siehe Abb. 2). Im Folgenden wird das Seminar anhand der vier Teilbereiche skizziert.

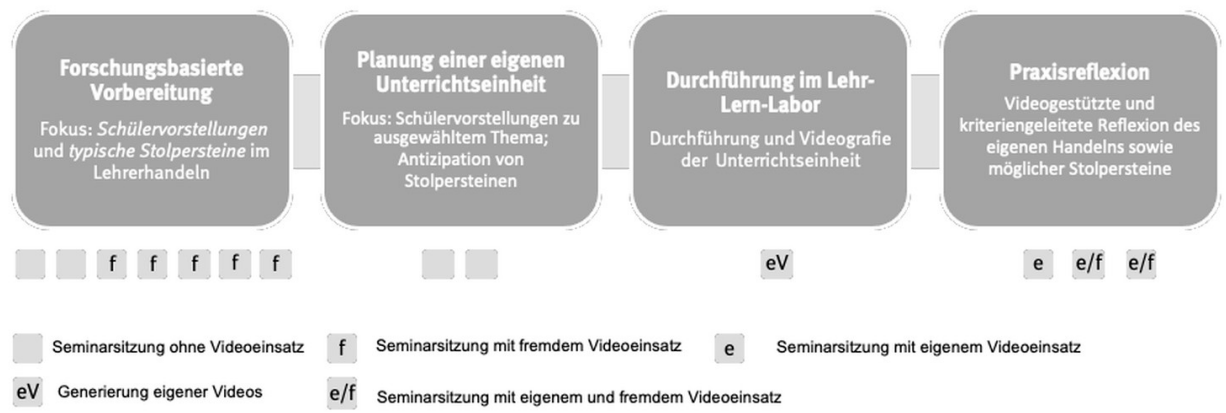

Abbildung 2: Linearer Aufbau des Seminars unter Kennzeichnung der einzelnen Sitzungen in den Teilbereichen und des Videoeinsatzes.

\subsubsection{Forschungsbasierte Vorbereitung}

In der Vorbereitung erwerben die Studierenden wesentliche Wissenselemente und Fähigkeiten im Kontext Schülervorstellungen. Als Grundlage dieser Phase dient das bereits in Kapitel 2.1 vorgestellte Modell von Marohn und Rohrbach (2013).

In den ersten beiden Seminarsitzungen werden die Studierenden in eine moderat-konstruktivistische Sichtweise des Lernens eingeführt und lernen auf Basis empirischer Studien typische Schülervorstellungen und deren vielfältige Ursachen kennen. Praxisnahe Beispiele und Übungen unterstützen die Studierenden dabei, einen Zugang zu den lerntheoretischen Ansätzen zu finden und den notwendigen Wechsel in die Perspektive und Denkweise von Lernenden zu vollziehen. 
Daran schließen sich drei Einheiten zur Diagnose von Schülervorstellungen an. Die Studierenden lernen durch eine Kleingruppenarbeit mit anschließendem Museumsrundgang unterschiedliche Möglichkeiten zur Diagnose von Schülervorstellungen kennen. Sie führen kleine Diagnosestudien in ihrem Umfeld durch und teilen ihre Ergebnisse mit den anderen Seminarteilnehmerinnen und -teilnehmern. In dieser Phase kommt zum ersten Mal das Medium der Videografie zum Einsatz: Die Studierenden sehen Videovignetten zur Diagnose von Schülervorstellungen und identifizieren typische Unterrichtsphasen, die im Verlauf einer Diagnose auftreten. Zudem wird der Blick auf den zweiten Seminarfokus gelenkt: Die Studierenden werden aufgefordert, aus ihrer subjektiven Sicht StolpersteinSituationen in den Videos zu benennen, mögliche Ursachen zu beschreiben und über Vermeidungs- und Bewältigungsstrategien nachzudenken. Die StolpersteinSituationen werden im Plenum gezeigt und gemeinsam reflektiert.

Die anschließende Seminarphase widmet sich im Umfang von drei Sitzungen der Veränderung von Schülervorstellungen. Nach der Erarbeitung der theoretischen Grundlagen (z. B. Conceptual-Change-Theorien) entwickeln die Studierenden am Beispiel „Lösen einer Brausetablette“ eigene Strategien zur Veränderung ausgewählter Schülervorstellungen. Sie lernen die Grundzüge des Unterrichtskonzepts choice 2 learn (Marohn, 2008a) kennen und setzen sich anhand einer digitalen Lernumgebung intensiv mit diesem auseinander. Die Lernumgebung beinhaltet Videos von Schülergruppen, anhand derer die Studierenden den Umgang mit anomalen Daten beobachten und analysieren können. In der nachfolgenden Sitzung werden anhand von Videovignetten weitere typische Stolpersteine reflektiert - diesmal bezogen auf die Veränderung von Schülervorstellungen. Die gemeinsam erarbeiteten Bewältigungs- und Vermeidungsstrategien werden dokumentiert, um als Handlungsrepertoire für die Durchführung der eigenen Unterrichtseinheit zur Verfügung zu stehen. Die Studierenden erarbeiten anhand der betrachteten Videovignetten zudem typische Unterrichtsphasen zur Veränderung von Schülervorstellungen. Diese sollen ihnen helfen, ihren eigenen Unterricht zu strukturieren.

\subsubsection{Planung und Durchführung einer Unterrichtseinheit}

Die Planungszeit beträgt ca. vier Wochen und umfasst zwei Planungssitzungen. Die Studierenden gestalten auf Basis des Seminars eine 45-minütige Unterrichtseinheit. Diese soll die zuvor erarbeiteten Phasen zur Diagnose und Veränderung von Schülervorstellungen beinhalten. Außerdem werden potenzielle Stolpersteine antizipiert sowie geeignete Strategien zur Vermeidung und Bewältigung dieser Situationen entwickelt. Im Anschluss an die Planung führen die Studierenden ihre Unterrichtsstunde mit Gruppen von 10 bis 15 Schülerinnen und Schülern der Sekundarstufe I durch. Die Schülerinnen und Schüler kommen dazu in die Universität, damit die Studierenden ihre Einheit in einer vertrauten Umgebung und einem „geschützten“ Rahmen durchführen können (Dohrmann \& Nordmeier, 2016). 
Den Studierenden werden für ihre Planung verschiedene lebensweltliche Kontexte zur Auswahl gestellt (z.B. Tintenkiller, Wärmekissen, Streusalz im Winter etc.). Diese sind bewusst offen formuliert, um ausreichend Freiheiten für die thematische Ausgestaltung zu gewähren. Um die Studierenden zu entlasten, finden Planung, Durchführung und Reflexion in Zweier-Teams statt; während der Planungszeit wird zudem der Betreuungsschlüssel von Dozierenden zu Studierenden hochgesetzt. Die Studierenden erhalten die Möglichkeit, ihre Experimente vor der Durchführung der Unterrichtseinheit im Labor auszuprobieren. Die Durchführung der Einheiten wird videografiert und die eingesetzte Kameratechnik währenddessen durch eine studentische Hilfskraft bedient und beaufsichtigt.

\subsubsection{Videobasierte Reflexion}

Unmittelbar nach der Durchführung wird eine schriftliche Reflexion durchgeführt, um die Innenperspektive eines jeden Studierenden direkt nach der Stunde festzuhalten. In der anschließenden „Hauptreflexion“ wird das Video der Durchführung unter vorgegebenen Schwerpunkten in den Studierendenteams analysiert. Ein Fokus der Reflexion liegt auf dem Vergleich des Stundenverlaufsplans mit dem tatsächlichen Verlauf der Stunde. Die Studierenden ziehen aus diesem Vergleich Rückschlüsse für nachfolgende Planungen - auch wenn im Rahmen dieses Seminars keine zweite Durchführung stattfinden kann. Ein weiterer Schwerpunkt liegt auf der Reflexion von Stolperstein-Situationen. Um die Studierenden mit dem Fokus Stolpersteine nicht zu frustrieren, reflektiert jedes Team zusätzlich eine „good practice"-Szene. Es werden wesentliche Faktoren herausgestellt, die zum Gelingen der Situation beigetragen haben und im Hinblick auf zukünftige Planungen und Handlungen dokumentiert. Die Stolperstein-Situationen werden anhand des in Kapitel 2.3 dargestellten Schemas reflektiert, um auch von diesen bei zukünftigen Unterrichtshandlungen profitieren zu können.

Den Abschluss der Reflexionsphase bildet das Vorstellen einer prägnanten Videoszene aus der eigenen Durchführung. Diese kann aus unterschiedlichen Motiven ausgewählt werden - z. B. weil sie als besonders gelungen erachtet wird, oder weil die Studierenden Optimierungspotenzial sehen. Wichtig dabei ist, dass die Studierenden eine Szene wählen, in der sie sich nicht „vorgeführt“ fühlen; Vorstellung und Diskussion sollen vielmehr als konstruktiv und gewinnbringend erlebt werden. Dies setzt voraus, dass im Seminar eine wertschätzende Atmosphäre herrscht.

\subsection{Komplexitätsreduktion}

Die Konzeption des Lehr-Lern-Labors C(LE) ${ }^{2}$ VER stellt Studierende vor diverse Herausforderungen im Bereich der Planung, Durchführung und Reflexion von 
Unterricht. Um eine Überforderung der Studierenden zu vermeiden, werden auf inhaltlicher und struktureller Ebene eine Vielzahl von unterstützenden Elementen eingesetzt, die die Komplexität des Settings reduzieren sollen. Die folgenden Abschnitte gehen exemplarisch auf sechs „Regler“ des Modells zur Komplexitätsreduktion ein, die in Abbildung 3 durch Fettdruck hervorgehoben sind.

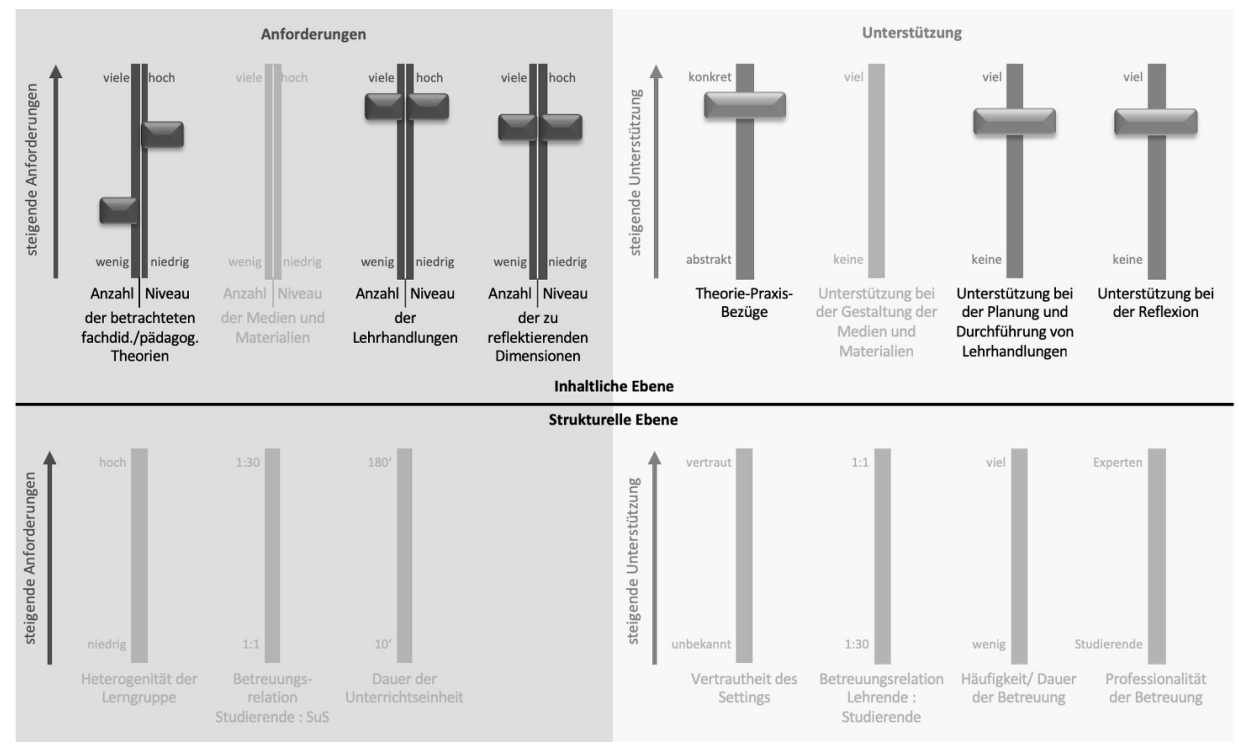

Abbildung 3: Modell „Komplexität in Lehr-Lern-Laboren“ (Marohn et al., in diesem Band). Hervorgehoben sind die „Regler“, auf die in diesem Beitrag näher eingegangen wird.

\subsubsection{Anforderungen}

Das hohe Anforderungsniveau des Lehr-Lern-Labors im Bereich der eigenständigen Planung und Durchführung von Unterricht resultiert im Wesentlichen aus zwei Dimensionen: dem Niveau der betrachteten fachdidaktischen Theorie sowie der Anzahl und dem Niveau der Lehrhandlungen. Aufgabe der Studierenden ist es, eine Unterrichtseinheit zu planen, die Schülervorstellungen zu einem ausgewählten fachlichen Kontext in den Blick nimmt. Das theoretische Konstrukt der Schülervorstellung erweist sich als komplex, da unterschiedliche theoretische Annahmen über Ursachen, Manifestation und Veränderbarkeit von Vorstellungen existieren. Darüber hinaus besteht kein Konsens, in welcher Weise sich Conceptual-Change-Ansätze praktikabel und lernwirksam in der Unterrichtspraxis realisieren lassen. Zudem lassen sich Schülervorstellungen zwar auf Basis empirischer Erhebungen mit einer gewissen Wahrscheinlichkeit antizipieren, jedoch nicht mit 100-prozentiger Sicherheit vorhersagen. Die Unterrichtsplanung ist daher stets mit 
einem gewissen Unsicherheitsmoment behaftet und erfordert die Integration von Handlungsoptionen für den Umgang mit unerwarteten Vorstellungen.

Hinzu kommt, dass die Studierenden eine Vielzahl an Lehrhandlungen planen und realisieren müssen, die mit einem hohen Anforderungsniveau verbunden sind. Hierzu zählt z. B. die Durchführung einer Diagnosephase, die in Abhängigkeit von der gewählten Methode entsprechend eingeleitet und ausgewertet werden muss. Die Lehrhandlungen umfassen zudem Aktivitäten zur Initiierung von kognitiven Konflikten, wie etwa die Durchführung von Lehrer- oder Schülerexperimenten oder den Einsatz von Modellen. Sie beinhalten zudem eine möglichst motivierende Einführung in den fachlichen Kontext zu Beginn der Stunde (z.B. über eine Geschichte, ein Bild etc.) wie auch Plenumsphasen, in denen Ideen gesammelt oder Schlussfolgerungen besprochen werden.

Ein hohes Anforderungsniveau zeigt sich überdies im Bereich der Reflexion, die verschiedene Dimensionen fokussiert. Zum einen wird der Stundenverlauf im Sinne der Generierung von Theorie-Praxis-Bezügen unter dem Fokus Schülervorstellungen analysiert: Welche Vorstellungen werden sichtbar, die nicht in der fachdidaktischen Literatur beschrieben sind bzw. nicht antizipiert wurden? Welche Anzeichen kognitiver Konflikte und Veränderungen von Vorstellungen können beobachtet werden? Welche Experimente oder Modelle erweisen sich aus Sicht der Lernenden nicht als überzeugend und welche Gründe lassen sich hierfür annehmen? Zum anderen richtet sich die Reflexion explizit auf eigene Lehrhandlungen: Welche Abweichungen vom geplanten Handeln lassen sich erkennen und wie wirken sich diese aus? Welche Ursachen haben zu der jeweiligen Situation geführt? Welche Planungsalternativen oder Bewältigungsstrategien lassen sich aus der gewonnenen Erfahrung ableiten? Dabei ist zu berücksichtigen, dass Reflexionen des eigenen Handelns nicht nur kognitive Anforderungen stellen, sondern stets emotional besetzt sind, wodurch sich das subjektiv empfundene Anforderungsniveau erhöhen kann.

\subsubsection{Unterstützung}

Um zu gewährleisten, dass die Studierenden das Lehr-Lern-Labor als gewinnbringende Erfahrung erleben und nicht in eine Überforderungssituation geraten, ist eine Vielzahl an Unterstützungsmaßnahmen erforderlich. Ein wesentliches Element der Komplexitätsreduktion auf inhaltlicher Ebene bildet der Einsatz von Unterrichtsvideos.

In der Vorbereitungsphase werden zur Unterstützung der nachfolgenden Planung einer eigenen Unterrichtseinheit Fremdvideos eingesetzt, die im Rahmen früherer Forschungsprojekte des Arbeitskreises zu Schülervorstellungen entstanden sind (Egbers, 2017; Egbers \& Marohn, 2014; Marohn \& Rott, 2018; Schillmüller \& Marohn, 2017). Weitere Videovignetten zeigen die Arbeit von Studierenden mit Schülerinnen und Schülern aus vorangegangenen Lehr-Lern-Labor-Durchgängen, was ihnen eine besondere Authentizität verleiht. Die Videos dienen in mehrfacher 
Hinsicht der Generierung von Theorie-Praxis-Bezügen: Zum einen machen sie im wörtlichen Sinne „anschaulich“, dass Schülervorstellungen - die den Studierenden bislang nur aus der fachdidaktischen Literatur bekannt sind - tatsächlich in großer Vielfalt in der Unterrichtspraxis auftreten und fachliche Lernprozesse maßgeblich beeinflussen. Die Videografien generieren damit Relevanz und Interesse als wesentliche Voraussetzungen für eine erfolgreiche Bewältigung der Anforderungen im Lehr-Lern-Labor. Zum anderen visualisieren sie, in welcher Weise (lern-)theoretische sowie empirische Erkenntnisse zu Schülervorstellungen konkret in die Praxis transferiert werden können. Die Studierenden lernen Möglichkeiten kennen, kognitive Konflikte zu erzeugen, beobachten Argumentations- und Aushandlungsprozesse innerhalb von Schülergruppen und analysieren den Umgang mit anomalen Daten in Form von Experimenten oder Modellen. Sie erhalten damit konkrete Anregungen und Hilfestellungen für ihre eigene Unterrichtsplanung.

Als komplexitätsreduzierend erweist sich dabei die Möglichkeit, durch die gezielte Auswahl von Szenen den Aufmerksamkeitsfokus lenken zu können. Der Fokus kann auf inhaltlichen Aspekten des Unterrichts liegen (z. B. Ursachen einer Schülervorstellung), auf methodischen (z.B. Diagnosemethoden), strukturellen (z.B. Phasierung des Unterrichts) oder auch personalen (z.B. Handlungen von Lernenden und Lehrenden). Auf diese Weise werden gezielt diejenigen Aspekte betrachtet, die für die spätere Planung, Durchführung und Reflexion des eigenen Unterrichts von Bedeutung sind. Durch eine sukzessive Erweiterung des Analysefokus sowie eine Zunahme des zeitlichen Umfangs der Videosequenzen wird die Komplexität gesteigert. Eingebettet werden die Videovignetten in eine Vielzahl praxisnaher Aufgaben und Übungen (z.B. Entwicklung und Anwendung eines Diagnoseinstruments), die einen erfolgreichen Theorie-Praxis-Transfer befördern sollen. Der Wissenserwerb im Rahmen der Vorbereitungsphase geht somit deutlich über ein rein deklaratives Wissen hinaus; die Studierenden erhalten zudem die Möglichkeit, prozedurales und reflexives Wissen (Schmelzing, Wüsten, Sandmann \& Neuhaus, 2010) zu erwerben, z. B. indem sie gelernte Inhalte auf neue Situationen übertragen.

Als unterstützend im Hinblick auf die Reflexion erweist sich darüber hinaus ein Reflexionsschema, das auf Basis des o.g. Modells (siehe Abb. 1) entwickelt wurde. Es dient dazu, Stolperstein-Situationen anhand von Videovignetten zu charakterisieren, potenzielle Ursachen zu ermitteln und gezielte Vermeidungs- und Bewältigungsstrategien zu entwickeln. Durch wiederholte Anwendung des Schemas werden die Studierenden für mögliche Stolpersteine sensibilisiert und erhalten die Möglichkeit, diese antizipierend in ihren Unterrichtsplanungen zu berücksichtigen. Das Schema unterstützt zudem die Reflexion der eigenen Unterrichtseinheit in der letzten Phase des Lehr-Lern-Labors; es kann darüber hinaus im Rahmen der späteren Lehrtätigkeit helfen, eigene Unterrichtspraxis im Sinne eines „Reflective Practitioner" permanent zu hinterfragen und weiterzuentwickeln.

Ergänzt werden die hier beschriebenen unterstützenden Faktoren durch eine Vielzahl weiterer Maßnahmen. Unter anderem werden die 20 Studierenden bei 
den zwei Planungssitzungen nicht nur durch drei fachdidaktische Expertinnen und Experten des Instituts, sondern auch zwei Laborantinnen sowie studentische Hilfskräfte begleitet. Sie erhalten Vorschläge für geeignete fachliche Kontexte, Tipps zur Auswahl und Durchführung von Experimenten und Hilfestellung bei der Zusammenstellung von Materialboxen.

\subsection{Die Sicht der Studierenden}

Um einen Einblick zu gewinnen, welche komplexitätsreduzierenden Maßnahmen die Studierenden tatsächlich als entlastend empfinden, wurden Items für den Post-Fragebogen sowie reflektierende Portfolioaufgaben entwickelt. Zudem wurden leitfadengestützte Interviews nach Ende des Seminars (elf Studierende, Sommersemester 2017) bzw. unmittelbar nach der Durchführung der Unterrichtseinheit (elf Studierende, Wintersemester 2018/19) geführt. Die Interviews wurden transkribiert, die Antworten kategorisiert und den Bereichen aus dem Modell zur Komplexitätsreduktion (siehe Abb. 3) zugeordnet, die in diesem Beitrag fokussiert werden.

Die Ergebnisse des Post-Fragebogens zeigen, dass die Studierenden die Unterstützung im Bereich der Planung und Reflexion insgesamt als hoch einschätzen (siehe Abb. 4).

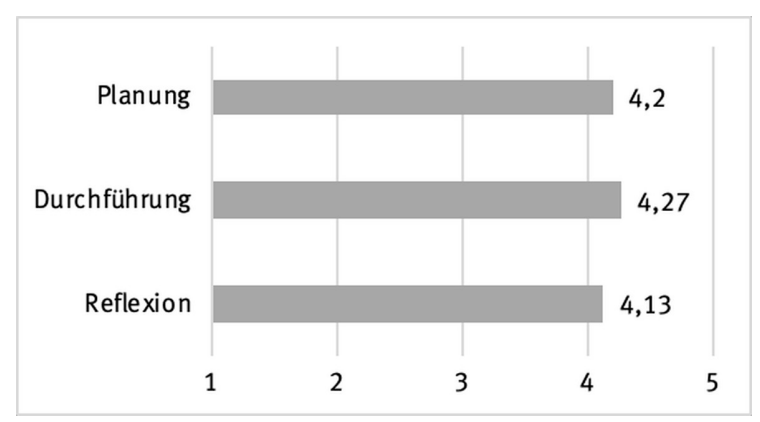

Abbildung 4: Einschätzung der Unterstützung in den verschiedenen Phasen durch die Studierenden, $1=$ keine bis $5=$ viel, $n=17$.

Die folgenden Abschnitte bieten einen zusammenfassenden Überblick über die Sichtweisen der Studierenden und veranschaulichen diese exemplarisch durch Zitate. 


\subsubsection{Theorie-Praxis-Bezüge}

Der inhaltliche Fokus Schülervorstellungen und Stolpersteine im Lehrerhandeln wird von den Studierenden als sehr praxisrelevant eingestuft.

Die Studierenden $(n=17)$ stufen die hergestellten Theorie-Praxis-Bezüge auf einer 5-stufigen Likert-Skala („abstrakt“ bis „konkret“; MW = 4,47) als konkret ein und benennen explizit Elemente des Seminars, die aus ihrer Sicht Bezüge zwischen den behandelten Theorien und der Unterrichtspraxis herstellen. Der Einsatz von Unterrichtsvideos in der Vorbereitungsphase wird dabei besonders hervorgehoben:

„Und die Videos, die wir ja dann immer gesehen haben, waren auch total hilfreich. Einfach mal zu sehen, wie sieht das denn jetzt auch in der Praxis aus? Wie kommt das dann bei Schülern an? Wie gehen die damit um? Und dieses choice ${ }^{2} l e a r n$, das fand ich auch richtig gut! Also in den anderen Videos waren das ja immer so kurze Sequenzen und da haben wir uns mal richtig eine ganze Reihe angeguckt und da konnte man RICHTIG sehen, wie bei den Schülern so ein AHA-Effekt eingesetzt hat. Und dann wurde einem auch so bewusst. Ja ok, das wollen wir dann auch erreichen." (S_3.13, Interview, WS 2018/19)

Das Lehr-Lern-Setting, das durch einen stetigen Wechsel von Theorie- und Praxiselementen gekennzeichnet ist, wird von den Studierenden als sinnvoll erachtet. Sie sprechen sich einheitlich gegen einen vorgeschalteten Theorie- und nachgeschalteten Praxis-Block aus:

"Ich finde so einen direkten Bezug eigentlich immer ganz gut, weil man das dann, falls die Theorie nicht ganz verstanden wurde, [...] mit so einem Beispiel leichter versteht. Also ich bin Fan davon, wenn man das direkt macht und nicht erst wochenlang nur Theorie. Weil dann ist es so viel, dass man meistens gar nicht das Richtige miteinander verknüpfen kann." (S_3.17, Interview, WS 2018/19)

Ergänzend zu den Videos wurden der variierende Methodeneinsatz sowie die praktischen Übungen als hilfreich für das Verständnis der Lerntheorien eingestuft:

„Also was mir gerade jetzt auf Anhieb einfällt, ist das mit der Ente [Anm. der Autoren: praktische Übung im Seminar zum Konstruktivismus]. Also, dass man wirklich sieht, wie viele verschiedene Vorstellungen irgendwie entstehen können. Und, dass man es einfach mal selber ausprobiert. Ich mein, wenn das jetzt irgendwie einfach nur gezeigt worden wäre, hätte das nicht diesen Effekt gehabt.“ (S_3.2, Interview, WS 2018/19)

\subsubsection{Unterstützung bei der Planung und Durchführung von Lehrhandlungen}

Die Auswertung der Items zur Unterstützung bei der Planung und Durchführung der eigenen Einheiten belegt, dass viele Faktoren, die als Mittel der Komplexitäts- 
reduktion eingesetzt wurden, auch von den Studierenden als unterstützend empfunden werden (siehe Abb. 5).

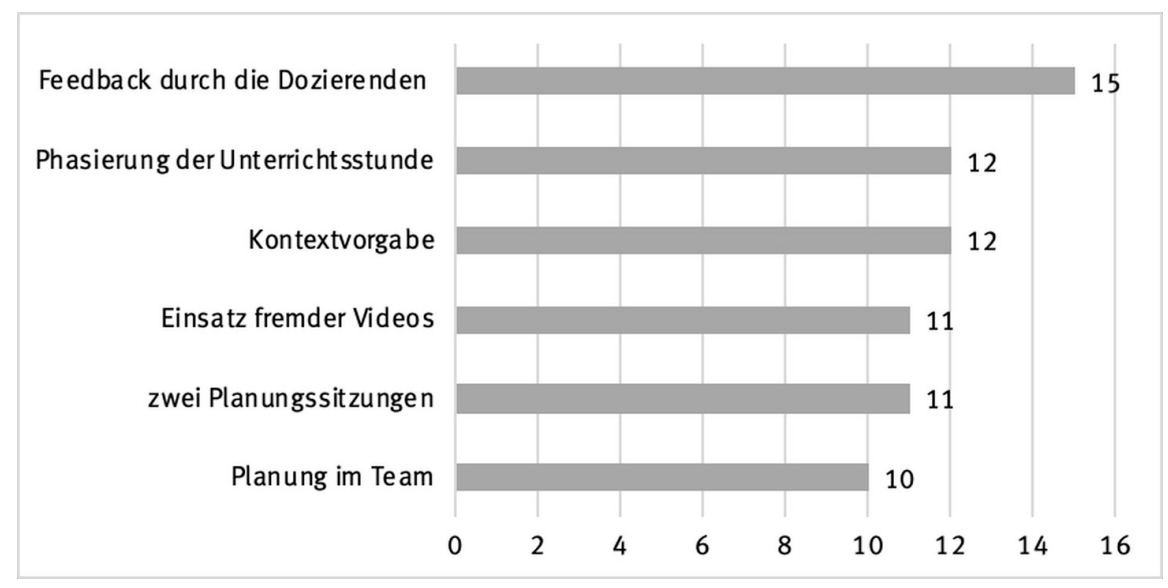

Abbildung 5: Häufigkeit der Nennung von Faktoren zur Unterstützung der Planungsphase, $n=16$ Studierende.

Besonders hilfreich empfinden die Studierenden die Unterstützung durch die Betreuenden im Hinblick auf die Besprechung der Stundenverlaufspläne, die gezielte Thematisierung von Unsicherheiten und Problemen sowie die Unterstützung bei der Experimententwicklung.

„Gerade das Besprechen der Stundenverlaufspläne. Als nicht nur Sie da waren, sondern auch Frau X. und Herr Y. durch die Reihen gegangen sind. Das hat sehr geholfen, weil man dann nochmal explizit Fragen anhand des Plans stellen konnte. Das war sehr gut! Also gerade in den letzten Zügen quasi das Planen." (S_3.9, Interview, WS 2018/19)

Einen weiteren wichtigen Punkt bildet aus Sicht der Studierenden die Bereitstellung einer Auswahl an Kontexten, auf die sie bei der Planung zurückgreifen können:

„Ich habe auch von jemandem gehört, der das [Seminar] vor einem Jahr gemacht hat. Die meinten auch, da [habt] ihr Glück gehabt, dass ihr Kontexte vorgegeben bekommen habt. Weil bei uns war es komplett frei und da war große Verwirrung. Also, das ist schon deutlich besser dann dadurch." (S_3.19, Interview, WS 2018/19)

„Das [Kontext überlegen] ist ja wie bei Hausarbeiten mit der Arbeitshypothese. Das dauert immer am allerlängsten." (S_3.2, Interview, WS 2018/19) 
Der Einsatz von fremden Unterrichtsvideos wird auch in Bezug auf die Planung eigener Lehrhandlungen hervorgehoben. Besonders häufig wird die Identifizierung von Unterrichtsphasen anhand der Videosequenzen als unterstützendes Element angeführt:

„Ja, da habe ich mich schon entlang gehangelt. Also, ich habe geguckt, welche Phasen haben wir. Und dann habe ich die Phasen erst notiert. Und dann überlegt: Ok, was machst du jetzt in jeder Phase. Und dann habe ich auch bei den Übergängen so geguckt: Wie war das in den Videos? Wie haben die das so sinnvoll übergeleitet? Und ja, da habe ich mich dann ein bisschen dran orientiert." (S_3.12, Interview, WS 2018/19)

„Ohne die Videos hätte man wahrscheinlich nicht so schnell so eine Vorstellung davon gehabt, wie so eine Stunde aufgebaut werden muss. [...] Also so eine Grundstruktur hat man dadurch kennengelernt und dadurch war es auf jeden Fall sehr hilfreich. Ohne die Videos wäre es viel viel schwieriger gewesen." (S_3.13, Interview, WS 2018/19)

Aber auch die vorangegangenen Reflexionen typischer Stolpersteine werden als hilfreich für die Planung empfunden. Die Antizipation möglicher Stolpersteine im eigenen Unterrichtsentwurf vermittelt den Studierenden ein Gefühl der Sicherheit:

„So haben wir sie [Stolpersteine] auch in die Planung mit reingenommen. Was wir machen können, damit das gar nicht erst passiert. Oder wenn es dann passiert, dann ist man ja schon ein bisschen sicherer und steht nicht vorne und fängt an zu zittern: Oh Gott was mach ich jetzt?" (S_3.2, Interview, WS 2018/19)

Ein Punkt, der von den Studierenden nicht in allen Phasen als positiv eingeschätzt wird, ist die Arbeit im Team (siehe Abb. 6). In der Planungsphase stufen 13 von 17 Studierenden die Teamarbeit als hilfreich ein:

„Also in der Vorbereitung habe ich es definitiv als erleichternd empfunden, weil man nicht selber auf Ideen kommen musste und sich alles überlegen musste, sondern die Möglichkeit hatte mit jemandem zu diskutieren, zu überlegen und einfach mal ein bisschen Brainstorming zu machen, was es überhaupt für Möglichkeiten gibt, wie man da rangehen könnte und dann dadurch auch einen Austausch hatte und direkt Ideen, die halt blöd waren, verwerfen konnte." (S_o.9, Interview, SS 2017)

In Bezug auf die Durchführung bewerten nur 6 Studierende die Teamarbeit positiv (siehe Abb. 6). Es werden unterschiedliche Gründe für Schwierigkeiten in der Teamarbeit angeführt:

„Ich finde, in der Durchführung macht es das gar nicht unbedingt einfacher, weil wir selber gemerkt haben, dass man sich manchmal so ein bisschen in die Quere kommt. Das heißt man muss halt wirklich auch ganz klar sagen: ,Okay, bis zu dem Punkt 
macht der Eine' und wenn er irgendwie was vergessen hat, ist es halt auch schwierig, das nochmal mit einzubringen." (S_o.6, Interview, SS 2017)

„Das Einzige, was mir aufgefallen ist, ist, dass Student $\mathrm{X}$ sehr viele Anteile übernommen hat - selbst teilweise dann die Anteile wo wir vorher gesagt haben, ich mache die. Also an der Stelle habe ich es nicht als schlimm empfunden, aber eventuell wäre es doch schöner gewesen, wenn ich dann ein bisschen mehr Material mit mir in der führenden Rolle gehabt hätte.“ (S_o.9, Interview, SS 2017)

Bei anderen Studierenden hingegen vermittelt die Teamarbeit ein Gefühl der Sicherheit:

„Also wenn der Eine eine Idee hatte, konnte man dann immer noch sagen ,Ja, find ich gut, aber' und wenn man alleine ist, hat man diesen Input nicht. Und da hilft es dann auf jeden Fall. Ja, und es ist natürlich auch eine totale psychische Entlastung, dass man weiß: ,Ok, ich bin da nicht alleine. Da ist noch jemand.“ (S_3.12, Interview, WS 2018/19)

Der kollegiale Austausch im Team wird somit vor allem in der Planungs- und Reflexionsphase als unterstützend empfunden; in Bezug auf die gemeinsame Durchführung der Unterrichtsstunde zeigt sich demgegenüber ein differenzierteres Bild.

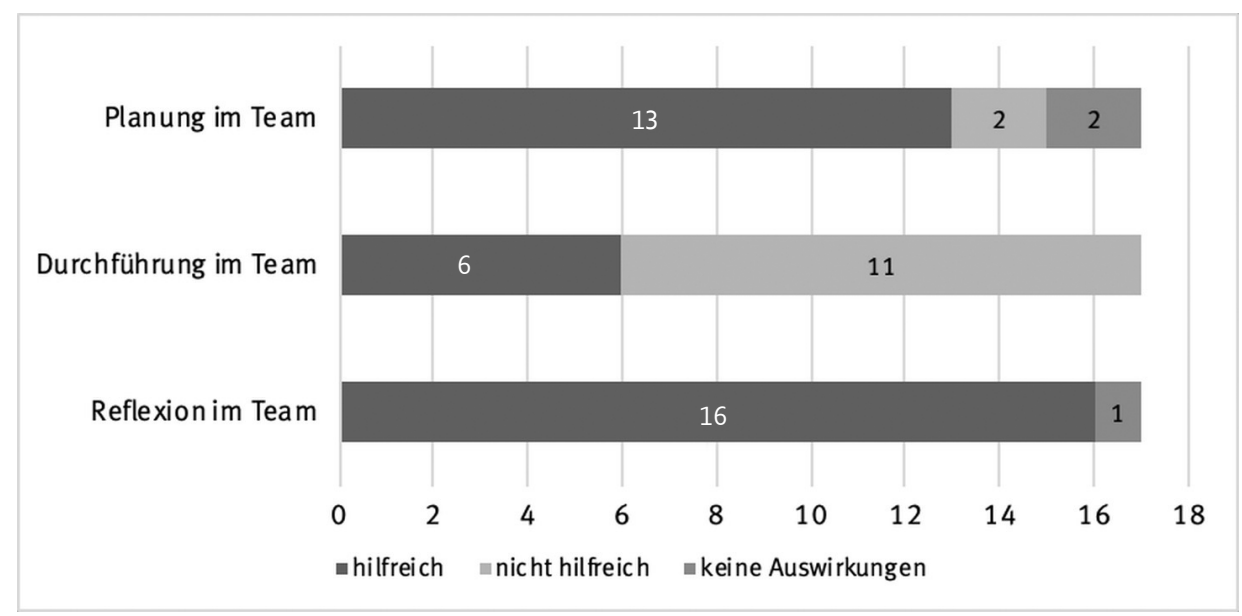

Abbildung 6: Einschätzung der Teamarbeit in den verschiedenen Phasen, $n=17$ Studierende. 


\subsubsection{Unterstützung bei der Reflexion}

Die Auswertung der Items zur Reflexion verdeutlicht, dass die überwiegende Zahl der Studierenden auch hier die antizipierten Unterstützungsmaßnahmen als hilfreich empfindet (siehe Abb. 7). Besonders der Einsatz eigener Videos wie auch die Reflexion anhand eines gegebenen Reflexionsschemas werden von den Studierenden hervorgehoben (siehe Abb. 7).

Die folgenden Studierenden beschreiben in ihren Portfolios, in welchen Aspekten die Betrachtung des eigenen Videos als gewinnbringend empfunden wurde:

„Ich finde das Video sehr hilfreich, da mir dadurch viele Aspekte aufgefallen sind, die mir vorher nicht bewusst waren." (S_3.15, Portfolio, WS 2018/19)

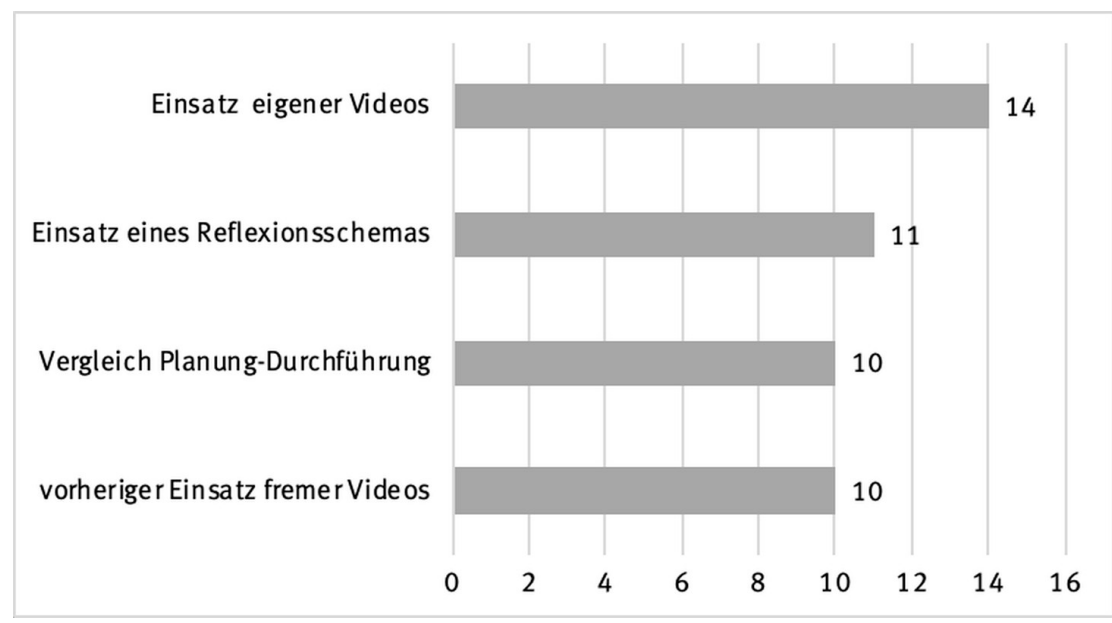

Abbildung 7: Häufigkeit der Nennung von Faktoren zur Unterstützung in der Reflexionsphase, $n=16$ Studierende.

„Die Bereitstellung meines eigenen Videos hat mir die Möglichkeit gegeben, mein Lehrerverhalten näher einzuschätzen und weitere Stolpersteine in der Retrospektive zu entdecken, die mir während der Stunde nicht aufgefallen sind." (S_3.8, Portfolio, WS 2018/19)

Das Reflexionsschema als nützliche Hilfestellung und Strukturierungsmittel wird auch im Interview deutlich. Es wird von zehn Studierenden explizit benannt:

„Ähm, dann mit dem genauen Identifizieren, was ist jetzt genau die Ursache, und was sind nur die Folgen der Ursache oder was ist dieses große Ganze? Also, das fand ich auch sehr gut, weil man da auch wirklich nochmal auf diese Kernprobleme zu sprechen kommt. [...] Okay, was war jetzt genau der Fehler? Und das war auch oft gar nicht so einfach. [...] Und da hilft so ein Schema vor allem am Anfang bei 
Videoanalysen und generell bei Analysen von Problemen. Weil man sonst nicht so ganz weiß, wie soll man da rein gehen. (S_3.6, Interview, WS 2018/19)

Insbesondere wird die Möglichkeit, anhand des Reflexionsschemas über Vermeidungs- und Bewältigungsstrategien nachzudenken, als unterstützende Maßnahme im Hinblick auf die Planung, Durchführung und Reflexion von Unterricht angesehen:

„Die Auseinandersetzung mit Stolpersteinen, auch in Form der Analyse von Videomaterial, inklusive der Planung von Bewältigungsstrategien und Planungsalternativen, hat mich auf die Berücksichtigung von möglichen Stolpersteinen als auch auf deren Erkennen in meinem eigenen Video vorbereitet.“ (S_3.8, Portfolio, WS 2018/19)

„Also ich fand's vor allem mit den Vermeidungs- und Bewältigungsstrategien sehr gut [...] Dass man darüber nachdenkt: Okay, wie kann man's [Stolperstein] dann in dieser Situation bewältigen, weil du kannst nicht so gut den Unterricht planen, dass nie sowas [Stolperstein] stattfindet. [...] Und, dass man da jetzt die Zeit geboten bekommt, darüber wirklich nachzudenken, damit man dann in der Situation oder auch in der Vorbereitung besser drauf vorbereitet ist. Das fand ich sehr hilfreich." (S_3.6, Interview, WS 2018/19)

Einige Studierende beschreiben eigene Lernfortschritte aufgrund der wiederholten Durchführung von Reflexionsprozessen im Verlauf des Seminars:

„Das Benennen der Situation. Das wurde mit der Zeit immer besser. Indem man das immer öfter gemacht hat, konnte man auch viel besser sagen: ,Ja ok, ähm, DAS läuft hier gerade schief.' und nicht nur: ,Ok, da läuft irgendwas schief.' und man weiß eigentlich gerade gar nicht warum.“ (S_3.13, Interview, WS 2018/19)

Einigkeit besteht darüber, dass sich die Reflexion der Fremdvideos in der Vorbereitungsphase auf die Reflexion des Eigenvideos auswirken wird (diese hatte zum Zeitpunkt der Interviews vom WS 2018/19 noch nicht stattgefunden). Die Studierenden skizzieren dabei unterschiedliche Erwartungen und Herangehensweisen:

„Ähm ja, dadurch, dass man schon sehr viele Videos gesehen hat, wird man, glaub ich, einen ganz anderen Blick darauf kriegen. Ich habe mir auch vorgenommen den Verlaufsplan daneben zu legen, um mal intensiver zu schauen, was die Stolpersteine, die ich selber aufgeschrieben habe, machen. Also man wird das Video ja so oder so mehrmals schauen. Von daher kann man durchaus öfter den Fokus auf verschiedene Dinge legen. Und da hat das Seminar auf jeden Fall Eindruck hinterlassen." (S_3.9, Interview, WS 2018/19) 
Während in der Durchführung die Arbeit im Team nicht bei allen Studierenden als unterstützend empfunden wird, wird sie in der Phase der Reflexion sehr geschätzt (siehe Abb. 6).

„Ja, da war es auch wieder sehr positiv. Eben weil ihm dann noch Punkte aufgefallen sind, die ich nicht im ersten Moment aufgeschrieben habe und umgekehrt. Da haben wir uns sehr stark ergänzt und sind dadurch auf mehr Punkte gekommen, als wenn ich das alleine gemacht hätte, und konnten uns dann auch direkt austauschen, inwieweit diese Punkte jetzt positiv oder negativ waren, inwieweit unsere Reaktion angemessen war oder halt eben auch nicht. Also da war es auch sehr schön, das nicht alleine machen $\mathrm{zu}$ müssen, sondern wirklich sich auszutauschen $\mathrm{zu}$ können und auch andere Meinungen - auch wenn es nur eine war - zu hören." (S_o.9, Interview, SS 2017)

\section{4. $\quad$ Fazit}

Vergleicht man die Einschätzungen der Studierenden mit den in der Seminarplanung antizipierten komplexitätsreduzierenden Faktoren, so zeigen sich viele Überschneidungen. Eine besondere Stellung über alle fokussierten „Regler“ des Modells hinweg nimmt der Einsatz von Fremdvideos ein: Durch ihn werden Theorie-Praxis-Bezüge geschaffen, Reflexionsanlässe generiert und Handlungsanregungen für den eigenen Unterricht geboten. Die Interviews deuten darauf hin, dass die positive Wahrnehmung des Videoeinsatzes unter anderem auf zwei Faktoren zurückzuführen ist: Zum einen besteht ein hoher Identifikationsgrad mit den gezeigten Situationen, da viele Videosequenzen im Rahmen vorangegangener LehrLern-Labor-Durchgänge entstanden sind. Das sichtbar werdende Lehrerhandeln erscheint somit für die Studierenden authentisch und erreichbar. Zum anderen wird durch die konstruktive Reflexion von Stolperstein-Situationen von Beginn an eine „positive Fehlerkultur“ aufgebaut. Die Studierenden erleben schwierige Unterrichtsmomente nicht als defizitär, sondern als selbstverständliche Bestandteile von Praxiserfahrung, die mit Hilfe zunehmender Reflexion sowie der Entwicklung von Vermeidungs- und Bewältigungsstrategien gemeistert werden können. Die Reflexion von Fremdvideos wird zudem als gute Vorbereitung auf die Auseinandersetzung mit dem eigenen Video empfunden; einige Studierende berichten von einem gefühlten „Trainingseffekt", der ihnen die Betrachtung des eigenen Videos erleichtert. Als hilfreich wird darüber hinaus die wiederholte Anwendung eines Reflexionsschemas eingeschätzt, das eine fokussierte und strukturierte Auseinandersetzung mit den Videosequenzen fördert.

Während die Betreuung durch Experten durchweg als positiv beschrieben wird, zeigt sich in Bezug auf die Arbeit in Studierenden-Teams ein differenzierteres Bild: Im Verlauf der Planung und Reflexion des eigenen Unterrichts wird diese als gewinnbringend empfunden, in der Durchführung dagegen teilweise als herausfordernd und schwierig erlebt. Hier zeigt sich der Nutzen des Modells zur 
Komplexität in Lehr-Lern-Laboren, das es möglich macht, „different erlebte Komplexität zu reflektieren“" (Marohn et al., in diesem Band), um daraus Konsequenzen für die Weiterentwicklung des Lehr-Lern-Labors zu ziehen.

\section{Literatur}

Abels, S. (2011). Lehrerinnen und Lehrer als „Reflective Practitioner": Reflexionskompetenz für einen demokratieförderlichen Naturwissenschaftsunterricht. Wiesbaden: VS Verlag für Sozialwissenschaften. https://doi.org/10.1007/978-3-531-92735-0

Biedermann, H. (2011). Gebrochene Übergänge: Durch professionelle Lernkerne zur professionellen Lehrtätigkeit. Habilitationsschrift. Fribourg: Universität Fribourg, Departement Erziehungswissenschaften.

Biedermann, H. \& Oser, F. (2011). Wirksame Lehrerausbildung: Globale Ausbildungskultur und/oder regionale Routinenschulung? TEDS-M - erste internationale Vergleichsstudie der IEA mit Fokus auf die Lehrerausbildung. Beiträge zur Lehrerinnenund Lehrerbildung, 29 (1), 66-81.

Brouwer, N. \& Korthagen, F. A. J. (2005). Can Teacher Education Make a Difference? American Educational Research Journal, 42 (1), 153-224. https://doi.org/10. 3102/00028312042001153

Combe, A. \& Helsper, W. (1996). Pädagogische Professionalität. Untersuchungen zum Typus pädagogischen Handelns. Frankfurt a. M.: Suhrkamp.

Copeland, W. D., Birmingham, C., De la Cruz, E. \& Lewin, B. (1993). The Reflective Practitioner in Teaching: Toward a Research Agenda. Teaching and Teacher Education, 9 (4), 347-359. https://doi.org/10.1016/0742-051X(93)90002-X

Day, C. (1993). Reflection: a necessary but not sufficient condition for professional development. British Educational Research Journal, 19 (1), 83-93. https://doi.org/10. 1080/0141192930190107

Denner, L. \& Gesenhues, D. (2013). Professionalisierungsprozesse im Lehramtsstudium eine explorative Studie zu Analyse, Interpretation und Handlungsoption. In R. Bolle (Hrsg.), Professionalisierung im Lehramtsstudium: Schulpraktische Kompetenzentwicklung und theoriegeleitete Reflexion (S. 59-119). Leipzig: Leipziger Universitätsverlag.

Dewey, J. (1951). Wie wir denken: eine Untersuchung über die Beziehung des reflektiven Denkens zum Prozeß der Erziehung. Zürich: Morgarten-Verlag.

Dohrmann, R. \& Nordmeier, V. (2016). Lehr-Lern-Labore (LLL) als Orte komplexitätsreduzierter Praxis: Erste Professionalisierungsschritte im Lehramtsstudium Physik. In V. Nordmeier \& H. Grötzebauch (Hrsg.), PhyDid B, Didaktik der Physik, Beiträge zur DPG-Frühjahrstagung. Verfügbar unter http://phydid.physik.fu-berlin.de/index.php/ phydid-b/article/view/732/860 [20.01.2019].

Doyle, W. (1986). Classroom Organization and Management. In M. C. Wittrock (Hrsg.), Handbook of Research on Teaching (3. Aufl., S. 392-431). New York: Macmillan.

Egbers, M. (2017). Konzeptentwicklungs- und Gesprächsprozesse im Rahmen der Unterrichtskonzeption "choice2learn“. In A. Marohn (Hrsg.), Lernen in Naturwissenschaften - verstehen und entwickeln (Band 1). Berlin: Logos.

Egbers, M. \& Marohn, A. (2014). Schülervorstellungen verändern - Konzeptentwicklungs- und Gesprächsprozesse im Rahmen der Unterrichtskonzeption „choice2learn“. In B. Ralle, S. Prediger, M. Hammann \& M. Rothgangel (Hrsg.), Lernaufgaben ent- 
wickeln, bearbeiten und überprüfen. Ergebnisse und Perspektiven fachdidaktischer Forschung (S. 120-128). Münster: Waxmann.

Fend, H. (1998). Qualität im Bildungswesen. Schulforschung zu Systembedingungen, Schulprofilen und Lehrerleistung. Weinheim: Juventa.

Gilbert, J. K., Justi, R., van Driel, J. H., de Jong, O. \& Treagust, D. F. (2004). Securing a future for chemical education. Chemistry education: research and practice, 5 (1), 5-14. https://doi.org/10.1039/B3RP90027D

Goeze, A., Hetfleisch, P. \& Schrader, J. (2013). Wirkungen des Lernens mit Videofällen bei Lehrkräften. Welche Rolle spielen instruktionale Unterstützung, Personen- und Prozessmerkmale? Zeitschrift für Erziehungswissenschaft, 16, 79-113. https://doi.org/ 10.1007/s11618-013-0352-X

Gropengießer, H. \& Marohn, A. (2018). Schülervorstellungen und Conceptual Change. In D. Krüger, I. Parchmann \& H. Schecker (Hrsg.), Theorien in der Naturwissenschaftsdidaktischen Forschung (S. 49-67). Berlin: Springer Spektrum. https://doi.org/ 10.1007/978-3-662-56320-5_4

Großebrahm, N. (2013). Elemente fachdidaktischen Wissens in der universitären Ausbildung angehender Chemielehrkräfte. Ein Beitrag zur Standardentwicklung. Universität Duisburg-Essen: Dissertation.

Gruber, H. (2007). Bedingungen von Expertise. In K. A. Heller \& A. Ziegler (Hrsg.), Begabt sein in Deutschland (93-112). Münster: Lit.

Gruber, H., Heid, H. \& Bauer, J. (2005). Forschungsprogramm Kompetenzentwicklung im Arbeitsprozess: Die Rolle von Arbeitsplatz-Veränderungen und Reflexion für Workplace Learning (Forschungsbericht Nr. 17). Regensburg: Universität Regensburg, Lehrstuhl für Lehr-Lern-Forschung. Verfügbar unter https://portal.uni-regensburg. de/50/1/050\%29\%20ubr17913.pdf [20.01.2019].

Gruber, H. \& Rehrl, M. (2005). Praktikum statt Theorie? Eine Analyse relevanten Wissens zum Aufbau pädagogischer Handlungskompetenz (Forschungsbericht 15). Regensburg: Universität Regensburg, Lehrstuhl für Lehr-Lern-Forschung. Verfügbar unter: http:// www.forschungsnetzwerk.at/downloadpub/fb15_2005_regensburg_uni_paed.pdf [20.01.2019].

Hattie, J. (2010). Visible Learning. A Synthesis of over 800 Meta-Analyses relating to Achievement. London: Routledge.

Hellermann, C. (2015). Fallbasiertes Lernen in der Lehrerausbildung. Förderung des strategischen Wissens und der professionellen Wahrnehmung der Klassenführung. Dissertation, Westfälische Wilhems-Universität Münster.

Hofer, M. (1986). Sozialpsychologie erzieherischen Handelns. Göttingen: Hogrefe.

Kelly, G. A. (1963). A theory of personality. A psychology of personal constructs. New York: Norton \& Company.

Kittelberger, R. \& Freisleben, I. (1991). Lernen mit Video und Film. Weinheim: Beltz.

Kleinknecht, M. \& Schneider, J. (2013). What do teachers think and feel when analyzing videos of themselves and other teachers teaching? Teaching and Teacher Education, 33, 13-23. https://doi.org/10.1016/j.tate.2013.02.002

Kohler, B., Prinz, E., Schneider, J. \& Syring, M. (2015). Ein neuer Blick auf die Praxis: Selbst- und Fremdreflexion mithilfe von Unterrichtsvideos. Schulmagazin 5-10 (1), $11-14$. 
Kultusministerkonferenz (2004). Beschluss der Kultusministerkonferenz vom 16.12.2004: Standards für die Lehrerbildung: Bildungswissenschaften. Bonn: Ständige Konferenz der Kultusminister der Länder in der Bundesrepublik Deutschland.

Kultusministerkonferenz (2008). Beschluss der Kultusministerkonferenz vom 16.10.2008 i.d.F. vom 08.12.2008: Ländergemeinsame inhaltliche Anforderungen für die Fachwissenschaften und Fachdidaktiken in der Lehrerbildung. Bonn: Ständige Konferenz der Kultusminister der Länder in der Bundesrepublik Deutschland.

Krammer, K., Hugener, I. \& Biaggi, S. (2012). Unterrichtsvideos als Medium des beruflichen Lernens in der Lehrerinnen- und Lehrerbildung - Formen und Erfahrungen. Beiträge zur Lehrerbildung, 30 (2), 261-272.

Krammer, K. \& Reusser, K. (2005). Unterrichtsvideos als Medium der Aus- und Weiterbildung von Lehrpersonen. Beiträge zur Lehrerbildung, 23 (1), 35-50.

Leonhard, T. \& Rihm, T. (2011). Erhöhung der Reflexionskompetenz durch Begleitveranstaltungen zum Schulpraktikum? Konzeption und Ergebnisse eines Pilotprojekts mit Lehramtsstudierenden. Lehrerbildung auf dem Prüfstand, 4 (2), 240-270.

Luhmann, N. (2002): Das Erziehungssystem der Gesellschaft. Frankfurt a. Main: Suhrkamp.

Marohn, A. (2008a). Choicezlearn - eine Konzeption zur Exploration und Veränderung von Lernervorstellungen im naturwissenschaftlichen Unterricht. Zeitschrift für Didaktik der Naturwissenschaften, 14, 57-83.

Marohn, A. (2008b). Schülervorstellungen zum Lösen und Sieden - Auf der Suche nach ,elementaren' Vorstellungen. Mathematisch Naturwissenschaftlicher Unterricht, 61 (8), 451-457.

Marohn, A. (2014). Von der Diagnose zur Veränderung - Zum Umgang mit Schülervorstellungen in Unterricht und Lehrerausbildung. In A. Fischer et al. (Hrsg.), Diagnostik für lernwirksamen Unterricht (S. 157-175). Baltmannsweiler: Schneider Verlag Hohengehren.

Marohn, A. \& Rohrbach, F. (2013). Professionalisierung in der Lehrerausbildung - Entwicklung und Evaluation eines am Forschenden Lernen orientierten Seminars zum Thema ,Schülervorstellungen im Chemieunterricht. Chimica et ceterae artes rerum naturae didacticae, 38 (105), 21-46.

Marohn, A. \& Rott, L. (2018). Naturwissenschaftliches Lernen im Inklusiven Unterricht. In A. Langner (Hrsg.), Inklusion im Dialog: Fachdidaktik - Erziehungswissenschaft Sonderpädagogik (S. 102-108). Bad Heilbrunn: Klinkhardt.

Mühlhausen, U. (1994). Überraschungen im Unterricht: Situative Unterrichtsplanung. Weinheim: Beltz.

Mühlhausen, U. (2007). Abenteuer Unterricht: Wie Lehrer/innen mit überraschenden Unterrichtssituationen umgehen. Baltmannsweiler: Schneider Verlag Hohengehren.

Rambow, R. \& Bromme, R. (2000). Was Schöns ,reflective practitioner' durch die Kommunikation mit Laien lernen könnte. In G. H. Neuweg (Hrsg.) Wissen - Können - Reflexion: Ausgewählte Verhältnisbestimmungen (S. 245-263). Innsbruck: Studienverlag.

Reinmann-Rothmeier, G. \& Mandl, H. (2001). Unterrichten und Lernumgebungen gestalten. In A. Krapp \& B. Weidenmann (Hrsg.), Pädagogische Psychologie. Ein Lehrbuch (5. Aufl., S. 601-646). Weinheim: Beltz.

Rohrbach-Lochner, F. (2019). Design-Based Research zur Weiterentwicklung der chemiedidaktischen Lehrerausbildung zu Schülervorstellungen: Entwicklung und Eva- 
luation eines an Forschendem Lernen orientierten Seminarkonzepts. In A. Marohn (Hrsg.), Lernen in Naturwissenschaften - verstehen und entwickeln (Band 3). Berlin: Logos. https://doi.org/10.30819/4944

Rohrbach-Lochner, F. \& Marohn, A. (2018). How research-based learning can increase teacher students' knowledge and abilities: a design-based research project in the context of pupils' (mis)conceptions in science. RISTAL. Research in Subject-matter Teaching and Learning, 1, 35-50.

Santagata, R. \& Guarino, J. (2011). Using video to teach future teachers to learn from teaching. ZDM The International Journal on Mathematics Education, 43 (1), 133-145. https://doi.org/10.1007/s11858-010-0292-3

Schillmüller, R. \& Marohn, A. (2017). Warum blubbert's in der Brause? - choicezlearn in der Sekundarstufe I. Naturwissenschaften im Unterricht Chemie, 28(159), 13-18.

Schmelzing, S., Wüsten, S., Sandmann, A. \& Neuhaus, B. (2010). Fachdidaktisches Wissen und Reflektieren im Querschnitt der Biologielehrerbildung. Zeitschrift für Didaktik der Naturwissenschaften, 16, 189-207.

Schneider, J. (2016). Lehramtsstudierende analysieren Praxis. Ein Vergleich der Effekte unterschiedlicher fallbasierter Lehr-Lern-Arrangements. Dissertation: Tübingen. Verfügbar unter https://publikationen.uni-tuebingen.de/xmlui/bitstream/handle/109oo/ 71843/Juergen $\% 20$ Schneider\%202016\%2oLehramtsstudierende\%20analysieren $\% 20$ Praxis.pdf? sequence $=1$ \&isAllowed $=y$ [20.01.2019].

Schön, D. A. (1983). The reflective practitioner. How professionals think in action. New York: Basic Books.

Seidel, T., Blomberg, G. \& Renkl, A. (2013). Instructional strategies for using video in teacher education. Teaching and Teacher Education, 34, 56-65. https://doi.org/10.1016/ j.tate.2013.03.004

Seidel, T., Stürmer, K., Blomberg, G., Kobarg, M. \& Schwindt, K. (2011). Teacher learning from analysis of videotaped classroom situations: Does it make a difference whether teachers observe their own teaching or that of others? Teaching and Teacher Education, 27 (2), 259-267. https://doi.org/10.1016/j.tate.2010.08.009

Terhart, E. (Hrsg). (2000). Perspektiven der Lehrerbildung Deutschland. Weinheim: Beltz.

Wahl, D. (1991). Handeln unter Druck - Der weite Weg vom Wissen zum Handeln bei Lehrern, Hochschullehrern und Erwachsenenbildnern. Weinheim: Deutscher Studienverlag.

Weidenmann, B. (2001). Lernen mit Medien. In A. Krapp \& B. Weidenmann (Hrsg.), Pädagogische Psychologie. Ein Lehrbuch (S. 417-465). Weinheim: Beltz PVU.

Wyss, C. (2013). Unterricht und Reflexion: Eine mehrperspektivische Untersuchung der Unterrichts- und Reflexionskompetenz von Lehrkräften (Empirische Erziehungswissenschaft, Bd. 44). Münster: Waxmann. 


\title{
Positive Einflussfaktoren im Zertifikat lehren.lernen
}

\author{
Entwicklung beruflicher Handlungskompetenz von \\ Lehramtsstudierenden in der Erziehungswissenschaftlichen \\ Lehr- und Forschungswerkstatt (ELF)
}

Im Beitrag werden positive Einflussfaktoren auf die Kompetenzentwicklung Studierender im Rahmen ihrer Teilnahme am Zertifikatsprogramm der ELF analysiert. Die Studierenden erprobten sich dort im Handeln, indem sie nicht nur aktiv an den Angeboten Kollegiale Fallberatung und Kompetenztrainings teilnahmen, sondern diese auch selbst konzipierten und durchführten und dabei intensiv begleitet wurden. In zwei Gruppendiskussionen wurden insgesamt zwölf der Zertifikatsabsolventinnen und-absolventen zu ihrer Kompetenzentwicklung befragt. Dabei zeigte sich, dass die Befragten insbesondere die folgenden Faktoren als förderlich für ihren Lernprozess wahrnahmen: (1) ELF als geschützter Raum, (2) Peer Learning, (3) sich ausprobieren, (4) Verantwortungsübernahme, (5) Begleitung, (6) Reflexion und (7) stringente Bearbeitung von Entwicklungszielen. Innerhalb eines Lehramtsstudiums bietet das Zertifikat zusätzliche Lernerfahrungen, die die Kompetenzentwicklung und das professionelle Selbst der zukünftigen Lehrkräfte anregen und stützen.

Schlüsselworter: Handlungskompetenz, positive Einflussfaktoren auf Kompetenzentwicklung, Peer Coaching

\section{Einleitung}

Im Hinblick auf die Förderung beruflicher Handlungskompetenz angehender Lehrkräfte werden bislang Praktika sowie das Praxissemester im Rahmen universitärer Lehrerbildung diskutiert und erforscht: Praxisphasen sollen den Studierenden nicht nur einen Einblick in das spätere Handlungsfeld Schule gewähren, sondern ihnen auch die Möglichkeit zur praktischen Erprobung eigener Fähigkeiten und Fertigkeiten bieten und so zur Kompetenzentwicklung beitragen (Gollub, Paulus, Rott, \& Veber, 2018; König, Rothland \& Schaper, 2018). Das im Fokus des Beitrags stehende Zertifikat lehren.lernen der Erziehungswissenschaftlichen Lehrund Forschungswerkstatt (ELF) der Westfälischen Wilhelms-Universität (WWU) Münster wählt mit seinen fall- und handlungsorientierten Angeboten einen anderen Ansatz: Studierende sollen sich bereits in der Hochschule selbst im Handeln erproben und reflektieren. Handlungskompetenz wird in der ELF in Anlehnung an Baumert und Kunter (2006) verstanden als Konglomerat aus berufsspezifischem Wissen und Können, motivationalen Orientierungen, Überzeugungen und 
selbstregulativen Fähigkeiten. Zur Kompetenzentwicklung wurde ein geschützter Lehr- und Lernraum geschaffen, der ausschließlich von und mit Studierenden der Erziehungs- und Bildungswissenschaft gestaltet wurde. Wenngleich sich das Konzept des Zertifikats auf bestehende pädagogische Konzepte (z. B. Ermöglichungsdidaktik, TZI) stützt und gute Bedingungen zur Entwicklung von Handlungskompetenz bereitstellen will, war bislang unklar, ob die teilnehmenden Studierenden eben diese Bedingungen auch aus ihrer Perspektive als förderlich wahrnehmen. Der vorliegende Beitrag widmet sich daher den Einflussfaktoren, die sich aus Sicht der Zertifikatsabsolventen positiv auf ihren Lernprozess innerhalb der ELF ausgewirkt haben. Dazu wird im Folgenden zunächst auf Faktoren eingegangen, die sich bislang in Forschungsarbeiten als die Kompetenzentwicklung beeinflussend herausgestellt haben (2). Anschließend wird das Konzept des Zertifikats lehren.lernen unter besonderer Berücksichtigung der Überlegungen zur Komplexitätsreduktion in Lehr-Lern-Laboren erörtert (3). In Abschnitt 4 wird das Forschungsdesign dargestellt und nachfolgend die Ergebnisse präsentiert (5). Der Beitrag schließt mit einem Fazit (6).

\section{Forschungsergebnisse zu Einflussfaktoren auf die Kompetenzentwicklung}

Die Forschungsergebnisse zu unterschiedlichen Einflussfaktoren, die die Kompetenzentwicklung beeinflussen können, sind bislang als überschaubar zu bezeichnen. $\mathrm{Zu}$ finden sind Studien mit unterschiedlichen Forschungsdesigns, in denen meist die Kompetenzentwicklung von Studierenden - häufig in Praktika oder Praxissemestern - erfasst und in diesem Zusammenhang auch die Einflussfaktoren auf die Kompetenzentwicklung untersucht wurden. Dabei handelt es sich in der Regel um Befragungen von Studierenden (z. B. Bach, Besa \& Arnold, 2014; Gröschner, Schmitt \& Seidel, 2013; Hascher \& Moser, 2001; Schubarth, Gottmann \& Krohn, 2014; Schubarth, Speck, Seidel, Gottmann, Kamm, \& Krohn, 2012a, 2012b; Schubarth, Speck, Seidel, Gottmann, Kamm, Kleinfeld \& Krohn, 2012).

Ein positiver Einfluss, der sich in mehreren Studien zeigt, wird der Begleitung von Praxiserfahrungen zugesprochen. Positiv wirkt sich hier vor allem eine gute Beziehungsqualität zur Mentorin oder zum Mentor, d.h. der betreuenden Lehrkraft aus, die in der Regel die Praxisphasen in der Schule begleitet (z. B. Beck \& Kosnik, 2002; Gröschner et al., 2013; Hascher \& Moser, 2001; Schubarth et al., 2014; Schubarth et al., 2012b; Schubarth et al., 2012). Bennewitz und Grabosch (2017) konnten dies zudem auch für die Begleitung von Praxiserfahrungen durch Peer Coaches feststellen. Eine positive Beziehungsqualität zwischen Studierenden und Begleitpersonen zeichnet sich laut Studien durch eine kollegiale Zusammenarbeit, einen respektvollen Umgang miteinander und gegenseitige Sympathie sowie Zeit für persönliche Treffen aus (Beck \& Kosnik, 2002; Hascher \& Moser, 2001; Hobson, Ashby, Malderez \& Tomlinson, 2009). 
Mayr (2007, 2009) konnte zwei weitere Faktoren herausarbeiten: In seiner Studie, in der er nicht nur Lehramtsstudierende, sondern auch Absolventen befragte, zielte er auf die Erfassung der Nützlichkeit und des Zusammenhangs verschiedener Lerngelegenheiten (eigene Praxiserfahrung, kollegiale Anregungen, Informationsinputs und Übungen) zum Kompetenzerwerb. Unter Kompetenzen versteht er dabei die Fähigkeit zur Gestaltung von Unterricht, zum Fördern sozialer Beziehungen, zum Eingehen auf spezifische Bedürfnisse, zur Kontrolle und Bewertung von Schülerverhalten sowie zur Zusammenarbeit mit Eltern und Kollegen. Im Ergebnis zeigt sich erstens, dass das Lernen durch eigene praktische Erfahrungen nicht nur von den Befragten als am nützlichsten beurteilt wird, sondern auch mit Entwicklungen in allen Kompetenzbereichen korreliert, insbesondere mit der Kompetenz ,Unterricht gestalten' (Mayr, 2007). Zweitens zeigt sich, dass die Kompetenzentwicklung in dem Bereich am höchsten ist, der von den einzelnen Befragten individuell als am nützlichsten bewertet wurde (Mayr, 2007). Eine Ausnahme bilde hier die Aneignung von Wissen: Diese scheint laut seiner Analysen keinen Einfluss auf die Kompetenzentwicklung zu haben, selbst dann nicht, wenn sie von den Befragten als nützlich eingestuft wurde (Mayr, 2007). Ähnliches zeigen auch die Befunde von Bach et al. (2014) aus der Studie ESIS: Weder eine pädagogische Vorerfahrung der befragten Studierenden noch das schulpädagogisch-didaktische Vorwissen haben einen positiven Einfluss auf die Kompetenzentwicklung im Praktikum. In den Studien stellte sich also heraus, dass sich die Einflussfaktoren ,praktische Erfahrungen sammeln', ,Begleitung' und ,Berücksichtigung subjektiver Präferenzen in der Auswahl des Lernsettings' positiv auf eine Kompetenzentwicklung auswirken, Vorerfahrungen oder die Wissensaneignung hingegen nicht.

\section{Das Konzept des Zertifikats lehren.lernen der Erziehungswissenschaftlichen Lehr- und Forschungswerkstatt (ELF)}

Das Zertifikat lehren.lernen wurde von 2007 bis 2018 von der ELF des Instituts für Erziehungswissenschaft der WWU Münster angeboten. Das von Hedda Bennewitz entwickelte Projekt stand allen Studierenden der Erziehungs- und Bildungswissenschaft offen und es konnte zu einem beliebigen Zeitpunkt im Studium begonnen und studienbegleitend durchgeführt werden. Bis zum Abschluss des Zertifikats wurden in der Regel drei Semester benötigt. Das Angebot setzte sich aus drei Bausteinen zusammen, die von drei bis vier studentischen Mitarbeitenden der ELF koordiniert und in Anteilen durchgeführt wurden: (1) Kollegiale Fallberatung (KFB), (2) Kompetenztrainings und (3) Portfolioarbeit.

Die Kollegiale Fallberatung ist ein strukturiertes Beratungsangebot, das in Kleingruppen stattfindet. Ziel der 6o-minütigen Beratungssitzung ist die systematische Bearbeitung von Handlungsproblemen aus der pädagogischen Praxis anhand eines standardisierten Ablaufschemas (Tietze, 2010). 
Unter Kompetenztrainings sind im Rahmen des Zertifikats zweitägige (12-stündige) Lehr-Lernsettings von und für Studierende zu wechselnden berufsbezogenen Themen wie Leitungskompetenz, Präsentationsfähigkeit, Inklusion etc. zu verstehen. Das Besondere der Kompetenztrainings liegt in dem Vorhandensein hoher handlungspraktischer sowie reflexiver Anteile. Gleichzeitig sind die theoretischen Anteile gering. Damit stellen die Kompetenztrainings eine Ergänzung zum regulären Lehrangebot der Universität dar.

Das ca. 25-seitige Entwicklungsportfolio bildet die Abschlussarbeit des Zertifikats. Es dient der Reflexion der einzelnen Bausteine in Bezug auf die persönliche Entwicklung der teilnehmenden Studierenden.

Nachfolgend werden die Anforderungen an die Studierenden sowie die Unterstützungsleistungen im Rahmen des Zertifikats unter besonderer Berücksichtigung des Modells der Komplexitätsreduktion (Marohn, Greefrath, Hammann, Hemmer, Kürten \& Windt, in diesem Band) dargestellt. Das Modell zeigt mit Hilfe von ,Reglern' zu unterschiedlichen Aspekten auf, wie die Anforderungen an Studierende in einem Lehr-Lern-Labor verringert oder erhöht werden können (siehe Abb. 1, linke Spalte). Zugleich zeigt es Maßnahmen zur Unterstützung der Studierenden im Lehr-Lern-Labor auf, die ebenfalls erhöht oder verringert werden können (siehe Abb. 1, rechte Spalte). In Abbildung 1 sind die Regler so eingestellt, dass sie die Anforderungen und Unterstützungsleistungen im Rahmen der ELF widerspiegeln. Diese werden in den beiden nachfolgenden Kapiteln erörtert.

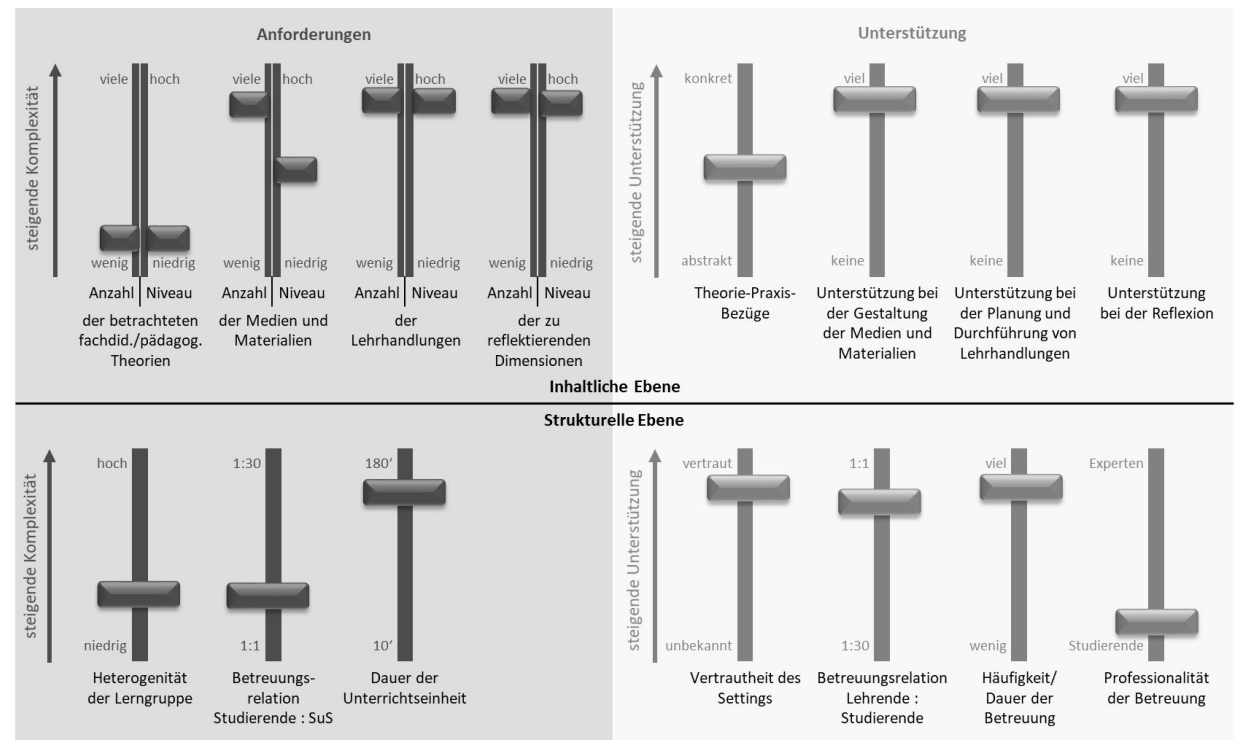

Abbildung 1: Anforderungen und Unterstützungsleistungen im Zertifikat lehren.lernen der ELF dargestellt anhand des Modells zur Komplexitätsreduktion von Marohn, Greefrath, Hammann, Hemmer, Kürten und Windt (in diesem Band) 


\subsection{Anforderungen an die Studierenden im Zertifikat lehren.lernen}

Die Anforderungen, die das Zertifikat an die Zertifikatsanwärterinnen und -anwärter stellt, können anschaulich anhand der Struktur des Zertifikats: TeilnahmeSchulung-Leitung-Reflexion erläutert werden:

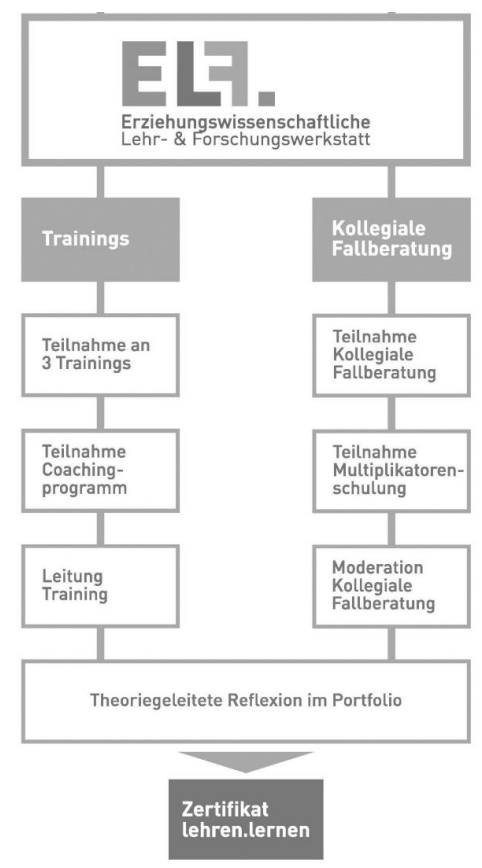

Abbildung 2: Ablauf des Zertifikats lehren.lernen der ELF

Das Zertifikatskonzept sieht zunächst die Teilnahme an acht Sitzungen Kollegialer Fallberatung sowie die Teilnahme an drei Trainings inklusive des obligatorischen ,Leitungskompetenztrainings' vor, in dem sich die Studierenden mit Aufgaben und Funktionen von Leitungspersonen auseinandersetzen. ${ }^{1}$ Anschließend sieht das Konzept die Teilnahme an je einer Schulung in den Bausteinen KFB sowie Trainings vor, in der die Studierenden auf die Moderation bzw. Leitung des jeweiligen Angebots vorbereitet werden. Daraufhin erfolgt schließlich die eigenständige Leitung - das Herzstück des Zertifikats - auf die mit den anderen Stationen hingearbeitet wird. Die Leitungsaufgabe besteht zum einen darin, dass die Studierenden acht Sitzungen KFB entsprechend des standardisierten Ablaufschemas (siehe Bennewitz \& Grabosch, 2017) moderieren und darüber hinaus zu Beginn jeder KFB-Sitzung eine kurze Kennenlern- bzw. Ankommensrunde sowie am Ende eine kurze Reflexionsrunde mit den teilnehmenden Studierenden gestalten. Zum an-

1 Die Teilnahme an den beiden Angeboten KFB und Trainings konnte sowohl parallel als auch nacheinander erfolgen, wobei die Reihenfolge frei wählbar war. 
deren besteht die Leitungsaufgabe darin, ein Training nach einem selbstgewählten Thema mit Berufsbezug zu konzipieren und im Trainertandem mit einem anderen Zertifikatsanwärter durchzuführen.

Bei der Konzeption des Trainings sind die Studierenden angehalten, einen kleinen Theorie-Input aufzubereiten und darauf aufbauend praktische Methoden (z.B. Rollenspiel oder Gruppenpuzzle) sowie reflexive Einheiten (z.B. in Einzeloder Gruppenarbeit) einzubauen. Außerdem sollen die Zertifikatsanwärterinnen und -anwärter eine Einstiegsrunde zum Kennenlernen der Lerngruppe sowie eine Ausstiegsrunde zur Reflexion des Trainings und ihres eigenen Leitungshandelns planen. Weitere Vorgaben bestehen im Vorbereiten ansprechender Lernmaterialien (dazu gehört z.B. die Gestaltung eines Handouts sowie eines Lerntagebuchs mit gezielten Reflexionsfragen zu einzelnen Übungen), die Auswahl geeigneter Medien sowie das Vorbereiten eines Buffets, an dem sich die teilnehmenden Studierenden an den zwei Tagen bedienen können. Zuletzt werden die Zertifikatsanwärterinnen und -anwärter angehalten, die Kommunikation mit den teilnehmenden Studierenden wertschätzend und konstruktiv zu gestalten, so wie es in den Schulungen zuvor reflektiert und geübt wurde (siehe zu den Anforderungen auch Grabosch \& Bennewitz, 2018). Nachdem die Leitung erfolgt ist, sollen abschließend die Erfahrungen der Studierenden während des Zertifikatserwerbs in einem Portfolio unter besonderer Berücksichtigung der individuellen Entwicklungsaufgaben reflektiert werden. Die Entwicklungsaufgaben wurden im Laufe des Zertifikatserwerbsprozesses von den Studierenden selbst gesetzt und standen immer in Bezug zu ihrer Handlungskompetenzentwicklung. Dabei konnte es sich um völlig unterschiedliche Inhalte handeln, wie beispielsweise die Verbesserung der eigenen Sicherheit im Auftreten oder die Verbesserung der eigenen Planungs- oder Kommunikationsfähigkeit etc. (siehe hierzu Grabosch \& Bennewitz, 2018).

Insgesamt können die hier dargestellten Anforderungen vor dem Hintergrund der meist fehlenden Erfahrung der Studierenden in der Planung und Durchführung von Lehr-Lernsettings als hoch und komplex bezeichnet werden. Die Komplexität wird in diesem Sinne nur in Bezug auf das Einbringen pädagogischer Theorien (der Theorieanteil ist vorhanden, aber gering), die Heterogenität der Lerngruppe (es dürfen nur Studierende der Erziehungs- und Bildungswissenschaft teilnehmen) und die Betreuungsrelation (es wird ausschließlich in Kleingruppen von 2-14 Personen gearbeitet) reduziert (vgl. Abb. 2). Damit die Studierenden die anspruchsvollen Aufgaben dennoch ohne Überforderung und zur Zufriedenheit aller Beteiligten bewältigen können, existiert ein umfangreiches Begleitkonzept zur Komplexitätsreduktion, das nachfolgend erörtert wird. 


\subsection{Komplexitätsreduktion durch Unterstützungsmaßnahmen im Zertifikat lehren.lernen}

Das Begleitkonzept zur Unterstützung der Zertifikatsanwärterinnen und -anwärter besteht aus den in Tabelle 1 dargestellten Maßnahmen und Begleitmaterialien.

Tabelle 1: Maßnahmen und Begleitmaterialien zur Unterstützung der Zertifikatsanwärterinnen und -anwärter

\begin{tabular}{|c|c|c|c|c|}
\hline & Maßnahme & Umfang & Ziel & Betreuungsschlüssel \\
\hline 1 & $\begin{array}{l}\text { Einführungs- } \\
\text { gespräch zu } \\
\text { Beginn des } \\
\text { Zertifikats }\end{array}$ & 1-stündig & $\begin{array}{l}\text { Besprechen und Festhalten } \\
\text { des Ablaufs des Zertifikats }\end{array}$ & $\begin{array}{l}1 \text { Peer Coach - } \\
1 \text { Zertifikats- } \\
\text { anwärter/in }\end{array}$ \\
\hline 2 & $\begin{array}{l}\text { Zwischenrefle- } \\
\text { xion während } \\
\text { der Moderation } \\
\text { der KFB }\end{array}$ & 4-stündig & $\begin{array}{l}\text { Reflexion der eigenen KFB } \\
\text { Moderation; } \\
\text { Bei Bedarf gemeinsame } \\
\text { Klärung von Handlungs- } \\
\text { problemen }\end{array}$ & $\begin{array}{l}1 \text { Peer Coach - } \\
10 \text { Zertifikats- } \\
\text { anwärter/innen, } \\
\text { die KFB moderieren }\end{array}$ \\
\hline 3 & $\begin{array}{l}\text { Abschlussre- } \\
\text { flexionssitzung } \\
\text { nach der Mo- } \\
\text { deration der } \\
\text { KFB }\end{array}$ & 4-stündig & $\begin{array}{l}\text { Reflexion der eigenen KFB } \\
\text { Moderation; } \\
\text { Bei Bedarf gemeinsame } \\
\text { Klärung von Handlungs- } \\
\text { problemen; } \\
\text { Abschließende Evaluation } \\
\text { der KFB Sitzungen }\end{array}$ & $\begin{array}{l}1 \text { Peer Coach - } \\
10 \text { Zertifikats- } \\
\text { anwärter/innen, } \\
\text { die KFB moderieren }\end{array}$ \\
\hline 4 & $\begin{array}{l}\text { Drei Peer- } \\
\text { Coaching- } \\
\text { Sitzungen: zwei } \\
\text { Sitzungen vor } \\
\text { und eine nach } \\
\text { der Trainings- } \\
\text { leitung }\end{array}$ & Je 4-stündig & $\begin{array}{l}\text { Unterstützung in der Pla- } \\
\text { nung und Strukturierung } \\
\text { des Kompetenztrainings; } \\
\text { Reflexion der persönlichen } \\
\text { Entwicklung in Bezug auf } \\
\text { die Handlungskompetenz; } \\
\text { Festhalten und Reflexion } \\
\text { von Entwicklungsaufgaben; } \\
\text { Evaluation der Trainings- } \\
\text { leitung und Trainingsbe- } \\
\text { gleitung }\end{array}$ & $\begin{array}{l}1 \text { Peer Coach - } \\
2 \text { Zertifikats- } \\
\text { anwärter/innen, } \\
\text { die zusammen ein } \\
\text { Training konzipieren } \\
\text { und leiten }\end{array}$ \\
\hline 5 & $\begin{array}{l}\text { Ein das Zerti- } \\
\text { fikat abschlie- } \\
\text { ßendes Portfo- } \\
\text { liogespräch }\end{array}$ & 1-stündig & $\begin{array}{l}\text { Reflexion des gesamten } \\
\text { Zertifikatserwerbs unter be- } \\
\text { sonderer Berücksichtigung } \\
\text { der persönlichen Entwick- } \\
\text { lungsaufgaben }\end{array}$ & $\begin{array}{l}1 \text { Peer Coach - } \\
1 \text { Zertifikats- } \\
\text { anwärter/in }\end{array}$ \\
\hline 6 & Präsenzzeit & variabel & $\begin{array}{l}\text { Pro Woche bieten alle 3- } 4 \\
\text { Mitarbeitenden der ELF je } \\
\text { eine offene Präsenzzeit für } \\
\text { alle Zertifikatsanwärter/in- } \\
\text { nen sowie alle am Zertifikat } \\
\text { interessierten Personen an, } \\
\text { in der sämtliche Anliegen } \\
\text { persönlich besprochen } \\
\text { werden können }\end{array}$ & $\begin{array}{l}1 \text { Peer Coach - } \\
1 \text { Zertifikats- } \\
\text { anwärter/in bzw. } \\
\text { interessierte Person }\end{array}$ \\
\hline
\end{tabular}




\begin{tabular}{|l|l|l|l|l|}
\hline & $\begin{array}{l}\text { Begleit- } \\
\text { materialien }\end{array}$ & Beschreibung & Ziel & Betreuungsschlüssel \\
\hline 7 & FiBEL & $\begin{array}{l}\text { Die FiBEl ist ein Sammel- } \\
\text { ordner mit einem Katalog } \\
\text { an Reflexionsfragen zu } \\
\text { den einzelnen Bausteinen } \\
\text { (KFB, Trainings, Portfo- } \\
\text { lio), den verschiedenen } \\
\text { Stationen (Teilnahme, } \\
\text { Schulung, Leitung) sowie } \\
\text { zu vier universitären } \\
\text { Lehrveranstaltungen des } \\
\text { Bildungswissenschaft- } \\
\text { lichen Studiums der } \\
\text { WwU. Die FiBEL enthält } \\
\text { auch die Arbeitsanwei- } \\
\text { sungen zur Erstellung des } \\
\text { aion des eigenen Handelns } \\
\text { im Zertifikat und der Bau- } \\
\text { steine. Hinzukommen Re- } \\
\text { flexionsfragen zu den vier } \\
\text { im Bildungswissenschaftli- } \\
\text { chen Studium der WWU zu } \\
\text { belegenden Veranstaltun- } \\
\text { gen. Diese sollen ebenfalls, } \\
\text { vor allem in Bezug auf die } \\
\text { eigene Entwicklung, reflek- } \\
\text { tiert werden }\end{array}$ & $\begin{array}{l}\text { Unbetreute, weil } \\
\text { freiwillige, nicht vor- } \\
\text { die zuhause bearbei- } \\
\text { tet werden kann }\end{array}$ \\
\hline 8 & Lerntagebücher \\
$\begin{array}{l}\text { Die Lerntagebücher } \\
\text { werden in den jeweiligen } \\
\text { Trainings an die Teil- } \\
\text { nehmenden ausgeteilt } \\
\text { und enthalten Reflexi- } \\
\text { onsfragen, die auf die im } \\
\text { Training durchgeführten } \\
\text { Methoden abgestimmt } \\
\text { sind }\end{array}$ & $\begin{array}{l}\text { Zielgerichtete Reflexion } \\
\text { ausgewählter Methoden im } \\
\text { Training sowie Selbstre- } \\
\text { flexion }\end{array}$ & $\begin{array}{l}\text { Die Bearbeitung } \\
\text { der Lerntagebücher } \\
\text { erfolgt zwar unter } \\
\text { Anleitung der Zerti- } \\
\text { fikatsanwärter/innen, } \\
\text { die das jeweilige } \\
\text { Training leiten, den- } \\
\text { noch sind die Inhalte } \\
\text { der Lerntagebücher } \\
\text { privat und werden } \\
\text { nicht eingesehen, } \\
\text { in der Regel nicht } \\
\text { reflektiert und auch } \\
\text { nicht gemeinsam } \\
\text { bearbeitet }\end{array}$ \\
\hline & & & \\
\hline
\end{tabular}

Bereits zu Beginn des Zertifikatserwerbs werden die Zertifikatsanwärterinnen und -anwärter in einem Einführungsgespräch von einem studentischen Mitarbeiter oder einer studentischen Mitarbeiterin (dem Coach) begleitet (siehe Tab. 1, Zeile 1). In dieser Sitzung wird der Zertifikatsablauf gemeinsam besprochen. Dabei wird festgelegt, ob die Zertifikatsanwärterin oder der -anwärter zuerst mit der Teilnahme an den Trainings bzw. der KFB oder zeitgleich mit beiden Angeboten startet. Auch Auslandssemester oder Praktika etc. finden Eingang in die Planung. Abschließend erhalten die Studierenden ihre persönliche FiBEL (siehe Tab. 1, Zeile 7), die sie über den gesamten Prozess des Zertifikatserwerbs bearbeiten können.

Neben den wöchentlichen Präsenzzeiten in der ELF, in der je ein studentischer Mitarbeiter oder eine studentische Mitarbeiterin der ELF für ein persönliches Gespräch oder Telefonat zur Verfügung steht (siehe Tab. 1, Zeile 6), existieren verpflichtende Face-to-Face-Treffen in der Phase der eigenständigen Leitung der Angebote: Zum einen existieren die Zwischen- sowie die Abschlussreflexion während der Moderation der KFB (siehe Tab. 1, Zeile 2), zum anderen die drei PeerCoaching-Sitzungen während der Konzeption und Leitung des eigenen Trainings 
(siehe Tab. 1, Zeile 3). Diese Treffen sind von besonderer Bedeutung, da die Leitung des jeweiligen Angebots ohne Peer Coaches abläuft, d.h. die Studierenden haben während der Leitung keinen direkten Ansprechpartner vor Ort und sind alleine für das Lehr-Lernsetting und die Teilnehmenden verantwortlich. Damit diese Verantwortung von den Zertifikatsanwärterinnen und -anwärtern übernommen werden kann, werden die Aufgaben sowohl in den Schulungen als auch den Begleitsitzungen ausführlich besprochen. Neben Unklarheiten und Handlungsproblemen werden in den mehrstündigen Coaching-Sitzungen auch Entwicklungsaspekte in Form der Entwicklungsaufgaben in sämtlichen Begleitsitzungen festgehalten, bearbeitet und reflektiert. Auf diese Weise soll die zielgerichtete Weiterentwicklung der Handlungskompetenz gesichert werden.

Mit Blick auf das Modell der Komplexitätsreduktion (siehe Abb. 2) erfolgt also in allen Bereichen eine hohe Unterstützungsleistung von Seiten der ELF. Ausnahmen bilden die Regler ,Theorie-Praxis-Bezüge' und 'Professionalität der Betreuung. Zwar erfolgt eine theoretische Fundierung der Inhalte in den Trainings, sodass die Studierenden sich auch theoretisch einarbeiten müssen und TheoriePraxis-Bezüge herstellen müssen - sie werden darin auch in den Coaching-Sitzungen unterstützt - allerdings liegt der Schwerpunkt in den Trainings nicht auf einer Theorievermittlung, sondern dem Ausprobieren und Reflektieren des eigenen Handelns. Hinsichtlich der ,Professionalität der Betreuung' sieht das Modell eine Einteilung in ,Studierende` und ,Experten 'vor. Da es sich bei der ELF um ein Peer-Learning-Projekt handelt, sind auch die betreuenden Personen Studierende. Aufgrund einer ausführlichen Schulung dieser - die auch mehrere Hospitationen bei Coaching-Sitzungen umfasst - sowie einer umfassenden Supervision durch Dozierende der Universität Münster wird hier die Ansicht vertreten, dass auch die Studierenden professionell arbeiten, aber eben im Rahmen eines Peer-LearningSettings. Demnach ist im Grunde die Unterstützungsleistung auch in diesem Bereich als hoch anzusehen.

\section{Forschungsfragen und forschungsmethodisches Vorgehen}

Dem Beitrag liegt ein Datenkorpus zugrunde, das im Rahmen des Dissertationsprojektes von Anna Grabosch mit dem Titel „Deutungsmuster und Handlungskompetenz - Empirische Analysen zur praktischen Elementen in der Lehrer_innenbildung" gewonnen wurde. Es handelt sich hierbei um zwei Gruppendiskussionen mit insgesamt zwölf Zertifikatsabsolventinnen und -absolventen: Die erste Gruppendiskussion fand mit fünf Personen statt, die das Zertifikat zu diesem Zeitpunkt vor bereits zwei Jahren beendet hatten und beruflich tätig waren. Die zweite Gruppendiskussion erfolgte mit Studierenden, die das Zertifikat zu diesem Zeitpunkt vor ca. zwei Wochen beendet hatten. Als Gesprächsimpuls der Gruppendiskussionen diente die Fragestellung: „Was zieht ihr für euch aus dem Zertifikatserwerb?“. Die Frage zielte in erster Linie auf die durch das Zertifikat 
gewonnenen Erfahrungen und erworbenen Lerninhalte der Befragten, auf die sie offen, d.h. ohne Vorgaben antworten sollten. Auf diese Weise sollte die Perspektive der Befragten erfasst werden. Inhaltlich wurden von den Befragten erlernte Kompetenzfacetten genannt. Darüber hinaus wurden aber auch Erfahrungen mit dem Zertifikat ausgetauscht. Es wurde benannt, was die Befragten damals dazu bewegte, das Zertifikat zu erwerben (insbesondere hier zeigt sich eine Kritik am Studium, die sie dazu bewegte, das Zertifikat zu erwerben) und welche Faktoren sie als für den Lernprozess im Zertifikat förderlich bewerten. Die Grundstimmung in den Gruppendiskussionen war dem Zertifikat positiv zugewandt und befürwortend, gegenüber dem regulären Studium jedoch eher kritisch eingestellt.

Im Weiteren erfolgt eine Fokussierung auf die Forschungsfrage: „Welche Faktoren werden von den Zertifikatsabsolventinnen und Zertifikatsabsolventen als förderlich für ihren Lernprozess beschrieben?“. Die Fragestellung wurde mit Hilfe der qualitativen Inhaltsanalyse in Anlehnung an Kuckartz (2016) bearbeitet. Entsprechend der Forschungsfrage wurde das Material codiert. Dazu wurden zunächst Sinneinheiten gebildet. Dabei handelt es sich um Sätze oder ganze Textabschnitte $\mathrm{zu}$ einem bestimmten Thema. Diese wurden wiederum inhaltlich systematisiert. Durch diese Systematisierung kristallisierten sich Kategorien heraus, die Auskunft über förderliche Faktoren geben und nachfolgend dargestellt werden.

\section{Ergebnisse}

Die Aussagen der Befragten zu dem, was sie als lernförderlich im Zertifikat wahrgenommen haben, konnten den folgenden sieben Faktoren zugeordnet werden: (1) ELF als geschützter Raum, (2) Peer Learning, (3) Sich ausprobieren, (4) Verantwortungsübernahme, (5) Begleitung, (6) Reflexion, (7) Stringente Bearbeitung von Entwicklungszielen. Nachfolgend werden die Faktoren in ihren Grundzügen zusammengefasst und mit einzelnen Textbeispielen aus den Gruppendiskussionen versehen:

1) ELF als geschützter Raum (10 von 66 Codiereinheiten):

Die Befragten beschreiben das Zertifikat - und auch die ELF als übergeordnete Instanz - in den Gruppendiskussionen als geschützten Raum, in dem sie sich und ihre Ängste offenbaren konnten. Teilnehmerin (TN) 1 aus der zweiten Gruppendiskussion (GD 2) formuliert dies folgendermaßen:

„du kannst halt auch mal sagen, ich habe Angst davor und das kann ich nicht so gut und was kann ich machen, ohne dass die anderen denken, ach was bist du denn für ein Loser, ne, sondern sich alle erstmal zusammensetzen und gemeinsam versuchen /ähm/ eine Lösung zu finden oder sich gegenseitig zu unterstützen und Tipps zu geben“ (TN 1, GD 2) 
Darüber hinaus fanden es die Befragten hilfreich, dass im Zertifikat keine Noten und dadurch auch keine Sanktionen in Form von schlechten Noten vergeben wurden: Statt des Erreichens von Credit Points oder guter Noten konnte die eigene Entwicklung ohne Leistungsdruck in den Fokus gerückt werden:

„TN 1: Genau und ich finde dafür war das Zertifikat einfach super (-) weil man da die Möglichkeit bekommen hat, das total frei, ohne jetzt durch irgendeinen Notendruck oder \#00:11:46-2\#

TN ?: Mh (bejahend) \#00:11:46-2\#

TN 1: Leistungsdruck oder so und auch wenn mal was nicht (-) /eh/ so gelaufen ist, wie man sich das vorher überlegt hat (-) war das nicht schlimm \#00:11:51-7\#

TN ?: Mh (bejahend)“ \#00:11:49-o\# (GD 1)

2) Peer Learning (14 von 66 Codiereinheiten):

Das Lernen von und mit anderen Peers wurde aus verschiedenen Gründen als förderlich für den Lernprozess bewertet: Zum einen wurden die Peers als kompetent und dadurch als Vorbilder bzw. Mentorinnen und Mentoren gesehen, die eine Zielgröße für die eigene Entwicklung darstellten:

„ja, also ich bin da zum ersten Mal auf so Menschen gestoßen, die total reflektiert waren, die so super wertschätzendes Feedback geben konnten und so und ich war einfach nur begeistert von den Leuten, die da waren und dachte so, boah so will ich auch sein und habe die so irgendwie so als Vorbild genommen" (TN 4, GD 2)

Zum anderen nahm die Zusammenarbeit mit den Peers aufgrund der Gleichrangigkeit erstens die Angst davor, Fehler zu begehen, da diese als ,auch nicht perfekt wahrgenommen wurden und zweitens fungierten sie als Ansporn, sich selbst auszuprobieren im Sinne von, wenn sie das können, kann ich das auch!

3) Sich ausprobieren (13 von 66 Codiereinheiten):

Das Ausprobieren wird als lernförderlich betrachtet, da die Studierenden sich so nicht nur theoretisch mit Methoden des Unterrichtens auseinandergesetzt haben, sondern diese auch selbst umsetzen und reflektieren mussten:

„also die einzigste Möglichkeit wo wir Methoden kennenlernen ist eigentlich bei den ELFen $^{2}$ und das geht dann leichter von der Hand, weil man die nicht nur gelesen hat, sondern die meisten auch schon ausprobiert hat" (TN 1, GD 2)

2 Als „ELFen“ werden die studentischen Mitarbeiterinnen und Mitarbeiter der ELF bezeichnet. 
Dabei konnten die Befragten auch feststellen, welche Art von Methoden ihnen gefällt und zu ihnen und ihren Vorlieben ,passt' und, welche sie lieber nicht umsetzen möchten, weil sie diese nicht vertreten können. ${ }^{3}$

4) Verantwortungsübernahme (3 von 66 Codiereinheiten):

Die Übernahme von Verantwortung für die Durchführung des jeweiligen LehrLernsettings und die teilnehmenden Studierenden wird von den Befragten positiv hervorgehoben. Sie konnten so an den Aufgaben „wachsen“ und sich selbst in der Rolle der Leitung erleben und reflektieren.

„Das fand ich auch super (-) also sowohl, ich habe glaube ich zwei Seminare ${ }^{4}$ letztendlich gemacht, weil das /eh/ Spaß gemacht hat /ähm/ und /eh/ ja einige KFB's auch und /ähm/ das fand ich gut da irgendwie so herangeführt zu werden und dann aber auch so (-) ja, das eigenständig durchführen zu können und da bin ich glaube ich sehr daran gewachsen“ ( $\left.\mathrm{TN}_{3}, \mathrm{GD} 1\right)$

\section{5) Begleitung (5 von 66 Codiereinheiten):}

Vor allem die Trainingsbegleitung, d.h. die Schulung und die Coaching-Sitzungen, werden von den Befragten als hilfreich betrachtet, und zwar nicht nur in Bezug auf die formale Ebene der Trainingskonzeption und -durchführung, sondern auch in Bezug auf das Teambuilding, das in den Sitzungen erfolgte. So haben sie zum Einen einen Leitfaden für die Reflexion eigener Lehr-Lernsettings (z. B. den eigenen Unterricht im Referendariat) erhalten und zum anderen stellten sie durch die Begleitung eine verbesserte Zusammenarbeit im Trainertandem fest.

„TN 2: [...] „/ähm/ Vielleicht nur noch ganz kurz ergänzend /eh/ als du das Stichwort gesagt hast /eh/ Referendariat; da vielleicht nur ganz (-)/eh/ kurz noch, im Referendariat mit Stichwort Reflexion /ähm/fand ich es auch gerade in der Ausbildung ${ }^{5}$ sehr, sehr hilfreich, auch diese /eh/ Begleittreffen /ähm/was hat gut geklappt, was hat vielleicht nicht so gut geklappt /ähm/ wie bewerte ich, wie sehe ich rückblickend meine eigene Unterrichtstunde, [meinen Unterricht] \#00:18:54-8\#

TN 4: [mh (bejahend)]\#00:18:58-5\#

TN ?: [ja] \#oo:18:55-3\#

TN 2: /ähm/ Wie baue ich das auf, wie spreche ich darüber mit Seminarleitern im Seminar /ähm/ während dieser Ausbildungsphase" (GD 1)

6) Reflexion (14 von 66 Codiereinheiten)

Die regelmäßigen Reflexionen in den unterschiedlichen Begleitsitzungen werden sowohl für die persönliche Entwicklung als auch für das Verständnis des Studiums

3 Hier wird meist Bezug genommen auf Methoden mit stark spielerischem Charakter, dem nicht alle zugetan waren.

4 Mit „Seminaren“ spricht der oder die Studierende hier die Trainings der ELF an.

5 Mit „Ausbildung“ spricht der oder die Studierende hier das Referendariat an. 
und den Zusammenhang der Inhalte im Studium (roter Faden) als sinnvoll erachtet.

„Ich habe jetzt gerade bei dir auch gedacht /ähm/ so, wie wenn man eine Biografiearbeit quasi über seinen Lebenslauf nachdenkt und so seinen roten Faden irgendwie findet, finde ich /eh/ hat man, durch diese Reflexion in der ELF irgendwie so den roten Faden in seinem Studium gefunden oder zumindest ging es /ähm/ mir so, dass ich dann wirklich sehen konnte, ok, das macht alles irgendwo Sinn, es baut aufeinander auf, da sind, die Veranstaltungen, die du gerne gemacht hast, die hängen alle irgendwo damit zusammen, klar, es gibt auch immer mal Veranstaltungen, die man da jetzt nicht so einordnen kann, weil es einfach Pflichtveranstaltungen waren, aber /ähm/ ja man erkennt so eine Art roten Faden irgendwie so, warum man das alles macht" ( $\left.\mathrm{TN}_{4}, \mathrm{GD}_{2}\right)$

Gleichzeitig wird davon berichtet, dass das Reflektieren als anstrengend, gar schmerzhaft empfunden wurde, weshalb die verpflichtenden Reflexionseinheiten in den Begleitsitzungen befürwortet werden: freiwillig hätten sich die Befragten den intensiven Prozessen der Reflexion wohl eher nicht ausgesetzt. Als guten Zeitpunkt für eine (Selbst-)Reflexion nennen sie einen Zeitpunkt, der weder zu nah, noch zu weit von der jeweiligen durchgeführten Aufgabe entfernt ist: Reflexionsprozesse benötigen aus ihrer Sicht Zeit, damit sich Erkenntnisse einstellen, gleichzeitig sind sie aber auch an Übungen gebunden, sodass mögliche Erkenntnisse in Vergessenheit geraten, wenn sie nicht zeitnah reflektiert werden.

7) Stringente Bearbeitung von Entwicklungszielen (7 von 66 Codiereinheiten) Auch die Bearbeitung der Entwicklungsziele wurde als förderlich betrachtet, da diese regelmäßig aufgegriffen und die Entwicklung zielgerichtet bearbeitet wurde.

„Ich wollte gerade nochmal kurz auf dieses Entwicklung oder auf die Entwicklungsziele zu sprechen kommen, weil ich /ähm/ auch finde, dass das dann, also das wir das dann auch immer so sehr intensiv uns wirklich dann auf ein dieses Entwicklungsziel quasi so auch eingestellt haben und dann wirklich auch /ähm/ versucht haben, daran aktiv zu arbeiten und sonst finde ich, ist es auch oft so, ja das könnte ich ein bisschen verbessern und das vielleicht auch und ich finde, so war das aber viel mehr im Fokus“ (TN, GD 2)

\section{Fazit}

Die hier (nur in einem Ausschnitt) präsentierten Ergebnisse der Studie zum Einfluss des Lernens in der ELF auf die berufliche Kompetenzentwicklung von Lehramtsstudierenden ordnet sich im Großen und Ganzen in die Ergebnistendenzen anderer empirischer Studien zu den Einflussfaktoren unterschiedlicher Formen von Praxiserfahrungen während des Studiums auf die Kompetenzentwicklung ein. 
Entscheidend ist die Beziehungsqualität während der Praxisphase, ebenso die erfahrene Unterstützung. Die Begleitung der Studierenden vor, während und nach dem Praktikum, oder allgemeiner gesprochen: Die Einbettung der Praktikumserfahrung in das gesamte Studium erweist sich als eine der zentralen Gelingensvoraussetzungen für produktive Praktikumserfahrungen im Lehramtsstudium. Der besondere Ansatz der ELF weist darüber hinaus spezielle Kennzeichen auf, die von den Zertifikatsabsolventinnen und -absolventen positiv bewertet werden: (1) ELF als geschützter Raum ohne Leistungsdruck, (2) Peer Learning steht im Vordergrund und wird als angstreduzierend und motivierend wahrgenommen, (4) Verantwortungsübernahme wird konkret erfahrbar, (6) Reflexion wird sozial geteilt und führt sowohl zur Selbsterkenntnis als auch zu Erkenntnisprozessen, die das Studium betreffen, (7) stringente Bearbeitung von Entwicklungszielen zur systematischen Kompetenzförderung. Wenngleich die Anforderungen der ELF an die Studierenden als hoch und komplex zu bezeichnen sind, scheinen die vielfältigen Unterstützungsmaßnahmen im Rahmen der engen Begleitung die hohen Anforderungen für die Studierenden bewältigbar werden zu lassen. Die Studierenden befürworten in ihrer Bewertung sogar die hohe Verantwortungsübernahme für ihre persönliche Entwicklung und nehmen die ELF als geschützten Raum war. Innerhalb eines regulären Lehramtsstudiums bietet die ELF mithin erweiterte, praxisgestützte und mit Peers geteilte Lernerfahrungen, die das professionelle Selbst der zukünftigen Lehrkräfte anregen und stützen.

\section{Literatur}

Bach, A., Besa, K.-S. \& Arnold, K.-H. (2014). Bedingungen von Lernprozessen in Schulpraktika: Ergebnisse aus dem Projekt ESIS (Entwicklung Studierender in Schulpraktika). Conditions of learning processes during interships: Results from the projekt TDSI (Teacher Student Development in Interships). In K-H. Arnold, A. Gröschner \& T. Hascher (Hrsg.), Schulpraktika in der Lehrerbildung: Theoretische Grundlagen, Konzeptionen, Prozesse und Effekte. Pedagogical field experiences in teacher education: Theoretical foundations, programmes, processes, and effects (S. 165-182). Münster: Waxmann.

Baumert, J. \& Kunter, M. (2006). Stichwort: Professionelle Kompetenz von Lehrkräften. Zeitschrift für Erziehungswissenschaft, 9 (4), 469-520. https://doi.org/10.1007/s11618oo6-0165-2

Beck, C. \& Kosnik, C. (2002). Components of a Good Practicum Placement: Pre-Service Teacher Perceptions. Teacher Education Quarterly, 29, 81-98.

Bennewitz, H. \& Grabosch, A. (2017). Peer Coaching in der universitären Lehrerbildung: Empirische Befunde zur Begleitung von Praxiserfahrungen. Sonderheft der Zeitschrift Lehrerbildung auf dem Prüfstand, 10, 50-61.

Gollub, P., Paulus, D., Rott, D. \& Veber, M. (2018). Studentische Forschung im Praxissemester. Frankfurt: Lang. https://doi.org/10.3726/b12655

Grabosch, A. \& Bennewitz, H. (2018). Peer Coaching im Kontext der Erziehungswissenschaftlichen Lehr- und Forschungswerkstatt (ELF) der Universität Münster. In T. 
Stroot \& P. Westphal (2018), Peer Learning an Hochschule: Elemente einer diversitysensiblen, inklusiven Bildung (S. 129-146). Bad Heilbrunn: Klinkhardt.

Gröschner, A., Schmitt, C. \& Seidel, T. (2013). Veränderung subjektiver Kompetenzeinschätzungen von Lehramtsstudierenden im Praxissemester. Zeitschrift für Psychologie, 27, 77-86. https://doi.org/10.1024/1010-0652/aoooo9o

Hascher, T. \& Moser, P. (2001). Betreute Praktika - Anforderungen an Praktikumslehrerinnen und -lehrer. Beiträge zur Lehrerinnen- und Lehrerbildung, 19 (2), 217-231.

Hobson, A., Ashby, P., Malderez, A. \& Tomlinson, P. (2009). Mentoring beginning teachers: What we know and what we don't. Teaching and Teacher Education, 25, 207-216. https://doi.org/10.1016/j.tate.2008.09.001

König, J., Rothland, M. \& Schaper, N. (Hrsg.). (2018). Learning to Practice, Learning to Reflect?: Ergebnisse aus der Längsschnittstudie LtP zur Nutzung und Wirkung des Praxissemesters in der Lehrerbildung. Wiesbaden: Springer. https://doi.org/10.1007/978-3658-19536-6

Kuckartz, U. (2016). Qualitative Inhaltsanalyse. Methoden, Praxis, Computerunterstützung (3. Aufl.). Weinheim: Beltz.

Marohn, A., Greefrath, G., Hammann, M., Kürten, R. \& Windt, A. (2020). Komplexitätsreduktion in Lehr-Lern-Laboren. Ein Planungs- und Reflexionsmodell. In R. Kürten, G. Greefrath \& M. Hammann (Hrsg.), Komplexitätsreduktion in Lehr-Lern-Laboren. Innovative Lehrformate in der Lehrerbildung zum Umgang mit Heterogenität und Inklusion (S. 17-31). Münster: Waxmann.

Mayr, J. (2007). Wie Lehrer/innen lernen - Befunde zur Beziehung von Lernvoraussetzungen, Lernprozessen und Kompetenz. In M. Lüders \& J. Wissinger (Hrsg.), Forschung zur Lehrerbildung. Kompetenzentwicklung und Programmevaluation (S. 73-90). Münster: Waxmann.

Mayr, J. (2009). Selektieren und/oder Qualifizieren? Empirische Befunde zu guten Lehrpersonen. In J. Abel \& G. Faust (Hrsg.), Wirkt Lehrerbildung? Antworten aus der empirischen Forschung (S. 73-89). Münster: Waxmann.

Schubarth, W., Gottmann, C. \& Krohn, M. (2014). Wahrgenommene Kompetenzentwicklung im Praxissemester und dessen berufsorientierende Wirkung: Ergebnisse der ProPrax-Studie. Perceived competency development during the student teaching semester and its impact on vocational orientation: Results from the ProPrax Study. In K.-H. Arnold, A. Gröschner \& T. Hascher (Hrsg.), Schulpraktika in der Lehrerbildung. Theoretische Grundlagen, Konzeptionen, Prozesse und Effekte. Pedagogical field experiences in teacher education. Theoretical foundations, programmes, processes, and effects (S. 201-219). Münster: Waxmann.

Schubarth, W., Speck, K., Seidel, A., Gottmann, C., Kamm, C., Kleinfeld, M. \& Krohn, M. (2012). Kompetenzentwicklung im Praxissemester: Ergebnisse einer Längsschnittanalyse zum „Potsdamer Modell der Lehrerbildung“. In T. Hascher \& G. H. Neuweg (Hrsg.), Forschung zur (Wirksamkeit der) Lehrer/innen/Bildung. Österreichische Beiträge zur Bildungsforschung (Band 8) (S. 201-220). Münster: LIT.

Schubarth, W., Speck, K., Seidel, A., Gottmann, C., Kamm, C. \& Krohn, M. (2012a). Das Praxissemester im Lehramt - ein Erfolgsmodell? Zur Wirksamkeit des Praxissemesters im Land Brandenburg. In W. Schubarth, K. Speck, A. Seidel, C. Gottmann, C. Kamm \& M. Krohn (2012), Studium nach Bologna: Praxisbezüge stärken?! - Praktika als Brücke zwischen Hochschule und Arbeitsmarkt (S. 137-169). Wiesbaden: VS. https://doi.org/10.1007/978-3-531-19122-5_8 
Schubarth, W., Speck, K., Seidel, A., Gottmann, C., Kamm, C. \& Krohn, M. (2012b). Praxisbezüge im Studium - Ergebnisse des ProPrax-Projektes zu Konzepten und Effekten von Praxisphasen unterschiedlicher Fachkulturen. In W. Schubarth, K. Speck, A. Seidel, C. Gottmann, C. Kamm \& M. Krohn (2012), Studium nach Bologna: Praxisbezüge stärken?! - Praktika als Brücke zwischen Hochschule und Arbeitsmarkt (S. 47-100). Wiesbaden: VS. https://doi.org/10.1007/978-3-531-19122-5_4

Tietze, K.-O. (2010). Wirkprozesse und personenbezogene Wirkungen von kollegialer Beratung: Theoretische Entwürfe und empirische Forschung. Wiesbaden: VS. https://doi. org/10.1007/978-3-531-92155-6 


\section{Schulischen Umgang mit Vielfalt verstehen und gestalten}

Konzentration auf Komplexität im erziehungswissenschaftlichen Lehr-Lern-Labor

In einem zweisemestrigen Seminar haben sich Lehramtsstudierende mit dem Themenfeld "Heterogenität und Schule" auseinandergesetzt. Zunächst wurden kleine Beobachtungsstudien in Kooperationsschulen durchgeführt, auf deren Grundlage dann Ideen für didaktisches Handeln ausgearbeitet wurden. Im folgenden Beitrag wird zunächst die Konzeption der Lehrveranstaltung vorgestellt, institutionell verortet und theoretisch begründet. Sodann wird mit Blick auf die Thematik des vorliegenden Bandes genauer untersucht, wie sich das Thema „Komplexität" im Seminarzusammenhang darstellt. Anhand von Beobachtungsprotokollen von Studierenden wird nachgezeichnet, inwiefern der hier thematisierte hochschuldidaktische Ansatz nicht nur mit einer Reduktion, sondern auch mit einer Steigerung von Komplexität verknüpft ist. Vor diesem Hintergrund wird mit Blick auf professionstheoretische Positionen vorgeschlagen, die Arbeit im Lehr-Lern-Labor als Konzentration auf Komplexität zu konzeptualisieren.

Schlüsselwörter: Aktionsforschung, Heterogenität, Konzentration auf Komplexität

\section{Das Lehr-Lern-Labor „Diagnose und Individuelle Förderung“}

Das Lehr-Lern-Labor „Diagnose und Individuelle Förderung“ im Institut für Erziehungswissenschaft der Westfälischen Wilhelms-Universität (WWU) Münster ist eine Lern- und Forschungswerkstatt, in der mit unterschiedlichen Konzepten und Formaten phasenübergreifend Professionalisierungsprozesse von (angehenden) Lehrpersonen unterstützt und begleitet werden. Dabei ist das Thema „Heterogenität in der Schule" der zentrale inhaltliche Fokus, der die einzelnen Vorhaben rahmt.

Das Lehr-Lern-Labor weist eine hybride Grundstruktur auf: Es ist sowohl als realer, materieller Raum in der Universität als auch als hochschuldidaktisches Prinzip konzeptualisiert. Dabei durchdringen sich beide Grundpfeiler, gehen aber nicht ineinander auf. Als realer Raum bildet das Lehr-Lern-Labor einen universitären Ort, an dem vielfältige Auseinandersetzungsprozesse mit dem Themenfeld „Heterogenität in der Schule“ stattfinden und unterstützt werden können. So wurde bspw. eine kleine Präsenzbibliothek mit bildungswissenschaftlichen Veröffentlichungen zum Themenfeld einschließlich wissenschaftstheoretischer und forschungsmethodischer Grundlagenliteratur aufgebaut. Die in der Arbeitsgruppe „Begabungsforschung/Individuelle Förderung" des Instituts für Erziehungswissen- 
schaft dokumentierten Test- und Diagnoseverfahren werden in das Lehr-LernLabor integriert und zur Verfügung gestellt. Darüber hinaus werden im LehrLern-Labor ausgewählte Unterrichtsmaterialien zur Diagnose und Individuellen Förderung systematisiert und bereitgestellt. Dabei handelt es sich um bereits vorhandene Materialien (z.B. Montessori-Material), aber auch um Material, das im Kontext von Arbeitszusammenhängen des Lehr-Lern-Labors entsteht.

Für Studierende, die sich im Rahmen von Studien- oder Prüfungsleistungen mit thematisch einschlägigen Fragestellungen beschäftigen, ist das Lehr-LernLabor ein Ort, an dem Beratungsangebote wahrgenommen werden können. Diese umfassen sowohl individuelle Beratungen als auch regelmäßig stattfindende Kolloquien zu ausgewählten Teilbereichen. Das Lehr-Lern-Labor richtet sich in seiner Angebotsstruktur aber nicht nur an Studierende, sondern auch an Wissenschaftlerinnen und Wissenschaftler, die in unterschiedlichen Zusammenhängen zu den Themenfeldern Heterogenität, Diagnose und Individuelle Förderung arbeiten. Es versteht sich als ein Ort, an dem ein multiperspektivischer und interdisziplinärer Austausch verfolgt wird und somit Fragestellungen, Forschungswege und Erkenntnisse nicht nur isoliert betrachtet, sondern in einen breiten Diskurs eingetragen werden.

Ziel ist die Entwicklung und Etablierung einer flexiblen universitären Lernumgebung, in der sich die o.g. Aspekte unter einem gemeinsamen Dach versammeln und die einen konkreten Ort für die Ausgestaltung verschiedener hochschuldidaktischer Konzepte eröffnet. Diese hochschuldidaktischen Konzepte, die den zweiten Grundpfeiler der universitären Lern- und Forschungswerkstatt bilden, können teils auf eine langjährige Erprobung und Weiterentwicklung zurückblicken. Andere Formate wurden neu entwickelt und mit dem Lehr-Lern-Labor assoziiert oder explizit als hochschuldidaktisches Konzept im Rahmen der Qualitätsoffensive Lehrerbildung erprobt und reflektiert. Die folgende Abbildung (Abb. 1) eröffnet einen Überblick über die verschiedenen (hochschuldidaktischen) Formate, die mit dem Lehr-Lern-Labor assoziiert (untere Ebene) oder direkt dort angesiedelt sind (obere Ebene).

Organisiert und verantwortet vom Institut für Erziehungswissenschaft der WWU werden regelmäßig Ringvorlesungen zum Themenfeld „Schule, Heterogenität und Individuelle Förderung" angeboten. Dabei wird ein breiter Blick auf unterschiedliche Heterogenitätsdimensionen eingenommen. Im Vordergrund steht die Frage, wie und mit welchen konkreten Ansätzen in verschiedenen Disziplinen und Fachdidaktiken das Themenfeld bearbeitet wird.

Das vom Landeskompetenzzentrum für Individuelle Förderung NRW entwickelte und evaluierte Qualifizierungskonzept zum Zertifikat „Experte Individuelle Förderung" (Fischer, 2013) soll Lehrkräfte aller Schulformen dabei unterstützen, ihre Kompetenzen zur individuellen Förderung aller Schülerinnen und Schüler zu erweitern. Im Mittelpunkt steht die Entfaltung individueller Interessen und Begabungen und die Persönlichkeitsentwicklung der Schülerinnen und Schüler. Die Teilnehmerinnen und Teilnehmer der Qualifizierung erwerben grundlegende Kompetenzen zur individuellen Förderung in Theorie und Praxis, die im eigenen 


\section{LEHR.LERN.LABOR \\ Diagnose \& Individuelle Förderung}

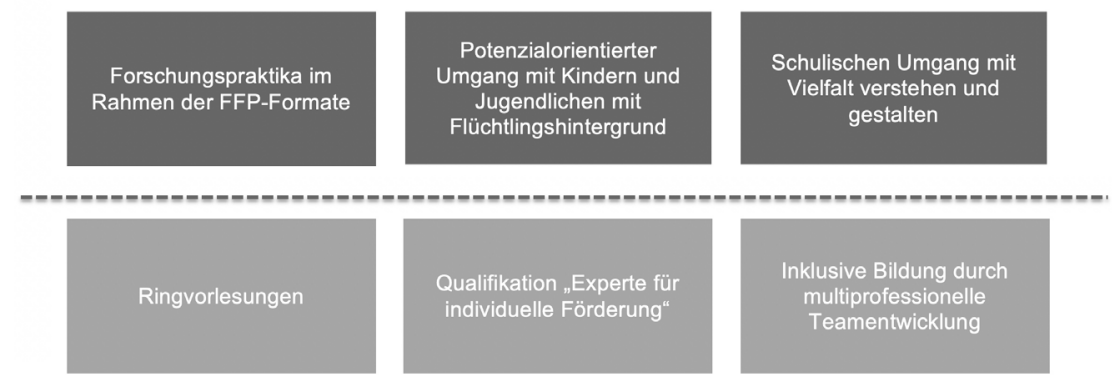

Abbildung 1: Formate und Konzepte im Lehr-Lern-Labor

Unterricht und in der systemischen Weiterentwicklung der Schule angewandt werden können. Verantwortlich für die Durchführung der Qualifizierung ist das Internationale Centrum für Begabungsforschung (ICBF). Der erfolgreiche Abschluss des Lehrgangs wird bestätigt durch ein Zertifikat der WWU Münster.

Der thematisch einschlägige Schwerpunkt „Multiprofessionelle Teamarbeit" wird sowohl im Bachelor als auch im Master in enger Anbindung an Begleitseminare zu den Praxisphasen bearbeitet (Bertels, 2018; Dexel, Käpnick \& Bertels, 2018). In inklusiven Settings treffen unterschiedliche Professionen an der Schnittstelle der Förderung einer heterogenen Schülerschaft zusammen. Mit dem Aufbau multiprofessioneller Teams bietet sich die Chance, unterschiedliche Wissensbereiche und fachliche Kompetenzen (nicht nur im Unterricht) zusammenfließen zu lassen. In der Praxis lässt sich dabei häufig eine klare Trennung der Verantwortlichkeiten und jeweiligen Kompetenzwahrnehmung innerhalb und außerhalb des Unterrichts feststellen. Folgende Fragen stehen mit Blick auf Forschendes Lernen der Studierenden zur multiprofessionellen Kooperation im inklusiven Klassenzimmer im Mittelpunkt: Welche Akteure spielen hierbei eine Rolle? Wie kann Kooperation im Sinne der Inklusion gelingen? Welche Herausforderungen stellen sich in der aktuellen Schullandschaft? Welche Perspektiven können im Sinne eines Forschenden Lernens auf die aktuelle Praxis entwickelt werden?

Das Forder-Förder-Projekt (FFP) (Fischer \& Fischer-Ontrup, 2016; Rott, 2017) ist ein Angebot für Schülerinnen und Schüler der Klassenstufen 2 bis 4 in der Grundschule und der Klassenstufen 5 bis 9 an weiterführenden Schulen. Kinder und Jugendliche erarbeiten eine Projektdokumentation $\mathrm{zu}$ einem Thema ihrer Wahl und halten darüber einen Expertenvortrag. In der Arbeit mit den Schülerinnen und Schülern sind Strategien des selbstgesteuerten Lernens ebenso Bestandteil wie Strategien des Forschenden Lernens. Beteiligte Studierende und Lehrpersonen übernehmen in diesem Zusammenhang die Mentorinnen- und Mentorenrolle, die auf die Lernbegleitung der Schülerinnen und Schüler zielt. Das Projekt erstreckt 
sich aus studentischer Perspektive insgesamt über knapp ein Jahr. Im Wintersemester erfolgt die Teilnahme an einem vorbereitenden Seminar, in dem es um die Vermittlung theoretischer Grundlagen geht. Zu Beginn des zweiten Schulhalbjahres (ca. Februar) startet die praktische Tätigkeit in der Schule mit einer Doppelstunde pro Woche, die sich bis etwa Ende Juni des jeweiligen Jahres erstreckt. Parallel dazu findet im Sommersemester eine Begleitveranstaltung statt, bei der der Schwerpunkt auf der Reflexion der Praxiserfahrungen liegt.

Das Seminarformat „Potenzialorientierter Umgang mit Kindern und Jugendlichen mit Flüchtlingshintergrund“ (Rott, 2019) fokussiert explizit „Migration“ als eine schulisch relevante Heterogenitätsdimension. Mit dem Seminar wird das Ziel verfolgt, Studierende vorzubereiten und zu begleiten, die sich im Kontext von Migration und Bildung engagieren möchten. Schwerpunkt ist dabei die Idee der Potenzialorientierung. In der Arbeit mit den Kindern und Jugendlichen sollen nicht die Probleme und Defizite im Fokus stehen, sondern vielmehr die individuellen Potenziale, Ressourcen und Entwicklungsperspektiven, die die Kinder und Jugendlichen mitbringen, sowie deren Förderung.

Schließlich ist noch das Seminarformat „Schulischen Umgang mit Vielfalt verstehen und gestalten" zu nennen. Dieses bildet den Ausgangspunkt für die folgenden Ausführungen und wird daher im folgenden Kapitel genauer vorgestellt. Insgesamt zielen die hier skizzierten Angebote darauf, den Studierenden einen interdisziplinären Blick auf das Thema „Schule und Heterogenität“ zu eröffnen, der sowohl konstruktiv-pragmatische als auch kritisch-reflexive Perspektiven in Beziehung zueinander setzt. Dieser Anspruch ist auch eine Leitperspektive für das Seminar „Schulischen Umgang mit Vielfalt verstehen und gestalten“, das im Rahmen der Qualitätsoffensive Lehrerbildung entwickelt, erprobt und reflektiert wurde.

\section{Das Seminar „Schulischen Umgang mit Vielfalt verstehen und gestalten“"}

Ausgangspunkt für das auf zwei Semester angelegte Seminar ist die in ausgewählten Kooperationsklassen anzutreffende Schul- und Unterrichtspraxis. Diese wird in einem ersten Schritt systematisch beobachtet und reflektiert und erst in einem zweiten Schritt durch die Entwicklung möglicher Handlungsalternativen pragmatisch gewendet. Damit unterscheidet sich das Vorgehen von anderen Lehr-LernLabor-Konzeptionen, bei denen zuerst Lehr-Lern-Situationen von den Studierenden didaktisch geplant und dann im Anschluss praktisch erprobt werden.

Das Vorgehen ist dadurch begründet, dass die Bearbeitung von Heterogenität in schulischen Zusammenhängen eine Reihe von handlungspraktischen und strukturellen Herausforderungen mit sich bringt (z.B. Bohl, Budde \& Rieger-Ladich, 2017; Rabenstein \& Wischer, 2016; Sturm \& Wagner-Willi, 2018; Trautmann \& Wischer, 2011). Die Frage aber, welche Unterrichtsformen und -konzepte, Handlungsmuster, Medien und Materialien am besten geeignet sind, diesen Herausforde- 
rungen zu begegnen, ist weitgehend ungeklärt bzw. lässt sich auch nicht universal klären. Mit Blick auf den schulpädagogischen Diskurs hinsichtlich Heterogenität und Inklusion kann vielmehr davon ausgegangen werden, dass es angesichts der strukturellen Komplexität und Widersprüchlichkeit des heterogenitätssensiblen pädagogischen Handelns in der Institution Schule nicht den „einen richtigen“ Weg im Umgang mit Heterogenität geben wird. Damit rücken situativ gebundene didaktische Strategien oder anders gesagt, die konkrete Schul- und Unterrichtspraxis in den Fokus. Aus hochschuldidaktischer Perspektive ergibt sich daraus die Überlegung, die Studierenden zunächst durch einen reflexiven Zugang hinsichtlich der Komplexität der Herausforderungen auch unter Berücksichtigung intersektionaler Perspektiven (Walgenbach, 2014) zu sensibilisieren. Erst im Anschluss an die Reflexion der Prozesse im konkreten Klassenzimmer wird der Blick auf die Planung alternativer Handlungsmöglichkeiten gerichtet.

Die Verbindung einer verstehenden und einer gestaltenden Perspektive im Seminarformat verweist auf weitere theoretische Bezüge. So verweisen Lindmeier und Lindmeier (2018) darauf, dass die „inklusionspädagogisch ausgerichtete Professionalisierung von Lehrerinnen und Lehrern ein komplexes Unterfangen darstellt" (S. 267) und die Berücksichtigung kompetenztheoretischer, strukturtheoretischer und berufsbiografischer Perspektiven erforderlich sei. Für die Lehrerinnen- und Lehrerbildung bedeutet das u.E., dass sowohl pragmatisch als auch reflexiv ausgerichtete hochschuldidaktische Angebote nötig sind, die die Reflexion struktureller und institutioneller Bedingungen des Lehrerinnen- bzw. Lehrerhandelns sowie die Auseinandersetzung mit der auch biografisch bedingten Standortgebundenheit des eigenen Denkens und Handelns einschließen (Feindt, 2019). Diese Position weist darüber hinaus Schnittstellen zum Konzept der „Reflexiven Inklusion" von Budde und Hummrich (2015) auf, das die schulische Ausgestaltung inklusiver pädagogischer Praxis sowohl mit Prozessen der Dramatisierung als auch der Entdramatisierung verbindet: Zum einen müssen schulisch relevante Differenzkategorien benannt und damit deren professionelle Bearbeitung ermöglicht werden (Dramatisierung), zum anderen muss das damit einhergehende, doing difference, müssen Zuschreibungen und Manifestationen auch im Hinblick auf die intersektionale Verwobenheit mit anderen Differenzkategorien kritisch reflektiert werden (Entdramatisierung). Schließlich verweist die Verbindung von Aktion und Reflexion auch auf den Ansatz der Praxisforschung (Altrichter, Feindt \& Zehetmeier, 2014), bei dem praktisches Handeln und wissenschaftliche Reflexion nicht maximal voneinander getrennt, sondern „Aktion und Reflexion eng und immer wieder aufeinander bezogen werden" (Altrichter, Posch \& Spann, 2018, S. 13).

Begründet durch den hier skizzierten theoretischen Hintergrund erarbeiten sich die Studierenden in kleinen, auf Kontinuität hin gebildeten Teams zunächst einen reflexiven Zugang zur Schul- und Unterrichtspraxis in ausgewählten Lerngruppen. Bei den Kooperationsschulen handelt es sich um solche Schulen, die ihre Entwicklungsprozesse schwerpunktmäßig und explizit auf heterogenitätssensible didaktische Konzepte hin ausgerichtet haben. Im Austausch mit den Lehrpersonen 
entwickeln die Studierenden konkrete, situative Erkenntnisinteressen. Dabei wird keine Konzentration auf bestimmte Heterogenitätsdimensionen vorgenommen, sondern der Blick der Studierenden in Absprache mit den beteiligten Lehrpersonen während der Beobachtungen fokussiert.

Damit verbunden ist das Ziel, dass nicht nur die Studierenden hinsichtlich ihres Professionalisierungsprozesses profitieren, sondern möglichst auch die Schulen einen Gewinn für ihre konkrete Schul- und Unterrichtsentwicklung haben (Bastian, Combe, Hellmer, Hellrung \& Roggatz, 2002; Feindt, 2005, 2007; Fichten, Gebken \& Obolenski, 2002; Meyer \& Feindt, 2000). Durch Unterrichtsbeobachtungen (z. B. de Boer \& Reh, 2012) wird ein Zugang zu den formulierten Erkenntnisinteressen und damit ein reflexiver Zugang zur Schul- und Unterrichtswirklichkeit eröffnet. Die im Seminar verfolgte gemeinsame perspektiventriangulierende Analyse der erhobenen Daten in Form einer Forschungswerkstatt ist dabei ein zentraler Bestandteil des Konzepts. Die Rückmeldung der Beobachtungen und Analyseergebnisse bildet den Abschluss der ersten Arbeitsphase im Seminar und damit auch den Abschluss des ersten Semesters (siehe Abb. 2).
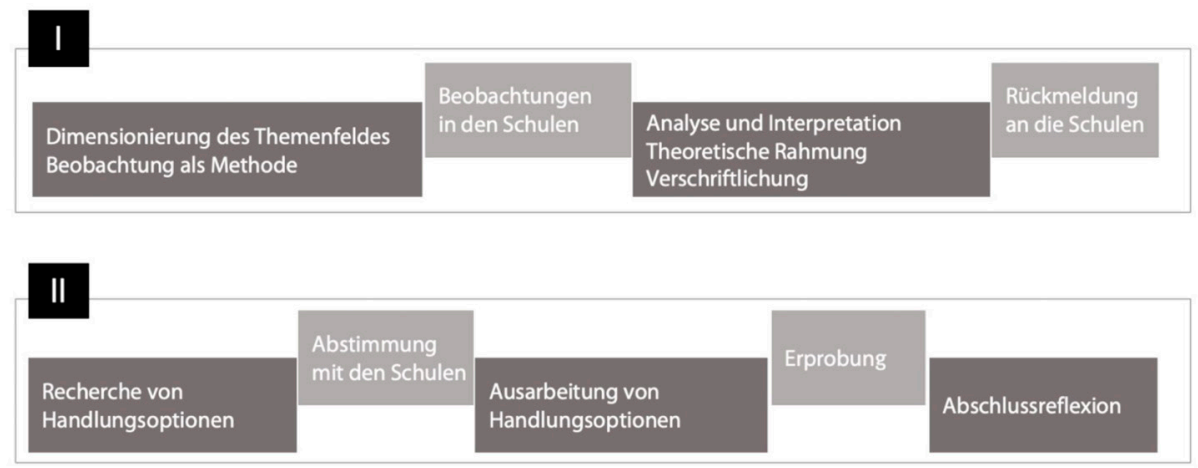

Abbildung 2: Verstehen und Gestalten im Rahmen der Lern- und Forschungswerkstatt

Auf Grundlage der Analyseergebnisse werden im zweiten Semester gemeinsam mit den verantwortlichen Lehrpersonen didaktische Optionen für die Weiterentwicklung der jeweils konkreten pädagogischen Praxis vor Ort entwickelt und erprobt. In einem ersten Schritt überlegen die Studierenden in ihren Teams, an welchen Punkten sie ansetzen würden, um eine heterogenitätssensible Didaktik in den jeweiligen Lerngruppen zu verändern bzw. zu entwickeln. Literaturrecherchen, Expertise aus den Fachdidaktiken, Diskussionen im Seminar und die bereits verfügbaren Kompetenzen der Studierenden bilden die Grundlage, um die Ideen zu schärfen, mit den beteiligten Lehrpersonen abzustimmen, zu fundieren und didaktisch zu konkretisieren. Erst dann werden die Handlungsoptionen in den Kooperationsklassen erprobt und die Erfahrungen im Seminar reflektiert (siehe Abb. 2). 


\section{Komplexitätsreduktion: ein Blick auf die ,Schieberegler}

Angesichts der Partikularität der situativen Gegebenheiten in den Lerngruppen und der damit verbundenen (thematischen) Offenheit in den Seminarverläufen ist eine vorab definierte und stets gültige Einstellung der ,Schieberegler' nicht möglich. Mit Blick auf den Aspekt „Komplexitätsreduktion“ werden für die folgenden Ausführungen die Bereiche „Anforderungen, inhaltliche Ebene" sowie „Unterstützung, inhaltliche Ebene" genauer in den Blick genommen (siehe Abb. 3). Hier hat sich in den bisherigen Seminarverläufen herauskristallisiert, dass eher wenig Theorien, Medien, Lehrhandlungen und Dimensionen in den Blick genommen werden. Das Niveau der damit verbundenen Anforderungen an die Studierenden kann hingegen als hoch eingeschätzt werden: Es geht um eine genaue, detaillierte Analyse ausgewählter Lehrhandlungen und/oder Medien und Materialien, die durch theoretische Bezüge fundiert und damit einer differenzierten Reflexion zugänglich gemacht werden sollen. Explizit wird dabei berücksichtigt, dass das Themenfeld „Heterogenität und Schule“ von einer Vielzahl widersprüchlicher Aspekte durchzogen ist, die sich nur begrenzt mit eindeutigen und linearen Perspektiven fassen lassen. So kommt es zu einem Bild der Schieberegler, das quantitative Begrenzung mit qualitativer Tiefe in Verbindung bringt. Auf der Seite der Unterstützung geht diese konzeptionelle Entscheidung damit einher, dass die Studierenden bei der Reflexion des beobachteten Unterrichts umfassend unterstützt werden. Diese Unterstützung erfolgt dadurch, dass geeignete Theorieperspektiven im Seminar angeboten werden und damit der Theorie-Praxis-Bezug konkretisiert werden soll. Darüber hinaus werden die Studierenden durch das Setting einer Forschungswerk-

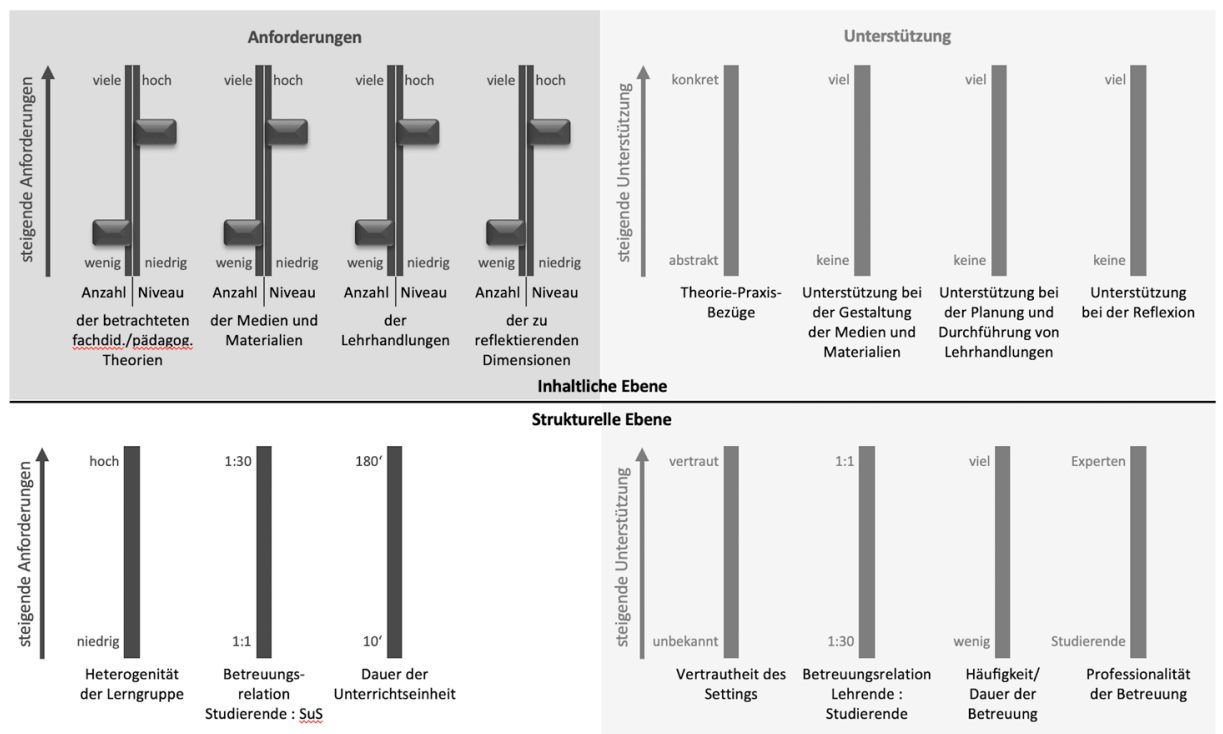

Abbildung 3: Schieberegler 
statt (Reim \& Riemann, 1997; Strauss, 1998) begleitet. Analyse und Reflexion der Beobachtungen erfolgen in gemeinsamen Sitzungen, in denen sowohl Studierende als auch Lehrende ihre Perspektiven auf das Material einbringen. Ziel ist es, die im Beitrag zur Komplexitätsreduktion (Marohn, Greefrath, Hammann, Hemmer, Kürten \& Windt, in diesem Band) angesprochene Balance von Anforderung und Unterstützung konstruktiv auszugestalten.

\section{Konzentration auf Komplexität vs. Reduktion von Komplexität}

Was bedeuten diese Ausführungen nun für den Blick auf Komplexität im Seminarkontext? Auf der linken Seite der Schieberegler im Bereich „inhaltliche Anforderungen“ ist deutlich zu erkennen: Es geht um ausgewählte Aspekte eines größeren Ganzen: Nicht der gesamte Unterricht ist Thema, sondern ausgewählte Teilaspekte, nicht alle denk- bzw. verfügbaren Theorien werden für die Analyse herangezogen, sondern einzelne, gezielt ausgewählte. Schaut man hingegen auf die rechte Seite der jeweiligen Schieberegler, dann wird deutlich, dass die ausgewählten Aspekte sehr differenziert analysiert und reflektiert werden sollen. Die Anforderung und, wie sich zeigen wird, auch die für die Studierenden zu bearbeitende Komplexität sind hoch.

Die folgenden Beispiele konkretisieren diesen Befund und zeigen, wie ausgewählte Theorien dazu beitragen können, eine differenziertere Perspektive auf den beobachteten Unterricht zu entwickeln. Die Auswahl der herangezogenen Theorien erfolgt dabei in enger Begleitung durch die Lehrenden. Deren Erfahrung und wissenschaftliche Expertise ermöglichen Orientierung in der oftmals als unübersichtlich erlebten Theorielandschaft und eine Konzentration auf Aspekte, die als bedeutsam für die Lernprozesse der Studierenden und die curricularen Ziele des Seminarformates erachtet werden. Hier zeigt sich, wie im Seminar Unterstützungsangebote für die Studierenden greifen. Darüber hinaus erfolgt die Auswahl theoretischer Orientierungen bisweilen auch durch die Studierenden selbst. Das ist dann der Fall, wenn Inhalte aus anderen Studienkontexten für die Studierenden eine Passung zu ihren Beobachtungen aufweisen und sie in diesem Fall eigenständig Studienhinhalte miteinander vernetzen.

Im ersten Beispiel geht es um eine Gruppe von Studierenden, die in ihren Beobachtungsprotokollen eine Situation beschrieben haben, in der eine Klassenarbeit mit einem heterogenen Notenspektrum zurückgegeben wird und dennoch die Anerkennung der Leistung aller Schülerinnen und Schüler gewährleistet werden soll: 
Nach der Einzelarbeit zeigt sich der Lehrer wieder präsent, indem er sich vorne vor die Klasse stellt. Er sagt, dass nun alles in die Mappe zu heften sei, wofür die Schüler eine Minute Zeit hätten und dass er anschließend die Arbeit zurückgeben würde. Nun wird es laut in der Klasse. Mit einem "pssst" bittet der Lehrer dann um Ruhe und sagt: "Ich verstehe auch, dass ihr alle ganz neugierig seid. Die Arbeit ist nicht so gut ausgefallen, einige haben auch ganz tolle Arbeiten geschrieben. Es gibt aber auch Arbeiten, die nicht so gut ausgefallen sind. Das ist dann ja immer nicht so schön. Man ist dann ja auch traurig, wenn man nicht so eine gute Arbeit geschrieben hat. Wie könnte man denn den Kindern, die jetzt gleich eine Arbeit wiederkriegen, die nicht so gut ausgefallen ist, was könnte man denen denn mal sagen?".

Diese Szene wurde von den Studierenden zunächst auf der Handlungsebene in den Blick genommen. Eine von Seiten des Lehrers sensible Wahrnehmung der Situation der Schülerinnen und Schüler, die keine gute Note bekommen haben, wurde betont. Die Wendung der Notenvergabe hin zu pädagogischen Interventionen zur Stärkung des Selbstwertgefühls von Schülerinnen und Schülern mit nicht so gut ausgefallenen Noten wurde als heterogenitätssensibles Handeln verstanden.

Unter Rückgriff auf die Ausführungen zu Heterogenität und Schule von Trautmann und Wischer (2011, S. 69ff.) wurde in einem zweiten Analyseschritt auch die schul- und organisationstheoretische Dimension des Beobachtungsprotokolls herausgearbeitet. So wurde deutlich, dass der Lehrer in dieser Situation die pädagogische Orientierung an den einzelnen Schülerinnen und Schülern irgendwie mit der Allokationsfunktion der Schule vermitteln muss. Oder mit Trautmann und Wischer (2011) gesagt, dass „die Schule als gesellschaftliche Institution keineswegs allein nur pädagogischen Interessen und Absichten folgt, also nur pädagogische Aufgaben wahrzunehmen hat, sondern auch in einen gesellschaftlichen Funktionszusammenhang eingebunden ist" (S. 101). Letztlich zeigt sich in diesem Beispiel, mit welchen Strategien in der schulischen Praxis versucht wird, die Selektionsfunktion der Schule mit einem heterogenitätssensiblen Unterricht zu vermitteln.

Darüber hinaus wurde herausgearbeitet, wie Schule konkret in diesem Beispiel an der Konstruktion von Heterogenität beteiligt ist. So wird eine Differenz zwischen "tollen“ und "nicht so gut ausgefallenen“ Arbeiten explizit von der Lehrperson benannt. Weiter wird das Gefühl von Traurigkeit mit den "nicht so guten“ Arbeiten verknüpft. Durch die Situation der Notenvergabe und die begleitenden Worte des Lehrers trägt die Schule in dieser Situation ganz konkret zur Herstellung von durch Noten ausgewiesene leistungsbezogene Differenz bei (Trautmann \& Wischer, 2011, S. 47f.).

Genau an dieser Stelle wird am praktischen Beispiel deutlich, dass die Formel „Komplexitätsreduktion = geringere Anforderungen“ zu kurz greift, wie Marohn et al. (in diesem Band) schreiben. Es handelt sich zwar um einen begrenzten Ausschnitt aus dem Gesamtgeschehen des Unterrichts, der im Rahmen der bisherigen 
Argumentation zu Lehr-Lern-Laboren als Komplexitätsreduktion beschreibbar wäre (Marohn et al., in diesem Band). Schaut man aber genauer hin, so wird deutlich, dass eine dem Ausschnitt innewohnende Komplexität erst durch die Fokussierung des Ausschnittes sicht- und bearbeitbar wird. Aus einer professionstheoretischen Perspektive, die sich an strukturtheoretischen Argumenten orientiert (z. B. Helsper, 1996, 2000, 2014), wäre eine Reduktion der Komplexität von Unterricht auch kontraproduktiv, denn dann würde es zu einer einseitigen Auflösung antinomischer Grundstrukturen kommen, die einem Zerrbild schulischer und unterrichtlicher Praxis Vorschub leisten und damit Professionalisierungsprozesse bei Studierenden eher behindern als ermöglichen würde. Im Rahmen einer strukturtheoretischen Perspektive sind Professionalisierungsprozesse gar nicht anders vorstellbar, als dass die Komplexität und Widersprüchlichkeit schulischen Handelns in den Blick geraten:

Aus diesen dilemmatischen Kernproblemen resultieren grundlegende Fehlerpotenziale für das professionelle Handeln, die dann gesteigert werden, wenn die professionellen Paradoxien nicht bearbeitet, sondern ausgeblendet, einseitig aufgelöst, nicht reflexiv gehandhabt, mit biografischen Verletzungspositionen der Professionellen kurzgeschlossen und zusätzlich durch starre organisationsförmige Rahmen in Spannung versetzt werden. (Helsper, 2016, S. 52)

Die Ausführungen zeigen, dass eine Konzentration auf ausgewählte Einzelaspekte (geringere Anforderungen auf quantitativer Ebene, Regler „Anzahl“) in diesem Fall mit der Möglichkeit der Reflexion unterrichtlicher Komplexität (höhere Anforderungen auf qualitativer Ebene, Regler „Niveau“) verbunden ist, weil ausgehend vom Einzelfall weitreichende Analysen eröffnet werden. Die o.a. Unterstützung, die den Studierenden im Seminar zur Seite gestellt wird, trägt dazu bei, dass eine Balance der „gestellten Anforderungen und angebotenen Unterstützungsmaßnahmen" (Marohn et al., in diesem Band) angebahnt wird.

Diese Argumentation lässt sich am folgenden Beobachtungsprotokoll weiter verdeutlichen. Die Beobachtung wurde ebenfalls im Rahmen des Seminars von einem Studierenden-Team in einer Schule gemacht und verschriftlicht. Es handelt sich um eine Phase der Gruppenarbeit in einer achten Jahrgangsstufe einer Gesamtschule. Die einzelnen Schülerinnen und Schüler sind im Beobachtungsprotokoll mit Nummern (1-5) maskiert. 
Der Lehrer (L) leitet die Stunde ein, dabei richten alle Schülerinnen und Schüler (SuS) der Tischgruppe 5 (TG) ihre Köpfe zum L hin. In einer kurzen Redepause des $L$ spricht 1 zu 2. 1 wird darauf von dem L indirekt angesprochen: „Es nervt, wenn hier dauernd gesprochen wird. "Der L führt eine sehr kurze Wiederholung durch, indem er der Klasse ein - zwei Fragen stellt. 1, 2 und 3 rufen Antworten in die Klasse. Daraufhin sprechen 1-4 untereinander (außerschulisch) und 1 gibt explizit einen Kommentar an 2. Der L hat der Klasse den neuen Arbeitsauftrag mitgeteilt und die Arbeitsphase beginnt.

2 holt ein Buch. 1 blättert in seinem Buch. 2 setzt sich nun mit dem Buch an den Tisch und sagt zu der gesamten TG: „So ihr kleinen Lümmel“. 1 hat eine Seite im Buch aufgeschlagen und deutet mit dem Finger auf die Seite und sagt: "Das ist ein Kondom". Nun steht 4 auf und holt sich ein Buch. 3 steht von seinem Stuhl auf und beugt sich zu 1 und 2 rüber (5 sek.). Dann setzt sich 3 wieder und steht direkt wieder auf und holt Blätter. 1 und 2 sprechen über außerschulische Themen, dabei lachen sie und schauen sich an. Ein $S$ einer anderen TG kommt zur TG5 und nimmt sich Stifte von 2. 4 spricht 3 an: „Warum sind Entwicklungsländer so arm?". 3 setzt zur Antwort an, bricht jedoch ab. 4: „Think (Methode). Du musst jetzt deine Vermutung aufstellen.".

Der L kommt zu der Tischgruppe und schlägt in dem Buch von 3 eine Seite auf. 1 steht auf und putzt sich die Nase. 2 spricht mit 3 über den Unterrichtsinhalt. 4 richtet an die gesamte Gruppe eine Frage: „So, was habt ihr?". Es kommen einige Antworten von 1, 2 und 3. 4 unterbricht und sagt: „Aber schau mal, hier steht [...]". 5 beteiligt sich nicht an der Diskussion und gähnt. 4 spricht wieder zu der gesamten TG: „So, wir hatten letztens dieses Beispiel.“. 3 unterbricht und sagt: "Ich weiß es“ [...]. 4 sagt: „Warte mal. Also was... Du musst einfach mal nachdenken... “. 1: „Die exportieren Rohstoffe!“. Nachdem 1 das gesagt hat, erklärt 4 den anderen etwas und schließt mit den Worten "also stimmt das, was 1 gesagt hat" ihre Erklärung ab.

Danach findet eine aktive Diskussion zwischen 1 und 4 statt (thematischer Inhalt). Die Diskussion ist beendet. Die TG spricht miteinander (außerschulisch) und sie lachen miteinander [...]. 5 spricht zu 4: "Ich hab kein Bock, 4. Ich hasse es. " 4 kippelt mit dem Stuhl und wiederholt im Folgenden einzelne Wörter der anderen, während sie sich unterhalten. Die SuS der TG bearbeiten weiter die Aufgaben und sind bei dem Arbeitsschritt, ihre Ergebnisse festzuhalten. 4 sagt zu dem Rest der TG: "Ihr könnt auch schreiben mit was..." 5 kippelt weiterhin mit dem Stuhl und beteiligt sich nicht an der Diskussion und macht schnalzende Geräusche mit ihrer Zunge. 4 richtet wieder eine Frage an die Gruppe: „So Leute, versteht ihr das denn jetzt?". 2 gibt eine Antwort und 4 antwortet darauf: „2, genau“.

Schaut man sich diese Beobachtungen genau an, dann wird deutlich, dass nur ein kleiner Ausschnitt aus dem Gesamtgefüge des Unterrichts in den Blick genommen 
wurde: Die Interaktion innerhalb einer Tischgruppe während einer Gruppenarbeitsphase. Mit dieser Fokussierung ist nun aber eben nicht eine Komplexitätsreduktion verbunden. Vielmehr wird durch den genauen beobachtenden Blick deutlich, wie komplex und vielschichtig sich die Interaktion der Schülerinnen und Schüler in der Kleingruppenarbeit darstellt. Wie mit einem Teleobjektiv wird ein Detail aus einem größeren Zusammenhang herausgelöst. Dadurch fällt eine ganze Reihe von Informationen jenseits des Ausschnittes weg. Aber innerhalb des Ausschnittes werden jetzt Details sichtbar, die vorher verborgen waren:

In der Analyse des Beobachtungsprotokolls stellte sich die Frage, wie diese Tischgruppe, zusammengesetzt aus Schülerinnen und Schülern mit unterschiedlichen Kompetenzen, Motivationen und Erfahrungen, den Arbeitsauftrag bearbeitet. Durch die Wahl einer Gruppenarbeit hat sich der Lehrer entschieden, auf eine frontale Unterrichtsorganisation zu verzichten. Das könnte daran liegen, dass er dadurch mehr Schülerinnen und Schülern Möglichkeiten zur Mitwirkung eröffnen möchte. Oder es könnte daran liegen, dass er das selbstständige Lernen befördern oder kooperative Lernprozesse unterstützen möchte. Vor dem Hintergrund des im Selbstverständnis dieser Gesamtschule dokumentierten Anspruches, das gemeinsame Lernen aller Schülerinnen und Schüler zu befördern, ist dieses Vorgehen in den Rahmen einer heterogenitätssensiblen Didaktik gestellt.

Im Beobachtungsprotokoll wird nun sichtbar, dass die Schülerin 4 die Verantwortung für den Arbeitsprozess der Gruppe übernimmt. Sie aktiviert Interaktionsmuster, die traditionell den Lehrpersonen in einem gelenkten Unterrichtsgespräch zugeschrieben werden:

- 4 spricht 3 an: „Warum sind Entwicklungsländer so arm?“.

- 4: „Think (Methode). Du musst jetzt deine Vermutung aufstellen.“

- 4 richtet an die gesamte Gruppe eine Frage: „So, was habt ihr?“.

- 4 unterbricht und sagt: „Aber schau mal, hier steht [...]".

- 4 spricht wieder zu der gesamten TG: „So, wir hatten letztens dieses Beispiel.“.

- 4 sagt: "Warte mal. Also was... Du musst einfach mal nachdenken..."

- Nachdem 1 das gesagt hat, erklärt 4 den anderen etwas und schließt mit den Worten "also stimmt das, was 1 gesagt hat" ihre Erklärung ab.

- 4 sagt zu dem Rest der TG: „Ihr könnt auch schreiben mit was..."

- 4 richtet wieder eine Frage an die Gruppe: „So Leute, versteht ihr das denn jetzt?“

Vor diesem Hintergrund wurde im Seminar die These diskutiert, ob es sich bei der beobachteten Sequenz nicht um ein gelenktes Unterrichtsgespräch auf hohem Niveau im Setting einer Kleingruppenarbeit handelt. Es gibt kognitiv aktivierende Nachfragen, Vernetzungen zwischen verschiedenen Unterrichtsinhalten, zusammenfassende Erklärungen und Handlungsaufforderungen. Mit Blick auf das Ziel des Seminars, Strategien und Prozesse des schulischen Umgangs mit Heterogenität zu verstehen, wurde diese These abstrahiert und als „Erweiterung der Ressource Lehrkraft" (Breidenstein \& Rademacher, 2017, S. 7of.) diskutiert. Die Vermutung liegt nahe, dass die Arbeit in dieser Tischgruppe funktioniert, weil eine Schülerin 
stellvertretend die Rolle der Lehrperson übernimmt. Mit Helsper (2014) ließe sich auch sagen, dass sie als „Koprofessionelle“ (S. 228) konstruktiv das Unterrichtsgeschehen unterstützt.

In diesem Detaillierungsgrad würde eine Lehrperson während des Unterrichts die Interaktionsdynamik innerhalb einer Tischgruppe kaum wahrnehmen. Erst durch die Konzentration auf ein aus dem Gesamtzusammenhang herausgelöstes Detail und dessen genaue Beobachtung kann es gelingen, nicht nur die Oberflächen-, sondern auch die Tiefenstruktur (Bohl, 2017; Reusser \& Halbheer, 2008) des Unterrichts in den Blick zu nehmen. Dieser Gewinn ist eng mit der Beobachtungsmethode verknüpft, die Distanzierung und Befremdung ermöglicht:

Das Protokoll der Szene kann man anschließend beliebig oft und beliebig genau und langsam lesen. Man kann das verschriftlichte Geschehen gewissermaßen in Zeitlupe betrachten oder unter das Mikroskop legen. (Breidenstein, 2012, S. 32)

Abschließend stellt sich erneut die Frage nach der Stellung der Komplexitätsreduktion im Lehr-Lern-Labor. An den hier geschilderten Beispielen lässt sich erkennen, dass eine Fokussierung von Einzelaspekten nicht mit der Reduktion von Komplexität gleichzusetzen ist. Vielmehr geht es vor dem Hintergrund der o.a. professionstheoretischen Perspektive darum, schulische Komplexität exemplarisch sicht- und bearbeitbar zu machen. Nicht die Reduktion von Komplexität steht im Vordergrund, sondern die Konzentration auf Komplexität. Damit diese gelingen kann und sich die Studierenden nicht überfordert fühlen, braucht es die geschilderte Balance von gestellten Anforderungen und angebotenen Unterstützungsmaßnahmen.

\section{Literatur}

Altrichter, H., Feindt, A. \& Zehetmeier, S. (2014). Lehrerinnen und Lehrer erforschen ihren Unterricht: Aktionsforschung. In E. Terhart, H. Bennewitz \& M. Rothland (Hrsg.), Handbuch der Forschung zum Lehrerberuf (S. 285-307, 2. überarbeitete und erweiterte Auflage). Münster: Waxmann.

Altrichter, H., Posch, P. \& Spann, H. (2018). Lehrerinnen und Lehrer erforschen ihren Unterricht (5. Auflage). Bad Heilbrunn: Klinkhardt (UTB).

Bastian, J., Combe, A., Hellmer, J., Hellrung, M. \& Roggatz, C. (2002). Forschungswerkstatt Schulentwicklung - Schulbegleitforschung in der Lehrerbildung. In U. Dirks \& W. Hansmann (Hrsg.), Forschendes Lernen in der Lehrerbildung. Auf dem Weg zu einer professionellen Unterrichts- und Schulentwicklung (S. 129-141). Bad Heilbrunn: Klinkhardt.

Bertels, D. (2018). Kooperation in multiprofessionellen Teams. Möglichkeiten und Probleme der Zusammenarbeit in der inklusiven Schule. In C. Fischer \& P. Platzbecker (Hrsg.), Auf den Lehrer kommt es an?! Unterstützung für professionelles Handeln angesichts aktueller Herausforderungen (S. 115-124). Münster: Waxmann. 
Bohl, T. (2017). Umgang mit Heterogenität im Unterricht: Forschungsbefunde und didaktische Implikationen. In T. Bohl, J. Budde \& M. Rieger-Ladich (Hrsg.), Umgang mit Heterogenität in Schule und Unterricht (S. 257-273). Bad Heilbrunn: Klinkhardt.

Bohl, T., Budde, J. \& Rieger-Ladich, M. (Hrsg.) (2017). Umgang mit Heterogenität in Schule und Unterricht. Bad Heilbrunn: Klinkhardt.

Breidenstein, G. (2012). Ethnographisches Beobachten. In H. de Boer \& S. Reh (Hrsg.), Beobachtung in der Schule - Beobachten lernen (S. 27-44). Wiesbaden: Springer VS. https://doi.org/10.1007/978-3-531-18938-3_2

Breidenstein, G. \& Rademacher, S. (2017). Individualisierung und Kontrolle - Empirische Studien zum geöffneten Unterricht in der Grundschule. Wiesbaden: Springer. https:// doi.org/10.1007/978-3-658-13805-9

Budde, J. \& Hummrich, M. (2015). Intersektionalität und reflexive Inklusion. Sonderpädagogische Förderung heute, 6o, 165-175.

de Boer, H. \& Reh, S. (Hrsg.) (2012). Beobachtung in der Schule - Beobachten lernen. Wiesbaden: Springer VS. https://doi.org/10.1007/978-3-531-18938-3

Dexel, T., Käpnick, F. \& Bertels, D. (2018). Diversität im Mathematikunterricht der Grundschule - Ein kooperatives Lehrprojekt. In D. Rott, N. Zeuch, C. Fischer, E. Souvignier \& E. Terhart (Hrsg.), Dealing with Diversity. Innovative Lehrkonzepte in der Lehrer*innenbildung zum Umgang mit Heterogenität und Inklusion (S. 75-88). Münster: Waxmann.

Feindt, A. (2005). Forschen für die Schulentwicklung. Eine sinnvolle Aufgabe für Lehramtsstudierende? PÄDAGOGIK, 57, 10-18.

Feindt, A. (2007). Studentische Forschung im Lehramtsstudium. Eine fallrekonstruktive Untersuchung studienbiografischer Verläufe und studentischer Forschungspraxen. Opladen: Barbara Budrich. https://doi.org/10.2307/j.ctvdfo193

Feindt, A. (2019). Reflektierende Praktiker*innen. Zur wissenssoziologischen Rahmung eines doppelten Leitbilds forschenden Lernens. In M. Schiefner-Rohs, G. Favella \& A.-C. Herrman (Hrsg.), Forschungsnahes Lehren und Lernen in der Lehrer*innenbildung. Forschungsmethodische Zugänge und Modelle zur Umsetzung (S. 67-85). Berlin: Peter Lang.

Fichten, W., Gebken, U. \& Obolenski, A. (2002). Entwicklung und Perspektiven der Oldenburger Team-Forschung. In U. Dirks \& W. Hansmann (Hrsg.), Forschendes Lernen in der Lehrerbildung. Auf dem Weg zu einer professionellen Unterrichts- und Schulentwicklung (S. 115-128). Bad Heilbrunn: Klinkhardt.

Fischer C. (2013). Qualifizierung für Lehrer und Lehrerinnen. Systematische Aus- und Weiterbildung der Pädagoginnen und Pädagogen in allen Schulformen. Pädagogische Führung. Zeitschrift für Schulleitung und Schulberatung, 24, 13-16.

Fischer C. \& Fischer-Ontrup C. (2016). Das Forder-Förder-Projekt am ICBF der Universität Münster. News \& Science. Begabtenförderung und Begabungsforschung, 42 (2), 22-24.

Helsper, W. (1996). Antinomien des Lehrerhandelns in modernisierten pädagogischen Kulturen. In A. Combe \& W. Helsper (Hrsg.), Pädagogische Professionalität (S. 521569). Frankfurt am Main: Suhrkamp.

Helsper, W. (2000). Antinomien des Lehrerhandelns und die Bedeutung der Fallrekonstruktion - Überlegungen zu einer Professionalisierung im Rahmen universitärer Lehrerausbildung. In E. Cloer, D. Klika \& H. Kunert (Hrsg.), Welche Lehrer braucht das 
Land? Notwendige und mögliche Reformen der Lehrerbildung (S. 142-177). Weinheim: Juventa.

Helsper, W. (2014). Lehrerprofessionalität - der strukturtheoretische Professionsansatz zum Lehrerberuf. In E. Terhart, H. Bennewitz \& M. Rothland (Hrsg.), Handbuch der Forschung zum Lehrerberuf (2. überarbeitete und erweiterte Auflage, S. 216-240). Münster: Waxmann.

Helsper, W. (2016). Antinomien und Paradoxien im professionellen Handeln. In M. Dick, W. Marotzki \& H. Mieg (Hrsg.), Handbuch Professionsforschung (S. 50-62). Bad Heilbrunn: Klinkhardt.

Lindmeier, C. \& Lindmeier, B. (2018). Professionalisierung von Lehrpersonen. In T. Sturm \& M. Wagner-Willi (Hrsg.), Handbuch Schulische Inklusion (S. 267-281). Opladen: Barbara Budrich.

Meyer, H. \& Feindt, A. (200o). Die Oldenburger Team-Forschung. Konkretion und Irritation eines Versuchs zur Professionalisierung in der LehrerInnenbildung. In M. Meri, K. Uusikylä, M. Talvio \& R. Jyrhämä (Hrsg.), Discussions on some pedagogical issues (S. 49-79). Helsinki: University of Helsinki.

Rabenstein, K. \& Wischer, B. (2016). Individualisierung schulischen Lernens. Mythos oder Königsweg?. Seelze: Klett, Kallmeyer.

Reim, T. \& Riemann, G. (1997). Die Forschungswerkstatt. Erfahrungen aus der Arbeit mit Studentinnen und Studenten der Sozialarbeit. In G. Jakob \& H.-J. Wensierski (Hrsg.), Rekonstruktive Sozialpädagogik. Konzepte und Methoden sozialpädagogischen Verstehens in Forschung und Praxis (S. 223-238). Weinheim: Juventa.

Reusser, K. \& Halbheer, U. (2008). Die Implementation von Bildungsstandards als Anstoß zur Qualitätsentwicklung in Schule und Unterricht. In LISUM/ bm:ukk/ EDK (Hrsg.), Bildungsmonitoring, Vergleichsstudien und Innovation. Von evidenzbasierter Steuerung zur Praxis. (S. 125-138). Berlin: Berliner Wissenschafts-Verlag.

Rott, D. (2017). Die Entwicklung der Handlungskompetenz von Lehramtsstudierenden in der Individuellen Begabungsförderung. Forschendes Lernen aufgezeigt am ForderFörder-Projekt Advanced. Münster: Waxmann.

Rott, D. (2019). Partizipation und Kompetenzorientierung - ein Widerspruch? Überlegungen zum Lehr-Lern-Labor Diagnose und Individuelle Förderung am Beispiel des Seminarformats ,Fragen einer diversitätssensiblen Schul- und Unterrichtsentwicklung. In R. Baar, A. Feindt \& S. Trostmann (Hrsg.), Struktur und Handlung in Lernwerkstätten (S. 254-264). Bad Heilbrunn: Klinkhardt.

Strauss, A. (1998). Grundlagen qualitativer Sozialforschung. München: Fink.

Sturm, T. \& Wagner-Willi, M. (Hrsg.) (2018). Handbuch Schulische Inklusion. Opladen: Barbara Budrich.

Trautmann, M. \& Wischer, B. (2011). Heterogenität in der Schule. Eine kritische Einführung. Wiesbaden: VS. https://doi.org/10.1007/978-3-531-92893-7

Walgenbach, K. (2014). Heterogenität, Intersektionalität, Diversity in der Erziehungswissenschaft. Opladen: Barbara Budrich (UTB). 



\section{Mit Vielfalt experimentieren}

\section{Professionalisierung angehender Lehrkräfte im GEO-Lehr-Lern-Labor}

Das GEO-Lehr-Lern-Labor der Westfälischen Wilhelms-Universität (WWU) Münster hat zum Ziel, Lehramtsstudierende hinsichtlich des Einsatzes von Experimenten in einem heterogenitätssensiblen Geographieunterricht zu professionalisieren. Bezüglich der Heterogenität wird ein besonderer Fokus auf die Leistungsheterogenität gelegt. Im begleitenden Forschungsprojekt wird untersucht, inwiefern es gelingt, die Entwicklung der situationsspezifischen Selbstwirksamkeitserwartung der Studierenden zu fördern und welche Rolle die getroffenen komplexitätsreduzierenden Maßnahmen hierbei einnehmen. Die Entwicklung der Selbstwirksamkeitserwartung und deren Bedingungen werden mittels eines quantitativen Fragebogens sowie mit qualitativen Interviews für vier Durchläufe erhoben. Erste Ergebnisse zeigen, dass die Selbstwirksamkeitserwartung nahezu aller Studierenden im Seminarverlauf gestiegen ist, wobei die komplexitätsreduzierenden Maßnahmen different bewertet werden.

Schlüsselwörter: Selbstwirksamkeitserwartung, Diagnose von Leistungsheterogenität, Experimentieraufgaben

\section{Einleitung}

Die Potenziale des Einsatzes von naturwissenschaftlichen Experimenten im Geographieunterricht werden seit Jahren in zahlreichen fachdidaktischen Publikationen thematisiert und hervorgehoben (Mönter \& Otto, 2017; Otto, Mönter, Hof \& Wirth, 2010; Peter, 2014; Wilhelmi, 2012). Dennoch werden Experimente nur selten von Geographielehrkräften eingesetzt (Hemmer \& Hemmer, 2010). Als Erklärung für die Diskrepanz zwischen Forderung und unterrichtlichem Einsatz werden in der Literatur verschiedene Herausforderungen genannt, die von (angehenden) Geographielehrkräften mit einem Einsatz von Experimenten verbunden werden (Höhnle \& Schubert, 2016; Otto, 2009). So existiere unter anderem eine vorherrschende Unsicherheit seitens der Lehrkräfte durch eine mangelnde Vorbereitung und Thematisierung experimenteller Arbeitsweisen in der universitären Lehrerbildung (Höhnle \& Schubert, 2016; Otto, 2009). Das im Rahmen der Qualitätsoffensive Lehrerbildung entwickelte GEO-Lehr-Lern-Labor an der Westfälischen Wilhelms-Universität (WWU) Münster setzt an diesem Punkt an und hat zum Ziel, angehende Geographielehrkräfte hinsichtlich des Einsatzes von Experimenten im Geographieunterricht zu professionalisieren. Im begleitenden Forschungsprojekt wird untersucht, inwiefern das Seminar dazu beiträgt, dass sich die 
Studierenden trotz wahrgenommener Herausforderungen den Einsatz von Experimenten im Geographieunterricht zutrauen. Hierfür wird die Selbstwirksamkeitserwartung als wichtiger Teil der motivationalen Orientierung (Baumert \& Kunter, 2006) in den Blick genommen. In diesem Beitrag werden nach der Darstellung des theoretischen Hintergrunds die Konzeption des GEO-Lehr-Lern-Labors sowie das begleitende Forschungsvorhaben vorgestellt.

\section{Theoretische Grundlagen}

Im Nachfolgenden werden in Kapitel 2.1 der Experimentbegriff, die Relevanz und empirische Befunde zum Einsatz von Experimenten im Geographieunterricht, in Kapitel 2.2 die im Projektkontext gewählte Professionalisierung von Lehrkräften im Rahmen von Lehr-Lehr-Laboren sowie in Kapitel 2.3 der in der fachdidaktischen Begleitforschung gewählte Fokus auf die Selbstwirksamkeitserwartung dargelegt.

\subsection{Experimente im Geographieunterricht}

Zur Beantwortung geographischer Fragen (z. B. Welche Faktoren bedingen die Bodenerosion und welche Maßnahmen können für den Bodenschutz ergriffen werden?) wird im Geographieunterricht sowohl auf naturwissenschaftliche als auch gesellschaftswissenschaftliche Theorien und Methoden zurückgegriffen (Deutsche Gesellschaft für Geographie [DGfG], 2017). Im Rahmen eines naturwissenschaftlichen Zugriffs stellt das Experiment eine Methode dar, an der die grundlegenden Prinzipien naturwissenschaftlichen Arbeitens aufgezeigt werden können. Laut Kaminske (2009) steht das Experimentieren beim anwendungsorientierten Arbeiten in den naturwissenschaftlichen Gegenstandsbereichen gar an vorderster Stelle. In der geographiedidaktischen Literatur wird ein Experiment als „eine planmäßige, grundsätzlich wiederholbare Beobachtung von natürlichen und auch gesellschaftlichen Vorgängen unter künstlich hergestellten, möglichst veränderbaren Bedingungen" definiert (Otto, 2003, S. 3). Das Experimentieren dient der Identifikation von kausalen Wirkungszusammenhängen zwischen an einem Prozess beteiligten Faktoren. Aus diesen lassen sich Regelmäßigkeiten und allgemeine GesetzmäBigkeiten hinsichtlich der Bedingungen eines Phänomens bzw. Objekts ableiten. Nach Otto et al. (2010) unterscheiden sich Experimente im Fach Geographie von Experimenten in anderen naturwissenschaftlichen Fächern zum einen durch das inhaltlich-thematische Spektrum und zum anderen durch einen obligatorischen Kontextbezug. Inhaltlich-thematisch stehen mineralogische, bodenkundliche und geomorphologische Experimente im Mittelpunkt, wobei diese einen Rückgriff auf biologische, chemische und physikalische Phänomene beinhalten können. Darüber hinaus ist die „Bezugnahme auf und die Untersuchung von Wechselwirkungen zwischen Geosphäre und Anthroposphäre" (S. 138) spezifisch für das experimen- 
telle Arbeiten im Geographieunterricht. Der angesprochene Aspekt des Kontextbezugs besagt, dass Experimente im Geographieunterricht in der Regel von einer Fragestellung zu gesellschaftlich relevanten Aspekten und Problemstellungen ausgehen (Wilhelmi, 2012). Da sich eine geographische Fragestellung auch durch ihren räumlichen Bezug auszeichnet, ergibt sich für das Experimentieren im Geographieunterricht stets die Notwendigkeit eines Transfers zwischen Realraum und Laborraum.

Dass die Methode des Experimentierens zum festen Methodenrepertoire im Geographieunterricht gehört, wird in den Positionspapieren der Deutschen Gesellschaft für Geographie und des Sekretariats der Ständigen Konferenz der Kultusminister der Länder in der Bundesrepublik Deutschland unterstrichen (DGfG, 2017; Otto, 2009; Sekretariat der Ständigen Konferenz der Kultusminister der Länder in der Bundesrepublik Deutschland [KMK], 2018). Im Geographieunterricht sollen bei Schülerinnen und Schülern die Fähigkeiten gefördert werden, Informationen zur Behandlung geowissenschaftlicher Fragestellungen aus Experimenten zu gewinnen sowie den Weg der Erkenntnisgewinnung zu beschreiben und zu reflektieren (DGfG, 2017). Hierfür sollen angehende Geographielehrkräfte im Lehramtsstudium für den Einsatz von Experimenten, v. a. auch im Hinblick auf didaktische und organisatorische Voraussetzungen für deren Durchführung mit heterogenen und inklusiven Lerngruppen, professionalisiert werden (KMK, 2018). Eine Modellierung der damit verbundenen und bei Lehramtsstudierenden zu fördernden Fähigkeiten liegt für das Fach Geographie bislang nicht vor.

Im Kontrast zur Würdigung von Experimenten auf theoriegeleiteter Ebene und dem hohen Interesse, welches dieser Erkenntnismethode von Schülerinnen und Schülern entgegengebracht wird, steht die mangelnde praktische Berücksichtigung im Geographieunterricht (Hemmer \& Hemmer, 2010). Die Wahrnehmung von angehenden Geographielehrkräften, dass die eigene didaktisch-methodische Qualifikation in diesem Bereich mangelhaft ist, bietet hierfür einen Erklärungsansatz (Höhnle \& Schubert, 2016). Forschungsergebnisse zur tatsächlichen Ausprägung von Kompetenzaspekten bei (angehenden) Geographielehrkräften in diesem Bereich liegen bislang nicht vor. Die laufende Studie von Schubert und Kreuziger (2018) widmet sich der Erforschung von beliefs (angehender) Geographielehrkräfte zu naturwissenschaftlichen Arbeitsweisen und wird hier erste Daten liefern. Das hier vorgestellte Forschungsprojekt leistet einen weiteren Beitrag, indem es die Selbstwirksamkeitserwartung von Geographielehramtsstudierenden hinsichtlich des Einsatzes von Experimenten in den Blick nimmt. Inwiefern der Einsatz von Experimenten im Geographieunterricht (sofern er erfolgt) wie gefordert kompetenzorientiert und unter Berücksichtigung heterogener Lerngruppen erfolgt (KMK, 2018), kann aufgrund der ungenügenden Forschungslage ebenfalls nicht beantwortet werden. Empirische Befunde zum naturwissenschaftlichen Unterricht in Deutschland deuten darauf hin, dass die Planung eigener Experimente und die Durchführung strukturierter Experimente sowie das Eingehen auf heteroge- 
ne Lernvoraussetzungen in diesem zu kurz kommen (Schiepe-Tiska, Rönnebeck, Schöps \& Neumann, 2016).

\subsection{Professionalisierung von Lehrkräften}

Aus den obigen Ausführungen lässt sich resümieren, dass sowohl der Stellenwert, der dem Experimentieren im alltäglichen Geographieunterricht beigemessen wird, als auch die anzunehmende Qualität des Experimenteinsatzes der deklarierten Relevanz der Methode nicht gerecht werden. Höhnle und Schubert (2016) schlagen resultierend aus den Ergebnissen ihrer Studie (siehe Kap. 2.1) die Schaffung eines (größeren) Angebots an geographiedidaktischen Lehrveranstaltungen zum Einsatz von naturwissenschaftlichen Arbeitsweisen, die Nutzung lehrplankonformer Unterrichtsbeispiele sowie einen Einbezug von Praxiselementen bzw. eine Verknüpfung mit Praxisphasen als mögliche Konsequenzen für die Lehrerbildung vor. Die vorgeschlagenen Maßnahmen zielen vor allem auf den Aufbau der didaktischmethodischen Qualifikation von (angehenden) Lehrkräften und damit den Abbau von wahrgenommenen Hindernissen bzw. von Unsicherheiten. Es ließ sich bereits in Studien empirisch nachweisen, dass Praxiserfahrungen das Zutrauen in die eigenen Fähigkeiten wirksam stärken können (Warner \& Schwarzer, 2009). Praxisphasen müssen hierzu allerdings so gestaltet sein, dass Erfolgserlebnisse ermöglicht werden (siehe Kap. 2.3). Ein Veranstaltungskonzept, welches die Forderungen nach einer ebenso gestalteten Praxisphase erfüllt, ist das Lehr-Lern-Labor. Dieses erlaubt es Studierenden, eine Unterrichtssequenz zu planen, diese mit Schülerinnen und Schülern zu erproben und eigene Lehrhandlungen zu reflektieren (Dohrmann \& Nordmeier, 2015). Eine Besonderheit des Veranstaltungsformats liegt in der vorgenommenen Komplexitätsreduktion, die auf unterschiedlichen Ebenen erfolgen kann (Marohn, Greefrath, Hammann, Hemmer, Kürten \& Windt, in diesem Band; Kap. 3.3). Erste Studien zur Erforschung von Professionalisierungsprozessen in Lehr-Lern-Laboren sind v.a. in den naturwissenschaftlichen Fachdidaktiken verortet (Dohrmann \& Nordmeier, 2015; Fischer, Niesel \& Sjuts, 2011; Völker \& Trefzger, 2011). Auch wenn es einzelne hochschuldidaktische Formate in der Geographiedidaktik gibt, die die Kriterien eines Lehr-Lern-Labors erfüllen (z. B. Hemmer \& Miener, 2013), wird der Begriff des Lehr-Lern-Labors im Fach Geographie bislang nur selten verwendet (u. a. bei Mönter, Lütje \& Schlitt, 2016). Entsprechend liegen kaum fachspezifische Erkenntnisse zu Bedingungen der Wirksamkeit dieses Veranstaltungsformats vor. 


\subsection{Selbstwirksamkeitserwartung als Teil der professionellen Handlungskompetenz von Lehrkräften}

Im Zentrum der Begleitforschung des hier vorgestellten Projekts steht die situationsspezifische Selbstwirksamkeitserwartung von angehenden Geographielehrkräften. Selbstwirksamkeitserwartungen (SWE), mitunter auch als Kompetenzerwartungen tituliert (Warner \& Schwarzer, 2009), gelten als Bestandteil der professionellen Handlungskompetenz von Lehrkräften und bezeichnen die „Überzeugung einer Person, über die Fähigkeiten und Mittel zu verfügen, um diejenigen Handlungen durchführen zu können, die notwendig sind, um ein definiertes Ziel zu erreichen - und zwar auch dann, wenn Barrieren zu überwinden sind“ (Baumert \& Kunter, 2006, S. 502; übersetzt nach Bandura, 1997). Für die Domäne des Lehrberufs definieren Tschannen-Moran, Hoy, und Hoy (1998) in ihrem Beitrag die Lehrer-Selbstwirksamkeitserwartung wie folgt: "the teacher's belief in his or her capability to organize and execute courses of action required to successfully accomplish a specific teaching task in a particular context" (S. 233). Aus den Definitionen wird bereits deutlich, dass Kompetenzerwartungen eine große Rolle bei selbstregulativen Zielprozessen spielen und handlungsrelevant sind (Bandura, 1997; Baumert \& Kunter, 2006; Schwarzer \& Jerusalem, 2002). So zeigen Lehrkräfte mit höherer Selbstwirksamkeitserwartung beispielsweise eine höhere Resilienz gegenüber beruflichen Anforderungen (Skaalvik \& Skaalvik, 2010), stehen neuen Unterrichtsmethoden offener gegenüber (Cousins \& Walker, 2000), setzen sich eher Ziele mit einem höheren Anspruchsniveau und verfolgen diese mit mehr Anstrengung und Ausdauer (Bandura, 1997; Kocher, 2011, S. 76; Schüle, Besa, Schriek \& Arnold, 2017). Im Projektkontext kann die Erforschung der situationsspezifischen Selbstwirksamkeitserwartung Auskunft darüber geben, inwiefern ein Abbau von Unsicherheiten hinsichtlich eines Einsatzes von Experimenten im Geographieunterricht durch das GEO-Lehr-Lern-Labor gelingt. Gerade in Hinblick auf die mit einem Einsatz von Experimenten verbundenen Hindernisse und die geringe Einsatzhäufigkeit von Experimenten im Geographieunterricht ist dies relevant (siehe Kap. 2.1).

Um die Entwicklungsbedingungen von Selbstwirksamkeitserwartungen analysieren zu können, bedarf es eines Verständnisses des Entstehungs- und Entwicklungsprozesses der Selbstwirksamkeitserwartung. Laut theoretischer Annahmen von Bandura (1997) können Selbstwirksamkeitserwartungen durch vier Quellen beeinflusst werden: (1) Handlungsergebnisse in Gestalt eigener Erfolge und Misserfolge; (2) stellvertretende Erfahrungen durch Beobachtung von Verhaltensmodellen; (3) sprachliche Überzeugungen (z. B. Fremdbewertung oder Selbstinstruktion) sowie (4) Wahrnehmungen eigener Gefühlserregung (Schwarzer \& Jerusalem, 2002). Dabei wird den eigenen Handlungserfahrungen der größte Einfluss zugesprochen, was sich in empirischen Studien bereits mehrfach bestätigt hat (Tschannen-Moran \& Hoy, 2007). Die genannten Informationsquellen beeinflussen allerdings nicht automatisch die Selbstwirksamkeitserwartungen. Vielmehr 
komme es auf die subjektive Einschätzung der Gesamtsituation bzw. der Rahmenbedingungen an (Schwarzer \& Jerusalem, 2002).

Die Forschungslage hinsichtlich der Entwicklung von Selbstwirksamkeitserwartung bei Lehrkräften ist noch recht unbefriedigend (Baumert \& Kunter, 2006; Kocher, 2011). Dies spiegelt sich ebenso in der geographiedidaktischen Forschung wider. Erst in den letzten Jahren gerät hier das Konstrukt der Selbstwirksamkeitserwartung und deren Entwicklungsbedingungen verstärkt in den Fokus (Lindau, 2018; Renner, 2018). Die bisherigen empirischen Befunde stellen daher lediglich erste Indizien für die Bedingungen einzelner Entwicklungsprozesse bereit. Ein Phänomen, welches Tschannen-Moran et al. (1998) beschreiben, ist das Absinken der Lehrer- Selbstwirksamkeitserwartung in Praxisphasen der universitären Lehrerbildung. Hoy und Spero (2005) berichten selbiges bei Berufsanfängern. Zurückgeführt wird dies auf die Konfrontation mit der (oft unerwarteten) Komplexität von realen Unterrichtssituationen und die Erkenntnis, dass die Bewältigung der Praxis schwieriger ist, als zunächst angenommen. Tschannen-Moran et al. (1998) schlagen daraus folgend für die Lehrerbildung vor, Lehramtsstudierenden mehr praktische Erfahrungen zu ermöglichen. Diese sollten sich in ihrer Komplexität und somit den Anforderungen sukzessiv steigern. Zudem soll eine Kopplung mit gezieltem Feedback vorgenommen werden, welches positive Leistungen hervorhebt und kontrollierbare Zuschreibungen betont. Die Einführung neuer Lehrmethoden solle durch erfahrene Kolleginnen und Kollegen begleitet (Modelllernen) und eigene Erfahrungen sollten diskutiert werden (verbale Überzeugung) (Warner \& Schwarzer, 2009).

Beim Betrachten der Charakteristika von Lehr-Lern-Laboren (Dohrmann \& Nordmeier, 2015) wird deutlich, dass diese die Forderungen nach (komplexitätsreduzierten) Übungen zum eigenen Lehren mit Unterstützungsmaßnahmen (Erfolgserfahrungen), einer Begleitung durch eine Mentorin oder einen Mentoren (Modelllernen) und einer intensiven Reflexionsphase (verbale Überzeugung und kognitive Bewertung) erfüllen. Erste Hinweise auf einen positiven oder zumindest stabilisierenden Einfluss des Formats auf die Selbstwirksamkeitserwartung liefern Studien der Universität Berlin (Dohrmann \& Nordmeier, 2018; Krofta \& Nordmeier, 2014).

\section{Konzeption des GEO-Lehr-Lern-Labors}

Das GEO-Lehr-Lern-Labor wurde im Wintersemester 2016/17 konzipiert und im Sommersemester 2017 erstmalig durchgeführt. Es stellt ein Wahlpflichtseminar mit zwei Semesterwochenstunden im Rahmen des Studiengangs Master of Education Geographie dar und trägt den Veranstaltungstitel: „Mit Vielfalt experimentieren Experimentelle Arbeitsweisen im Geographieunterricht unter besonderer Berücksichtigung heterogener Lerngruppen “. 


\subsection{Zielsetzung und inhaltliche Schwerpunkte}

Ziel des GEO-Lehr-Lern-Labors ist die Professionalisierung von Lehramtsstudierenden hinsichtlich fachlicher und fachdidaktischer Kompetenzen für den Einsatz von Experimenten im Geographieunterricht. Mit Abschluss der Veranstaltung sollen die Studierenden fähig sein, die Methode des Experimentierens ziel-, schülerund fachgerecht im Geographieunterricht und unter besonderer Berücksichtigung heterogener Lernvoraussetzungen einzusetzen (KMK, 2018). Im Zentrum des GEOLehr-Lern-Labors steht der Aufbau von Wissen und Fähigkeiten in den folgenden Bereichen:

1. Experimentieraufgaben im Geographieunterricht

- Erkennen des didaktischen und diagnostischen Potenzials von Experimentieraufgaben

- Erstellung/Abwandlung von Experimentieraufgaben

2. Diagnose und Förderung der Experimentierkompetenz

- Erkennen und Beurteilen der Experimentierleistung von Schülerinnen und Schülern anhand von Kompetenzmodellen

- Formulieren möglicher Handlungsableitungen zur Förderung

Beide Bereiche lassen sich den Facetten des fachdidaktischen Wissens nach Baumert und Kunter (2006) zuordnen. Auf der Ebene der motivationalen Orientierung der professionellen Handlungskompetenz von Lehrkräften (Baumert \& Kunter, 2006) soll das GEO-Lehr-Lern-Labor vor allem zu einer Steigerung der situationsspezifischen Selbstwirksamkeitserwartung der Studierenden hinsichtlich des Einsatzes von Experimenten im Geographieunterricht beitragen (siehe Kap. 2.3). Angestrebt wird zudem die Ausbildung realistischer Vorstellungen über die eigenen Fähigkeiten im Abgleich mit den Anforderungen unterrichtlicher Situationen.

\subsection{Aufbau}

Die Strukturierung des GEO-Lehr-Lern-Labors erfolgte anhand der gesetzten inhaltlichen Schwerpunkte, der Definition von Lehr-Lern-Laboren (Dohrmann \& Nordmeier, 2015) und der vorliegenden Rahmenbedingungen. Der Aufbau ist charakterisiert durch vier Phasen: (1) Theoriephase, (2) Planungsphase, (3) Praxisphase und (4) Reflexionsphase (siehe Abb. 1).

Im Zentrum der (1) Theoriephase steht die Vermittlung von Professionswissen. Fußend auf einer Einführung in das Thema der experimentellen Arbeitsweisen der Geographie (Definitionen der Arbeitsweisen Beobachtung, Untersuchung, Experiment, Modellieren; Chancen und Herausforderungen für einen Einsatz im Geographieunterricht) wird der Schwerpunkt auf den Erwerb anwendungsfähigen Wissens in den Facetten ,Experimentieraufgaben' und ,Diagnose und Förderung 


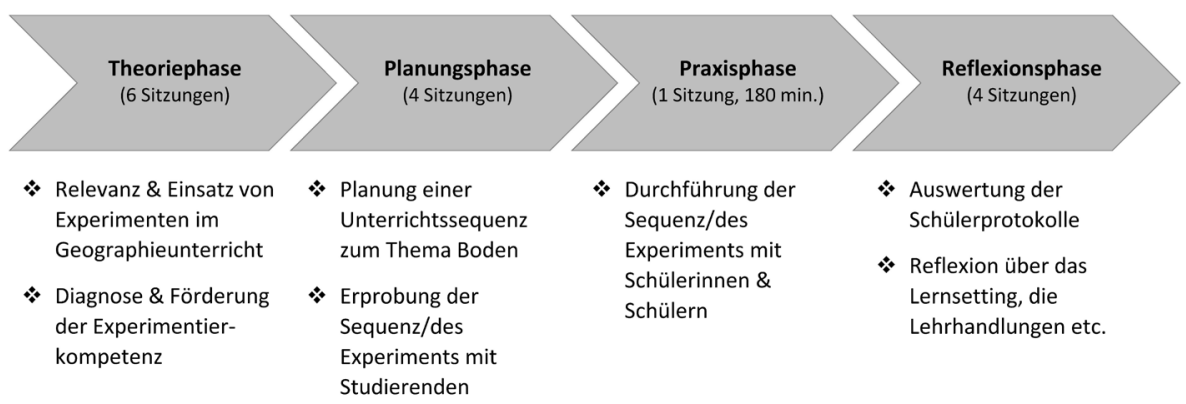

Abbildung 1: Struktur und Inhalte des GEO-Lehr-Lern-Labors

der Experimentierkompetenz' gelegt (siehe Kap. 3.1). Methodisch ist die Theoriephase so angelegt, dass sie den Studierenden bereits frühzeitig eine praktische Durchdringung der theoretischen Inhalte ermöglicht (z. B. durch die Durchführung und Analyse von Experimenten oder das Erleben von diagnostischen Tools). Die vermittelten theoretischen Grundlagen bilden die Basis für die (2) Planungsphase. In dieser sollen die Studierenden das erworbene Wissen anwenden, indem sie zunächst ein ausgearbeitetes Lernsetting zu einem geographischen Experiment kennenlernen, um es anschließend zu bewerten und ggf. zu optimieren. Hierfür wurden Experimente zum Thema Bodenerosion (durch Wasser und Wind) gewählt, da sie lehrplankonform, anschaulich und mit einfachen Mitteln durchführbar sind. Zudem lassen sich an diesem Thema verschiedene Wechselwirkungen von Mensch-Umwelt-Beziehungen problemorientiert erarbeiten sowie bereits publizierte Unterrichtsvorschläge miteinander vergleichen. Anschließend planen die Studierenden auf dieser Grundlage die Praxisphase mit den Schülerinnen und Schülern. Ziel ist es, eine Unterrichtseinheit von ca. 180 Minuten unter Einbeziehung eines Schülerexperiments zu entwickeln. Die sich anschließende (3) Praxisphase dient der Durchführung der geplanten Unterrichtseinheit sowie dem Sammeln von Erfahrungen im Umgang mit Schülerinnen und Schülern. Während des Praxistermins leiten die Studierenden die Schülerinnen und Schüler an und begleiten sie in ihren Lernprozessen. Durch die eigenständige Planung der Unterrichtseinheit und die praktische Durchführung derselben mit Schülerinnen und Schülern soll der Aufbau eines realistischen Bildes von den Anforderungen unterrichtlicher Handlungen und Situationen unterstützt werden. Die abschließende (4) Reflexionsphase des GEO-Lehr-Lern-Labors hat zum Ziel, Raum für die Diagnose der Schülerleistungen zu bieten sowie eine Reflexion über das Lernsetting, Lehrhandlungen und eigene Fähigkeiten anzuregen. 


\subsection{Komplexitätsreduktion}

Im GEO-Lehr-Lern-Labor wurden verschiedene Maßnahmen umgesetzt, um eine Reduzierung der Komplexität und damit des Anforderungsniveaus zu erreichen (siehe Abb. 2). Wenngleich alle in Abbildung 2 aufgeführten Maßnahmen im GEOLehr-Lern-Labor Berücksichtigung finden, werden im Folgenden die fünf in der Abbildung durch Fettdruck hervorgehobenen Maßnahmen näher erläutert, da diese auch Teil der Evaluationsforschung sind. Die auf der Basis der Evaluation erfolgten Änderungen werden ebenfalls thematisiert.

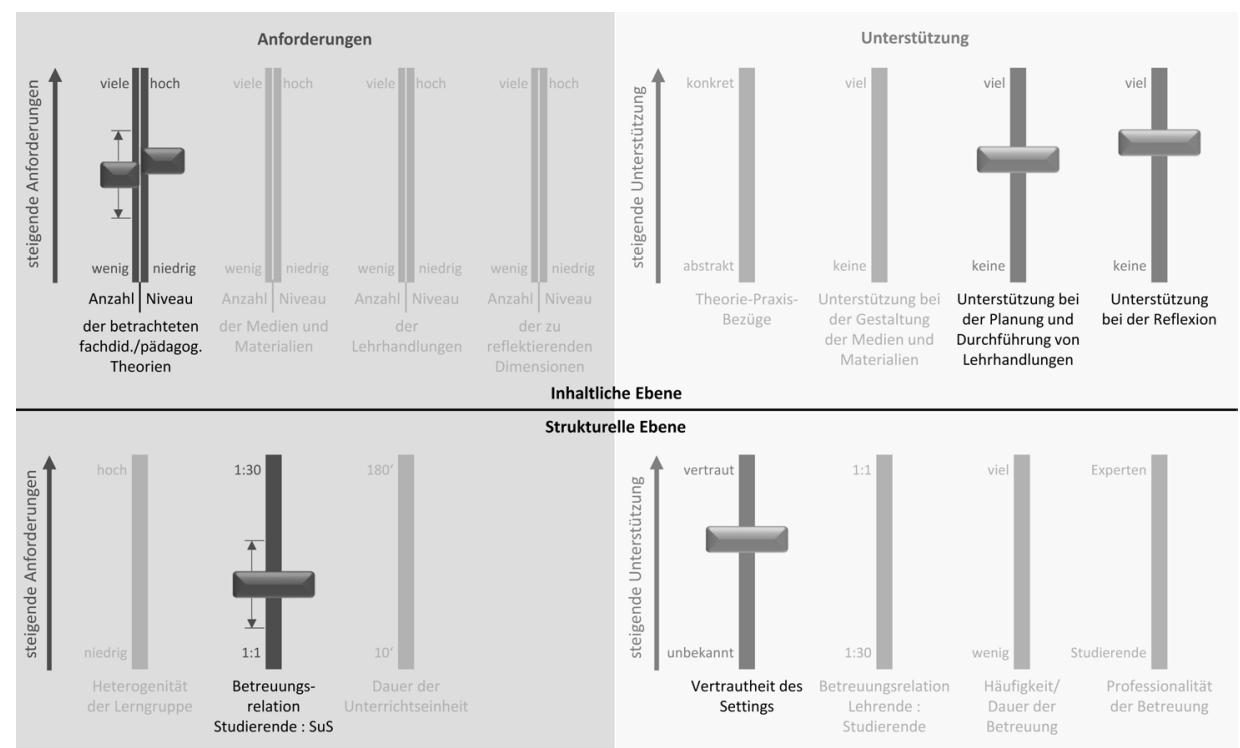

Abbildung 2: Maßnahmen zur Komplexitätsreduktion des GEO-Lehr-Lern-Labors im Wintersemester 2018/19

Anzahl und Niveau der betrachteten fachdidaktischen/pädagogischen Theorien Wie bereits aus der Zielstellung der Veranstaltung (siehe Kap. 3.1) zu entnehmen ist, steht das Erlangen von Wissen und Fähigkeiten in den Bereichen ,Experimentieraufgaben' sowie ,Diagnose und Förderung der Experimentierkompetenz' im Fokus des GEO-Lehr-Lern-Labors. Die Festlegung der in der Lehrveranstaltung zu fördernden Einzelfähigkeiten erfolgte unter Rückgriff auf formulierte Standards und theoretische Modelle zur Professionalisierung von (angehenden) Lehrkräften (Baumert \& Kunter, 2006; Buholzer, Joller-Graf, Kummer Wyss \& Zobrist, 2014; KMK, 2018) sowie unter Sichtung der fachdidaktischen Literatur zum Thema experimentelle Arbeitsweisen. Eine Eingrenzung der zu behandelnden Inhalte wurde mit Blick auf die Rahmenbedingungen des GEO-Lehr-Lern-Labors vorgenommen. Hinsichtlich des Umgangs mit Heterogenität erfolgte eine Fokussierung auf die Experimentierleistung der Schülerinnen und Schüler als Heterogenitätsdimension. 
Neben dem Aufzeigen von Möglichkeiten für eine entsprechend binnendifferenzierte Gestaltung von Experimentieraufgaben wurde ein besonderes Augenmerk auf die Förderung diagnostischer Fähigkeiten gelegt. Sie gelten als Voraussetzung für die Gestaltung adaptiven Unterrichts und eines reflektierten Umgangs mit $\mathrm{He}$ terogenität (Ohl \& Mehren, 2016). In den ersten Durchgängen erfolgte aufgrund der Vielschichtigkeit der Experimentierkompetenz (Hammann, 2004; Mayer et al., 2009) eine weitere Komplexitätsreduktion auf inhaltlicher Ebene derart, dass die Studierenden zu Beginn der Planungsphase Expertengruppen für je eine Teilkompetenz der Experimentierkompetenz bildeten und im weiteren Verlauf der Veranstaltung kooperativ arbeiteten. Hierdurch sollte einer Überforderung aufgrund der Komplexität und Menge der zu berücksichtigen Einzelfacetten der Experimentierkompetenz entgegengewirkt und zugleich die individuelle Verantwortlichkeit für den Lernprozess erhöht werden. Durch den Austausch der Studierenden untereinander und die gegenseitige kriteriengestützte Beobachtung der Kommilitoninnen und Kommilitonen während des Praxistermins sollten dennoch allen alle Inhalte zugänglich gemacht werden. Da bei der Auswertung der Begleitforschung vermehrt zu Tage trat, dass eine zu starke Arbeitsteilung in diesem Punkt jedoch eher hinderlich für die Entwicklung des Zutrauens in die eigenen Kompetenzen wirkt (siehe Kap. 4), wurde dieser Teil der Komplexitätsreduktion modifiziert. Ab dem Wintersemester 2018/19 können die Studierenden zu Beginn der Planungsphase selbstständig entscheiden, ob sie eine derartige Arbeitsteilung und Spezialisierung in den Gruppen vornehmen oder ob sie die Diagnose der Schülerleistung für alle Facetten der Experimentierkompetenz gemeinsam durchführen.

\section{Betreuungsrelation Studierende-Schülerinnen und Schüler}

Eine Möglichkeit, die Anforderungen für die Studierenden in der Praxisphase auf struktureller Ebene zu verringern, ist die Erhöhung der Betreuungsrelation zwischen Studierenden und Schülerinnen und Schülern. Es kann davon ausgegangen werden, dass hierdurch der Aufbau von Lehr-Lern-Prozessen störungsfreier gelingt und die Studierenden sich auf andere Aspekte des Unterrichts konzentrieren können. Bezüglich der Heterogenität von Lerngruppen wird angenommen, dass sich auch bei wenigen Lernenden eine Leistungsheterogenität hinsichtlich experimenteller Fähigkeiten zeigen wird (Schubert, 2016). Eine Beschränkung auf kleine Schülergruppen scheint vor allem dann sinnvoll, wenn diagnostische Handlungen wie die Wahrnehmung und Bewertung eines individuellen Schülermerkmals im Fokus stehen und das Eingehen auf einzelne Verständnisprobleme durch Mikroadaption ermöglicht werden soll. Zudem entsteht eine erhöhte Anforderung dadurch, dass die Studierenden nicht mit der Lerngruppe vertraut sind, sodass eine Anpassung des Unterrichtssettings an die individuellen Lernbedürfnisse der Lernenden durch zuvor geplante Maßnahmen weitestgehend eingeschränkt ist. Im ersten Durchlauf besuchten sechs Schülerinnen und Schüler das GEO-Lehr-LernLabor. Dabei betreute abwechselnd eine Studentin oder ein Student eine Schülergruppe von zwei Lernenden, während andere Studierende die Lehr-Lern-Prozesse 
kriteriengeleitet beobachteten. Das Unterrichtssetting war dabei offen gestaltet, sodass die Studierenden stärker die Rolle eines Lernbegleiters einnahmen. Lediglich der von der Dozentin geleitete Einstieg der Praxisphase wurde im Klassenunterricht abgehalten. Als Reaktion auf die Ergebnisse der Begleitforschung (siehe Kap. 4) wurde die Praxisphase mit dem Ziel modifiziert, eine stärkere Berücksichtigung der individuellen Lernbedürfnisse der Studierenden und damit eine stärkere Unterstützung des Professionalisierungsprozesses zu ermöglichen. In einem ersten Schritt wurde die Anzahl der Schülerinnen und Schüler auf insgesamt zwölf erhöht (Wintersemester 2017/18). Hierauf wurde die Gestaltung und Durchführung der Einstiegsphase an die Studierenden abgegeben sowie der Einbau von Plenumsphasen ermöglicht. Die Studierenden können fortan eigenständig entscheiden, welche Lehrhandlungen (eher anleitend vs. eher begleitend) sie mit welchem Betreuungsverhältnis in der Praxisphase übernehmen möchten.

\section{Unterstützung bei der Planung und Durchführung von Lehrhandlungen}

Während die Sitzungen zu den theoretischen Grundlagen von der Dozentin vorbereitet und gehalten wurden, überwog die Aktivität der Studierenden ab der Planungsphase. Sie wurden dabei in ihren Tätigkeiten weiterhin von der Dozentin begleitet und unterstützt. So wurden alle benötigten Experimentiermaterialien, didaktische Materialien zur Binnendifferenzierung, eine Auswahl an diagnostischen Tools, Arbeitsblätter zu den wesentlichen Seminarinhalten, zusätzliche Literatur und Übungsaufgaben zur Vertiefung bereitgestellt. Die Studierenden erhielten zu ihren Planungsskizzen Feedback von der Dozentin und Peer-Feedback. In der Praxisphase erhielten die Studierenden außer dem Materialaufbau nur in seltenen Fällen und auf ausdrücklichen Wunsch hin Unterstützung seitens der Dozentin. Diese bezog sich allerdings ausschließlich auf Hilfen beim Umgang mit technischen Geräten und der spontanen Bereitstellung weiterer Labormaterialien.

\section{Unterstützung bei der Reflexion}

Der Reflexionsprozess wurde durch verschiedene Aufgaben strukturiert. In den ersten drei Durchgängen der Veranstaltung standen vor allem diagnostische Lehrhandlungen im Zentrum der Reflexion. Als Reaktion auf die Erfahrungen aus diesen Seminardurchläufen wurde ab dem Wintersemester 2018/19 zudem Raum für andere Reflexionsgegenstände gegeben. So waren es zum Beispiel weitere Aspekte der Unterrichtsdurchführung oder Unsicherheiten bezüglich der Deutung einer Lehr-Lern-Situation, die die Studierenden zusätzlich bearbeiten und besprechen wollten. Eine Nichtbeachtung dieser als dringlich empfundenen Entwicklungsbedürfnisse könnte zu einer wachsenden Unsicherheit führen und würde den Zielen der Veranstaltung nicht gerecht werden. Die methodische Umsetzung erfolgte mit einer zusätzlichen Reflexionsaufgabe, in der eine selbstgewählte Situation aus dem Praxistermin anhand festgelegter Reflexionsschritte (Gibbs, 1988; Hilzensauer, 2008) zunächst schriftlich reflektiert und anschließend innerhalb des Seminars in Gruppen besprochen wurde. 


\section{Vertrautheit des Settings}

Da die Studierenden das einzusetzende Experiment für die Praxisphase bereits zu Beginn der Planungsphase kennenlernen, durchführen, reflektieren und optimieren konnten, war das Unterrichtssetting recht vertraut. Vor der Durchführung mit den Schülerinnen und Schülern erhielten die Studierenden ein Feedback und Hinweise für eine mögliche Optimierung ihrer Unterrichtsplanung. Diese Maßnahme sollte neben einer Qualitätssicherung der Arbeitsmaterialien auch der Unterstützung der Selbstsicherheit der Studierenden dienen. Zur Erhöhung der Vertrautheit mit der eigenen Planungsskizze, wurde ab dem Wintersemester 2018/19 ein zusätzlicher Probedurchlauf mit Studierenden eingeführt. Diese Maßnahme resultierte ebenfalls aus den Erkenntnissen der Begleitforschung (siehe Kap. 4).

\section{Begleitende Forschung}

\subsection{Zielsetzung \& Forschungsfragen}

Da gerade das Zutrauen in die eigenen Fähigkeiten für den Einsatz von bisher selten eingesetzten und als herausfordernd empfundenen Methoden handlungsrelevant $\mathrm{zu}$ sein scheint (siehe Kap. 2.3), wurde der Fokus der Begleitforschung auf die Kompetenzerwartung als Teil der professionellen Handlungskompetenz von Lehrkräften gelegt. Ausgangspunkt für die Forschung ist die praxisrelevante Frage: Wie kann das GEO-Lehr-Lern-Labor gestaltet werden, um bei Lehramtsstudierenden die situationsspezifische Selbstwirksamkeitserwartung zum Einsatz von Experimenten im Geographieunterricht zu fördern? Für die Beantwortung dieser Frage ist es notwendig, die Entwicklungen der situationsspezifischen Selbstwirksamkeitserwartung sowie deren Bedingungen aufzudecken. Resultierend ergeben sich folgende Forschungsfragen:

1) Wie verändert sich die situationsspezifische Selbstwirksamkeitserwartung angehender Geographielehrkräfte während des GEO-Lehr-Lern-Labors?

2) In welchen Bereichen nehmen die Studierenden eine Kompetenzentwicklung durch das GEO-Lehr-Lern-Labor wahr und worauf führen sie diese zurück?

3) Welche Verständnisprobleme/Schwierigkeiten haben Studierende im Seminar und inwieweit können diese überwunden werden?

4) Welche komplexitätsreduzierenden Designelemente des GEO-Lehr-LernLabors erleben die Studierenden als förderlich bzw. hinderlich für ihre Kompetenzentwicklung?

Ziel ist es, anhand der Forschungsergebnisse konkrete Hinweise zur Optimierung des GEO-Lehr-Lern-Labors zu gewinnen und einen Beitrag zur lokalen Theoriebildung zu leisten. 


\subsection{Material \& Methode}

Forschungsdesign

Ausgehend von der Zielsetzung wurde das Forschungsdesign nach dem DesignBased-Research-Ansatz (DBR) angelegt. Dieser stellt ein Konzept zur nutzungsorientierten Grundlagenforschung dar, in der sowohl die (Weiter-)Entwicklung einer Lernumgebung als auch das Gewinnen von empirischen Erkenntnissen für eine lokale Theoriebildung inkludiert werden. Dabei ist die Entwicklung Bestandteil des Forschungsprozesses: „Erkenntnis erzielt man mit DBR über die Entwicklung oder Gestaltung von Interventionen, die in authentischen Kontexten zyklisch auch mehrfach implementiert, evaluiert und einem Re-Design unterzogen werden. Dies wiederholt man so lange, bis man die angestrebten Ergebnisse erlangt, nämlich praktisch relevante Problemlösungen bzw. praxistaugliche Interventionen und wissenschaftlich relevante Theorien z.B. in Form von Gestaltungsprinzipien" (Reinmann, 2019, S. 129). In Anlehnung an die Ausführungen von Krüger (2010) und Feulner, Ohl, und Hörmann (2015) wurde der Forschungsprozess in vier Phasen unterteilt (siehe Abb. 3). Die ersten drei Phasen wurden dabei in kontinuierlichen Zyklen aus Entwurf, Umsetzung, Analyse und Re-Design bis zum Sommersemester 2019 durchlaufen.

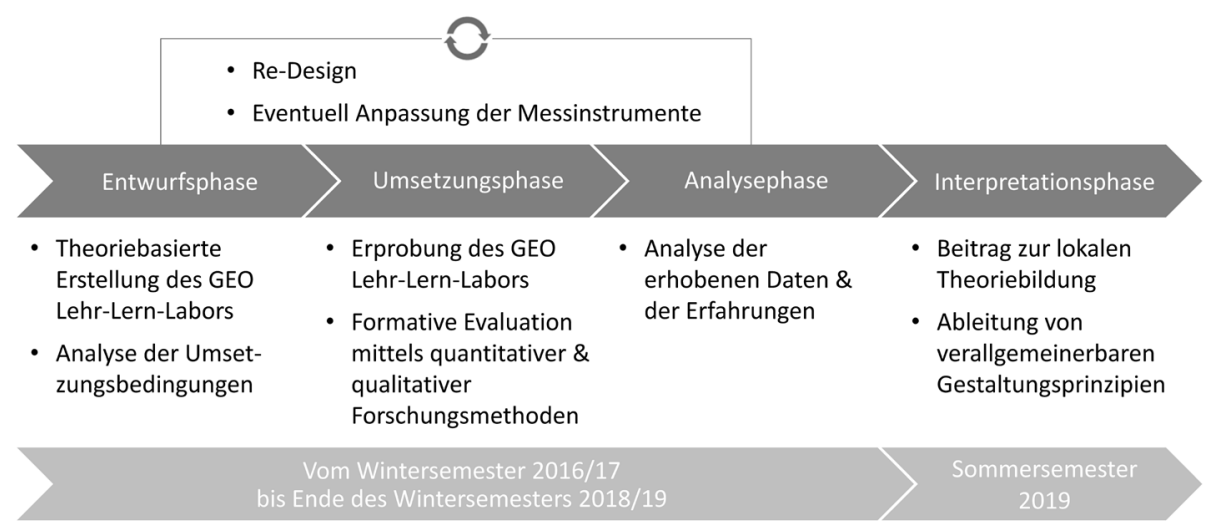

Abbildung 3: Forschungsdesign (in Anlehnung an Feulner et al., 2015; Krüger, 2010)

Erhebung, Aufbereitung und Analyse der Daten

Die Entwicklung der situationsspezifischen Selbstwirksamkeitserwartung wurde mittels eines quantitativen Fragebogens jeweils vor und nach den Seminaren erhoben (Forschungsfrage 1). Dabei sollen die Ergebnisse des Fragebogens v.a. eine Vergleichbarkeit ermöglichen sowie als Anknüpfungspunkte für die qualitative Erhebung dienen. Der Fragebogen wurde auf der Grundlage der formulierten Lehrveranstaltungsziele (siehe Kap. 3.1) und der Skala von Meinhardt, Rabe, und Krey (2016) erstellt und bildet die im Seminar fokussierten Bereiche der situationsspezi- 
fischen Selbstwirksamkeitserwartung ab: das Beurteilen/Erstellen von Experimentieraufgaben (Beispielitem: „Ich kann bei Bedarf eine offene Experimentieraufgabe mit mehreren Lösungswegen für meinen Geographieunterricht entwickeln.") und Erkennen/Beurteilen von Experimentierleistungen (Beispielitem: „Ich kann Experimentierleistungen von Schülerinnen und Schülern anhand transparenter Kriterien beurteilen."). Der Testentwurf der Skala wurde im Sommer 2017 (n=120) zur Evaluation mit Lehramtsstudierenden der Geographie pilotiert (Bühner, 2011) und im Wintersemester 2017/18 erstmalig eingesetzt. Nach Überarbeitung ergab sich eine Skala mit 22 geschlossenen Items, die eine hohe interne Konsistenz aufweist (Cronbachs $\alpha=.95$ ). Daneben wurden im Fragebogen weitere personenbezogene Daten (Geschlecht, Fachsemester etc.) und die Vorerfahrung hinsichtlich des Einsatzes von Experimenten im Unterricht erhoben. Nach der explorativen Faktorenanalyse kann von einer einfaktoriellen Lösung ausgegangen werden. Die Auswertung beschränkt sich in Anbetracht der Fragestellung, der kleinen Stichprobe und der Ergänzung durch die qualitative Erhebung auf die deskriptive Statistik.

Für die Erhebung wahrgenommener Kompetenzentwicklungsprozesse und deren Bedingungen als Indikatoren für Veränderungsprozesse der Selbstwirksamkeitserwartung wurden leitfadengestützte Interviews im Anschluss an die Veranstaltung geführt (Forschungsfragen 2-4). Die Fragen des Leitfadens wurden deduktiv aus theoretischen Modellen und empirischen Erkenntnissen zur Selbstwirksamkeitserwartung sowie den Designelementen des GEO-Lehr-Lern-Labors abgeleitet (siehe Tab. 1). Nach der Pilotierung des Leitfadens wurden die ersten Interviews im Sommersemester 2017 geführt. Die Auswertung basiert auf der zusammenfassenden Inhaltsanalyse nach Mayring (2015), wobei die Kategorienbildung sowohl deduktiv anhand des Interviewleitfadens (siehe Tab. 1) und des theoretischen Modells zur professionellen Kompetenz von Lehrkräften (Baumert \& Kunter, 2006) als auch induktiv aus dem Material heraus erfolgte. In einem letzten (noch ausstehenden) Schritt werden die Daten der Teilnehmenden, zu denen sowohl quantitative als auch qualitative Daten vorliegen, fallbasiert analysiert. Hierdurch sollen die Gründe für unterschiedlich hohe Veränderungen der Selbstwirksamkeitserwartung offengelegt werden. 
Tabelle 1: Struktur und Inhalte des Interviewleitfadens

\begin{tabular}{|c|c|}
\hline Abschnitt & Teilabschnitte \\
\hline 1) Einstieg & $\begin{array}{l}\text { - Bisherige Erfahrungen mit dem Thema Experimentelle Arbeitswei- } \\
\text { sen im Geographieunterricht }\end{array}$ \\
\hline $\begin{array}{l}\text { 2) Wahrgenommene Kompe- } \\
\text { tenzentwicklung während } \\
\text { des Seminars und deren } \\
\text { Quellen }\end{array}$ & $\begin{array}{l}\text { - Persönlicher Output allgemein (offener Impuls) } \\
\text { - Fähigkeitsentwicklung konkret (auf die Seminarziele bezogen) } \\
\text { mit Abfrage möglicher Ankersituationen } \\
\text { - Erfolgserfahrungen im Seminar und deren Attribuierung } \\
\text { - Schwierigkeiten/Probleme im Seminar, deren möglichen Über- } \\
\text { windung und Attribuierung }\end{array}$ \\
\hline $\begin{array}{l}\text { 3) Bewertung der Design- } \\
\text { elemente des Seminars für } \\
\text { die Kompetenzentwicklung }\end{array}$ & $\begin{array}{l}\text { - Bewertung der Praxisphase } \\
\text { - Bewertung der Elemente der Komplexitätsreduktion (mit Vorga- } \\
\text { be der Elemente) } \\
\text { - Verbesserungsvorschläge für das GEO-Lehr-Lern-Labor }\end{array}$ \\
\hline 4) Ausstieg & - Raum für weitere Aspekte je nach Wunsch \\
\hline
\end{tabular}

\section{Stichprobe}

Da es sich beim GEO-Lehr-Lern-Labor um eine Wahlpflichtveranstaltung handelt, besteht die Stichprobe nur aus Studierenden, die sich aus unterschiedlichen Gründen für eine Teilnahme am Seminar entschieden haben. Während der quantitative Fragebogen von allen Teilnehmenden auszufüllen war, nahmen an den qualitativen Interviews nur einzelne Studierende auf Freiwilligenbasis teil. In den ersten drei Durchläufen haben insgesamt 43 Studierende die Veranstaltung durchlaufen. Nach der Datenaufbereitung liegen hieraus 29 (vollständige und zusammengehörige Pre-Post-)Fragebögen (der Durchläufe 2 und 3) und 20 Interviewtranskripte (der Durchläufe 1, 2 und 3) vor.

\subsection{Erste Ergebnisse und Diskussion}

1) Wie verändert sich die situationsspezifische Selbstwirksamkeitserwartung angehender Geographielehrkräfte während des entwickelten GEO-Lehr-Lern-Labors?

Aus den Daten der Fragebögen lässt sich bisher bei fast allen Studierenden ein Anstieg der situationsspezifischen Selbstwirksamkeitserwartung feststellen (Messung ab Wintersemester 17/18). Lediglich bei zwei Studierenden des Durchlaufs im Sommersemester 2018 ist ein leichter Abfall zu verzeichnen (siehe Abb. 4). Aus den Daten lässt sich ebenso ablesen, dass sowohl die Ausgangslagen als auch die Entwicklungsprozesse bzgl. der situationsspezifischen Selbstwirksamkeitserwartung recht heterogen sind. Die Streuung der Werte des Posttests ist dabei geringer als die des Pretests.

Insgesamt lässt sich aus den bisherigen Ergebnissen schlussfolgern, dass eine Stärkung der situationsspezifischen Selbstwirksamkeitserwartung im Lehr-LernLabor die Regel war. Im Vergleich zur Studie von Krofta und Nordmeier (2014) 


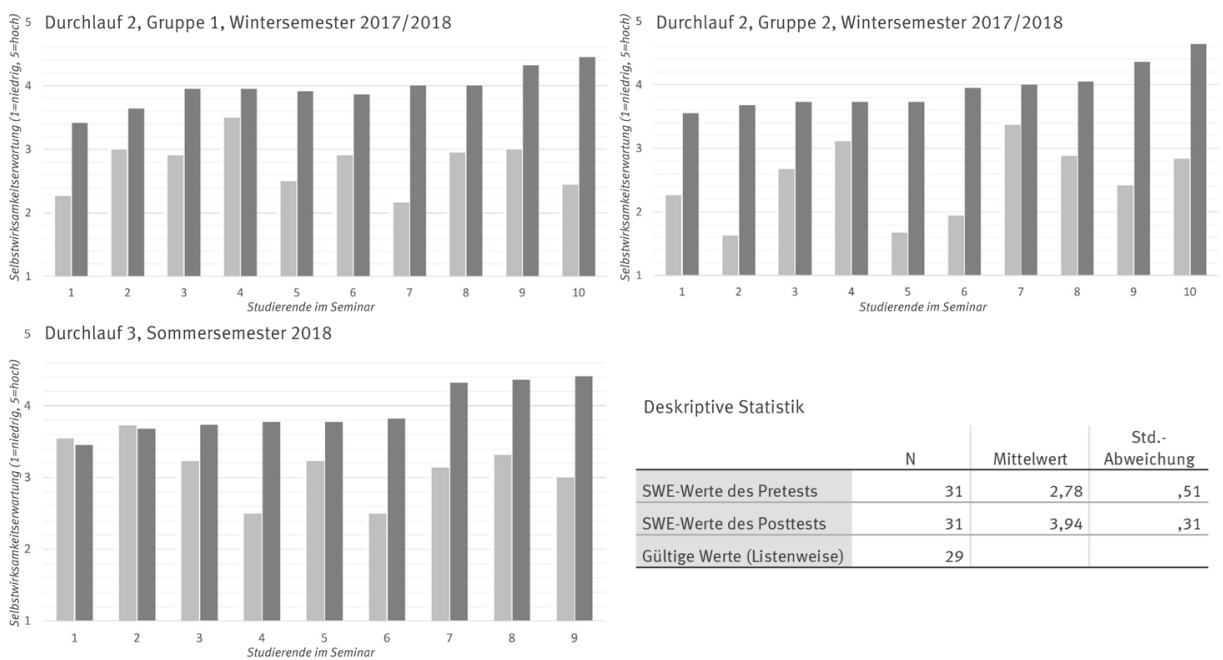

Abbildung 4: Entwicklung der situationsspezifischen Selbstwirksamkeitserwartung der Studierenden (Durchläufe 2 und 3, n=29); Pretest = hellgrau, Posttest = dunkelgrau

lässt sich ein Zuwachs der Selbstwirksamkeitserwartung verzeichnen. Möglicherweise liegt dies an der höheren Spezifität des Messinstruments. Die Abnahme der Streuung der Werte kann so gedeutet werden, dass auch bei unterschiedlichen Ausgangslagen relativ hohe Werte der Selbstwirksamkeitserwartung im Seminar erreicht werden können. Die Höhe des Zuwachses hängt dabei von verschiedenen Faktoren ab, die mit den gesammelten Erfahrungen und deren kognitiven Verarbeitung einhergehen. Es ist davon auszugehen, dass die Veranstaltung in ihrer Konzeption nicht dazu beitragen kann, die Selbstwirksamkeitserwartung auf ein Maximalmaß anzuheben. Ein Blick auf die theoretischen Grundlagen zur Selbstwirksamkeitserwartung sowie auf die qualitativen Daten bestätigt dies. Abschließend gilt es zu bedenken, dass diese Daten keine Aussage darüber zulassen, wie stabil die Selbstwirksamkeitserwartung sind bzw. inwiefern sich weitere praktische Erfahrungen, die komplexere Anforderungen an die Studierenden stellen, auf sie auswirken.

\section{2) In welchen Bereichen nehmen die Studierenden eine Kompetenzentwicklung} durch das GEO-Lehr-Lern-Labor wahr und worauf führen sie diese zurück?

Aufgrund des laufenden Auswertungsprozesses können folgend nur erste Einblicke die Interviewergebnisse aus den ersten beiden Durchläufen $(n=16)$ gegeben werden. Hinsichtlich der Kompetenzentwicklung werden die Antworten auf die offene Frage „Was nimmst du aus dem Seminar mit?" dargestellt und diskutiert (siehe Tab. 1: Interviewabschnitt 2, Teilabschnitt 1). Zur Clusterung der Antworten wurden diese zunächst den einzelnen Bereichen der professionellen Kompetenz von Lehrkräften zugeordnet (Baumert \& Kunter, 2006). Bei der Beschreibung der 
eigenen Kompetenzentwicklung wurde in den Interviews auf die offene Impulsfrage hin von allen Probanden v. a. der empfundene Zuwachs in den Bereichen des fachdidaktischen und pädagogischen Wissens aufgeführt. Sieben Studierende betonten hierbei, dass sie gelernt hätten, wie sie Experimente praktisch im Unterricht einsetzen können. Im Bereich der motivationalen Orientierungen wurde von vier Interviewten explizit eine gesteigerte Motivation für einen Einsatz von Experimenten genannt. Sie berichteten von dem festen Vorsatz, diese in ihrem späteren Unterricht einbringen zu wollen. Acht Studierende gaben an, während des Seminars erkannt zu haben, dass für sie das Experimentieren eine lohnenswerte Methode für den Geographieunterricht darstelle. Die Hälfte von ihnen erwähnte in diesem Zusammenhang zugleich die Einsicht, dass mit dem Experimentieren ein hoher Aufwand verbunden sei, im Abwägungsprozess jedoch die positiven Aspekte überwögen. Alle befragten Studierenden empfanden die Praxisphase als bedeutend für ihre Kompetenzentwicklung und sprachen ihr einen positiven Einfluss zu. Begründet wurde dies u.a. mit einem verbesserten Theorieverständnis und damit einhergehend einem größeren Lerneffekt. Auch weitere Situationsbeschreibungen, die laut den Teilnehmenden zur Kompetenzentwicklung beitrugen, verweisen auf die Relevanz, etwas selbst durchgeführt und erprobt zu haben.

\footnotetext{
Beispielzitate:

„[...] ich weiß auf jeden Fall jetzt, wie man Experimente durchführt. Also mit dem experimentellen Algorithmus und welche Schritte man machen muss, um überhaupt ein Experiment zu machen, ich weiß jetzt, was eine abhängige und unabhängige Variable ist (lachend). Habe ich jetzt auch verstanden. Ja (...) ach ja wie man binnendifferenzieren kann mit den Hilfekarten zum Beispiel, welche Gütekriterien erfüllt werden müssen. Auf jeden Fall das Experiment mit den Schülern. Da ist mir da habe ich die Zusammenhänge erst richtig verstanden $[\ldots]$ “. (I:2_3)

„[...] dass man mehr Experimente im Geounterricht durchführen sollte und sich trauen sollte, sowas auch anzugehen. Also es ist ja auch immer mit sehr viel Aufwand und so verbunden, aber ich glaube, wie wir es ja auch im Seminar erlebt haben, eigentlich durch den Kontakt Student/Schüler nimmt man nochmal viel mit, auch für die spätere Praxis und das würde ich für mich für die Zukunft mitnehmen, dass man sich auch die Mühe macht, sowas zu planen und ich glaube, der Mehrwert für einen selbst ist da auch sehr groß [...]"(I:1_6)
}

Es ist davon auszugehen, dass die Wahrnehmung des Zuwachses von Wissen als Kompetenzfacette zur positiven Entwicklung der situationsspezifischen Selbstwirksamkeitserwartung beigetragen hat (siehe Abb. 4). Die erwähnte Einsicht, dass sich der Einsatz von Experimenten im Geographieunterricht lohne, zielt auf die motivationalen Orientierungen, steht aber nur bedingt im Zusammenhang mit der Selbstwirksamkeitserwartung. So spielt die wahrgenommene Attraktivität von Zielen neben der Selbstwirksamkeitserwartung eine wichtige Rolle, wenn es um die Wahl anspruchsvoller Aufgaben geht (Atkinson, 1957). Die erwähnte hohe Bedeutung der Praxisphase für die Kompetenzentwicklung steht im Einklang mit der 
Theorie und den bisherigen empirischen Befunden, dass eigene Erfolgserfahrungen eine starke Quelle der Selbstwirksamkeitserwartung darstellen (siehe Kap. 2.3).

3) Welche Verständnisprobleme/Schwierigkeiten haben Studierende im Seminar und inwiefern können diese überwunden werden?

Bei den in den Interviews $(\mathrm{n}=16)$ geschilderten Schwierigkeiten zeichnet sich ein heterogenes Bild ab. Auch die Ursachenzuschreibungen für diese sind unterschiedlich und geben Hinweise auf die kognitiven Prozesse, die die Selbstwirksamkeitserwartung beeinflussen. Zur Clusterung der Schwierigkeiten wurden diese den Bereichen des Professionswissens zugeordnet (Baumert \& Kunter, 2006). Probleme im Bereich des pädagogischen Wissens betrafen v. a. die Aufarbeitung der Theorie zur pädagogischen Diagnostik und diagnostische Handlungen (Beobachtung und Bewertung von Experimentierleistungen). Insgesamt sahen nur vier von $16 \mathrm{Stu}-$ dierenden hier keine Probleme. Erklärt wurden die Probleme u. a. dadurch, dass das Thema Diagnostik insgesamt als anspruchsvoll wahrgenommen wurde und wenige bis keine Vorkenntnisse auf dem Gebiet vorhanden waren. Im Bereich des fachdidaktischen Wissens berichteten neun Studierende von einzelnen schwierigen Situationen, die sich vor allem auf Lehrhandlungen in der Planungs- und Praxisphase beziehen. So fiel es einigen Studierenden schwer, Experimentieraufgaben zu gestalten oder während des Schülerbesuchs einzuschätzen, wann und wie Hilfestellungen gegeben werden sollten. Begründet wurde dies u.a. damit, dass auf keinerlei Erfahrungen zurückgegriffen werden konnte. Ein/e Studierende/r äußerte, sich nicht genug auf die Praxisphase vorbereitet gefühlt zu haben, während eine weitere Person von starker Nervosität während der Unterrichtsdurchführung berichtete. Beim Fachwissen gab es weniger Problemdarstellungen als in den anderen Bereichen. Wenn Probleme angeführt wurden, dann bezogen sie sich v.a. auf die eigenen experimentellen Fähigkeiten und auf den Umgang mit Begrifflichkeiten (Definitionen, Variablenunterscheidung). Zwei Studierende gaben an, keine Schwierigkeiten im Seminar bemerkt zu haben. 
Beispielzitate:

„[...] Also ich glaube, wenn dann gab es einzelne Teile bei der Diagnose, die ich schwierig fand. Also sowas wie die Gütekriterien oder Diagnoseinstrumente im Allgemeinen [...] Aber so ein bisschen diese Begrifflichkeiten alle neu. [...] Aber danach, als wir das einmal durchgesprochen haben und am Ende auch angewendet haben, da war es dann auf jeden Fall auch besser so. [... ]“ (I:1_2)

„[...] während dieses Schülerexperiments hat man, also habe ich mich da auch nicht komplett sicher gefühlt, also ich glaube ich habe da eher ein bisschen zu viel eingegriffen. [...] Also ja, wenn man dann diese Schwierigkeiten erkennt, wie, wie lässt man die Schüler dann trotzdem noch frei arbeiten und versucht irgendwie nur wenig Hilfe zu leisten so. Das wäre so eine Schwierigkeit noch. [...]“"(I:2_7)

„[...] Das war einer der prägenden Momente insofern, dass ich mir selbst nochmal bewusstwurde, dass mir experimentieren gar nicht liegt, auch nicht in der Praxis. Das Schlimme ist, wir wussten ja, was rauskommen muss, aber das ist einfach bei uns nicht herausgekommen. [...] Deshalb fing es da schon mal an, dass mir klar wurde, dass man über Experimentieren ein bisschen mehr Hintergrundwissen haben muss. Deshalb war das etwas ernüchternd für mich. [...]“(I:2_1)

Insgesamt spiegeln die Antworten die Heterogenität der Lernvoraussetzungen der Studierenden wider. Zudem ließen sich Unterschiede hinsichtlich des Umgangs mit den angeführten Problemen sowie deren potentiellen Überwindung feststellen. Es gilt zu beachten, dass die Lehrveranstaltung nur begrenzt dazu beitragen kann, Studierenden ein Gefühl der Handlungssicherheit zu geben. Die Äußerungen zum Gefühl der mangelnden Vorbereitung und der starken Nervosität weisen auf eine emotionale Erregung hin, die sich, je nach Attribuierung, negativ auf die Entwicklung der Selbstwirksamkeitserwartung auswirken hätte können (siehe Kap. 2.3). Die Erkenntnisse, die sich aus den Antworten dieser Forschungsfrage ergaben, konnten zum Teil direkt in Re-Design-Prozesse einfließen.

4) Welche komplexitätsreduzierenden Designelemente des GEO-Lehr-Lern-Labors erleben die Studierenden als förderlich bzw. hinderlich für ihre Kompetenzentwicklung?

Aus den Ergebnissen der Tabelle 2 wird zunächst deutlich, dass die Studierenden die einzelnen Elemente der Komplexitätsreduktion im Interview unterschiedlich stark thematisierten. Bei einem (rein quantitativen) Vergleich der positiven und negativen Bewertungen zeigt sich, dass die Komplexitätsreduktion insgesamt eher positiv bewertet wurde. Lediglich in den Bereichen, geringe Schülerzahl' und ,Zeitumfang' wurden reine Negativäußerungen von einzelnen Studierenden getätigt. 
Tabelle 2: Häufigkeiten der aus den Transkripten abgeleiteten Bewertung der Elemente der Komplexitätsreduktion $(\mathrm{n}=16)$

\begin{tabular}{l|c|c|c}
\multirow{2}{*}{ Element der Komplexitätsreduktion } & \multicolumn{3}{|c}{ Bewertung } \\
\cline { 2 - 4 } & Positiv & Teils, teils & Negativ \\
\hline Inhaltliche Fokussierung & 10 & 4 & - \\
\hline Unterstützung durch Kommilitonen & 7 & 1 & - \\
\hline Unterstützung durch Dozentin & 6 & 1 & - \\
\hline Geringe Schülerzahl & 5 & 4 & - \\
\hline Vertrautheit des Raums/Materials & 1 & - & 3
\end{tabular}

Vor allem die Unterstützung durch andere Studierende und die Dozentin wurden im Seminar als überwiegend hilfreich und unterstützend wahrgenommen. Die inhaltliche Fokussierung wurde ebenfalls überwiegend positiv bewertet. Negativ wurde hierzu angemerkt, dass eine zu starke Fokussierung durch Arbeitsteilung (z.B. auf nur eine Phase der Experimentierkompetenz) dazu führe, dass sich die Studierenden hinsichtlich der anderen (nicht behandelten) Inhalte ungenügend vorbereitet fühlen und ihnen die Beobachtung der Kommilitonen zum Erlangen von Sicherheit nicht ausreiche. Different bewertet wurde die reduzierte Schüleranzahl. So wird einerseits berichtet, dass es entlastend und hilfreich war, wenige Schüler zu unterrichten. Andererseits gab es Äußerungen, dass dadurch die Authentizität gegenüber der schulischen Situation nicht gegeben sei. Ein gedanklicher Transfer der Lehrhandlungen in die schulische Anforderungssituation gelänge daher nicht und somit könne kein Gefühl von Sicherheit erlangt werden.

Beispielzitate:

"[...] Ich würde sagen, dass das sehr gut war, grade WEIL man nicht so auf sich alleine gestell direkt ins kalte Wasser geworfen wird und „Ja, macht mal!", sondern weil in der Gruppe konnte man sich gut austauschen, finde ich, und auch das man sich erstmal nur auf einen Schüler erstmal konzentriert [...]“" (I:1_2)

„[...] hätten wir da jetzt dreißig Schüler gehabt, wäre ich überfordert gewesen. Ich habe das noch nie gemacht, deshalb wäre das zu viel gewesen für mich. Deswegen finde ich das gut, dass wir das mit wenig Schülern gemacht haben [...]“ (I:1_3)

„[...] aber ich hätte es glaube ich noch, schon, also reduziert aber nicht so stark, dass nicht mehr so dieses. Ich bin ja als Lehrer nicht verantwortlich für eine oder für zwei Personen, sondern für eine Klasse von 30 Leuten, zumindest für eine kleine Gruppe aber eine Gruppe verantwortlich zu sein, hätte ich glaube ich ein bisschen hilfreicher gefunden [...]" (I:2_7) 
Der unterschiedlich starken Thematisierung der Elemente kann eine implizite Relevanzangabe unterstellt werden. So scheint die Vertrautheit der Umgebung für die Studierenden nicht so bedeutsam wie die inhaltliche Fokussierung und die zu betreuende Schüleranzahl. Hinsichtlich der Quellen der Selbstwirksamkeitserwartung (siehe Kap. 2.3) könnte die als positiv wahrgenommene Unterstützung durch die Konsemester und die Dozentin neben einer reinen Arbeitserleichterung auch Möglichkeiten für verbale Überzeugungen (via Feedback) geschaffen und somit zur Entwicklung der Selbstwirksamkeitserwartung beigetragen haben. Das Modelllernen als eine mögliche Quelle der Selbstwirksamkeitserwartung fand sich nur in einer Äußerung konkret wieder, in der expliziert wird, dass die stellvertretende Erfahrung durch die Beobachtung der Konsemester eben gerade nicht ausgereicht habe. Bezüglich der Anzahl der zu betreuenden Schülerinnen und Schüler scheint sich ein Spannungsfeld zwischen Komplexitätsreduktion und Authentizität zu eröffnen, welches für die Entwicklung der situationsspezifischen Selbstwirksamkeitserwartung von Bedeutung sein dürfte.

\section{Fazit \& Ausblick}

Das GEO-Lehr-Lern-Labor hat sich bislang als Veranstaltungsformat am Institut für Didaktik der Geographie in Münster bewährt und konnte aufgrund der Evaluationen mit Studierenden iterativ im Sinne des Design-Based-Research weiterentwickelt werden. Ein Blick in die Ergebnisse der ersten drei Seminardurchläufe zeigt eine überwiegend positive Entwicklung der situationsspezifischen Selbstwirksamkeitserwartung während der Veranstaltungen. Die Analyse der qualitativen Daten zeigt, dass im Seminar wahrgenommene Kompetenzentwicklungen und Schwierigkeiten heterogen ausfallen. Insgesamt wurde der Praxisphase durchgängig eine hohe Bedeutung zugemessen, während einzelne Elemente der Komplexitätsreduktion different bewertet wurden. Die Datenerhebungsphase wurde mit dem Ende des Wintersemesters 2018/19 abgeschlossen. In einem angelegten Dissertationsvorhaben wird die Begleitforschung weitergehend bearbeitet und vertieft. Auf der strukturellen Ebene wird eine dauerhafte Implementierung des Lehrveranstaltungsformats angestrebt. Angedacht ist des Weiteren eine Erweiterung des Angebots für Schulen sowie der Einsatz von Videografie, um verstärkte Einblicke in die Lernprozesse sowohl bei den Studierenden als auch bei Schülerinnen und Schülern zu erhalten. Für die weitere Erforschung des GEO-Lehr-Lern-Labors sollte die Entwicklung anderer Kompetenzdimensionen (v.a. das Professionswissen) in den Fokus genommen werden, um weitergehende Aussagen über die (fachspezifische) Wirkung des Formats tätigen zu können. 


\section{Literatur}

Atkinson, J. W. (1957). Motivational determinants of risk-taking behavior. Psychological Review (359-372).

Bandura, A. (1997). Self-efficacy: The exercise of control. New York: Freeman.

Baumert, J. \& Kunter, M. (2006). Stichwort: Professionelle Kompetenz von Lehrkräften. Zeitschrift Für Erziehungswissenschaft, 4, 469-520. https://doi.org/10.1007/s11618-oo60165-2

Bühner, M. (2011). Einführung in die Test- und Fragebogenkonstruktion. München: Pearson Studium.

Buholzer, A., Joller-Graf, K., Kummer Wyss, A. \& Zobrist, B. (2014). Kompetenzprofil zum Umgang mit heterogenen Lerngruppen. Wien, Zürich, Berlin: LIT.

Cousins, J. B. \& Walker, C. A. (2000). Predictors of Educators' Valuing of Systematic Inquiry in Schools. Canadian Journal of Program Evaluation, 15, 25-52.

Deutsche Gesellschaft für Geographie (2017). Bildungsstandards im Fach Geographie für den Mittleren Schulabschluss: Mit Aufgabenbeispielen. Bonn.

Dohrmann, R. \& Nordmeier, V. (2015). Schülerlabore als Lehr-Lern-Labore (LLL): Ein Projekt zur forschungsorientierten Verknüpfung von Theorie und Praxis in der MINT-Lehrerbildung: Förderung von Professionswissen, professioneller Unterrichtswahrnehmung und Reflexionskompetenz im LLL Physik. PhyDid B - Didaktik Der Physik - Beiträge Zur DPG-Frühjahrstagung 2015, 1-7.

Dohrmann, R. \& Nordmeier, V. (2018). Praxisbezug und Professionalisierung im Lehr-Lern-Labor-Seminar (LLLS) - ausgewählte vorläufige Ergebnisse zur professionsbezogenen Wirksamkeit. In C. Maurer (Hrsg.), GDCP: Vol. 38. Qualitätsvoller Chemie- und Physikunterricht - normative und empirische Dimensionen: Gesellschaft für Didaktik der Chemie und Physik, Jahrestagung in Regensburg 2017 (S. 515-518). Regensburg: Universität Regensburg.

Feulner, B., Ohl, U. \& Hörmann, I. (2015). Design-Based Research - ein Ansatz empirischer Forschung und seine Potenziale für die Geographiedidaktik. Zeitschrift Für Geographiedidaktik (ZGD), 205-231.

Fischer, A., Niesel, V. \& Sjuts, J. (Hrsg.) (2011). Lehr-Lern-Labore und ihre Bedeutung für Schule und Lehrerbildung: Bestandsaufnahme im Verbundprojekt OLAW. Tagungsband; Fachtagung vom 31. März 2011 an der Carl-von-Ossietzky-Universität Oldenburg. Oldenburg: BIS-Verlag der Carl-von-Ossietzky-Universität Oldenburg.

Gibbs, G. (1988). Learning by doing: A guide to teaching and learning methods. London: FEU.

Hammann, M. (2004). Kompetenzentwicklungsmodelle: Merkmale und ihre Bedeutung - dargestellt anhand von Kompetenzen beim Experimentieren. MNU, 196-203.

Hemmer, I. \& Hemmer, M. (Hrsg.) (2010). Schülerinteresse an Themen, Regionen und Arbeitsweisen des Geographieunterrichts: Ergebnisse der empirischen Forschung und deren Konsequenzen für die Unterrichtspraxis (Geographiedidaktische Forschungen: Bd. 46). Weingarten: HGD.

Hemmer, M. \& Miener, K. (2013). Schülerexkursionen konzipieren und durchführen lernen: Förderung exkursionsdidaktischer Kompetenzen in der Geographielehrerausbildung an der Universität Münster. In K. Neeb, U. Ohl \& J. Schockenmöhle (Hrsg.), Hochschullehre in der Geographiedidaktik: Wie kann die Ausbildung zukünftiger Leh- 
rerinnen und Lehrer optimiert werden? (Gießener geographische Manuskripte: Bd. 7, S. 130-137). Gießen: Shaker.

Hilzensauer, W. (2008). Theoretische Zugänge und Methoden zur Reflexion des Lernens. Ein Diskussionsbeitrag. Bildungsforschung, 5 (2), 1-18.

Höhnle, S. \& Schubert, J. C. (2016). Hindernisse für den Einsatz naturwissenschaftlicher Arbeitsweisen im Geographieunterricht aus Studierendenperspektive - Ausgewählte Ergebnisse einer empirischen Studie mit Lehramtsstudierenden. GW-Unterricht, 142/143, 153-161. https://doi.org/10.1553/gw-unterricht142/143s153

Hoy, A. W. \& Spero, R. B. (2005). Changes in teacher efficacy during the early years of teaching: A comparison of four measures. Teaching and Teacher Education, 21, 343-356. https://doi.org/10.1016/j.tate.2005.01.007

Kaminske, V. (2009). Experimentelles Arbeiten in der Geographie: Durchführbarkeit und Lerneffizienz. Geographie Und Schule, 31, 21-30.

Kocher, M. (2011). Selbstwirksamkeit und Unterrichtsqualität: Unterricht und Persönlichkeitsaspekte im Berufsübergang von Lehrpersonen (Dissertation). Zürich.

Krofta, H. \& Nordmeier, V. (2014). Bewirken Praxisseminare im Lehr-Lern-Labor Änderungen der Lehrerselbstwirksamkeitserwartung bei Studierenden? PhyDid B - Didaktik Der Physik - Beiträge Zur DPG-Frühjahrstagung 2014, 1-12.

Krüger, M. (2010). Das Lernszenario VideoLern: Selbstgesteuertes und kooperatives Lernen mit Vorlesungsaufzeichnungen: Eine Design-Based-Research Studie. Universität der Bundeswehr München, München.

Lindau, A.-K. (2018). Selbstwahrnehmungen von Aspekten der professionellen Handlungskompetenz sowie Überzeugungen zu geographischen Exkursionen bei Lehramtsstudierenden. In M. Hemmer, A.-K. Lindau, C. Peter \& G. Schrüfer (Hrsg.), "Auf den/die Geographielehrer/in kommt es an!?" - Lehrer/innenprofessionalität und Lehrer/innenbildung im Fokus von Theorie, Empirie und Praxis. Abstractband zum HGD-Symposium 2018 (S. 21-22). Münster: Westfälische Wilhelms-Universität Münster.

Mayer, J., Grube, C. \& Möller, A. (2008). Kompetenzmodell naturwissenschaftlicher Erkenntnisgewinnung. In U. Harms \& A. Sandmann (Hrsg.), Forschungen zur Fachdidaktik: Band 10. Ausbildung und Professionalisierung von Lehrkräften: Internationale Tagung der Fachsektion Didaktik der Biologie im VBiO, Essen 2007 (S. 63-79). Innsbruck [u.a.]: Studien Verlag.

Mayring, P. (2015). Qualitative Inhaltsanalyse: Grundlagen und Techniken (Beltz Pädagogik, 12., überarb. Aufl.). Weinheim: Beltz. Retrieved from http://content-select.com/ index.php?id=bib_view\&ean=9783407293930

Meinhardt, C., Rabe, T. \& Krey, O. (2016). Selbstwirksamkeitserwartungen in physikdidaktischen Handlungsfeldern. Skalendokumentation. Frankfurt.

Mönter, L., Lütje, S. \& Schlitt, M. (2016). „Biogas“ im geographischen Lehr-Lernlabor. In K.-H. Otto (Hrsg.), Geographie und naturwissenschaftliche Bildung - der Beitrag des Faches für Schule, Lernlabor und Hochschule: Dokumentation des 21. HGD-Symposiums im März 2015 in Bochum (Geographiedidaktische Forschungen: Bd. 63). Münster: Verlagshaus Monsenstein und Vannerdat OHG.

Mönter, L. \& Otto, K.-H. (2017). Experimentelles Arbeiten im Geographieunterricht: Grundlagen, Erkenntnisse und Konsequenzen. In L. Mönter, K.-H. Otto \& C. Peter (Hrsg.), Diercke. Experimentelles Arbeiten: Beobachten, untersuchen, experimentieren (S. 5-9). Braunschweig: Westermann. 
Ohl, U. \& Mehren, M. (2016). Diagnose - Grundlage gezielter Förderung im Geographieunterricht. Geographie aktuell und Schule, 38, 4-13.

Otto, K.-H. (2003). Experimentieren im Geographieunterricht. Geographie Heute, 24, $2-7$.

Otto, K.-H. (2009). Experimentieren als Arbeitsweise im Geographieunterricht. Geographie Und Schule, 31, 4-15.

Otto, K.-H., Mönter, L., Hof, S. \& Wirth, J. (2010). Das geographische Experiment im Kontext empirischer Lehr-/Lernforschung. Zeitschrift für Geographiedidaktik, 38, 133-145.

Peter, C. (2014). Erkenntnisgewinnung in der geographiedidaktischen Forschung: Konzeption und Ergebnisse einer Studie zur Experimentierkompetenz. In S. Haffer \& C. Peter (Hrsg.), Herausforderungen in der Geographiedidaktik: Neue Medien, Kompetenzen, Leitbilder, Realbegegnungen (Gießener geographische Manuskripte: Bd. 8, S. 55-69). Aachen: Shaker.

Reinmann, G. (2019). Die Selbstbezüglichkeit der hochschuldidaktischen Forschung und ihre Folgen für die Möglichkeiten des Erkennens. In T. Jenert, G. Reinmann \& T. Schmohl (Hrsg.), Hochschulbildungsforschung: Theoretische, methodologische und methodische Denkanstöße für die Hochschuldidaktik (S. 125-148). Wiesbaden: Springer VS. https://doi.org/10.1007/978-3-658-20309-2_8

Renner, T. (2018). Geographische Exkursionen: So kompetent fühlen sich Lehramtsstudierende. In M. Hemmer, A.-K. Lindau, C. Peter \& G. Schrüfer (Hrsg.), „Auf den/ die Geographielehrer/ in kommt es an!?" - Lehrer/innenprofessionalität und Lehrer/ innenbildung im Fokus von Theorie, Empirie und Praxis. Abstractband zum HGDSymposium 2018. (S. 23-24).

Schiepe-Tiska, A., Rönnebeck, S., Schöps, K. \& Neumann, K. (2016). Naturwissenschaftliche Kompetenz in PISA 2015 - Ergebnisse des internationalen Vergleichs mit einem modifizierten Testansatz. In K. Reiss, C. Sälzer, A. Schiepe-Tiska, E. Klieme \& O. Köller (Hrsg.), PISA 2015: Eine Studie zwischen Kontinuität und Innovation (S. 45-98). Münster, New York: Waxmann.

Schubert, J. C. (2016). Kognitiv aktivierend und eigenständig experimentieren: Schülerinnen und Schüler erforschen das Wasserhaltevermögen von Böden. Geographie Aktuell Und Schule, 38, 24-34.

Schubert, J. C. \& Kreuziger, T. (2018). Beliefs von (angehenden) Geographielehrpersonen zu naturwissenschaftlichen Arbeitsweisen. In M. Hemmer, A.-K. Lindau, C. Peter \& G. Schrüfer (Hrsg.), „Auf den/die Geographielehrer/ in kommt es an!?" - Lehrer/ innenprofessionalität und Lehrer/innenbildung im Fokus von Theorie, Empirie und Praxis.: Abstractband zum HGD-Symposium 2018. (S. 76).

Schüle, C., Besa, K.-S., Schriek, J. \& Arnold, K.-H. (2017). Die Veränderung der Lehrerselbstwirksamkeitsüberzeugung in Schulpraktika. Zeitschrift Für Bildungsforschung, 7, 23-40. https://doi.org/10.1007/s35834-016-0177-9

Schwarzer, R. \& Jerusalem, M. (2002). Das Konzept der Selbstwirksamkeit. Zeitschrift Für Pädagogik, 28-51.

Sekretariat der Ständigen Konferenz der Kultusminister der Länder in der Bundesrepublik Deutschland (2018). Ländergemeinsame inhaltliche Anforderungen für die Fachwissenschaften und Fachdidaktiken in der Lehrerbildung. Bonn. 
Skaalvik, E. M. \& Skaalvik, S. (2010). Teacher self-efficacy and teacher burnout. A study of relations. Teaching and Teacher Education, 26, 1059-1069. https://doi.org/10.1016/j. tate.2009.11.001

Tschannen-Moran, M. \& Hoy, A. W. (2007). The differential antecedents of self-efficacy beliefs of novice and experienced teachers. Teaching and Teacher Education, 23, 944-956. https://doi.org/10.1016/j.tate.2006.05.003

Tschannen-Moran, M., Hoy, A. W. \& Hoy, W. K. (1998). Teacher Efficacy: Its Meaning and Measure. Review of Educational Research, 68, 202-248. https://doi.org/10.3102/ 00346543068002202

Völker, M. \& Trefzger, T. (2011). Ergebnisse einer explorativen empirischen Untersuchung zum Lehr-Lern-Labor im Lehramtsstudium. PhyDid B - Didaktik Der Physik - Beiträge Zur DPG-Frühjahrstagung 2011 (1-9).

Warner, L. M. \& Schwarzer, R. (2009). Selbstwirksamkeit bei Lehrkräften. In O. ZlatkinTroitschanskaia (Hrsg.), Lehrprofessionalität: Bedingungen, Genese, Wirkungen und ihre Messung. Weinheim: Beltz.

Wilhelmi, V. (2012). Die experimentelle Lernform: Herausforderung des kompetenzorientierten Geographieunterrichts. Praxis Geographie (4-8). 



\title{
Komplexitätsreduktionen im Bereich Medien und Materialien
}

\author{
Entwicklung förderdiagnostischer Kompetenz im \\ mathematikdidaktischen Lehr-Labor MiRA ${ }^{+}$
}

Modellierungsaufgaben eignen sich durch ihre selbstdifferenzierenden Eigenschaften für einen produktiven Umgang mit Heterogenität. Dabei müssen zukünftige Lehrkräfte über verschiedene kognitive Fähigkeiten und Fertigkeiten verfügen, um mathematisches Modellieren adäquat zu unterrichten. Im Kontext des Lehr-Labors MiRR $A^{+}$ wird in der gymnasialen Lehrerbildung die Entwicklung professioneller Kompetenz zum Lehren mathematischen Modellierens bei angehenden Lehrkräften in komplexitätsreduzierten Lehr-Lern-Arrangements fokussiert. So wird in der vorliegenden Studie untersucht, wie sich Komplexitätsreduktionen im Bereich Medien und Materialien auf die Entwicklung bereichsspezifischer professioneller Kompetenz auswirken. Dazu wurde das Lehr-Labor MiRA $A^{+}$im Vergleich zu einem anderen Lehr-LernLabor zum mathematischen Modellieren mit Hilfe eines quasi-experimentellen PräPost-Designs evaluiert.

Schlüsselwörter: förderdiagnostische Kompetenz, mathematisches Modellieren, selbstdifferenzierende Aufgaben

\section{Einleitung}

Während in klassischen Schülerlaboren die teilnehmenden Schülerinnen und Schüler die eindeutig auszumachende Zielgruppe darstellen, bieten Lehr-LernLabore im MINT-Bereich zugleich hervorragende Bedingungen für die Lehrerbildung (Dohrmann \& Nordmeier, 2015; Lengnink \& Roth, 2016). Dabei sollen „in einem iterativen Prozess [...] insbesondere die professionelle Unterrichtswahrnehmung, die Reflexionskompetenz, der Erwerb von Professionswissen und Handlungskompetenz" (Dohrmann \& Nordmeier, 2015, S. 2) gefördert werden. So werden im Rahmen der Qualitätsoffensive Lehrerbildung an der Westfälischen Wilhelms-Universität (WWU) Münster Lehr-Lern-Labore implementiert, weiterentwickelt und evaluiert, die Studierende durch reflektierte Praxiserfahrungen auf einen produktiven Umgang mit heterogenen Lerngruppen vorbereiten (Greefrath \& Hammann, in diesem Band). Aus Sicht der Mathematikdidaktik erlauben insbesondere Modellierungsaufgaben durch ihr großes Potenzial im Bereich natürlicher Differenzierung individuelle Zugänge sowie Bearbeitungsmöglichkeiten und bilden somit die Basis der in diesem Beitrag vorgestellten Seminarkonzepte (Maaß, 2004). Ungeachtet dessen stellt das mathematische Modellieren sowohl für 
Lernende als auch für (angehende) Lehrkräfte eine große Herausforderung dar (Blum, 2015), weshalb die genaue Betrachtung professioneller Kompetenzen im Bereich des mathematischen Modellierens notwendig erscheint (Borromeo Ferri \& Blum, 2018).

Neben Elementen wie dem theoriegeleiteten Erproben praktischen didaktischen Handelns (Völker \& Trefzger, 2011) oder der Analyse und Reflexion der Lehr-Lern-Prozesse aus den Praxisphasen (Krofta, Fandrich \& Nordmeier, 2012) stellt die Komplexitätsreduktion ein weiteres wesentliches Element von LehrLern-Laboren dar. Diese ist auf verschiedene Weisen realisierbar, sei es durch die Arbeit mit kleinen Lerngruppen, die Unterstützung durch Mitstudierende und Lehrende, die Lokalisation der Lerngelegenheit in vertrauter Umgebung, die Beschränkung der Beobachtungsaufgaben auf ausgewählte Aspekte etc. (Dohrmann \& Nordmeier, 2015; Marohn, Greefrath, Hammann, Hemmer, Kürten \& Windt, in diesem Band). Der Fokus dieses Beitrags liegt auf den Komplexitätsreduktionen im Bereich Medien und Materialien sowie auf den hieraus resultierenden Implikationen für die Entwicklung der - für den kompetenten Umgang mit Heterogenität bedeutsamen (Fischer, Rott, Veber, Fischer-Ontrup \& Gralla, 2014) - förderdiagnostischen Kompetenz. Dafür wird neben dem am Standort Münster entwickelten noch ein weiteres an der Qualitätsoffensive Lehrerbildung beteiligtes Lehr-LernLabor zum mathematischen Modellieren am Standort Koblenz der Universität Koblenz-Landau betrachtet, welches sich hinsichtlich einzelner Dimensionen der Komplexitätsreduktion unterscheidet und in der vorliegenden Studie somit als Vergleichsgruppe dient.

\section{Mathematikdidaktische Lehr-Lern-Labore zum Modellieren}

Das münstersche Lehr-Labor MiRA ${ }^{+}$ist im Master of Education, das Lehr-LernLabor aus Koblenz (Klock \& Siller, 2017) im Bachelor of Education für das Lehramt an Gymnasien verortet. Beide Lehrveranstaltungen bestehen aus einer theoriebasierten Vorbereitungsphase, einer Praxisphase sowie einer Reflexionsphase (vgl. Abb. 1). Den inhaltlichen Kern aller Phasen bilden Modellierungsprozesse auf der einen sowie die Sensibilisierung für und der potentialorientierte Umgang mit Heterogenität auf der anderen Seite. Aufgrund der Konzeption des Formats aus der Qualitätsoffensive Lehrerbildung heraus wurde am Standort Münster die Bezeichnung „Lehr-Labor“ verwendet. Selbstverständlich finden aber Lehr-LernProzesse von Studierenden sowie Lern-Prozesse von Schülerinnen und Schülern gleichermaßen statt.

Die Vorbereitungsphase beinhaltet neben den theoretischen Grundlagen des Modellierens (vgl. Kapitel 3) auch eine eigene Modellierung (vgl. Abb. 4) sowie die damit einhergehende Diskussion individueller Modellierungsrouten (Borromeo Ferri, 2011). Diese bilden den Übergang zur Heterogenität und zur individuellen Förderung, welche im Zuge eines produktiven Umgangs mit jener thematisiert 

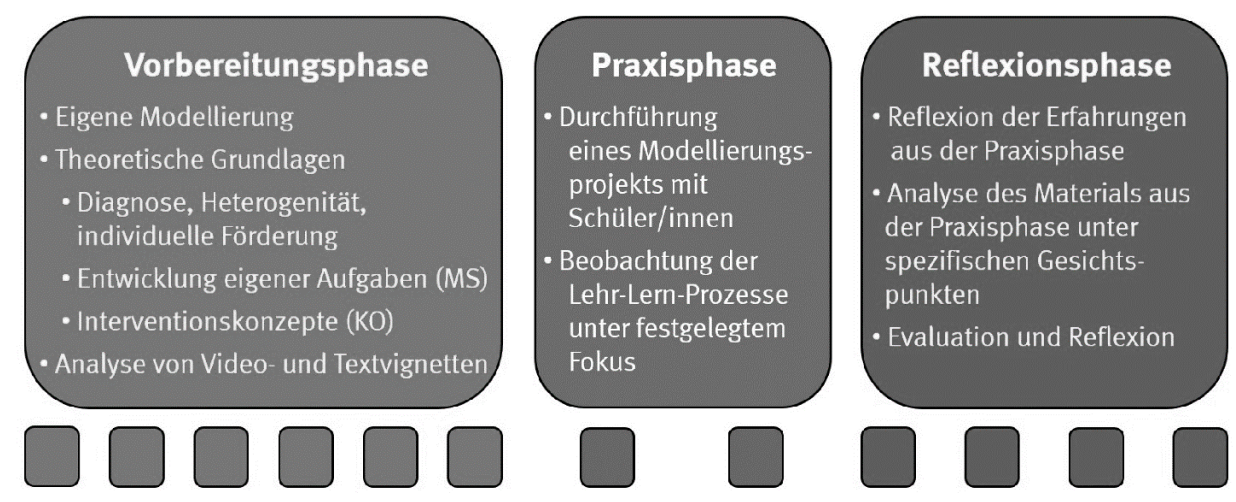

Abbildung 1: Konzeption der Seminare an den beteiligten Standorten.

wird. Hieran anschließend werden am Standort Münster Kriterien für geeignete Modellierungsaufgaben erstellt und solche von den Studierenden unter Einbezug verschiedener Feedbackzyklen für den Einsatz in der Praxisphase entwickelt, während am Standort Koblenz Interventionskonzepte im Kontext mathematischen Modellierens diskutiert werden. Abschließend werden Indikatoren zu festgelegten Teilprozessen des Modellierens formuliert, welche der Beobachtung und Diagnose von Lehr-Lern-Prozessen dienen. Insbesondere die Auseinandersetzung mit Modellierungsaufgaben weist standortübergreifend ein hohes inhaltliches Anforderungsniveau auf. Diesem Niveau wird sowohl im Lehr-Labor MiRA ${ }^{+}$als auch im Lehr-Lern-Labor am Standort Koblenz im Sinne des Gleichgewichtsprinzips (Marohn et al., in diesem Band) begegnet. Dabei ist die inhaltliche Unterstützung am Standort Koblenz durch den Einsatz etablierter Aufgaben maximal, während die strukturellen Unterstützungen im Bereich der Betreuung etwas geringer ausfallen. Dem gegenüber werden die Anforderungen am Standort Münster durch eine weniger starke inhaltliche Unterstützung im Bereich der Gestaltung, jedoch durch eine intensivere und vielschichtigere strukturelle Betreuung bei der Entwicklung der Aufgaben in ihrer Komplexität reduziert (vgl. Abb. 2). Dieser Unterschied gestaltet sich in der vorliegenden Studie als besonders relevant, da im Zuge der empirischen Untersuchung Ergebnisse zur Kompetenzentwicklung der Studierenden in Abhängigkeit differenter Zugänge zur Komplexitätsreduktion berichtet werden.

In der Praxisphase gestalten die Studierenden jeweils zwei Lehr-Lern-LaborTermine und wiederholen somit ihre didaktischen Handlungen mit der Möglichkeit, die Instruktionen zu variieren. Während dieser Projektsitzungen betreut je ein Team aus Studierenden eine Kleingruppe von Schülerinnen und Schülern bei der Bearbeitung der am Standort Münster selbsterstellten bzw. am Standort Koblenz aus der Literatur entnommenen Modellierungsaufgaben. Dabei liegt der Fokus der Beobachtungen auf den im Prozess gezeigten Teilkompetenzen mathematischen Modellierens, welche mit dem zuvor erstellten Beobachtungsbogen protokolliert werden. Diese Fokussierung auf einzelne Kompetenzaspekte während der Bearbeitung trägt insbesondere vor dem Hintergrund einer fachlichen und di- 


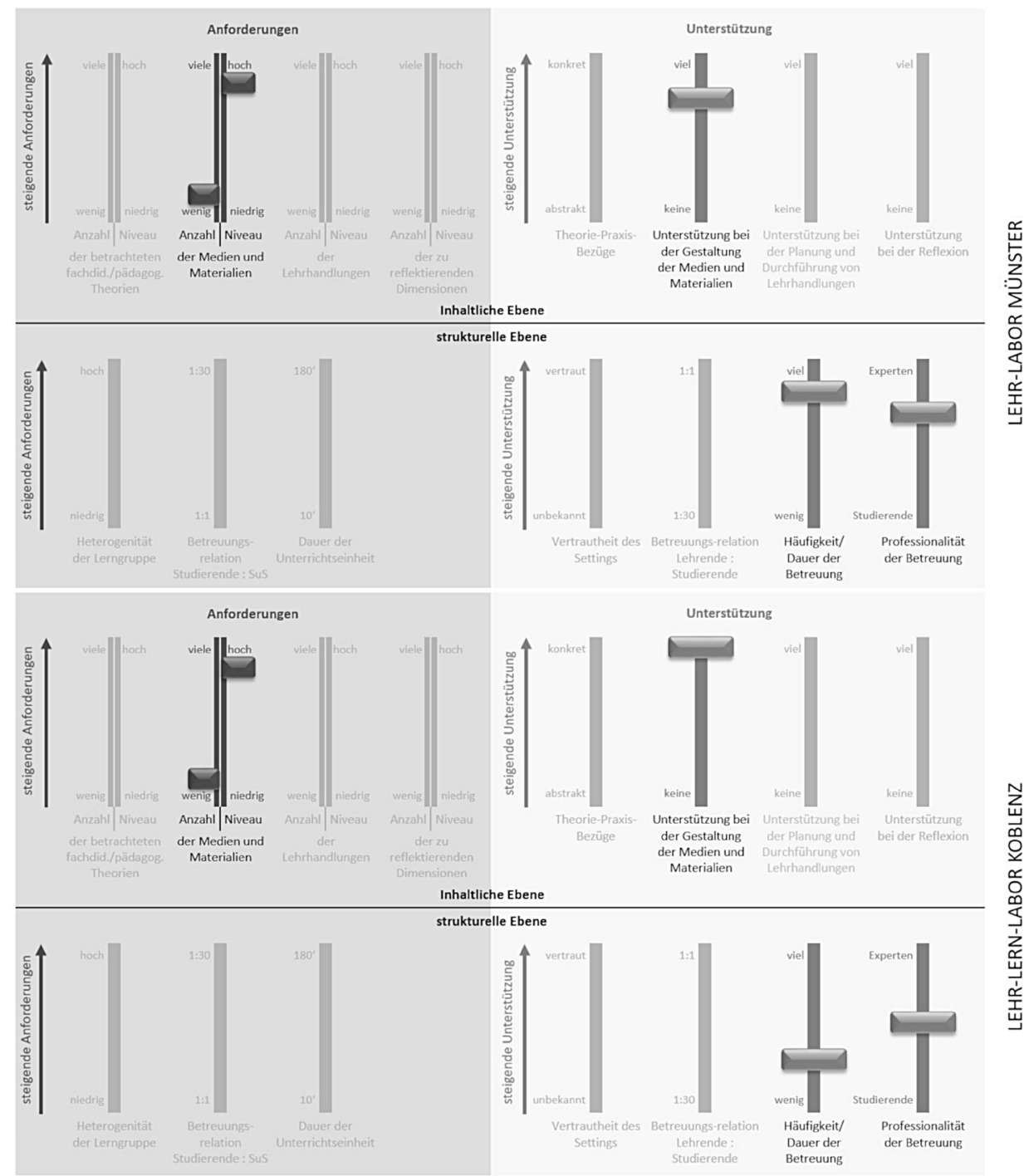

Abbildung 2: Komplexitätsreduktionen im Bereich Medien und Materialien der beteiligten Lehr-Lern-Labore. (Hervorgehobene Regler stellen unterschiedliche Ausprägungen der Unterstützung bei gleichen Anforderungen in den beteiligten Lehr-Lern-Laboren dar)

daktischen Durchdringung der Modellierungsaufgaben ebenso zur Komplexitätsreduktion in dieser Phase bei, wie die Wiederholung der Praxiserfahrung.

In der Reflexionsphase stehen die beobachteten Lehr-Lern-Prozesse ebenso im Mittelpunkt wie der Umgang mit Heterogenität und die Konsequenzen für die Konzeption eigener Modellierungsaufgaben bzw. die Auswahl geeigneter Interventionen im Modellierungsprozess. Hierfür finden aufgabenübergreifende theoriebasierte Gruppenreflexionen zu den jeweiligen Beobachtungsschwerpunkten statt, 
in welchen die Teilnehmenden ihre diagnostischen Einschätzungen anhand des kollegialen Feedbacks ihrer Kommilitoninnen und Kommilitonen vertiefen. Zum Abschluss werden die gewonnenen Erkenntnisse für die Professionalisierung der eigenen Lehrtätigkeit sowie zur Evaluation der am Standort Münster selbsterstellten Modellierungsaufgabe bzw. der am Standort Koblenz ausgewählten Interventionen genutzt und in einem Reflexionsbericht gesammelt. Diese vielschichtigen Zugänge und Unterstützungsmaßnahmen in der Reflexionsphase reduzieren die Komplexität und fördern zudem die Praxisreflexion der Studierenden.

Zusammenfassend verfolgt die dargestellte Konzeption der mathematikdidaktischen Seminare mit Praxiseinbindung insbesondere das Ziel der Professionalisierung angehender Lehrkräfte im Bereich des Lehrens mathematischen Modellierens. So wird das erworbene didaktische und fachliche Wissen bei der Erstellung von Aufgaben sowie bei der Arbeit mit Schülerinnen und Schülern miteinander verzahnt und umgesetzt. Die Komplexitätsreduktionen im Bereich Medien und Materialien unterstützen diese Theorie-Praxis-Bezüge und fördern somit maßgeblich den Professionalisierungsprozess angehender Lehrkräfte.

\section{Theoretische Hintergründe zum mathematischen Modellieren}

\subsection{Mathematisches Modellieren}

Mathematisches Modellieren bildet einen zentralen fachdidaktischen Fokus der Lehr-Lern-Prozesse im Lehr-Labor MiRA ${ }^{+}$. Blum (1985) sieht den gesamten Modellierungsprozess und die damit verbundene, für mathematische Anwendungen charakteristische Unterscheidung zwischen Mathematik und Realität (bzw. dem „Rest der Welt“ (Pollak, 1979)) als grundlegend für die Auffassung des Modellierens an. So liefern reale Kontexte eine Fülle an Problemstellungen, welche beim Modellieren individuell - durch geeignete Übersetzung in die Mathematik - gelöst sowie schließlich wieder auf den Kontext bezogen werden können. Demnach wird in diesem Beitrag unter mathematischem Modellieren der „Prozess des Lösens von [authentischen] Problemen aus der Realität" (Greefrath, Kaiser, Blum \& Borromeo Ferri, 2013, S. 11) verstanden. Dabei stellt mathematisches Modellieren ein sowohl nationales als auch internationales fachdidaktisches Forschungsfeld dar, welches besondere Beachtung erhält (Borromeo Ferri, Greefrath \& Kaiser, 2013). So hielt das Modellieren als allgemeine Kompetenz nicht nur Einzug in die deutschen Bildungsstandards und somit auch in die Lehrpläne für den Mathematikunterricht der Bundesländer, sondern findet sich auch in den Curricula anderer Nationen (z.B. Niss, 2003). In den folgenden Unterkapiteln werden ausgewählte theoretische und didaktische Hintergründe mathematischen Modellierens betrachtet, die für die weiteren Ausführungen grundlegend sind. 


\subsection{Modellierungskreisläufe}

Betrachtet man den gesamten Prozess des mathematischen Modellierens, lässt sich dieser in unterschiedlicher Form als idealisierter Kreislauf darstellen und bildet somit selbst wieder ein Modell des Modellierungsprozesses. Diese Modelle werden zielgerichtet erstellt und unterscheiden sich in ihren Facetten demnach bewusst voneinander. Der in Abbildung 1 dargestellte 7-schrittige Kreislauf nach Blum (2010) eignet sich beispielweise durch seine kognitive Perspektive besonders als Diagnoseinstrument für (angehende) Lehrerinnen und Lehrer.

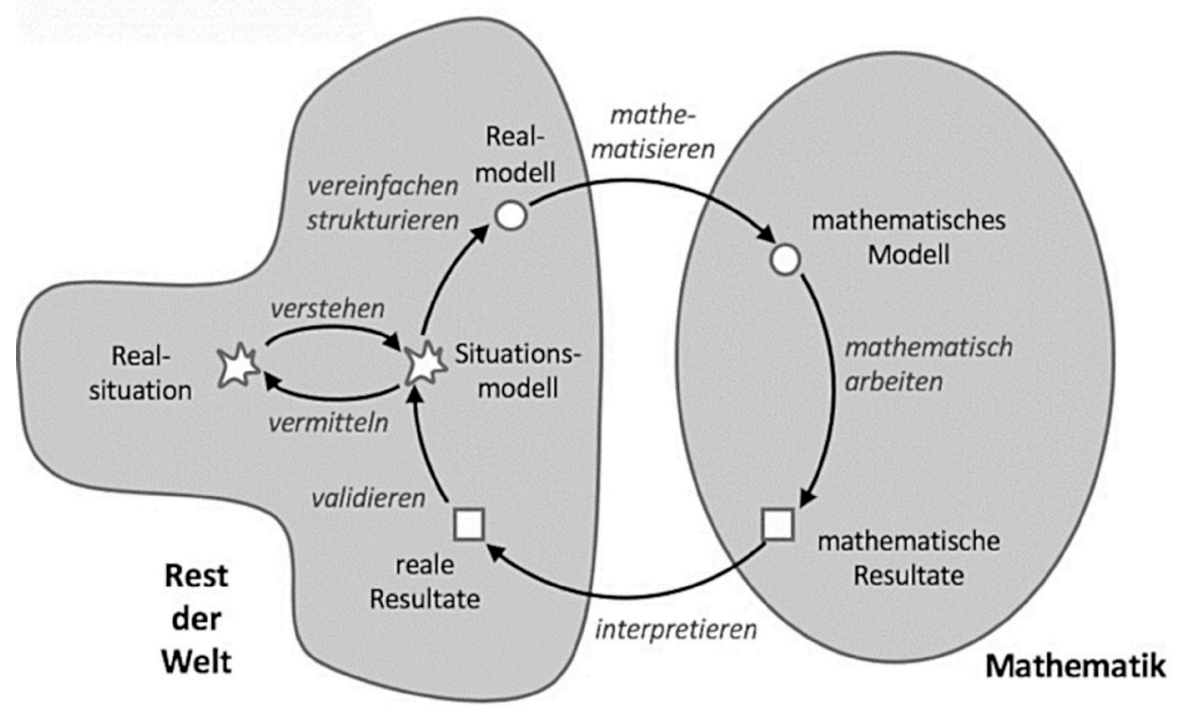

Abbildung 3: Modellierungskreislauf nach Blum (2010, S. 42).

Durch ihre umfangreiche theoretische Fundierung stellen Modellierungskreisläufe einen eigenen Lerninhalt in Schule und Hochschule dar und werden darüber hinaus als Grundlage für empirische Untersuchungen genutzt. Im Folgenden wird der obige Kreislauf am Beispiel der nachstehenden Modellierungsaufgabe (vgl. Abb. 3) idealisiert beschrieben.

Der Heißluftballon bildet den Ausgangspunkt für den Modellierungsprozess und entstammt der Realität bzw. dem sogenannten „Rest der Welt“ (Pollak, 1979). Weiter beinhaltet die obige Aufgabe eine authentische Fragestellung, die mit mathematischen Hilfsmitteln bearbeitet wird. Diese Situation wird nun unter Einbezug des Vorwissens und der Interessen der Modellierenden in ein Situationsmodell überführt. Durch Vereinfachungen und das Treffen von Annahmen, wie z. B. der Annahme einer „glatten“ Ballonoberfläche oder der Schätzung der Ballonkorbhöhe auf 1,40 m, wird aus dem kognitiven Situationsmodell ein vereinfachtes reales Modell bzw. eine Spezifikation des Problems gebildet (Siller, 2008). Die getroffenen 


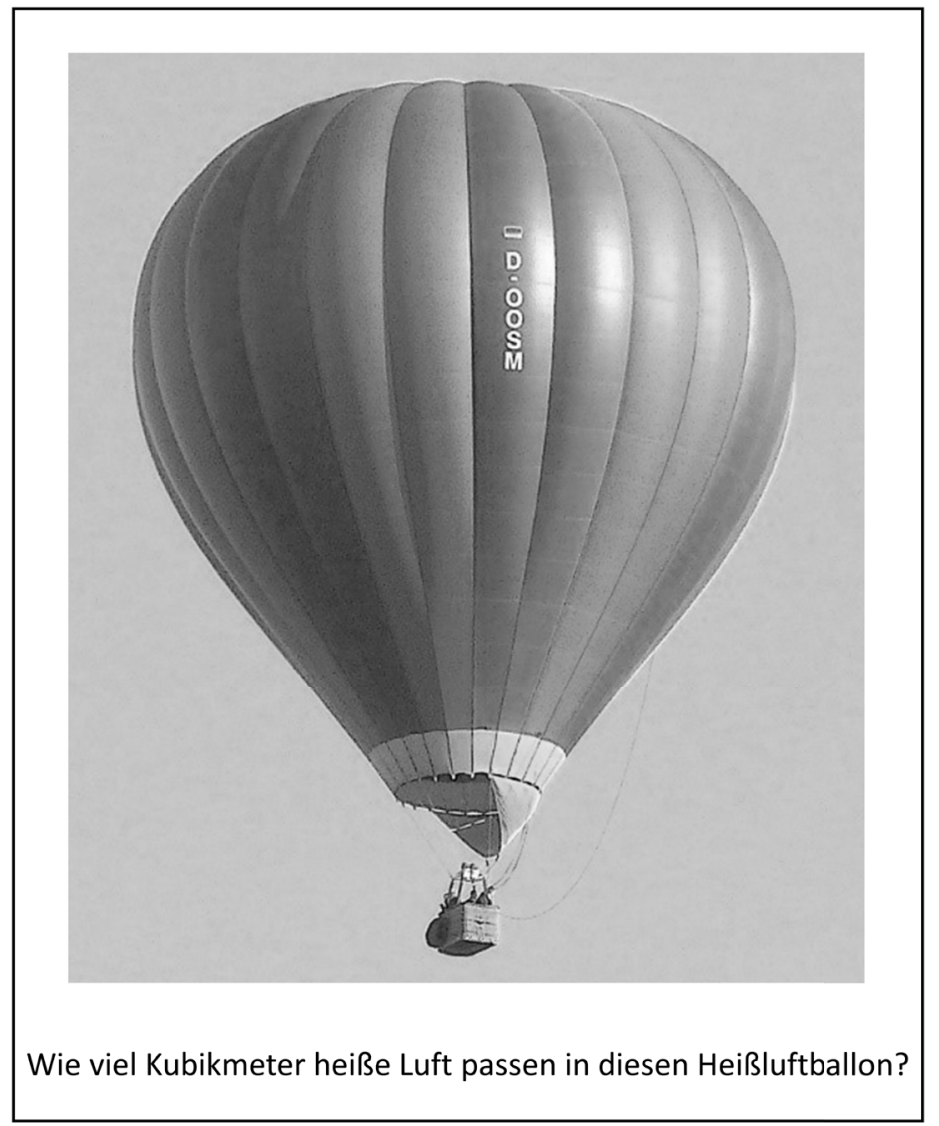

Abbildung 4: Modellierungsaufgabe - Heißluftballon nach Herget (200o).

Annahmen und zentralen Zusammenhänge werden durch einen Mathematisierungsprozess aus dem „Rest der Welt“ in die Mathematik übersetzt, was zu einem mathematischen Modell führt (vgl. Abb. 5). Hier lassen sich auf Basis des gewählten Maßstabes die Abmessungen - in diesem Fall eines Kegelstumpfes sowie einer Kugelschale - ableiten und ein Ergebnis für das Volumen berechnen. Natürlich sind auch andere Modelle auf unterschiedlichen Niveaustufen wie beispielsweise eine Zusammensetzung aus Kegel und Kugel oder das Rotationsvolumen einer Funktion dritten Grades denkbar. Das derart ermittelte mathematische Resultat wird in Bezug auf den realen Kontext als Volumen des betrachteten Heißluftballons interpretiert. Anschließend wird der gesamte Prozess validiert, z. B. ergibt eine Recherche, dass Größen von 2.000 bis 10.000 Kubikmeter für Heißluftballone gängig sind, weshalb 5.00o Kubikmeter Volumen ein plausibles Ergebnis für den abgebildeten Heißluftballon darstellt, und somit auch das Modell hinsichtlich der resultierenden Lösung als geeignet erscheint. Im letzten Schritt wird die Lösung des Problems dargelegt und gegebenenfalls an andere weitergegeben (Blum, 2007). 


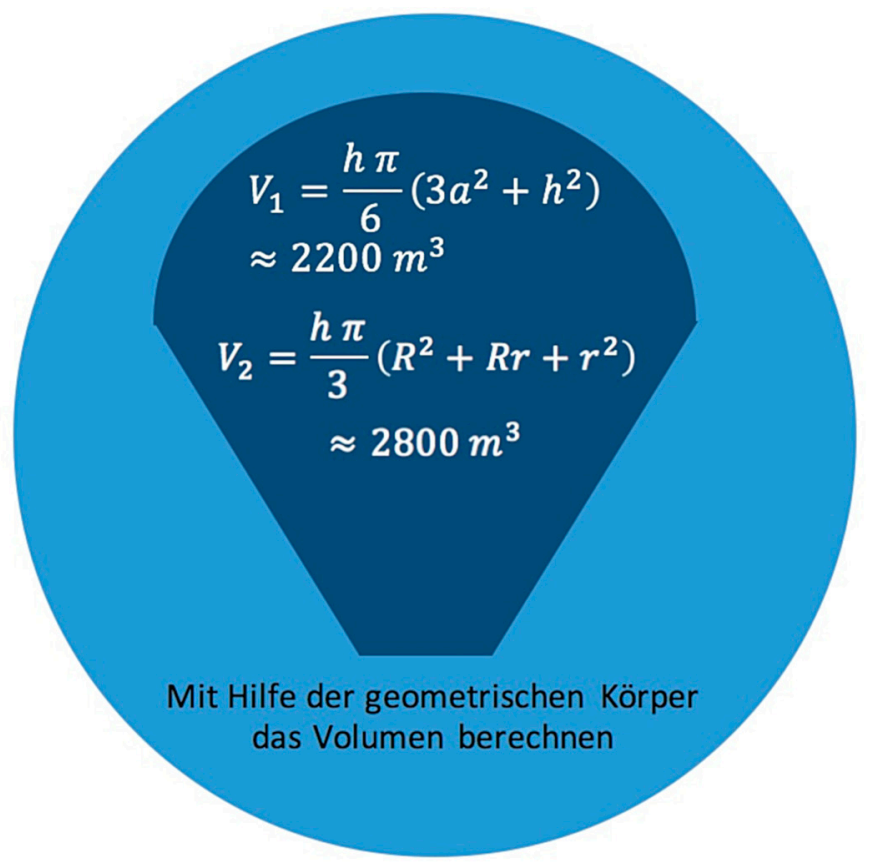

Abbildung 5: Mathematisieren und mathematisches Arbeiten.

Andere Idealisierungen des Modellierungsprozesses sind ebenfalls möglich. Zum Beispiel könnte die Datenbeschaffung separat betrachtet oder es könnte auf Zwischenschritte bei der Konstruktion des mathematischen Modells verzichtet werden. Daher gibt es viele weitere Kreislaufdarstellungen von Modellierungsprozessen.

\subsubsection{Modellieren als Kompetenz}

Mathematisches Modellieren wird als eine allgemeine mathematische Kompetenz in den Bildungsstandards aller Schulstufen beschrieben (z. B. Kultusministerkonferenz [KMK], 2003). Blum (2015) sieht dabei Modellierungskompetenz als die Fähigkeit, mathematische Modelle zu konstruieren, zu nutzen oder anzupassen, indem die Prozessschritte der einzelnen Modellierungsphasen adäquat und problemangemessen ausgeführt werden. Die Fähigkeit, eben solche Teilprozesse auszuführen, kann als eine Teilkompetenz des Modellierens angesehen werden (Kaiser, 2007). Diese Teilkompetenzen kann man, in Anlehnung an den 7-Schrittigen Modellierungskreislauf, aus Abb. 3, wie in Tab. 1 charakterisieren und die zugehörigen Modellierungsphasen auf Basis dieser Indikationen diagnostizieren. 
Tabelle 1: Teilkompetenzen des Modellierens (z. B. Greefrath et al., 2013; Maaß, 2004).

\begin{tabular}{|c|c|}
\hline Teilkompetenz & Indikator \\
\hline Verstehen & $\begin{array}{l}\text { Die Schülerinnen und Schüler konstruieren ein eigenes mentales Modell zu } \\
\text { einer gegebenen Problemsituation und verstehen so die Fragestellung. }\end{array}$ \\
\hline Vereinfachen & $\begin{array}{l}\text { Die Schülerinnen und Schüler trennen wichtige und unwichtige Informationen } \\
\text { einer Realsituation. }\end{array}$ \\
\hline Mathematisieren & $\begin{array}{l}\text { Die Schülerinnen und Schüler übersetzen geeignet vereinfachte Realsituationen } \\
\text { in mathematische Modelle (z. B. Term, Gleichung, Figur, Diagramm, Funktion). }\end{array}$ \\
\hline $\begin{array}{l}\text { Mathematisch } \\
\text { Arbeiten }\end{array}$ & $\begin{array}{l}\text { Die Schülerinnen und Schüler arbeiten mit dem mathematischen Modell und } \\
\text { nutzen dabei mathematische Regeln und Verfahren. }\end{array}$ \\
\hline Interpretieren & $\begin{array}{l}\text { Die Schülerinnen und Schüler beziehen die im Modell gewonnenen Resultate } \\
\text { auf die Realsituation und erzielen damit reale Resultate. }\end{array}$ \\
\hline Validieren & $\begin{array}{l}\text { Die Schülerinnen und Schüler überprüfen die realen Resultate im Situationsmo- } \\
\text { dell auf Angemessenheit. }\end{array}$ \\
\hline Vermitteln & $\begin{array}{l}\text { Die Schülerinnen und Schüler beziehen die im Situationsmodell gefundenen } \\
\text { Antworten auf die Realsituation und beantworten so die Fragestellung. }\end{array}$ \\
\hline
\end{tabular}

Die Orientierung an anderen Modellen des Modellierens kann entsprechend auch $\mathrm{zu}$ anders akzentuierten Teilkompetenzen führen.

\subsubsection{Modellierungsaufgaben}

Aufgaben sind im Mathematikunterricht allgegenwärtig. „Sie konkretisieren Lernsituationen und Leistungsanforderungen und rahmen so das Denken und Handeln von Lehrenden und Lernenden" (Leuders, 2015, S. 435). Konkret lassen sich Modellierungsprozesse im Mathematikunterricht also durch geeignete Aufgabenstellungen anregen. Dabei findet sich eine Fülle von Kategorien zur Analyse und Entwicklung von Modellierungsaufgaben. So berücksichtigt Maaß (2010) in ihrem Klassifikationsschema neben der Art der Beziehung zur Realität, auch die Offenheit und den Fokus auf die Modellierungsaktivität als Kriterien für Modellierungsaufgaben. Dabei unterscheiden wir bezüglich der Beziehung zur Realität die Authentizität und die Relevanz des Kontextes genauer und nehmen mit Blick auf den Fokus der Modellierungsaktivität die Teilkompetenzen des Modellierens in den Blick. Auf Basis dieser zentralen Eigenschaften von Modellierungsaufgaben (z.B. Bruder, 1988; Greefrath, Siller \& Ludwig, 2017; Greefrath \& Vorhölter, 2016; Maaß, 2004) haben wir den nachstehenden Kriterienkatalog zur Entwicklung und Evaluation von Modellierungsaufgaben zusammengestellt. 
Tabelle 2: Kriterienkatalog zur Analyse und Entwicklung von Modellierungsaufgaben.

\begin{tabular}{ll}
\hline Kriterium & Konkretisierung \\
\hline Realitätsbezug & $\begin{array}{l}\text { Die Problemstellung weist einen außermathematischen Sachbezug auf. } \\
\text { Delevanz }\end{array}$ \\
$\begin{array}{l}\text { Die Problemstellung wird von Schülerinnen und Schülern als interessant, } \\
\text { eng verbunden mit oder relevant für ihr tägliches Leben betrachtet. } \\
\text { Die Problemstellung ist authentisch mit Blick auf den außermathematischen } \\
\text { Sachbezug. } \\
\text { Die Problemstellung ist authentisch mit Blick auf die Verwendung von Ma- } \\
\text { thematik in der konkreten Situation. } \\
\text { Die Problemstellung ermöglicht verschiedene Lösungen. } \\
\text { Die Problemstellung ermöglicht Lösungswege auf unterschiedlichen Ni- } \\
\text { veaus. } \\
\text { Förderung von }\end{array} \quad \begin{array}{l}\text { Die Problemstellung fördert kognitive Elemente in Form von Teilkompeten- } \\
\text { zen mathematischen Modellierens. }\end{array}$ \\
\hline
\end{tabular}

\subsubsection{Professionelle Kompetenz zum Lehren mathematischen Modellierens}

Aus Sicht der ersten Phase der Lehrerbildung ist eine genaue Betrachtung notwendiger professioneller Kompetenzen zum Lehren mathematischen Modellierens mit Blick auf die Qualitätsentwicklung der Lehrerbildung erforderlich. Zur Messung dieser Kompetenzen nutzen wir ein Modell, welches im Zuge eines Kooperationsprojekts entwickelt wurde (Klock, Wess, Greefrath \& Siller, 2019) und eine erste strukturelle Antwort im Bereich professioneller Kompetenz zum Lehren mathematischen Modellierens gibt (Wess, Klock, Greefrath \& Siller, im Druck; vgl. Abb. 6). Auf Basis dieses Strukturmodells wurde ein Instrument konzipiert und pilotiert (Klock \& Wess, 2018; Klock et al., 2019), welches ausgewählte zentrale Aspekte dieser bereichsspezifischen professionellen Kompetenz messbar macht.

Insgesamt orientieren wir uns dazu in erster Linie am COACTIV-Modell zur professionellen Kompetenz von Lehrkräften (Baumert \& Kunter, 2011). Darin setzt sich die professionelle Kompetenz aus den übergeordneten Aspekten Überzeugungen, motivationale Orientierungen, Selbstregulation und Professionswissen zusammen. Das Professionswissen unterteilt sich dabei in Anlehnung an Shulman $(1986,1987)$ in die Kompetenzbereiche Fachwissen, fachdidaktisches Wissen, pädagogisch-psychologisches Wissen, Organisationswissen und Beratungswissen. Im Kontext des vorliegenden Beitrags sowie mit Blick auf das Lehren mathematischen Modellierens erscheint der Bereich des fachdidaktischen Wissens besonders bedeutsam. Dieser zeichnet sich durch modellierungsspezifische Inhalte aus (Borromeo Ferri \& Blum, 2010), welche sich in die Facetten Wissen über Interventionen, Wissen über Modellierungsprozesse, Wissen über Modellierungsaufgaben sowie Wissen über Konzeptionen / Dimensionen / Ziele des Modellierens auffächern lassen (Klock et al., 2019; Wess et al., im Druck). 


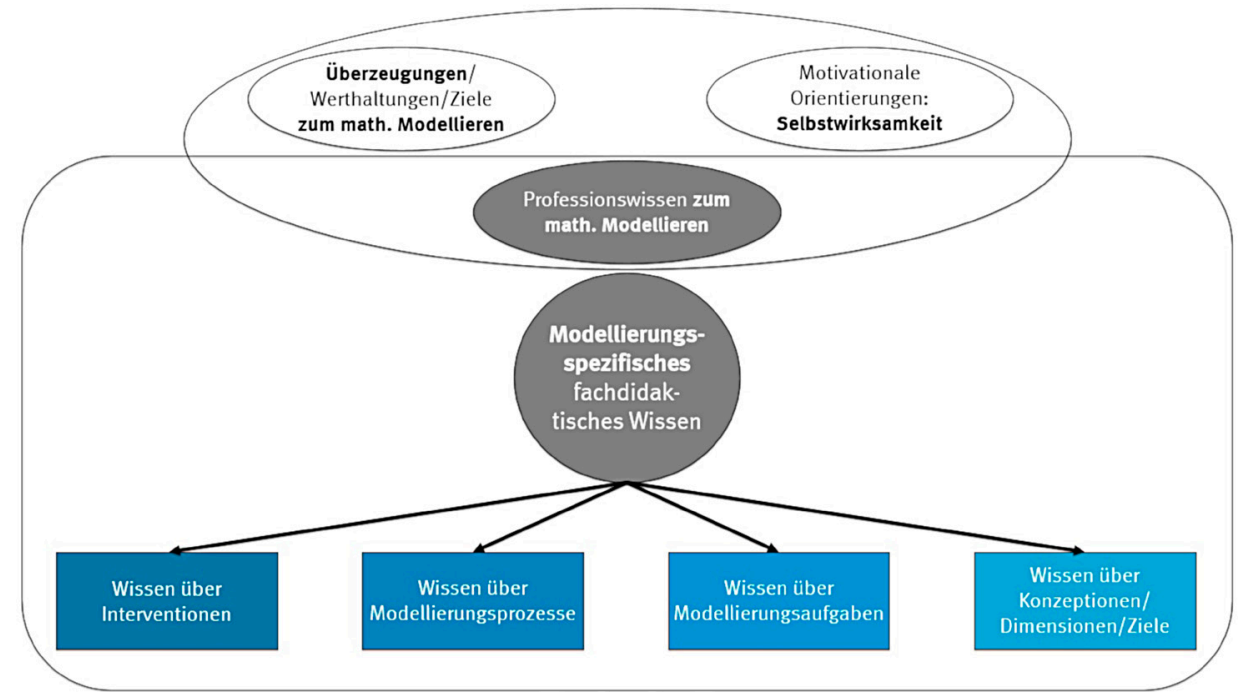

Abbildung 6: Professionelle Kompetenz zum Lehren mathematischen Modellierens.

\subsubsection{Modellierungsspezifische förderdiagnostische Kompetenz}

Eine empirische Studie zur Analyse der Qualität des aktuellen Bildungssystems weist darauf hin, dass die Professionalisierung von (angehenden) Lehrkräften in Deutschland noch nicht überzeugend gelingt (Felbrich, Müller \& Blömeke, 2008). Hinsichtlich der Frage, wie angehende Lehrkräfte professionelle Kompetenzen effektiver aufbauen können, steht somit nicht nur der Erwerb theoretischen Wissens, sondern auch der Praxisbezug im Fokus. Dabei kann im Kontext der professionellen Wahrnehmung die Analysekompetenz als Bindeglied von Theorie und Praxis sowie als eine notwendige Voraussetzung für professionelles Handeln (Schoenfeld, 2011) verstanden und weiter als Voraussetzung für das flexible Reagieren auf Schüleräußerungen angesehen werden (Friesen, Dreher \& Kuntze, 2014). Vor dem Hintergrund des kompetenten Umgangs mit Heterogenität (Fischer et al., 2014) gehören darüber hinaus die Diagnose der Kompetenzen von Lernenden, die Anpassung der Lernangebote an die Lernvoraussetzungen von Lernenden in alltäglichen Unterrichtssituationen sowie die differenzierte Förderung der Lernenden zum Tätigkeitsfeld von Lehrkräften und erfordern spezifische professionelle Kompetenzen (Hoffmann, 2015). Bezieht man nun die professionellen analytischen Wahrnehmungsprozesse darauf, eben jene individuellen Lernvoraussetzungen zutreffend einschätzen und die Lernumgebungen an diesen ausrichten zu können, spricht man von pädagogischer, förderorientierter diagnostischer Kompetenz oder nach Sengenberger, Lange-Schubert und Hartinger (2015) kurz von förderdiagnostischer Kompetenz. Moser Opitz (2006) konkretisiert dies für den Mathematikunterricht über das fachliche und fachdidaktische Wissen von Lehrkräften über das Entwickeln und Einsetzen von Aufgaben, welche der Erfassung, Beobachtung und Überprüfung der Lernprozesse und Kompetenzen ihrer Schülerinnen und Schüler 
dienen, sowie das Geben von hieran anschließenden und passenden Hilfestellungen bzw. Herausforderungen. Ziel dieses Handelns ist eine adäquate Förderung, wobei aufgrund der wechselseitigen Beziehung von Diagnose und Förderung die theoretische Expertise als grundlegend und handlungsleitend identifiziert werden kann. Darüber hinaus weisen Studien darauf hin, dass die Förderung dieser vielschichtigen Kompetenzfacetten durch möglichst authentische Lehr-Lern-Situationen unterstützt wird (Fölling-Albers, Hartinger \& Mörtl-Hafizović, 2004).

In Bezug auf das Lehren mathematischen Modellierens zeigen sich Aspekte förderdiagnostischer Kompetenz insbesondere in den Facetten Wissen über Modellierungsaufgaben und Wissen über Modellierungsprozesse. So dienen das Wissen über die förderdiagnostische Wirkung von Aufgaben, die Identifikation der Modellierungsphasen bei Schülerinnen und Schülern, das Erkennen von Schwierigkeiten während des Modellierungsprozesses sowie das Formulieren von Förderzielen als Charakteristika modellierungsspezifischer förderdiagnostischer Kompetenz (vgl. Abb. 7).

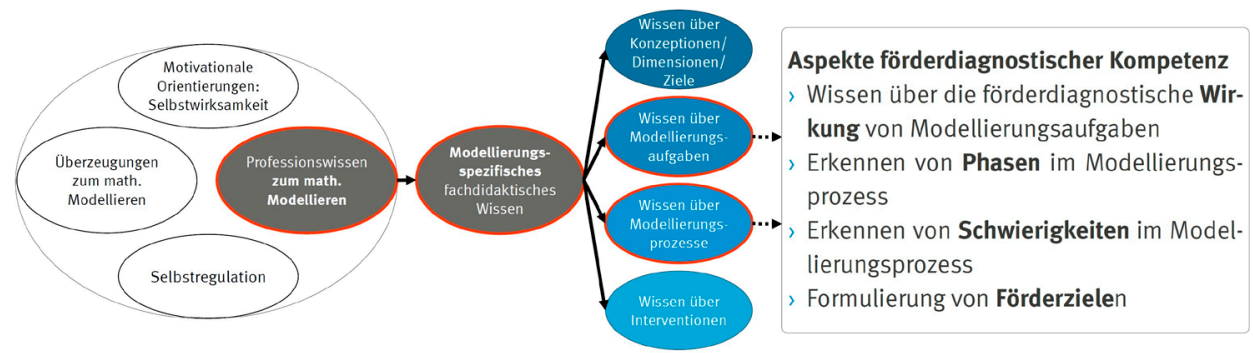

Abbildung 7: Aspekte förderdiagnostischer Kompetenz im Bereich des Lehrens mathematischen Modellierens.

\section{Forschungsfrage}

Die vorliegende Untersuchung befasst sich mit Aspekten professioneller Kompetenzen von angehenden Lehrkräften zum Lehren mathematischen Modellierens unter besonderer Berücksichtigung der Komplexitätsreduktion im Bereich des Lernarrangements im Lehr-Labor $\mathrm{MiRA}^{+}$. Demnach stellt sich die übergeordnete Frage, inwieweit sich die Kompetenzen der angehenden Lehrkräfte im Kontext mathematischen Modellierens durch die Teilnahme am Lehr-Labor mit seinen spezifischen komplexitätsreduzierenden Elementen fördern lassen. Insbesondere interessiert vor dem Hintergrund der theoretischen Überlegungen die Entwicklung der modellierungsspezifischen förderdiagnostischen Kompetenz, sodass wir die obige Fragestellung wie folgt konkretisieren wollen:

Inwieweit unterscheidet sich die Entwicklung der modellierungsspezifischen förderdiagnostischen Kompetenz bei Studierenden in mathematikdidaktischen 
Lehr-Lern-Laboren zum Modellieren in Abhängigkeit von der Komplexitätsreduktion im Bereich Medien und Materialien?

\section{Methodischer Ansatz}

Im Rahmen zweier Dissertationsprojekte wurden Lehr-Lern-Labore in Form von fachdidaktischen Seminaren zum mathematischen Modellieren mit Praxiseinbindung an den Hochschulstandorten Koblenz und Münster konzipiert. Am Standort Münster entwickeln die teilnehmenden Studierenden dabei eigene Modellierungsaufgaben und setzen diese in der Praxis ein, während am Standort Koblenz Interventionskonzepte in den Blick genommen und etablierte Modellierungsaufgaben in der Praxis eingesetzt werden. Die betrachteten Gruppen unterscheiden sich demnach in Abhängigkeit von der hier fokussierten Komplexitätsreduktion im Bereich Medien und Materialien (vgl. Kapitel 2). Nachstehend werden die Stichprobe, das Design sowie das verwendete Testinstrument der vorliegenden Studie beschrieben.

\subsection{Stichprobe und Untersuchungsdesign}

Zur Beantwortung der genannten Fragestellung wurden in einer quasi-experimentellen Interventionsstudie mithilfe eines Paper-Pencil-Fragebogens im Prä-PostDesign Daten von 78 Lehramtsstudierenden für das Lehramt an Gymnasien/Gesamtschulen an den Universitäten Koblenz-Landau und Münster erhoben. Dabei stellen die Studierenden aus dem Lehr-Labor $\mathrm{MiRA}^{+}(\mathrm{N}=35)$ die Experimentalgruppe (EG) und die Studierenden aus dem Lehr-Lern-Labor in Koblenz $(\mathrm{N}=43)$ die Vergleichsgruppe (VG) dar.

\subsection{Testinstrument}

Zur Erfassung der modellierungsspezifischen förderdiagnostischen Kompetenz (vgl. Abb. 7) wurden einzelne Skalen eines Testinstruments zu Lehrerkompetenzen beim mathematischen Modellieren (Klock \& Wess, 2018) genutzt. Dieses Instrument wurde im Sommersemester 2017 anhand der Daten von 156 Lehramtsstudierenden für das Lehramt an Gymnasien/Gesamtschulen an den Universitäten Koblenz-Landau, Duisburg-Essen und Münster pilotiert (Klock et al., 2019). Das Wissen über Modellierungsprozesse wurde dabei mit Textvignetten erhoben, die jeweils ein Schülergespräch während der Bearbeitung einer vorgegebenen Modellierungsaufgabe zeigen (vgl. Abb. 8). Nachstehend findet sich je ein Beispiel-Item der betrachteten Skalen Erkennen von Phasen (vgl. Abb. 9), Erkennen von Schwierigkeiten (vgl. Abb. 10), Formulieren eines Förderziels (vgl. Abb. 11). Das Wissen über 
Modellierungsaufgaben wurde mit Combined-Single-Choice-Items zusammengetragen. Ein Beispiel-Item für das Erfassen des Wissens über die förderdiagnostische Wirkung von Aufgaben findet sich in Abbildung 12.

\subsection{Stau (9. Klasse)}

Zu Beginn der Sommerferien kommt es oft zu Staus. Christina steckt für 6 Stunden in einem $20 \mathrm{~km}$ langen Stau fest. Es ist sehr warm und sie hat großen Durst. Es kursiert das Gerücht, dass ein kleiner Lastwagen die Leute mit Wasser versorgen soll, aber sie hat bisher noch nichts erhalten. Wie lange wird der Lastwagen benötigen, um alle Leute mit Wasser zu versorgen?

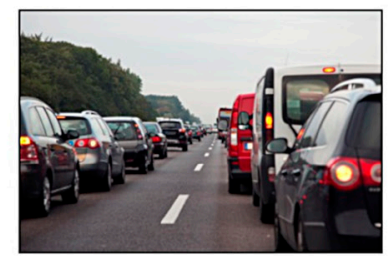

SCHÜLER 1: Wir müssten ja eigentlich wissen wie viele Autos überhaupt in dem Stau stehen.

SCHÜLERIN 2: Hä? Stimmt!

SCHÜLER 1: Wie sollen wir dann ausrechnen wie lange der braucht? Da fehlen doch voll viele Sachen in der Aufgabe!

SCHÜLER 3: Ja, wir wissen ja auch nicht wie lange der für jedes Auto braucht.

SCHÜLERIN 2: Voll die dumme Aufgabe.

SCHÜLER 1: Wir können ja mal die 20 km durch die 6 Stunden teilen, dann wissen wir wie schnell der sein muss.

SCHÜLER 3: Genau! Mehr haben wir ja eh nicht gegeben.

Abbildung 8: Textvignette zum Erfassen des Wissens über Modellierungsprozesse (Klock \& Wess, 2018).

\begin{tabular}{|l|l|l|}
\hline 7.1 .1 & $\begin{array}{l}\text { In welcher Phase des Lösungsprozesses befinden sich die Schülerinnen und Schüler } \\
\text { hauptsächlich? Bitte setzen Sie eine Markierung. }\end{array}$ \\
\hline Konstruieren/Verstehen & $\square$ \\
\hline Vereinfachen/Strukturieren & $\square$ \\
\hline Mathematisieren & $\square$ \\
\hline Interpretieren & $\square$ \\
\hline
\end{tabular}

Abbildung 9: Beispiel-Item zum Erkennen von Phasen im Modellierungsprozess (Klock \& Wess, 2018). 


\begin{tabular}{|l|l|l|}
\hline 7.1 .2 & $\begin{array}{l}\text { Diagnostizieren Sie das Problem der Schülerinnen und Schüler bei der Bearbeitung der } \\
\text { Aufgabe in dieser Situation. Bitte setzen Sie eine Markierung. }\end{array}$ \\
\hline Die Lernenden... & $\square$ \\
\hline$\ldots$ haben Probleme mit dem Treffen von Annahmen. & $\square$ \\
\hline$\ldots$ ziehen einen falschen Schluss aus Ihrem mathematischen Ergebnis. & $\square$ \\
\hline$\ldots$ haben Probleme beim Verstehen des Kontextes. & $\square$ \\
\hline$\ldots$ verwenden ein ungeeignetes mathematisches Modell.
\end{tabular}

Abbildung 10: Beispiel-Item zum Erkennen von Schwierigkeiten im Modellierungsprozess (Klock \& Wess, 2018).

\begin{tabular}{|l|l|l|}
\hline 7.1.7 & $\begin{array}{l}\text { Kreuzen Sie bitte an, welches Förderziel Sie im Anschluss an diese Situation für die } \\
\text { Gruppe formulieren würden. Bitte setzen Sie eine Markierung. }\end{array}$ \\
\hline Selbstständige Beschaffung und Bewertung von Informationen. & $\square$ \\
\hline Kritisches Hinterfragen von Ergebnissen im Modellierungsprozess. & $\square$ \\
\hline Selbstständige Konstruktion mentaler Modelle zu gegebenen Problemsituationen. & $\square$ \\
\hline Sichere Übersetzung vereinfachter realer Situationen in mathematische Modelle. & $\square$ \\
\hline
\end{tabular}

Abbildung 11: Beispiel-Item zum Formulieren eines Förderziels (Klock \& Wess, 2018).

\begin{tabular}{|l|l|c|c|}
\hline 5.3.2 & Modellierungsaufgaben... & wahr & falsch \\
\hline$\ldots$ sollen das Einüben von kurz zuvor behandelten Lösungsschemata fördern. & $\square$ & $\square$ \\
\hline ... dienen dem Erfassen von Phänomenen der realen Welt. & $\square$ & $\square$ \\
\hline$\ldots$ können Teilkompetenzen individuell fördern. & $\square$ & $\square$ \\
\hline
\end{tabular}

Abbildung 12: Beispiel-Item zum Erfassen des Wissens über die förderdiagnostische Wirkung von Aufgaben (Klock \& Wess, 2018).

\section{Ergebnisdarstellung}

Im Folgenden werden die Ergebnisse der Entwicklungen der förderdiagnostischen Kompetenz bei den teilnehmenden Studierenden in Abhängigkeit von der Komplexitätsreduktion im Bereich Medien und Materialien präsentiert. Dabei lassen sich zum ersten Messzeitpunkt keine signifikanten Unterschiede zwischen den Testergebnissen der beteiligten Gruppen feststellen $(t(76)=1.403, p=.165)$.

Abbildung 13 zeigt die Entwicklung der beiden Gruppen über die einsemestrige Teilnahme am Lehr-Lern-Labor hinweg. Sowohl die EG aus dem Lehr-Labor in Münster $(\mathrm{t}(34)=-13.562, \mathrm{p}=.001 ; \mathrm{d}=1.79)$ als auch die VG aus Koblenz 


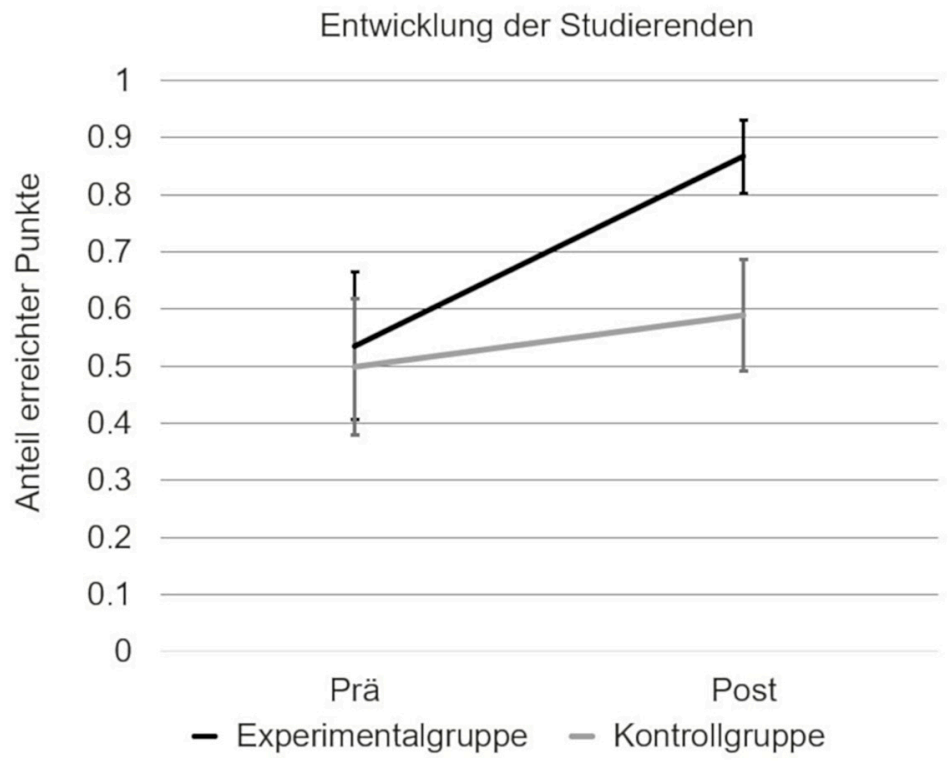

Abbildung 13: Entwicklung der modellierungsspezifischen förderdiagnostischen Kompetenz.

$(t(42)=-4.709, p=.001 ; d=.68)$ verbesserten sich signifikant mit großem bzw. mittlerem Effekt.

Da keine Ausreißer identifiziert werden konnten (beurteilt mit dem Box-Plot), die Daten für beide Gruppen normalverteilt sind (Shapiro-Wilk-Test, p > .05) und Varianzhomogenität gemäß dem Levene-Test gegeben ist $(\mathrm{F}(1,76)=.688, \mathrm{p}=$ .409), wurde eine zweifaktorielle ANOVA mit Messwiederholung auf einem Faktor berechnet, um zu untersuchen, ob es einen Unterschied der Kompetenzentwicklung in Abhängigkeit von der Gruppenzugehörigkeit und der damit verbundenen Komplexitätsreduktion gibt. Demnach lässt sich einerseits ein signifikanter Interaktionseffekt über den Teilnahmezeitraum zwischen den Seminargruppen $(F(1,76)$ $\left.=58.985, \mathrm{p}=.001 ; \eta^{2}=.437 ; 1-\beta=.997\right)$, andererseits ein signifikanter Effekt des nicht messwiederholten Faktors Versuchsgruppe feststellen $(F(1,76)=73.448$, $\left.\mathrm{p}=.001 ; \eta^{2}=.492 ; 1-\beta=.999\right)$. Die Effektstärke $d$ nach Cohen (1988) liegt für den Zwischensubjekteffekt bei 1.96 und entspricht einem großen Effekt. Die Entwicklung der modellierungsspezifischen förderdiagnostischen Kompetenz unterscheidet sich somit statistisch signifikant in Abhängigkeit von der Umsetzung der Komplexitätsreduktion in den betrachteten Lehr-Lern-Laboren. 


\section{Diskussion und Ausblick}

Im Rahmen eines Prä-Post-Vergleichs der modellierungsspezifischen förderdiagnostischen Kompetenz beider Gruppen wurde untersucht, ob sich Unterschiede im Kompetenzaufbau finden lassen, die auf die Komplexitätsreduktion im Bereich Medien und Materialien zurückzuführen sind. Um jedoch den Vergleich dieser beiden Gruppen besser einordnen zu können, wurde zunächst der Frage nachgegangen, wie sich die Kompetenzen der Lehr-Lern-Labor-Teilnehmenden über die Zeit hinweg verändert haben. Dazu wurden die durchschnittlichen förderdiagnostischen Kompetenzen innerhalb der beiden Gruppen zwischen den jeweiligen Messzeitpunkt mit Hilfe eines t-Tests für abhängige Stichproben verglichen. Die Analysen zeigen anhand signifikanter Zuwächse in beiden Untersuchungsgruppen, dass sich die förderdiagnostische Kompetenz, die einen starken Einfluss auf den Lernzuwachs der Schülerinnen und Schüler hat (Moser Opitz \& Nührenbörger, 2015), im Kontext von Lehr-Lern-Laboren zum mathematischen Modellieren fördern lässt.

In Bezug auf die Forschungsfrage nach der Abhängigkeit der Entwicklung förderdiagnostischer Kompetenz im Bereich mathematischen Modellierens von der Komplexitätsreduktion lässt sich durch die zweifaktorielle Varianzanalyse mit Messwiederholung auf einem Faktor, bei welcher die Effekte des Zwischensubjektfaktors Versuchsgruppe auf Signifikanz geprüft wurden, ein statistisch signifikanter Unterschied zwischen den Entwicklungen der beiden Gruppen feststellen. Demnach zeigt sich, dass die Komplexitätsreduktion im Bereich Medien und Materialien innerhalb der Experimentalgruppe mit großem Effekt bei hoher Teststärke wirksamer gewesen ist. Insbesondere vor dem Hintergrund eines hohen inhaltlichen Anforderungsniveaus im Kontext der Auseinandersetzung mit Modellierungsaufgaben weisen die Ergebnisse auf einen deutlichen Zusammenhang des Kompetenzzuwachses mit der Komplexitätsreduktion in den strukturellen und inhaltlichen Unterstützungsdimensionen hin. So zeigt sich sowohl in der intensiven Auseinandersetzung mit als auch in der vielschichtigen Begleitung bei der Konzeption eigener selbstdifferenzierender Aufgaben in Lehr-Lern-Laboren eine adressatengerechte Passung hinsichtlich des Leistungsniveaus der Studierenden im Sinne des Gleichgewichtsprinzips (Marohn et al., in diesem Band). Diese äußert sich zum einen in der kontinuierlichen professionellen Betreuung, welche es ermöglicht den Entstehungsprozess der Modellierungsaufgaben wie den Lernprozess der Studierenden permanent zu begleiten und adaptiv auf individuelle Schwierigkeiten einzugehen sowie zum anderen in der vielschichtigen Betreuung durch Mitstudierende, im Rahmen kollegialer Beratungen während der verschiedenen Feedbackzyklen zur Konzeption eigener selbstdifferenzierender Aufgaben. Die Implementation von Theorie-Praxis verbindenden Strukturen mit eigener Materialentwicklung kann also zum effektiven Aufbau professioneller Kompetenzen angehender Lehrerkräfte sowie zur Qualitätssteigerung in der Lehrerbildung beitragen. 
Es ist jedoch anzumerken, dass sich der Befund der vorliegenden Studie nur auf die gemessene Quantität förderdiagnostischer Kompetenz beschränkt und beide Gruppen qualitativ unterschiedliche Fähigkeiten erworben haben können, für welche die ausgewählten Skalen des Testinstruments aber nicht sensitiv waren. So erscheint es lohnenswert, den engen Blick auf die Komplexitätsreduktion im Bereich Medien und Materialien beizubehalten und die kognitiven Prozesse, die bei der Auseinandersetzung mit den Modellierungsaufgaben ablaufen, genauer zu untersuchen. Insbesondere ist noch nicht klar, an welchen Stellen im Lehr-Labor diese Reduktionen der Komplexität zu einem tieferen Verständnis im Sinne der Konkretisierung förderdiagnostischer Kompetenz von Moser Opitz (vgl. Kapitel 3.3) beitragen, ob es bestimmte Typen gibt, die unterschiedlich stark von den Unterstützungsmaßnahmen profitieren und wie eine solche Typisierung mit der Aufgabenentwicklung zusammenhängt. Diese differenzierte Betrachtungsweise ist gerade vor dem Hintergrund einer Neukonzeption und Evaluation von Lehr-LernLaboren wünschenswert.

\section{Literatur}

Baumert, J. \& Kunter, M. (2011). Das Kompetenzmodell von COACTIV. In M. Kunter, J. Baumert, W. Blum, U. Klusmann, S. Krauss \& M. Neubrand (Hrsg.), Professionelle Kompetenz von Lehrkräften - Ergebnisse des Forschungsprogramms COACTIV (S. 29-54). Münster: Waxmann.

Blum, W. (1985). Anwendungsorientierter Mathematikunterricht in der didaktischen Diskussion. Mathematische Semesterberichte, 32 (2), 195-232.

Blum, W. (2007). Mathematisches Modellieren - zu schwer für Schüler und Lehrer? In H.-W. Henn \& K. Maaß (Hrsg.), Beiträge zum Mathematikunterricht 2007 (S. 3-12). Berlin: Franzbecker.

Blum, W. (2010). Modellierungsaufgaben im Mathematikunterricht. Herausforderung für Schüler und Lehrer. Praxis der Mathematik, 34 (52), 42-48.

Blum, W. (2015). Quality Teaching of Mathematical Modelling: What Do We Know, What Can We Do? In S. J. Cho (Hrsg.), The Proceedings of the 12th International Congress on Mathematical Education (S. 73-96). Cham: Springer International. https:// doi.org/10.1007/978-3-319-12688-3_9

Borromeo Ferri, R. (2011). Wege zur Innenwelt des mathematischen Modellierens. Wiesbaden: Vieweg + Teubner. https://doi.org/10.1007/978-3-8348-9784-8

Borromeo Ferri, R. \& Blum, W. (2010). Mathematical modelling in teacher education experiences from a modelling seminar. In V. Durand-Guerrier, S. Soury-Lavergne \& F. Arzarello (Hrsg.), Proceedings of CERME 6 (S. 2046-2055). Lyon, France.

Borromeo Ferri, R. \& Blum, W. (Hrsg.). (2018). Lehrerkompetenzen zum Unterrichten mathematischer Modellierung: Konzepte und Transfer. Wiesbaden: Springer Fachmedien. https://doi.org/10.1007/978-3-658-22616-9

Borromeo Ferri, R., Greefrath, G. \& Kaiser, G. (2013). Einführung. In R. Borromeo Ferri, G. Greefrath \& G. Kaiser (Hrsg.), Mathematisches Modellieren für Schule und Hochschule; Theoretische und didaktische Hintergründe (S. 1-7). Wiesbaden: Springer Spektrum. https://doi.org/10.1007/978-3-658-01580-o 
Bruder, R. (1988). Grundfragen mathematikmethodischer Theoriebildung unter besonderer Berücksichtigung des Arbeitens mit Aufgaben. Potsdam: Pädagogische Hochschule Potsdam.

Cohen, J. (1988). Statistical power analysis for the behavioral sciences (2. Auflage). Hillsdale, NJ: Erlbaum.

Dohrmann, R. \& Nordmeier, V. (2015). Schülerlabore als Lehr-Lern-Labore (LLL): Ein Projekt zur forschungsorientierten Verknüpfung von Theorie und Praxis in der MINT-Lehrerbildung. PhyDid B - Didaktik der Physik - Beiträge zur DPG-Frühjahrstagung.

Felbrich, A., Müller, C. \& Blömeke, S. (2008). Lehrerausbildnerinnen und Lehrerausbildner der ersten und zweiten Phase. In S. Blömeke, G. Kaiser \& R. Lehmann (Hrsg.), Professionelle Kompetenz angehender Lehrerinnen und Lehrer. Wissen, Überzeugungen und Lerngelegenheiten deutscher Mathematikstudierender und -referendare. Erste Ergebnisse zur Wirksamkeit der Lehrerausbildung (S. 363-389). Münster: Waxmann.

Fischer, C., Rott, D., Veber, M., Fischer-Ontrup, C. \& Gralla, A. (2014). Individuelle Förderung als schulische Herausforderung. Netzwerk Bildung. Berlin: Friedrich-EbertStiftung.

Fölling-Albers, M., Hartinger, A. \& Mörtl-Hafizović, D. (2004). Situiertes Lernen in der Lehrerbildung. Zeitschrift für Pädagogik, 50(5), 727-747.

Friesen, M., Dreher, A. \& Kuntze, S. (2014). Aspekte fachdidaktischer Analysekompetenz bezogen auf den Umgang mit Repräsentationen im Mathematikunterricht. In J. Roth \& J. Ames (Hrsg.), Beiträge zum Mathematikunterricht 2014 (S. 381-384). Münster: WTM.

Greefrath, G., Kaiser, G., Blum, W. \& Borromeo Ferri, R. (2013). Mathematisches Modellieren - Eine Einführung in theoretische und didaktische Hintergründe. In R. Borromeo Ferri, G. Greefrath \& G. Kaiser (Hrsg.), Mathematisches Modellieren für Schule und Hochschule; Theoretische und didaktische Hintergründe (S. 11-37). Wiesbaden: Springer Spektrum. https://doi.org/10.1007/978-3-658-01580-o_1

Greefrath, G., Siller, H.-S. \& Ludwig, M. (2017). Modelling Problems in German Grammar School Leaving Examinations (Abitur) - Theory and Practice. In T. Dooley \& G. Gueudet (Hrsg.), Proceedings of the Tenth Congress of the European Society for Research in Mathematics Education (CERME1o, February 1 - 5, 2017) (S. 932-939). Dublin, Ireland: DCU Institute of Education \& ERME.

Greefrath, G. \& Vorhölter, K. (2016). Teaching and Learning Mathematical Modelling: Approaches and Developments from German Speaking Countries [ICME-13 Topical Survey]. Cham: Springer Open. https://doi.org/10.1007/978-3-319-45004-9

Herget, W. (2000). Wie groß? Wie hoch? Wie schwer? Wie viele? Mathe-Welt. mathematik lehren, 100, 23-46.

Hoffmann, M. (2015). Förderdiagnostische Kompetenzen von Grund- und Förderschullehrkräften im inklusiven Mathematikunterricht. In F. Caluori, H. Linneweber-Lammerskitten \& C. Streit (Hrsg.), Beiträge zum Mathematikunterricht 2015 (S. 396-399). Münster: WTM.

Kaiser, G. (2007). Modelling and modelling competencies in school. In C. Haines, P. Galbraith, W. Blum \& S. Khan (Hrsg.), Mathematical modelling: Education, engineering and economics (ICTMA 12) (S. 110-119). Chichester: Horwood.

Klock, H. \& Siller, H.-S. (2017). Förderung des modellierungsspezifischen Professionswissens von Lehramtsstudierenden im Rahmen eines Mathe-Labors. In U. Korten- 
kamp \& A. Kuzle (Hrsg.), Beiträge zum Mathematikunterricht 2017 (S. 1393-1394). Münster: WTM.

Klock, H. \& Wess, R. (2018). Lehrerkompetenzen zum mathematischen Modellieren - Test zur Erfassung von Aspekten professioneller Kompetenz zum Lehren mathematischen Modellierens. Universität Münster: MIAMI. Abgerufen von https://nbn-resolving.org/ urn:nbn:de:hbz:6-35169679459.

Klock, H., Wess, R., Greefrath, G. \& Siller, H.-S. (2019). Aspekte professioneller Kompetenz zum Lehren mathematischen Modellierens bei (angehenden) Lehrkräften Erfassung und Evaluation. In T. Leuders, E. Christophel, M. Hemmer, F. Korneck, \& P. Labudde (Hrsg.), Fachdidaktische Forschungen zur Lehrerbildung (S. 135-146). Münster: Waxmann.

Kultusministerkonferenz (2003). Bildungsstandards im Fach Mathematik für den Mittleren Schulabschluss. Beschluss vom 4.12.2003.

Krofta, H., Fandrich, J. \& Nordmeier, V. (2012). Professionalisierung im Schülerlabor: Praxisseminare in der Lehrerbildung. PhyDid B - Didaktik der Physik - Beiträge zur DPG-Frühjahrstagung.

Lengnink, K. \& Roth, J., (2016). „Lehr-Lern-Labor Mathematik“ als Ort der Forschung. In Institut für Mathematik und Informatik der Pädagogischen Hochschule Heidelberg (Hrsg.), Beiträge zum Mathematikunterricht 2016, 3 (S. 1267-1268). Münster: WTM.

Leuders, T. (2015). Aufgaben in Forschung und Praxis. In R. Bruder, L. Hefendehl-Hebeker, B. Schmidt-Thieme, H.-G. Weigand (Hrsg.), Handbuch der Mathematikdidaktik (S. 435-460). Berlin Heidelberg: Springer Spektrum. https://doi.org/10.1007/978-3642-35119-8_16

Maaß, K. (2004). Mathematisches Modellieren im Unterricht: Ergebnisse einer empirischen Studie. Hildesheim: Franzbecker. https://doi.org/10.1007/BF03339002

Maaß, K. (2010). Classification Scheme for Modelling Tasks. Journal für MathematikDidaktik, 31(2), 285-311. https://doi.org/10.1007/s13138-010-0010-2

Moser Opitz, E. (2006). Förderdiagnostik: Ziele, Leitideen, Beispiele. In M. Grüßing \& A. Peter-Koop (Hrsg.), Die Entwicklung mathematischen Denkens in Kindergarten und Grundschule: Beobachten - Fördern - Dokumentieren (2. Auflage; S. 10-28). Offenburg: Mildenberger.

Moser Opitz, E. \& Nührenbörger, M. (2015). Diagnostik und Leistungsbeurteilung. In R. Bruder, L. Hefendehl-Hebeker, B. Schmidt-Thieme \& H.-G. Weigand (Hrsg.), Handbuch der Mathematikdidaktik (S. 491-512). Berlin: Springer Spektrum. https:// doi.org/10.1007/978-3-642-35119-8_18

Niss, M. (2003). Mathematical competencies and the learning of mathematics: the danish KOM project. In A. Gagatsis \& S. Papastavridis (Hrsg.), Mediterranean Conference on Mathematical Education (S. 115-124). Athen: 3rd Hellenic Mathematical Society and Cyprus Mathematical Society.

Pollak, H. O. (1979). The interaction between mathematics and other school subjects. In UNESCO (Hrsg.), New trends in mathematics teaching IV (S. 232-248). Paris: OECD. Schoenfeld, A. H. (2011). Noticing matters. A lot. Now what? In M. Sherin, V. Jacobs \& R. Philipp (Hrsg.), Mathematics Teacher Noticing. Seeing Through Teachers' Eyes (S. 223-238). New York: Routledge.

Sengenberger, E., Lange-Schubert, K. \& Hartinger, A. (2015). Zum Aufbau förderdiagnostischer Kompetenzen bei Lehramtsstudierenden im Schriftspracherwerb durch 
videogestützte Lehr-Lern-Umgebungen. In K. Liebers, B. Landwehr, A. Marquardt \& K. Schlotter (Hrsg.), Lernprozessbegleitung und adaptives Lernen in der Grundschule: Forschungsbezogene Beiträge (S. 273-274). Wiesbaden: Springer Fachmedien. https:// doi.org/10.1007/978-3-658-11346-9_37

Shulman, L. S. (1986). Those who understand: Knowledge growth in teaching. Educational Researcher, 15(2), 4-14. https://doi.org/10.3102/0013189X015002004

Shulman, L. S. (1987). Knowledge and teaching: Foundations of the new reform. Harvard Educational Review, 57(1), 1-22. https://doi.org/10.17763/haer.57.1.j463w79r56455411

Siller, H.-S. (2008). Modellbilden - eine zentrale Leitidee der Mathematik. Aachen: Shaker. https://doi.org/10.1007/BFo3339053

Völker, M. \& Trefzger, T. (2011). Ergebnisse einer explorativen empirischen Untersuchung zum Lehr-Lern-Labor im Lehramtsstudium. PhyDid B - Didaktik der Physik - Beiträge zur DPG-Frühjahrstagung.

Wess, R., Klock, H., Greefrath, G. \& Siller, H.-S. (im Druck). Aspekte professioneller Kompetenz zum Lehren mathematischen Modellierens bei (angehenden) Lehrkräften - Theoretische und empirische Fundierung. In M. Zimmermann \& W. Paravicini (Hrsg.), Hanse-Kolloquium zur Hochschuldidaktik der Mathematik 2016/2017. Münster: WTM. 



\section{Unsichere Daten beim Experimentieren}

Förderung des Lehrerhandelns in ungeplanten Situationen im physikdidaktischen Lehr-Lern-Labor La:gune

Im naturwissenschaftlichen Unterricht spielen Experimente eine zentrale Rolle (Harlen, 1999; Tesch \& Duit, 2002). Viele Experimente bringen allerdings bekanntermaßen eine gewisse Instabilität mit sich: Sie produzieren nicht immer zuverlässig die theoretisch erwarteten Ergebnisse. Dies stellt vor allem Novizinnen und Novizen im Lehrberuf vor Herausforderungen.

Im Lehr-Lern-Labor „La:gune" steht der Umgang mit eben solchen Situationen im Fokus. Durch drei videographierte Zyklen (Iterationen) von Unterrichtsplanung, Praxiserfahrung und Reflexion erhalten Studierende in diesem Lehr-Lern-Labor die Möglichkeit, ihren Umgang mit unerwarteten Situationen zu reflektieren.

Der Beitrag beschreibt die notwendigen Rahmenbedingungen einer solchen Lernumgebung für die Studierenden. Neben unterschiedlichen Facetten der Komplexitätsreduktion im Lehr-Lern-Labor werden die Auswirkung mehrfacher Iterationen beschrieben und anhand empirischer Ergebnisse diskutiert.

Schlüsselwörter: Lehrerhandeln, Videobasierte Reflexion, Umgang mit ungeplanten Situationen

\section{Motivation und Einleitung}

Unterricht ist ein komplexes System von Wechselwirkungen, in dem sich eine Lehrkraft erst einmal zurechtfinden muss und welches eine angehende Lehrkraft schnell überfordern kann. Der Einsatz von Experimenten stellt dabei für die Physikdidaktik einen großen Schwerpunkt dar, da Experimente eine essenzielle Rolle im Physikunterricht spielen (Harlen, 1999; Tesch \& Duit, 2002). Besonders für Novizinnen und Novizen im Lehrerberuf und Studierende öffnet sich eine große Problemstelle, wenn Experimente unsichere Daten ${ }^{1}$ hervorbringen (Heinicke \& Holz, 2018; Ruhrig \& Höttecke, 2015). So haben wir beispielsweise festgestellt, dass Studierende beim Einsatz von Experimenten besonders an diesen Stellen Überforderung zeigen und damit einhergehend Verunsicherung berichten (Holz \& Heinicke, 2019). Dies ist von physikdidaktischer Seite betrachtet besonders elementar, da gerade bei quantitativen Experimenten kein Ergebnis wirklich „sicher“ ist. Ein Umgang mit den unsicheren Daten ist damit nicht nur ein Muss, sondern

1 Der Begriff „unsichere Daten“ wird im Folgenden weit gefasst und kann so z. B. für schwankende Messwerte, aber auch für von den Erwartungen abweichende experimentelle Ergebnisse stehen. 
sogar physikalischer Fachinhalt im Sinne von nature of science: Er ist elementar für ein Verständnis physikalischer Erkenntnisgewinnung (Buffler, Lubben \& Ibrahim, 2009) und der Bewertung von Daten. Das Aufkommen unsicherer Daten bietet demnach große Chancen für Lerngelegenheiten physikalischer Fachmethoden (Heinicke, Glomski, Priemer \& Rieß, 2010; Kelly, Brown \& Crawford, 2000). Ein fachmethodisch inadäquater Umgang mit experimentellen Daten seitens der Lehrkraft hat dagegen das Potenzial ein falsches Bild von der Art und Weise zu vermitteln (Modelllernen; Bandura, 1976), mit der Physik mit experimentellen Daten umgeht. Das Handeln der Lehrkraft spielt daher besonders in Situationen, in denen unsichere Daten auftreten, eine große Rolle.

Um das Lehrerhandeln im Umgang mit unsicheren Daten zu fördern, wurde das Lehr-Lern-Labor „La:gune“ konzipiert. Studierende erhalten in dieser Lernumgebung die Möglichkeit, Praxiserfahrung in einem geschützten Raum zu sammeln. Dies geschieht, indem Unterrichtsminiaturen (bezüglich der enthaltenen Unterrichtsphasen vollständige Einheiten mit einer Länge von ca. 12 Minuten; Korneck, Kunter, Oettinghaus, Lamprecht \& Sach, 2014) geplant, durchgeführt und anhand von Videoaufnahmen in Gruppen reflektiert werden. Innerhalb einer Durchführung der konzipierten Lernumgebungen durchlaufen Studierende dabei drei Iterationen von Planung, Durchführung und Reflexion, sodass reflektierte Einheiten optimiert und erneut durchgeführt und dabei erarbeitete Handlungsalternativen ausprobiert werden können.

In diesem Beitrag wird zunächst näher auf die Zielsetzung des Lehr-LernLabors La:gune eingegangen (Kapitel 2). Dabei wird deutlich, dass über die Betrachtung unsicherer Daten im Experiment hinaus eine allgemeine Betrachtung von ungeplanten Situationen notwendig ist. In Kapitel 3 werden Anforderungen (A1 bis $A_{3}$ ) hergeleitet, die durch die gewählte Zielsetzung an die Lernumgebung gestellt werden. Abgeschlossen werden diese Herleitungen jeweils mit der praktischen Umsetzung dieser Anforderungen innerhalb der Lernumgebung. Im vierten Kapitel wird schließlich der Aufbau der konzipierten Lernumgebung selbst dargestellt. Dabei liegt der Fokus auf den umgesetzten Facetten von Komplexitätsreduktion innerhalb der Lernumgebung. Eine spezifische Eigenart des konzipierten Lehr-Lern-Labors ist die dreifache Iteration der Praxiserfahrung. Besonders in Bezug auf die Facetten der Komplexitätsreduktion ist die Auswirkung dieser konzeptionellen Entscheidung stark sichtbar. Kapitel 5 beschreibt diesen Einfluss und diskutiert daraus erwachsende Erkenntnisse bezüglich der gezielten Steuerung einzelner Facetten der Komplexitätsreduktion bei wiederholender Praxiserfahrung. Der Beitrag fährt fort mit einem Rückblick auf die hergeleiteten Bedingungen an die Lernumgebung und gibt Erfahrungen sowie empirische Befunde der vergangenen Durchführungen bezüglich dieser Bedingungen wieder. Abschließend werden ein kurzes Fazit und ein Ausblick gegeben. 


\section{Ziele der Lernumgebung}

Mögliche Problematiken beim Umgang mit unsicheren Daten können in zwei Schwerpunkte unterteilt werden, die jedoch ineinander übergehen: Zunächst ergibt sich ein physikdidaktischer Schwerpunkt (der fachmethodische adäquate Umgang mit unsicheren Daten im Physikunterricht), aus welchem dann ein allgemeiner didaktischer Schwerpunkt entsteht (die Handlung bei ungeplanten Situationen im Unterricht). In den folgenden zwei Unterkapiteln werden beide Schwerpunkte kurz beschrieben.

\subsection{Physikdidaktischer Schwerpunkt}

Das Experiment nimmt im modernen Physikunterricht eine sehr zentrale Rolle ein und ist elementar für das Verständnis von Physik (z. B. Harlen, 1999; Tesch \& Duit, 2002). Unter der Vielfalt möglicher Ziele, die der Experimenteinsatz im Physikunterricht innehält, ist an dieser Stelle besonders das Lehren von nature of science zu nennen.

Ein bei der Betrachtung von nature of science oft unbeachteter Bereich ist die Bewertung und Interpretation experimenteller Daten, obwohl gerade dieser einen Großteil der physikalischen Erkenntnisgewinnung ausmacht (Buffler et al., 2009; Kanari \& Millar, 2004). Das Ergebnis eines physikalischen Experimentes kann ohne Informationen über dessen Genauigkeit zu keinerlei Interpretation genutzt werden. Auch darüber hinaus spielen Messdaten und deren Unsicherheit eine große Rolle für den Physikunterricht: Dort, wo Experimente stattfinden, muss auch mit (begrenzt genauen) experimentellen Daten umgegangen werden (Heinicke, 2014; Heinicke \& Holz, 2018). Das bedeutet, dass ein moderner Physikunterricht nicht um diesen Umgang herumkommt. Die Physiklehrkraft vermittelt also in jedem Fall im Minimum ein implizites Bild vom fachmethodischen Umgang mit Daten (Modelllernen, Bandura 1976). Die Art und Weise, mit der Lehrkräfte diesen Umgang vorleben, ist daher zentral für die Vermittlung physikalischer Fachmethoden und von nature of science.

Besonders der Umgang mit unsicheren Daten ist bezüglich des Umgangs mit experimentellen Daten hervorzuheben. Dass Experimente nicht das theoretisch erwartete Ergebnis liefern, ist im Physikunterricht häufig der Fall. Dies ist nicht etwa auf qualitativ minderwertige Experimente oder eine schlecht ausgestattete Schulsammlung zurückzuführen, sondern fußt auf der endlichen Genauigkeit, die eine den experimentellen Daten inhärente Eigenschaft ist. Streuungen und systematische Effekte sind stets vorhanden und beeinflussen die Ergebnisse von Experimenten, sodass eine exakte Entsprechung von Experiment und (auf Idealisierungen basierender) Theorie in der Regel eher zum Zweifel Anlass gäbe als eine gewisse Diskrepanz zwischen beiden. Jedoch erfordert eine solche Diskrepanz zwischen physikalischer Theorie und Experiment eine Handlung der Lehrkraft 
und birgt die Gefahr inadäquates fachmethodisches Vorgehen (z. B. wegdiskutieren experimenteller Messdaten) zu vermitteln. Andererseits können besonders solche Vorkommnisse effektiv zur Vermittlung von physikalischen Fachmethoden und nature of science genutzt werden (Höttecke, 2013), da naturwissenschaftliche Arbeitsweisen angewandt werden und damit vermittelt werden können.

Wie aber sollte eine Lehrkraft damit umgehen, wenn ein Experiment nicht das Ergebnis zeigt, welches die Theorie vorhersagt? Wie soll gehandelt werden, wenn Messgeräte ungeplant schwanken?

Die konzipierte Lernumgebung zielt darauf ab, angehende Lehrkräfte zur Erhaltung ihrer Handlungsfähigkeit in solchen kritischen Situationen im Umgang mit unsicheren Daten hinzuleiten.

Dazu werden sie angeleitet, diese Situationen in Videoreflexionen wahrzunehmen, sie zu verstehen, alternative Strategien für die nächste Durchführung sowie langfristigere Entwicklung zu generieren und diese in Iterationen der Praxiserfahrungen umzusetzen.

\subsection{Allgemeiner didaktischer Schwerpunkt}

Die Problematik, die sich bei ungeplanten experimentellen Daten ergibt, ist keine rein fachdidaktische. Vielmehr scheint eine Betrachtung des allgemeinen Umgangs mit ungeplanten Ereignissen in Unterrichtssituationen angebracht.

Ein Ereignis, das das momentane Unterrichtsziel scheinbar angreift, fordert ein schnelles Handeln der Lehrperson. Dies setzt die Lehrperson unter Handlungsdruck (Wahl, 1991) und kann zu negativer emotionaler Belastung führen (Peterson, Driver-Linn \& Deyoung, 2002). Dies ist besonders wahrscheinlich, wenn die Situation unvorhergesehen geschieht (Gray, 1987). Durch die begrenzte Zeit bedingt bleibt auch für das Erfassen der Situation, das Abwägen möglicher Handlungsoptionen sowie deren möglichen Folgen nur begrenzt Zeit. Der Rückgriff auf implizite Theorien und Handlungsroutinen ist die Folge (Wahl, 1991). Dies stellt besonders für angehende und wenig routinierte Lehrkräfte eine große Problematik dar: Fehlende Handlungsroutinen, fehlende Handlungsoptionen und wenig ausgeprägte Coping Mechanismen spitzen die Situation zunehmend zu. Stress und negative emotionale Belastung können die Folge sein (Lazarus, 1999). Das Auftreten unsicherer Daten kann durchaus als eine solche Situation gefasst werden, die somit auch die genannten Schwierigkeiten beinhaltet. Der reine Fokus auf den Umgang mit unsicheren Daten scheint daher für eine unterrichtsnahe Betrachtung zu spezifisch, da ein wesentlicher Teil der Problematik außer Acht gelassen wird. Eine Ausweitung der Zielsetzung, die mithilfe des Lehr-Lern-Labors La:gune erreicht werden soll, ist angebracht: 
Neben dem fachdidaktischen Schwerpunkt auf den Umgang mit unsicheren Daten zielt das konzipierte Lehr-Lern-Labor auf den Umgang mit von der Unterrichtsplanung abweichenden Situationen bzw. die Erhaltung der Handlungsfähigkeit in diesen $a b$.

\section{Herleitung der Anforderungen an die Lernumgebung}

Ausgehend von der beschriebenen Zielsetzung für das Lehr-Lern-Labor können verschiedene Anforderungen ( $\mathrm{A}_{1}$ bis $\mathrm{A}_{3}$ ) theoretisch herausgearbeitet werden, die bei der Konzeption bedacht werden. Die Herleitung folgt in diesem Kapitel. Die Gesamtheit dieser Spezifikationen macht den Rahmen der Lernumgebung aus, die im darauffolgenden Kapitel genauer und im Hinblick auf umgesetzte Aspekte der Komplexitätsreduktion beschrieben wird.

\subsection{Diskrepanzen zwischen Theorie und Praxis (A1)}

Die Diskrepanz zwischen „wissenschaftlicher Ausbildung und praktischem beruflichen Handeln" (Wahl, 1991, S. 5) ist eine in der Lehrerbildung häufig genannte Problematik. Im expliziten Rahmen (beispielsweise in Seminaren oder Lehrerfortbildungen) erarbeitete und erlernte Theorien, Methoden usw. sind nicht unmittelbar im tatsächlichen unterrichtlichen Handeln anwendbar, selbst wenn diese als sinnvoll und hilfreich eingeschätzt werden (Wahl, 1991). Kurz gefasst besteht eine Diskrepanz zwischen einer Auseinandersetzung mit unterrichtlichen Handlungen (on action) und tatsächlicher Handlung im Unterricht (in action). Besonders deutlich tritt diese Diskrepanz im Handeln unter Druck auf, d.h. zum Beispiel in kritischen Situationen, in denen Handlungen bzw. Entscheidungen seitens der Lehrkraft zeitnah erfordert werden. Wahl (1991) stellte hierzu insbesondere fest, dass Lehrkräfte unter Druck zunehmend auf implizite Theorien zurückzugreifen scheinen. Bereits aus neuropsychologischer Sicht leuchtet dies ein, da die Nutzung impliziten Wissens und Affekthandlungen die kognitiv „kostengünstigere“ Variante darstellt und als solche wesentlich schneller stattfinden kann (Ledoux, 1996). Auch Baumert und Kunter (2011) wiesen bereits darauf hin, dass sich das Professionswissen unterrichtlichen Handelns oft auf impliziter Ebene bewegt. Dies umrahmt ein grundlegendes Problem praxisorientierter Lernumgebungen: Soll die Handlungsfähigkeit der Studierenden (in action) beeinflusst werden, so genügt es nicht, explizite Theorien und Ansichten genügend praxisorientiert zu vermitteln (on action), vielmehr müssen die implizit gehaltenen Theorien und Ansichten aufgedeckt werden. Worin liegen die Ursachen dieser Diskrepanzen und wie kann für einen konkreten Aspekt eine Brücke zwischen Theorie und Praxis geschlagen werden?

Erreicht werden kann dies durch Selbstreflexion des eigenen Verhaltens (Wahl, 1991). Eigler (1983) beschreibt hierzu das Vermögen von Selbstreflexionen, selbst 
stark automatisierte Handlungsmuster aufzudecken. Durch eine Bewusstmachung dieser Muster kann deren Veränderung vereinfacht werden (Cranach, 1983). Damit eine konzipierte Lernumgebung die Brücke zwischen on action und in action schaffen kann, muss die Lernumgebung demnach zunächst praktische Elemente beinhalten, die reflektiert und analysiert werden können. Studierende müssen die Chance haben, eigenen Unterricht durchzuführen und diesen zu reflektieren.

Eine in diesem Zusammenhang etablierte Methode ist die des „Stoppens“ oder „Innehaltens“ und „Optionen Analysierens“ (Wahl, 1991). Becker (1981) beschreibt diese Methode beispielsweise als einen notwendigen „Handlungsaufschub“. Die Situation (in action) wird aufgeschoben um außerhalb des Zeitdruckes (on action) nach Lösungen $\mathrm{zu}$ suchen. Im unterrichtlichen Fall entspricht diese Suche nach Lösungen einer reflektiven Analyse der Situation: Was war die Ursache? Wie trat diese zu Tage? Welche Handlungsoptionen hätte ich gehabt? Welche Folgen hätten diese gehabt? Wurden im zeitlich entzerrten Rahmen „Vorbereitungen getroffen, so ist der Akteur in der Lage, in einer definierten Situation vorgeplant zu agieren“ (Wahl, 1991, S. 191). Tritt diese oder eine ähnliche Situation erneut auf, so kann wie geplant agiert und die gefundene Handlung gefestigt oder ggfs. optimiert werden (Wahl, 1991). Dies ist selbstverständlich eine sehr idealisierte Beschreibung, die nicht frei von Störungen und Problemen ist, dennoch scheint sie eine sinnvolle grundlegende Betrachtungsweise zu sein.

Treten während der Praxiserfahrung ungeplante Situationen auf, so sollten diese tiefgreifend analysiert und reflektiert werden. Ein „Stoppen“ der Situation ist nur schwer umsetzbar, ein „Handlungsaufschub“ kann jedoch durch die Reflexion mit Hilfe von Videos realisiert werden, da Videoaufnahmen eine große Immersion schaffen (Goldman, 2007). Sie ermöglichen es Studierenden also, sich möglichst gut in die vergangene Situation hineinzuversetzen. Die Analyse innerhalb der Reflexion soll ermöglichen Handlungsalternativen für ähnliche Situationen zu generieren. Um diese Art der Auseinandersetzung zu unterstützen, finden die Reflexionen theoriegeleitet statt. Dies geschieht im konzipierten Lehr-Lern-Labor anhand einer vereinfachten Adaption des Stress-Modells nach Lazarus und Folkman (1984, für nähere Beschreibung siehe Kasten 1). Zusätzlich zu dieser tiefgreifenden Analyse müssen Gelegenheiten geschaffen werden, erarbeitete Handlungsalternativen ausprobieren zu können. Dies wird innerhalb des konzipierten Lehr-LernLabors mithilfe von Wiederholungen der Einheiten (Iterationen) im Anschluss an die Reflexionsphasen realisiert.

\section{Resultierende konzeptuelle Entscheidungen:}

Die Lernumgebung beinhaltet ...

- die praktische Durchführung eigenen Unterrichtes,

- eine tiefgreifende, theoriegeleitete Reflexion, um Handlungsalternativen herauszuarbeiten,

- Videounterstützung der Reflexionen für immersive Reflexion und

- eine Wiederholung der Unterrichtsminiaturen (Iterationen), um geschaffene Handlungsalternativen einzusetzen. 
Das Stressmodell von Lazarus und Folkman (siehe Abb. 1) bildet einen theoretischen Rahmen zur Beschreibung der Entscheidungsprozesse und Handlungen, die zu stresserzeugenden Situationen führen. Es bezieht sowohl vorlaufende Prozesse als auch den Umgang mit vorliegendem Stress (Coping) ein. Als grundlegende Prozesse dienen hier die subjektiven „Bewertungen“ (Appraisals).

Wird ein Reiz wahrgenommen, so findet in der primären Bewertung eine grobe Einschätzung des Reizes statt. Zugrunde liegende Fragen sind „Hat das negative, positive oder keine Folgen für mein verfolgtes Ziel?" bzw. „Muss ich etwas tun?“.

Ein als negativ eingeschätzter Reiz erfordert eine Handlung. In der sekundären Bewertung wird daher abgewägt, ob Handlungsoptionen zur Verfügung stehen, die Adaption des Stressmodells von zufriedenstellend sind. Analog zur ersten Lazarus und Folkman (1984); freie Bewertung ist auch diese subjektiv und damit Übersetzung. von emotionalem Zustand, Zeitdruck usw. geprägt.

Stellt sich heraus, dass keine zufriedenstellenden Handlungsoptionen vorliegen (zu scheinen), entsteht Stress und es folgt eine Stressbewältigung (Coping). Letztlich besteht noch die Möglichkeit retrospektiv aus diesen zu lernen (Neubewertung).

Die Nutzung dieses Modells bietet sich für die Strukturierung der Reflexion von kritischen Situationen an, da mit diesem ein Rahmen geschaffen wird, der sowohl

- zur Situation hinführende oder vorab beitragende Ereignisse,

- in der Situation stattgefundene Prozesse als auch

- auf die Situation folgende Handlungen und Ereignisse und

- alternative Handlungsstränge

einbezieht.

Kasten 1: Modell von Lazarus und Folkman (1984) mit zugehörigen Erklärungen zum Einsatz als Reflexionsunterstützung 


\subsection{Emotionale negative Konnotation und Blockade kritischer Reflexion (A2)}

Allgemein scheint die Nutzung von Videos für die Ausbildung von Lehrkräften von großem Nutzen zu sein. So können für den mathematisch-naturwissenschaftlichen Bereich neben vielen anderen Effekten eine Steigerung effektiver Lehrhandlungen (Hougham, 1992) sowie der Zuwachs neuer Perspektiven auf Unterricht (Sherin \& van Es, 2005) festgestellt werden. Für die Nutzung (insbesondere eigener) Videos in der Lehramtsausbildung wurde ein positiver Einfluss auf die Motivation und die Aktivierung von Vorwissen und Erfahrung nachgewiesen (Baum \& Gray, 1992; Borko, Jacobs, Eiteljorg \& Pittman, 2008). Gerade die Nutzung eigener Videos kann allerdings auch eine negative Auswirkung auf die Motivation haben. Der Einsatz von eigenen Videos und die Betrachtung von diesen kann den Fokus der Studierenden sehr selbstbezogen werden lassen (die „self-attention“ wird gesteigert; Fiske, 1995). Dies wiederum kann eine kritische Reflexion blockieren. Ein Grund sind negative Emotionen, die Lernprozesse blockieren können (vgl. Oser \& Spychiger, 2005 für eine pädagogische oder beispielsweise Ledoux, 1996 für eine neuropsychologische Sichtweise). Diese Eigenschaft von Videoreflexionen tritt nach Krone, Hamborg und Gediga (2002) besonders in Fehlersituationen zu Tage, zu denen auch „ungeplante Situationen“ zu zählen sind. Bestätigt wird dies beispielsweise durch Zapf (1993) in einer quantitativen Untersuchung. Auch Krone et al. (2002) bestätigen diesen Zusammenhang in einer qualitativen Untersuchung zu Teilen, wobei eine genauere Differenzierung in „Hilflosigkeit" und „Ärger" als notwendig vermerkt wird.

$\mathrm{Zu}$ beachten ist jedoch, dass eine Steigerung der self attention zunächst nicht als negativ zu bewerten ist. Im Zentrum der Reflexionsprozesse innerhalb des konzipierten Lehr-Lern-Labors stehen die Studierenden selbst und deren Handeln in Lehrsituationen. Es soll also eine Auseinandersetzung mit dem eigenen Handeln und der eigenen Lehrperson stattfinden, sodass eine hohe self attention zunächst wünschenswert ist. $\mathrm{Zu}$ vermeiden ist dabei allerdings die Blockade einer konstruktiven (Selbst-)Reflexion durch eine negative emotionale Konnotation der Reflexionen. Es muss also eine Umgebung geschaffen werden, die eben dies begünstigt. Grundlegend dafür ist die Schaffung klarer Regeln und eine vertraute Gruppe von reflektierenden Teilnehmern (Borko et al. 2008; Sherin \& van Es, 2009).

Umgesetzt wird dies durch klare Reflexionsregeln, die neben konstruktivem Vorgehen vor allem den Studierenden selbst die Kontrolle über die eigenen Reflexionen geben. Studierende suchen sich beispielsweise Schwerpunkte sowie zu reflektierende Videoszenen vorab selbst aus. Die curriculare Einbindung des Seminars sorgt für vertraute Gruppen und für einen wertungsfreien Rahmen. 
Resultierende konzeptuelle Entscheidungen:

- Schaffung eines komplexitätsreduzierten und wertungsfreien Raums

- Klare Reflexionsregeln für konstruktive Reflexionssitzungen

- Hoher Anteil an Selbstbestimmung der Studierenden in Reflexionsphasen (z. B. durch eine eigene Auswahl der zu reflektierenden Videoszenen)

\subsection{Praxisschock und Realitätsnähe (A3)}

Besonders bei einer Lernumgebung, die kritische unterrichtliche Situationen in den Fokus setzt, erscheint die Gefahr eines Praxisschock beachtlich. Zur Vermeidung desselben bietet sich daher die Schaffung eines geschützten und komplexitätsreduzierten Raums an. Diese Reduktion hat zwar das Potenzial negative Erlebnisse zu vermindern, allerdings findet damit auch eine Verminderung der Realitätsnähe der von den Studierenden betrachteten und erlebten Situationen statt. Dies wiederum stellt sich erneut gegen den gesetzten Fokus.

Untersuchungen wie beispielsweise die von Peuser, Szogs, Krüger und Korneck (2017) weisen darauf hin, dass ein Praxisschock im Rahmen eines Lehrkonzeptes mit Unterrichtsminiaturen (microteaching) und Videoreflexionen vermieden werden kann. Sie konnten dabei zeigen, dass die Selbstwirksamkeitserwartung (SWE) bezüglich verschiedener Facetten des Physikunterrichtes sogar über den Verlauf der Lernumgebung ansteigt. Die Selbstwirksamkeitserwartung wurde in Peuser et al. (2017) ähnlich wie in vielen Studien dieser Art zu Beginn und nach Abschluss des Seminars erhoben, allerdings nicht speziell in einer weiteren Praxisphase wie dem Referendariat. Damit kann zwar gezeigt werden, dass ein Praxisschock durch das Seminar selbst ausbleibt, ein Ausbleiben in realen Unterrichtssituationen wie dem Referendariat ist jedoch nicht nachgewiesen.

Der größte (aber bei weitem nicht einzige) Faktor zur Beeinflussung der Selbstwirksamkeitserwartung ist eigenes Kompetenzerleben (Bandura, 1997; Usher \& Pajares, 2008). Wird eine deutliche Diskrepanz zwischen Selbstbild (in Bezug auf eigene Kompetenzen) und tatsächlichen Kompetenzen in einer Selbstreflexion deutlich, so besteht damit eine große Möglichkeit zur Veränderung der Selbstwirksamkeitserwartung - jedoch in positiver wie auch negativer Richtung. Letzteres würde, sofern es in entsprechender Stärke auftritt, als Praxisschock beschrieben, jedoch findet eine Veränderung des Selbstbildes zu Gunsten einer besseren Realitätspassung statt. In einem solchen Fall (beispielsweise bei deutlicher Überschätzung der eigenen Kompetenzen) kann ein solcher Praxisschock als wünschenswert bezeichnet werden, da die Notwendigkeit der Kompetenzsteigerung deutlich wird. Cranach (1983) und Mutzeck (1988) beschreiben einen solchen Wunsch nach einer Änderung des eigenen Handelns als grundlegende Voraussetzung, die der erfolgreichen gezielten Reflexion sowie dem wiederholten Praktizieren vorausgehen muss. Sofern eine konstruktive und unterstützende Lernumgebung geschaffen wird, kann damit eine im herkömmlichen Sinne als Praxisschock gedeutete „Kri- 
se“ der Selbstwirksamkeitserwartung als positiver Grundstein für weiteres Lernen angesehen werden, dessen Vermeidung sogar die Kompetenzentwicklung hemmen könnte. Ein solcher „Sprung ins kalte Wasser“ kann demnach sehr nützlich sein, sofern dieser auch innerhalb des Seminars aufgefangen wird und nur zu einer zeitweisen Verringerung der Selbstwirksamkeitserwartung führt. Im Lehr-Lern-Labor La:gune wird ein solcher Sprung ins kalte Wasser umgesetzt, indem die Studierenden (im geschützten Raum) für die Praxiserfahrung der ersten Iteration nur sehr begrenzte Vorbereitungszeit besitzen.

Eine schrittweise Öffnung des geschützten Raumes durch Veränderung verschiedener Facetten der Komplexitätsreduktion schafft zudem im Verlauf der Iterationen eine zunehmende Realitätsnähe, um ein Kompetenzerleben der Studierenden im Seminar zu ermöglichen. Gesteuert wird diese Öffnung im Wesentlichen durch die Lerngruppe. Während die erste Iteration innerhalb der Seminargruppe stattfindet, wird die Praxisphase der dritten Iteration in einer Regelschulklasse durchgeführt.

\section{Umsetzung:}

- „Sprung ins kalte Wasser“ durch Praxiserfahrung bereits nach kurzer Vorbereitungszeit in erster Iteration

- Schrittweise Öffnung des geschützten Raumes durch Veränderung der komplexitätsreduzierenden Faktoren im Laufe der Iterationen

\section{Aufbau der Lernumgebung und Reduktion der Komplexität}

Der Konzeption der Lernumgebung liegen wesentliche Entscheidungen bezüglich der Komplexitätsreduktion zu Grunde. Es ist daher sinnvoll, sowohl Aufbau und Konzeption der Lernumgebung als auch die getroffenen Entscheidungen zur Reduktion der Komplexität gemeinsam aufzugreifen. Eine Übersicht der beschriebenen Regler findet sich in Abbildung 2. Die Facetten Heterogenität der Lerngruppe und Vertrautheit des Settings werden dabei erst im nächsten Kapitel genauer beschrieben, da sich diese innerhalb des Verlaufs des Lehr-Lern-Labors ändern. Angegeben wurde daher in Abbildung 2 ein „Mittel“ der Lernumgebung.

Die folgende Beschreibung beschränkt sich auf Merkmale und Faktoren, die für die gesetzten Schwerpunkte des Seminars (die Erhaltung von Handlungsfähigkeit bei ungeplanten Situationen im Allgemeinen sowie im Rahmen von Experimenten) und damit für die Konzeption einer dahinzielenden Lernumgebung von besonderer Bedeutung sind. Neben dem generellen Aufbau der Lernumgebung werden dazu ...

1. die Art der Praxiserfahrungen und

2. die Reflexionsphasen

aufgegriffen und erläutert. 


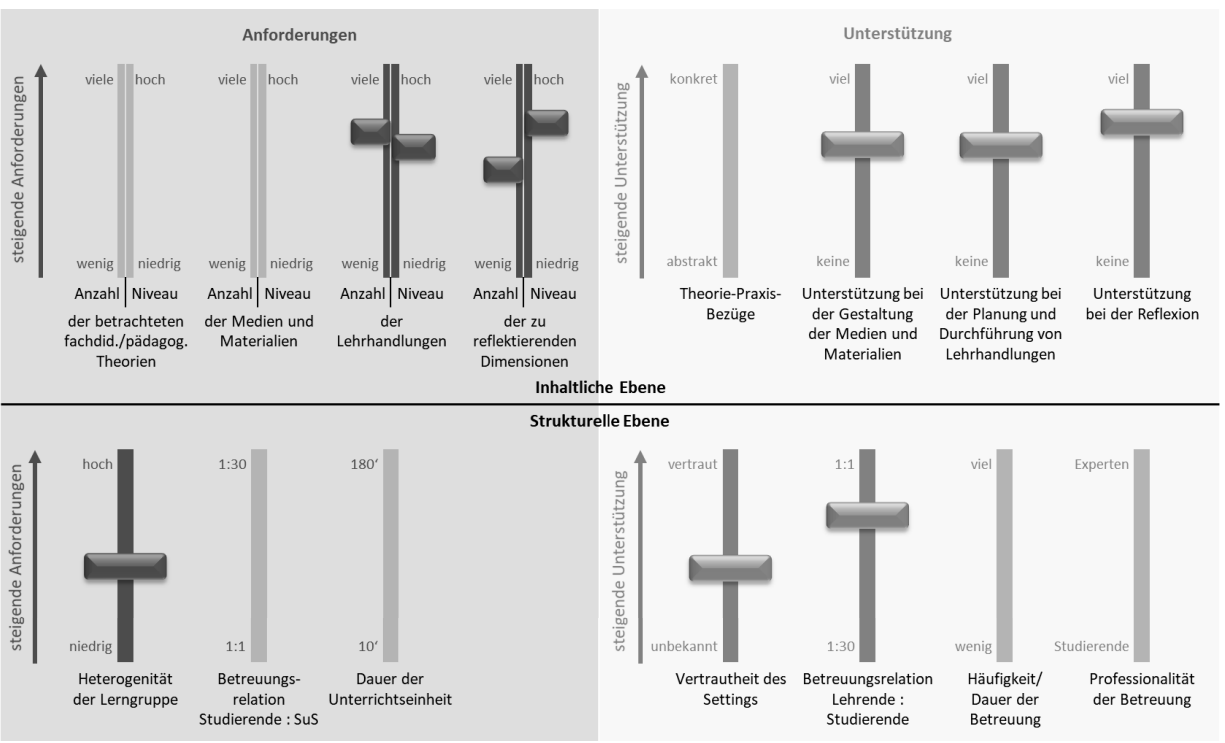

Abbildung 2: Übersicht über die beschriebenen Regler des Planungs- und Reflexionsmodells

\subsection{Ablauf}

Dem Lehr-Lern-Labor La:gune liegt eine für solche Lernumgebungen typische Abfolge von Vorbereitungsphase, Praxisphase und Reflexionsphase zu Grunde (siehe Abb. 3). Weiterführend wird diese Abfolge jedoch dreifach wiederholt (Iterationen). Die von Studierenden in der Vorbereitungsphase geplanten Unterrichtseinheiten bleiben dabei zu großen Teilen bestehen, sodass die Vorbereitungsphasen der zweiten und dritten Iteration der Optimierung der Einheiten gewidmet werden können. Die jeweiligen Praxisphasen werden

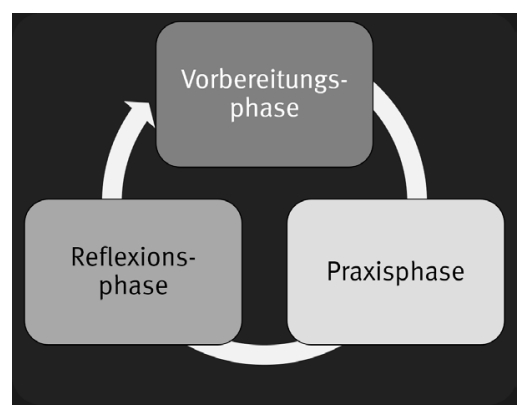

Abbildung 3:

Ablauf einer Iteration im Lehr-LernLabor La:gune auf Video aufgezeichnet, um eine videogestützte Reflexion zu ermöglichen. Zwischen erster und zweiter Iteration findet zudem ein Zwischenblock statt, in dem Inputs zu beispielsweise physikalischen Fachmethoden und zur Unterrichtsplanung gegeben werden. Zu Gunsten einer steigenden Realitätsnähe wird der geschützte Raum der Lernumgebung mit steigender Iteration schrittweise geöffnet (siehe Abb. 4). Dies geschieht im Wesentlichen durch eine Änderung der Lerngruppe. Die erste der drei Iterationen findet dabei nur mit den Studierenden des eigenen Seminars statt, die hier als Lerngruppe dienen. In der zweiten Iteration wird diese Lerngruppe verändert, nun dienen Studierende eines 


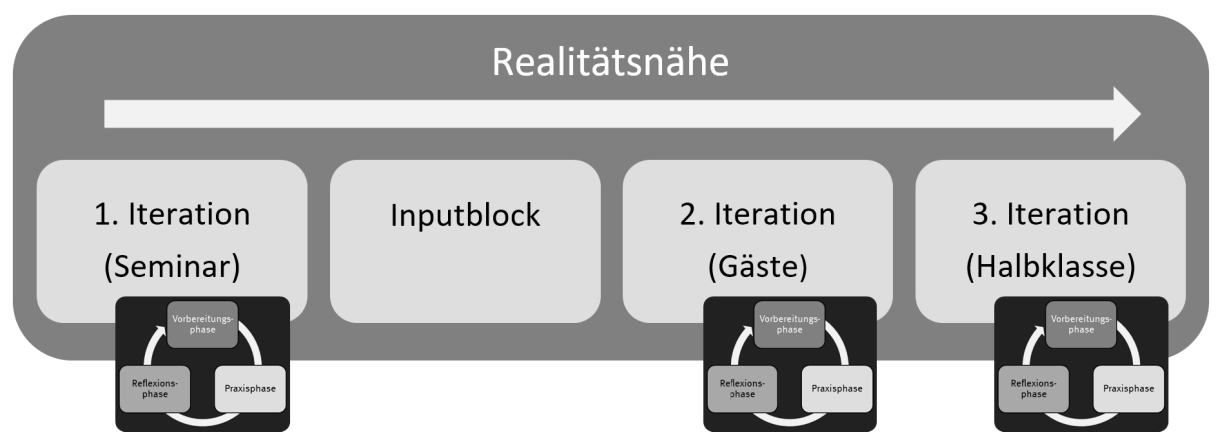

Abbildung 4: Übersicht über den Ablauf des Lehr-Lern-Labors La:gune

anderen Seminars als Lernende. In der letzten Iteration werden die Unterrichtsminiaturen schließlich in einer Regelschulklasse durchgeführt.

\subsection{Art der Praxiserfahrung}

Angelehnt an das Konzept der „Unterrichtsminiatur“ von beispielsweise Korneck et al. (2014) umfasst die Praxiserfahrung des Lehr-Lern-Labors La:gune auf 12 Minuten reduzierte Unterrichtseinheiten. Eine Unterrichtsminiatur ist eine solche kurze Unterrichtseinheit, die trotz ihrer zeitlichen Kürze eine geschlossene Lehreinheit bildet. Dem physikdidaktischen Schwerpunkt entsprechend beinhaltet jede der Unterrichtsminiaturen ein einfaches, quantitatives Experiment.

Durch die Wahl einer vollständigen Unterrichtsminiatur steigen die Anforderungen auf inhaltlicher Ebene deutlich an (vgl. Abb. 2): Die Anzahl verschiedener Lehrhandlungen ist hoch. Auch das Niveau dieser Lehrhandlungen wird durch den kurzen Zeitrahmen stark angehoben. Aufgefangen werden diese hohen Anforderungen durch ein hohes Maß an Unterstützung bei der Planung dieser Lehrhandlungen sowie bei der Gestaltung von Medien und Materialien. Dies kann insbesondere durch Planungsphasen innerhalb der Seminarzeit und eine kleine Betreuungsrelation Lehrende zu Studierende von ca. zwei zu ca. zehn erreicht werden.

Begründung der Wahl: Die äußerst kurze Unterrichtszeit von 12 Minuten wird durch die Notwendigkeit der Iteration sowie einer tiefgreifenden Reflexion bedingt. Im Gegensatz zu vielen anderen Formaten mit kurzer Unterrichtszeit handelt es sich um „vollständige“ Unterrichtseinheiten statt eines Lehrens einzelner Unterrichtsphasen (beispielsweise Hinführung). Besonders durch den Schwerpunkt auf das physikalische Experiment ist dies notwendig, da diese in wesentlichen Teilen durch die Vor- und Nachbereitung im Unterricht ausgemacht werden (Tesch \& Duit, 2002). Außerdem wird somit keine genaue Absprache mit anderen Studierenden im Sinne eines Teamteachings notwendig, da die Unterrichtsminiaturen für sich stehen können. 


\subsection{Reflexionsphasen}

Die Reflexion macht den weitaus größten und wichtigsten Teil des Lehr-LernLabors La:gune aus. Innerhalb der theoretischen Betrachtung wurden zwei große Anforderungen an die Reflexionsphasen deutlich:

a) Eine ausführliche und tiefgehende Analyse, die Handlungsoptionen und -möglichkeiten sowie Ursachen und Entstehung der Situationen aufzeigt:

Um eine solche Art der Analyse zu ermöglichen, wird diese anhand des in Kasten 1 beschriebenen Stressmodells von Lazarus und Folkman (1984) durchgeführt. Der Stressbegriff wird dabei innerhalb des Seminars vermieden, da er sowohl negativ konnotiert als auch in der psychologischen Literatur uneinheitlich definiert ist (Lazarus \& Folkman, 1984). Der Bezug des Modells erfordert nicht nur die Analyse verfügbarer Handlungsoptionen im Rahmen der „sekundären Bewertung", sondern bezieht vorausgehende Ereignisse sowie die tatsächliche Handlung und daraus ableitbare Folgerungen mit ein. Anhand der Videos und zugehöriger Transkripte können die Situationen mithilfe des theoretischen Rahmens kleinschrittig analysiert und Handlungsalternativen entwickelt werden.

Diese tiefgreifende Reflexion sorgt für hohe Anforderungen durch Anzahl und Niveau der zu reflektierenden Dimensionen. Das hohe Niveau entsteht durch die tiefgreifende Natur der Reflexion, während durch die Betrachtung verschiedener Ursachen und Aspekte auch die Anzahl der zu reflektierenden Dimensionen eine mehr als niedrige Anforderung darstellt (vgl. Abb. 2).

b) Eine Reflexion, die in einem geschützten Raum stattfindet, sodass negativ konnotierte Situationen dennoch zu effektiven Reflexionen führen können:

Der strukturelle Rahmen der Lernumgebung ist wertungsfrei und die Reflexion findet stets in kleineren Gruppen mit hohem Betreuende-zu-Studierenden-Verhältnis statt. Die Reflexionen werden in kleineren Gruppen unter Dozentenanleitung durchgeführt. Neben diesen strukturellen Maßnahmen und gängigen Reflexionsregeln wie ausbleibende Rechtfertigungen und das Beharren auf konstruktive Rückmeldungen findet eine letzte wesentliche Maßnahme statt, um eine eventuelle negative Konnotation zu kompensieren: Die Studierenden erhalten bereits im Vorfeld auf die Reflexionsphasen ein hohes Maß an Mitbestimmung. Zu reflektierende Situationen werden von den „Betroffenen“ selbst bewertet und zur Analyse ausgewählt. Dies sorgt dafür, dass

- unangenehme Situationen nicht reflektiert werden müssen,

- Situationen, die analysiert werden, vorab bekannt sind und

- von Studierenden als wichtig empfundene Situationen analysiert werden.

Durch diesen geschaffenen Rahmen sowie klare Reflexionsregeln und unterstützende Materialien entsteht das äußerst hohe Maß an Unterstützung bei der Refle- 
xion (vgl. Abb. 2), mit dem Ziel die hohe Anforderung in den Reflexionen auszugleichen.

\section{Veränderung der Komplexität - Dreischritt}

Durch mehrfache Iteration und zwischenzeitliche Reflexion sowie Optimierung der Unterrichtsminiaturen wird die Anwendung und ggfs. Optimierung herausgearbeiteter Handlungsalternativen ermöglicht. Voraussetzung ist dafür, dass sich die Unterrichtsminiaturen durch die Überarbeitungen nicht zu sehr von der ursprünglichen Form entfernen. So besteht trotz leichter Veränderungen die Möglichkeit in Reflexionen ausgearbeitete Handlungsweisen auszuprobieren. Während sich die drei Iterationen thematisch höchstens leicht im Rahmen der von Studierenden umgesetzten Optimierungen unterscheiden, ändern sich einzelne Facetten der Komplexitätsreduktion über die drei Iterationen deutlich. Dabei ist grundlegend zu unterscheiden zwischen Änderungen, die gezielt stattfinden, und Änderungen, die abhängig von primären Änderungen (reaktiv) stattfinden. Besonders durch die Iteration der Einheiten tritt auch letzterer Fall sichtbar auf.

In diesem Kapitel werden beide Fälle beispielhaft an den Facetten Heterogenität der Lerngruppe und Vertrautheit des Settings verdeutlicht. Im Falle der Vertrautheit des Settings ist dabei eine feinere Aufteilung der Facette notwendig, um die Auswirkungen hinreichend genau beschreiben zu können.

\subsection{Heterogenität der Lerngruppe - gezielte Änderung der Komplexität}

Die Gruppe der Lernenden wird über die drei Iterationen stetig verändert. Dies stellt eine wesentliche Möglichkeit zur Steuerung der Komplexität dar. Im Reglermodell kann diese Veränderung am besten am Beispiel des Reglers Heterogenität der Lerngruppe festgemacht werden. Dieser steigt über die verschiedenen Iterationen der Lernumgebung an (siehe Abb. 5). Diese Veränderung stellt eine gezielte Entscheidung bei der Konzeption des Lehr-Lern-Labors dar und wurde als solche gesteuert, um den geschützten Raum schrittweise $\mathrm{zu}$ Gunsten steigender Realitätsnähe abzubauen.

1. Iteration: Hier besteht die Lerngruppe ausschließlich aus Teilnehmerinnen und Teilnehmern

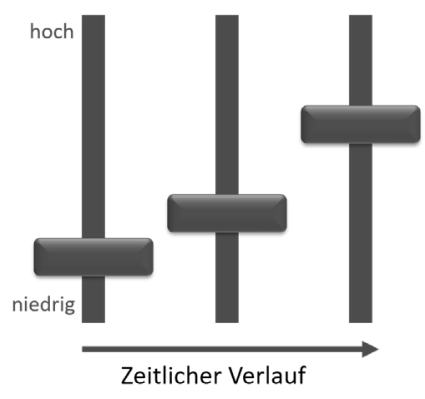

Abbildung 5:

Veränderung des Reglers „Heterogenität der Lerngruppe“ im Verlauf der drei Iterationen des eigenen Seminars. Diese Gruppe ist der jeweils lehrenden Person bekannt und durch den Hintergrund des einheitlich vor- 
handenem physikdidaktischen Studiums sehr homogen (niedrige Heterogenität der Lerngruppe).

2. Iteration: Die Gruppe der Lernenden besteht hier noch immer aus Studierenden der Physikdidaktik. Die diesbezügliche Homogenität bleibt daher größtenteils bestehen. Im Gegensatz zur ersten Iteration handelt es sich nun aber nicht mehr um Studierende, die zum Zeitpunkt der Durchführung dieselbe Lernumgebung durchlaufen, sondern um (junge) Studierende einer anderen Veranstaltung. Diese Lerngruppe kennt weder die Themen Unterrichtsminiaturen noch die Studierenden selbst. Die Heterogentität der Lerngruppe steigt somit gering an.

3. Iteration: Die letzte Iteration findet mit einer halben Schulklasse in einer Regelschule statt. Neben einer Änderung der Vertrautheit des Settings durch andere Räumlichkeiten (s.u.) steigt die Heterogenität der Lerngruppe damit weiter zu Gunsten einer realitätsnäheren Situation.

\subsection{Vertrautheit des Settings - reaktive Änderung der Komplexität}

Hier finden sich im Rahmen einer mehrfachen Iteration von Unterrichtsminiaturen Besonderheiten in der Auswirkung auf die Komplexität der Lehrsituation, die nur bedingt gesteuert werden können. Sie werden durch die strukturelle Entscheidung der mehrfachen Iteration ausgelöst. Der Regler Vertrautheit des Settings kann dabei in zwei Unterkategorien unterteilt werden, um getroffene Entscheidungen besser zu verdeutlichen: Die Vertrautheit mit der Praxiserfahrung (siehe Abb. 6) und die Vertrautheit mit dem Material und den Räumlichkeiten (siehe Abb. 7).

\subsubsection{Vertrautheit mit der Praxiserfahrung}

Durch die wiederholte Beschäftigung mit einer Unterrichtsminiatur zur selben Thematik werden die Studierenden zunehmend vertrauter mit dieser Art der Praxiserfahrung. Dies umfasst unter anderem ungeplante Ereignisse, die beispielsweise bei einer früheren Durchführung auftraten. Diese wurden ggfs. reflektiert und können damit vermieden werden. Durch längere Auseinandersetzung mit dem fachlichen Inhalt können außerdem eigene fachliche Wissenslücken und Fehlkonzepte ausgebessert werden. Letztlich spielt hier auch das Format der Praxiserfahrung selbst eine sehr große Rolle. Die begrenzte Zeit (in diesem Fall 12 Minuten) und die daraus resultierende Reduktion der Unterrichtsinhalte stellte

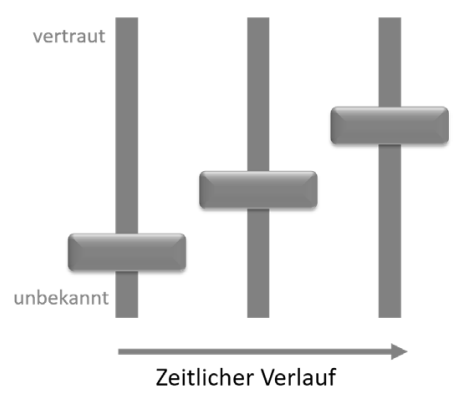

Abbildung 6:

Schieberegler für „Vertrautheit des Settings“ Teilaspekt „Vertrautheit mit der Praxiserfahrung " über den Verlauf der drei Iterationen 
beispielsweise in vielen Iterationen ein Problem für Studierende dar. Im Laufe der Veranstaltung konnten diese oftmals zunehmend besser eingeschätzt werden. Eine zunehmende Gewöhnung führt damit zu einer Reduzierung dieser Facette der Komplexität, die Vertrautheit mit der Praxiserfahrung steigt stetig an.

\subsubsection{Vertrautheit mit dem Material und den Räumlichkeiten}

Ähnlich der Vertrautheit mit Praxiserfahrung wächst über drei Iterationen auch die Vertrautheit mit dem Material. Arbeitsblätter oder Tafelbilder etwa werden von den Studierenden im Anschluss an die Reflexionen weiter optimiert. Hervorzuheben ist hier vor allem auch die Vertrautheit mit den genutzten Experimenten. Als wesentlicher Bestandteil werden auch diese von den Studierenden zwischen den einzelnen Iterationen weiter optimiert und damit ggfs. stabilisiert. Gerade eine solche tiefere Auseinandersetzung mit Experimenten führt zu besserer Bekanntheit von deren Eigenheiten, Einflussfaktoren, Genauigkeit und vielem mehr. Die dritte Iteration findet in den Räumlichkeiten der Schule

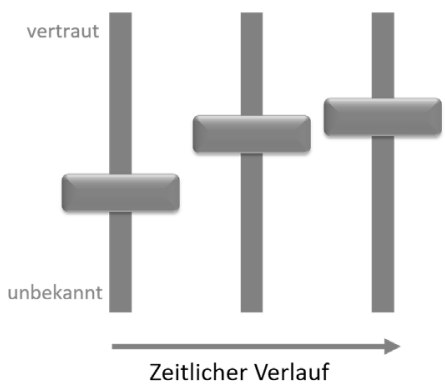

Abbildung 7:

Schieberegler für „Vertrautheit des Settings“ Teilaspekt „Vertrautheit mit dem Material und den Räumlichkeiten“ über den Verlauf der drei Iterationen statt. Dadurch steigt die Komplexität leicht an, da beispielsweise ein spontanes Nutzen der angrenzenden Sammlung erschwert wird. Die von den Studierenden genutzten Materialien und Versuchsaufbauten bleiben jedoch auch in dieser Iteration erhalten. Die Vertrautheit mit dem Material und den Räumlichkeiten steigt somit über die drei Iterationen an, wobei zwischen zweiter und dritter Iteration nur ein kleiner Anstieg vorliegt.

\subsection{Diskussion}

Die Veränderung der Komplexität über mehrere Iterationen erscheint (zumindest bei dem gewählten Schwerpunkt dieses Lehr-Lern-Labors) sinn- und wirkungsvoll. Die Iteration selbst zieht allerdings schon einige Änderungen der Komplexität mit sich, die in der Konzeption und „Einstellung der Regler“ mitbedacht werden sollten. Genannt wurde hier beispielsweise die Vertrautheit des Settings. Diese steigt im diskutierten Beispiel bereits durch das Stattfinden der Iteration selbst. Die „Einstellung“ eines Reglers suggeriert eine aktive Entscheidung der Wahl des Reglers. Dies ist in vielen Fällen auch der Fall: Die gelungene und gewollte Verschiebung einzelner Regler wie die Heterogenität der Lerngruppe demonstriert dies. 
Im Falle der konzipierten Lernumgebung wurde das Steigen der Vertrautheit durch Wiederholung aktiv genutzt: Die Anforderungen durch beispielsweise die Heterogenität der Lerngruppe konnte bei späteren Iterationen angehoben werden, ein Gleichgewicht zwischen Anforderungen und Unterstützung wurde dennoch gewahrt.

Ebenso können im Rahmen der Komplexität der Lernumgebung sehr subjektiv empfundene Faktoren eine Rolle spielen: Eine Unterrichtseinheit unter Studierenden, die jedoch nicht im Seminar selbst sind (2. Iteration), wurde von einzelnen Studierenden beispielsweise als "fordernder" angesehen als eine Unterrichtseinheit vor einer unbekannten Schülergruppe (3. Iteration). Als Gründe wurden von Studierenden in Interviews unter anderem die fachliche Kenntnis der Studierenden und die hohe Wahrscheinlichkeit, die Schülerinnen und Schüler „nie wieder zu sehen", genannt.

\section{Erfahrungen und empirische Ergebnisse}

Die Umsetzung des konzipierten Lehr-Lern-Labors wurde durch ein breites Spektrum von empirischen Instrumenten begleitet. Um das Gelingen der Implementation des Lehr-Lern-Labors zu prüfen, kann dazu im Wesentlichen auf zwei der Instrumente zurückgegriffen werden:

- Die Selbstwirksamkeitserwartung bezüglich des Experimenteinsatzes im Physikunterricht (Instrument nach Meinhardt, 2018) wurde zu verschiedenen Zeitpunkten erhoben. Neben Beginn und Ende der Lernumgebung wurde diese Skala auch zwischen den einzelnen Iterationen eingesetzt, um eine detailliertere Betrachtung der Entwicklung der Selbstwirksamkeitserwartung zu ermöglichen.

- Zum Abschluss des Lehr-Lern-Labors wurden leitfadengestützte Interviews mit 27 teilnehmenden Studierenden geführt. Neben Einschätzungen zu verschiedenen konzeptionellen Entscheidungen wurden hier - gestützt durch kurze Videosequenzen - einzelne kritische Unterrichtssituationen betrachtet um eine Einschätzung bezüglich der emotionalen Wahrnehmung der Situation zu erlangen.

Mithilfe dieser zwei Instrumente kann nachvollzogen werden, inwiefern die Anforderungen $\mathrm{A}_{1}$ bis $\mathrm{A}_{3}$ in der Umsetzung des Lehr-Lern-Labors erfolgreich erfüllt wurden:

\section{Diskrepanzen zwischen Theorie und Praxis (A1)}

Für eine Brücke zwischen Theorie und Praxis im Aspekt des Umgangs mit ungeplanten Ereignissen wurden im Wesentlichen zwei Anforderungen gestellt: a) eine Reflexion von Praxiserfahrungen, die eine tiefgehende Analyse der Situation und die Ausarbeitung von Handlungsalternativen beinhaltet, und b) die Erprobung dieser Handlungsalternativen in ähnlichen Situationen. 
Auf Rückfrage nach dazu hilfreichen Aspekten innerhalb der Reflexionssitzungen wurde in vielen der Interviews auf die Nutzung von Videos hingewiesen. Hervorgehoben wurde dabei insbesondere ein vereinfachtes Hineinversetzen in die Situation, im Vergleich zu anderen von den jeweiligen Studierenden erlebten Reflexionsprozessen. Die erhoffte starke Immersion, die der Nutzung von Videoaufnahmen zugesprochen wird (Goldman, 2007), scheint auch hier vorfindbar zu sein. Auch die Möglichkeit, eine Videosequenz mehrfach und unter anderen Gesichtspunkten anschauen zu können, wurde in den Interviews aufgeführt.

Die tiefgehende Analyse einzelner Unterrichtsszenen empfanden alle befragten Studierenden als hilfreich, vor allem die Kombination mit der Möglichkeit, die Unterrichtsminiaturen zu optimieren und erneut zu unterrichten, wurde als positiv hervorgehoben. In wenigen Fällen wurde angemerkt, dass das Vorgehen teilweise zu kleinschrittig war oder eine zusätzliche, allgemeinere Betrachtung erwünscht war. Auch die Iteration der Durchführungen wurde als hilfreich eingeschätzt, da die in Reflexionen erarbeiteten Punkte so ausgetestet werden konnten.

Als Stütze für die tiefgehende Analyse wurde das Stressmodell von Lazarus und Folkman (1984) eingesetzt. Das Modell diente dabei der in relevante Teile strukturierten Aufarbeitung einer Situation. Besonders für die Analyse der Situation wurde es dabei von den Studierenden nur manchmal als hilfreich beschrieben. Viele der Befragten gaben an, dass die Nutzung innerhalb der Reflexionssitzungen - angeleitet durch Dozenten - als hilfreich empfunden wurde, das Modell jedoch für den eigenen Einsatz in Vor- und Nachbereitung der Unterrichtsminiaturen zu komplex und kleinschrittig sei. Eine Adaption des Modells, die es auf wesentliche, für die Reflexion wichtige Komponenten reduziert, scheint angebracht.

Den Einschätzungen der Studierenden nach wurden demnach die spezifischen Anforderungen, die für eine erfolgreiche Verknüpfung von Theorie und Praxis hergeleitet wurden, erfüllt. Für eine effektivere Gestaltung der Reflexionsanlässe scheint jedoch die Reduktion des genutzten Modells erforderlich.

\section{Emotionale negative Konnotation und Blockade kritischer Reflexion (A2)}

Die Nutzung eigener Unterrichtsvideos kann dazu führen, dass eine kritische Selbstreflexion im Gegensatz zur Nutzung fremder Videos gehemmt wird (vgl. Ergebnisse aus Seidel, Stürmer, Blomberg, Kobarg \& Schwindt, 2011). Ein wesentlicher Grund dafür ist die mögliche emotional negative Konnotation, die eine kritische Auseinandersetzung mit dem eigenen Handeln mit sich ziehen kann. Besonders der Fokus auf ungeplante Situationen könnte dies verstärken, da die Situation selbst bereits als negativ empfunden werden kann. Der gewählte Schwerpunkt der Lernumgebung bedingt jedoch dieses Nutzen eigener Videos. Um dennoch kritische und damit hilfreiche Selbstreflexionen zu initiieren, wurden mehrere strukturelle Maßnahmen getroffen, die eine mögliche Blockade verhindern sollten.

Keine Studierenden gaben in den Interviews an, sich innerhalb der Reflexionssitzungen unwohl gefühlt zu haben. Als Gründe gaben die Studierenden hier eine positive Atmosphäre, kleine Gruppen und die Nutzung der Videos an. Letzterer 
Grund wurde damit ergänzt, dass durch die Videonutzung der Fokus von der Person abgelenkt und auf das Video gesetzt wird. Auch der Fokus auf ungeplante Situationen wurde nach Angabe der Studierenden nicht als negativ empfunden, da die Vorauswahl der Szenen durch die Studierenden selbst getroffen werden konnte. Eine Blockade von Reflexionen scheint auch bei Betrachtung schriftlicher Selbstreflexionen der Studierenden nicht der Fall sein. Eine negative Konnotation der betrachteten Situationen selbst (in action) wird dagegen in einzelnen Fällen sowohl durch schriftliche Reflexionen als auch durch Aussagen in den Interviews deutlich. Demnach waren die Situationen selbst durchaus mit Unwohlsein verknüpft.

Durch die umgesetzten konzeptuellen Maßnahmen im Lehr-Lern-Labor La:gune scheint eine mögliche Blockade von kritischen Selbstreflexionen erfolgreich verhindert worden zu sein. Dies scheint trotz des gewählten Fokus und des rückgemeldeten Unwohlseins während der kritischen Situationen der Fall zu sein.

\section{Praxisschock und Realitätsnähe ( $\left.\mathrm{A}_{3}\right)$}

Die Selbstwirksamkeitserwartung bezüglich der Durchführung von Experimenten in Unterrichtssituationen (Instrument nach Meinhardt, 2018) wurde zwischen den drei Iterationen sowie vorab und im Nachhinein erhoben. Eine signifikante Aussage kann auf Grund der zu geringen Stichprobe zu diesem Zeitpunkt nicht gemacht werden, erkennbare Trends sprechen aber für eine positive Entwicklung durch das Seminar, wie sie z. B. in Peuser et al. (2017) gefunden wurde. Weiter wird durch die Erhebung der Selbstwirksamkeitserwartung zu verschiedenen Zeitpunkten deutlich, dass der Trend innerhalb einer Durchführung des Lehr-Lern-Labors nicht rein positiv ist. Aus schriftlichen Reflexionen direkt im Anschluss an die einzelnen Iterationen der Unterrichtsminiaturen geht ebenso hervor, dass insbesondere die erste Iteration (der „Sprung ins kalte Wasser“) teilweise zu Verunsicherung geführt hat. Diese anfängliche Verunsicherung und das damit verbundene Absinken der Selbstwirksamkeitserwartung kann in einigen Fällen durch die Erhebungen nachvollzogen werden, wird aber besonders in den abschließenden Interviews deutlich. Hier wurden Studierende gezielt nach einer ggfs. vorliegenden Verunsicherung durch die erste Iteration befragt. In einigen Fällen gaben Studierende eine (teilweise als "sehr groß" bezeichnete) zweitweise Verunsicherung an. Ohne Ausnahme wurde diese zeitweise Verunsicherung jedoch auf den ganzen Seminarverlauf gesehen nicht als negativ angesehen und in einigen Fällen sogar als äußerst hilfreich bewertet.

Auch die schrittweise Veränderung der Komplexität durch die Änderung der Lerngruppe wurde zu Gunsten einer realitätsnäheren Praxiserfahrung als hilfreich eingeschätzt. Als realitätsfern und daher als wenig hilfreich wurde die kurze Länge der Unterrichtsminiaturen genannt. In den meisten Fällen wurde jedoch geäußert, dass die Länge dennoch gar nicht oder nur leicht geändert werden sollte, da die Wiederholung und ausgiebige Reflexion als wichtiger erachtet würden. Der in einigen Fällen als hilfreich vermutete "Wurf ins kalte Wasser" scheint gelungen und 
von Studierenden als positiv wahrgenommen zu werden. Auch die Steigerung der Realitätsnähe scheint erfolgreich zu sein.

\section{Fazit und Ausblick}

Rückblickend kann zusammengefasst werden, dass eine Lernumgebung mit Fokus auf ungeplante Lehrsituationen unter der Berücksichtigung der hergeleiteten Anforderungen erfolgreich umsetzbar ist. So kann beispielsweise die Blockierung kritischer Selbstreflexionen durch geeignete strukturelle Maßnahmen vermindert werden, um konstruktive Reflexionsprozesse trotz des gewählten Fokus zu initiieren. Insbesondere die dreifache Iteration der Unterrichtsminiaturen und die daraus entstehende Möglichkeit, die in Reflexionen entwickelten Handlungsalternativen zu erproben, sind diesbezüglich hervorzuheben. Selbst bei einem „Wurf ins kalte Wasser", der durchaus Verunsicherung und Unwohlsein hervorzurufen scheint, kann eine solche Reflexion in Kombination mit geeigneten Maßnahmen erfolgreich stattfinden.

Bedacht werden sollte jedoch bei einer mehrfachen Iteration von Unterrichtseinheiten, dass diese Iteration in sich bereits eine Veränderung der Komplexität in verschiedenen Facetten mit sich bringen kann, ohne dass diese gezielt initiiert wird. Als Beispiel für eine solche Änderung wurde die Facette Vertrautheit des Settings diesbezüglich dargestellt. Das diskutierte Beispiel demonstriert, dass diese Änderung nicht unbedingt als negativ anzusehen ist, sondern zu Gunsten einer Lernumgebung genutzt werden kann. So kann die Komplexität an anderer Stelle somit gesteigert werden.

\section{Literatur}

Bandura, A. (1976). Lernen am Modell: Ansätze zu einer sozial-kognitiven Lerntheorie. Stuttgart: Klett.

Bandura, A. (1997). Self-efficacy: The exercise of control. New York: W.H. Freeman.

Baum, B. E. \& Gray, J. J. (1992). Expert modelling, self-observation using videotape, and acquisition of basic therapy skills. Professional Psychology Research and Practice, 23 (3), 220-225. https://doi.org/10.1037/0735-7028.23.3.220

Baumert, J. \& Kunter, M. (2011) Das Kompetenzmodell COACTIV. In M. Kunter, J. Baumert, W. Blum, U. Klusmann, S. Krauss \& M. Neubrand (Hrsg.), Professionelle Kompetenz von Lehrkräften. Ergebnisse des Forschungsprogramms COACTIV (S. 29-53). Münster: Waxmann.

Becker, G. E. (1981). Lehrer lösen Konflikte: Ein Studien- und Übungsbuch. Weinheim: Beltz.

Borko, H., Jacobs, J. K., Eiteljorg, E. \& Pittman, M. E. (2008). Video as a tool for fostering productive discussions in mathematics professional development. Teaching and Teacher Education, 24 (2), 417-436. https://doi.org/10.1016/j.tate.2006.11.012 
Buffler, A., Lubben, F. \& Ibrahim, B. (2009). The Relationship between Students' Views of the Nature of Science and their Views of the Nature of Scientific Measurement. International Journal of Science Education, 31 (9), 1137-1156. https://doi. org/10.1080/09500690802189807

Cranach, M. v. (1983). Über die bewusste Repräsentation handlungsbezogener Kognitionen. In L. Montada, K. Reusser \& G. Steiner (Hrsg.), Kognition und Handeln (S. 64-76). Stuttgart: Klett-Kotta.

Eigler, G. (1983). Die Rückkehr des Lehrers. In H. Wollenweber, (Hrsg.), Schule im Brennpunkt (S. 326-346). Paderborn: Schöningh.

Fiske, S. T. (1995). Social cognition. In A. Tesser (Hrsg.), Advanced social psychology (S. 149-193). Boston, MA: McGraw Hill.

Goldman, R. (2007). Video representations and the perspectivity framework: Epistemology, ethnography, evaluation, and ethics. In R. Goldman, R. Pea, B. Barron \& S. J. Derry (Hrgs.), Video research in the learning sciences (S. 3-38). New Jersey: Lawrence Erlbaum Associates.

Gray, J. A. (1987). The psychology of fear and stress (Problems in the behavioural sciences, Bd. 5, 2. Aufl.). New York: Cambridge University Press.

Harlen, W. (1999). Effective teaching of science: A review of research. Glasgow: Scottish Council for Research in Education.

Heinicke, S. (2014). Experimentieren geht nicht ohne (Mess-)Unsicherheiten. Naturwissenschaft im Unterricht Physik: Experimentieren Gestalten, 144, 29-31.

Heinicke, S., Glomski, J, Priemer, B. \& Rieß, F. (2010). Aus Fehlern wird man klug Über die Relevanz eines adäquaten Verständnisses von „Messfehlern“ im Physikunterricht. Praxis der Naturwissenschaften - Physik in der Schule, 59(5), 26-33.

Heinicke, S. \& Holz, C. (2018). Mit Messfehlern umgehen und Messungen evaluieren. Neue Wege der Fehlerbetrachtung am Beispiel der e/m-Bestimmung. Naturwissenschaft im Unterricht Physik, 168, 18-23.

Holz, C. \& Heinicke, S. (2019). Messunsicherheit - ein ungeliebter Gast im Physikunterricht? In C. Maurer (Hrsg.), Naturwissenschaftliche Bildung als Grundlage für berufliche und gesellschaftliche Teilhabe, Gesellschaft für Didaktik der Chemie und Physik. Jahrestagung in Kiel 2018 (S. 89-92). Regensburg: Universität Regensburg.

Höttecke, D. (2013). A sketch of the problem of authentic inquiry-based learning form a history of science perspective. Paper presented at the Twelfth International History, Philosophy, Sociology \& Science Teaching Conference (IHPST), Pittsburgh, PA.

Hougham, P. (1992). Improving student teachers' strategies for asking a range of both high and low level questions through video evaluations. Ed. D. Practicum, Nova Southeastern University, Fort Lauderdale, FL.

Kanari, Z. \& Millar, R. (2004). Reasoning from data: How students collect and interpret data in science investigations. Journal of Research in Science Teaching, 41(7), 748-769. https://doi.org/10.1002/tea.20020

Kelly, G. J., Brown, C. \& Crawford, T. (2000). Experiments, contingencies, and curriculum: Providing opportunities for learning through improvisation in science teaching. Science Education, 84 (5), 624-657. https://doi.org/10.1002/1098-237X(200009) 84:5<624::AID-SCE5>3.0.CO;2-S

Korneck, F., Kunter, M., Oettinghaus, L., Lamprecht, J. \& Sach, M. (2014). Analyse von Unterrichtshandeln in komplexitätsreduzierten Sequenzen. In S. Bernholt (Hrsg.), 
Naturwissenschaftliche Bildung zwischen Science- und Fachunterricht. Jahrestagung der GDCP 2013 (S. 138-140). Kiel: IPN.

Krone, A., Hamborg, K. C. \& Gediga, G. (2002). About error-related emotional reactions in humane-computer interaction. Zeitschrift für Arbeits- und Organisationspsychologie, 46(4), 185-200. https://doi.org/10.1026//0932-4089.46.4.185

Lazarus, R. (1999). Stress and Emotion. A New Synthesis. New York: Springer.

Lazarus, R. \& Folkman, S. (1984). Stress, appraisal, and coping. New York: Springer.

LeDoux, J. E. (1996). The Emotional Brain: The Mysterious Underpinnings of Emotional Life. New York: Simon \& Schuster.

Meinhardt, C. (2018). Entwicklung und Validierung eines Testinstruments zu Selbstwirksamkeitserwartungen von (angehenden) Physiklehrkräften in physikdidaktischen Handlungsfeldern. https://doi.org/10.30819/4712

Mutzeck, W. (1988). Von der Absicht zum Handeln. Weinheim: Dt. Studien Verl.

Oser, F. \& Spychiger, M. (2005). Lernen ist schmerzhaft: Zur Theorie des negativen Wissens und zur Praxis der Fehlerkultur. Weinheim: Beltz.

Peterson, J. B., Driver-Linn, E. \& Deyoung, C. G. (2002). Self-deception and impaired categorization of anomaly. Personality and Individual Differences, 33(2), 327-340. https://doi.org/10.1016/So191-8869(01)oo158-1

Peuser, M., Szogs, M., Krüger, M. \& Korneck, F. (2017). Veränderung von Selbstwirksamkeitserwartungen durch Microteaching. In C. Maurer (Hrsg.), Implementation fachdidaktischer Innovation im Spiegel von Forschung und Praxis. Jahrestagung der GDCP 2016. (S. 780-783). Regensburg: Universität Regensburg.

Ruhrig, J. \& Höttecke, D. (2015). Components of Science Teachers' Professional Competence and Their Orientational Frameworks when Dealing with Uncertain Evidence in Science Teaching. Int J of Sci and Math Educ, 13(2), 447-465. https://doi.org/10.1007/ s10763-015-9628-3

Seidel, T., Stürmer, K., Blomberg, G., Kobarg, M. \& Schwindt, K. (2011). Teacher learning from analysis of videotaped classroom situations: Does it make a difference whether teachers observe their own teaching or that of others? Teaching and Teacher Education, 27(2), 259-267. https://doi.org/10.1016/j.tate.2010.08.009

Sherin, M. G. \& van Es, E. A. (2005). Using video to support teachers' ability to notice classroom interactions. Journal of Technology and Teacher Education, 13(3), 475-491.

Sherin, M. G. \& van Es, E. A. (2009). Effects of video club participation on teachers' professional vision. Journal of Teacher Education, 6o, 20-37. https://doi.org/10.1177/ 0022487108328155

Tesch, M. \& Duit, R. (2002). Zur Rolle des Experiments im Physikanfangsunterricht. In CD zur Frühjahrstagung des Fachverbandes Didaktik der Physik in der DPG, Leipzig.

Usher, E. L. \& Pajares, F. (2008). Sources of self-efficacy in school: Critical review of the literature and future directions. Review of Educational Research, 78(4), 751-796. https://doi.org/10.3102/0034654308321456

Wahl, D. (1991). Handeln unter Druck: Der weite Weg vom Wissen zum Handeln bei Lehrern, Hochschullehrern und Erwachsenenbildern. Weinheim: Deutscher Studien Verl.

Zapf, D. (1993). Fehler in der Mensch-Computer Interaktion. Zum Einfluss von Komplexität, Kontrolle und Stress auf Fehler und Fehlerbewältigung. Habilitationsschrift, JustusLiebig-Universität, Gießen. 


\title{
Naturwissenschaftlichen Unterricht planen lernen
}

\author{
Professionalisierung durch Unterrichtserprobungen im \\ Lehr-Lern-Labor der Sachunterrichtsdidaktik
}

Im Rahmen der Qualitätsoffensive Lehrerbildung an der Westfälischen WilhelmsUniversität (WWU) Münster wurde eine Lehrveranstaltung implementiert, in der Grundschulstudierende lernen, naturwissenschaftlichen Sachunterricht zu planen. Als Lehrformat wurde das Lehr-Lern-Labor eingesetzt, um den Studierenden neben einem theoretischen auch einen praktischen Zugang zum Lernziel zu ermöglichen. So setzen sie in einem komplexitätsreduzierten Setting ihre theoretisch erarbeiteten Unterrichtsplanungen mit Grundschülerinnen und -schülern um, reflektieren diese Erprobung auf Grundlage der Theorie und vertiefen ihre Erfahrungen dann durch eine erneute Erprobung der überarbeiteten Planungen mit wiederum anschließender, theoriebasierter Reflexion. Die Besonderheit der wiederholten praktischen Erprobung wird im Beitrag bei der Analyse der Komplexität durch den Vergleich der Anforderungen und Niveaus einzelner Elemente zwischen erster und zweiter Erprobung verdeutlicht. Darüber hinaus werden erste interviewbasierte Erkenntnisse zur Einschätzung der intendierten Komplexitätsreduktion rund um die Unterrichtserprobung im Lehr-Lern-Labor durch Studierende dargestellt.

Schlüsselwörter: Planungskompetenz, videobasierte Reflexion, Schülervorstellungen

\section{Einleitung}

Im Rahmen der Qualitätsoffensive Lehrerbildung an der Westfälischen WilhelmsUniversität (WWU) Münster werden Lehr-Lern-Labore als neue Lehrformate in Lehrveranstaltungen implementiert, die Lehramtsstudierenden den Erwerb von Praxiserfahrung im universitären Kontext ermöglichen. Eine besondere Qualität dieser speziellen Art der Praxiserfahrung, im Rahmen derer authentische LehrLern-Situationen mit Schülerinnen und Schülern an der Universität für Studierende geschaffen werden, wird in den Möglichkeiten der Komplexitätsreduktion gesehen. In diesem Zusammenhang wurde ein Modell entwickelt, das die Planung und Analyse der Komplexität einer Praxiserfahrung im Lehr-Lern-Labor erleichtert (Marohn, Greefrath, Hammann, Hemmer, Kürten \& Windt, in diesem Band). Im vorliegenden Beitrag steht eine Lehrveranstaltung im Fokus der Betrachtung, in der zur Förderung der Planungskompetenz von Lehramtsstudierenden die tatsächliche Erprobung einer eigenen Unterrichtsplanung im Lehr-Lern-Labor in den Seminarverlauf eingebunden wird. Die Lehrveranstaltung zeichnet sich durch verschiedene komplexitätsreduzierende Merkmale auf inhaltlicher und strukturel- 
ler Ebene aus. Teilnehmende sind Masterstudierende im Rahmen des Sachunterrichtstudiums für das Grundschullehramt.

Im Folgenden werden zunächst die theoretische Verortung und die Ziele der Veranstaltung verdeutlicht sowie das Konzept und der Ablauf der Veranstaltung detailliert erläutert. Anschließend folgt eine Beschreibung der komplexitätsreduzierenden Elemente in dieser Veranstaltung, indem ausgewählte Aspekte der Anforderungen und Unterstützungsmaßnahmen auf inhaltlicher und struktureller Ebene dargestellt werden. In einem weiteren Abschnitt werden erste Erkenntnisse zu den komplexitätsreduzierenden Maßnahmen im Lehr-Lern-Labor dargestellt, die aus Interviews mit Studierenden abgeleitet wurden. Die Studierenden wurden dahingehend befragt, wie hilfreich sie einzelne Aspekte der Erprobung im Lehr-Lern-Labor wie z. B. die Wiederholung, die Aufnahme eines Videos oder das Agieren in einer Kleingruppe für die Weiterentwicklung ihrer Planungskompetenz einschätzen. Diese Erkenntnisse werden abschließend und mit Erfahrungen aus der Durchführung der Lehrveranstaltung angereichert diskutiert.

\section{Theoretische Verortung und Ziele der Veranstaltung}

Die Kernaufgabe von Lehrkräften ist „die gezielte und nach wissenschaftlichen Erkenntnissen gestaltete Planung, Organisation und Reflexion von Lehr- und Lernprozessen sowie ihre individuelle Bewertung und systematische Evaluation“ (Kultusministerkonferenz [KMK], 2004, S. 2). In den von der KMK (2004) formulierten Standards für die Lehrerbildung findet sich das Unterrichten, von der KMK als die Organisation von Lehr-Lern-Prozessen bezeichnet, neben dem Erziehen, Beraten und Innovieren als ein von Lehrkräften zu beherrschender Kompetenzbereich und wird oftmals als das „,Kerngeschäft' einer Lehrkraft“ (Zierer, Werner \& Wernke, 2015, S. 376) bezeichnet. Zentrale Merkmale des Unterrichts sind dabei, dass er institutionalisiert, professionalisiert, in besonderem Maße intendiert und weitgehend planmäßig erfolgt. Auch wenn es keinen kausalen Zusammenhang zwischen der Effektivität von Unterricht und der Qualität einer vorherigen Planung zu geben scheint, so kann die Planung dennoch als Schlüsselstelle des Unterrichtens betrachtet werden, die über Erfolg oder Misserfolg entscheiden kann: Eine gute Planung kann die Wahrscheinlichkeit für das Gelingen von Unterricht erhöhen (Zierer et al., 2015). Da die Unterrichtsplanung eine zentrale Bedeutung für die Ausübung des Lehrerberufs trägt, muss Planungskompetenz während der Lehrerausbildung erworben werden, was bereits in der universitären Phase beginnen kann. Studierende müssen für den Erwerb von Planungskompetenz wissenschaftlich ausgebildet und in der Praxis geschult werden (König, Buchholtz \& Dohmen, 2015). Kiper und Mischke (2009) postulieren neben theoretischen Kenntnissen folgende Schritte als wichtig zum Erwerb von Planungskompetenz: 
1. Analyse der Planungsaufgabe

2. Durchführung der Planung

3. Ausführung der geplanten Handlungen

4. Evaluation der Ausführung

5. Reflexion der Zusammenhänge von Planung und Erfolg der Ausführung

Mit dem dritten Schritt wird demnach eine praktische Erfahrung als sinnvoll für den Erwerb von Planungskompetenz erachtet. Eine solche praktische Erfahrung und damit das Durchführen von Unterricht lässt sich im universitären Rahmen z.B. durch den Einsatz eines Lehr-Lern- Labors umsetzen. Dies erleichtert durch die Möglichkeit komplexitätsreduzierender Elemente zum einen ein behutsames Heranführen der Studierenden an die Praxis ohne eine Überforderung (Krofta \& Nordmeier, 2014) und zum anderen eine intensive Verzahnung zwischen Theorie und Praxis (Mansholt \& Komorek, 2016) durch die direkte Einbindung der Praxiserfahrung in die Lehrveranstaltung.

In der konzipierten Veranstaltung werden verschiedene Teilziele verfolgt, die das Erreichen des übergeordneten Ziels - die Entwicklung von Planungskompetenz der Studierenden - unterstützen. (1) Als Ausgangspunkt sollen die Studierenden Wissen erwerben über zentrale Elemente und Schritte eines Planungsprozesses, über den fachlichen Inhalt des Unterrichtsthemas, über Schülervorstellungen zum Unterrichtsthema sowie über Möglichkeiten der kognitiven Aktivierung mit besonderem Fokus auf der Gestaltung kognitiv aktivierender Lernaufgaben. (2) Darüber hinaus sollen die Studierenden dieses Wissen in einer praktischen Situation für eine eigene Unterrichtsplanung anwenden können, um einen kognitiv aktivierenden Unterricht zu entwickeln. (3) Unterstützt durch die unterrichtspraktische Erfahrung im Lehr-Lern-Labor sowie (Peer-)Feedback-Elemente sollen die Studierenden in die Lage versetzt werden, über Unterrichtsplanungen reflektieren und diese optimieren zu können.

\section{Vorstellung des Veranstaltungskonzepts}

Ausgehend von den eben genannten Zielen wurden im Rahmen der Sachunterrichtsausbildung von Studierenden des Grundschullehramts am Institut für Didaktik des Sachunterrichts die Phasen der Unterrichtsvorbereitung (Planung), der praktischen Durchführung im Lehr-Lern-Labor und der Reflexion in eine bestehende Lehrveranstaltung implementiert. Es handelt sich dabei um eine vierstündige Pflichtveranstaltung im ersten Mastersemester. Die Studierenden haben sich während ihres Bachelorstudiums bereits mit vorgegebenen Unterrichtsplanungen und der Anpassung solcher Planungen beschäftigt. Im Sinne einer sich steigernden Komplexität im Studium werden die Studierenden in der hier beschriebenen Veranstaltung zu Beginn ihres Masterstudiums vor die Herausforderung gestellt, eine eigene Unterrichtsplanung anzufertigen, ohne dabei auf 
bestehende Elemente zurückgreifen zu können. Dabei erhalten sie entsprechende Anleitung und Unterstützung, welche im weiteren Verlauf des Beitrags noch genauer erläutert werden.

Zunächst werden mit den Studierenden drei Planungsmodelle und weitere zentrale theoretische Grundlagen erarbeitet. Bei den Modellen handelt es sich um das Modell der didaktischen Rekonstruktion nach Kattmann (2007), die Didaktische Analyse nach Klafki (1958) und das Berliner Modell nach Heimann, Otto und Schulz (1979). Diese drei Modelle ergänzen sich einerseits und bilden insgesamt den gesamten Verlauf eines Planungsprozesses ab. Andererseits gibt es auch Überschneidungen zwischen den Modellen, die die Wichtigkeit der jeweiligen Aspekte hervorheben und für die Studierenden verdeutlichen. Da keines der Modelle den gesamten Planungsprozess ausreichend im Detail abbildet, wurde eine verbindende und vergleichende Betrachtung dieser Modelle gewählt. Auf die konkreten Inhalte der Modelle kann in diesem Beitrag nicht näher eingegangen werden. Wohl aber werden im Folgenden über die Modelle hinausgehende zentrale, theoretische Grundlagen zur Unterrichtsplanung näher ausgeführt, da diese Aufklärung darüber verschaffen, wie die Studierenden in der vorgestellten Lehrveranstaltung während ihres Planungsprozesses vorgehen. Die Besonderheit der Herangehensweise bei der Unterrichtsplanung liegt darin, dass die Studierenden die Lernprozesse der Schülerinnen und Schüler fokussieren, ihre gesamte Planung damit beginnen und nicht etwa mit vom Lernen der Schülerinnen und Schüler losgelösten Unterrichtsschritten bzw. Handlungen der Lehrkraft. Es wird erwartet, dass diese besondere Perspektive den Planungsprozess für die Studierenden stark strukturiert und die Fokussierung auf die Lernprozesse es ihnen erleichtert, einen kognitiv aktivierenden, auf Schülerinnen und Schüler abgestimmten Unterricht zu entwickeln. Gleichzeitig stellt dies allerdings für Studierende häufig einen neuen Ansatz der Unterrichtsplanung dar, so dass dieser explizit erarbeitet werden muss.

\subsection{Fokussierung auf Lernprozesse von Schülerinnen und Schülern bei der Unterrichtsplanung}

Für die Strukturierung des Planungsprozesses oder genauer für die Fokussierung auf den Lernprozess von Schülerinnen und Schülern wird in der Lehrveranstaltung auf den Ansatz der „kooperativen Planung“ (Giest, 2010, S. 187) zurückgegriffen, der sich wie im Folgenden kurz erläutert als konstruktive Synthese aus verschiedenen anderen Ansätzen darstellt. Bei der Planung von Unterricht lassen sich verschiedene Perspektiven einnehmen. Im traditionellen Unterricht in den 196oerund 1970er-Jahren dominierte ein Lehren-ohne-Lernen-Modell, bei dem durch eine möglichst perfekte Vorplanung des Lehr-Lern-Geschehens, Schülerinnen und Schüler im Unterricht zu den angestrebten Zielen geführt werden sollten (Giest, 2010). Dabei wurden die einzelnen Akteure und insbesondere die Schülerinnen und Schüler nicht in den Blick genommen. In der konstruktivistisch orientierten 
Folgezeit kehrte sich die Perspektive ins Gegenteil und hin zu einem Lernen-ohneLehren-Modell (Giest, 2010). Unter der Annahme, dass Lernen ausschließlich vom Individuum abhängig und damit nicht planbar ist, bestand das Ziel von Unterrichtsplanung darin, möglichst anregende Lernumgebungen zu entwickeln, die von den Lernenden im Unterricht genutzt werden sollten. Die Lehrkraft fungierte in dieser Art von Unterricht lediglich als Moderatorin bzw. Moderator des Lernens. Beide Ansätze führten aufgrund der Vernachlässigung einer der beiden Komponenten Lernen und Lehren nicht zum Erfolg. Daher schlägt z. B. Giest (2010) mit dem Ansatz der kooperativen Planung eine konstruktive Synthese der beiden Ansätze vor, die sich durch eine dialektische Einheit aus Lernen und Lehren, Führung und Selbsttätigkeit auszeichnet. Ein zentraler Grundgedanke dieses Ansatzes ist es, bei der Unterrichtsplanung den Lernprozess der Schülerinnen und Schüler als Ausgangspunkt zu nutzen und davon ausgehend förderliches Lehrverhalten für den Unterricht abzuleiten. Dabei spielt dann auch die Berücksichtigung von Vorstellungen, mit denen Schülerinnen und Schüler in den Unterricht kommen, eine zentrale Rolle. Die Theorieansätze zum Conceptual-Change (z.B. Möller, 2010; Posner, Strike, Hewson, \& Gertzog, 1982), zur Zone der nächsten Entwicklung (Vygotzsky, 1978) sowie zum Scaffolding (z.B. Van de Pol, Volman \& Beishuizen, 2010; Wood, Bruner \& Ross, 1976) sind in diesem Zusammenhang relevant.

Das Ziel des Planungsprozesses in der Lehrveranstaltung, der sich an den Lernprozessen der Schülerinnen und Schüler orientiert und ihre Vorstellungen aufgreift, ist es im allgemeinen, möglichst guten, erfolgreichen Unterricht zu entwickeln: Für die Qualität von Unterricht kommt es insbesondere auf seine Tiefenstrukturen an (Kunter \& Ewald, 2016), sodass diesen auch bei der Planung besondere Aufmerksamkeit gewidmet werden sollte. Mit Tiefenstrukturen ist zum einen die Interaktion der Lernenden mit den Lehrenden und zum anderen die Beschäftigung der Lernenden mit dem Lerninhalt gemeint (Kunter \& Trautwein, 2013). Es werden drei Dimensionen von Tiefenstrukturen unterschieden: Klassenführung, Potenzial zur kognitiven Aktivierung und konstruktive Unterstützung. Im Rahmen des Seminars erfolgt für den Planungsprozess eine Fokussierung auf das Potenzial zur kognitiven Aktivierung und damit auf Aufgaben und die Aufgabenimplementation im Unterricht, da diese Facette am stärksten fachspezifisch ist. Ihre Bedeutung ist für Studierende im Zusammenhang der Unterrichtsplanung sehr hoch und sie bedarf somit einer speziellen Thematisierung im Rahmen der Sachunterrichtsausbildung. Als Hilfe für die konkrete Umsetzung der Dimension der kognitiven Aktivierung bei der Planung von Unterricht erfolgt eine literaturbasierte Auseinandersetzung mit der Gestaltung guter Lernaufgaben und die gemeinsame Erarbeitung einer Kriterienliste. Die Erstellung einer Lernaufgabe ausgehend von vorhandenen Vorstellungen der Schülerinnen und Schüler wird bei der gewählten Vorgehensweise als Kern der Unterrichtsplanung angesehen. Um die bestehende Aufgabe herum werden anschließend weitere Unterrichtsschritte wie ein Einstieg und eine Reflexion sowie das begleitende Lehrverhalten geplant. 
Zusammenfassend kann festgehalten werden, dass die theoretischen Grundlagen für den Planungsprozess im Seminar insgesamt darauf abzielen, die Planung von Unterricht von den Lernprozessen der Schülerinnen und Schüler aus zu denken und die Handlungen der Lehrkraft daran auszurichten. Erfahrungsgemäß wird die Ebene der Tiefenstrukturen oft von Studierenden vernachlässigt und die Planung von Unterricht stark auf das Lehrverhalten bzw. die Beschreibung von Unterrichtsschritten beschränkt. Durch den Fokus auf die Lernprozesse von Schülerinnen und Schülern, d.h. durch die Entwicklung einer Lernaufgabe als Ausgangspunkt der gesamten Planung, kann in der Veranstaltung eine stärkere Auseinandersetzung mit den Tiefenstrukturen von Unterricht während des Planungsprozesses gefördert werden.

\subsection{Ablauf der Lehrveranstaltung}

Die folgende Abbildung 1 zeigt die Struktur der Lehrveranstaltung und die Sequenzierung der einzelnen Sitzungen, was im weiteren Verlauf ausführlich erläutert wird.

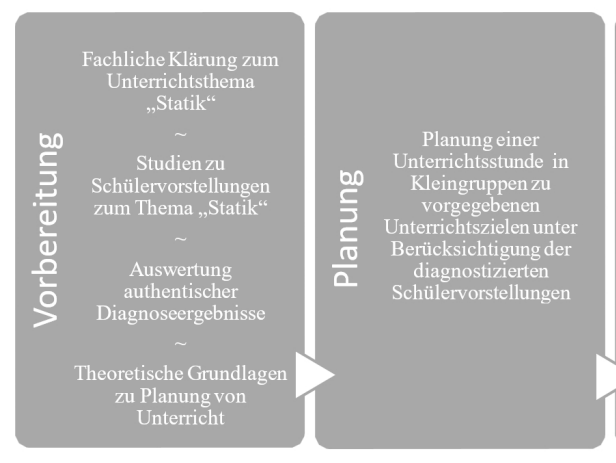

3 Sitzungen

2 Sitzungen

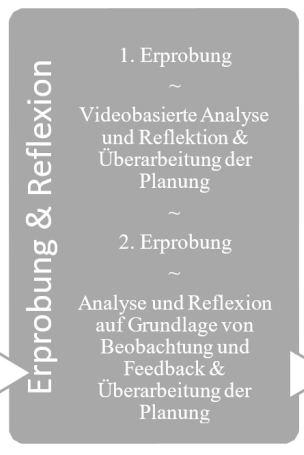

4 Sitzungen

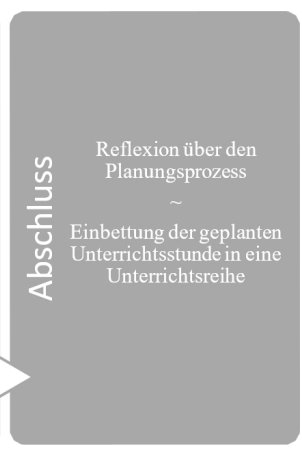

2 Sitzungen

Abbildung 1: Überblick über den Ablauf der Lehrveranstaltung

Die Veranstaltung beginnt mit der Erarbeitung theoretischer Grundlagen. Zunächst erfolgt die Vorstellung der didaktischen Modelle zur Unterrichtsplanung sowie ein Vergleich dieser Modelle, sodass zentrale Schritte bzw. Elemente einer Unterrichtsplanung deutlich werden. Das Modell der didaktischen Rekonstruktion, welches den Planungsprozess in die Schritte der fachlichen Klärung, des Erfassens von Lernerperspektiven und der didaktischen Strukturierung gliedert (Kattmann, 2007), dient dabei als Grundstruktur des weiteren Planungsprozesses. Gemäß dieser drei Schritte steht in der folgenden Sitzung die fachliche Klärung des Unterrichtsinhalts „Statik - Kippverhalten von Bauklötzen“ im Mittelpunkt, zu 
dem die Studierenden im weiteren Verlauf ihren Unterricht planen. Die Auswahl genau dieses Themas begründet sich zum einen in der Tatsache, dass es im vorherigen Studienverlauf noch nicht behandelt wurde und die Studierenden aufgrund kaum vorhandener Vorlagen und Materialien unvoreingenommen an die Planung des Unterrichts herangehen können. Zum anderen liegen zum Thema Statik bereits Studien zu Schülervorstellungen vor, die als eine wichtige Grundlage für gute Unterrichtsplanung im Seminar benötigt werden. Darüber hinaus lässt sich das Thema mit einer Kompetenzerwartung zur Untersuchung technischer Problemstellungen des Lehrplans vereinen und stellt als nicht häufig umgesetztes Thema in der schulischen Praxis eine sinnvolle Ergänzung zu üblichen Themen dar. Da im Vorfeld Unterrichtsziele für den von den Studierenden zu planenden Unterricht festgelegt wurden, erfolgt die fachliche Klärung in der Veranstaltung sehr fokussiert im Hinblick auf die für diese Ziele relevanten Inhalte. Zum Erfassen von Lernerperspektiven lernen die Studierenden die erwähnten Studien kennen, in denen Konzepte von Schülerinnen und Schülern zum Kippverhalten von Bauklotzkonstruktionen untersucht wurden. Anknüpfend daran werden Diagnosebögen ausgewertet, die im Vorfeld des Seminars entwickelt und von den Schülerinnen und Schülern, die im späteren Verlauf der Veranstaltung von den Studierenden unterrichtet werden, ausgefüllt wurden, was eine hohe Passung des geplanten Unterrichts und der Lerngruppe ermöglicht. Es folgt in den nächsten beiden Sitzungen die Erarbeitung der theoretischen Grundlagen für die eigene Unterrichtsplanung, wie es im vorherigen Abschnitt dargestellt wurde. Dabei lernen die Studierenden kognitive Lernschritte von Schülerinnen und Schülern zu formulieren und kriteriengeleitet gute Lernaufgaben zu entwickeln. Beides ist zentrale Grundlage für die anschließende Planung des Unterrichts. Diese findet in den folgenden beiden Sitzungen statt. Die Studierenden werden dazu angeregt, ihre Planung mit der Formulierung von kognitiven Lernschritten der Schülerinnen und Schüler und der Entwicklung einer Lernaufgabe, die diese Lernschritte auslösen kann, zu beginnen. Erst im zweiten Schritt soll das gesamte Unterrichtsgeschehen und das Lehrerhandeln geplant werden. Während des Planungsprozesses entstehen in Kleingruppen zwei aufeinander aufbauende Unterrichtsstunden, wobei jede Gruppe eine Unterrichtsstunde plant. Durch die klare thematische Abstimmung zwischen den beiden Unterrichtsstunden und im Planungsprozess zwischen den Studierendengruppen einerseits und die Abgrenzung durch die vorgegebenen Unterrichtsziele andererseits fügen sich die beiden geplanten Stunden erfahrungsgemäß unproblematisch zu einer kurzen, stringenten Unterrichtseinheit zusammen. Den eigenständig geplanten Unterricht führen die Studierenden dann mit Schülerinnen und Schülern in dem mit Videotechnik ausgestatteten Lehr-Lern-Labor des Instituts durch, wobei die beiden Unterrichtsstunden von zwei unterschiedlichen Studierendengruppen mit derselben Schülergruppe nacheinander umgesetzt werden. Dabei werden Videoaufnahmen des Unterrichts angefertigt, die den Studierenden in der anschließenden Reflexionssitzung als Grundlage zur Analyse des Gelingens ihrer Unterrichtsplanung dienen. Auch bei der Analyse liegt der Fokus weiterhin auf 
den Lernprozessen der Schülerinnen und Schüler zur Bewertung der Qualität der Unterrichtsplanung. Nach der videobasierten Analyse und Reflexion überarbeiten die Studierenden ihre Unterrichtsplanung, denn es folgt im Anschluss eine zweite Erprobung der eigenen Planung mit anderen, aber hinsichtlich der Leistungsstärke und dem sozialen Verhalten vergleichbaren Schülerinnen und Schülern (eine Klasse wird für die beiden Erprobungen in zwei Hälften aufgeteilt). Bei dieser Unterrichtsdurchführung beobachten sich die Studierenden gegenseitig mit Hilfe eines Beobachtungsbogens. Auf dieser Grundlage geben sie sich in der anschließenden Sitzung gegenseitig Feedback und überarbeiten ihre Planung ein zweites Mal. Zum Abschluss des Seminars lernen die Studierenden didaktische Netze (Kahlert, 2009) als ein sachunterrichtsspezifisches Planungsinstrument kennen, mit dem inhaltliche Möglichkeiten eines Themas erörtert werden können, und sie nehmen eine Einbettung der beiden geplanten und durchgeführten Unterrichtsstunden in eine Unterrichtsreihe vor. Verschiedene Ideen der Studierendengruppen werden präsentiert und diskutiert.

Als Prüfungsleistung verfassen die Studierenden begleitend zum Seminar ein Portfolio, in dem sie über ihre eigene Kompetenz Unterricht zu planen und über die didaktischen Modelle reflektieren, sowie ihre eigene Unterrichtsplanung theoriebasiert analysieren. Im folgenden Kapitel werden die Gegebenheiten des Planungsprozesses und der Unterrichtserprobungen genauer in den Blick genommen und hinsichtlich ihrer Komplexitätsreduktion beschrieben.

\section{Komplexitätsreduktion bei der Erstellung und Erprobung einer eigenen Unterrichtsplanung im Lehr-Lern-Labor}

Bezugnehmend auf das Modell zur Komplexitätsreduktion (Marohn et al., in diesem Band) stehen in den folgenden Abschnitten die Vorbereitungs- sowie die Praxisphase im Fokus. Dabei werden einzelne Anforderungen und Unterstützungsmaßnahmen auf inhaltlicher und struktureller Ebene herausgestellt. Darüber hinaus werden in einem dritten Abschnitt Veränderungen einzelner Facetten im Vergleich der ersten und zweiten Erprobung thematisiert. Als Maßstab zur Einschätzung der Komplexität diente stets die Überlegung, welche anderen Optionen im Rahmen dieser Veranstaltung auch denkbar und potentiell möglich gewesen wären. Die Abbildung 2 zeigt die Ausprägung ausgewählter Facetten der Komplexität, die in den folgenden beiden Abschnitten aufgegliedert nach inhaltlicher und struktureller Ebene, erläutert werden. 


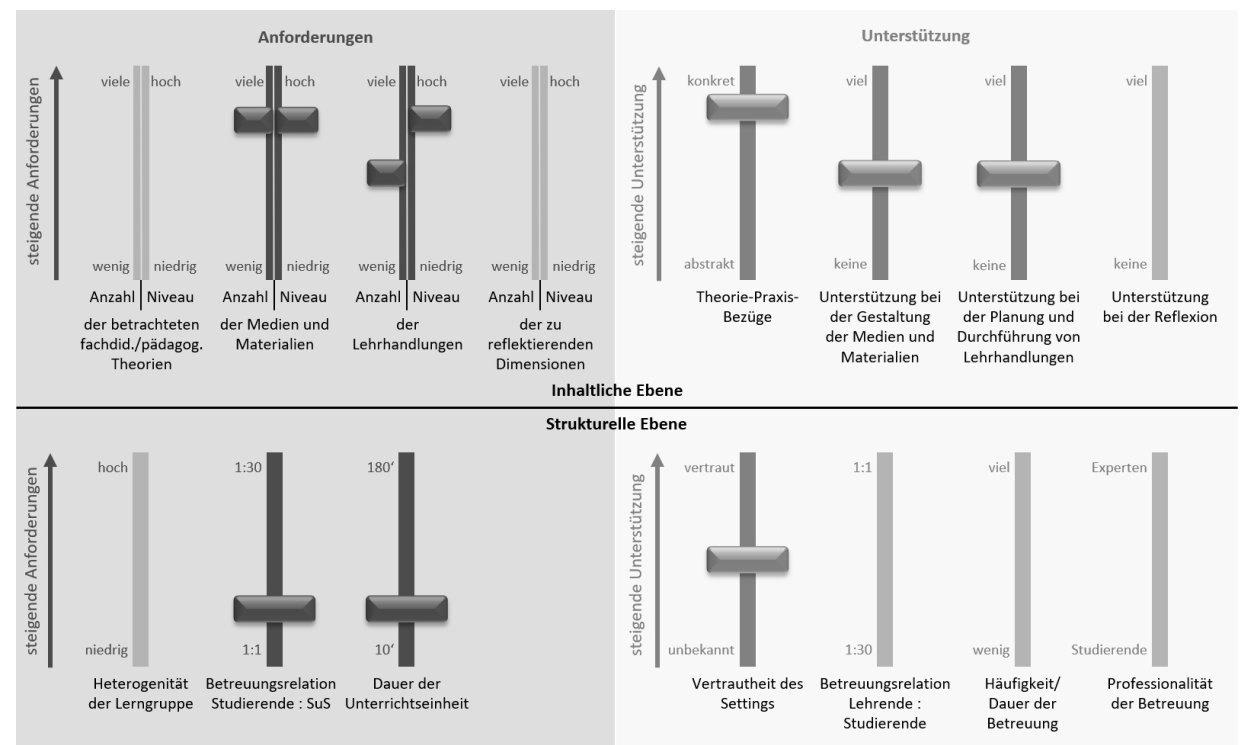

Abbildung 2: Komplexitätseinschätzung hinsichtlich der ersten Unterrichtserprobung

\subsection{Anforderungen und Unterstützung auf inhaltlicher Ebene}

Die folgenden Einschätzungen beziehen sich auf die Anforderungen der Planungsphase vor der ersten Erprobung sowie die Anforderungen der ersten Erprobung. Die Studierenden erhalten die Aufgabe, die Planung einer vollständigen Unterrichtsstunde vorzunehmen. Das inhaltliche Thema für den Unterricht wird den Studierenden vorgegeben und ist so ausgewählt, dass es den Studierenden nicht aus früheren Veranstaltungen bekannt ist. So haben sie noch keine konkreten Unterrichtsverläufe zum Thema im Kopf, die eine eigenständige Planung behindern könnten. Sie haben sich jedoch in ihrem Bachelorstudium bereits mit vorgegebenen Unterrichtsplanungen und der Anpassung solcher befasst, sodass die eigenständige Planung einer gesamten Unterrichtsstunde in dieser Veranstaltung zu Beginn des Masterstudiums eine angemessene Herausforderung für die Studierenden darstellt. Die Anforderungen für die Studierenden bezüglich der Medien, Materialien und Lehrhandlungen sind demnach sowohl für die Planungs- als auch für die Praxisphase recht hoch: Alle Medien und Materialien müssen selbstständig konzipiert und hergestellt und alle in einer Unterrichtsstunde relevanten Lehrhandlungen müssen geplant und in der Praxisphase durchgeführt werden. Da gewisse Lehrhandlungen wie z.B. das Diagnostizieren oder das Prüfen und Bewerten von Lernergebnissen keine Rolle in der Veranstaltung spielen und die Studierenden sich die Durchführung der eigenen Planung in Kleingruppen aufteilen (jeder Studierende führt nur einen Teil des Unterrichts durch), sind die Anzahl und das Niveau der Lehrhandlungen nicht maximal hoch. 
Da die Studierenden sich bei der Planung stark auf den Lernprozess der Schülerinnen und Schüler fokussieren sollen, bekommen sie zur Unterstützung von Schülerinnen und Schülern bearbeitete Diagnoseaufgaben zur Verfügung gestellt, aus denen sie erste Erkenntnisse zu fachlichen Konzepten und Vorstellungen der Schülerinnen und Schüler als Grundlage für ihren Planungsprozess ziehen. Ein von der Klassenlehrerin bzw. dem Klassenlehrer ausgefüllter Informationsbogen zu bekannten Sozialformen, Arbeitsweisen und relevanten Besonderheiten einzelner Schülerinnen und Schüler liefert den Studierenden zusätzlich hilfreiche Informationen für ihren Planungsprozess. Vorgegeben werden außerdem die Unterrichtsziele und -themen für die beiden Unterrichtsstunden, die von den Studierenden geplant werden. Durch die Vorgabe kann eine Konzentration auf die Ausgestaltung der Unterrichtsstunde in der Planung erfolgen, was dem Ziel der Veranstaltung entspricht. Eine weitere Unterstützung für die Gestaltung der Medien und Materialien, insbesondere für die Gestaltung der Unterrichtsplanung, erhalten die Studierenden durch die zu Beginn der Veranstaltung behandelten theoretischen Grundlagen. Hier werden konkrete Aspekte erarbeitet und direkt im Anschluss in Übungsphasen praktisch angewendet bzw. umgesetzt. Dadurch weist die Veranstaltung enge Theorie-Praxis-Bezüge auf, die den Studierenden einerseits das Verstehen der Theorie und andererseits das Handeln in der Praxis, das tatsächliche Planen und Durchführen von Unterricht, erleichtern. Dies wird auch durch den Einsatz des Portfolios als Prüfungsleistung unterstützt, da hierfür die Verknüpfung von Theorie und Praxis von den Studierenden gefordert wird. Unterstützung bei der Erstellung der Unterrichtsplanung und allen Materialien für den geplanten Unterricht erhalten die Studierenden außerdem in Form von Beratung durch Hochschullehrende sowie aufgrund der Arbeit in einer Kleingruppe durch die anderen Studierenden. Die Studierenden können gemeinsam Ideen entwickeln und sich gegenseitig beraten. Gleiches gilt für die Unterstützung bei der Planung von Lehrhandlungen: Während der Planungsphase können sich die Studierenden gegenseitig unterstützen sowie von Lehrenden Hilfestellungen erhalten. Bei der Durchführung der Lehrhandlung, also während der Praxisphase, unterstützen sich die Studierenden gegenseitig in aktiver Weise, da sie den Unterricht in der Kleingruppe durchführen und dabei der bzw. die jeweils als Lehrperson aktive Studierende zwei weitere Studierende zur Unterstützung an der Seite hat. Die Lehrenden sind während der Praxisphase bei Problemen ansprechbar, nehmen jedoch nicht an den Unterrichtserprobungen teil und unterstützen somit in dieser Phase nicht mehr aktiv.

\subsection{Anforderungen und Unterstïtzung auf struktureller Ebene}

Die von den Studierenden geplante Unterrichtsstunde hat einen Umfang von 45 Minuten und wird auch in diesem Umfang im Lehr-Lern-Labor mit den Schülerinnen und Schülern erprobt. Dabei unterrichten die Studierenden i.d.R. zu dritt 
- in derselben Gruppe, in der sie auch den Unterricht geplant haben - eine halbe Klasse, d.h. je nach Klassengröße 10-15 Schülerinnen und Schüler. Dabei ist, wie bereits im vorherigen Abschnitt erwähnt wurde, jeweils eine bzw. einer der Studierenden als Lehrperson aktiv, die anderen beiden nehmen am Unterricht teil und unterstützen bei Bedarf. Ein zentraler Aspekt bei der Einschätzung der Komplexität hinsichtlich der Praxisphase ist die Vertrautheit des Settings, die in diesem Fall eher hoch ist. Dabei sind verschiedene Elemente relevant. Zunächst sind die räumlichen Gegebenheiten und damit alle eingesetzten Medien und Materialien den Studierenden gut bekannt, es handelt sich um Räume, die auch im Vorfeld als Seminarraum genutzt werden. Auch der durchgeführte Unterricht ist den Studierenden mit allen dazugehörigen Elementen bis ins kleinste Detail vertraut, da sie ihn vollständig selbst geplant haben. Nicht vertraut ist den Studierenden hingegen die unterrichtete Schülergruppe. Abgesehen von Diagnoseergebnissen und Informationen durch die Klassenlehrkraft über die Klasse sind die Schülerinnen und Schüler bei der Erprobung unbekannt, was eine Herausforderung für die Studierenden bedeutet. Die Komplexität wird dann dadurch wieder reduziert, dass die Studierenden keine ganze Klasse, sondern nur die halbe Klasse unterrichten und damit eine geringere Anzahl unbekannter Schülerinnen und Schüler, auf die sie sich spontan einstellen müssen.

\subsection{Komplexitätsreduktion durch Wiederholung der Erprobung}

Wie bereits bei der Beschreibung der Veranstaltung deutlich wurde, finden zwei Erprobungen des eigenen Unterrichts mit einer dazwischen stattfindenden Reflexions- und Überarbeitungsphase statt. In diesem Zusammenhang lohnt es sich zu betrachten, inwiefern sich die Komplexität der Planungs- und Praxisphase durch die Wiederholung verändert. Die Abbildung 3 stellt die Dimensionen dar (eingekreist), die bei der zweiten Erprobung anders sind als bei der ersten.

In der Planungsphase vor der zweiten Erprobung bleibt zwar die Anzahl der Medien und Materialien, mit denen die Studierenden umzugehen haben, gleich, das Anforderungsniveau jedoch ist im Vergleich zur ersten Erprobung geringer. Die Studierenden müssen für die zweite Erprobung nicht mit einer vollständig neuen Planung beginnen, sondern alle vorhandenen Medien und Materialien auf ihre Eignung prüfen und gegebenenfalls überarbeiten und anpassen. Auf vorhandenes Material zurückgreifen zu können, reduziert hier somit das Anforderungsniveau für die Studierenden. Da die Anpassung jedoch keine triviale Aufgabe ist, ist das Anforderungsniveau auch nicht ganz gering. Gleiches gilt für die Planung der Lehrhandlungen. In Bezug auf die Praxisphase ist ebenfalls das Anforderungsniveau geringer, da die Studierenden sowohl den Einsatz aller Medien und Materialien sowie ihre eigenen Lehrhandlungen in der ersten Erprobung bereits einmal geübt haben und somit mehr Sicherheit haben können. Die Unterstützung hinsichtlich dieser beiden Facetten von Komplexität fällt für 


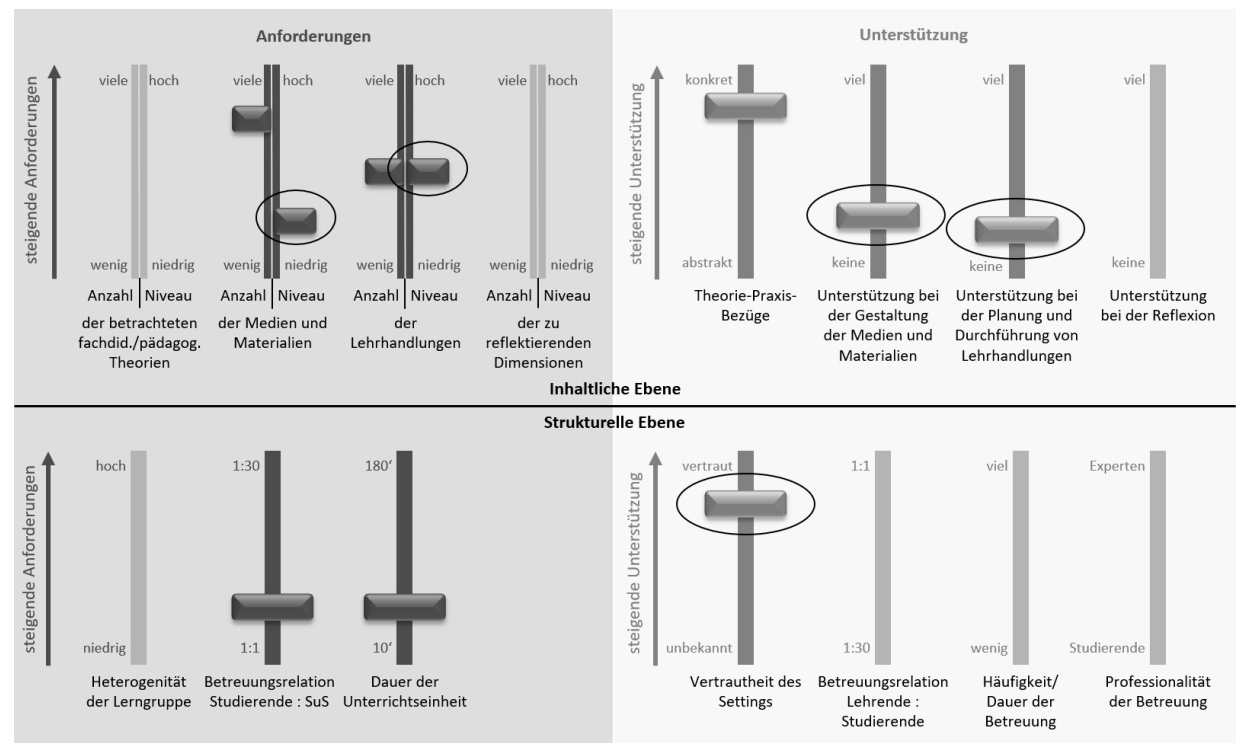

Abbildung 3: Komplexitätseinschätzung hinsichtlich der zweiten Unterrichtserprobung

die Planungsphase geringer aus, da hier die Verantwortung für die Erarbeitung stärker bei den Studierenden liegt. Es gibt keinen neuen Input und keine weiterführenden theoretischen Hilfen.

Die zweite Erprobung läuft ebenso ab wie die erste. Die Studierenden unterrichten zu dritt die von ihnen geplante Unterrichtsstunde mit einer halben Klasse. Dabei ist die Vertrautheit des Settings bei dieser zweiten Erprobung höher als bei der ersten, da die Studierenden zum einen den genauen Ablauf kennen und sich aufgrund der Erfahrung in der ersten Durchführung sicherer hinsichtlich aller Unterrichtsaktivitäten und -elemente fühlen. Zum anderen sind die Schülergruppen der beiden Erprobungen sehr ähnlich, da es sich jeweils um die Hälften einer Klasse handelt. Auch wenn die Studierenden die Schülerinnen und Schüler bei der zweiten Erprobung ebenfalls nicht kennen, so können sie dennoch auf bereits gemachte Erfahrungen zurückgreifen und die Schülerinnen und Schüler hinsichtlich allgemeiner Aspekte wie Sozialform, Arbeitsweisen und Sozialverhalten besser einschätzen. Im Verlauf der Entwicklung der Lehrveranstaltung wurden zwei verschiedene Varianten erprobt: Einmal haben die Studierenden die beiden Hälften einer Klasse, einmal Hälften zweier unterschiedlicher Klassen (von verschiedenen Schulen) unterrichtet. Hier zeigte sich durch Rückmeldung von Studierenden, dass die Komplexität für Studierende geringer ist, wenn sie die Hälften einer Klasse unterrichten, da diese beiden Gruppen sehr vergleichbar sind. So kann die Anpassung und Überarbeitung der eigenen Planung erfolgsversprechender vorgenommen werden.

Es wird deutlich, dass sich im Vergleich der ersten und zweiten Unterrichtserprobung nicht nur die Anforderungen, sondern auch die Unterstützungsmaßnah- 
men verändern, da die Studierenden die von ihnen erarbeitete Unterrichtsplanung zweimal erproben und diese zwischendurch nur überarbeiten und anpassen. Da also die Anforderungen bei der zweiten Erprobung geringer sind, kann das Ausmaß der Unterstützung ebenfalls geringer ausfallen. Mit der zweiten Erprobung ist ein höheres Ausmaß an Erkenntnis zur eigenen Unterrichtsplanung intendiert und somit ein höherer Kompetenzzuwachs angestrebt, als er mit nur einer Erprobung erreicht werden würde. Eine stärkere Konzentration auf die inhaltliche Umsetzung der Planung bei der zweiten Erprobung sowie das direkte Ausprobieren der Überarbeitungen nach der ersten Erprobung werden zu diesem Zwecke als relevant erachtet.

\section{Erkenntnisse zur Komplexitätsreduktion}

Im Folgenden werden erste Erkenntnisse dargestellt, wie Studierende die intendierte Komplexitätsreduktion rund um die Unterrichtserprobung im Lehr-LernLabor einschätzen. Dabei wird zunächst kurz beschrieben, wie diese Erkenntnisse entstanden sind.

\subsection{Methode}

Im Anschluss an die Veranstaltung wurden mit $\mathrm{N}=14$ Studierenden Leitfadeninterviews durchgeführt: Aufgrund weiterer Fragestellungen im begleitenden Projekt des Instituts waren die Studierenden in zwei Teilstichproben aufgeteilt, die zwei leicht unterschiedliche Varianten der Lehrveranstaltung besuchten. Der Ablauf der einen Gruppe entspricht mit einer wiederholten Erprobung der selbst erarbeiteten Unterrichtsplanung der vorangegangenen Beschreibung in diesem Beitrag, der Ablauf der anderen Gruppe unterscheidet sich insofern davon, als dass nur eine Erprobung und diese zu einem späteren Zeitpunkt stattfand. Die Stichprobe der Interviews umfasst je sieben Studierende beider Gruppen. Da in beiden Teilstichproben eine Erprobung der erarbeiteten Unterrichtsplanung mit Schülerinnen und Schülern zentrales Element der Veranstaltung war, können die Interviewdaten der Studierenden beider Gruppen zur Ermittlung erster Erkenntnisse zur Gestaltung der Unterrichtserprobung im Lehr-Lern-Labor genutzt werden. Lediglich zum Aspekt der wiederholten Erprobung können nur Einschätzungen der einen Teilstichprobe herangezogen werden. In den Interviews wurden die Seminarteilnehmerinnen und -teilnehmer u. a. nach ihrer Einschätzung der Praxiserfahrung im Lehr-Lern-Labor gefragt. Nach einer zunächst offenen Frage, welche besonderen Begebenheiten der Erprobung im Lehr-Lern-Labor aus Sicht der Studierenden wichtig und hilfreich waren, um die eigene Kompetenz Unterricht zu planen weiterzuentwickeln, wurden ausgewählte Aspekte gezielt abgefragt. Diese sollten von den Studierenden auf einer Skala $(1=$ nicht hilfreich, $2=$ eher nicht hilf- 
reich, 3 = hilfreich, $4=$ hilfreich bis sehr hilfreich, $5=$ sehr hilfreich) eingeordnet werden. Diese Einschätzungen auf der Skala wurden bei der Auswertung ausgezählt und können in Verbindung mit zusätzlich eingeforderten Begründungen der Studierenden erste Erkenntnisse liefern, wie die intendiert komplexitätsreduzierenden Maßnahmen von Studierenden wahrgenommen und eingeschätzt werden.

\subsection{Ergebnisse der Interviews}

Zunächst werden die Ergebnisse der offenen Frage an die Studierenden präsentiert, welche Merkmale der Erprobung im Lehr-Lern-Labor sie als hilfreich und wichtig für ihre Planungskompetenz einschätzen. Am häufigsten wurden dabei der Einsatz des Unterrichtsvideos bzw. die Videoanalyse genannt $(\mathrm{n}=10)$. Die zweithäufigste Antwort war die verringerte Größe der Schülergruppe, also das Unterrichten einer halben Klasse bei der Erprobung $(n=5)$. Die Vertrautheit der Umgebung war ein weiterer Aspekt, der von mehreren Studierenden genannt wurde $(\mathrm{n}=3)$. Vereinzelt wurden außerdem folgende weitere Aspekte genannt: Die Unterstützung in der Kleingruppe $(n=1)$, die Wiederholung der Erprobung $(n=2)$ sowie die Möglichkeit, die eigene Unterrichtsplanung zu erproben und dadurch die Qualität zu prüfen $(\mathrm{n}=1)$.

Die folgende Abbildung 4 zeigt die quantifizierte Einschätzung der befragten 14 Studierenden zu den einzelnen vorgegebenen Aspekten der Unterrichterprobung im Lehr-Lern-Labor, wobei zum letzten Aspekt der Wiederholung aufgrund der beiden Teilstichproben, wie bereits erläutert, nur Angaben von sieben der vierzehn Studierenden vorliegen.

Die Vorbereitung, also die Erarbeitung der Unterrichtsplanung in der Kleingruppe, wurde von fast allen befragten Studierenden mindestens als hilfreich eingeschätzt, von der Hälfte der Studierenden sogar als sehr hilfreich. Insbesondere der Austausch und das Vorhandensein verschiedener Ideen und Impulse

\section{Einschätzung der Unterrichtserprobung \\ im Lehr-Lern-Labor}

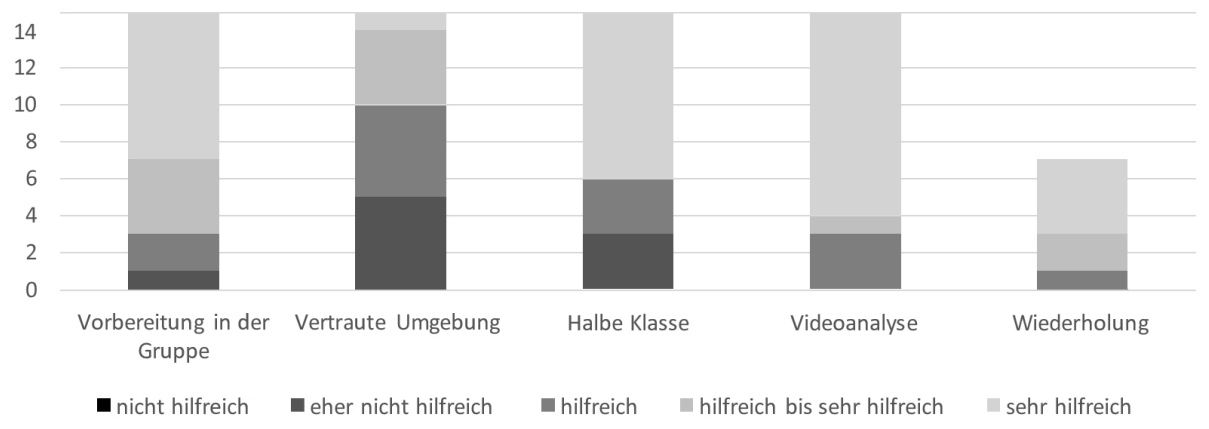

Abbildung 4: Ergebnisse zur Einschätzung der Unterrichtserprobung aus den Interviews 
wurden als Gründe hierfür genannt. Außerdem führten die Studierenden an, dass es hilfreich gewesen sei, gemeinsam Entscheidungen zu treffen, und dass man sich durch den Austausch nicht auf eine Sache versteift habe. In diesem Zusammenhang wurde im Seminarverlauf außerdem deutlich, dass die Studierenden das gemeinsame Durchführen als gewinnbringend empfanden, da man nicht alleine die Verantwortung getragen habe und sich gegenseitig unterstützen konnte - auch auf emotionaler Ebene.

Das Erproben der eigenen Unterrichtsplanung in vertrauter Umgebung wurde von den Studierenden recht unterschiedlich eingeschätzt: Fünf Befragte schätzten die vertraute Umgebung zwischen nicht hilfreich und hilfreich ein, weitere fünf Befragte als hilfreich, die Übrigen positiver. Kritische Begründungen hoben hervor, dass die Umgebung in der Universität für die Schülerinnen und Schüler unbekannt sei und es für sie in der Schule leichter gewesen wäre. Positive Argumente für die vertraute Umgebung zielten insgesamt häufig darauf $a b$, dass die Organisation nicht in der Verantwortung der Studierenden gewesen sei und sie sich so besser auf die Schülerinnen und Schüler einlassen und sich stärker auf ihren Unterricht konzentrieren konnten. Zu wissen, wo sich alle Materialien befinden, vorher alles in Ruhe aufbauen zu können sowie die Sitzordnung und die sonstige Ausstattung des Raumes detailliert zu kennen, sei eine Erleichterung gewesen, so argumentierten einige der Befragten.

In Bezug auf die Einschätzung der Größe der Schülergruppe ist ebenfalls kein einheitliches Bild zu erkennen: Acht der vierzehn Studierenden hielten es für sehr hilfreich die eigene Unterrichtplanung mit nur einer halben Klasse zu erproben. Die übrigen Befragten schätzten diesen Aspekt zwischen nicht hilfreich und hilfreich bzw. als hilfreich ein. Als Begründungen für die positiven Einschätzungen wurde angeführt, dass es ein Herantasten gewesen sei und die Studierenden nicht „ins kalte Wasser geworfen“ worden wären. Es habe durch die kleine Gruppengröße weniger Störfaktoren gegeben, der Kontaktaufbau zu den Schülerinnen und Schülern und das Eingehen auf ihre Ideen und Bedürfnisse sei leichter gewesen. Sich selbst in der Rolle der Lehrkraft kennenzulernen, ausprobieren und zu üben, dafür sei eine kleinere Schülergruppe hilfreich gewesen - so die Begründungen einzelner Studierender. Begründungen der kritischer eingestellten Befragten waren, dass die kleine Schülergruppe praxisfern sei und bestimmte Aspekte wie der Umgang mit einer ganzen Klasse bei der Unterrichtsplanung so nicht geübt werden könne. Eine befragte Person führte außerdem eine geringe Beteiligung der Schülerinnen und Schüler in seinem Unterricht auf die kleine Gruppengröße zurück und argumentierte, dass in einer größeren Gruppe mehr Ideen hätten zusammengetragen werden können.

Der Einsatz eines Videos vom eigenen Unterricht für eine Videoanalyse wurde von allen Befragten mindestens als hilfreich, von einer Mehrheit (10 von 14) sogar als sehr hilfreich für die Weiterentwicklung der eigenen Kompetenz Unterricht zu planen eingeschätzt. Das Video ermöglichte die Analyse von Aspekten, die während des Unterrichts gar nicht wahrgenommen oder im Anschluss nicht gut aus 
dem Gedächtnis reflektiert und bewertet werden könnten. Mithilfe des Videos ließen sich bereits gelungene Aspekte sowie Verbesserungspotenziale der eigenen Planung erkennen und im weiteren Planungsprozess berücksichtigen. Eine befragte Person begründete die Einschätzung als weniger hilfreich damit, dass der gemeinsame Austausch und das Erproben der eigenen Planung auch gereicht und das Video nicht notwendig gewesen sei. Ihre Gruppe hätte es daher auch nicht so viel benutzt.

Die Einschätzung der zweimaligen Erprobung durch die Studierenden der einen Teilstichprobe fiel insgesamt sehr positiv aus. Alle Befragten hielten die Wiederholung mindestens für hilfreich, die Mehrzahl (4 von 7) hielt sie für sehr hilfreich. Gründe dafür seien die Möglichkeit des direkten Vergleichs zwischen den beiden praktischen Erfahrungen mit der eigenen Planung gewesen und Schlussfolgerungen daraus ziehen zu können, verschiedene Ideen oder Anpassungen der Planung beim zweiten Mal noch einmal auszuprobieren.

\section{Zusammenfassung und Fazit}

Abschließend werden die Erkenntnisse aus den Interviews mit bisherigen Erfahrungen der Dozentinnen und Dozenten aus der konzipierten Veranstaltung mit der implementierten Vorbereitung, Erprobung und Reflexion im Lehr-Lern-Labor angereichert, zusammengefasst und diskutiert. In einer Veranstaltung, in der Studierende lernen sollen, Unterricht zu planen und durch die Erprobung die Eignung ihrer Planung zu prüfen, scheint die Durchführung des geplanten Unterrichts in einem Lehr-Lern-Labor als geeignete Möglichkeit, eine intensive Auseinandersetzung der Studierenden mit der eigenen Unterrichtplanung zu fördern. Unterrichten ist eine sehr komplexe Aufgabe, sodass es besonders für Novizinnen und Novizen sinnvoll scheint, Fokussierungen vorzunehmen und somit nicht alle relevanten Aspekte von Unterricht gleichzeitig in den Blick zu nehmen. Die Fokussierung auf Lernprozesse von Schülerinnen und Schüler in der Veranstaltung ermöglicht dies. Wird wie in der beschriebenen Veranstaltung eine herausfordernde Aufgabe, z. B. die völlig eigenständige Planung einer ganzen Unterrichtstunde, an die Studierenden gestellt, scheint eine gelungene Art der Unterstützung die Zusammenarbeit und der Austausch zwischen den Studierenden in Kleingruppen zu sein. Auch das gemeinsame Unterrichten und damit die Verringerung der durchzuführenden Lehrhandlungen scheint den Studierenden Sicherheit zu geben und die für sie neue und noch ungewohnte Situation zu erleichtern. In der Einschätzung der Größe der Schülergruppe sind sich die Studierenden jedoch nicht einig. Möglicherweise wird dies durch unterschiedliche Erwartungen verursacht, wenn Studierende sich entweder offen auf die Praxissituation im Lehr- Lern-Labor einlassen oder aber mit dem Blick auf den Schulalltag eine sehr realistische Situation erwarten. Es wäre denkbar, dass auch diese Studierenden den Umgang mit einer kleineren Schülergruppe als hilfreich wahrnehmen könnten, wenn ihnen 
direkt zu Beginn bei der Vorstellung des Seminarverlaufs die Intention der Erprobung bewusster gemacht und explizit verdeutlicht würde, welche Chancen ganz konkret in den Unterschieden zur Durchführung von Unterricht in der Schule liegen können. Die Reaktionen der befragten Studierenden geben Grund zur Annahme, dass der Umgang mit einer fremden, ganzen Klasse für eine Unterrichtserprobung eine zu hohe Anforderung an die Studierenden stellen könnte und den Fokus von den Lernprozessen der Schülerinnen und Schüler weg auf z. B. Aspekte der Klassenführung verschieben könnte. Hier könnte es hilfreich sein, den Fokus und die Möglichkeiten der Erprobung, sich auf diesen Fokus zu konzentrieren, den Studierenden stärker bewusst zu machen. Die Wiederholung der Erprobung und damit die Überarbeitung der eigenen Planung sowie der Einsatz eines Videos bei der Reflexion, die der Überarbeitung vorausgeht, scheinen wichtige Elemente zu sein, um die Planungskompetenzen der Studierenden zu fördern. In Bezug auf die Wiederholung der Unterrichtsdurchführung wurde von Studierenden in der Lehrveranstaltung immer wieder rückgemeldet, dass sie sich beim zweiten Mal stärker auf die inhaltlichen statt die organisatorischen Aspekte im Unterricht konzentrieren konnten, da sie den Ablauf bereits einmal erlebt hatten. Da die intensive Auseinandersetzung mit dem Unterricht auf inhaltlicher Ebene, z. B. mit den Tiefenstrukturen, ein wichtiges Element für die Zielerreichung der Lehrveranstaltung ist, kann besonders das wiederholte Erproben als gewinnbringend für das Erreichen der Ziele eingeschätzt werden. Die Videoanalyse scheint ebenfalls zu einer intensiveren inhaltlichen Auseinandersetzung mit dem geplanten Unterricht beizutragen und somit die angestrebte Verknüpfung von Theorie und Praxis maßgeblich zu unterstützen.

Um insbesondere solche Aspekte herausfinden zu können, bezüglich derer Studierende eine andere Wahrnehmung haben als bei der Konzeption einer Veranstaltung erwartet, scheint es wichtig, Studierende zu befragen. Mithilfe der Einschätzung durch Studierende dürfte sich das Verhältnis zwischen Anforderungen und notwendigen Unterstützungsmaßnahmen und damit insgesamt das Komplexitätsniveau besser austarieren lassen. Dies scheint der zentrale Aspekt für die Konzeption einer erfolgreichen Lehrveranstaltung zu sein - ein ausgeglichenes Verhältnis von Herausforderung und unterstützenden Maßnahmen zur Komplexitätsreduktion. Um weitere Erkenntnisse über die Qualität der Lehrveranstaltung zu erhalten, werden sowohl die Planungskompetenz als auch die Selbstwirksamkeitserwartungen der Studierenden hinsichtlich dieser Kompetenz vor und nach der Teilnahme an der Veranstaltung gemessen: die Planungskompetenz mit einem Performanztest, die Selbstwirksamkeitserwartungen mit einem Fragebogen. Es wird erwartet, dass sich die Planungskompetenz durch die Teilnahme an der Lehrveranstaltung steigert und die Selbstwirksamkeitserwartung zumindest nicht durch einen Praxisschock absinkt (Tschannen-Moran, Woolfolk-Hoy \& Hoy, 1998). So wird evaluiert, ob die Ziele der Veranstaltung mithilfe der komplexitätsreduzierenden Maßnahmen erreicht werden können. 


\section{Literatur}

Giest, H. (2010). Sachunterrichtsplanung als Planung des Lernprozesses der Schülerinnen und Schüler. In S. Tänzer \& R. Lauterbach (Hrsg.), Sachunterricht begründet planen. Bedingungen, Entscheidungen, Modelle (S. 179-202). Bad Heilbrunn: Klinkhardt.

Heimann, P., Otto, G. \& Schulz, W. (1979). Unterricht: Analyse und Planung (10., unveränd. Aufl.). Hannover: Schroedel.

Kahlert, J. (2009). Der Sachunterricht und seine Didaktik (3. Aufl.). Bad Heilbrunn: Klinkhardt.

Kattmann, U. (2007). Didaktische Rekonstruktion - eine praktische Theorie. In D. Krüger \& H. Vogt (Hrsg.), Theorien in der biologiedidaktischen Forschung. Ein Handbuch für Lehramtsstudenten und Doktoranden (S. 93-104). Berlin: Springer. https://doi. org/10.1007/978-3-540-68166-3_9

Kiper, H. \& Mischke, W. (2009). Unterrichtsplanung. Bachelor, Master. Weinheim: Beltz.

Klafki, W. (1958). Die Erziehung im Spannungsfeld von Vergangenheit, Gegenwart und Zukunft. Göttingen: Vandenhoeck \& Ruprecht.

König, J., Buchholtz, C. \& Dohmen, D. (2015). Analyse von schriftlichen Unterrichtsplanungen: Empirische Befunde zur didaktischen Adaptivität als Aspekt der Planungskompetenz angehender Lehrkräfte. Zeitschrift für Erziehungswissenschaft, 18 (2), 375-404. https://doi.org/10.1007/s11618-015-0625-7

Kultusministerkonferenz (2004). Beschluss der Kultusministerkonferenz vom 16.12.2004: Standards für die Lehrerbildung: Bildungswissenschaften. Bonn: Ständige Konferenz der Kultusminister der Länder in der Bundesrepublik Deutschland.

Kunter, M. \& Ewald, S. (2016). Bedingungen und Effekte von Unterricht: Aktuelle Forschungsperspektiven aus der pädagogischen Psychologie. In N. McElvany, W. Bos, H. G. Holtappels, M. M. Gebauer \& F. Schwabe (Hrsg.), Bedingungen und Effekte guten Unterrichts (Dortmunder Symposium der Empirischen Bildungsforschung: Bd. 1, S. 9-31). Münster: Waxmann.

Kunter, M. \& Trautwein, U. (2013). Psychologie des Unterrichts. Paderborn: Schöningh.

Krofta, H. \& Nordmeier, V. (2014). Bewirken Praxisseminare im Lehr-Lern-Labor Änderungen der Lehrerselbstwirksamkeitserwartung bei Studierenden? In V. Nordmeier \& H. Grötzebauch (Hrsg.), PhyDid B, Didaktik der Physik, Beiträge zur DPG-Frühjahrstagung. Verfügbar unter http://phydid.physik.fu-berlin.de/index.php/ phydid-b/index [23.01.2017].

Mansholt, M. \& Komorek, M. (2016). Adaptive Lehrprozesse im Lehr-Lern-Labor. In C. Maurer (Hrsg.), Authentizität und Lernen - das Fach in der Fachdidaktik. Gesellschaft für Didaktik der Chemie und Physik, Jahrestagung in Berlin, 2015 (S. 101-103). Regensburg: Universität Regensburg.

Möller, K. (2010). Lernen von Naturwissenschaften heißt: Konzepte verändern. Fachdidaktik Naturwissenschaft, 1 (9), 57-72.

Posner, G. J., Strike, K. A., Hewson, P. W. \& Gertzog, W. A. (1982). Accommodation of a scientific conception: Toward a theory of conceptual change. Science Education, 66(2), 211-227. https://doi.org/10.1002/sce.3730660207

Tschannen-Moran, M., Woolfolk-Hoy A. \& Hoy, W. K. (1998). Teacher Efficacy: Its Meaning and Measure. Review of Educational Research, 68, 202-248. https://doi.org/ 10.3102/00346543068002202 
Vygotsky, L. S. (1978). Mind in society: The development of higher mental process. Cambrigde, MA: Harvard University Press.

Wood, D., Bruner, J. S. \& Ross, G. (1976). The role of tutoring in problem solving. Journal of Child Psychology and Psychiatry, 17(2), 89-10o. https://doi.org/10.1111/j.14697610.1976.tboo381.x

Van de Pol, J., Volman, M. \& Beishuizen, J. (2010). Scaffolding in Teacher-Student Interaction: A Decade of Research. Educational Psychology Review, 22(3), 271-296. https://doi.org/10.1007/s10648-010-9127-6

Zierer, K., Werner, J. \& Wernke, S. (2015). Besser planen? Mit Modell! Empirisch basierte Überlegungen zur Entwicklung eines Planungskompetenzmodells. DDS - Die Deutsche Schule, 107 (4), 375-395. 



\section{Professionalisierung von Studierenden des Lehramts durch Komplexitätsreduktion in Lehr-Lern-Laboren}

Schlüsselwörter: Reflexionskompetenz, Selbstwirksamkeitserwartung, Professionalisierung von Lehramtsstudierenden

\section{Motivation}

Die in diesem Sammelband beschriebenen Lehr-Lern-Laborformate der Fachdidaktiken und Erziehungswissenschaften zielen darauf ab, in komplexitätsreduzierten und dennoch authentischen Settings unterschiedliche Facetten der Lehrerprofessionalisierung zu fördern. Im Rahmen des Teilprojektes der Qualitätsoffensive Lehrerbildung an der Westfälischen Wilhelms-Universität (WWU) Münster wurden dementsprechend Untersuchungen durchgeführt, um den diesbezüglichen Erfolg der Lehr-Lern-Labore zu überprüfen. Ein wichtiges Augenmerk lag dabei auf der Entwicklung der Selbstwirksamkeitserwartung der Studierenden in Bezug auf unterrichtliches Handeln und auch spezieller in Bezug auf den Umgang mit heterogenen Lerngruppen.

Dieser Beitrag gibt einen Einblick in die Ergebnisse genannter Studien. Als Grundlage dienen exemplarisch die Seminarformate der Lehr-Lern-Labore in den Fächern Mathematik und Physik, die im Rahmen der Qualitätsoffensive Lehrerbildung Dealing with Diversity an der WWU Münster etabliert bzw. weiterentwickelt wurden. Im Fokus steht dabei die Kompetenz professioneller Reflexion von Unterricht: Welche Bedeutung messen die Studierenden dieser Kompetenz bei? Wie entwickelt sich in ihrer Selbsteinschätzung ihre Reflexionskompetenz im Laufe und aufgrund der Seminare? Inwiefern trägt ihrer Ansicht nach die Komplexitätsreduktion innerhalb der Lehr-Lern-Laborformate zu dieser Entwicklung bei? Diesen Fragen geht der folgende Beitrag nach. Die Ergebnisse der Studie können der weiteren Optimierung von Lehr-Lern-Laboren dienen.

\section{Theoretischer Rahmen}

\subsection{Selbstwirksamkeitserwartungen}

Die professionelle Kompetenz von Lehrkräften umfasst neben Fach- und fachdidaktischem Wissen auch motivationale Orientierungen sowie Einstellungen und Überzeugungen (Baumert \& Kunter, 2011). $\mathrm{Zu}$ den motivationalen Orientierungen gehören dabei unter anderem die Selbstwirksamkeitserwartungen. Dabei handelt 
es sich um die Gewissheit, eine gegebene Handlung erfolgreich durchführen zu können (Bandura, 1997).

Selbstwirksamkeitserwartungen können sich in Bezug auf die Schwierigkeit der Handlung, die Stabilität der Überzeugung gegenüber Widerständen oder Misserfolgen und die Allgemeinheit der Einschätzung unterscheiden (Bandura, 1997). Obgleich in der ursprünglichen Definition von Bandura situations- und handlungsspezifische Selbstwirksamkeitserwartungen beschrieben sind, sind auch allgemeinere Varianten sinnvoll, wenn die zusammengefassten Handlungen gemeinsame Charakteristika aufweisen (Bandura, 1999). So lassen sich bei Lehrkräften beispielsweise Selbstwirksamkeitserwartungen in Bezug auf einzelne Anforderungen des Unterrichtens betrachten (Kunter \& Pohlmann, 2009).

Selbstwirksamkeitserwartungen können durch die Verarbeitung von Informationen aus vier unterschiedlichen Quellen beeinflusst werden: Erfolgs- und Misserfolgserlebnisse, Modelllernen, verbale Einflüsse und physiologische Reaktionen. Dabei üben diese Quellen einen absteigenden Einfluss (von den eigenen Erfahrungen zu den beobachteten physiologischen Reaktionen) auf die Selbstwirksamkeitserwartung aus, der darüber hinaus durch die jeweilige mentale Verarbeitung der Informationen beeinflusst wird (Bandura, 1997).

Selbstwirksamkeitserwartungen spielen bei (angehenden) Lehrkräften in zahlreichen Bereichen eine wichtige Rolle: Einerseits beeinflussen sie Leistung, Motivation und Selbstregulation in Lernsituationen und damit die eigene Weiterentwicklung (Bandura, 1997; Lent, Brown \& Larkin, 1986; Schunk \& Pajares, 2002). Andererseits beeinflussen sie den Erfolg der eigenen Lehre. Hohe Selbstwirksamkeitserwartungen gehen mit höherer Effizienz (Klassen \& Tze, 2014) und höherem Anspruch des durchgeführten Unterrichts bei gleichzeitig hoher Unterstützung der Schülerinnen und Schüler (Schwarzer \& Jerusalem, 2002) einher. Die Schülerinnen und Schüler selbstwirksamer Lehrkräfte zeigen bessere Leistungen (Caprara, Barbaranelli, Steca \& Malone, 2006) und die Lehrkräfte fühlen sich weniger stark durch ihre Arbeit belastet (Subellok, Lüke \& Ritterfeld, 2013; Urton, Wilbert \& Hennemann, 2014). Im Umgang mit heterogenen Lerngruppen gehen Lehrkräfte mit einer hohen Selbstwirksamkeitserwartung feinfühliger mit schwächeren Schülerinnen und Schülern und normabweichendem Verhalten um (Meijer \& Foster, 1988; Ross, 1995).

Die Bedeutung von positiven Selbstwirksamkeitserwartungen für guten Unterricht ist vor diesem Hintergrund offensichtlich. In der Realität empfinden jedoch viele Studierende des Lehramts gerade den Übergang in die Schulpraxis als Bruch zur an der Hochschule erlernten Theorie und fühlen sich von der Situation überfordert. Bedingt durch diesen "Praxisschock“ kommt es zu einem Absinken der Selbstwirksamkeitserwartung (Tschannen-Moran, Woolfolk Hoy \& Hoy, 1998). Universitäre Lehrveranstaltungen, die Praxisphasen angeleitet und theoriebasiert vorbereiten sowie reflektieren, helfen den Studierenden dabei, ihre Erfahrungen geeignet zu verarbeiten und eine stabile zuversichtliche Einschätzung der eigenen Wirksamkeit zu erreichen. Darüber hinaus gelingt es den Studierenden durch der- 
artige Veranstaltungen besser, Theorie und Praxis in Beziehung zu setzen (Korthagen, 2002).

Die in diesem Band vorgestellten Lehr-Lern-Labore verfolgen neben individuellen Schwerpunkten als gemeinsames Ziel die Entwicklung einer positiven Selbstwirksamkeitserwartung durch Reflexion von Praxisphasen. Damit sollen durch das Hinterfragen der eigenen Vorstellungen und Theorien die positiven und negativen Praxiserfahrungen für die eigene Professionalisierung nutzbar gemacht werden (Schön, 1983). Eine komplexitätsreduzierte Umgebung soll dabei helfen „kognitive Überlastung zu vermeiden und den Studierenden selbstständiges Handeln und zeitgleiche kognitive Aktivität zu ermöglichen“" (Zucker \& Leuchter, 2018, S. 365).

\subsection{Reflexionskompetenz - ein multidimensionales Konstrukt}

Reflexion repräsentiert im Wesentlichen eine besondere, auf dem Konzept des „reflective thinkings“ (Dewey, 1933) basierende Form des Denkens, welche sich auf ein bewusstes und zielgerichtetes Nachdenken stützt und stets zu einer Schlussfolgerung und einer daraus resultierenden Erweiterung des individuellen Handlungswissens führt (Roters, 2012). In diesem Kontext besteht weitgehend Einigkeit darüber, dass Reflexionskompetenz eine bedeutende Rolle für die berufliche Entwicklung von Lehrpersonen spielt sowie einen wesentlichen Beitrag zur Erlangung von Professionalität leisten kann (Wyss, 2008). Dennoch besteht auch Uneinigkeit darüber, was genau unter Reflexion und Reflexionskompetenz zu verstehen ist, wie sich Reflexionskompetenz adäquat fördern und die Wirksamkeit von Reflexion empirisch nachweisen lässt (Abels, 2011). Demnach zeigt sich die Vielschichtigkeit der Reflexionskompetenz bereits in der Unterscheidung hinsichtlich des Einflusses auf die Professionalisierung von Lehrpersonen sowie als Bestandteil derselben.

\subsubsection{Professionalisierung durch Reflexion}

Ein zentrales Ziel der Lehrerbildung ist die Entwicklung professioneller Kompetenzen (Baumert \& Kunter, 2006). Hier liegt der Fokus neben fachlichen, fachdidaktischen und pädagogisch-psychologischen Wissensaspekten (Shulman, 1986) auch auf Überzeugungen und motivationalen Orientierungen (Baumert \& Kunter, 2011). Diesen Komponenten wird insgesamt eine bedeutende Rolle für erfolgreiches Unterrichten zugesprochen (Baumert \& Kunter, 2006). Jedoch haben Studierende häufig Schwierigkeiten theoretische Inhalte des Studiums auf konkretes Unterrichtshandeln zu beziehen (Hößle, Hußmann, Michaelis, Niesel \& Nührenbörger, 2017), weshalb vermehrt Theorie-Praxis verbindende Strukturen in das Lehramtsstudium implementiert werden. Die Integration dieser Praxiselemente führt jedoch nicht automatisch zur Generierung handlungsfähigen Wissens aus den gemachten Erfahrungen (Krofta, Fandrich \& Nordmeier, 2012). Es reicht also 
nicht aus, integrierte Praxisphasen als Orte der Erfahrungsbildung zu begreifen, vielmehr schaffen sie konkrete Gelegenheiten pädagogische und fachdidaktische Wissensbestände zu reflektieren und auf diese Weise mit wissenschaftlich fundiertem Wissen zu verknüpfen (Herzog \& Felten, 2001). So können angehende Lehrpersonen durch reflektierte Praxiserfahrungen, welche häufig Bestandteil der komplexitätsreduzierten, authentischen Lehr-Lern-Settings im Kontext von LehrLern-Laboren sind (vgl. auch Greefrath \& Hammann, in diesem Band), professionelle Kompetenzen erwerben (Park \& Oliver, 2008; Putnam \& Borko, 2000).

\subsubsection{Reflexion als Bestandteil professioneller Kompetenz}

Reflexionsprozesse und -kompetenzen dienen nicht nur auf einer Metaebene dem Erwerb professioneller Kompetenzen, sondern lassen sich ebenso in Form gezielter Reflexionen von Unterrichtspraxis als integraler Bestandteil der Lehrerprofessionalität selbst auffassen (Gutzwiller-Helfenfinger, Aeppli \& Lötscher, 2017). Durch diese verstehen und erweitern Lehrpersonen ihre professionellen Aktivitäten, erwerben professionelles Unterrichtswissen und gewinnen neue Erkenntnisse für die Praxis (Darling-Hammond \& Richardson, 2009). Demnach kann Reflexionskompetenz als bedeutender Faktor effektiven und adäquaten Lehrerhandelns identifiziert werden. Jedoch herrscht aufgrund der zum Teil sehr differenten Auffassungen von Reflexionskompetenzen hinsichtlich einer klaren Definition dieser sowie eines Ablaufs von Reflexionsprozessen wenig Einigkeit (Collin, Karsenti \& Komis, 2013).

In Anlehnung an die Arbeiten Deweys (1933) wurden zahlreiche Definitionsversuche von Reflexionskompetenz vorgenommen. Dabei stellt das Modell der „Reflective Practice“ von Schön (1983) den Ausgangspunkt der Forderungen nach reflexiver Lehrerbildung dar. Dieses differenziert zwischen zwei unterschiedlichen Reflexionsprozessen:

- Reflection-in-action bezieht sich auf die Analyse und Reflexion der Adaptivität verschiedener professioneller Handlungen während einer konkreten Situation.

- Reflection-on-action bezieht sich auf die Reflexion der in einer konkreten Situation ausgeführten professionellen Handlungen, nachdem diese stattgefunden haben.

Insbesondere der Prozess der reflection-in-action ist sehr umstritten, „da es eine hohe Kompetenz abverlangt, parallel zur Handlung zu reflektieren und augenblicklich Veränderungen einzuleiten“ (Abels, 2011, S. 53). Aus diesem Grund werden in vorliegendem Beitrag unter Reflexionskompetenz die kognitiven Fähigkeiten und Fertigkeiten verstanden,

das eigene didaktische Handeln und die eigenen didaktischen Entscheidungen im Kontext einer pädagogischen Situation im Nachhinein zu überdenken und explizit $\mathrm{zu}$ begründen, um bewusst daraus zu lernen, mit dem Ziel eines persönlichkeitswirksamen Bildungsprozesses. Dafür sollte rückblickend Bezug genommen werden 
auf die eigenen Erfahrungen im didaktischen Feld, die Kommunikation mit Dritten (Schüler, Kommilitonen, Seminarleitung), das eigene Vorwissen und Faktenwissen aus der Literatur im Sinne einer Theorie-Praxis-Relationierung. (Abels, 2011, S. 56)

\subsection{Komplexitätsreduktion}

Komplexitätsreduktion stellt einen wesentlichen Aspekt von Lehr-Lern-Laboren dar (u. a. Dohrmann \& Nordmeier, 2015). Das Anforderungs-, aber auch das Unterstützungsniveau der Lernumgebung werden angepasst, um eine möglichst gute Passung an das Leistungsniveau der Studierenden, aber auch an das gesetzte Ziel des Lehr-Lern-Labors zu schaffen (Marohn, Greefrath, Hammann, Hemmer, Kürten \& Windt, in diesem Band).

Hinsichtlich der Anforderungen innerhalb der Reflexionsphasen spielen hier die Breite und Tiefe der Reflexionen eine offensichtliche Rolle, jedoch sind auch weitere Aspekte zu berücksichtigen. So ist auch die Einflussnahme von beispielsweise der Art der Praxiserfahrung, der Rolle der Studierenden innerhalb der Praxisphasen, aber auch des Modus der Reflexion auf das Anforderungsniveau der Reflexionsphasen denkbar. Vielseitige Faktoren hinsichtlich des Anforderungsniveaus sind aber auch mögliche Unterstützungsmaßnahmen, die getroffen werden können. So sind Betreuung während der Reflexionen, Regeln, mediale Unterstützung durch Videoaufnahmen oder auch die Wiederholung oder Übung der Reflexionsphasen zu nennen.

Diese starke Abhängigkeit von Konzeption, aber auch Umsetzung der LehrLern-Labore verspricht bezüglich einer gemeinsamen Evaluation durch die Heterogenität der verschiedenen Lernumgebungen eine Herausforderung. Neben übergreifenden quantitativen Auswertungen beschäftigt sich dieser Beitrag daher in wesentlichen Teilen mit zwei konkreten Lehr-Lern-Laboren des Teilprojekts.

\subsection{Ziele der Lehrveranstaltungen}

Den im Teilprojekt Lehr-Lern-Labore realisierten Lehrformaten liegen unterschiedliche Dimensionen und Ausprägungen von Komplexitätsreduktion zugrunde. Die beiden hier betrachteten Seminarformate der Mathematik und Physik unterscheiden sich dabei in Bezug auf die berücksichtigten Dimensionen der Komplexität.

Im Lehr-Lern-Labor der Physik spielt die Reflexionskompetenz eine eher implizite, aber dennoch zentrale Rolle. Eine tiefgreifende Reflexion einzelner Unterrichtssequenzen macht hier einen wesentlichen Teil der Seminarzeit aus, wobei der Begriff „Reflexionskompetenz“ allerdings nicht explizit verwendet und besprochen wird. Reflexionen finden hier gestützt durch Videoaufnahmen der mehrfachen Praxiserfahrung statt und werden sehr tiefgreifend geführt. Zentral ist dabei die 
Entwicklung möglicher Handlungsalternativen im direkten Bezug auf die Lehrhandlungen der jeweiligen Studierenden.

Erwartet wird hier neben einer sehr handlungs- und selbstbezogenen Reflexionskompetenz auch der Einbezug der Reflexion anderer. Zwar ist der Fokus einer einzigen Reflexion im Rahmen des Lehr-Lern-Labors auf die Person selbst gerichtet, sie findet jedoch in Gruppen im Sinne einer kollegialen Fallberatung statt. In der Regel stehen dabei die Rolle der anderen Personen und die Wirkung auf Außenstehende im Fokus.

Die Komplexitätsreduktionen sind im Wesentlichen durch die dreifache Wiederholung der Praxiserfahrung, kleine Lerngruppen in meist vertrauter Umgebung und eine hohe Unterstützung bei Planung der Praxiserfahrung realisiert. Andererseits ist zu erwarten, dass die mit 12 Minuten sehr kurze Dauer der Lehrsequenzen von den Studierenden eher als komplexitätssteigernd bewertet wird.

Im Lehr-Lern-Labor der Mathematik spielt die Reflexionskompetenz eine ebenso zentrale wie auch implizite Rolle in Bezug auf die Professionalisierungsprozesse der teilnehmenden Studierenden. Eine tiefgreifende Reflexion der Beobachtungen der Lehr-Lern-Prozesse unter festem fachdidaktischem Fokus sowie der selbsterstellten Aufgabenstellungen nimmt einen Großteil der Seminarzeit in Anspruch. Diese Reflexionsprozesse werden in Form von Aufgabenbewertungen, Schreibgesprächen, Pecha-Kucha-Vorträgen und kollegialen Beratungen auf Basis der wiederholten Praxiserfahrungen initiiert. Dabei wird der Begriff der Reflexionskompetenz als solcher nicht expliziert. Der Fokus richtet sich auf die Diagnose von Modellierungsprozessen bei Schülerinnen und Schülern sowie die Konsequenzen für die Anpassung der selbsterstellten Aufgaben und das eigene didaktische Handeln.

Erwartet wird eine praxisorientierte und handlungsbezogene auf den konkreten Seminarerfahrungen basierende und in erster Linie selbstbezogene Reflexionskompetenz. So wurden durch die kollegialen Beratungen einerseits gruppenbasierte Reflexionsprozesse angestoßen und die Möglichkeit des Einbezugs von Fremdwahrnehmungen gegeben. Da die eigenen Instruktionen der Studierenden im Rahmen der Praxisphase jedoch konzeptbedingt eingeschränkt waren, standen andererseits eher die indirekten Lehrhandlungen in Form der Aufgabenstellungen im Fokus und weniger die direkten Instruktionen.

Die Komplexitätsreduktion wird im Mathematikseminar unter anderem durch die wiederholte Durchführung der Lehrhandlungen realisiert. Hinzu kommt ein ausgeglichenes Betreuungsverhältnis von Studierenden zu Lernenden (i.d.R. 3:4) sowie eine primär hochschulische Verortung der Praxiserprobungen. Die Studierenden werden dadurch insgesamt durch eine intensive und vielschichtige strukturelle Betreuung bei der Entwicklung der Aufgaben unterstützt, welche die hohen Anforderungen der fachdidaktischen Theorien im Bereich Medien und Materialien ausgleichen soll. 


\section{Forschungsfragen}

Die Konzeption der Lehr-Lern-Labor-Seminare zielt darauf ab, ein für die Studierenden angemessenes Gleichgewicht zwischen Anforderungen und Unterstützungen umzusetzen. Die erste Forschungsfrage beschäftigt sich folglich damit, ob dies aus Sicht der Studierenden erreicht wurde. In diesem Beitrag beschränken wir uns auf die strukturellen Dimensionen der Komplexitätsreduktion, da diese zwischen den unterschiedlichen Fächern eher vergleichbar sind als die inhaltlichen Dimensionen.

F1: Wie schätzen die Studierenden die Angemessenheit der Komplexitätsreduktion in den unterschiedlichen strukturellen Dimensionen ein?

Ziel ist es dabei die Einschätzung aller Studierender, die ein Lehr-Lern-LaborSeminar im Rahmen des Projekts besucht haben, zu erfassen. Dazu wird die Einschätzung mithilfe von Fragebögen erhoben und quantitativ ausgewertet.

Wie bereits oben geschildert, sollen Lehr-Lern-Labore durch die Komplexitätsreduktion den Studierenden ein geeignetes Umfeld für die Entwicklung einer starken Selbstwirksamkeitserwartung in Bezug auf die eigene Lehrhandlung bieten. Ob dies in der hier vorgestellten Studie erfolgreich ist, wird in den nächsten Forschungsfragen thematisiert.

F2.1: Wie entwickelt sich die Selbstwirksamkeitserwartung in Bezug auf den Umgang mit Heterogenität bei den Studierenden, die ein Lehr-Lern-Labor-Seminar besucht haben, im Vergleich zu anderen Studierenden?

F2.2: Wie entwickelt sich die Selbstwirksamkeitserwartung in Bezug auf Lehrhandlungen im Lehr-Lern-Labor bei den Studierenden, die ein Lehr-Lern-Labor-Seminar besucht haben, im Vergleich zu anderen Studierenden?

Auch hier soll die Einschätzung aller Studierender erhoben werden, die ein LehrLern-Labor-Seminar besucht haben. Dazu werden ebenfalls Fragebögen verwendet, die in den Lehr-Lern-Labor-Seminaren sowie vergleichbaren Veranstaltungen ohne Lehr-Lern-Labor eingesetzt werden, sodass eine quantitative Auswertung angeschlossen werden kann.

In einem nächsten Schritt soll der Blick über die statistischen Zusammenhänge hinaus vertieft werden. Ziel ist es, die Sicht der Studierenden auf die Bedeutung von Reflexionskompetenz und die Einschätzung ihrer diesbezüglichen Entwicklung durch das Seminar zu beleuchten.

F3: Welche Definition und Bedeutung schreiben die Studierenden als angehende Lehrkräfte der Reflexionskompetenz in ihrem zukünftigen Berufsfeld zu? 
F4.1: Wie bewerten die Studierenden ihre eigene Reflexionskompetenz zu Beginn und nach Durchlaufen der jeweiligen Lehrveranstaltung in Bezug auf unterrichtliches Handeln?

F4.2: Wie bewerten die Studierenden ihre eigene Reflexionskompetenz zu Beginn und nach Durchlaufen der jeweiligen Lehrveranstaltung in Bezug auf die den Handlungen zugrunde liegenden kognitiven Prozesse?

Da diese Fragen auf Grundlage der quantitativen Daten nicht beantwortet werden können, wurden zwei Seminare ausgewählt, deren Studierende im Anschluss an die Lehrveranstaltung vertieft in Interviews befragt wurden. Die Lehr-Lern-LaborSeminare in den Fächern Mathematik („MiRA+“) und Physik („La:gune“) im münsterschen Projekt der Qualitätsoffensive Lehrerbildung weisen einerseits Parallelen in ihrer Struktur und andererseits Unterschiede durch die verschiedenen fachbedingten didaktischen Inhalte und unterrichtsmethodischen Schwerpunkte auf (vgl. Holz \& Heinicke, in diesem Band; Wess \& Greefrath, in diesem Band). Dadurch soll es möglich sein, einen differenzierten Blick auf die im Projekt durchgeführten Lehr-Lern-Labore zu werfen.

Anhand weiterer Daten kann abschließend der Zusammenhang zwischen den für F1 erhobenen Bewertungen der Komplexitätsreduktionen durch die Studierenden und den in $\mathrm{F}_{3}-\mathrm{F}_{5}$ erhobenen Selbsteinschätzungen ihrer Professionalisierung betrachtet werden.

F5: Welchen Einfluss schreiben die Studierenden der in den Lehrveranstaltungen vorgenommenen Komplexitätsreduktionen in Bezug auf ihre Entwicklung von Reflexionskompetenz zu?

\section{Methodik}

\subsection{Quantitative Studie}

\subsubsection{Fragebögen}

Alle Studierenden der Lehr-Lern-Labor-Seminare wurden zu Beginn (Pretest) und am Ende (Posttest) des Semesters gebeten einen Fragebogen auszufüllen. Dieser Bogen umfasst neben demographischen Daten und einer anonymisierten Kennung unter anderem Skalen zur Messung von Selbstwirksamkeitserwartungen in Bezug auf den Umgang mit Heterogenität bzw. Lehrhandlungen im Lehr-Lern-Labor. Zum zweiten Messzeitpunkt wurde zusätzlich erhoben, inwieweit die Studierenden die Umsetzung der Komplexitätsreduktion in den einzelnen strukturellen Dimensionen als angemessen einschätzen. Die Fragebögen wurden in dieser Form im Sommersemester 2018 sowie im Wintersemester 2018/2019 eingesetzt.

Die Einschätzung der Angemessenheit der Umsetzung der Komplexitätsreduktion wurde mit sieben Items (je eines für jede strukturelle Dimension) erfasst. Zu 
jeder Dimension konnten die Studierenden auf einer fünfstufigen Skala die Angemessenheit einschätzen (Beispiel: „Die Heterogenität der Lerngruppe, die ins Lehr-Lern-Labor gekommen ist, war ..." mit den Einschätzungsmöglichkeiten: $z u$ gering, eher zu gering, angemessen, eher zu hoch, zu hoch). Für die Einschätzung der Selbstwirksamkeitserwartung in Bezug auf den Umgang mit Heterogenität wurde der Selbstwirksamkeitserwartungsteil des MESS-H-Fragebogens verwendet [MESS-H_SWE] (Junker, Zeuch, Rott, Bartsch, Henke \& Kürten, ang.). Die Skala ist fünfstufig (trifft überhaupt nicht $z u$ bis trifft voll $z u$ ) und umfasst je zwei Items zur Diagnostik, Förderung und Evaluation (siehe Tabelle 1).

Die Skala zur Einschätzung der Selbstwirksamkeitserwartung in Bezug auf Lehrhandlungen im Lehr-Lern-Labor [SWE_L] ist eine siebenstufige Likertskala (trifft überhaupt nicht $z u$ bis trifft voll $z u$ ) und umfasst je fünf Items zur Planung, Durchführung und Reflexion von Lernarrangements im Lehr-Lern-Labor (siehe Tabelle 1). Die Skala wurde im Rahmen des Projekts entwickelt und pilotiert (Kürten, Wess \& Greefrath, 2018).

Tabelle 1: Verwendete Skalen zur Erfassung der Selbstwirksamkeitserwartung mit Kennwerten aus dem Pretest und jeweils einem Beispielitem

\begin{tabular}{llll}
\hline Skala & Items & $\begin{array}{l}\text { Interne Konsistenz } \\
(\text { Cronbachs } \alpha)\end{array}$ & Beispielitem \\
\hline MESS-H_SWE & 6 & 0.76 & $\begin{array}{l}\text { Ich fühle mich kompetent, unterschiedliche Facetten der } \\
\text { Lernausgangslagen von Lernenden zu erkennen. }\end{array}$ \\
SWE_L & 15 & 0.88 & $\begin{array}{l}\text { Bei der Durchführung von Lernarrangements traue ich } \\
\text { mir zu, auf individuelle Präkonzepte der Lernenden } \\
\text { einzugehen. }\end{array}$ \\
\hline
\end{tabular}

Für die Beantwortung der zweiten Forschungsfrage wurden zusätzlich zu den Lehr-Lern-Labor-Seminaren Veranstaltungen ausgewählt, die von vergleichbaren Studierendengruppen (in Bezug auf Studienfach und Fachsemester) besucht wurden, bei denen jedoch kein Lehr-Lern-Labor eingebunden war. Diese Studierenden wurden ebenfalls zu Beginn und am Ende des Semesters befragt, wobei die Einschätzung der Angemessenheit der Komplexitätsreduktion nicht abgefragt wurde.

\subsubsection{Beschreibung der Stichprobe der quantitativen Studie}

In den Lehr-Lern-Labor-Seminaren (Treatmentgruppe) haben in den beiden Semestern zusammen 256 (Pretest) bzw. 212 (Posttest) Studierende den Fragebogen bearbeitet. Davon haben 197 zu beiden Zeitpunkten den Bogen ausgefüllt.

Von diesen Studierenden sind ca. $74 \%$ weiblich und $24 \%$ männlich, $2 \%$ haben keine Angabe zum Geschlecht gemacht. Von den Teilnehmenden sind $56 \%$ Studierende für das Lehramt an Gymnasien und Gesamtschulen, 32 \% für das Lehramt 
an Grundschulen, 8 \% für das Lehramt an Haupt-, Real-, Sekundar- und Gesamtschulen und $2 \%$ für das Lehramt an Berufskollegs.

In den Veranstaltungen der Vergleichsgruppe haben 195 (Pretest) bzw. 141 (Posttest) den Fragebogen bearbeitet. Davon haben 122 an beiden Testzeitpunkten teilgenommen. Die Verteilung der Geschlechter ist mit der der Treatmentgruppe vergleichbar. Von ihnen sind $65 \%$ Studierende für das Lehramt an Gymnasien und Gesamtschulen, $23 \%$ für das Lehramt an Grundschulen, $7 \%$ für das Lehramt an Haupt-, Real-, Sekundar- und Gesamtschulen und $6 \%$ für das Lehramt an Berufskollegs.

\subsection{Qualitative Studie}

\subsubsection{Interviews}

Da die Forschungsfragen 3, 4 und 5 auf Basis der quantitativen Befragung nicht beantwortet werden können, wurden im Rahmen einiger Lehrveranstaltungen des Teilprojektes zusätzlich qualitative Daten in Fragebögen und Interviews erhoben. Basierend auf den Skalenitems der Fragebögen zur Reflexionskompetenz und Komplexitätsreduktion wurde dafür ein Befragungsinstrument für ein leitfadengestütztes Interview entwickelt. Der Leitfaden enthält sowohl Items zur quantitativen Einschätzung der eigenen Reflexionskompetenz zu Beginn und am Ende der Lehrveranstaltung als auch zu der Angemessenheit der Ausprägungen der einzelnen Dimensionen von Komplexität. Nachfolgend wurden die Befragten jeweils gebeten, ihre Einschätzung zu erläutern und gegebenenfalls mit Beispielen aus dem jeweiligen Seminar zu illustrieren. Dazu wurden weitere Leitfragen zum jeweiligen Seminar gestellt.

Die Daten ermöglichen vor allem in ihrer Kombination einen vertieften Einblick in die Bewertung der eigenen Lernprozesse aus Sicht der Studierenden. Sie bieten daher Ansatzpunkte für die Weiterentwicklung der Lernformate durch eine Stärkung der als positiv bewerteten Aspekte einerseits sowie eine Optimierung der noch bestehenden Herausforderungen andererseits.

\subsubsection{Auswertung der Interviews}

In den beiden hier betrachteten Seminaren der Mathematik und Physik wurden leitfadengestützte Interviews mit insgesamt 24 Teilnehmenden (11 aus der Physik und 13 aus der Mathematik) durchgeführt, die jeweils ca. 30 Minuten dauerten. Die Interviews wurden vollständig wortgetreu transkribiert und nach kategorienbildenden Verfahren in Anlehnung an die Qualitative Inhaltsanalyse ausgewertet. Dabei wurden die oben genannten drei Aspekte in Fragen des Leitfadens explizit aufgegriffen. Weitere Antworten der Befragten, die im Kontext anderer Fragen 
Informationen zu den drei Aspekten enthielten, wurden ebenfalls analysiert und kategorisiert.

Bei der Auswertung der Interviewdaten stand eine Quantifizierung der Aussagen nicht im Fokus. Die Leitfragen der Interviews regten die Befragten zu einer nachträglichen Auseinandersetzung und Bewertung der Seminarkonzepte an, daher kamen situationsbedingt teils sehr unterschiedliche Aspekte zur Sprache. Es scheint demnach nicht angemessen, aus diesen situativ entstandenen Schwerpunkten der Interviewthemen Aussagen zu den Häufigkeiten abzuleiten. Es wird darum in den folgenden Darstellungen auf prozentuale Angaben verzichtet. Solche Aussagen könnten in einer folgenden Studie anhand von Fragebögen erhoben werden.

\section{Auswertung}

\subsection{F1: Angemessenheit der Komplexitätsreduktion}

Die in diesem Band vorgestellten Lehr-Lern-Labore zeichnen sich insbesondere durch die große Heterogenität der gewählten Ansätze aus. Bei der Auswertung der Antworten der Studierenden bei den Skalenitems zu den strukturellen Aspekten der Komplexitätsreduktion wurde deutlich, dass diese überwiegend als angemessen eingestuft wurde, obwohl sich die Lehr-Lern-Labore so grundlegend unterscheiden. Neben der Waage, die sich Anforderungen und Unterstützungsmaßnahmen halten sollten (Marohn et al., in diesem Band), wird hier besonders deutlich, dass Facetten der Komplexitätsreduktion dann angemessen sind, wenn sie als passend zur Zielsetzung des jeweiligen Lehr-Lern-Labors empfunden werden.

Die Studierenden schätzen die Komplexitätsreduktion auf den verschiedenen Items im Mittel zwischen 2.7 und 3.1 und somit als angemessen ein. Dabei zeigen sich insbesondere hohe Standardabweichungen in den Bereichen Heterogenität der Lerngruppe, Verhältnis von Schülerinnen und Schülern zu Studierenden und Zeit der Unterrichtssequenz (siehe Abb. 1). 


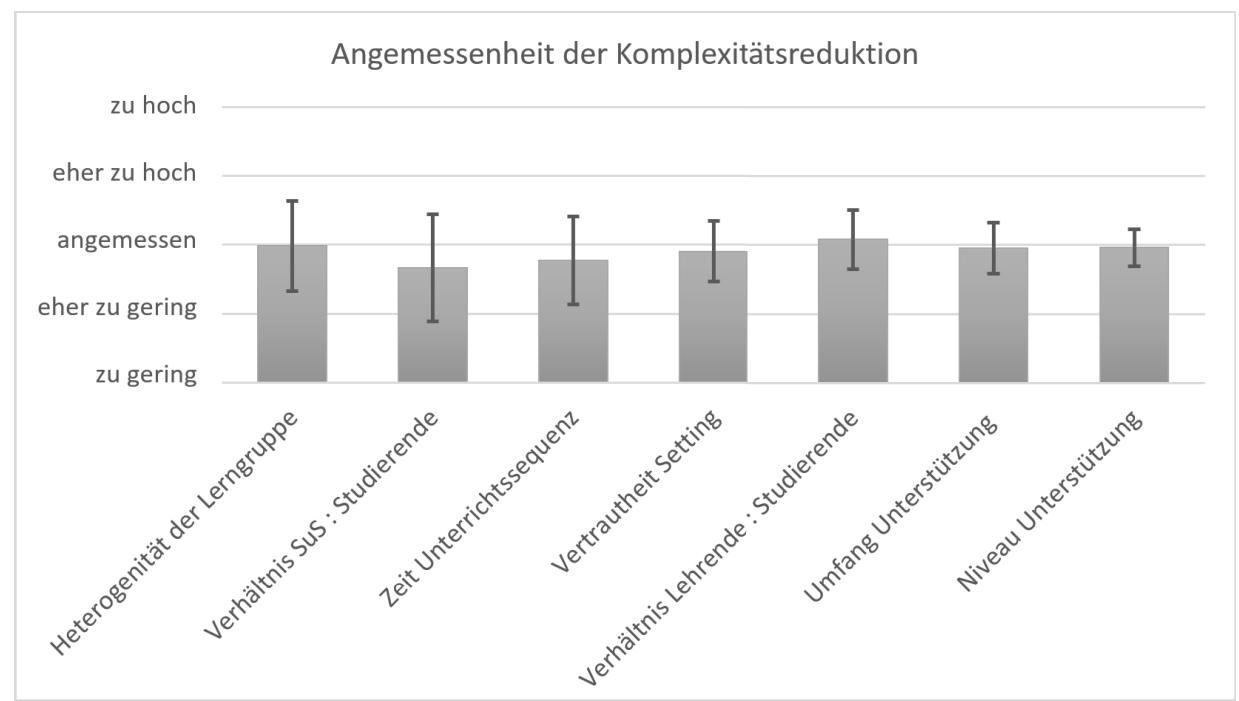

Abbildung 1: Mittelwerte und Standardabweichungen bezüglich der Angemessenheit der Komplexitätsreduktion

\subsection{F2: Entwicklung der Selbstwirksamkeitserwartungen von Studierenden im Lehr-Lern-Labor-Seminar und anderen Studierenden}

In Tabelle 2 sind die Mittelwerte und Standardabweichungen der Konstrukte im Pre- und Posttest für die Treatment- und Vergleichsgruppe dargestellt.

Tabelle 2: Mittelwerte und Standardabweichungen (in Klammern) der Skalen zur MESSH_SWE und SWE_LLL von Studierenden der Vergleichs- und Treatmentgruppe im Pre- und Posttest.

\begin{tabular}{|c|c|c|c|c|c|}
\hline & & \multicolumn{2}{|c|}{ Treatmentgruppe } & \multicolumn{2}{|c|}{ Vergleichsgruppe } \\
\hline & & Pretest & Posttest & Pretest & Posttest \\
\hline MESS-H_SWE & MW (SD) & $3.23(0.62)$ & $3.68(0.48)$ & $3.05(0.68)$ & $3.28(0.68)$ \\
\hline SWE_L & MW (SD) & $4.88(0.63)$ & $5.34(0.63)$ & $4.73(0.73)$ & $4.75(0.74)$ \\
\hline
\end{tabular}

Zum Vergleich der Entwicklung zwischen Studierenden der Treatment- und der Vergleichsgruppe wurde eine zweifaktorielle Varianzanalyse mit Messwiederholung auf einem Faktor gerechnet. 


\subsubsection{Selbstwirksamkeitserwartung in Bezug auf den Umgang mit Heterogenität}

Für die Auswertung konnten 300 der 319 Datensätze genutzt werden. Es wurden keine Ausreißer identifiziert (beurteilt mit dem Box-Plot) und Varianzhomogenität ist gemäß dem Levene-Test gegeben $(F(1,298)=0.806, p=0.37)$. Die Daten beider Gruppen sind jedoch weder für den ersten noch für den zweiten Testzeitpunkt normalverteilt (Shapiro-Wilk-Test, $\mathrm{p}<0.05$ ). Da sich die Varianzanalyse sehr robust gegenüber Verletzungen dieser Voraussetzung zeigt (Glass, Peckham \& Sanders, 1972), wurde dennoch eine zweifaktorielle ANOVA mit Messwiederholung auf einem Faktor berechnet, um zu untersuchen, ob es einen Unterschied der Entwicklung der Selbstwirksamkeitserwartung zum Umgang mit Heterogenität zwischen den betrachteten Gruppen gibt.

Es zeigt sich ein signifikanter Effekt für den Faktor Zeit x Gruppe, d.h. die Selbstwirksamkeitserwartung verändert sich innerhalb des Semesters bei Studierenden, die ein Lehr-Lern-Labor-Seminar besucht haben, stärker als bei Studierenden der Vergleichsveranstaltungen. Die Effektstärke liegt im niedrigen Bereich $(\mathrm{F}(1,298)=8.546, \mathrm{p}=0.004)$.

Bei Betrachtung der Mittelwerte der beiden Gruppen zeigt sich, dass die Selbstwirksamkeitserwartung in beiden Gruppen ansteigt.

\subsubsection{Selbstwirksamkeitserwartung in Bezug auf Lehrhandlungen im Lehr-Lern-Labor}

Für diese Auswertung konnten 298 der 319 Datensätze genutzt werden. Da keine Ausreißer identifiziert werden konnten (beurteilt mit dem Box-Plot), die Daten für beide Gruppen und Zeitpunkte normalverteilt sind (Shapiro-Wilk-Test, $\mathrm{p}>0.05$ ) und Varianzhomogenität gemäß dem Levene-Test gegeben ist $(\mathrm{F}(1,296)=3.315$, $\mathrm{p}=0.067$ ), wurde eine zweifaktorielle ANOVA mit Messwiederholung auf einem Faktor berechnet, um zu untersuchen, ob es einen Unterschied der Entwicklung der Selbstwirksamkeitserwartung zu Lehrhandlungen im Lehr-Lern-Labor zwischen den betrachteten Gruppen gibt.

Es zeigt sich ein signifikanter Effekt für den Faktor Zeit x Gruppe, d.h. die Selbstwirksamkeitserwartung verändert sich innerhalb des Semesters bei Studierenden, die ein Lehr-Lern-Labor-Seminar besucht haben, stärker als bei Studierenden der Vergleichsveranstaltungen. Die Effektstärke liegt im mittleren Bereich $(\mathrm{F}(1,296)=2.745, \mathrm{p}<0.001)$.

Bei Betrachtung der Mittelwerte der beiden Gruppen zeigt sich, dass sich die Selbstwirksamkeitserwartung bei den Studierenden der Vergleichsgruppe nicht verändert haben, während die Studierenden der Treatmentgruppe einen Anstieg der Selbstwirksamkeitserwartung zeigen. 


\subsection{F3: Definition und Bedeutung von Reflexionskompetenz aus Sicht der Studierenden}

In den Interviews wurden die Studierenden explizit nach ihrem Verständnis von Reflexionskompetenz und der Relevanz der Reflexionskompetenz für den Lehrerberuf gefragt. Für die Beantwortung der Forschungsfragen wurden darüber hinaus auch weitere Stellen der Interviews genutzt.

\subsubsection{Was verstehen die Studierenden unter Reflexionskompetenz?}

Eine Definition von Reflexionskompetenz wurde in den Seminaren der Physik und der Mathematik nicht explizit thematisiert, daher handelt es sich bei den Antworten der Studierenden nicht um Reproduktionen, sondern um eigene Formulierungen. Dabei besteht unter den Befragten weitgehende Einigkeit darin, dass Reflexion eine rückblickende Analyse einer Situation bezeichnet. Rund die Hälfte der Antworten schränkt dies explizit auf Handlungen im Unterrichtsgeschehen ein. Für einen Großteil der Befragten umfasst die Definition von Reflexion außerdem explizit den zielgerichteten Aspekt, zukünftiges Handeln zu verändern bzw. $\mathrm{zu}$ verbessern. (Kategorien: Reflexion bedeutet über sich selbst und sein Handeln nachdenken und alternative Handlungsoptionen für die Zukunft zu entwickeln. / Reflexion ist Handlungskorrektur.) Entsprechend schränkt die Mehrzahl der Befragten den Fokus der Reflexion auf Negativaspekte ein. (In der Reflexion geht es v. a. um das, was nicht geklappt hat.)

Nur wenige der Befragten fassen hierunter neben den optimierungsbedürftigen Aspekten auch solche, die positiv bewertet werden und beibehalten bzw. bestärkt werden sollten. In Bezug auf die als erfolgreich und als nicht erfolgreich bewerteten Handlungen und Ereignisse formulieren sie, Reflexionskompetenz bedeute, das eigene Handeln rückblickend zu bewerten, um es eventuell in Zukunft ändern oder wiederholen zu können, bzw. Positives wie Negatives zu analysieren und daraus konstruktiv Schlüsse für zukünftiges Handeln zu ziehen. So äußert sich eine Person diesbezüglich, „... dass man auch konkreter verfolgen kann, das war gut, das kann ich nochmal so machen." (M8, Z. 14)

\subsubsection{Welche Bedeutung hat Reflexionskompetenz für die Studierenden?}

Die Mehrheit der interviewten Studierenden halten Reflexionskompetenz für zentral wichtig für ihren zukünftigen Beruf, ein weiteres Drittel für eher wichtig. Die Befragten äußerten, ohne Reflexion könne „man keinen guten Unterricht gestalten“ (P9, Z. 41) oder Reflexionskompetenz sei die Grundlage für „eine effiziente Unterrichtsentwicklung“ (M7, Z. 36).

Auf der anderen Seite äußerten sich aber einige Befragte auch kritisch: Reflexionskompetenz werde zu sehr "gehypt“ oder beworben im Lehramtsstudium. 
Andere kritische Stimmen äußerten die Befürchtung, dass zu viel Reflexion sich negativ auswirken könnte und es daher darum gehe, ein gutes Maß für kritische Selbstreflexion zu finden. Konkret hatten die Kritiken folgende Kategorien zum Inhalt:

- Reflexion kostet Zeit und Nerven und sollte nicht zu viel erfolgen.

- Reflexion wird überbewertet und sollte nicht zu viel erfolgen.

- Reflexionskompetenz ist für den Lehrerberuf zwar zentral wichtig, aber in Maßen zu verwenden.

- Zu viel Reflexion ist nicht hilfreich.

Ein etwas größerer Teil der Studierenden konnotierte Reflexion als ein wesentliches Werkzeug primär für Novizen im Lehrberuf. Dabei schloss rund die Hälfte von ihnen hieran die Vermutung an, dass mit zunehmender Erfahrung die Reflexion Schritt für Schritt durch Routinen ersetzt würde. Die andere Hälfte vermutete, dass umgekehrt eine Zunahme an Routinen die Reflexion des Unterrichts nach und nach ablöse und die Unterrichtsqualität daher gerade von berufserfahreneren Lehrkräften stagnieren könne.

In Bezug auf das jeweilige Seminar bewerteten alle Befragten die durchgeführten Reflexionseinheiten als persönlich besonders wertvoll, beispielsweise:

- „Ich fand das richtig, richtig hilfreich, dass wir das gemacht haben und das werde ich auch im Praxissemester machen, so für mich selbst." (P2, Z. 18)

- „Ich fand das richtig gut so. Ich brauche das nicht noch tiefer, aber auch oberflächlicher nicht, nee. Ich glaube das war so ein gutes Maß.“ (P2, Z. 30)

- „Dass die Reflexion eigentlich hätte ich die viel länger gewünscht, aber [...] weil die war wirklich hilfreich." (P6, Z. 61-63)

\subsection{F4: Wie schätzen die Studierenden ihre Reflexionskompetenz und deren Veränderung ein?}

In den Interviews wurden die Studierenden explizit nach ihrer Einschätzung der Entwicklung ihrer Reflexionskompetenz und damit verbundenen Elementen des besuchten Seminars gefragt.

\subsubsection{F4.1: Einschätzung in Bezug auf unterrichtliche Handlungen}

Die Befragten nannten aus den Seminaren heraus Aspekte, die sie in der Weiterentwicklung ihrer Reflexionskompetenz unterstützt hatten. Im Fall des Lehr-LernLabors der Physik wurde von fast allen Befragten der Einsatz von Videographie hervorgehoben. Die Videos stützten die Reflexion diesen Aussagen zufolge in mindestens zwei Punkten: Sie boten zum einen die Möglichkeit, die Szene im Nachhinein noch einmal zu betrachten und so viele weitere Aspekte wahrnehmen und 
reflektieren zu können. Sie unterstützten demnach die differenzierte Analyse der Situation. Dabei stand bei fast allen der Befragten die Betrachtung und Wirkung der eigenen Person im Vordergrund, da sie sich selbst noch nie auf einem Video in einer unterrichtlichen Handlung betrachtet hatten:

- „Hilfreich war, dass man sich, also für mich war es das erste Mal, dass ich das auch auf Video hatte, dass ich selbst mich quasi beobachten konnte, hatte ich vorher nicht. Dann merkt man anders wie man wirkt und kann sich das in Ruhe nochmal angucken." ( $\left.\mathrm{P}_{3}, \mathrm{Z} .12\right)$

- „... einfach mal von außen den Blick hat, wie man eigentlich wirkt.“ ( $\left(P_{4}, Z\right.$. 10)

- „Ja, sehr viel, weil da hat man auch Sachen gesehen, die man nie drauf achtet, wie, dass ich leise rede, das haben wir gesagt." (P6, Z. 4)

- „... aber vor allem fand ich jetzt wichtig oder sinnvoll auch, dass mit diesen Videos zu machen, weil ich mich dann nochmal anders reflektiert habe. [...] ist das nochmal was anderes, als wenn ich das im Video natürlich nochmal sehe, wie habe ich mich überhaupt verhalten, wo sind vielleicht meine Macken und so. Es war auch mal eine andere Art und Weise wie ich reflektiere und das hat mir ziemlich viel doch irgendwo geholfen." (P5, Z. 6)

Zum zweiten unterstützten die Videos nach Ansicht der Befragten eine Objektivierung der Diskussion, da Szenen noch einmal gemeinsam betrachtet werden konnten und nicht nur aus der (subjektiven) Erinnerung heraus diskutiert werden mussten. Für einzelne Befragte führte dies allerdings auch zu einer überwältigenden Menge an Information, vor allem, da die gemeinsame Betrachtung der Videos mitunter auch eine Reihe von kritischen Detailanmerkungen hervorbrachte:

- „Nicht hilfreich war für mich, dass viel Information, glaube ich, gesammelt kam. Also ich/ am ersten Reflexionstag, das weiß ich noch, da kam so viel Input oder so viel was ich falsch gemacht habe. Natürlich subjektiv falsch gemacht habe, dass ich in meinem Handeln relativ unsicher geworden bin. Dass ich mir danach gedacht habe ,oh scheiße, was ist denn jetzt los' ich habe ja alles falsch gemacht gefühlt. Ich habe gar nichts wirklich richtig gemacht, obwohl ich das Gefühl hatte bei der ersten Stunde ,es ging eigentlich, ich fand es gar nicht so schlecht' ${ }^{t}$ und das Bild wurde einfach komplett zerschmettert." (P13, Z. 19)

Diese Wahrnehmung der Wertung in den Rückmeldungen der Gruppe variierte von Person zu Person. Andere beschrieben es im Gegensatz dazu als konstruktives und weitgehend wertfreies Feedback:

- „Also, man hat nicht das Gefühl von einer Bewertung, sondern eher von einem Feedback, ein konstruktives Feedback, was natürlich auch wieder in gewisser Weise einer Reflexion spiegelt, die er einem gibt.“(M6, Z. 10)

Diese Dimension der empfundenen Konstruktivität von Rückmeldungen wäre anhand weiterer Studien und der entsprechenden theoretischen pädagogischen Theorien noch genauer zu beleuchten. 
Im Physikseminar wurde die Wiederholung der Reflexionsphasen diskutiert. Dabei bewertete die Mehrheit sie als positiv oder wünschte sich sogar noch mehr als drei Durchläufe (Iterationen). Manche äußerten hingegen, auch eine gemeinsame Reflexion hätte bereits ausgereicht.

\subsubsection{F4.2: Einschätzung in Bezug auf den Handlungen zugrundeliegende kognitive Prozesse}

In den Interviews der Befragten des Mathematikseminars stand hingegen die Reflexion der kognitiven Prozesse der Beteiligten im Vordergrund. Dadurch, dass die Studierenden in den Durchführungsphasen in erster Linie die Lehr-Lern-Prozesse unter festgelegtem Fokus beobachteten und weniger aktiv in die Lernprozesse eingriffen, entstanden hier andere Lernsituationen, die die meisten durch den Abgleich der eigenen Denkprozesse mit denen der Schülerinnen und Schüler beschrieben.

- „Also in dem Moment habe ich drüber nachgedacht, dass es verrückt ist, dass ich, ich konnte nicht nachvollziehen wie die dann so denken konnten während ich ganz anders gedacht hab ..." (M1, Z. 23)

- „Aber auch mich selber in dieser Unterrichtssituation quasi sehen zu können und zu beobachten, wie die Schüler arbeiten, das habe ich auch mitgenommen, weil ich doch in der Aufgabe, weil ich doch gedacht habe die ist perfekt, aber dann haben wir die durchgeführt und dann war sie das leider nicht." (M10, Z. 10)

- „Genau, weil in dem Moment, wo ich die Schüler beobachtet habe und gesehen habe, die kommen nicht weiter. Die haben ein Problem, ein Problem, von dem ich nicht dachte, dass es das gibt. Das war der Moment, wo ich darüber nachgedacht habe, ist unsere Aufgabe wirklich gut. Haben wir vielleicht oder habe ich vielleicht mir das falsch vorgestellt, bin mit falschen Erwartungen daran herangegangen." (M1O, Z. 24)

In den Interviews mit den Studierenden des Mathematikseminars zeigt sich allerdings auch deutlich, dass Reflexion, wie oben bereits beschrieben, für die meisten Befragten mit einer vorausgegangenen eigenen aktiven Handlung einhergehen muss. Nun bestanden die in diesem Seminarformat vollzogenen didaktischen Handlungen neben der Erprobung semiformeller Diagnostik vor allem auch aus der Konzeption einer Modellierungsaufgabe, während in der aktiven Phase diese Aufgabe allein durch die Schülergruppe bearbeitet wurde und die Studierenden nur beobachteten. Viele der Befragten werteten dies aufgrund der fehlenden eigenen unterrichtlichen Handlung nicht als Reflexion im engeren Sinne. Es lassen sich daher aus einigen Interviews nur indirekte Aussagen über den persönlichen Nutzen der Reflexionsphasen im Seminar ableiten, die von den Studierenden nicht immer explizit als solche deklariert wurden. 
Die Reflexion in der Gruppe fand in diesem Lehrformat auch vor allem im Vorfeld der Umsetzung statt, indem die konzipierten Aufgabenformate mit den Kommilitonen vorab diskutiert wurden.

- „In der Vorbereitungsphase haben wir auch mal uns gegenseitig Feedbacks gegeben, wie wir die Aufgaben der anderen finden oder auch was wir noch überarbeiten sollen und haben quasi darüber immer wieder die Aufgabe überarbeitet, was ja in dem Sinne eine Reflexion teilweise darstellt, weil man doch darüber nachdenkt, was nicht gut ist." (M6, Z. 20)

Demgegenüber wurde von den Befragten jedoch die abschließende Sitzung des Seminars als wesentlich für die Reflexion genannt, in der die Kriterien von Modellierungsaufgaben noch einmal gemeinsam mit der jeweils selbst konzipierten Aufgabe abgeglichen wurden.

- „In der allerletzten Sitzung haben wir darüber reflektiert, was wir vorher für Erwartungen an unserer Aufgabe hatten. Also welche Kriterien die erfüllt, welche Kompetenzen, die am meisten anspricht. Und wir haben darüber reflektiert, welche Kompetenzen wirklich am meisten angesprochen wurden." (M6, Z. 14)

\subsection{F5: Hilfreiche (strukturelle) Rahmenbedingungen des Lehr-Lern- Labors aus Sicht der Studierenden und zugehörige Dimensionen der Komplexität}

Die Aussagen und Einschätzungen, die die befragten Studierenden der beiden Lehr-Lern-Labore $\mathrm{MiRA}^{+}$und La:gune in den geführten Interviews machten, erlauben eine Gegenüberstellung ausgewählter, im Zuge der Kategorienbildung entstandener struktureller Facetten. Diese können zwar im Modell zur Komplexitätsreduktion (Marohn et al., in diesem Band) verortet werden, entsprechen diesem jedoch abhängig vom Antwortverhalten der Befragten nicht in allen Fällen. Diskutiert werden im Folgenden die Dimensionen Unterstützung durch Dozierende, Größe der Lerngruppe, Dauer der Praxiserfahrung und Vertrautheit mit Räumlichkeiten und Material.

\subsubsection{Unterstützung durch Dozierende}

Die Qualität und vor allem Quantität von Hilfestellungen seitens der Dozentinnen und Dozenten kann in der Konzeption und Umsetzung von Lernumgebungen gesteuert werden. In einer Vorbereitungsphase, in der Unterricht oder Unterrichtsmaterialien entworfen und geplant werden, kann beispielsweise von Anfang an oft und intensiv Hilfestellung gegeben werden. Genauso kann es dem Ziel der Lernumgebung aber auch dienlich sein, Studierende zunächst eigenverantwortlich und ohne große Eingriffe arbeiten zu lassen. 
Bei der Einschätzung der Unterstützung durch Dozentinnen und Dozenten wurde dabei von den Studierenden meist nicht die Unterstützung und deren Qualität oder Quantität als zentral gesehen und gewertet, vielmehr wurde die Möglichkeit Unterstützung anzufordern genannt. Ein eigenverantwortliches Einfordern von Hilfestellungen wurde beispielsweise oftmals als Begründung für angemessene Unterstützung angegeben. In vereinzelten Fällen wurde sogar darauf hingewiesen, dass eine zu starke Hilfestellung teilweise in eine vorgegebene Richtung drängt und damit als negativ einzuschätzen ist. Nur in einem Fall wurde eine Unterstützung als positiv definiert, wenn sich Dozenten eigenständig einschalten, sobald sie merken, "dass man hängt" (M4, Z. 43). Auch ein sukzessiver Aufbau von Unterstützung wurde im Falle des Lehr-Lern-Labors La:gune als positiv beschrieben (vgl. Holz \& Heinicke, in diesem Band).

Im Rahmen dieses Faktors zeigt sich eine weitestgehende Einigkeit der Befragten beider Lehr-Lern-Labore. Unterstützung wird in den meisten Fällen dann als positiv eingeschätzt, wenn sie eingefordert werden kann. Ein leichter Unterschied zeigt sich in den einzelnen Fällen, die den Wunsch nach stärkerer Unterstützung äußern. Diese finden sich ausschließlich unter den Befragten des Lehr-LernLabors La:gune.

\subsubsection{Größe der Lerngruppe}

Diese Facette beschreibt das Verhältnis zwischen Studierenden und Schülerinnen und Schülern während der Praxiserfahrung. Hier ist eine besonders große Differenz der Einschätzungen zwischen den beiden befragten Lehr-Lern-Laboren festzustellen, daher werden beide Gruppen von Befragten zunächst getrennt beschrieben. Zudem wird die Art der Praxiserfahrung in beiden Fällen zunächst in für die Auswertung relevanten Merkmalen kurz angerissen (für detaillierte Beschreibungen der Lehr-Lern-Labore siehe Holz \& Heinicke, in diesem Band; Wess \& Greefrath, in diesem Band).

\subsubsection{Physik}

Die Praxiserfahrungen umfassen hier unterrichtsähnliche Lehrsituationen mit allen zugehörigen Handlungen. Der Fokus liegt dabei klar auf der Lehrkraft und wie diese handelt. Die Größe der Lernendengruppe liegt je nachdem, welche der drei gestuften Praxiserfahrungen betrachtet wird, zwischen 5 und 10 Personen, die von einem/einer Studierenden unterrichtet werden.

Diese Größe der Lerngruppe wurde von den Interviewten in sehr vielen Fällen als zu gering eingeschätzt. Als Grund wurde hier vorwiegend die sinkende Authentizität genannt, die sich beispielsweise durch Antwortverhalten in Unterrichtsgesprächen oder Brainstorming-Phasen sowie in Gruppenarbeiten bemerkbar 
macht. Als angemessen wurde die Größe nur in Bezug auf das Lernziel des LehrLern-Labors bezeichnet. Eine Umsetzung mit größeren Lerngruppen sei damit nur schlecht umsetzbar. Die unterrichtsähnliche Art der Praxiserfahrung erfordert nach Meinung der Studierenden auch eine unterrichtsähnliche Anzahl von Schülerinnen und Schülern, um eine authentische Lehr-Lern-Situation zu erzeugen.

\subsubsection{Mathematik}

Die Praxiserfahrung fokussiert hier auf die diagnostische Beobachtung der LehrLern-Prozesse durch die Studierenden unter festgelegtem Fokus, ohne bspw. durch adaptive Interventionen in diese einzugreifen. Die wesentlichen Lehrhandlungen (beispielsweise das Entwerfen der Arbeitsmaterialien) wurden bereits vorab vollzogen. Der Fokus liegt auf den Bearbeitungsprozessen der Schülerinnen und Schüler und wie diese bezogen auf einzelne Teilkompetenzen konkret agieren. Hier wird innerhalb einer Praxiserfahrung eine Schülergruppe mit 3-4 Schülerinnen und Schülern von einer Studierendengruppe mit 3 Studierenden beobachtet.

Von der überwiegenden Zahl der Befragten wird das Verhältnis von Studierenden zur Größe der Lerngruppe als angemessen bezeichnet. In einigen Fällen wurde sogar angemerkt, dass die Beobachtung einer größeren Anzahl von Schülerinnen und Schülern mit dem gegebenen starken und detaillierten Fokus nicht möglich gewesen wäre. So wurde in zwei Fällen geäußert, dass zu einzelnen Beobachtungsphasen auch diese Relation bereits sehr schwierig sei. Angemerkt wurde jedoch auch, dass eine 1:1-Betreuung die Schülerinnen und Schüler unter Druck setzen kann.

\subsubsection{Vergleich}

An dieser Stelle wird die Abhängigkeit der Angemessenheit einzelner Facetten der Komplexitätsreduktion von dem gesetzten Ziel und Fokus des Lehr-Lern-Labors sehr deutlich. Am Beispiel der beiden Lehr-Lern-Labore zeigt sich, dass eine Relation von nahezu 1:1 als annähernd zu hoch beschrieben werden kann, während eine Relation von 1:5-10 für die durchgeführten Aktivitäten durchaus auch als zu niedrig eingeschätzt wird.

Studierende im Lehr-Lern-Labor La:gune vollführen eine authentische Einheit, sie stehen im Fokus und sollen hier praxisnah unterrichten. Dies erfordert, dass eine realitätsnahe Interaktion zwischen Lerngruppe und Lehrkraft ermöglicht wird. Bei einer zu geringen Größe der Lerngruppe ist dies nicht mehr gegeben. Das Lehr-Lern-Labor MiRA ${ }^{+}$setzt dagegen einen sehr konzentrierten Fokus auf die Handlungen einer Lerngruppe. Die Studierenden beobachten sehr fokussiert und damit aber auch detailliert. Ein geringeres Verhältnis von Studierenden zu Lerngruppe ist mit diesem Fokus nicht effektiv umsetzbar. Das Ausmaß der 
„Komplexität einer Facette“ verändert sich demnach stark in Bezug auf den gesetzten Fokus.

\subsubsection{Dauer der Praxiserfahrung}

Ähnlich wie im oben beschriebenen Beispiel demonstriert auch der Vergleich der Zeit, die die Praxiserfahrung in den Lehr-Lern-Laboren La:gune und $\mathrm{MiRA}^{+}$einnimmt, die Abhängigkeit von „angemessenen“ strukturellen Einschätzungen vom Ziel des Lehr-Lern-Labors. Gründe für die Einschätzung dieser Zeit bezogen sich innerhalb der Interviews meist auf die Realitätsnähe, die durch die Zeit gegeben oder verhindert wird. Auch hier werden beide Lehr-Lern-Labore zunächst getrennt betrachtet und darauffolgend verglichen.

\subsubsection{Physik}

Im Lehr-Lern-Labor La:gune werden Unterrichtsminiaturen eingesetzt. Dies bedeutet, dass Unterrichtseinheiten durchgeführt werden, die zwar alle typischen Phasen von Unterricht enthalten, jedoch zeitlich stark reduziert sind. Im Falle des beschriebenen Lehr-Lern-Labors werden die Einheiten auf 12 Minuten komprimiert.

Die Studierenden schätzten die Dauer der Praxiserfahrung in fast allen Fällen als zu kurz ein, da die Situation damit gekünstelt wirke und diese somit gegenüber längeren Praxisphasen realitätsferner wird. Gesondert genannt wird meist die Kompression der verschiedenen Unterrichtsphasen in diese geringe Zeit. Von mehreren Studierenden wird diese sinkende Realitätsnähe als „Zwang zu einem Vortrag" geäußert, da durch eine solche starke Vorstrukturierung das Einhalten der Zeitvorgabe vereinfacht wird. In einigen Fällen wird die Zeit jedoch auch als „angemessen“ bezeichnet. Begründet wird letzteres in der Regel durch die Passung an die Zeit, die für ein Seminar zur Verfügung steht. Es wird geäußert, dass die mehrfache Durchführung und Reflexion (die Grundlage der Lernumgebung sind) nur durch diese Kürzung ermöglicht werden und sie daher hinzunehmen sei. Vereinzelt wird die kurze Zeit auch als Herausforderung und daher positiv bezeichnet.

\subsubsection{Mathematik}

Im Lehr-Lern-Labor $\mathrm{Mi} \mathrm{A}^{+}$werden innerhalb der Praxiserfahrungen Schülergruppen durch Studierende bei der Bearbeitung vorbereiteter Aufgabenstellungen beobachtet. Die Dauer dieser Praxiserfahrungen beläuft sich dabei auf etwa 90 Minuten. 
Die Dauer der Praxiserfahrungen wird von den meisten befragten Studierenden als angemessen eingeschätzt. Dabei wird jedoch häufig in Bezug auf die Passung zwischen Aufgabenstellung und Bearbeitungszeit, nicht aber auf die persönliche Praxiserfahrung geantwortet. Auch in Bezug auf den gewählten Fokus des Lehr-Lern-Labors wird die Zeit von ca. 9o Minuten meist als angemessen und notwendig bezeichnet, um die entsprechenden Kompetenzen ausreichend beobachten zu können. Als negativ eingeschätzt wurde weniger die Dauer einer einzigen Praxiserfahrung, jedoch die Anzahl solcher Praxiserfahrungen. Durch die Betrachtung einzelner Gruppen sei nur ein kleiner Ausschnitt der Realität betrachtet worden. In vielen Fällen wurde daher der Wunsch geäußert, zu Gunsten einer steigenden Realitätsnähe noch weitere Praxiserfahrungen durchzuführen.

\subsubsection{Vergleich}

Obwohl sich die beiden Lehr-Lern-Labore in der Dauer der Praxisphase stark unterscheiden (9o Minuten vs. 12 Minuten), werden beide zu Teilen als angemessen oder zu gering eingeschätzt. Dabei ist jedoch zu beachten, dass letztere Einschätzung im Falle des Lehr-Lern-Labors La:gune einen überwiegenden Teil der Befragten ausmacht, im Falle des Lehr-Lern-Labors MiRA ${ }^{+}$jedoch nur einen kleinen Teil. Gemeinsam haben beide Lehr-Lern-Labore, dass die Dauer basierend auf dem Fokus, der gesetzt wird, jeweils als angemessen eingeschätzt wird. Beide Ansätze werden also mit Blick auf das jeweilige Lernziel des Lehr-Lern-Labors als legitim bzw. angemessen angesehen, die Einschränkungen durch kurze Zeit (Physik) und kleine Anzahl (Mathe) wird als ökonomisch sinnvoll beziehungsweise alternativlos wahrgenommen.

\subsubsection{Vertrautheit mit Räumlichkeiten und Material}

Durch die Vertrautheit mit Räumlichkeiten und Material soll im Kontext eines Lehr-Lern-Labors ein geschützter Rahmen geschaffen werden. Studierende werden somit bei den Praxiserfahrungen, aber auch deren Vorbereitung entlastet, was einen wichtigen Aspekt eines komplexitätsreduzierenden Lehr-Lern-Labors darstellt.

Seitens der befragten Studierenden wurde diese Vertrautheit jedoch in vielen Fällen nicht als relevant angesehen, so werden Schulräume gleichzeitig als „gänzlich unvertraut" beschrieben, die Vertrautheit des erlebten Settings jedoch auch als angemessen bewertet. Wurden die Räumlichkeiten selbst nicht als „angemessen“ beschrieben, so war das Ausmaß der Unvertrautheit entweder nicht groß oder wurde durch vorbereitetes und mitgebrachtes Material (beispielsweise iPads) nach Meinung der befragten Studierenden ausgeglichen. 
Auch hier unterscheiden sich die Einschätzungen leicht zwischen den beiden Lehr-Lern-Laboren, jedoch nicht in einem so großen Ausmaß wie bisher beschrieben.

\subsubsection{Mathematik}

Die Vertrautheit mit Räumlichkeiten wurde im Falle des Lehr-Lern-Labors MiRA ${ }^{+}$ vereinzelt als nicht relevant bezeichnet. Einher mit dieser Einschätzung geht ein oftmaliges Beantworten der Frage im Sinne einer Vertrautheit für die Schülerinnen und Schüler. Dies unterstützt den geringen Stellenwert, der dieser Vertrautheit zugeschrieben wird. Die Vertrautheit für die Studierenden selbst wird nicht in Erwägung gezogen. Als Grund für diese Irrelevanz wurde meist der Fokus auf Beobachtung herangezogen. Die Studierenden als Lehrende stehen so während der Praxisphase nicht im Fokus ihrer Einschätzungen bezüglich komplexitätsreduzierender Elemente. Es spielt demnach für die am Lehr-Lern-Labor Teilnehmenden keine Rolle, ob die Räumlichkeiten bekannt oder vertraut sind.

\subsubsection{Physik}

Die Vertrautheit wurde im Lehr-Lern-Labor La:gune meist auch als angemessen bezeichnet. Vereinzelt wird jedoch eine geringere Vertrautheit der Umgebung während der Praxiserfahrung innerhalb der Schule beschrieben (nur die dritte der drei gestuften Praxisphasen findet außerhalb der Universität und mit tatsächlichen Schülerinnen und Schülern statt). Eine meist hohe Vertrautheit mit „unbekannten Klassenräumen" scheint also auch hier sichtbar zu sein, jedoch ist diese nicht so stark ausgeprägt wie im Lehr-Lern-Labor MiRA ${ }^{+}$.

Ein möglicher Grund für diese Abweichung kann erneut durch die Art der Praxiserfahrungen gegeben sein. Im Lehr-Lern-Labor La:gune stehen die Studierenden als Lehrkräfte im Zentrum und müssen sowohl mit der Klasse als auch mit dem Klassenraum interagieren. Die Vertrautheit mit dieser Umgebung wird relevanter. Innerhalb des Lehr-Lern-Labors La:gune wurde besonders die Vertrautheit mit den Materialien als „steigend“ beschrieben, da es ermöglicht wurde, sich über längere Zeit mit Materialien und Räumlichkeiten auseinanderzusetzen. Besonders die Experimentiermaterialien wurden zudem innerhalb der Praxiserfahrung für die Durchführung in der Schule aus den Universitätsräumen mitgenommen und blieben somit vertraut. 


\subsubsection{Vergleich}

Im Vergleich der beiden Lehr-Lern-Labore wird deutlich, dass die Vertrautheit mit Materialien und Räumlichkeiten eine generell niedrige Relevanz für die Studierenden zu besitzen scheint. Kleine Unterschiede werden dennoch im Ausmaß der beschriebenen Relevanz deutlich: Studierende, die während der Praxiserfahrungen im Fokus stehen und mit Räumlichkeiten und Material interagieren müssen (vgl. Physik), scheinen dieser Vertrautheit einen höheren Stellenwert beizumessen. Studierende, die eine eher beobachtende Rolle während der Praxiserfahrung einnehmen (vgl. Mathematik), schätzen die Vertrautheit als weniger relevant ein.

\subsubsection{Diskussion des Vergleichs}

Die beschriebenen Beispiele und damit einhergehenden Vergleiche der beiden Lehr-Lern-Labore zeugen von den vielfältigen Wechselwirkungen nicht nur zwischen Anforderungen und unterstützenden Facetten, sondern auch zwischen den Zielen, die innerhalb der Lehr-Lern-Labore gesetzt werden.

So wurde dargelegt, inwiefern sich zwei in der Art der Praxiserfahrung grundsätzlich unterscheidende Lehr-Lern-Labore dennoch in den resultierenden Einschätzungen der Studierenden stark ähneln können. Die mit der unterschiedlichen Art der Praxiserfahrung einhergehenden stark differenten strukturellen Faktoren (beispielsweise Größe der Lerngruppe oder Zeit der Praxiserfahrung) wurden dahingehend gegenübergestellt. Je nach gesetztem Fokus kann beispielsweise eine Studierenden-zu-Lerngruppe Relation von 1:1 fast als zu niedrig eingeschätzt werden, während bei einem unterschiedlichen Konzept eine Relation von 1:10 noch als hoch empfunden wird. Ebenso wird deutlich, dass der Stellenwert, der einzelnen Faktoren seitens der Studierenden zugeschrieben wird, sehr unterschiedlich sein kann. Die Vertrautheit der Räumlichkeiten beispielsweise nimmt einen nur sehr geringen Stellenwert ein. Eine relevante Einschätzung wird fast ausschließlich in einzelnen Fällen sichtbar, in denen Studierende jedoch auch mit dieser Umgebung interagieren müssen.

\section{Diskussion der Ergebnisse}

Das Rahmenkonzept der Lehr-Lern-Labore in diesem Sammelband enthält einerseits die Phasierung der Seminare in Vorbereitungs-, Durchführungs- und Reflexionsphase(n) und andererseits die Berücksichtigung der in der Literatur häufig genannten Gestaltungselemente wie z. B. Authentizität, Komplexitätsreduktion, Berücksichtigung von Heterogenität und Förderung von Kompetenzen mit Relevanz für den Lehrerberuf (Haupt et al., 2013; Dohrmann \& Nordmeier 2015). Der Fokus dieses Beitrages lag einerseits auf der Umsetzung der Komplexitätsre- 
duktion und der Wahrnehmung dieser Umsetzung durch die Studierenden und andererseits auf der Förderung der Reflexionskompetenz sowie weiterer für den Lehrerberuf relevanter Kompetenzaspekte wie der Selbstwirksamkeitserwartung.

Sowohl in der quantitativen Erhebung als auch in den Interviews zeigt sich, dass die Studierenden die Umsetzung der Komplexitätsreduktion in den verschiedenen Dimensionen als angemessen wahrnehmen. Dies gibt einen Hinweis darauf, dass in den sehr unterschiedlichen Seminarkonzepten der beteiligten Fächer jeweils eine für die Lernziele und die Zielgruppe passende Konzeption gewählt wurde.

Diese angemessene Balance zwischen Anforderungen und Unterstützungsmaßnahmen in den Seminaren hat die Professionalisierung der Studierenden durch eine gemeinsame Reflexion der im Lehr-Lern-Labor gemachten Praxiserfahrungen ermöglicht (Putnam \& Borko, 200o). In den Interviews zeigt sich dabei, dass die Reflexionskompetenz bei den Studierenden durchaus einen sehr hohen Stellenwert für den Beruf als Lehrkraft einnimmt. Allerdings ist das Verständnis, was mit Reflexionskompetenz gemeint ist, noch auf einzelne Aspekte im Sinne der Definition nach Abels (2011) beschränkt. So verstehen die Studierenden Reflexion in der Regel als handlungsbezogen und beziehen vorwiegend ihre eigene Person in den Reflexionsprozess ein. Darüber hinaus sind die Vorstellungen der Studierenden von Reflexionskompetenz sehr divers. Dies zeigt sich beispielsweise an den uneinheitlichen Aussagen über den Zweck von Reflexionssitzungen.

Für eine erfolgreiche Reflexion muss daher einerseits das Loslösen von der eigenen Person und die Erweiterung des Blickfeldes auf Situationsaspekte wie z.B. die Unterrichtsmaterialien gezielt unterstützt werden. Andererseits bedarf auch die Berücksichtigung kollegialer Reflexion im Sinne der Kommunikation mit Dritten nach Abels (2011) einer entsprechenden Anleitung im Seminar. Hierfür ist die Einigung auf ein gemeinsames Reflexionsverständnis notwendig.

Zur Realisierung solcher Rahmenbedingungen erwiesen sich die eingesetzten Videographien als zentrales Hilfsmittel. Videos der Unterrichtsminiaturen bieten die Möglichkeit der Wiederholung und der Einnahme einer Außenperspektive und unterstützen dadurch eine objektivere und tiefgreifende Reflexion. Auf der anderen Seite geht mit dem Einsatz von Videos allerdings auch eine Informationsflut einher, die die Studierenden bewältigen müssen. Diese Informationsflut kann die Komplexität der Reflexionsprozesse wieder erhöhen. Daher ist die Unterstützung der Studierenden im Umgang mit den Videos essentiell für eine gelungene Reflexion der Studierenden. Diese kann, wenn sie angemessen angeleitet wird, den Studierenden bei der Entwicklung einer stabilen und zuversichtlichen Einschätzung der eigenen Selbstwirksamkeit helfen (Schön, 1983).

Weiterhin zeigt sich in den Lehr-Lern-Labor-Seminaren trotz Praxisphase kein Absinken der Selbstwirksamkeitserwartungen. So kann möglicherweise durch die Reduktion der Anforderungen bzw. Erhöhung von Unterstützungsmaßnahmen verschiedener Dimensionen des Modells zur Komplexität in Lehr-Lern-Laboren dem von Tschannen-Moran, Woolfolk Hoy und Hoy (1998) berichteten Praxis- 
schock begegnet werden. Die signifikant positive Entwicklung der Selbstwirksamkeitserwartungen in Bezug auf Lehrhandlungen im Lehr-Lern-Labor und den Umgang mit Heterogenität zeigt, dass die hier untersuchten Lehr-Lern-Labore diese Komplexitätsreduktion erfolgreich umsetzen und damit einen wesentlichen Beitrag zur Professionalisierung angehender Lehrkräfte leisten können.

\section{Ausblick}

Die hier dargestellte Studie hat aufgezeigt, wie die Komplexitätsreduktion in LehrLern-Laboren eine positive Entwicklung von Reflexionskompetenz und Selbstwirksamkeitserwartung durch vielfältige Zugänge unterstützen kann. Diesbezüglich gilt es, in weiteren Forschungsvorhaben zu klären, inwieweit Lehr-Lern-Labore hinsichtlich der Förderung von Reflexionskompetenz über den Selbstbericht der Studierenden hinaus wirksam werden. In diesem Kontext scheinen insbesondere die beiden im Zuge der Analyse entdeckten Studierendentypen der, positiven Reflektierer' (die negative und positive Aspekte ihrer Praxiserfahrung reflektieren) und der ,negativen Reflektierer' (die sich ausschließlich auf negative Aspekte fokussieren) interessant (siehe Abschnitt 4.3.1). Hier würden sich weitere Erhebungen und ein Abgleich mit der entsprechenden psychologischen Literatur anbieten, um genauer zu analysieren, welche Bedeutung diese explizite um die positiven Aspekte erweiterte subjektive Definition von Reflexion auf das Lernverhalten und die Entwicklung der Selbstwirksamkeitserwartungen der Personen hat.

Für die Konzeption oder Überarbeitung von Lehr-Lern-Labor-Seminaren kann diese Beobachtung darüber hinaus in Bezug auf die Anleitung von Reflexionsprozessen gewinnbringend eingesetzt werden. Insbesondere sollte sichergestellt werden, dass auch positive Aspekte der Praxiserfahrungen in die Reflexion aufgenommen und expliziert werden.

Die Konzeption von Lehr-Lern-Laboren mit von den Studierenden durchschnittlich als angemessen wahrgenommener Komplexität kann bereits als Erfolg gewertet werden. Interessant ist jedoch, ob Unterschiede in der Einschätzung der Angemessenheit einen Einfluss auf die Reflexionsprozesse und dadurch die Entwicklung von Reflexionskompetenz und Selbstwirksamkeitserwartungen haben. Dieser Zusammenhang bedarf noch weiterer Erforschung.

\section{Literatur}

Abels, S. (2011). LehrerInnen als „Reflective Practitioner“. Reflexionskompetenz für einen demokratieförderlichen Naturwissenschaftsunterricht. Wiesbaden: VS Verlag für Sozialwissenschaften. https://doi.org/10.1007/978-3-531-92735-O

Bandura, A. (1997). Self-efficacy. The exercise of control. New York: Freeman. 
Bandura, A. (1999). Social cognitive theory of personality. In L. A. Pervin \& O. P. John (Hrsg.), Handbook of Personality. Theory and Research (2. Aufl., S. 154-196). New York: The Guilford Press.

Baumert, J. \& Kunter, M. (2006). Stichwort: Professionelle Kompetenz von Lehrkräften. Zeitschrift für Erziehungswissenschaft, 9 (4), 469-520. https://doi.org/10.1007/s11618006-0165-2.

Baumert, J. \& Kunter, M. (2011). Das Kompetenzmodell von COACTIV. In M. Kunter, J. Baumert, W. Blum, U. Klusmann, S. Krauss \& M. Neubrand (Hrsg.), Professionelle Kompetenz von Lehrkräften. Ergebnisse des Forschungsprogramms COACTIV (S. 29-53). Münster: Waxmann.

Caprara, G. V., Barbaranelli, C., Steca, P. \& Malone, P. S. (2006). Teachers' self-efficacy beliefs as determinants of job satisfaction and students' academic achievement: A study at the school level. Journal of School Psychology, 44 (6), 473-49o. https://doi. org/10.1016/j.jsp.2006.09.001.

Collin, S., Karsenti, T. \& Komis, V. (2013). Reflective practice in initial teacher training. critiques and perspectives. Reflective Practice, 14 (1), 104-117. https://doi.org/10.1080/ 14623943.2012.732935.

Darling-Hammond, L. \& Richardson, N. (2009). Teacher learning: What matters? Educational Leadership, 66 (5), 46-53.

Dewey, J. (1933). How we think. A restatement of the relation of reflective thinking to the educative process. Boston: Health \& Co Publishers.

Dohrmann, R. \& Nordmeier, V. (2015). Schülerlabore als Lehr-Lern-Labore (LLL): Ein Projekt zur forschungsorientierten Verknüpfung von Theorie und Praxis in der MINT-Lehrerbildung. PhyDid B - Didaktik der Physik - Beiträge zur DPG-Frühjahrstagung.

Glass, G. V., Peckham, P. D. \& Sanders, J. R. (1972). Consequences of Failure to Meet Assumptions Underlying the Fixed Effects Analyses of Variance and Covariance. Review of Educational Research, 42 (3), 237-288. https://doi.org/10.3102/00346543042003237.

Gutzwiller-Helfenfinger, E., Aeppli, J. \& Lötscher, H. (2017). Lehramtsstudierende reflektieren eine Praxiserfahrung im Bereich „Beurteilen \& Fördern“. Qualität der Erfahrungsdarstellung und Multiperspektivität. In C. Berndt, T. H. Häcker \& T. Leonhard (Hrsg.), Reflexive Lehrerbildung revisited. Traditionen - Zugänge - Perspektiven (Studien zur Professionsforschung und Lehrerbildung). Bad Heilbrunn: Julius Klinkhardt.

Haupt, O. J., Domjahn, J., Martin, U., Skiebe-Corrette, P., Vorst, S., Zehren, W. \& Hempelmann, R. (2013). Schülerlabor - Begriffsschärfung und Kategorisierung. Der mathematische und naturwissenschaftliche Unterricht, 66 (2013), 324-330.

Herzog, W. \& Felten, R. von (2001). Erfahrung und Reflexion. Zur Professionalisierung der Praktikumsausbildung von Lehrerinnen und Lehrern. Beiträge zur Lehrerinnenund Lehrerbildung, 19 (1), 17-28.

Hößle, C., Hußmann, S., Michaelis, J., Niesel, V. \& Nührenbörger, M. (2017). Fachdidaktische Perspektiven auf die Entwicklung von Schlüsselkenntnissen einer förderorientierten Diagnostik. In C. Selter, S. Hußmann, C. Hößle, C. Knipping, K. Lengnink \& J. Michaelis (Hrsg.), Diagnose und Förderung heterogener Lerngruppen. Theorien, Konzepte und Beispiele aus der MINT-Lehrerbildung (S. 19-38). Münster: Waxmann.

Junker, R., Zeuch, N., Rott, D., Bartsch, C., Henke, I. J. \& Kürten, R. (ang.). Zur Veränderbarkeit von Heterogenitäts-Einstellungen und -Selbstwirksamkeitsüberzeugungen 
von Lehramtsstudierenden durch diversitätssensible hochschuldidaktische Lehrmodule. Empirische Sonderpädagogik.

Klassen, R. M. \& Tze, V. M. C. (2014). Teachers' self-efficacy, personality, and teaching effectiveness. A meta-analysis. Educational Research Review, 12, 59-76. https://doi. org/10.1016/j.edurev.2014.06.001.

Korthagen, F. A. J. (2002). Eine Reflexion über Reflexion. In J. Kessels, B. Koster, B. Lagerwerf \& T. Wubbels (Hrsg.), Schulwirklichkeit und Lehrerbildung. Reflexion der Lehrertätigkeit (S. 55-73). Hamburg: EB-Verlag.

Krofta, H., Fandrich, J. \& Nordmeier, V. (2012). Professionalisierung im Schülerlabor. Praxisseminare in der Lehrerbildung. PhyDid B - Didaktik der Physik - Beiträge zur DPG-Frühjahrstagung. Verfügbar unter phydid.physik.fu-berlin.de/index.php/phydidb/article/viewFile/412/550.

Kunter, M. \& Pohlmann, B. (2009). Lehrer. In E. Wild \& J. Möller (Hrsg.), Pädagogische Psychologie (Springer-Lehrbuch, S. 261-282). Berlin: Springer. https://doi.org/10.1007/ 978-3-540-88573-3_11

Kürten, R., Wess, R. \& Greefrath, G. (2018). Potentialorientierter Umgang mit Heterogenität durch reflektierte Praxiserfahrung: Professionalisierung von Lehramtsstudierenden im mathematikdidaktischen Lehr-Labor. In Fachgruppe Didaktik der Mathematik der Universität Paderborn (Hrsg.), Beiträge zum Mathematikunterricht 2018 (S. 1119-1122). Münster: WTM-Verlag. https://doi.org/10.17877/DE29oR-19488.

Lent, R. W., Brown, S. D. \& Larkin, K. C. (1986). Self-Efficacy in the Prediction of Academic Performance and Perceived Career Options. Journal of Counseling Psychology, 33 (3), 265-269. https://doi.org/10.1037/0022-0167.33.3.265

Meijer, C. J. W. \& Foster, S. F. (1988). The Effect of Teacher Self-Efficacy on Referral Chance. The Journal of Special Education, 22 (3), 378-385. https://doi.org/10.1177/ 002246698802200309.

Park, S. \& Oliver, J. S. (2008). Revisiting the Conceptualisation of Pedagogical Content Knowledge (PCK). PCK as a Conceptual Tool to Understand Teachers as Professionals. Research in Science Education, 38 (3), 261-284. https://doi.org/10.1007/s11165007-9049-6.

Putnam, R. T. \& Borko, H. (200o). What Do New Views of Knowledge and Thinking Have to Say About Research on Teacher Learning? Educational Researcher, 29 (1), 4-15. https://doi.org/10.3102/0013189X029001004.

Ross, J. A. (1995). Strategies for Enhancing Teachers' Beliefs in Their Effectiveness. Research on a School Improvement Hypothesis. Teachers College Record, 97 (2), 227251.

Roters, B. (2012). Professionalisierung durch Reflexion in der Lehrerbildung. Eine empirische Studie an einer deutschen und einer US-amerikanischen Universität (Studien zur international und interkulturell vergleichenden Erziehungswissenschaft, Bd. 12). Münster: Waxmann.

Schön, D. A. (1983). The reflective practitioner. How professionals think in action. New York: Basic Books.

Schunk, D. H. \& Pajares, F. (2002). The Development of Academic Self-Efficacy. In A. Wigfield \& J. S. Eccles (Hrsg.), Development of achievement motivation (S. 16-31). San Diego: Academic Press. https://doi.org/10.1016/B978-012750053-9/50003-6

Schwarzer, R. \& Jerusalem, M. (2002). Das Konzept der Selbstwirksamkeit. Zeitschrift für Pädagogik, 44 (Beiheft), 28-53. 
Shulman, L. S. (1986). Those who understand: Knowledge growth in teaching. Educational Researcher, 15 (2), 4-14. https://doi.org/10.3102/0013189X015002004

Subellok, K., Lüke, T. \& Ritterfeld, U. (2013). Förderbedingungen von Schülerinnen im Förderschwerpunkt Sprache. Zeitschrift für Heilpädagogik, 64, 144-154.

Tschannen-Moran, M., Woolfolk Hoy, A. \& Hoy, W. K. (1998). Teacher Efficacy: Its Meaning and Measure. Review of Educational Research, 68 (2), 202-248. https://doi. org/10.3102/00346543068002202.

Urton, K., Wilbert, J. \& Hennemann, T. (2014). Der Zusammenhang zwischen der Einstellung zur Integration und der Selbstwirksamkeit von Schulleitungen und deren Kollegien. Empirische Sonderpädagogik, 6 (1), 3-16.

Wyss, C. (2008). Zur Reflexionsfähigkeit und -praxis der Lehrperson. bildungsforschung, 5 (2). https://doi.org/10.25539/bildungsforschun.v2io.8o.

Zucker, V. \& Leuchter, M. (2018). Lehr-Lern-Labore als Orte der fachdidaktischen MINT-Lehramtsausbildung. Förderung von Kompetenzen Lehramtsstudierender hinsichtlich des Diagnostizierens und Rückmeldens. MNU Journal, 71 (6), 364-369. 



\section{Autorinnen und Autoren}

Andreas Feindt, Dr., ist Studienrat im Hochschuldienst am Institut für Erziehungswissenschaft der Westfälischen Wilhelms-Universität Münster. In der ersten Förderphase der Qualitätsoffensive Lehrerbildung hat er als abgeordneter Lehrer im Teilprojekt „Lehr-Lern-Labore, Lernwerkstätten und Learning-Center“ an einem Lehr-Lern-Labor ,Diagnose und Individuelle Förderung' zum Themenfeld „Heterogenität in der Schule“ gearbeitet. Seine Arbeits- und Forschungsschwerpunkte liegen im Bereich der Handlungs- und Aktionsforschung, der Schul- und Unterrichtsentwicklung, der Professionsforschung und der schulischen Bearbeitung von Heterogenität.

Kontakt: andreas.feindt@uni-muenster.de

Christian Fischer, Dr., ist Professor für Erziehungswissenschaft mit dem Schwerpunkt Schulpädagogik: Begabungsforschung und Individuelle Förderung am Institut für Erziehungswissenschaft der Westfälischen Wilhelms-Universität Münster. Im Rahmen der qualitätsoffensive Lehrerbildung der Universität Münster beteiligt er sich an den Teilprojekten „Curriculare Maßnahmen - Heterogenität als durchgängiges Thema im Curriculum“ und „Lehr-Lern-Labore, Lernwerkstätten und Learning-Center“. Im letztgenannten arbeitet er in seinem Einzelprojekt an einem Lehr-Lern-Labor ,Diagnose und Individuelle Förderung zum Themenfeld "Heterogenität in der Schule“. Seine Forschungsschwerpunkte liegen in den Bereichen Individuelle Begabungsförderung, Pädagogische Diagnostik, Selbstreguliertes Lernen, Lernstrategien, Lernschwierigkeiten, Lehrkräftebildung und Umgang mit Diversität.

Kontakt: ch.fischer@uni-muenster.de

Katharina Fricke, Dr., ist seit August 2019 Lehrerin an der Käthe-KollwitzGrundschule in Marl. Von 2015 bis 2019 war sie wissenschaftliche Mitarbeiterin am Institut für Didaktik des Sachunterrichts der Westfälischen Wilhelms-Universität Münster. In der Qualitätsoffensive Lehrerbildung arbeitete sie im Teilprojekt "Lehr-Lern-Labore, Lernwerkstätten und Learning-Center“. Ihre Forschungsschwerpunkte liegen bei der Planung und Reflexion von Sachunterricht, dem Einsatz digitaler Medien im Sachunterricht und der Ausbildung von Sachunterrichtslehrkräften.

Kontakt: kfricke@uni-muenster.de

Anna Grabosch war bis Dezember 2018 wissenschaftliche Mitarbeiterin am Institut für Erziehungswissenschaft der Westfälischen Wilhelms-Universität Münster. Seit Januar 2019 arbeitet sie als wissenschaftliche Mitarbeiterin an der Professur für Schulpädagogik mit dem Schwerpunkt Sekundarstufe I an der Universität Kassel. In der Qualitätsoffensive Lehrerbildung arbeitete sie im Teilprojekt „Lehr- 
Lern-Labore, Lernwerkstätten und Learning-Center“ an der Weiterentwicklung und Erforschung von Peer-Learning-Angeboten für Studierende im Rahmen des Projektes „ELF“. Ihre Forschungsschwerpunkte liegen im Bereich der Kompetenzentwicklung in der Lehrkräftebildung, Praxisphasen in der Lehrkräftebildung und des Peer Learnings und Coachings.

Kontakt: anna.grabosch@uni-kassel.de

Gilbert Greefrath, Dr., ist Professor am Institut für Didaktik der Mathematik und der Informatik der Westfälischen Wilhelms-Universität Münster. In der Qualitätsoffensive Lehrerbildung an der Universität Münster ist er gemeinsam mit Marcus Hammann Leiter des Projekts „Lehr-Lern-Labore, Lernwerkstätten und LearningCenter“ im Rahmen der Qualitätsoffensive an der Universität Münster. In seinem Einzelprojekt arbeitet er an der Entwicklung eines mathematikdidaktischen LehrLabors zur Professionalisierung angehender Lehrpersonen. Im Fokus stehen dabei die Sensibilisierung für und der produktive Umgang mit Heterogenität im Mathematikunterricht. Seine Forschungsschwerpunkte liegen in den Bereichen mathematisches Modellieren, Einsatz digitaler Mathematikwerkzeuge, Übergang Schule - Hochschule und Entwicklung von Test- und Prüfungsaufgaben.

Kontakt: greefrath@uni-muenster.de

Helge Gresch, Dr., ist Juniorprofessor am Zentrum für Didaktik der Biologie der Westfälischen Wilhelms-Universität Münster. Er ist assoziiertes Mitglied der Qualitätsoffensive Lehrerbildung an der Universität Münster. Seine Forschungsschwerpunkte liegen in den Bereichen des Umgangs mit Schülervorstellungen im Biologieunterricht, der Unterrichtsforschung sowie in der videovignettenbasierten Professionalisierung von Lehrkräften im Kontext von Evolution.

Kontakt: helgegresch@uni-muenster.de

Marcus Hammann, Dr., ist Professor am Zentrum für Didaktik der Biologie der Westfälischen Wilhelms-Universität Münster. Er beteiligt sich am Teilprojekt „Curriculare Maßnahmen - Heterogenität als durchgängiges Thema im Curriculum“ und ist zusammen mit Gilbert Greefrath Leiter des Teilprojekts „Lehr-Lern-Labore, Lernwerkstätten und Learning-Center“ im Rahmen der Qualitätsoffensive an der Universität Münster. Seine Forschungsschwerpunkte liegen bei Vorstellungen, Interessen und Einstellungen von Schülerinnen und Schülern zu verschiedenen Themen des Biologieunterrichts.

Kontakt: hammann.m@uni-muenster.de

Susanne Heinicke, Dr., ist Professorin am Institut für Didaktik der Physik der Westfälischen Wilhelms-Universität Münster. In der Qualitätsoffensive Lehrerbildung an der Universität Münster arbeitet sie im Teilprojekt „Lehr-Lern-Labore, Lernwerkstätten und Learning-Center“ an der Entwicklung eines Lehr-Lern-Labors zur Förderung der Handlungsfähigkeit angehender Physiklehrer in ungeplan- 
ten Unterrichtssituationen. Ihre Forschungsschwerpunkte liegen beim Umgang mit Heterogenität im Physikunterricht, der Fehlerkultur im Lernen von Physik, dem Umgang mit Messdaten und Messunsicherheiten und der wechselseitigen Integration von digitalen und real-experimentellen Zugängen im naturwissenschaftlichen Lernen und Experimentieren.

Kontakt: susanne.heinicke@uni-muenster.de

Michael Hemmer, Dr., ist Professor am Institut für Didaktik der Geographie der Westfälischen Wilhelms-Universität in Münster. In der Qualitätsoffensive Lehrerbildung leitet er in den Teilprojekten „Videobasierte Lehrmodule als Mittel der Theorie-Praxis-Integration“, „Praxisprojekte in Kooperationsschulen“ sowie „LehrLern-Labore, Lernwerkstätten und Learning-Center" die jeweiligen Fachprojekte. Im letztgenannten arbeitet er mit einem Fokus auf die naturwissenschaftliche Grundbildung im Fach Geographie an der Entwicklung und Evaluation eines GEO-Lehr-Lern-Labors zur Professionalisierung von Studierenden unter besonderer Berücksichtigung heterogener Lerngruppen. Seine Forschungsschwerpunkte liegen im Bereich des geographischen Schülerinteresses, der räumlichen Orientierungskompetenz, der Lehrerprofessionalität und Lehrerprofessionalisierung, der Exkursionsdidaktik und der geographiedidaktischen Bildungsmedienforschung. Kontakt: michael.hemmer@uni-muenster.de

Christoph Holz ist wissenschaftlicher Mitarbeiter am Institut für Didaktik der Physik der Westfälischen Wilhelms-Universität Münster. In der Qualitätsoffensive Lehrerbildung arbeitet er im Teilprojekt „Lehr-Lern-Labore, Lernwerkstätten und Learning-Center" an der Entwicklung eines Lehr-Lern-Labors zur Förderung der Handlungsfähigkeit angehender Physiklehrer in ungeplanten Unterrichtssituationen. Seine Forschungsschwerpunkte liegen in dem Umgang angehender Lehrkräfte mit experimentellen Daten in diesen kritischen Unterrichtssituationen und der Hervorhebung und Untersuchung diesbezüglicher Handlungstypen.

Kontakt: christoph.holz@uni-muenster.de

Ronja Kürten ist wissenschaftliche Mitarbeiterin am Institut für Didaktik der Mathematik und der Informatik der Westfälischen Wilhelms-Universität Münster. In der Qualitätsoffensive Lehrerbildung arbeitet sie als Koordinatorin des Teilprojekts „Lehr-Lern-Labore, Lernwerkstätten und Learning-Center“. Ihre Forschungsschwerpunkte liegen im Bereich der Hochschuldidaktik Mathematik, der Entwicklung und Evaluation von Vorkursen und der Selbstwirksamkeitserwartung von Studierenden.

Kontakt: ronja.kuerten@uni-muenster.de

Annette Marohn, Dr., ist Professorin am Institut für Didaktik der Chemie der Westfälischen Wilhelms-Universität Münster. In der Qualitätsoffensive Lehrerbildung arbeitet sie im Teilprojekt „Lehr-Lern-Labore, Lernwerkstätten und Lear- 
ning-Center" an der Weiterentwicklung des chemiedidaktischen Lehr-Lern-Labors $\mathrm{C}(\mathrm{LE})^{2}$ VER mit den Schwerpunkten „Umgang mit Heterogenität“, „Schülervorstellungen“, „Stolpersteine im Lehrerhandeln“ und „Digitale Medien“. Ihr Forschungsfokus liegt in der Entwicklung innovativer Konzepte für den naturwissenschaftlichen Unterricht und die Lehrerbildung sowie der empirischen Erforschung von Lernprozessen. Thematische Schwerpunkte liegen unter anderem im Bereich des inklusiven und sprachsensiblen Unterrichts sowie in den Feldern Schülervorstellungen, Bewertungskompetenz, Nature of Science und der Nutzung digitaler Werkzeuge.

Kontakt: a.marohn@uni-muenster.de

Yvonne Rath ist wissenschaftliche Mitarbeiterin am Institut für Didaktik der Chemie der Westfälischen Wilhelms-Universität Münster. In der Qualitätsoffensive Lehrerbildung arbeitet sie im Teilprojekt „Lehr-Lern-Labore, Lernwerkstätten und Learning-Center“ an der Weiterentwicklung des chemiedidaktischen Lehr-LernLabors $\mathrm{C}(\mathrm{LE})^{2} \mathrm{VER}$ mit dem Forschungsschwerpunkt „Stolpersteine im Lehrerhandeln“.

Kontakt: y.rath@uni-muenster.de

Annika Rochholz war bis März 2019 Mitarbeiterin am Institut für Didaktik des Sachunterrichts der Westfälischen Wilhelms-Universität Münster. In der Qualitätsoffensive Lehrerbildung arbeitete sie im Teilprojekt „Lehr-Lern-Labore, Lernwerkstätten und Learning-Center“ an der Entwicklung und Evaluation eines Lehr-Lern-Labors, in dem Studierende des Masterstudiengangs die Planung von Sachunterricht erlernen.

Kontakt über das Institut für Didaktik des Sachunterrichts

Nadine Rosendahl ist wissenschaftliche Mitarbeiterin am Institut für Didaktik der Geographie der Westfälischen Wilhelms-Universität Münster. In der Qualitätsoffensive Lehrerbildung arbeitet sie im Teilprojekt „Lehr-Lern-Labore, Lernwerkstätten und Learning-Center" an der Entwicklung und Evaluation eines GEO-Lehr-Lern-Labors zur Professionalisierung von Lehramtsstudierenden im Themenbereich „heterogenitätssensibler Einsatz von Experimenten im Geographieunterricht“. Ihre Forschungsschwerpunkte liegen im Bereich der naturwissenschaftlichen Grundbildung, experimenteller Arbeitsweisen, Lehrerprofessionalität und Lehrerprofessionalisierung und dem Umgang mit Heterogenität im Geographieunterricht.

Kontakt: nadine.rosendahl@uni-muenster.de

Gabriele Schrüfer, Dr., ist Professorin am Institut für Didaktik der Geographie der Westfälischen Wilhelms-Universität in Münster. In der Qualitätsoffensive Lehrerbildung arbeitet sie im Teilprojekt „Curriculare Maßnahmen - Heterogenität als durchgängiges Thema im Curriculum“ sowie im Teilprojekt „Lehr-Lern-Labore, 
Lernwerkstätten und Learning-Center" an der Entwicklung eines GEO-Lehr-LernLabors zur Professionalisierung von Studierenden im Umgang mit Heterogenität im Geographieunterricht. Ihre Forschungsschwerpunkte liegen vor allem im Globalen Lernen/Bildung für nachhaltige Entwicklung, Interkulturellen Lernen, Lernen im digitalen Zeitalter sowie „Afrika“ im Geographieunterricht.

Kontakt: gabriele.schruefer@uni-muenster.de

Jens Steinwachs ist Lehrer für Gymnasien und Gesamtschulen mit den Unterrichtsfächern Biologie und Sozialwissenschaften und derzeit abgeordnete Lehrkraft am Zentrum für Didaktik der Biologie der Westfälischen Wilhelms-Universität Münster. Er ist assoziiertes Mitglied der Qualitätsoffensive Lehrerbildung an der Universität Münster. Seine Forschungsschwerpunkte liegen in den Bereichen der Wahrnehmung von Schülervorstellungen und dem Umgang mit ihnen im Evolutionsunterricht durch Studierende sowie in der videovignettenbasierten Professionalisierung von Lehrkräften.

Kontakt: jens.steinwachs@uni-muenster.de

Ewald Terhart, Dipl. Päd., Dr., war bis Juli 2018 Professor für Schulpädagogik und Allgemeine Didaktik am Institut für Erziehungswissenschaft der Universität Münster. In der Qualitätsoffensive Lehrerbildung hatte er von Beginn bis Februar 2018 die Position des Fachlichen Koordinators des „Dealing with Diversity“-Projekts der Universität Münster inne. In der Qualitätsoffensive Lehrerbildung arbeitete er in den Teilprojekten „Curriculare Maßnahmen - Heterogenität als durchgängiges Thema im Curriculum“ und im Teilprojekt „Lehr-Lern-Labore, Lernwerkstätten und Learning-Center“. Seine Forschungsschwerpunkte liegen im Bereich der Allgemeinen Didaktik, der Lehrkräftebildung und des Berufs der Lehrkraft.

Kontakt: ewald.terhart@uni-muenster.de

Raphael Wess ist wissenschaftlicher Mitarbeiter am Institut für Didaktik der Mathematik und der Informatik der Westfälischen Wilhelms-Universität Münster. In der Qualitätsoffensive Lehrerbildung arbeitet er im Teilprojekt „Lehr-Lern-Labore, Lernwerkstätten und Learning-Center" an der Entwicklung eines mathematikdidaktischen Lehr-Labors zur Professionalisierung angehender Lehrpersonen. Im Fokus stehen dabei die Sensibilisierung für und der produktive Umgang mit Heterogenität im Mathematikunterricht. Seine Forschungsschwerpunkte liegen im Bereich des mathematischen Modellierens, der Implementation und Evaluation von Lehr-(Lern-)Laboren und der Entwicklung professioneller Kompetenz in der ersten Phase der Lehrerbildung.

Kontakt: r.wess@uni-muenster.de

Anna Windt, Dr., ist Professorin am Institut für Didaktik des Sachunterrichts der Westfälischen Wilhelms-Universität Münster. In der Qualitätsoffensive Lehrerbildung arbeitet sie im Teilprojekt „Lehr-Lern-Labore, Lernwerkstätten und Lear- 
ning-Center" an der Entwicklung und Evaluation eines Lehr-Lern-Labors, in dem Studierende des Masterstudiengangs die Planung von Sachunterricht erlernen. Ihre Forschungsschwerpunkte liegen in den Bereichen Naturwissenschaftliches Lernen im Primar- und Elementarbereich, Ausbildung von Sachunterrichtslehrkräften, Qualität von Sachunterricht, Planung und Reflexion von Sachunterricht und Einsatz digitaler Medien im Sachunterricht.

Kontakt: anna.windt@uni-muenster.de

Johannes Zang ist wissenschaftlicher Mitarbeiter am Zentrum für Didaktik der Biologie der Westfälischen Wilhelms-Universität Münster. In der Qualitätsoffensive Lehrerbildung arbeitet er im Teilprojekt „Lehr-Lern-Labore, Lernwerkstätten und Learning-Center" an der Konzeptualisierung und Realisierung des Lehr-LernLabors LEO. Seine Forschungsschwerpunkte liegen in den Bereichen der Vorstellungen zur Merkmalsentstehung mit einem Fokus auf Genetik, Gen-UmweltInteraktionen und Kausalitätsannahmen, Vorstellungen zu Emotionen, Förderung diagnostischer Kompetenz im Kontext der Professionalisierung von Lehrkräften sowie den molekularen Auswirkungen von Stress und aversiven Erfahrungen in der Kindheit.

Kontakt: johannes.zang@uni-muenster.de

Horst Zeinz, Dr., ist Professor für Erziehungswissenschaft mit dem Schwerpunkt Grundschulpädagogik am Institut für Erziehungswissenschaft der Westfälischen Wilhelms-Universität Münster. In der Qualitätsoffensive Lehrerbildung arbeitet er im Teilprojekt „Lehr-Lern-Labore, Lernwerkstätten und Learning-Center" an einem Lehr-Lern-Labor zum Themenfeld „Heterogenität in der Schule“. Seine Forschungsschwerpunkte liegen im Bereich der empirischen (Grund-)Schulforschung, der Professionalisierung von Lehrkräften und der Schulentwicklung.

Kontakt: horst.zeinz@uni-muenster.de 